






\section{Lea's Series \\ of Pocket Text-Books.}

\section{Diseases of the Eye, Ear Nose and Throat.}

By W. L. BALLENGER, M.D., Lecturer on Rhinology and Laryngology, and A. G.WIPPERN, M.D., Clinical Instructor in Diseases of the Nose and Throat, College of Physicians and Surgeons, Chicago.

\section{Anatomy.}

By FREDERICK J. BROCKWA Y, M.D., Assistant Demonstrator of Anatomy, College of Physicians and Surgeons, New York.

\section{Bacteriology and Hygiene.}

By W. E. CoAtes, JR., M.D., Instructor in Bacteriology and Pathology, college of Physicians and Surgeons, Chicago.

\section{Diagnosis.}

By C. P. Collins, M. D., Attending Physician to St. Luke's Hospital, New York.

\section{Physiology.}

By H. D. Collins, M. D., Assistant Demonstrator of Anatomy, and W. H. RockWELL, JR., A. B , M. D., Assistant Demonstrator of Anatomy, College of Physicians and Surgeons, New York.

\section{Gynecology.}

By Montgomery A. Crockett, A. B. M. D. Adjunct Professor of Obstetrics and Clinical Gynecology, Medical Department, University of Buffalo, New York.

\section{Obstetrics.}

By David J. Evans, M. D., Demonstrator of Obstetrics, McGill University, Faculty of Medicine, Montreal.

\section{Surgery.}

By Bern B. Galla UdeT, M.D., Demonstrator of Anatomy, and Clinical Lecturer on Surgery, College of Physicians and Surgeons, New York.

\section{Dermatology.}

By JosEPH GRINDON, M. I., Professor of Dermatology, St. Louis and Missouri Medical College, St. Louis.

\section{Genito-U rinary and Venereal Diseases.}

By SylvaN H. LikES, M.D., Demonstrator of Pathology and Genito-Urinary Surgery at the College of Physicians and Surgeons, Baltimore.

\section{Chemistry and Physics.}

By Walton Martin, M. D., Assistant Demonstrator of Anatomy, and WILliaM H. RockWell, JR., A. B., M. D., Assistant Demonstrator of Anatomy, College of Physicians and Surgeons, New York.

\section{Practice of Medicine.}

By George E. Malsbary, M. D. Assistant to the Chair of Theory and Practice of Medicine, Medical College of Ohio, Cincinnati.

\section{Histology and Pathology.}

By ЈонN B. Nichols, M. D., Assistant in Pathology, Medical Department, University of Georgetown, and F. P. VALE, M. D., Demonstrator of Normal Histology, Medical Department, Columbian University, Washington, D. C.

\section{Nervous and Mental Diseases.}

By Charles S. Potrs, M. D., Instructor in Electro-Therapeutics and Nervous Diseases in the University of Pennsylvania, Philadelphia.

\section{Materia Medica.}

By William Schleif, Ph. G., M.D., Instructor in Pharmacy in the University of Penusylvania, Philadelphia. Cloth, \$1.50, net.

\section{Diseases of Children.}

By George M. TUTtLe, M. D., Attending Physician to St. Luke's Hospital, Martha Parsons' Hospital for Children and Bethesda Foundling Asylum, St. Louis, Mo. 


\section{bea's Series of Pocket Text=Books.}

\section{HISTOLOGY AND PATHOLOGY. ।}

A MANUAL FOR STUDENTS AND PRACTITIONERS.

BY

JOHN BENJAMIN NICHOLS, M.D.,

Demonstrator of Histology, Medical Department Columbian University, Washington, D. C.,

AND

FRANK PALMER VALE, M. D., Assistant in Pathology, Medical Department University of Georgetown, Washington, D. C.

SERIES EDITED BY

BERN B. GALLAUDET, M.D.,

Demonstrator of Anatomy and Instructor in Surgery, College of Physicians and Surgeons, Columbia University, New York; Visiting Surgeon, Bellevue Hospital, New York.

ILLUSTRATED WITH TWO HUNDRED AND THIRTEEN ENGRAVINGS.

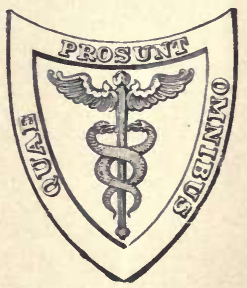

LEA BROTHERS \& CO., PHILADELPHIA AND NEW YORK. 


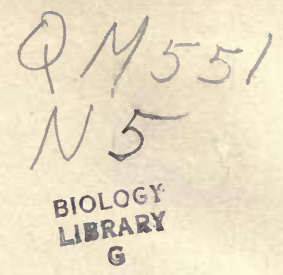

Entered according to Act of Congress, in the year 1899, by LEA BROTHERS \& CO.,

In the Office of the Librarian of Congress, at Washington. All rights reserved. 


\section{PREF A CE.}

IT is the purpose of this work to present all the essential facts relating to the normal histology and the pathological anatomy and histology of man in a systematic and concise way. In subjects so well established as these, originality of data is little to be expected, and the chief object to be aimed at is arrangement and treatment of the subject in a manner convenient and clear to students and practitioners of medicine, for whom this treatise is intended. It is attempted to present the subject up to the most recent discoveries and developments, avoiding points unsettled and in doubt.

The part on normal histology is written entirely by Dr. Nichols, that on pathology entirely by Dr. Vale. Each is fully responsible for the statements in his own part, and for those only.

J. B. NICHOLS.

FRANK P. VALE.

WASHington, D. C. 



\section{CONTENTS.}

\section{NORMAL HISTOLOGY.}

CHAPTER I.

INTRODUCTION

CHAPTER II.

Cells and Extra-cellullar Elements . . . . . . . . . 22

CHAPTER III.

REPROdUCtion and DeVELoPMENT . . . . . . . . . . . 33

CHAPTER IV.

The Tissues . . . . . . . . . . . . . . . . . . 42

CHAPTER V.

Glands; Mucous and Serous Membranes; Organs . . . . . 67

CHAPTER VI.

The Circulatory System 75

CHAPTER VII.

The Lymphatic System

CHAPTER VIII.

BLOOD AND LYMPH . . . . . . . . . . . . . . . . . 91

CHAPTER IX.

Blood-glands and Ductless Glands . . . . . . . . 104 
CHAPTER XI.

The Respiratory System . . . . . . . . . . 141

CHAPTER XII.

$\mathrm{T}_{\text {HE }} \operatorname{SkIN} \ldots \ldots \ldots \ldots \ldots \ldots$

CHAPTER XIII.

The Urinary Organs . . . . . . . . . . . 158

CHAPTER XIV.

The Reproductive System . . . . . . . . 172

CHAPTER XV.

Muscular and Skeletal Structures $\ldots \ldots \ldots$

CHAPTER XVI.

The Nervous System . . . . . . . . . 202

\section{PATHOLOGY.}

INTRODUCTORY . . . . . . . . . . . . . 243

GENERAL PATHOLOGY.

CHAPTER I.

Pathology of the Blood and Circulation . . . . . . 247

CHAPTER II.

Pathology of Nutrition ................. 274 


\section{SPECIAL PATHOLOGY.}

CHAPTER III.

DiseAsES OF THE BLOOD . . . . . . . . . . . . 309

CHAPTER IV.

Diseases of the Circulatory System . . . . . . . . . . . 318

CHAPTER V.

Diseases of the Respiratory Organs ... . . . . . . . 332

CHAPTER VI.

Diseases of the Gastro-intestinal Tract . . . . . . . . 359

CHAPTER VII.

Diseases of the Urinary Organs . . . . . . . . . . 403

CHAPTER VIII.

Diseases of the Nervous System . . . . . . . . . . 420 

NORMAL HISTOLOGY. 



\section{NORMAL HISTOLOGY.}

\section{CHA P TER I.}

\section{INTRODUCTION.}

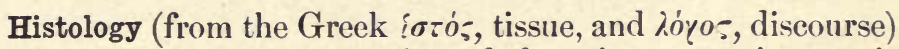
is a branch of anatomy treating of the minute or microscopical structure of living organisms. In its full significance it relates to the entire organic kingdom, all plants and animals alike. Within large classes of animals or plants the structure of the tissues is quite uniform; but on comparing one large group of organic types with another, differences in the structure appear, although there are a fundamental unity and similarity throughout.

The histologic structure of man, with which the present treatise deals, is substantially the same as that of all the mammalia, the points of variation being few and slight; hence human histology and mammalian histology are almost identical. In passing to the classes of birds and reptiles, however, marked differences begin to appear, as in the forms of the red blood-corpuscles, while the general structure remains similar.

Analysis of histologic structures: In analyzing the organism into its anatomic and histologic elements we find that they are arranged in an ascending series of aggregations. The ultimate elements of all are the atoms and molecules; as the scope of histologic science does not extend beyond the limits of microscopic vision, the atomic and molecular structure of tissues and cells does not come within its field, but is covered by organic chemistry and physics, with different methods of investigation.

The smallest elements with which histology deals are masses: 
hence chemistry and physics cover the atomic and molecular structure; histology, the minute molar structure of organisms.

The ultimate and smallest perceptible histologic elements of distinct individual character may be divided into two classes, cells and non-cellular or extracellular elements. By the union of these elements tissues are formed, as fibrous tissue, muscular tissue; by the union of tissues organs are formed; a combination of organs all working together for the accomplishment of some general function eonstitutes a system or tract, as the reproductive system, the alimentary tract; while all the systems together make up the complete organism.

The ascending grades of aggregation in organic structure are, then, after atoms and molecules, cells and extracellular elements, tissues, organs, systems, the organism.

The subject will here be elaborated in the order corresponding to this analysis, beginning with the fundamental elements, cells, etc., then taking up their aggregations in tissues, and finally studying the structure of the organs making up the various systems of organic activity.

Histologic technique: The histologic examination of tissues is carried out entirely by microscopic methods. To gain an adequate idea of histology, actual examination of the tissues under the microscope is necessary. The use of the microscope and the preparation of specimens of tissues for microscopic examination involve numerous delieate procedures, the mastery of which requires much special study and experience. Objects cannot be examined in bulk under the microscope, but require special treatment before their structure can be made visible. For one thing they have to be very thin, so thin as to be transparent; hence, solid tissues have to be cut into thin slices, or sections, about $\frac{1}{100}$ th millimetre thick. In their natural state most tissues are colorless, so that the details of the structure cannot well be made out. To make the details more visible it is customary to stain the sections with certain dyes, of which a considerable number are available for various purposes. Some stains have an affinity for some parts of the tissues, other stains for other parts; thus by the employment of various materials and methods different structures can be brought plainly into view and differentiated. The nuclear 
dyes, for instance, such as hæmatoxylin, carmine, methyleneblue, constitute a class of stains very much used, which impart to the cell-nuclei a deep color, while they leave the bodies of the cells uncolored; the general stains, as eosin, on the contrary, stain the cell-protoplasm, but not the nuclei. There are numerous other processes to be carried out; but the limits of this work do not permit the consideration of practical methods, for which reference may be made to the numerous special works on that subject. 


\section{CHA PTER II。}

\section{CELLS AND EXTRACELLULAR ELEMENTS.}

Elements of tissues: 'The ultimate elements of which tissues are composed are cells and extracellular (or intercellular) substances. Of these the cells comprise by far the bulk of the tissues and play the chief part in the phenomena of life. The body protoplasm, which is the seat and source of all vital phenomena, is located in the cells.

The non-cellular elements, produced by the vital activities of the cells, provide a connection, support, and framework for the cells, which, composed as they are of semifluid protoplasm, have little consistency of their own and are scarcely capable of self-support. The cells are alive and the seat of life; the function of the extracellular elements is merely mechanical, and they do not possess any real vitality of their own.

The cell: A cell is the smallest perceptible organization of protoplasm having a definite and complete individuality, structure, and vitality of its own. It manifests in miniature all the characteristics of life exhibited by the entire organism. It is a unit of organic structure and organic activity. It is the source and the seat of vital manifestations.

The name "cell" literally means a small inclosed space. This meaning does not express a correct conception of the nature of cells. 'The term arose from the fact that vegetable cells, with their prominent walls and their transparent though vital contents, appear like a mass of cavities separated by partitions. The term "corpuscle," often used synonymously with cell, better expresses the idea.

Classes of cells : All cells have a fundamental similarity in origin, structure, and nature; but as they actually occur they vary much in form and function in different situations. Two classes, in general, can be distinguished:

1. Generalized or undifferentiated, independent, free-living 
cells, each endowed with all the attributes of vitality and capable of complete existence singly and by itself. They are typical cells. Of this kind are $(a)$ the single cells of which some of the lowest (unicellular) forms of living beings consist, as the bacteria, desmids, and diatoms among plants ; and the protozoa - amøebæ, foraminifera, infusoria-among animals; (b) the ova and spores from which all the higher organisms develop, each being an independent and undifferentiated cell, and derived from the union of two other free though not typical cells, the female ovule and the male sperm-cell; (c) certain actively vital cells living free in the fluids and tissues of the highest organisms, exemplified by the white blood-corpuscle, or leukocyte.

2. Differentiated or specialized cells. In unicellular animals and plants the single cell carries on all the operations of life. In the forms that through evolution have become more highly developed, instead of a single cell the organism is composed of many cells; and instead of each cell in the multicellular organism carrying on all the functions of life, those operations are divided among different sets of cells, some serving one purpose, some another. This is differentiation, or specialization; it is a true division or organization of labor, and by it a maximum result is accomplished with the minimum and most economical expenditure of energy. In such a differentiated organism the cells in various situations and devoted to various purposes vary accordingly in form, structure, and function. Each great class of plant and animal forms has its own characteristic types of cells.

The specialized fixed cells of mammalian tissues may be in general divided into four classes : epithelial cells, connectivetissue cells, nerve-cells, and muscle-cells.

It is worthy of note that the young or embryonal forms of cells that are specialized when mature often partake of the more generalized character, thus exemplifying the principle that the different phases through which an organisn passes in the course of its genesis and development are indicative of the different forms through which its species passed in the course of its evolution.

Structure of cells: All cells have a body of protoplasm, which in most cases contains a nucleus (Fig. 1). The centro- 
some is an important feature of most cells. Many cells are surrounded by a cell-wall, while some have processes and other special features.

The cell-body, or cytosome: As a general thing, the body or

FIG. 1.

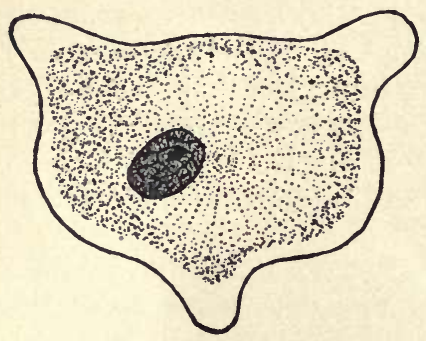

Diagrammatic representation of an amœboid cell, showing the nucleus, nuclear reticulum, nucleolus, the ccntrosome and attraction-sphere, the spongioplasm, hyaloplasm, and pseudopodia.

reticulum. The granular portion usually occupies the central portion of the cell, when it is called the endoplasm, or endosarc; the hyaline portion then forms the outer part of the cell, and is called the ectoplasm, or ectosarc.

Sometimes the protoplasm contains granules, as of pigment, or foreign particles which have been taken into the soft substance of the cell from without, as bacteria and other bodies absorbed by leukocytes, or food-particles taken in by the small unicellular organisms. Some cells contain vacuoles, minute rounded cavities or spaces; in some of the protozoa these are contractile, being alternately distended with fluid and then emptied by the contraction of the surrounding protoplasm, thus accomplishing some sort of circulatory process ; certain human cells are vacuolated. Cells often contain substances elaborated by their own activity and stored up in their bodies in granules or small masses. One of the commonest of these is fat; cells, as in the liver, secreting mammary gland, the vitellus of the ovum, often contain small fatty globules. In some cases, as in adipose tissue, this process is carried to an extreme degree and the cell becomes com- 
pletely filled and distended with a solid mass of fat, the original protoplasm remaining only as a thin atrophied shell. In plant-cells the body of the cell is largely occupied by sap, starch-granules, and the like. In some cases the protoplasm becomes transformed, so that little vitality is left to the cell ; as in the horny layer of the skin, in which the cells have been converted into a tough horny substance well adapted to purposes of protection.

Cell-granules: The granules which occur in the bodies of many cells are of various kinds. Some are particles of pigment produced by the action of the cell or absorbed from without. Some are produced artificially by the reagents employed in the preparation of the specimen, not occurring in the natural state; even water will cause the appearance of granules in some cells. Other granules, which are colorless, appear to be specializations of the body-protoplasm. Among such granules important distinctions are made out, especially by their behavior with staining-reagents; these distinctions are especially serviceable in the study of leukocytes. Some granules will become stained by one kind of dye, others by another kind, these staining-peculiarities corresponding to differences in the chemical constitution of the granules.

Stains in which the coloring-agent is the acid part of the compound, as eosin, are called acid stains; those in which the basic portion of the dyestuff is the staining-principle, as methylene-blue, are called basic stains; while stains composed of a mixture of acid and basic dyes are called neutral stains.

Granules which are colored by neutral stains are called "neutrophile"; those that take basic stains are called "basophile"; those that take acid stains are best called "oxyphile" ('os's, acid, and ciléc $\omega$, to love), though they are most commonly called "eosinophile" (eosin being one of the most potent acid stains), also sometimes "acidophile" (a barbarous term).

Ehrlich's much-quoted division of the granules of lenkocytes and other cells into five classes is as follows : o-granules, coarse oxyphilic or eosinophilic granules, found in certain human leukocytes; $\beta$-granules, amphophilic granules (taking both acid and basic stains), occurring in the blood of some animals ; $\gamma$-granules, coarse basophilic granules, found in mastcells; $\delta$-granules, fine basophilic granules, at times found in 
human leukocytes; and $\varepsilon$-granules, fine neutrophilic granules (by some considered oxyphilic), very common in human leukocytes.

The nucleus: Most cells contain in their interior a nucleus, a rounded structure distinctly marked off from the cell-body. It appears especially prominent when stained by nuclear and basic dyes, which impart to the nucleus a deep color, while the body of the cell is colored little or not at all. Nuclei are inclosed and separated from the rest of the cell by a membrane, the nuclear membrane. The nucleus consists of two portions: the nuclear fibrils, or reticulum, a network of fibers; and the nuclear matrix. a semifluid substance in the meshes of the reticulum.

The fibrillar substance, again, is composed of two parts, a homogeneous ground-substance called linin, supported in which are granules, fibres, and filaments of a substance called chromatin. In stained specimens the deep color shown by the nucleus is taken by the chromatin (whence the name), while the ground-substance, or linin, and the nuclear matrix are less colored.

From the phenomena of karyokinesis, the chromatin is supposed to be of the highest significance and importance in vital phenomena, especially in connection with reproduction and heredity. Chromatin has been conjectured to be identical with nuclein, a chemical constituent of cells which is regarded from physiological and chemical considerations as playing a highly important part in the economy of cell-activity.

In many cases, as in ova and nerve-cells, the nucleus contains a little nucleus of its own, or more than one, called the nucleolus. Most cells are uninucleated, or contain but a single nucleus each; certain classes of cells, however, are multinucleated, containing several or numerous nuclei each, as the voluntary muscle-cells, osteoclasts, and the giant cells present in certain pathological processes.

In shape, the nuclei are usually spheroidal, oval, or discoid; but in different cases the shape varies greatly, and may be exceedingly irregular.

The location of the nuclei in the colls varies, being sometimes in the centre, sometimes excentric, sometimes at the very periphery of the cell. 
Their size, in proportion to that of the entire cell, also varies; usually the nucleus comprises but a small part of the corpuscle; but in some cases, as in the mononuclear leukocyte, the nucleus occupies the largest part of the cell.

Most animal and vegetable eells are nucleated. Nuclei have not been demonstrated in some of the unicellular organisms, however, though they are probably present. Among mammalian cells one important class, the red blood-corpuscles, is not nucleated. The nucleus seems such an essential feature in the life and reproduction of cells that cells, like the red blood-corpuscles, that are not nucleated are probably lacking in the full attributes of vitality.

The precise function of the nucleus is not clear; but it is evidently of high importance, especially in connection with reproduction and heredity. It is probably the most vital part of the cell, the body largely serving nutritive and mechanical purposes.

The centrosome, or polar corpuscle, is a minute round refractive body or point, situated in the interior of the cell. The protoplasmic granules around it are arranged in delicate radiating lines, forming the attraction-sphere. In resting or non-dividing cells it may be within the nucleus, or just outside; but in this stage it is not conspicuous and is usually invisible. During the process of karyokinetic cell-division it appears prominently in the protoplasm of the cell-body, and it plays an important part in this process, apparently exercising some sort of polar, or directive, or attractive influence in the division.

The cell-wall, or cytolemma: Many cells are surrounded with a covering or wall, which gives support and protection to the soft protoplasm. This may be a distinct membrane, or a condensation and hardening of the outermost layer of protoplasm. Some of the lower unicellular organisms secrete around themselves a silicious or calcareous covering. The cell-walls of plants consist of cellulose, a firm substance belonging to the starch group, to which the hardness of wood is due. Some cells, as the leukocytes, appear to be devoid of a distinct cellwall.

Cilia and flagella: Certain kinds of cells have fine, hair-like processes or filaments projecting from their surface, capable 
of active vibratory or undulatory movements. They are composed of protoplasm like that of the cell-body, and their motility is of the same nature as that of protoplasm generally. When they are comparatively short and thickly crowded on the surface, or a part of the surface, of the cell, they are called cilia; when they are long and occur singly or only in small numbers on the cell, they are called flagella. They occur not only on low organisms, like bacteria and infusoria, but are of wide distribution and great importance in the human body; e. g., where cilia occur extensively on ciliated epithelium, and flagella on the spermatozoa.

The motion of cilia is vibratory, and, as they act in unison, a wave-like motion results, which, acting from a fixed surface, as in mammalia, carries particles along the surface or creates currents in the fluids adjacent.

The motion of flagella is undulatory, and when attached to free cells this movement rapidly propels the cells through the fluid in which they occur, acting in the same manner as the tail of a tadpole or fish. Flagella therefore are a means for rapid locomotion of spermatozoa, monads, and other freeswimming cells provided with them.

Other cell-processes: Besides motile processes like those just described, some cells possess permanent and non-motile projections, which may be very various in form and size, coarse or fine, long or short, branching or very irregular. Good examples are furnished by nerve-cells, bone-cells, connectivetissue cells.

Forms of cells: Cells vary widely in shape, according to the situation in which they occur and the purposes they serve. The typical shape is perhaps spheroidal or oval. The leukocytes, which are typical active cells, are spherical when at rest. Many cells which when mature are of diverse shapes are spheroidal when young. In a differentiated multicellular organism there is a great diversity of form in different localities. On exposed surfaces they become flattened or diskshaped; in other situations they are spheroidal, or columnar, or elongated, or branching, sheath-like, or tubular, or altogether irregular. But however diverse their form, their fundamental type is identical throughout; they are all definite protoplasmic bodies, usually nucleated. 
Size of cells: The size of cells varies much. Some of the bacteria are only about 1 micromillimetre ${ }^{1}$ in diameter ; these are very small cells. The diameter of ordinary vegetable cells ranges from 20 to $100 \mu$; while some plant-cells are fairly visible to the naked eye, nearly $1 \mathrm{~mm}$. in diameter. The mammalian ovum is a very large cell, about $0.2 \mathrm{~mm}$. $(200 \mu)$ in diameter. Human red blood-corpuscles are about 7 or $8 \mu$ in diameter, and leukocytes 7 to $15 \mu$. Human voluntary muscle-cells range from 10 to $40 \mu$ in diameter, and 2 to $4 \mathrm{~cm}$. in length. The ordinary cells of human tissues range from about 5 to $40 \mu$ in diameter.

Vital properties and functions of cells: The study of function pertains especially to the science of physiology ; in histology it is, however, necessary to pay some regard to functions, since function and structure are intimately related, and a consideration of the former throws light on the latter, as a full understanding of the latter is essential to an adequate understauding of the former. The function expresses the meaning of the structure.

The properties and manifestations of life and organisms in general reside in the individual cells. The functions and activities of cells are the same as those of life itself. These activities are usually divided into three classes, nutrition, sensibility, and motion.

Nutrition broadly comprehends the ingestion of food-material ; its elaboration and assimilation into the organic structure; the rejection of waste; the formation of the structural organization; the preserving of it intact; development to maturity; reproduction; decline; the passage through a definite life-cycle.

Independent free-living cells, as the unicellular organisms, leukocytes, etc., carry on all these vital operations alonenourish themselves through their life-cycle, manifest irritability, and possess the power of quasi-spontaneous movement. The specialized cells of differentiated organisms each devote their respective energies to some particular part of the work of life, instead of each performing all the vital functions.

1 The linear unit employed in microscopic measurements is the micromillimetre, or micron, briefly designated by the Greek letter $\mu$; it is $\frac{1}{1000}$ th of a millimetre, or about $\frac{1}{25000}$ th of an inch. 
Thus the functions of such cells become distributed and differentiated: one set of cells, in the muscular system, exercises the power of contractility ; others, in the nervous system, are specially arranged as seats of sensibility and consciousness; other cells have chemical functions, in the secretory apparatus; some build up the framework of the body, some serve as a covering to the surface, others provide for reproduction.

Movements of cells: The chief visible vital movements, of automatic or quasi-spontaneous character, of which cells are capable are : 1, amoboid movement; 2 , contraction ; 3 , ciliary movement ; 4 , cyclosis, or internal circulation. These are all probably varying manifestations of one fundamental mode of motion. Besides these, a purely physical, not vital, microscopic motion is sometimes observed in cells, namely, 5, Brownian movement.

Amœboid movement: Many free cells, as the leukocytes in the blood and some of the protozoa, possess the power of spontaneously protruding and withdrawing processes of the body-protoplasm. These processes are called pseudopodia; and this kind of movement is called amoboid, from the fact that it is characteristic of the protozoon known as the amoba. At rest, the cells capable of amoboid movement are usually spheroidal ; but when in motion their shape may become very irregular.

The pseudopodia have no definite forms or size, but are very variable and irregular. They may be broad, or they may be slender and filamentary ; they are always temporary and capable of retraction, unlike pernanent celi-processes. By means of them the cells are enabled to seize food-particles, or to move themselves about, extending a pseudopodium in one direction, and then by enlarging it the body gradually follows.

The hyaloplasm, or clear outer part of the protoplasm, is the more actively concerned in the amœboid movements ; it is the first to be protruded in the pseudopodia, while the granular portion of the protoplasm more slowly follows. The throwing out of the pseudopodia is said to be due to currents of the fluid protoplasm, which, impinging on the periphery of the cell, unbounded by a stiff wall, cause it to bulge and protrude at that point.

Contraction is a mode of movement, closely allied to amœ- 
boid movement, which is manifested by many cells. The protoplasm surrounding contractile vacuoles exhibits it. It is especially the function of muscle-cells, which decrease in length and at the same time increase in thickness. This movement is also said to be accomplished by the flowing of the fluid protoplasm.

Ciliary movement: The vibratory movement of cilia and the undulatory movements of flagella have been already described. It is a vital protoplasmic movement.

Cyclosis : In certain vegetable cells currents of liquid and minute particles are observable coursing about in definite channels entirely within the cell. This movement is called cyclosis, or internal cell-circulation, and is perhaps due to spaces and channels in the cell-substance, along which the contained fluid is forced by contractions of the protoplasm.

Brownian movement: Fine microscopic particles of solid matter, if mixed with water or certain slightly viscid fluids, exhibit under the microscope a characteristic constant dancing or oscillating movement. This was first described in 1826, by Robert Brown, from whom it has been called the Brownian movement; it is also called pedesis. Its cause is not understood. It is a purely physical phenomenon, not vital; but granules within cells sometimes exhibit this movement.

Intimate structure of cells: The description of cells above given covers the features observable by means of the microscope. When we contemplate how from a single microscopic cell, the ovum, a large organism can develop, composed of millions of cells of a highly differentiated and vastly complicated structure; and how ova indistinguishable from one another, though belonging to different species, produce their own kind with unerring fidelity : we are forced to conclude that the germ-cells must contain elements or mechanisms corresponding to and capable of developing all the various parts of the full grown organism - that is, that they have a vastly complex structure that is hidden from us beyond the limits of microscopic vision, and that what we see are but the coarsest features of their construction. Structural elements not ordinarily visible become manifest during karyokinesis. Between the atomic and molecular structure of which we gain some idea through chemical science, and the elements visible to us, 
there is in cells a large field of structural factors that is beyond our powers of observation. If we could penetrate within this invisible field, we might find much of histologic and structural organization that would be of the greatest interest and importance in explaining the mysteries of vital phenomena. But we cannot as yet penetrate it, and the various vital structures and units which eminent biologists have assumed as entering into the structure of cells, such as the hypothetical pangenes, plasomes, plastidules, biophors, determinants, and the like, have at present little more than a speculative value.

Extracellular elements: The non-cellular, intercellular, or extracellular constituents of tissues serve to connect, support, and provide a mechanical framework for the cells, which of themselves do not possess the firmness requisite for a large organism. These elements are not the seat of any real vitality, which pertains exclusively to the cells; they are produced by the vital activities of the latter.

The intercellular substances exhibit considerable variety, and in different tissues occur in varying proportions to the total amount of the cells.

The simplest form is the intercellular cement, a scanty substance which cements cells together. The plasma of the blood and the serum of lymph may be regarded as a fluid intercellular substance in which the cells are suspended.

The greatest variety and quantity of extracellular elements occur in the connective tissues, where the fibres, the hyaline matrix of cartilage, and the mineral matter deposited in bones are of this character. 


\section{CHA PTER III.}

\section{REPRODUCTION AND DEVELOPMENT.}

Origin of cells : Every cell is produced by a preëxisting cell -omnis cellula e cellula. A generation ago it was supposed that under certain circumstances some of the lower organisms, as the bacteria, could develop directly out of non-living matter. The generation of life de novo in such a manner was called abiogenesis, or spontaneous generation. The incorrectness of that supposition has been shown, and it is now universally believed that abiogenesis never occurs. Under all circumstances life is always produced by preëxisting lifeomne vivum e vivo; all protoplasm is elaborated by preëxisting protoplasm.

Modes of reproduction of cells : There are several modes in which cells reproduce themselves, namely : 1 , direct division ; 2 , budding, or gemmation ; 3 , segmentation ; 4 , indirect division, or karyokinesis.

FI's. 2.
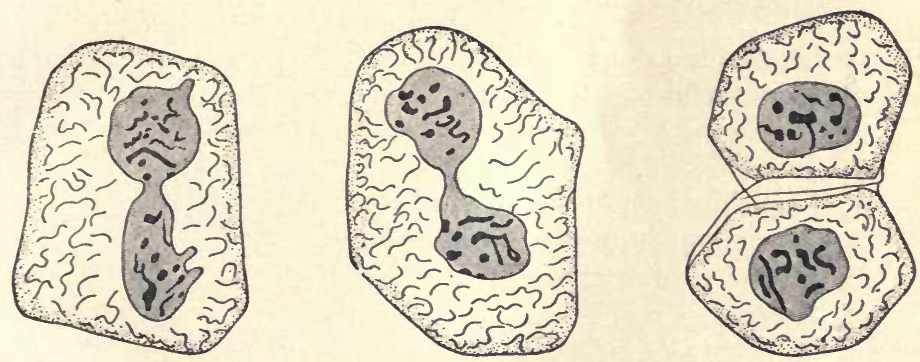

Direct cell-division (Flemming).

Direct division: In this mode of cell-division a constriction appears in the nucleus and in the cell-body; this constriction gradually deepens, until the two parts become completely 3-Hist. 
separated, and the one parent-cell has become two daughtercells. This process is called direct or amitotic division or fission (Fig. 2). This method of division sometimes occurs with some cells, but it is believed to be far less common than the indirect or karyokinetic method.

Budding, or gemmation: Occasionally this mode of cellreproduction occurs among the protozoa. A small protrusion of protoplasm appears at the margin of the cell, gradually enlarges, and is finally separated from the parent and becomes independent.

Segmentation: This consists in the simultaneous breaking up of the parent-cell into a number of small rounded cells, which separate, and in their turn develop to maturity. It occurs among some protozoa, and in human medicine is easily observed in the parasite of malaria, which at the end of its life-period rather abruptly breaks up into from eight to twenty minute hyaline spheres.

Indirect division, karyokinesis, or mitosis, is the usual and chief mode by which all cells reproduce themselves. The process is accomplished by a series of definite changes, affecting especially the chromatin-filaments and centrosomes (Figs. 3-8.) The stages of typical karyokinesis may for purposes of description be capitulated as follows :

1. Resting stage.

2. Continuous convolution $\left\{\begin{array}{l}a, \text { close skein, } \\ b, \text { open skein. }\end{array}\right.$

3. Segmented convulution.

4. Equatorial or aster stage.

5. Division of chromosomes and metakinesis.

6. Diaster stage.

7. Double segmented convolution.

8. Double continuous convolution.

9. Division of cell-body.

10. Resting stage.

In the intervals between karyokinetic division, or the resting stage, the chromatin of the nucleus is arranged in granules, filaments, or a network, but not in any definite manner.

The mitotic process begins with an enlargement of the nucleus and an increase of the chromatin; at the same time the centrosome appears in the body-protoplasm and enlarges. 
Fig. 3.

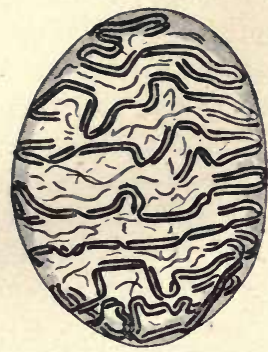

Continuous convolution.

Fis. 5.

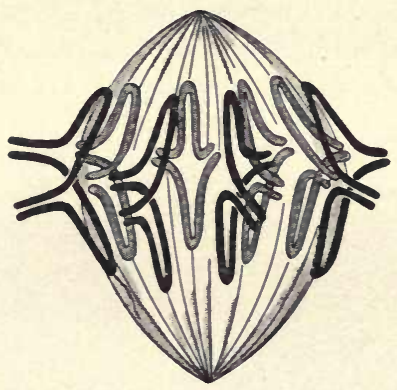

Metakinesis, earlier stage.

Fig. 7.

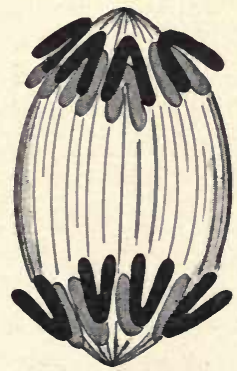

Diaster stage.
FIG. 4.

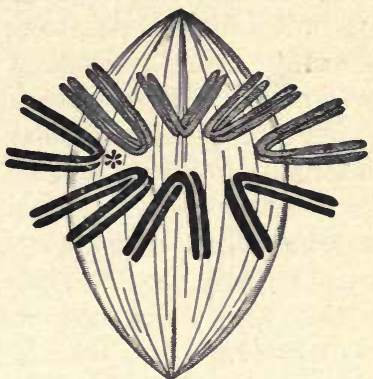

Equatorial or aster stage. Fig. 6.

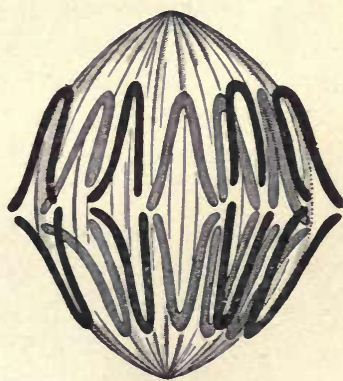

Metakinesis, later stage. FIr. 8.

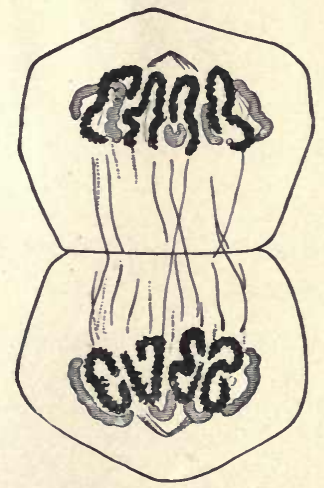

Double segmented convolution.

Diagrammatic representation of stages of karyokinesis (Flemming). 
The chromatin develops into an apparently continuous and singlle long filament in a tangled skein, or dense convolution, or "spirem." This is the stage of the continuous convolution. At first the chromatin-filament is fine, and is arranged in close, crowded turns; this formation is called the close skein. Later, the filament becomes coarser, and the loops and windings of the convolution more open, forming the open skein. The nuclear membrane and nucleoli become gradually transformed into the chromatin-filament and absorbed into the skein, and thus disappear, so that no wall now separates the nucleus from the cell-body.

The chromatin-filament, which heretofore has apparently been one continuous thread, then breaks up into segments, forming the segmented convolution. Each of these segments is called a chromosome. The chromosomes form V-shaped or $\mathrm{U}$-shaped loops, and tend to arrange themselves with the

FIG. 9.

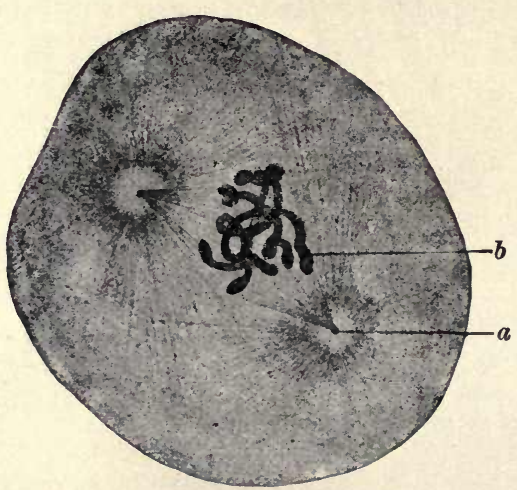

Cell (ovum of ascaris megalocephalus) undergoing karyokinetic division (Kostanecki and Siedlecki). $a$, centrosome; $b$, chromosomes.

angles toward a common centre and the arms radiating outward. The number of chromosomes in different species is usually from four to thirty-six in each cell ; one crustacean, artemia, has 168. Human cells contain about sixteen chromosomes each. The number of chromosomes is uniform in all the cells of the same species. In the meantime, during the 
segmentation of the spirem, the centrosome has divided into two, which have separated and become located at opposite points or poles, one on each side of the nuclens.

In the equatorial or aster stage the process is at its height. The achromatic spindle has developed, consisting of delicate striæ, uncolored by the stain (whence the term achromatic), which are arranged in the form of two cones having their apices in the two centrosomes, and their bases directed toward each other; or, in other words, the achromatin-fibres form a fusiform spindle, with the extremities in the centrosomes or poles (Fig. 9). The chromosomes become arranged in the equatorial plane of this spindle-that is, in the plane bisecting at right angles the axis or line connecting the two poles; their loops are directed inward toward the common centre; their arms and free extremities extend outward in a radiating manner, presenting the appearance, from the side, of a star, or aster.

The next stage is the division of the chromosomes and migration of their segments, or metakinesis. The chromosome-filaments split in two longitudinally, beginning at the apex or bend, and the apices of the separated halves then travel along the threads of the achromatic spindle toward the two centrosomes.

Thus the double aster or diaster stage develops. Around each centrosome the daughter-chromosomes become arranged in radiating or the aster form. The chromatin of the single original nucleus has been divided into two equal parts, to form two new daughter-nuclei; and it is to be observed that the number of chromosomes in the daughter-cells is precisely the same as in the parent-cell, and remains constant throughout for each species.

The free ends of the chromosome-fibers become connected by achromatic filaments, and the chromatin-fibers now undergo a change precisely the reverse of that of developing karyokinesis. The double segmented convolution, or disconnected chromosomes, is succeeded by the double continuous convolution: first the open skein appearing, then the close skein. The nuclear membrane and nucleoli appear, and ultimately the nuclei resume the condition of the resting stage. Simultaneous with these retrograde processes in the nuclei, division 
of the cell-body occurs; the body has become indented, a line of cleavage has appeared across it, and it divides entirely into two parts. Thus the original cell has divided into two cells, each like the parent in all respects.

Conjugation: In contrast to the division of one cell into two, the contrary process occurs, namely, the coalescence of two cells to form one. This is called conjugation. It is observed among some of the protozoa, two of these organisms becoming completely fused to form one individual. A form of conjugation also occurs throughout the greater part of the entire animal and plant kingdoms in connection with sexual reproduction. The fusion of the female and male germ-cells, the ovum and spermatozoon in animals, the ovule and pollen in plants, is a case of conjugation, although here the uniting cells are somewhat different from each other. This form of conjugation is evidently of profound significance in the vital economy, though not well understood. Even in the protozoa conjugation is perhaps connected with reproduction.

Sexual reproduction: Multicellular animals are developed from a female and a male cell, which coalesce and form a single cell, the fecundated ovum. The spores from which cryptogamous plants develop are likewise fertilized single cells. Phenogamous plants are developed in essentially the same way; the fully formed seeds are, however, multicellular, though they can be traced back to a single fertilized ovulecell. The female element before fecundation is termed the ovule; after union with the male element it becomes the ovum. The male elements in animals are the spermatozoa; in flowering plants, the pollen.

The ovule: The mammalian ovule, as fully developed in the female ovary, is a comparatively large spherical cell about 0.2 millimetre in diameter. It is surrounded by a delicate membranous wall, the vitelline membrane. The body of the cell consists of protoplasm infiltrated with a large number of minute fat-globules, for the early nourishment of the developing embryo; this is the vitellus, or yolk. Within the vitellus, located excentrically, is the nucleus of the cell, or the germinal vesicle; within this, again, is the nucleolus, or germinal spot.

Maturation of the ovule: Before or while the ovule receives 
the male element it undergoes certain changes, called maturation. The nucleus of the ovule divides and gives off a portion of its substance, which forms a small body, the polar body, which proceeds to the surface of the cell and is there extruded. This process is repeated, a second polar body being given off from the nucleus and expelled from the cell in the

Fig. 10.

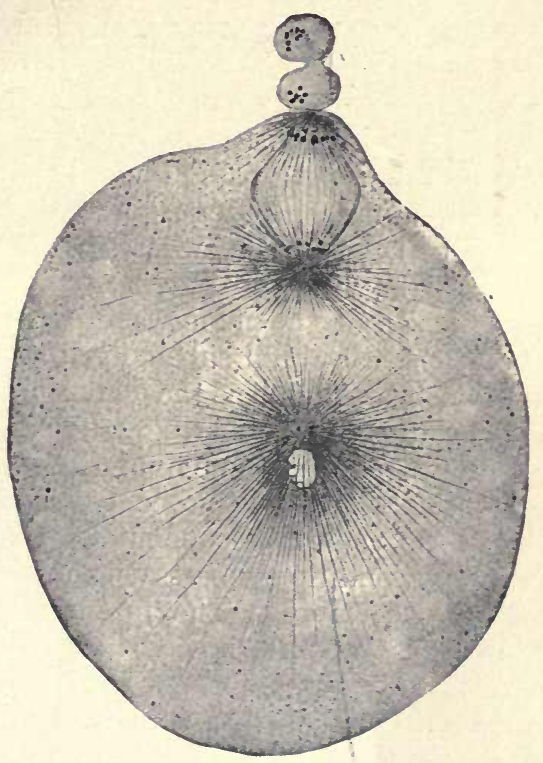

Maturing ovum of physa (fresh-water snail) (Kostanecki and Wierzejski). Above is the first polar body extruded from the ovule and dividing into two bodies. Just beneath it is the nucleus of the ovule, dividing to form the second polar body. At the centre of the ovule is the male pronucleus with its centrosome and attraction-sphere, derived from the spernatozoon.

same way. By this process the nucleus is "reduced," or divested of a portion of its chromatin, so that when the chromatin of the spermatozoon, also reduced, is united with it the number of chromosomes normal to the cells of the species is present. The portion of the original nucleus now remaining in the ovule is called the female pronucleus.

Fertilization of the ovum: The ovule is not capable of under- 
going further development until the male element, or sperm-cell, is added to it. This process is called the fertilization, impregnation, or fecundation of the ovum. During or after the process of maturation the spermatozoon finds its way to the surface of the ovule, passes through the vitelline membrane, or through a small opening in it called the micropyle, and enters the protoplasm, where it forms a nucleus, the male pronucleus (Fig. 10). The male and female pronuclei then coalesce, or at least become apposed, and temporarily disappear, reappearing when the cell undergoes division. The ovum is now fully formed and capable of further development. It is a typical cell, endowed potentially with all the attributes of vitality, and contains within its minute compass almost inconceivable powers of definite and intricate expansion and evolution. It might seem at first view that the largest part of the substance of the fertilized ovum was contributed by the female parent, while the contribution of the male was comparatively insignificant; but of the really vital substance, the chromatin, the male element furnishes as much as the female, and hence makes an equal impression on the offspring. To explain the inability of the unimpregnated female ovule to undergo division, it has been suggested that it has no centrosome, and that this is supplied by the spermatozoon, the addition of which therefore supplies all the conditions necessary for division.

Development of the embryo: The further development of the embryo proceeds by mitotic cell-division. The ovum divides into two cells; each of these into two more, and so on. Thus a mass of cells is formed, constituting the blastoderm. Soon the cells of the blastoderm develop so as to form two layers, the outer being called the epiblast, the inner the hypoblast. Later another layer of cells appears between these two, and is called the mesoblast. Up to a certain point the cells produced by repeated cleavage are similar to one another; but afterward differences of form and purpose appear in different situations according to the organs and tissues into which they are about to develop. This development of cells having similar origins into dissimilar cells is the process of differentiation. By the continued production and differentiation of the cells of the epiblast, hypoblast, and mesoblast, the embryo develops, and the various organs gradually 
come into existence. The details of this process it is the province of the science of embryology to consider.

Well-defined tracts of the adult body may be traced back to the three primary blastodermic layers, and the distinctions of origin thus made are commonly emphasized as of importance. Without entering into minutiæ, the tissues derived from the primary layers are in general as follows:

From the epiblast are derived the epithelium of the epidermis ; the nerve-centres and axis-cylinders, and the enamel of the teeth.

From the hypoblast is derived the epithelium of the mucous membranes lining the alimentary and respiratory tracts and bladder, and of the glands tributary thereto.

From the mesoblast are derived the corium of the skin and the tunica propria of mucous membranes ; the connective-tissue structures; cartilage, bone, the vascular and lymphatic systems, the blood, the muscular tissues, the reproductive organs, and the kidneys and ureters. 


\section{CHAPTER IV.}

\section{THE TISSUES.}

Classes of tissues: Cells and intercellular elements make up tissues; tissues make up organs. A full knowledge of the different varieties of tissues is essential to an adequate and easy understanding of the structure of organs. The simple tissues of the human body may be divided into five classes:

Epithelium and endothelium;

Connective tissue ;

Muscle ;

Nerve-tissue ;

Free cells and body-fluids.

Of these the first four are made up of fixed elements; the last comprises the free and fluid parts of the organism. These classes are sharply distinguished from one another by morphological and functional characters and by the fact that each class, in general, is developed from and in turn produces its own kind of cell and tissue exclusively.

\section{Endothelium and Epithelium.}

Location: The cells lining the exposed surfaces of the body form the class of epithelium and endothelium.

Those lining the closed internal cavities, as the vessels and serous saes, and derived from the mesoblast, are called endothelial cells.

Those lining the "external" surface of the body and the channels and tracts continuous with it (that is, those lining the skin, mucous membranes, and tributary glands), also the closed thyroid and other ductless glands, central canal of the spinal cord, and ventricles of the brain, are called epithelium.

Epithelium consists almost entirely of cells, there being only a slight amount of intercellular substance to cement them together. It is derived from the epiblast and hypoblast, except 
the epithelium of the kidneys, ureters, and sexual organs, which is mostly of mesoblastic origin.

Its function is, over large areas, protective; in the glands it is secretory ; in some tracts, as the intestine, it is not only protective, but has absorbent functions; while ciliated and other epithelium serve special purposes.

Varieties of epithelium: Epithelium-cells may be divided into the following classes: squamous, columnar, ciliated, goblet, glandular, and specialized epithelium. Some of these, again, are divisible into simple epithelium, in which the cells form a single layer; and stratified, in which they are arranged in several layers.

Simple squamous epithelium: Squamous epithelium-cells are large, thin, flat, disc-shaped cells, each with a large discoid nucleus. In simple squamous epithelium the cells form but a single layer, and meet edge to edge. It is not extensively distributed, occurring in the alveoli of the lungs, the capsule of the Malpighian bodies of the kidney, and a few other situations. Endothelium is very similar to it.

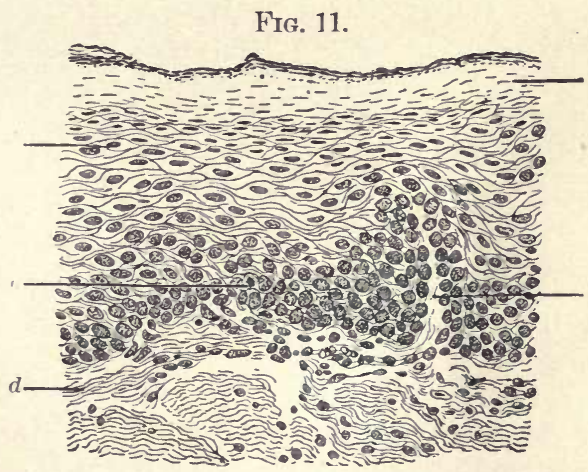

Stratified squamous epithelium, œesophagus of rabbit (Dunham). a, deeper layers of rounded germinal cells; $b$, more superficial layers of flattened cells; $c$, surface layer of horny cells; $d$, underlying fibrous tissue; $e$, papillary projection from the fibrous layer.

In stratified squamous epithelium the cells form several layers (Fig. 11). Those on the surface are flattened and squamous, overlapping one another at the edges. The cells of the lower layers are cuboidal or spheroidal; they become 
gradually flattened toward the surface, and are germinal cells which ultimately become squamous. This variety is of extensive distribution, forming the epidermis of the skin and the epithelial layer of the conjunctiva, mouth, part of the pharynx and larynx, œsophagus, vagina, and portions of the urethra.

The epithelium lining the bladder, ureters, and pelvis of the kidneys is a variety of stratified squamous epithelium sometimes called transitional epithelium (Fig. 12). The number of layers of cells is few, forming a sort of intermediate

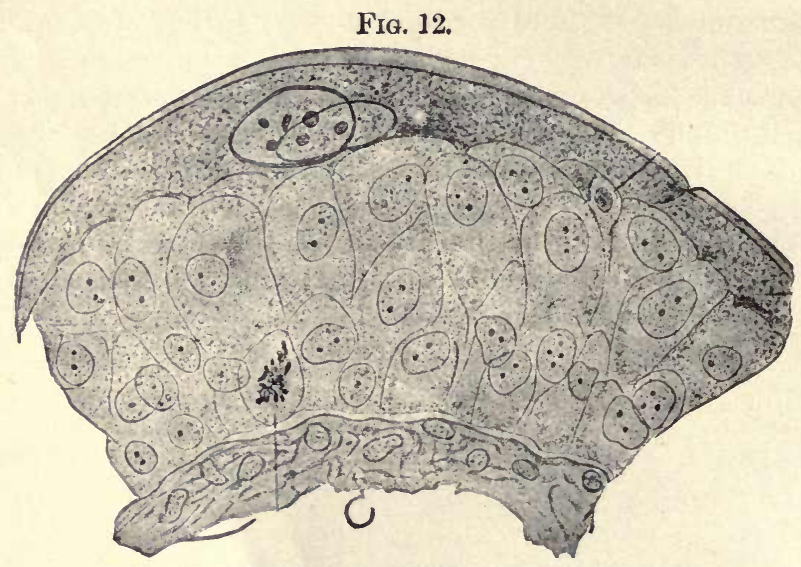

Transitional epithelium, bladder of mouse (Dogiel).

or transitional variety between the simple and stratified types. The lower cells become quickly changed into the flattened surface cells.

Columnar epithelium-non-ciliated: Columnar epitheliumcells are elongated, columnar, or prismatic in shape, arranged on a basement-membrane perpendicular to it, with one end directed outward. Sometimes they are shorter and more cuboidal.

When they form a single layer they constitute simple colunnar epithelium (Fig. 13), which occurs throughout the stomach and intestine, in some gland-ducts, covering the ovary, and in portions of the male urethra and seminal tracts.

Non-ciliated stratified columnar epithelium, in which the cells 
are in several layers (the outer only being columnar), occurs in the olfactory nasal membrane and a portion of the vas deferens.

Columnar epithelium-ciliated : A variety of epithelium in

FIG. 12.

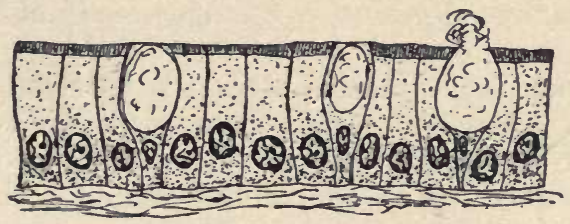

Simple columnar epithelium and goblet cells, from the intestine. Shows the striated free cuticular margin of the cells in this situation.

which the exposed surfaces or ends of the cells are covered with cilia, which in life are in characteristic wave-like motion.

Simple ciliated epithelium occurs in the Fallopian tubes, uterus, central canal of the spinal cord, and brain ventricles.

In stratified ciliated epithelium (Fig. 14) the cells form several layers. Only those of the outer layer are columnar and ciliated; those of the lower layers, the germinal cells, are rounded or pear-shaped, below and between the columnar cells, into which they ultimately develop. This variety covers the respiratory tract, Eustachian tubes, and epididymis.

Goblet epithelium-cells (Fig. 13) a re large elliptical or pyriform cells, with their larger ends outward and their nuclei FIG. 14.

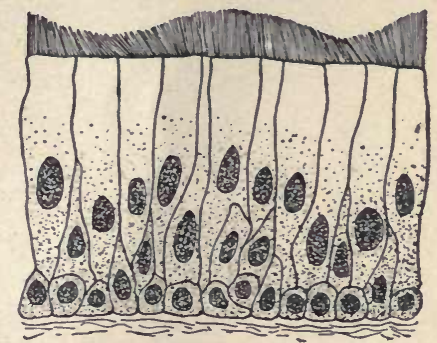

Ciliated columnar epithelium (stratified), from trachea, showing ciliary wave.

near their bases. They nccur scattered among columnar epithelium-cells. Their bodies have a clear unstained, distended appearance, being filled with a mucinous substance which they secrete and discharge on the surface of the mucous membrane.

Glandular epithelium is that which forms the secreting 
portion of glands. The cells are in general spheroidal or polyhedral, but vary in shape in different situations.

Specialized epithelium: In some situations epithelium-cells become modified in various ways different from those above mentioned, as the pigmented epithelium of the retina and elsewhere, and the so-called neuro-epithelium occurring in certain sensory organs, the retina, ear, tongue, and olfactory membrane.

Endothelium: The closed internal cavities of the bodythe vascular channels, serous cavities (as the pleura, peritoneum, and pericardium), synovial membranes, and lymphspaces-are lined with endothelium (Fig. 15). Endothelium

FIG. 15.

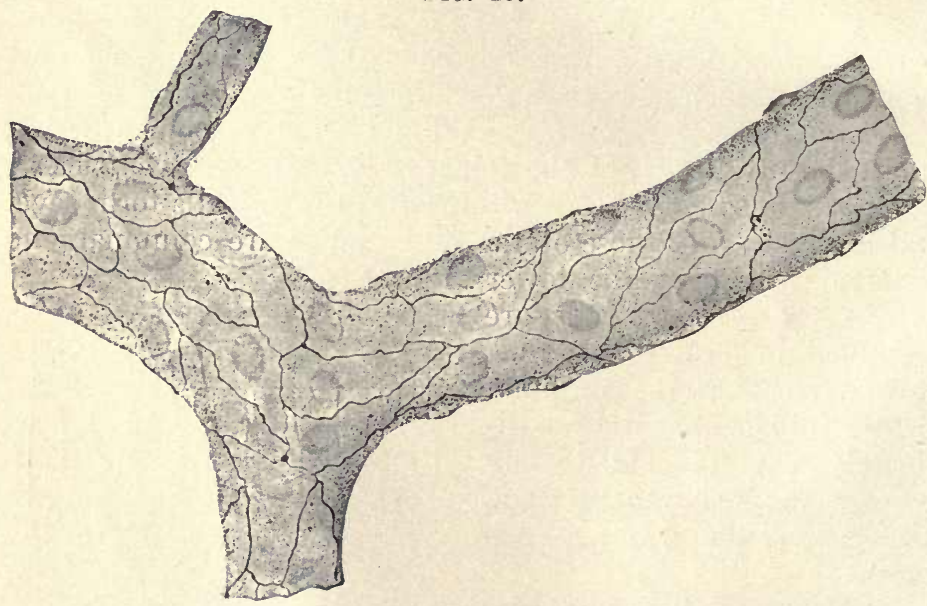

Endothelial lining of small vein and capillary (Engelmann).

is distinguished from epithelium mainly in being of mesoblastic origin. Morphologically it is similar to simple squamous epithelium, consisting of a single layer of polygonal flat nucleated cells, smooth and shiny, which meet edge to edge by oftentimes sinuous or serrated margins. Endotheliumcells merge into the flattened type of connective-tissue cells, with which they are genetically related. 


\section{Connective Tissue.}

Connective tissue is distributed everywhere throughout the body beneath the outer epithelial layer. It forms a supporting and connecting framework for the parts of the organism, and to this end it envelops and penetrates all the interior organs and structures. It permeates the organism so completely that if all the other tissues were removed the connective-tissue framework would still form a complete mould of the borly and its organs. It originates from the mesoblast, except the neuroglia of the nerve-centres, which is epiblastic. Like other tissues, it is made up of cells and non-cellular elements, but is peculiar in that the non-cellular portions largely predominate, as naturally follows from their constituting the main framework of the body.

The connective-tissue cells, while differing in various situations, have a fundamental morphological and genetic similarity.

The extracellular elements serve mechanical purposes, and are not endowed with vitality; they are produced by the connective-tissue cells. The most specialized and generally distributed of the extracellular elements are certain fibres, which are of two kinds, white fibres and yellow elastic fibres. Other intercellular materials are an interstitial mucinous or gelatinous substance especially found in young connective tissues (as mucous tissue), the matrix of hyaline cartilage, and the mineral matter in bone.

Connective-tissue cells are of one general type, though varying much in different situations. The forms associated with fibrous tissues are sometimes called "fibroblasts." Connective-tissue cells are sometimes round or spherical, small and prominently nucleated; such cells occur only in young, growing, or embryonal forms of connective tissues.

The mucous cell is another form of young or embryonal connective-tissue cell; it is a flat nucleated cell of irregular stellate shape, with its pointed processes, three or four in number, prolonged into fine filaments which anastomose with similar processes from neighboring cells.

In some situations, especially in fibrous tissues, the connective-tissue cells are fusiform in shape; in some they are stellate; in others, as in basement-membranes and the cells 
lining retiform tissue and lymph-spaces, they are flattened like endothelium, with which they are closely related; sometimes they are lamellar, as in tendon-cells ; or they may-be altogether irregular.

In general, connective-tissue cells are small flattened or elongated cells, with prominent nuclei and relatively small bodies, and often wirh processes; they are polymorphous and adaptable to the situations in which they are placed. Sometimes they are pigmented, such cells, stellate or irregular, occurring in the choroid, pia mater, and in amphibia. Some connective-tissue cells are granular, as precursors of fatcells.

Fat-cells are large spherical connective-tissue cells distended with fat. Odontoblasts, cartilage-cells, and bone-cells are forms of connective-tissue cells, and neuroglia-cells differ chiefly in their epiblastic origin. Plasma-cells occurring in areolar tissue contain numerous vacuoles and vary in form.

Mast-cells are large cells

Fig. 16.

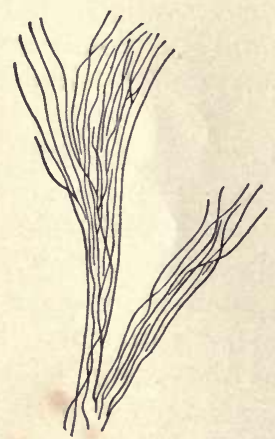

White tibres, teased to show individual fibrillæ (Dunham).

FIG. 17.

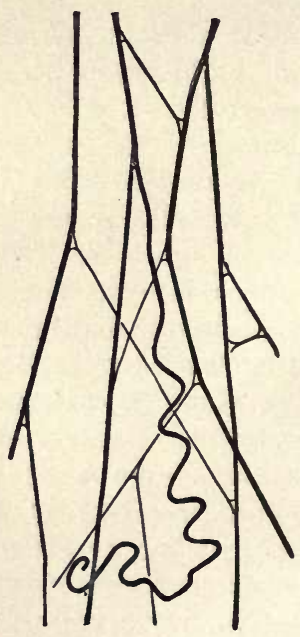

Yellow elastic fibres from subcutaneous areolar tissue (Schäfer).

twenty to thirty micromillimetres in diameter, containing coarse basophile $(\gamma)$ granules, and irregular pale-staining nuclei; they are occasionally found in areolar tissue. 
Wandering cells, cells which are free and capable of moving about in areolar tissue, are really leukocytes or lymph-cells, and belong to the class of free cells.

The white fibres consist of very fine colorless fibrillæ cemented together in coarser fibres (Fig. 16). They may be arranged in an interlacing network or parallel in bundles, and often present a wavy appearance. The fibrils do not branch. They swell and become indistinct when treated with acetic acid, often showing constrictions. They yield gelatin on boiling. They are characterized by unyielding strength, and occur in tendons, ligaments, etc., where tenacity and firmness are required.

The yellow elastic fibres (Fig. 17) are yellowish, highly refractive, branching fibres, fine or coarse, forming bundles or membranous interlacing networks. They yield elastin, not gelatin, and stain differentially with orcein. When divided, the freed ends curl up. They are characterized by elasticity, and are widely distributed, occurring in especial abundance in the ligamentum nuchæ of animals, ligamenta subflava, bloodvessels, and in yellow elastic cartilage.

Two theories are held as to the development of the fibres, white and yellow: one is that they are produced by a transformation of the protoplasm of the connective-tissue cells; the other, probably more generally true, is that they are not transformed cells, but develop in the mucinous intercellular matrix by a sort of secretory process, under the agency of the cells.

Varieties of connective tissues: According to the varying forms and proportions of the constituent elements, there are many varieties of connective tissues, merging imperceptibly into one another without sharp dividing lines. The following best-marked types may be mentioned:
Mucous tissue;
Tendons;
Areolar tissue;
White fibrous tissue;
Retiform tissue ;
Yellow elastic tissue;
Membranes;
Cartilage ;
Bone ;
Adipose tissue ;
Dentine ;
Neurnglia.

Mucous tissue (Fig. 18) consists of mucous cells, flattened 4--Hist. 
gradually into fibrous, adipose, or other varieties of connective tissue.

White fibrous tissue is that in which the white fibres predominate, forming a tough, dense, inelastic tissue. Connective-tissue celis, fusiform, or flat and clasping the fibrous fasciculi, are scattered among the wavy fibres, more numerous

FIG. 19.

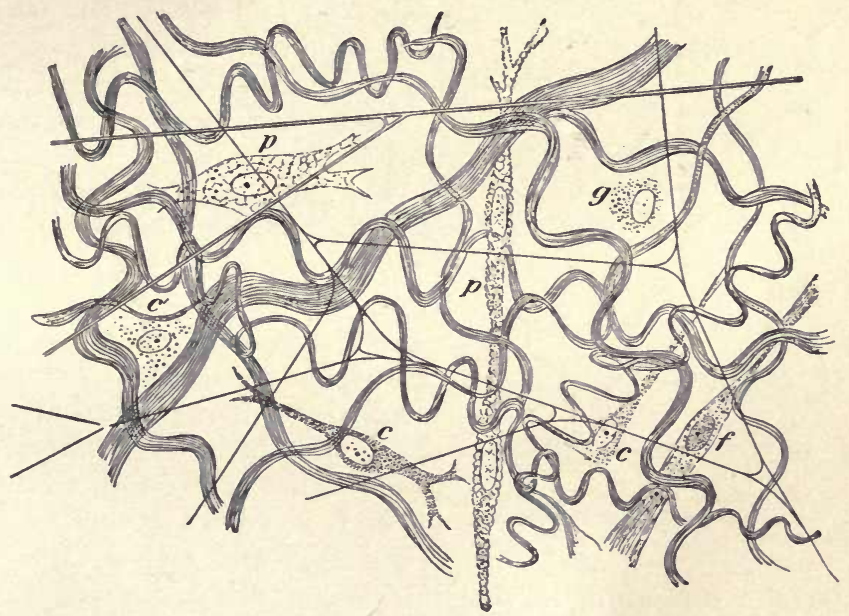

Subcutaneous areolar tissue, from rabbit, showing white and elastic fibres and connective-tissue cells (Schäfer). $c$, developing fusiform and stellate cells; $c^{\prime}$, tlattened cell; $f$, elongated cell; $g$, wandering cell; $p$, plasma-cells.

when the tissue is young, less numerous or even almost absent in old tissues. It is widely distributed, forming tough fasciæ, aponeuroses, ligaments, capsules and trabeculæ of organs, and the like.

Yellow elastic tissue is that in which elastic fibres predominate, as in the ligamenta subflava and bloodvessels, structures characterized by elasticity and resiliency. The ligamentum nuchæ of certain animals consists mainly of coarse polygonal yellow fibres, with a few scattered white fibres or cells. Arteries contain much elastic tissue, largely in the form of elastic plates, fibrous networks, or perforated membranes.

Membranes: Connective tissue often forms distinct mem- 
branes, as in the capsules surrounding organs and the corium of the skin. 'These are often fibrous tissue, made up of interlacing fibers; in other cases, especially in the basement-membranes which support epithelium, they consist of flattened connective-tissue cells joined edge to edge in a single layer.

Adipose or fatty tissue (Fig. 20) occurs in large masses under the skin, in the omentum, about the viscera, especially the kidneys and heart, and elsewhere. The cells are large,

FrG. 20.

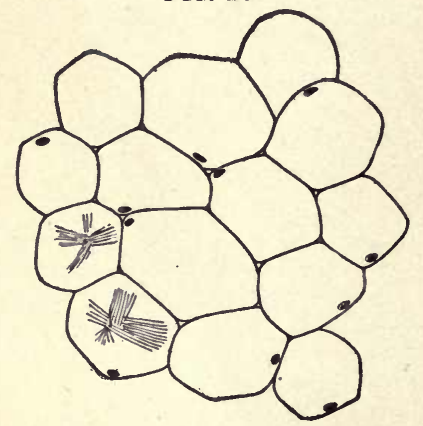

Adipose tissue. Crystals of fatty acids are represented in two of the cells. distended spheres of fat, which has been accumulated in the cell-body until the original protoplasm has been compressed into a thin atrophied membrane enveloping the fat, and the nucleus has been pushed out into the periphery. The cells are spherical in shape, and are packed together in masses, with inconspicuous fibres between. Fatty tissue merges into areolar tissue in which the connectivetissue cells become filled with fat and crowded together.

Retiform tissue (Fig. 21) consists of a reticulum of fine interlacing fibres, covered, especially at the intersections, with flat stellate endothelioid connective-tissue cells. This tissue forms the framework of lymphoid or adenoid tissue, as in lymph-follicles and glands, in which the open spaces of the reticulum are filled with lymph and lymphoid corpuscles, which crowd the meshes and obscure the network; these lymphoid corpuscles belong to the class of free cells and are not fixed in the spaces. The framework of the spleen is of a similar character. Retiform tissue may be regarded as an open sustentacular tissue for containing lymph-corpuscles and lymph. The term "retiform" as here used applies only to the connective-tissue framework, and is not taken to include the lymph-corpuscles or as a synonym of lymphoid tissue.

Cartilage is a specialized form of connective tissue consisting of cartilage-cells, which are modified connective-tissue 
cells, imbedded in an abundant matrix. According to the nature of the matrix, cartilage is of four kinds, hyaline carti-

FIG. 21.

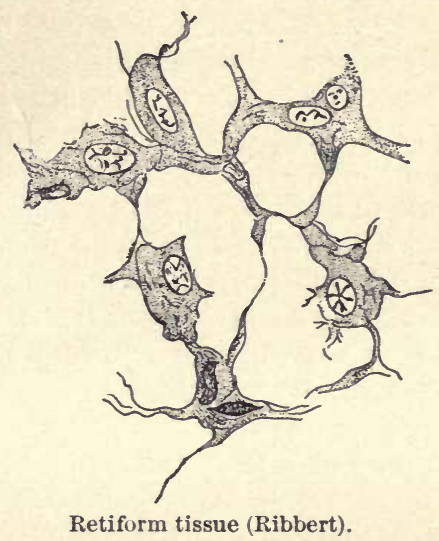

lage, white fibro-cartilage, yellow elastic cartilage, and cellular cartilage.

FIG. 22.
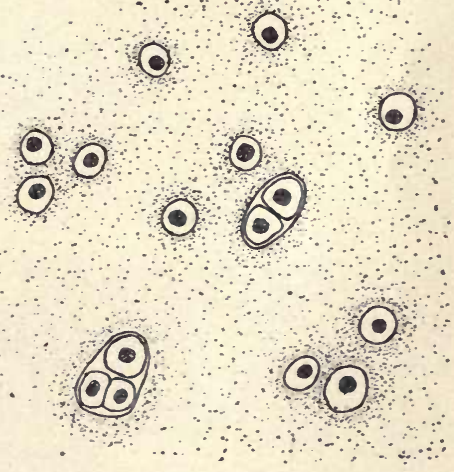

Hyaline cartilage.

Hyaline cartilage (Fig. 22) occurs in the costal cartilages, the articular ends of bones, the nasal cartilaginous septum, 
the larger cartilages of the larynx, the trachea and bronchi, and in the embryo is the precursor of most of the bones. The cells are prominent and rounded, with large nuclei and distinct capsules, and are imbedded in an apparently homogeneous, hyaline, tough matrix, which yields chondrin. Instead of being really homogeneous, the matrix is said to have a delicate fibrillar structure. The means or channels by which nutrient fluids can penetrate to the interior of hyaline cartilage for its nutrition are not known. The cartilage-cells divide in two within the capsule of the parent-cell, which is thus as it were cast off, and each daughter-cell then acquires a new capsule. By the repetition of this process two or three generations of cells may sometimes be found within the remains of the castoff capsule of the single original parent-cell. The capsules thus cast off are gradually transformed into the matrix, which is partly developed in this way. The ground-substance near the cells stains differently from that more remote. Owing to their mode of division the cells in costal cartilage tend to occur in groups ; in articular cartilage they form rows perpendicular to the surface.

Some of the cartilages are surrounded by a fibrous membrane, the perichondrium, containing fusiform or flattened connective-tissue cells. The lowermost of these cells take on the characters of cartilage-cells, become thicker and more rounded, and the edge of the cartilage shows a gradual transition from these cells to the typical cartilage-cells in the interior. In this manner the cartilage is produced or grows from the lower layer of the perichondrium.

Fit. 23.

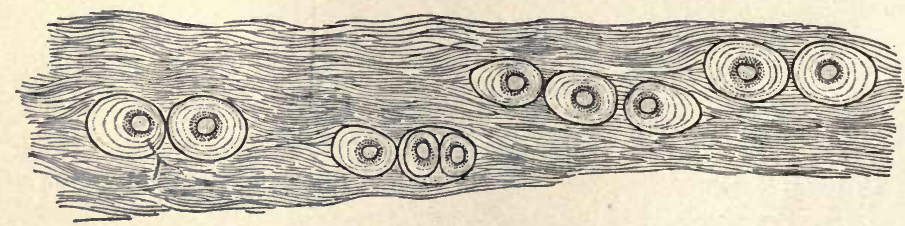

Fibro-cartilage, from human intervertebral disc (Schäfer).

White fibro-cartilage (Fig. 23) forms the intervertebral dises and other cartilages entering into the construction of certain 
joints. It consists of a dense white fibrous matrix in which cartilage-cells are imbedded. According to the character of the cells, it exhibits all gradations to ordinary white fibrous tissue.

Yellow elastic cartilage (Fig. 24) occurs in the auricles, Eustachian tubes, epiglottis, portions of the arytenoid cartilages, and the small cartilaginous bodies near the latter. It

Fig. 24.

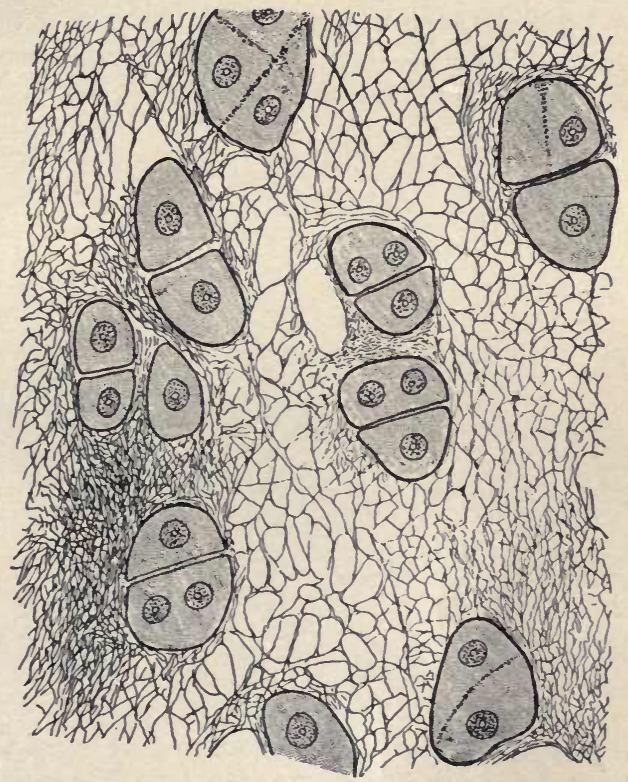

Elastic cartilage, from human auricle (Böhm and Davidoff).

is characterized by elasticity and resiliency. Its matrix mainly consists of a network of elastic fibres the meshes of which contain the cartilage-cells.

Cellular cartilage is a primitive form of cartilage, and consists of cartilage-cells cemented together in a dense cellular mass with scarcely any intercellular matrix. It occurs in man only at an embryonic period, making up the primitive vertebrce; it is found in the ear of mice and some other animals. 
Bone (Fir. 25) is another specialized form of connective tissue, made up of bone-cells or corpuseles and an intercellular matrix impregnated with calcareous salts. It occurs in two forms, compact, making up the greater part of long bones and the outer part of all bones, dense and solid ; and cancellous, in the ends and lining the medullary canal of long bones and in the interior of all other bones; it is loose and spongy, consisting of spaces (cancelli) separated by thin osseous walls.

The material of which bone is composed is divisible into two kinds, organic and mineral. By burning, the organic portion is destroyed, leaving the mineral portion; while on

FIG. 25.

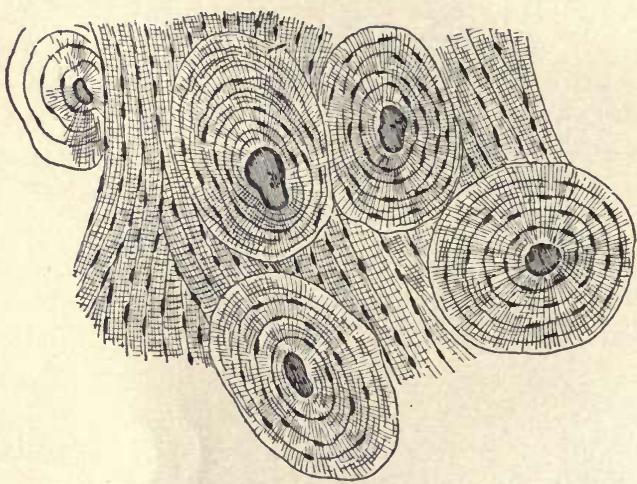

Cross-section of compact bone, showing Haversian systems, bony lamellæ, lacunæ, and canaliculi (Klein).

treatment with acid the mineral matter is removed, or the bone is "decalcified," the organic material remaining as a tough flexible substance.

The bone-cells are modified connective-tissue cells, of a flattened shape, with numerous fine processes projecting from them in all directions. The bodies of the cells occupy the spaces called the lacunce, while the processes occupy the canaliculi.

The matrix consists of dense fibrous organic matter (such as appears in decalcified bone) impregnated with calcareous salts. It is arranged in series of concentric lamella, which form four 
kinds of systems-Haversian, peripheral, peri-medullary, and intermediate.

A Haversian system consists of a series of concentric lamellæ surrounding a Haversian canal, a small round canal containing blood-and lymphatic-vessels and marrow, running generally lengthwise the bone, and communicating and continuous with the interior marrow-cavity. Between the adjacent lamellæe at short intervals are spaces called lacuno, occupied by the bone-cells; connecting the lacunæ are numerous minute passages called canaliculi, occupied by the processes of the cells. The canaliculi afford communication for the passage of nutrient fluids from the Haversian eanals throughout the adjacent lamellæ.

The peripheral or circumferential lamello are a series of subperiosteal lamellæ, similar to the Haversian lamellæ, surrounding the entire bone. In places there are fibres piercing the peripheral lamellse at right angles-perforating fibres, or fibres of Sharpey.

The peri-medullary lamello are a similar series surrounding the marrow-cavity.

The intermediate or interstitial lamellae are scattered irregularly about in the bone substance, and are the remains of old systems that in the course of the growth of the bone have been partially removed.

The periosteum, the covering membrane of bone, is composed of two layers: an outer firm fibrous protective layer; and an inner loose layer well supplied with blood and connective-tissue cells (or osteoblasts), which has important functions in the growth of bone and is hence called the osteogenetic layer.

Bone-marrow: The eavities in bone-the cancelli of spongy bone, the medullary or marrow-cavities of long bones, and the Haversian canals-contain a soft vascular tissue, the marrou, which is of two kinds, red and yellow.

Red marrow occurs in cancellous bone in the adult, in all bone in the young. It consists of delicate connective tissue, a rich vascular network including large venous sinuses, quantities of cells called marrow-cells, and fat-cells. The murrow-cells are of several kinds, chicf among which are: (a) plastic rounded connective-tissue cells, many of which in 
growing bone become osteoblasts and aid in bone-formation ; (b) large multinucleated giant cells, or myeloplaxes, which are probably osteoclasts and effect bone-absorption; $(c)$ erythroblasts, free nucleated cells, colored by and containing hæmoglobin, probably connected with the development of red bloodcorpuscles; and $(d)$ cells containing oxyphile granules, resembling the eosinophile lenkocytes of normal blood; other cells resembling the myelocytes which appear in the blood in certain abnormal conditions ; and other forms of leukocytes.

Yellow marrow oceurs in the medullary eavities of long bones and larger cancelli of the adult. It is similar to red marrow, except that it contains large quantities of fat-cells, or marrow-cells distended with fat, to which it owes its oiliness and straw color.

The function of marrow is partly to form bone-substance, partly to form red blood-corpuscles. Genetically the marrow is a continuation or offshoot of the osteogenetic periosteal layer.

The development of bone takes place by two methods, cartilaginous and membranous.

Cartilaginous bone-formation (Fig. 26): Most of the bones, especially the long bones, are in the embryo first formed of hyaline cartilage. The conversion of this cartilage into bone constitutes the cartilaginous method of bone-formation. In these embryonic cartilages bone-formation begins at definite points, the "centres of ossification." The cartilage-cells enlarge and proliferate abundantly, and arrange themselves in longitudinal rows, with septa between the rows composed of the hyaline matrix. The longitudinal spaces inclosed by these septa and filled with cartilage-cells are called the primary areoloe. These septa soon become calcified by the deposition in them of lime salts. From the osteogenetic layer of the periosteum loops of bloodvessels then grow into the cartilage, accompanied by connective-tissue cells, and by large multinucleated cells called osteoclasts, which have the power of absorbing the cartilage and bone-substance and thus open passages and spaces. The cartilage-cells filling the primary areolæ disappear, and communications are made between the areolæ by perforations through their calcified walls, though 
these walls are only partially absorbed. The larger spaces thus formed are termed secondary areola. Connective-tissue cells now deposit themselves on the calcified walls of the secondary areolæe and become osteoblasts, or bone-formers. They secrete a layer of bony substance, in which they and their processes become buried ; they thus become bone-cells, in lacunæ, with their processes in canaliculi. When one layer of bone is thus deposited, other osteoblasts become fixed on the new surface and form another layer. This process proceeds from the periphery of the spaces inward, until only a small passage is left, the Haversian canal, the surrounding lamellæ constituting a Haversian system. The formation of bone proceeds in this manner away from the centres of ossification; hence there are layers of growing cartilage intervening between the different ossifying areas, as between the shaft and the epiphyses of long bones. Not until the bone attains its full growth are these intervening cartilagi-

FIG. 26.

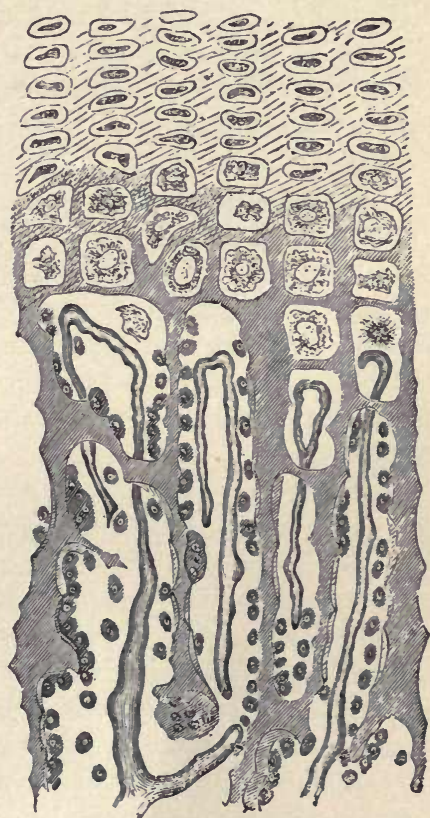

Development of bone, cartilaginous method (Klein).

nous portions fully converted into bone. The bone formed by the cartilaginous method is temporary, being subsequently removed and renewed by the nembranous method; while the interior of long bones becomes excavated to form the marrow cavities.

Membranous bone-formation: Some of the bones, as the flat bones of the skull, are preceded in the embryo by white fibrous membranes. The conversion of these into bony tissue (intramembranous bone-formation) and the formation of bone 
by the osteogenetic layer of the periosteum (subperiosteal boneformation), which are substantially identical processes, con-stitute the membranous method of bone-formation. All permanent bone is formed in this way. The process begins with a deposition of osseous matter upon some of the fibres, a portion of the connective-tissue cells assuming the function of osteoblasts. A bony retieulum is thus formed, in the meshes of which bone-formation is continued by the osteoblasts. Along with the formation of bone, absorption, replacement, and remodelling of the bone constantly go on. Osteoclasts excavate spaces in the bone previously laid down, in which new Haversian systems are formed. The remains of old Haversian and peripherie lamellæ thus partially destroyed constitute the interstitial lamellæ. The spaces occupied by osteoclasts are called Howship's lacuno. In the bones which develop from membrane a membranous portion persists and eontinues to grow between the ossifying areas or at the margins, until the bones attain their full growth, when they are eompletely ossified.

\section{Muscle.}

The muscle-cells are specialized cells having the property of contractility highly developed. Their function is motor, to effect the motions of the body. Muscle is derived from the mesoblast. Musenlar tissue is mainly made up of the cellular elements, the intercellular substance being seanty and inconsiderable. There are three varieties of muscle-cells:

Striated, or voluntary ;

Cardiae;

Involuntary, or non-striated.

Striated or striped muscle (Figs. 27, 28) makes up all the voluntary muscles of the body. The cells are very much elongated protoplasmic fibers, generally 10 to 50 micromillimetres in diameter, and 20 to 40 millimetres long, or even longer. In shape the cells or fibres are cylindrical or prismoidal, slightly fusiform, with tapering or blunt rounded extremities ; in cross-section they are usually rather polygonal. Each cell is inclosed in a delicate homogeneous separable envelope, the sarcolemma. The fibres are usually straight and 
unbranched, but in occasional situations they branch and anastomose. Each cell has many oval nuclei, arranged with their long axis longitudinal to the cell, situated in mammals just underneath the sarcolemma. The body of the cell under moderate magnification exhibits characteristic transverse striæ or stripes, consisting of delicate alternately dark and light lines.

The fibres are arranged parallel to one another, with overlapping ends. They are grouped in bundles or fasciculi, all of which bound together in fibrous sustentacular tissue make up an entire muscle. The connective tissue surrounding and enclosing the entire muscle is called the epimysium; that enveloping the separate fasciculi is called the perimysium; while the delicate and scanty connective tissue within the fasciculi among the individual muscle-cells is the endomysium.

The sarcolemma of each fibre is connected with the endomysial tissue, and through this with the tendon. Thus each cell when it contracts exerts more or less direct traction on the tendon. The muscle-fibres are richly supplied with blood by a capillary network in the endomysium. On the surface of each fibre is a flattened nerve-terminal.

The minute structure of striated muscle is very difficult to make out and has not been satisfactorily determined, and authorities are not agreed in their interpretations of the appearances presented.

The appearance of cross-striation is produced by alternating dark and light discs or segments in the substance of the muscle-cells. By treatment with weak acids these segments can be separated from one another in the form of transverse discs. The individual fibres also exhibit a longitudinal striation as well as transverse, and by treatment with alcohol and teasing can be broken up into fine longitudinal fibrilloe; these fibrillæ are arranged in bundles, called fields of Cohnheim. The protoplasm of the muscle-cell is made up of two parts, a dark sarcous substance ("anisotropic," or doubly refracting) and a colorless, hyaline, probably semifluid substance (" isotropic," or singly refracting), sometimes called sarcoplasm. 
These two substances make up the dark and light portions, respectively, of voluntary muscle. On close examination the light or clear transverse segments are found to be divided into two discs by a delicate transverse line, by some considered to be a definite membrane and called the membrane of Krause, and by others regarded as a row of minute granules, called Dobie's granules.

The transverse dises separated by the "membrane of Krause" are called sarcous dises or compartments; each is

Fig. 28.

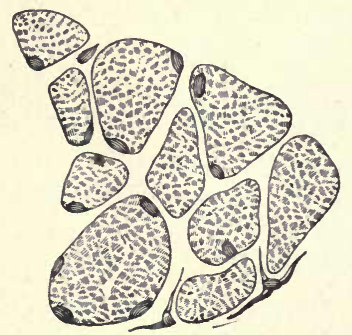

Striated muscle-cells in crosssection (Klein). composed of a dark segment with half of the adjacent light segment on each side. The dark portion of each sarcous disc, again, is not homogeneous, but is made up of minute dark longitudinal rods, or sarcous elements, separated by the light substance, or sarcoplasm. The sarcous elements of adjacent dises correspond to one another and with the intervening granules of Dobie, so as to form the continuous longitudinal fibrillæ. The appearances above described are those

of relaxed or extended muscle.

When the muscle contracts the fibres become shorter and thicker; the sarcous elements seem to divide in their middle, forming a clear transverse line or segment, called the line of Hensen; while the ends of the elements approach the ends of the corresponding elements of the adjacent dises, and tend to obliterate the clear segment between. Thus, in contracted muscle the light and the dark striæ seem to occupy positions precisely reverse to those of relaxed muscle. The mechanism of muscular contraction is not, however, well made out.

Cardiac muscle: The muscular substance of the heart has an individual character of its own (Fig. 29). The individual cells, much shorter than those of the striated variety, are cylindrical in shape with square or serrated ends; they send off branches which unite with neighboring cells. Adjuining cells meet end to end, the ends being so closely cemented together that the points of junction cannot be detected with- 
out special preparation of the specimen. Laterally the cells are not closely crowded, but are separated by slight intervals filled with delicate connective tissue and vascular channels. The cells have no separable sarcolemma. Each cell has a single nucleus, situated in the centre of its protoplasm. The cells are somewhat striated both longitudinally and transversely.

Involuntary or non-striated muscle (Fig. 30) is widely distributed throughout the viscera, being found in especial abundance in the alimentary canal, bloodvessels, bladder, and uterus. Its movements are not under the control of the will, but are of an automatic nature and are more slow and continuous than those of the voluntary muscles.

Involuntary, non-striped, or smooth mus-

Fig. 29.

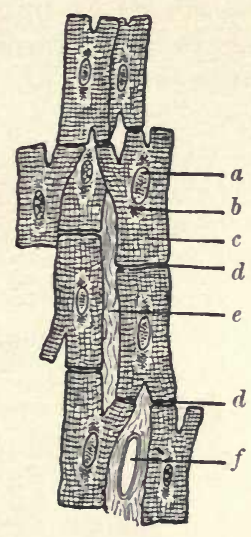

Cardiac muscle (Dunham). $a$, nucleus: $b, c$, cytoplasm; $d$, cement at junction of cells : $e$, a reolar or "interstitial" tissue between the cells; $f$, capillary.

FiG. 30.

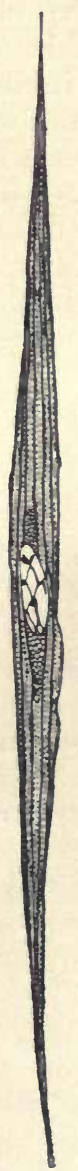

Involuntary musclecell (Schäfer.) The nucleus is not represented sufficiently long.

cle-cells are slender and fusiform in shape, each with a single 
characteristic elongated or rod shaped nucleus in its centre. The cells have a parallel and overlapping arrangement, held together by a scanty cement-substance, and form sheets, rings, or tubes of muscle. Indistinct longitudinal and transverse markings are sometimes observed, the latter being probably folds in the cell-walls. The cells have an envelope but no separable sarcolemma. Some authorities describe involuntary muscle-cells as having longitudinal ridges, or "intercellular bridges," on their surface, which unite with the ridges of the adjacent cells.

\section{Nerve-tissue.}

The elements of the nervous structures are greatly extended cells, called neurons, which for practical convenience may be divided into nerve-cells, nerve-fibres, and nerve-terminals. Their description will be deferred to that of the nervous system in general.

\section{Free Cells and Body-fluids.}

The tissues considered hitherto are all fixed. The present class is a somewhat miscellaneous assemblage of those bodyelements that are not fixed, but free or fluid.

They may be grouped as follows: 1 . Unattached cells, free in the solid tissues. 2. Cellular fluids-that is, fluids in which free living cells are suspended, as the blood. 3. Simple homogeneous fluids, mainly secretions. 4. Emulsions.

The free cells, situated for the most part in the solid tissues, but not attached to them, are represented mainly by the wandering cells, the lymphoid cells of lymphoid tissue, and ova. The so-called wandering cells are leukocytes or lymphcorpuscles which have escaped from the bloodvessels or lymphatic structures and exist free and migratory, especially among the meshes of the opener or areolar forms of connective tissue. The small spherical lymphoid cells, with large prominent nuclei, which occur crowded in the meshes of retiform or lymphoid tissue, are very similar to and closely related to the corpuscles of lymph. Some of the cellular elements of bone-marrow might also perhaps be included in this group. The ova of the lower animals are free cells; and 
in mammals they are free from their departure from the ovary until their attachment to the uterus, and even afterward they are in a sense independent.

The cellular fluids of the body are the blood, lymph, and semen. They consist of actively vital cells free in a fluid matrix. They can be regarded as liquid tissues analogous in all respects to the solid tissues, being composed of cells and an abundant intercellular substance, which in this case is fluid. The latter differs from the matrix of the solid tissues, however, in that it is not produced by the vital activities of the cells suspended in it.

The blood consists of cells or corpuscles of three kinds, red corpuscles, white corpuscles or leukocytes (of which there are several varieties), and blood-plates.

Lymph consists of a fluid portion, or serum, containing lymph-corpuscles, which are similar to or identical with some forms of leukocytes. Leukocytes and lymph-corpuscles often find their way into the fixed tissues or on mucous surfaces.

Semen is a thick opaque fluid entaining large numbers of spermatozoa, together with some loosened cells and granules.

The simple or homogeneous fluids are mainly the secretions of the various glands, consisting usually of clear, transparent solutions without any proper cellular or formed elements. Under the microscope they exhibit no structural features, and the study of their composition is a matter for chemical or microchemical methods. Among them may be mentioned the urine, perspiration, saliva, bile, gastric juice, etc.; also the fluids moistening the mucous surfaces, of which mucus is a prominent constituent.

Although these fluids contain no cellular elements as proper or essential ingredients, yet they sometimes, notably the saliva and urine, contain a few adventitious cells derived from the mucous membrane lining the surface of the cavities where they occur. The chief cells thus found are leukocytes or lymph-cells (as the "salivary corpuscles") which have worked their way through the mucous membrane to the surface; also ordinary epithelium-cells cast off from the epithelial covering; and "mucous corpuscles," or "mueocytes," young germinal epithelinm-cells from the lower epithelial lavers (especially in the bladder) which have grown rapidly and been thrown off 5-Hist. 
prematurely as free spherical corpuscles, usually larger than leukocytes.

Emulsions: Milk and chyle are emulsions, as are also the yolk of birds' eggs and the milky juice of certain plants. By means of certain substances, as albumin, oil may be caused to form a permanent mixture, or emulsion, with water. The oil forms minute spherules or granules enveloped in a viscid substance, which causes them to retain their shape, and suspended in the watery vehicle. Milk and chyle contain large numbers of such fat-granules, which are very minute and are suspended in an abundant aqueous fluid. Their opacity and whiteness are due to the reflection of the rays of light from the granules. In artificial emulsions the spherules of fat are much coarser and the proportion of water that can be introduced far less than is the case with these natural emulsions. 


\section{CHA PTER V.}

GLANDS; MUCOUS AND SEROUS MEMBRANES; ORGANS.

BeFore beginning the consideration of the organs and systems in detail, it will be convenient to consider briefly : (1) certain composite structures of wide distribution, namely, glands, mucous membranes, and serous membranes; and (2) organs in general.

Glands are of two entirely distinct types : (a) lymphoid and blood-glands; and (b) secretory or epithelial glands.

The lymphoid glands, as the lymphatic glands, thymus, tonsils, and lymphoid tissue generally, consist of an open network of retiform tissue the meshes of which are filled with lymph-corpuscles and lymph. The lymph-stream passes directly through them. They have no proper secretion, possess no outlet-ducts, and communicate only with lymphatic vessels. Their function is not completely understood, but they partly serve to develop some of the corpuscular elements of the blood and lymph, and partly to arrest noxious substances in the circulation.

The blood-glands, as the spleen, bear the same relation to the blood-stream that the lymphoid glands do to the lymphstream. In general structure they resemble the lymphoid glands.

The secretory glands remove or secrete from the blood various substances and fluids needed for special purposes. The active agent in this process is the glandular epithelium, which forms a layer supported on a basement-membrane; beneath and in close relation with the epithelium is a rich capillary blood-supply; on the free surface of the epithelium is an open space or cavity in which the secreted product collects and is carried away. Such is the general structure of all secretory glands : a sac of epithelium with blood-supply on the outside and a collecting cavity inside. The epithelium 
of the secreting portion of glands is usually different from that of the ducts.

Secretory glands are of two kinds : closed or ductless glands ; and the ordinary open glands with ducts.

The secretory ductlesis glands are the thyroid, parathyroids, adrenals, pituitary body, and pineal body. 'They have no outlets nor ducts, and hence their secretions are not discharged elsewhere except by reabsorption into the blood or lymph circulation. The lymphoid and blood-glands are also "ductless."

The open glands are the ordinary variety, as the kidney, salivary glands, pancreas, with outlet-ducts through which their secretions are discharged.

In addition to these secreting bodies, certain endothelial structures may be mentioned, as bursæ, synovial sacs, etc., which contain glairy lubricating fluids produced by the endothelium also by a true secretory process.

Internal and external secretions: The purpose and mechanism of the ductless glands, closed as they are, have long been obscure, but recent theories afford some explanation of the subject. According to these theories, the secretions elaborated by glands are of two kinds, internal and external.

The external secretions are those of the ordinary kind, discharged through the ducts of the glands and conveyed to the places where their functions are carried out, as the tears, the bile; their action is easily comprehensible.

The internal secretion, however, is not discharged by glandducts, but is absorbed into the circulation and there serves purposes useful in the animal economy. This is the only kind of secretory activity which the ductless glands manifest. The secretion of the thyroid, for instance, when in the blood, increases oxidation, promoting metabolism, and increasing the rapidity of cell-life; stimulates cerebral activity, makes the heart action more rapid, causes palpitation, and dilates the bloodvessels. That of the suprarenals is a powerful vasomotor constrictor and probably serves to maintain vascular tone.

The effects of these internal secretions are most markedly manifested when they are absent from the blood, as when the glands producing them are removed or impaired by disease; 
thus, removal or impairment of the thyroid causes myxœedema; of the suprarenals, Addison's disease ; of the pituitary body, acromegaly. It would seem as if the presence of the internal secretions in the blood afforded stimuli necessary to preserve

FIG. 31 .

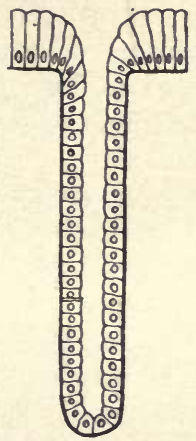

Simple tubular gland.

FIG. 33.

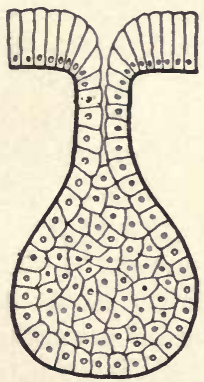

simple saccular gland.
FIG. 32.

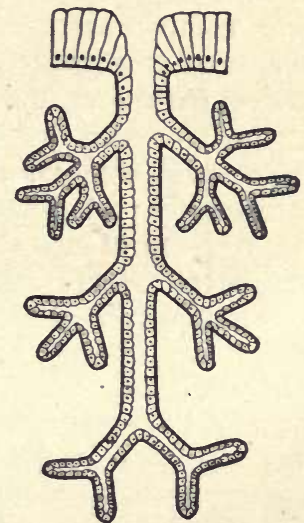

Compound tubular gland.

FIG. 34 .

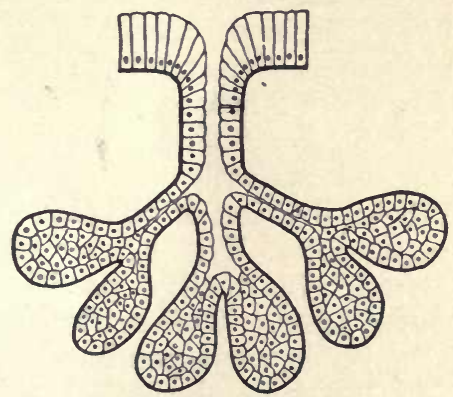
Compound or racemose saccular
gland.

Morphologic types of secretory glands (Dunham).

a certain tonus and produce definite reactions of the bodily cells.

Some of the glands with ducts, moreover, seem to produce 
internal as well as external secretions. Thus, the glycogenic and urea-forming functions of the liver and the relation of the pancreas to glycosuria are instances of internal secretory action.

Morphology of secretory glands (Figs. 31-34): The secreting glands, according to their form, are divisible into two classes: tubular and saccular; each of these, again, may be simple or compound.

Simple tubular glands consist of single tubes lined with epithelium, with one end closed and blind and the other opening on the skin or mucous surface. The glands of Lieberkühn and some of the gastric and uterine glands are typical examples; the sweat-glands are single tubes, modified in having the secreting portion much coiled.

In compound tubular glands the tubular structure is still present, but the tubes branch and divide, discharging into a common duct. Some of the gastric and uterine glands exhibit the simplest gradations into this type. The kidneys, testicles, and liver are compound tubular glands.

Simple saccular glands consist of a single rounded or spherical cavity or glandular sac with a small outlet to the surface. Sume of the simplest sebaceous glands may be of this type, but they scarcely occur otherwise in mammals.

Compound saccular or racemose glands, as the salivary, mucous, lachrymal, sebaceous, and mammary glands, consist of numbers of saccules opening into common ducts, and these again uniting in larger ducts, much like a bunch of grapes.

In compound glands the ultimate saccules or alveoli which communicate with common ducts form well-marked groups, so that a division of the gland into lobes and lobules is easily recognizable. The lobular divisions of glands are usually separated by distinct partitions of connective tissue, interlobular septa. Well-marked divisions of the ducts are also recognizable, corresponding to their degree of division and to their situation and relation to the lobules. Within a lobule is a small division of the ducts, the intralobulur duct, into which the ultimate alveoli of that lobule all empty. The short duct by which each individual saccule or alveolus empties into the intralobular duct is called an intermediate duct; the union of the intermediate ducts forms the intralobular ducts. The intralobular ducts unite to form the interlobular ducts, which 
are larger and lie in the connective tissue separating the lobules. By the union of the interlobular ducts the large common duct or ducts of the whole gland are formed.

According to the nature of their secretion and corresponding characteristics of the gland-cells, most secretory glands are divisible into serous glands, mucous

Fig. 35.

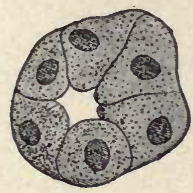

Alveolus of serous gland (Schiefferdecker). glands, and fatty glands.

Serous glands (Fig. 35), as the parotid and pancreas, secrete limpid watery fluids or solutions. The secreting-cells are spheroidal or polyhedral, granular, dark and opaque, stain deeply, and have rounded nuclei nearly centrally located. The appearance of the cells varies somewhat according to the stage of secretion. When, after resting for a time, they are charged with secretion, they are distended, clearer, less opaque, stain less deeply, and contain an abundance of granules. After being exhausted by prolonged secretion they are shrunken or diminished in size; darker, more opaque, and more deeply stained (from condensation of the protoplasm); and less granular, having discharged their granular contents into the lumen of the gland.

Mucous glands (Fig. 36), as the sublingual, secrete a viscid, mucinous fluid, which lubricates the mucous surfaces. Goblet epithelium-cells secrete a similar substance, and the characteristics of these cells are very similar

Fig. 36.

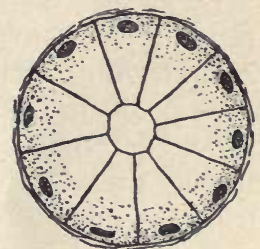

Alreolus of mucous gland, from lip. to those of the cells of mucous glands. Small racemose mucous glands are widely distributed in mucous membranes, having their alveoli in the submucosa and their ducts opening on the surface. The secreting-cells of mucous glands, when in the resting stage and distended with their mucinous secretion, are large and swollen, unstained, clear, and transparent, especially in the superficial portion ; their nuclei and bodyprotoplasm are crowded well toward the bases of the cells. When exhausted by continued activity they are smaller, less clear, and more granular in appearance. 
Fatty glands: The sebaceous and mammary glands produce the fatty constituents of their secretions by a fatty degeneration or infiltration of the protoplasm of the gland-cells. The cell-bodies become swollen and infiltrated with globules of fat, which are later discharged into the lumen.

Mucous membranes: The mucous membranes are those lining the spaces within the body that communicate directly or indirectly with the exterior. There are three distinct continuous tracts of mucous membrane: the respiratory-alimentary, the genito-urinary, and the mammary. At the orifices and along portions of these tracts the mucous membrane is a continuation of and similar in structure to the skin. In some situations the function of mucous membrane, like that of the skin, is chiefly protective, and in these places it most resembles the skin. In other situations mucous membrane has special functions, and its structure varies accordingly.

Mucous membrane is usually described as being, in general, composed of two layers, the mucosa and the submucosa, which are commonly situated on a muscular or fibrous bed.

The mucosa consists of : (a) a superficial layer of epithelium, stratified squamous, columnar, or ciliated, corresponding to the epidermis of the skin. Beneath the epithelium is $(b)$ the tunica propria, a layer of connective tissue, white-fibrous or elastic, corresponding in general to the corium of the skin, but varying greatly in different situations. Its upper or outer surface is condensed to form a basement-membrane for the support of the overlying epithelium. Beneath the tunica propria in many places is (c) the muscularis mucoso, a thin sheet of involuntary muscle-cells, some longitudinally arranged, some transverse ; it separates the mucosa from the submucosa, and has no analogue in the skin.

The submucosa is a layer of areolar tissue underneath the mucosa, corresponding to the areolar subcutaneous tissue ; it contains the larger vascular, lymphatic, and nerve branches, which send smaller twigs and capillaries into the tunica propria. The looseness of the submucosa permits some play or movement of the mucous layer on the layers beneath. Iymphoid tissue, diffuse or circumscribed, is present in many situations in the submucosa or tunica propria. 
Secretion: Mucous membranes are bathed and lubricated in a mucinous fluid, secreted mostly by goblet-cells or special small mucous glands. Most mucous membranes lined with stratified squamous or stratified ciliated epithelium are provided with large numbers of mucous glands; in the stomach and intestines (lined by simple columnar epithelium) mucus is secreted by large numbers of goblet-cells, no mucous glands being present. Some mucous membranes lined with stratified squamous epithelium, however, lack both goblet-cells and mucous glands, as in the vagina and bladder, whose mucous secretion is produced by the mucinogenous action of the squamous cells themselves. The mucous glands when present have their alveoli in the submucosa and their ducts penetrate the mucosa to empty on the surface; the cells of the secreting portions of these glands are of the mucous glandular type, while the ducts are lined with a different form of cell, usually cuboidal or columnar cells similar to or merging into those of the epithelial surface.

These are the general features of the structure of mucous membranes, but the details vary greatly in different situations.

Serous membranes, as the pleura, pericardium, peritoneum, tunica vaginalis, and the allied synovial membranes, line certain closed cavities or sacs. The portion of serous membranes covering the viscera is called the visceral portion, that reflected over the outer walls of the cavity being the parietal portion. Serous membranes consist of an interlacing fibrous connective-tissue membrane or basis, lined on the free surface by endothelium, while beneath is a variable amount of areolar and often adipose tissue, the subserous tissue, connected with the subjacent structures and analogous to the submucosa. They contain blood and lymphatic vessels. Between the endothelium-cells in some localities are occasional minute orifices or lymph-stomata, guarded by small spheroidal cells ; these open into lymphatic vessels within the membrane. Thus the serous sacs form large lymph-spaces, communicating directly with the lymphatic channels. The function of serous membranes is partly associated with that of the lymphatic system, and is largely to obviate friction and to permit and facilitate the gliding movement of viscera upon opposing surfaces. 
Organs are aggregations of the elementary cells and tissues in varying proportions and varying modes of arrangement. The tissues of many organs can be conveniently divided into parenchymatous tissue and sustentacular tissue.

The parenchyma of organs consists of the cells or portions that are the active and essential agents in carrying on the functions of the organ, such as the glandular epithelium of glands, the air-vesicles of the lungs, etc. The sustentacular tissue (often called interstitial tissue) of organs consists of their connective-tissue framework, permeating and supporting all their parts. It is usually a fibro-elastic or condensed areolar tissue. Different parts or divisions of the sustentacular tissue can often be distinguished, such as the membranous envelopes or capsules surrounding organs; trabeculæe or septa (interlobular tissue) traversing the interior of organs and dividing them into lobes and lobules; tracts of fibrous tissue (perivascular tissue, etc.) surrounding and supporting the vascular and nerve branches, and ducts, in their ramifications through the organ; and a delicate all-pervading network of fibrous tissue (intralobular tissue) embracing and sustaining the minutest subdivisions of the visceral and parenchymatous substance. In addition to their sustentacular and parenchymatous elements organs possess as a matter of course a blood, lymphatic, and nerve supply. 


\section{CH A P TER VI.}

\section{THE CIRCULATORY SYSTEM.}

THE apparatus by which the circulation of the blood throughout the body for the nourishment of the tissues is accomplished consists of the heart, covered by the pericardium; and the bloodvessels: arteries, veins, and capillaries.

The heart is a hollow muscular organ, whose walls are made up of a thick mass of muscle, the myocardium, lined on the inside by a delicate endothelial membrane, the endocardium; and on the outside by a serous membrane, the visceral portion of the pericardium.

The endocardium, which lines the interior of the heart, is similar to the lining of the bloodvessels, of which it is a continuation. It is thinner than the pericardium. Its free surface is lined by polygonal endothelium-cells, which lie on a firm connective-tissue basis of interlacing white and elastic fibres. The lowermost tissue of the endocardium merges gradually into the delicate connective tissue which occupies the interstices between the muscle-cells of the myocardium.

The valves of the heart and arterial orifices consist of folds or reduplications of the endocardium, fortified and strengthened by additional white and elastic fibrous tissue. The tissues at the bases of the valves are strengthened into wellmarked fibrous rings, which serve as a basis for the attachment or insertion of the valves and muscular bundles of the myocardium.

The myocardium is a muscular mass making up the main substance of the heart. The cardiac muscle-cells of which it is composed are of a kind peculiar to the heart, and have been already described (Fig. 29). The muscular fibres are arranged in bundles or layers which in different places run in different directions, transverse, longitudinal, oblique, spiral, in an intricate manner. 
Cardiac muscle-tissue is not compact, but the sides of the muscle-cells are separated by slight intervals or interstices through which delicate connective tissue (the interstitial tissue), capillaries, lymphaties, and nerves permeate the entire myocardium. The blood-supply of the heart, including the endocardium and visceral pericardium, is derived from the coronary vessels; the muscle-cells are richly supplied with capillaries.

The pericardium is a typical serous sac, consisting of a fibroelastic membrane(fibrous pericardium) lined on the free interior surface by a single layer of polygonal endothelium-cells (serous

FIG. 37.

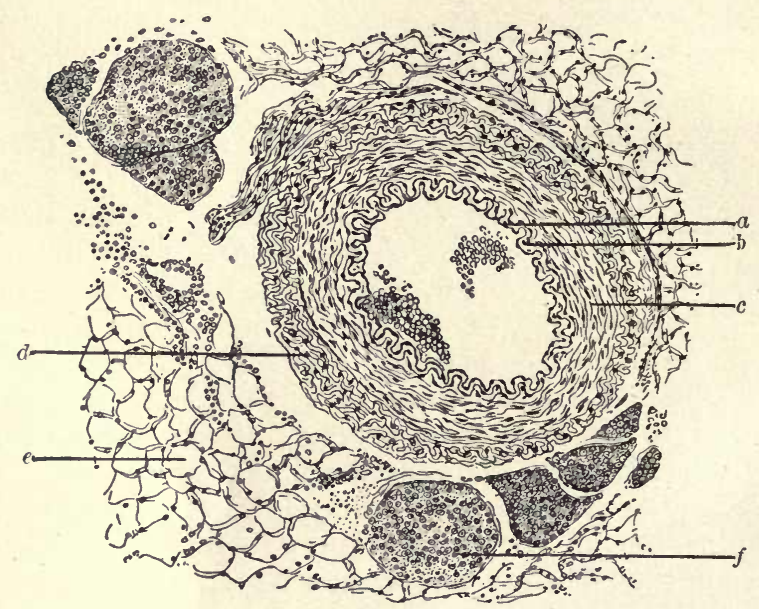

Cross-section of artery (Dunham). $a$, endothelial lining; $b$, internal elastic lamina ; $c$, tunica media; $d$, tunica adventitia; $e$, adipose tissue; $f$, small nerve.

pericardium); beneath which is the subserous or subpericardial areolar tissue, which in this situation often includes masses of fatty tissue. The visceral portion of the serous pericardium, sometimes called the epicardium, envelops the outside of the heart, while its parietal portion is reflected on and lines the inner surface of the fibrous pericardium. Under the portion of the pericardium which covers the heart the subpericardial tissue, areolar and fatty, is continuous with and merges into the interstitial connective tissue of the myocardium. 
Arteries: The arteries vary in structure somewhat-that is, in the relative proportions of their several constituents-according to their size and degree of subdivision (Fig. 37). The coats of arteries are usually described as consisting of three divisions, the tunica intima, tunica media, and tunica adventitia. 'The structure of medium-sized arteries, or all but the largest and the smallest, is as follows :

The tunica intima, or internal coat, consists of three layers. It is lined internally by endothelium, a single layer of flat squamous cells uniting by somewhat sinuous edges, oval and elongated in shape and with oval nuclei; the cells and nuclei lie with their long axes parallel with the course of the artery. These cells present a smooth surface to the bloodstream.

Beneath the endothelium is the subendothelial connective tissue, a thin delicate layer of white and elastic fibrous tissuc with stellate connective-tissue cells. In the smallest arteries this layer becomes thinner and finally disappears.

The lowest layer of the tunica intima, separating it from the tunica media, is the internal elastic lamina, a thin but prominent layer of interlacing yellow elastic fibres forming a perforated or sometimes almost continuous membrane; it is sometimes called the "fenestrated membrane of Henle." In empty and contracted arteries this elastic lamina is thrown into longitudinal folds, and in cross-sections of arteries it consequently presents a very conspicuous and characteristic appearance as a clear sinuous yellow line surrounding the lumen of the ressel.

The tunica media is a thick middle coat, between the intima and adventitia, consisting chiefly of intermingled yellow elastic fibres and involuntary-muscle cells, along with a small amount of white fibres. The muscle-cells are for the most part arranged circularly or transversely, but in a few arteries longitudinal cells also occur sparingly. The elastic tissue occurs as sinuous fibres, networks, and plates. In relative quantity the elastic and muscular elements vary inversely to each other according to the size of the artery.

- In the targe arteries the elastic tissue greatly predominates in amount over the muscle; as the arteries decrease in size these proportions are gradually reversed, until in the small 
arteries the media is mainly composed of muscle-cells, with elastic tissue only scantily represented.

This arrangement confers great elasticity on the largest arteries, so that they convert the spurts of blood intermittently pumped from the heart into a more even and continuous current; while the smaller arteries, by the action of the vaso-constrictor and vaso-dilator nerves upon their muscular walls and their calibre, are kept well under the control of the nervous system, which in this way exercises a full and highly coördinated control over the circulation and amount of blood-supply in all parts of the body.

In some arteries an external elastic lamina, similar to but less well marked than the internal elastic membrane, can be distinguished, marking the outer limit of the tunica media and separating it from the tunica adventitia.

The tunica adventitia, the outer coat of the arterial wall, is a layer of fibrous and elastic tissue, containing perivascular lymphatics and the vasa and nervi vasorum, or the small bloodvessels and nerve-filaments that supply the walls of the artery itself. The adventitia is of indefinite and variable thickness in different situations; the portion next to the media is a firm, strong tissue, while the more remote portions become looser and more areolar and merge gradually into the neighboring connective tissues.

In the largest arteries, as the aorta and pulmonary artery, the subendothelial tissue is thick and firm, and the internal elastic lamina is ill defined. The tunica media is made up mainly of elastic plates and reticula, the muscle-cells being present in relatively small proportion.

As the arteries decrease in size, the subendothelial tissue and the elastic elements of the media diminish and ultimately disappear, the muscle-cells become fewer and scattered, the adventitia decreases in amount, and the internal elastic lamina becomes thinner and finally absent. The endothelium persists throughout.

The smallest terminal arteries, then, are lined with endothelium, resting on the thin remnants of the subendothelial connective-tissue and elastic layers ; outside of this are transverse involuntary muscle-cells in a single layer or scat-. tered and separated by intervals; and surrounding all is 
scanty connective tissue representing the remains of the adventitia.

In small arteries, viewed longitudinally (or from the side), the transverse muscle-cells, with their conspicuous rod-shaped nuclei, present a very characteristic appearance by which vessels of this size are easily recognized.

When, finally, all the coats have disappeared except the endothelium, the artery has merged into a capillary.

Veins : The structure of veins is much the same as that of arteries; but they have a larger calibre, thinner walls, more white fibrous tissue, and less muscle than the corresponding arteries, and they are provided with valves.

The walls of veins, as of arteries, consist of three coats, the tunica intima, tunica media, and tunica adventitia. These coats, however, are often indistinctly marked off and merge into one another without well-marked dividinglines.

The tunica intima is lined internally with a single layer of endothelium-cells, which are more polygonal and less elongated in shape than are those of arteries. The subendothelial layer consists of white and elastic tissue. The internal elastic lamina in veins is often poorly defined.

The tunica media consists of white and elastic fibrous tissue and involuntary muscle-cells, which are mostly arranged circularly, but in some situations partly longitudinally also. The media is much thinner and contains less muscle than in arteries.

The tunica adventitia is a fibro-elastic outer sheath, often thick and well marked, containing vasa vasorum and in some situations longitudinal non-striated muscle-cells.

The valves of veins are folds of the tunica intima, strengthened by additional connective tissue.

At the junction of the large veins with the cardiac auricles the peculiar muscle of the heart is continued for a short distance in the venous walls. The walls of the veins of the lower extremities are thicker and more muscular than those of the upper part of the body.

The venous sinuses of the interior of the skull consist of an endothelial layer resting on fibrous tissue, and occupy 
spaces formed by separation of the layers of the dura mater.

Some veins are devoid of muscle-tissue.

Capillaries: The capillaries are minute tubes formed of a single layer of elongated flat squamous nucleated endotheliumcells, with their long axes arranged longitudinal to the course of the vessels. They are, therefore, continuations of the endothelial linings of the arteries and veins, but divested of all other coverings, except that whatever traces of connective tissue may surround the endothelial tubes may be regarded as vestiges of the adventitia.

At the junction of the endothelial cells in places are small points, which are perhaps stomata or openings, by which the escape or migration of leukocytes through the wall of the capillary is effected or facilitated.

The capillaries form a system of exceedingly numerous short minute passages connecting the terminal arteries on the one side with the initial veins on the other. They branch and anastomose so as to form reticula or networks, varying in the closeness, form, and arrangement of the meshes in different organs and situations, aceording to the blood-supply locally required. The eapillaries lie in intimate relation with all the tissues, and nearly all parts of the body are closely permeated by these channels. From the capillaries the blood accomplishes its metabolic and nutritional action on the body-tissues. The capillaries are therefore the ultimate active functional elements of the circulatory system, to which all the rest of the circulatory apparatus serves a subordinate and tributary purpose.

The thin walls of the capillaries are highly endowed with the vital properties of protoplasm, and are well fitted for the exosmosis of nutrient fluids; the endosmosis of carbon dioxide and other waste products of tissue-katabolism ; and the passage of leukocytes through them.

Vascular sinuses: In some situations the vascular passages are dilated or distended in the form of cavernous spaces or sinuses (often called venous sinuses), lined with endothelium and communicating with arteries and veins, and with one 
another. Such sinuses may be interposed between the terminal arteries and the commencing veins, taking the place of capillaries, as in the spleen and bone-marrow.

The erectle tissue of the male and female genitals consists of similar vascular sinuses so arranged that when the exit of the blood is checked by muscular compression of the outletveins the spaces are distended with blood and the organ becomes turgid and erect.

6- Mi:t. 


\section{CHA PTER VII.}

\section{THE LYMPHATIC SYSTEM.}

In the main the lymphatic system consists of a series of vessels or channels distributed throughout the entire organism and serving chiefly for draining away superfluous fluids in the tissues and returning them into the blood.

The fluid contained in these channels is the lymph.

The absorptive action of the lymphaties, as in the intestinal walls and the removal of substances introduced hypodermically, is allied to their drainage function. In some situations, as in the cornea and bone, lymph-channels afford the only passages by which fluids can permeate the tissues for the purpose of nourishing them.

In the course of lymphatic vessels are also interposed glandular structures, which among other functions have the power of arresting noxious and toxie bodies in the lymphatic circulation.

The morphologic elements of the lymphatic system may be stated to be lymph-spaces and origins, lymphatic vessels, lymphoid tissue, and lymphatic glands.

Lymph-spaces: The interstices or empty spaces in tissues, especially connective tissues, form cavities which may contain lymph and communicate directly or indirectly with lymphatic vessels. Cell-spaces, the intervals between (connective-tissue) cells and their processes and the walls of the cavities or lacumx in which they are situated (as in bone and the cornea), often afford sufficient room for the passage of lymph. In some instances separations or fissures in tissues form lymphspaces, withont any specialization of the walls; in other cases the walls of the spaces are lined partially or wholly with flattened connective-tissue cells or endothelium. The lacunæ and canaliculi of bone, the cell-spaces and channels in the cornea, the minute intervals between the prickle-cells of the epidermis, form communicating passages by which lymph can 
percolate through these tissues, which are not supplied by blood-capillaries, and afford pabulum for their nutrition which would otherwise be unprovided.

Cell-spaces are also especially observable in the fibrous tissue of the central tendon of the diaphragm, irregular stellate lymph-spaces corresponding to connective-tissue cells, with branches communicating with one another and perhaps with lymph-vessels.

The spaces in areolar tissue also serve as lymph-spaces, communicating freely with the lymph-vessels, and from their looseness are capable of holding large accumulations of lymph and fluid, as in oedematous conditions and subcutaneous injections. These spaces are simply fissures in the substance of the connective tissue, without any specialized walls.

In certain regions, notably the nervous centres, occur wellmarked perivascular and perineurial lymphatics, consisting of longitudinal passages or clefts in the tunica adventitia of the bloodvessels and fibrous tissue surrounding the nerves; these passages when well developed may be lined with endothelium.

The open meshes of lymphoid tissue also constitute lymphspaces, partially lined with flat connective-tissue or endothelioid cells.

The serous sacs, as the pleura, pericardium, peritoneum, synovial sacs, bursæ, etc., may be regarded as representing highly developed lymph-spaces. They are large cavities completely and definitely lined by serous membranes, which have been already described as fibrous membranes lined on the free surface by endothelium. The cavity of the serous sacs communicates with the lymph-vessels by stomata. The function of serous membranes is, however, apparently more to obviate friction and facilitate motion between opposing surfaces than to serve as components of the lymphatic system. Ordinarily the serous saes contain only a small amount of fluid, which acts as a lubricant; in the synovial sacs and bursæ this fluid is much different from ordinary lymph, being glairy and viscid and having the character of a special secretion.

Origins of the lymphatic vessels: The lymph-spaces just considered constitute the chief portion of the ultimate origins 
of the lymph-vessels. The nutritive fluids, after exuding from the blood-capillaries and bathing the tissues, accumulate in the interstices between the cells and in the spaces in the tissues. These spaces open directly into the smallest radicles or capillaries of the lymphatic vessels; or the fluids may enter the vessels by osmosis through their thin walls. The lacteals form another important class of lymphatic origins; as elsewhere described, they are small blind tubes occupying the axis of the villi of the small intestine, and emptying into the larger lymphatic vessels in the submucosa. They are really lymphatic radicles or capillaries, and their special function is to absorb into the circulation the fatty and other food-materials digested and elaborated in the alimentary canal. The lymph-stomata, minute openings guarded by small spheroidal cells, occurring at the points of junction of the endotheliumcells of serous membranes, also form a sort of origin or opening in to lymph-vessels.

Lymphatic vessels: The lymph-vessels form a series of channels ramifying through the entire body. The fluids from the lymph-spaces and lacteals are taken up by the small lymphatic branches, these combine to form larger channels, and finally they are all united into two vessels, the thoracie duct and the smaller right lymphatic duct, which empty into the blood at the junction of the jugular and subclavian veins on each side.

The smallest lymphatic vessels, the so-called lymph-capillaries, including the lacteals, are delicate protoplasmic tubes made up of a single layer of polygonal endothelium-cells. As these vessels unite and become somewhat larger, their endothelial lining, now made up of more elongated cells, beeomes surrounded with a certain amount of connective tissue. The smaller lymphatic channels are very delicate, inconspicuous structures difficult to distinguish.

The larger lymph-vessels have thicker walls, and in structure somewhat resemble the veins. Their walls are composed of three coats. The inner coat is lined with elongated endotheliumcells, beneath which is a thin layer of connective and elastic tissue. The middle coat consists mainly of elastic tissue and involuntary muscle-cells arranged transversely. The external coat, or adventitia, is an investment of fibrous and areolar tissue. 
Lymphatic vessels contain numerous valves, formed by transverse folds of the inner tunic. Associated with the valves are alternate dilatations and constrictions of the lymphatic vessels, which give them a characteristic beaded appearance.

The thoracic duct is the largest and best developed of the lymphatic vessels. Its inner tunic contains a considerable amount of subendothelial connective tissue and longitudinally arranged elastic fibres. Its outer coat contains scattered bundles of longitudinal involuntary muscle.

Lymphoid or adenoid tissue consists of an open connectivetissue network, the meshes of which are crowded with free cells, called lymphoid cells, the whole communicating with lymphatic vessels. The connective-tissue reticulum which forms the basis of typical lymphoid tissue has already been described under the name retiform tissue; it consists of fine interlacing fibrils covered or lined at their intersections with flat stellate connective-tissue or endothelioid cells. The meshes formed by this structure are filled with lymphoid cells, small free spherical cells with relatively large and prominent deeply staining round nuclei and small cell-bodies. These are often packed in so densely as to obscure and hide the reticular basis. The meshes communicate freely with one another and with the interior of entering or afferent and departing or efferent lymphatic vessels, so that the tissue forms a lymphspace, and through it lymph constantly flows. Adenoid tissue is also supplied with blood-capillaries.

Lymphoid tissue exhibits all gradations from diffuse masses without definite-boundaries to sharply circumscribed nodules. Diffuse lymphoid tissue occurs imbedded in connective tissue, and merges insensibly into the surrounding tissues. It presents all grades from ordinary connective or areolar or even epithelial tissue infiltrated scantily or densely with wandering lymphoid cells to typical dense adenoid tissue with a retiform basis.

In other cases lymphoid tissue forms well-defined nodules, more or less spherical in shape, often quite sharply circumscribed by a condensation of the surrounding fibrous tissue. Such nodules are often called lymph-follicles (Fig. 38). The afferent lymph-vessels are said to open into the periphery of such nodules or follicles. The tissue in the centre of the 
follicles is often less dense than that at the periphery. Lymphatic glands may be regarded as highly specialized or compound lymph-follicles.

Fig. 38.

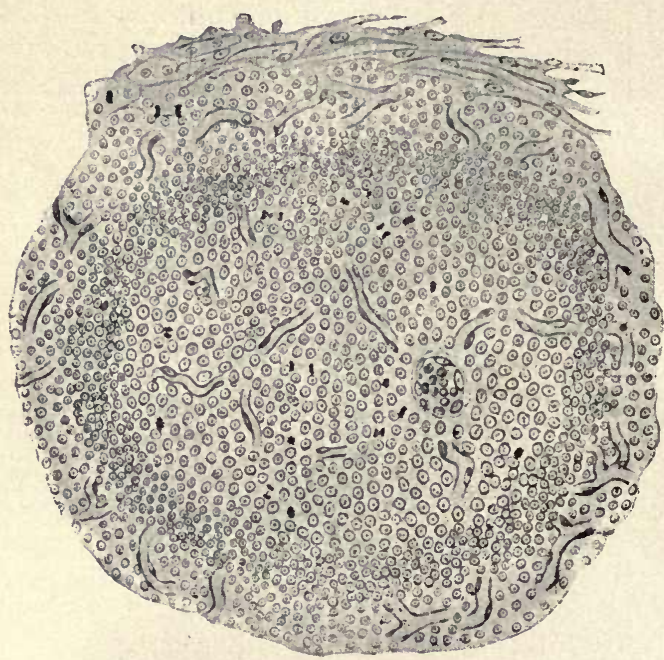

Lymph-follicle (Flemming).

Lymphoid tissue is widely distributed through the body. In a diffuse form it is common in mucous membranes in many situations, especially the alimentary canal. Circumscribed lymph-follicles are most typically represented by the solitary and agminate glands of the intestines; also by the tonsils and Malpighian corpuscles of the spleen. The lymphatic glands and thymus are also composed of lymphoid follicles.

Lymphatic glands: Interposed in the course of the lymphatic vessels in many situations, notably in the mesenteries, mediastina, and under the skin in certain parts, are numerous lymphatic glands. These are oval or kidney-shaped bodies, of small size, with a depression at one side, the hilum, where the bloodvessels and efferent lymph-vessels enter and leave the gland.

The elements of lymphatic glands are sustentacular tissue, 
afferent lymph-vessels, lymph-sinuses, dense lymphoid tissue, efferent lymph-vessels, and bloodvessels. Two regions can be distinguished in them, a dense peripheral region, the cortex, and an opener central region, the medulla, which is continuous with the hilum.

The sustentacular tissue forms a firm fibrous capsule enveloping the gland, which sends trabeculæ or septa inward toward the centre, dividing the gland into a number of radiating rounded lobules. Delicate retiform tissue, also a portion of the sustentacular framework, fills the entire gland and forms the basis of the lymphoid structures.

The afferent lymph-vessels are a number of vessels or branches which enter the gland at scattered points over the periphery, emptying into the peripheral lymph-sinuses.

The cortical portion of the gland is mainly made up of typical lymphoid tissue, densely packed with lymphoid cells. It is divided into lobules by the trabeculæ, and each lobular mass of lymphoid tissue is surrounded by a lymph-sinus. The adenoid tissue in the outer or cortical part of the lobules forms dense continuous masses; but toward the centre it breaks up into branches or columns of lymphoid tissue, called lymph-cords, which project into the medulla and are separated by the medullary lymph-sinuses and vessels.

The lymph-sinuses are open channels and spaces in the retiform tissue where the lymph is more free to flow than in the dense adenoid tissue. They consist of the same open reticulum which forms the basis of the lymphoid tissue; but very few lymphoid cells are contained in the meshes, so that the lymph is afforded free passage through the open network. Into these sinuses the afferent and efferent lymph-vessels freely open.

These sinuses may be divided into two groups, the peripheral sinuses and the medullary sinuses.

The peripheral lymph-sinuses surround the periphery of the lymphoid tissue which makes up the various lobules, and the afferent lymph-vessels open into them. They consist of a narrow space intervening between the capsule and trabeculæ and the dense lobular adenoid tissue.

The medullary lymph-sinuses are located in the central portion of the gland, and consist of branching open sinuses in- 
tervening between the columns or cords of dense lymphoid tissue, and uniting in the centre to empty into the efferent lymph-vessels.

The cortex of lymphatic glands consists of the lobules of dense lymphoid tissue surrounded by their lymph-sinuses.

The medulla is a smaller region in the eentre, continuous with the hilum, in which the tissue is opener. It is composed of the medullary lymph-sinuses, the columns or cords of lymphoid tissue, the branching bloodvessels and efferent lymph-vessels, and some connective tissue.

The efferent lymph-vessels form in the medulla by union of the smaller channels continuous with the medullary lymphsinuses, and leave the gland by the hilum.

The bloodvessels enter and leave mostly by the hilum, and in the medulla break up into networks; some vessels also lie in the trabeculæ.

The course of the circulation through lymphatic glands is as follows: the lymph enters by the afferent vessels at various points in the periphery, passes into the peripheral lymphsinuses, then percolates through the lymphoid tissue, is collected in the medullary lymph-sinuses, and is carried away by the efferent lymph-vessels. At the same time the blood circulates through the gland, and the serum which transudes through the capillary walls also joins the efferent stream of lymph.

Thymus gland (Fig. 39): This is an infantile organ, situated at the base of the neck and in the upper mediastinum. In the embryo it first appears as a downgrowth of hypoblastic epithelium, which, dividing and subdividing, takes on the character of a compound or racemose epithelial gland. A round this epithelial structure soon develops from the mesoblast a mass of lymphoid tissue in such abundance as to encroach on the epithelial growths, causing them to atrophy and cutting them off in detached masses, which in the mature state of the gland appear as scattered rudimentary epithelial nodules, the concentric corpuscles. The organ usually reaches it maximum development at the second or third year of age, and then in the course of ten or fifteen years gradually atrophies and finally disappears, being in its turn replaced by fatty and fibrous tissue

The thymus exhibits a medullary and a cortical region, 
and consists of a sustentacular framework, lymphoid tissue, the concentric corpuscles of Hassall, and blood and lymphatic vessels.

The sustentacular tissue consists of a fibrous capsule enveloping the gland, trabeculæ extending inward dividing the cortical portion into lobes and lobules, and a retiform framework filling the entire organ as a basis for the lymphoirl tissue.

The body of the gland consists throughout of lymphoid

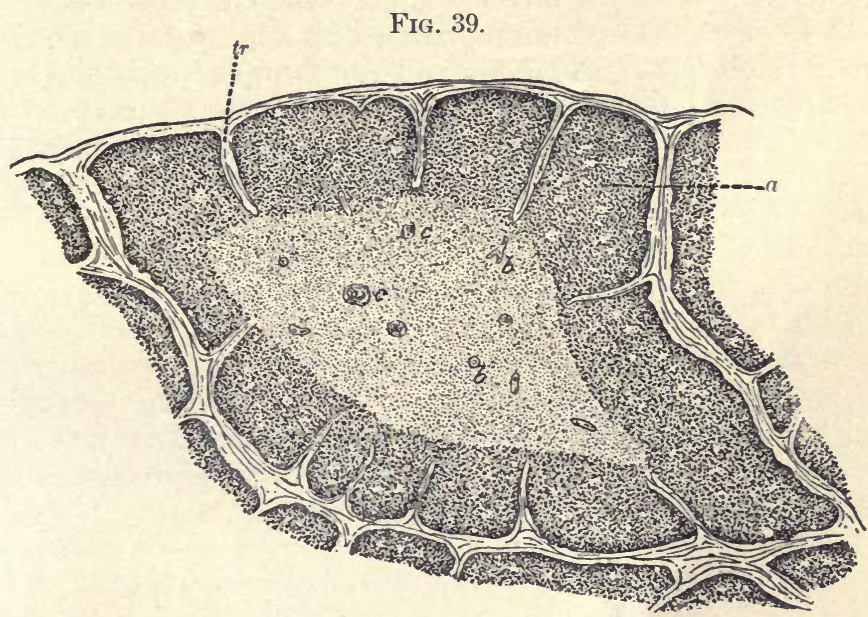

Lobule of thymus gland (Schäfer). $a$, cortex; $t r$, trabeculæ: $b$, bloodressels in the medulla ; $c$, corpuscles of Hassall.

tissue, open and clearer in the central portion, thus constituting the medullary region, and dense in the outer or peripheral portion forming the cortex.

The cortical region is divided into lobes and lobules by the trabeculæ extending inward from the capsule.

The lobular divisions do not extend into the medulla, which thus forms a common core from which all the lobules project.

Scattered about in the medulla are the concentric corpuscles, or corpuscles of Hassall, small spherical bodies having a granular core surrounded concentrically by flat squamous epi- 
thelioid cells, remains of the original epithelial structure of the gland. The thymus is abundantly supplied with blood and lymphatic vessels.

The tonsils and solitary and agminate glands of the intestines are lymphoid glands, and are described in conneetion with the alimentary system.

The functions of lymphoid tissue and glands are not entirely understood, but some important purposes which they serve are known. They are the souree of the lymph-corpuscles and leukocytes of the blood. The lymphoid cells of adenoid tissues, after undergoing enlargement and certain changes, enter the efferent lymph-stream as lymph-corpuscles, and after passing into the blood they form leukocytes. Lymphatic glands also serve to arrest toxic and foreign bodies which enter the lymph-stream; their tissue acts as a sort of filter in this respect. Thus, in eases of vaccination, carcinoma, chancre, and many similar conditions, the virus from the affected spot is taken up by the lymph-ressels, but is arrested in its passage at the next group of lymphatic glands, which swell up and become inflamed, thus for a time retarding the progress of the disease. The interchange between the blood and lymph eireulations in the lymphatic glands may also be associated with some sort of internal secretory process. The special purpose of the thymus is obscure. 


\section{CHAPTER VIII.}

\section{BLOOD AND LYMPH.}

The blood and lymph are the cellular fluids circulating about the body in the circulatory and lymphatic systems and providing for body-nutrition. They convey to the tissues the gaseons, fluid, and solid pabulum required in their anabolism or upbuilding, and bear away from them the wasteproducts of their katabolism or downbreaking.

Blood: On examining fresh blood under the microscope we observe large numbers of free straw-colored cells, with here and there a colorless cell, suspended in a clear homogeneous fluid. The blood, then, consists of two portions-a fluid portion, the plasma, and free blood-cells or corpuscles suspended in it.

The corpuscles, again, are of three kinds-red blood-corpuscles, white blood-corpuscles, and blood-plates.

The plasma comprises about three-fifths of the blood; the corpuscles about two-fifths.

The blood may be regarded as a fluid tissue, the corpuscles being the cellular elements and the plasma the intercellular substance. It differs from other tissnes, however, in that the intercellular portion is not produced by the activity of the blood-cells themselves, but is derived from absorption. The blood belongs to a class of tissues that is sui generis, different from and coördinate with the classes of epithelium, connective tissue, etc., which make up the fixed tissues. Owing to the facility with which it can be examined and the significant changes which it exhibits in various diseases, the histology of the blood is a matter of great importance in practical medicine.

Blood-plasma, or liquor sanguinis : This is a clear, homogeneous, colorless fluid comprising about three-fifths of the volume of the entire blood. It consists of water holding in solution 
about 9 per cent. of albuminous substances, mineral salts, and other constituents. These substances serve as pabulum for the nourishment of the tissues. It readily undergoes coagulation, separating into clot and serum. 'The plasma originates by absorption from the alimentary tract. Being homogeneous, it ordinarily exhibits no structural features to the microscope; its composition is a matter for chemical study.

Under certain circumstances, however, substances or particles visible to the microscope appear in the plasma. After standing for some time specimens of fresh blood exhibit in the plasma numerous delicate colorless straight filaments of fibrin interlacing and running in different directions; they often radiate from points or centres, and at these centres blood-plates are often situated. These filaments consist of fibrin which has undergone coagulation. The plasma under the mieroscope often has a yellow tinge, due to hæmoglobin dissolved out of the red corpuseles.

Rhombic crystals of homoglobin appear in blood under proper conditions or treatment. Small particles of fat are sometimes present in the plasma, especially after a fatty diet.

In the blood-plasma are frequently to be seen minute granules, or "blood-dust," both in normal and abnormal conditions. Ordinarily they are not numerous, but occur singly here and there. Some of these may be particles of fat, or foreign particles accidentally introduced. In most cases, however, they seem to be minute bits of protoplasm, or neutrophile or oxyphile granules extruded from leukocytes; their nature is not yet fully determined. These are very minute in size, variable in shape, colorless and refractile, and are in constant very active oscillating or Brownian movement.

Red blood-corpuscles (Fig. 40) are also called erythrocytes, and, most appropriately, colored blood-corpusiles.

The red blood-corpuscles of man are circular disc-shaped cells, between 7 and $8 \mu$ in diameter (averaging $7.5 \mu$ ), and about $2 \mu$ thick. They are biconcave, and thinner in the centre than at the edges, so that their color is lighter and paler in the middle and deepens toward the periphery. In appearance 
they are homogeneous throughout, and they possess no cellenvelope. They contain no nuclei, except in early embryonic life and in abnormal conditions. Their color, when seen singly or in thin layers, is not red, as the name indicates, but a characteristic pale yellow with a slight greenish tinge; it is only in mass that they produce the effect of red color. They are soft, elastic, pliable, almost gelatinous. As they flow through the capillaries or move in currents in the microscopical specimen they change shape with the least pressure, and may become greatly distorted ; but on release from restraint they resume their normal discoid shape.

The number of red corpuscles in given volumes of blood under similar normal conditions is practically constant, and near the sea-level in adult males is about $5,000,000$ in each cubic millimetre of blood; in females, about $4,500,000$. In very vigorous individuals the number of red corpuscles may be increased, even up to $6,000,000$; while with increase of altitude above sea-level there is a very marked increase in their number.

When a drop of blood is placed under the microscope the red corpuscles are observed to exhibit, for a time, a tendency to form rouleaux-that is, they become arranged or adherent together evenly side by side, like a pile of coins.

Crenation: The red corpuscles FIg. 40.

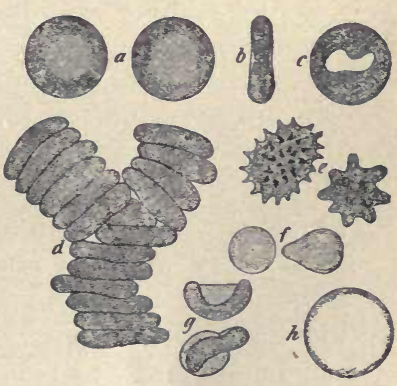

Red blood-corpuscles of man. $a$, surface view of normal corpuscles; $b$, edge view ; $c$, corpuscle with its substance shrunken, forming a vacuole; $d$, rouleau formation; $e$, crenated corpuscles; $f$, fragments of broken corpuscles; $g$, bent and distorted corpuscles; $h$, swollen and decolorized corpuscle.

are so delicately adjusted to the blood-plasma that they are very easily affected as to shape by any alteration in the density and composition of the medium in which they occur. One of the commonest of these changes is crenation. This consists of a shrinkage of the corpuscle, which loses its discoid shape and becomes spheroidal or irregularly distorted, while at the same time minute rounded or spiny projections appear on the surface; of these spines there may be only one 
on the entire corpuscle, or only a few; or a large number studding the surface of the much-shrivelled spherical cell. The more the cell is contracted the greater is the number of spines.

Crenation is caused by contraction of the corpuscle from loss of part of its fluid contents through osmosis, and occurs when the density of the plasma is increased by evaporation from exposure to the air or by the addition of saline or other substances.

Other changes of form: When the density of the plasma is diminished, as by the addition of water, the red corpuscles through osmosis absorb fluid, swell, and become spherical, and at the same time lose their color, the hromoglobin in the corpuscles leaving them and passing into solution in the plasma outside.

Sometimes the substance of the corpuscles shrinks, leaving artificial empty spaces or vacuoles.

In squeezing blood from a puncture in the skin, portions of the corpuscles are often broken off, and, assuming a rounded form, appear like diminutive corpuscles.

At other times the corpuscles appear flexed, twisted, or doubled up in the form of a hemispherical bowl.

Familiarity with the changes of form which red corpuscles undergo under changed conditions is necessary to avoid mistaking them for morphologic or pathologic abnormalities.

In various disease-conditions interesting variations from the normal are exhibited by red corpuscles, as the presence of nuclei, and abnormalities in size, coloration, and consistency.

The function of the red blood-corpuscles is to convey oxygen from the lungs, where it is taken up by the hæmoglobin, to the tissues, where it is given out for nutritive purposes.

In structure they appear to consist of a pliable and elastic colorless proteid stroma, in which hæmoglobin is suspended in solution; the form and consistency of the cells are due to the stroma, the color, to the hæmoglobin. They do not exhibit amoboid movement or cell division. Considering their lack of nuclei, and of other vital properties, the red blood-corpuscles must be regarded as a very degenerate form of cell, exhibiting few characteristics of vitality and serving the purely mechanical purpose of carrying oxygen. 
The origin of red blood-corpuscles is not fully determined. The earliest red corpuscles of embryonic life originate along with the bloodvessels from mesoblastic cells ("angioblasts") which form a protoplasmic network, in the substance of which, and especially at the intersections, nuclei proliferate and accumulate. These masses of multinucleated protoplasm break up, becoming converted into cavities filled with separated cells; the peripheral protoplasm and nuclei form an endothelial lining to the cavity, which is now an incipient bloodvessel, while the central mass of nucleated protoplasm separates into amœboid nucleated cells, which then acquire hæmoglobin and become nucleated red blood-corpuscles. From these "blood-islands" the process of vessel and corpuscle formation proceeds along the protoplasmic strands.

Thus the earliest embryonic red corpuscles are nucleated, and capable of self-reproduction by indirect division; these, however, soon disappear, and are replaced during the remainder of life by non-nucleated corpuseles.

The most probable source of red corpuseles in the adult seems to be the bone-marrow. As described above, bonemarrow contains an ample network of expanded vascular sinuses, and one class of the cells of the marrow consists of the erythroblasts, small nucleated hæmoglobincontaining cells capable of amoboid movement. The red corpuscles are probably produced from these cells, but in what manner is not known; the erythroblasts themselves may perhaps be descendants of the nucleated red corpuscles of the embryo, continued in existence by repeated division.

Other theories of the origin of red corpuscles have been presented, as that they are produced from leukocytes, or bloodplates, or in the spleen, lymphatic glands, or thymus, but these suppositions have not been well substantiated. There is a constant destruction of erythrocytes, and a means somewhere in the borly by which they can be actively renewed. They cannot reproduce themselves by division; and the only known seat for their formation supported by any great probability is the bone-marrow.

Decay of red blood-corpuscles: Erythrocytes, like all other living cells, pass through a life-cycle of development, maturity, decay, and death. The red corpuscles seen in normal 
blood are all perfect and show no visible signs of degeneration. What becomes of them when they decay and how they are removed from the circulating blood is not entirely known, but it is supposed that the spleen and liver, especially the former, are important agents in their removal and destruction.

Differences in vertebrate red blood-corpuscles: Red corpuscles occur only in the blood of vertebrates, that of invertebrates containing only colorless corpuscles. The different classes of vertebrates exhibit differences in the characters of the erythrocytes with regard to shape, nucleation, and size. All mammals, like man, have non-nucleated biconcave circular red corpuscles, except the camel family, in which they are elliptical (and non-nucleated). In the birds, reptiles, amphibians, and fishes, the red corpuscles are elliptical, nucleated, and biconvex (the nucleus causing an expansion of the corpuscle), except in the cyclostomata or lampreys among fishes, whose corpuscles are circular.

As to size, the erythrocytes of mammals are the smallest, those of amphibians the largest.

Among mammals the red cell of the elephant is the largest (9.2 $\mu$ in diameter), that of the musk-deer the smallest; and it is the smallest known of all animals $(2.5 \mu)$. Human erythrocytes are among the largest of those of mammalia. The erythrocytes of birds and fishes are much the same in size; the corpuscle of the fowl is about $12 \mu$ long, of the pigeon $15 \mu$, of the carp $13 \mu$. The frog's red corpuscles are about 16 by $22 \mu$; those of the amphibian proteus 35 by 58 $\mu$; while those of the amphibian amphiuma are about $46 \mu$ wide by $77 \mu$ long, the largest known, and visible to the naked eye.

The number of the erythrocytes is in general in an inverse proportion to their size, the smaller cells occurring in greater numbers; thus, the red corpuscles of the frog number only 404,000 , those of the proteus 36,000 to the cubic millinetre. Owing to this inverse relation of number and size the total mass of corpuscles in equal volumes of blood is somewhat equalized; but the greater the number of corpuscles the greater is their total surface; and the greater their surface (as in birds) the more active is the metabolism. 
White blood-corpuscles (Fig. 41) or leukocytes, are most appropriately, perhaps, called colorless blood-corpuscles. The leukocytes of man are of several varieties, differing in some particulars but similar in their general characteristics.

In shape when at rest they are spherical, but during their amœboid movements they are irregular and changing in form.

In size the different varieties range from about 7 to $15 \mu$ in diameter, averaging about $10 \mu$; they are thus somewhat larger than the erythrocytes. 'They are colorless, refractile, and granular in appearance.

Their surface is somewhat adhesive (unlike the red corpuscles), so that they adhere to the glass in microscopical preparations, and in the blood-current they roll slowly along at the edge of the stream on the wall of the bloodvessel.

They are of firmer consistency than the red corpuscles, and their form is not so easily affected by mechanical influences or changes in their environment. If the density of the fluid in which they occur be much increased, as by the addition of salts, they shrivel somewhat; while if the density be diminished by dilution with water, they assume the resting spherical form, become swollen, and within them appear coarse protoplasmic granules which often exhibit the Brownian movement.

The number of leukocytes under normal conditions is about 7500 or 8000 in each cubic millimetre of blood ; the number is greater in young children and during pregnancy; and during digestion, as three or four hours after a proteid meal, their number is normally increased about one-third (the "digestion leukocytosis"). The leukocytes are therefore far less numerous than the red corpuscles, the ratio being normally about 1 white to 600 red. In pathological conditions the number of leukocytes may vary widely. Leukocytes do not occur in circulating blood alone, but are also met with as lymph-corpuscles in the lymphatic system; as wandering cells in the connective tissues; in enormous accumulations in pus as puscorpuscles, etc.

The structure of leukocytes is that of typical actively vital cells. They have a cell-body of active protoplasm, wellmarked nuclei, and are said to be provided with centrosomes. 7-Hist, 
They have no observable cell-wall, other than some condensation of the peripheral protoplasm.

The nuclei vary in the different varieties of leukocytes as to number, form, size, and staining properties. The "mononuclear" leukocytes contain a single nucleus each. Other varieties when stained appear to contain about three separate rounded nuclei ; but some authorities at present consider that in the living state these nuclei are in most cases connected by strands of the nuclear substance, thus forming a single very irregular nucleus rather than three separate nuclei. The possession by a leukocyte of several distinct (or multiple) nuclei is indicated by the term "polynuclear"; while the possession of single variable and irregular (or multiform) nuclei is designated by the term "polymorphonuclear."

In form the nuclei range from spherical, through horseshoeshaped forms, to exceedingly irregular and variable coiled and twisted shapes. Their size varies from those that are large and occupy the largest part of the cell to those that are relatively smaller.

The nuclei take nuclear and basic stains with more or less avidity; the smaller nuclei, in which the chromatin is more concentrated, usually stain intensely; while the larger nuclei, whose chromatin is more diffused, are less deeply colored.

The cell-bodies of leukocytes consist of actively vital protoplasm. Sometimes, especially during amœboid movement, two parts can be distinguished in the body-protoplasm, a clear homogeneous hyaline peripheral portion, the hyaloplasm, and a less clear granular portion in the interior of the cell, the granuloplasm. Well-marked granules are present in the bodies of some varieties of leukocytes, absent from others. The granules mostly found are fine neutrophile granules and coarse oxyphile granules" $(\varepsilon$ and $\alpha$ granules of Ehrlich's classification); fine basophile (o) granules also occur but are inconspicuous. In the living state the granular appearance of the leukocytes often obscures the nuclei; but on treatment with acetic acid the granules disappear, the cell-body becomes clear, and the nuclei stand out prominently as about three spherical bodies.

Varieties of leukocytes: According to variations in size, nuclei, granules, and staining-properties, the leukocytes of 
normal human blood are divided into five classes, namely: small mononuclear, large mononuclear, transitional, polynuclear (or polymorphonuclear), and eosinophile.

FIG. 41.
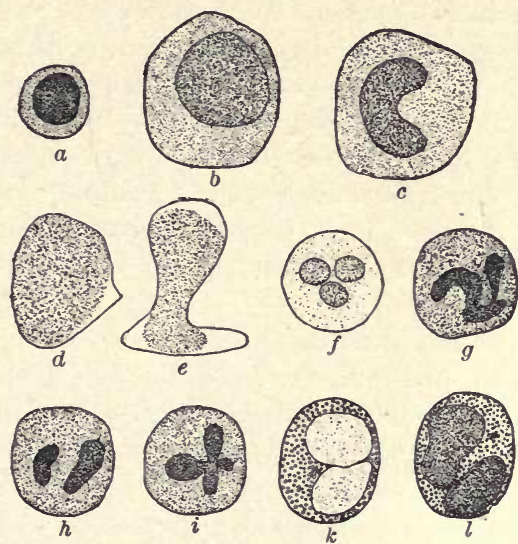

Leukocytes of human blood. $a$, small mononuclear leukocyte, stained; $b$, large mononuclear leukocyte, stained; $c$, transitional leukocyte, stained; $d, e$, polymorphonuclear or neutrophile leukocyte, living appearance, showing distinction of hyaloplasm and granuloplasm and amœboid movement ; $f$, polymorphonuclear leukocyte, in fresh state, treated with acetic acid; $g, h, i$, polymorphonuclear leukocyte, stained; $k$, eosinophile leukocyte, living appearance; $l$, eosinophile leukocyte, stained.

The small mononuclear leukocyte, or small lymphocyte, is about 6 to 8 micromillimetres in diameter (about the same as red corpuscles), and has a single large spherical, deeply staining nucleus, surrounded by a small amount of faintly-staining non-granular protoplasm, which forms a small cell-body. Morphologically and genetically it is similar to or identical with lymph-corpuscles.

The large mononuclear leukocyte, or large lymphocyte, the largest corpuscle of normal blood, is 12 to 15 micromillimetres in diameter, contains a single, very large, rounded nucleus that does not stain very deeply, and has a large body of nongranular, faintly staining protoplasm. Sometimes no sharp line can be drawn between this and the preceding class, all intermediate gradations of size and coloration being present.

The transitional leukocyte is like the large mononuclear 
form, except that its single large nucleus, instead of being spherical, is more or less indented at one side, tending toward a horseshoe shape; the body-protoplasm also sometimes contains a few fine granules. In the shape of its nucleus it is an intermediate form between the large mononuclear and the polymorphonuclear variety. It is, however, closely related to the former, all gradations being present so that a sharp line can scarcely be drawn between the large mononuclear and the transitional ; on the contrary (especially with respect to the absence of granules), there is a sharp gap between the transitional and polymorphonuclear varieties not filled by intermediate gradations. Transitional leukocytes are often to be regarded simply as a variety of the large mononuclear group.

The polymorphonuclear, polynuclear, or neutrophile leukocyte is abont 10 micromillimetres in diameter, has a large cellbody of active protoplasm, containing large numbers of fine neutrophile $(\varepsilon)$ granules, and a very variable, irregularly shaped, deeply staining nucleus. Often, especially after the action of reagents, the nucleus appears to consist of about three separate, rounded nuclei; whence until recently the term polynuclear was the prevalent designation of this leukocyte. At present the opinion is gaining ground that in most cases, instead of three separate nuclei, the living corpuscle contains one long twisted and irregular nucleus, so that the name polymorphonuclear is now considerably used. This is the most active and much the most abundant variety of leukocyte in the blood, and is also the form found in pus. It exhibits active amoboid movements, and the distinction of hyaloplasm and granuloplasm in its body can often be made out.

The eosinophile (or oxyphile) leukocyte is about $10 \mu$ in diameter; its nucleus, except that it stains less deeply, is similar to that of the polymorphonuclear variety, being polymorphous and irregular, or perhaps multiple; the body-protoplasm is crowded with conspicuous coarse spherical oxyphile or eosinophile $(\alpha)$ granules. In microscopical specimens the granules surround but do not overlie the nuclei, leaving the latter as clear spaces. This leukocyte has a striking appearance mpt is easily recognized, both in the living and stained sley Qbondalofich punfis the least common variety in the blood. Its 
wall is weak and easily ruptured, as the granules often appear spreading out into the surrounding plasma without any definite boundary. Intermediate forms between the eosinophile, the polymorphonuclear, and the mononuclear leukocytes are conspicuously absent from the blood.

These different varieties of leukocytes occur in normal blood in tolerably constant proportions to one another, averaging about as follows :

Small mononuclear leukocytes .

Large mononuclear and transitional leukocytes Polymorphonuclear leukocytes . . . . . . Eosinophile leukocytes

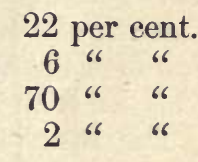

100

Occasionally a stray mast-cell finds its way into the blood. Variations from the normal occur in morbid conditions; the total number of leukocytes and the relative number of the different varieties may be altered, or entirely new forms of leukocytes (myelocytes) may appear.

Vital properties and functions of leukocytes: Leukocytes are typical cells, undifferentiated and independent, actively endowed with all the essential attributes of vitality. They are capable of undergoing division, direct and indirect. They. (especially the polymorphonuclears) exhibit active amœboid movements, which enable them to make their way through the walls of the capillaries and wander about in the tissues. By means of their amœboid movements they also have the power of surrounding and engulfing within their protoplasm foreign particles and living parasites, bacteria and protozoa. This process is called phagocytosis, and cells which engage in it are called phagocytes.

The act of phagocytosis can sometimes be observed under the microscope, as in connection with the malarial protozoön, and is a remarkable and interesting sight. The leukncyte seems capable of recognizing the presence and whereabouts of the parasite at a distance of many times its own diameter, and moves upon it directly and with distinct purpose, pushing the red corpuscles aside. By protruding pseudopodia toward 
the parasite and drawing itself up behind, it advances with a mode of locomotion resembling that of a snail. Finally, it sends processes around the parasite and engulfs the latter in its body, where it is disintegrated. The leukocyte seems endowed with intelligence, purpose, and a separate individuality. of its own, vividly analogous to the psychic manifestations of macroseopic animals.

Chemical substances are supposed to have an influence on leukocytes, some attracting them, others repelling them ; this is called chemotaxis. Positive chemotaxis consists in the attraction of leukocytes toward certain substances, negative chemotaxis in their repulsion from other substances.

The functions of leukocytes are not entirely understood. One important purpose which they are believed to subserve is the removal and destruction of foreign noxious substances and parasites, by phagocytosis ; they also play an active part in the repair of injuries, in immunity, and the resistance and reaction of the body to morbid processes.

Origin and development of leukocytes: The small mononuclear leukocytes are doubtless derived from the free lymphoid cells which crowd adenoid tissues; these undergo development, enter the lymph-stream, and are thence carried into the blood.

The large mononuclear and transitional leukocytes appear to develop from the small mononuclears, as intermediate gradations between them are to be found in the blood.

The source of the polymorphonuclear and eosinophile leukocytes is not so obvious. They may develop from the mononuclear variety-or, as has been suggested, the eosinophiles ("old" forms) may develop from the polynuclears, and the latter ("adult" forms) from the mononuclears ("young" forms); as, however, there are no intermediate forms between these varieties in the circulating blood, their development from one another must take place, if at all, somewhere in the viscera. Or perhaps the polynuclear and eosinophile leukocytes originate independently in some of the viscera, as the spleen or bone-narrow.

The eosinophiles, especially, may not improbably develop in the marrow, as in the latter are found similar cells containing oxyphile granules. Division of the developed leukocytes themselves may be a partial means of their production. 
Blood-plates are also called blood-plaques or platelets, corpuscles of Bizzozero, and hæmatoblasts (Fig. 42). These are small, colorless, hyaline, homogeneous bodies, of spherical or discoid shape, between 2 and 4 micromillimetres in diameter, numbering 200,000 to 400,000 in each cubic millimetre of normal blood. On exposure to the air they quickly disintegrate and disappear, so that special precautions are necessary in taking a specimen of blood to preserve FIG. 42.
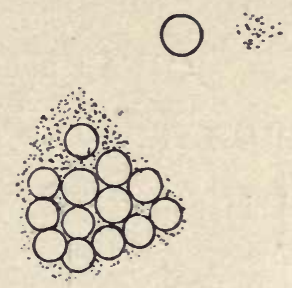

them. They occur singly, or often grouped together, in the vicinity of granular matter, probably the debris of disintegrated plaques. They often form centres from which filaments of coagulating fibrin radiate, and they may have some relation to the coagulation of the blood. They have been called hrematoblasts under the idea that they are developing forms of red blood-corpuscles, a supposition that has not been confirmed. Their origin, purpose, and significance are not definitely known.

Lymph, the fluid which flows in the lymphatic system, consists of a fluid, the serum or plasma, suspended in which are free cells, the lymph-corpuscles, and particles of fat. The plasma, or liquor lymphæ, is similar to that of the blood. The lymph-corpuscles are leukocytes, like those of the blood. Some of them are white corpuscles that have migrated from the capillaries and been taken up from the tissues by the lymph-stream; large numbers of them, however, are of the small mononuclear type, and are lymphoid cells carried out of the adenoid tissues through which the lymph-currents pass. The chyle is that portion of the lymph that is collected in the lacteals and intestinal lymphatics; it is of the nature of an emulsion, opaque and white, from the presence in the plasma of large numbers of minute particles of fat absorbed from the intestines. 


\section{CHAPTER IX.}

\section{BLOOD-GLANDS AND DUCTLESS GLANDS.}

THE lymphoid glands pertaining to the lymphatic system (lymphatic glands, tonsils, thymus, solitary and agninate glands, etc.), are described elsewhere.

Other structures, "blood-glands," bear the same relation to the blood-stream that lymphatic glands do to the lymph-stream.

Of these the chief representative is the spleen, an important gland partaking somewhat of the lymphoid type. In some mammals other blood-glands occur, the "hæmal glands." Bone-marrow, in structure and function, forms a sort of "bloodgland." Of similar nature, perhaps, are the small coccygeal and carotid glands.

The active ductless glands of the secretory or epithelial type are the thyroid, parathyroid, adrenal, and pituitary bodies; besides these are the ovary (which is of unique type), the rudimentary pineal body, and certain atrophic foetal structures about the genital organs, as the paradidymis and parovarium.

The functions of all these structures are more or less obscure, but (especially if the theory of internal secretion is true) they may be considered as probably belonging, in common, to the hæmapoietic (blood-forming) and hæmolytic (blood-destroying, blood-purifying) system, contributing corpuscular and chemical constituents to the blood or removing from it effete materials.

\section{The Spleen.}

General structure : The spleen (Fig. 43) consists of a pulpy parenchyma supported in a reticular sustentacular tissue; contains numerous lymphoid follicles (Malpighian corpuscles), and is freely supplied with bloodvessels opening directly into the reticular spaces. The outermost covering of the spleen is formed by the peritoneum. 
The sustentacular or connective-tissue framework of the spleen consists of a firm fibrous capsule enveloping the organ; of fibrous trabeculæ or septa, continuous with the capsule, traversing the organ in all directions and dividing it into small compartments or lobules; and of an open reticular tissue filling these compartments. This reticular tissue, which is of similar character to the retiform basis of lymphoid tissue, consists of fibres interlacing to form open communicating spaces or sinuses, which are incompletely lined with stellate flat endothelioid connective-tissue cells. These spaces open directly into the bloodvessels, and, filled with free cells of various kinds, form the splenic pulp.

Scattered involuntary muscle-cells occur in the capsule and larger trabeculæ.

The ample bloodvessels of the spleen enter at the hilum,

FIG. 43.

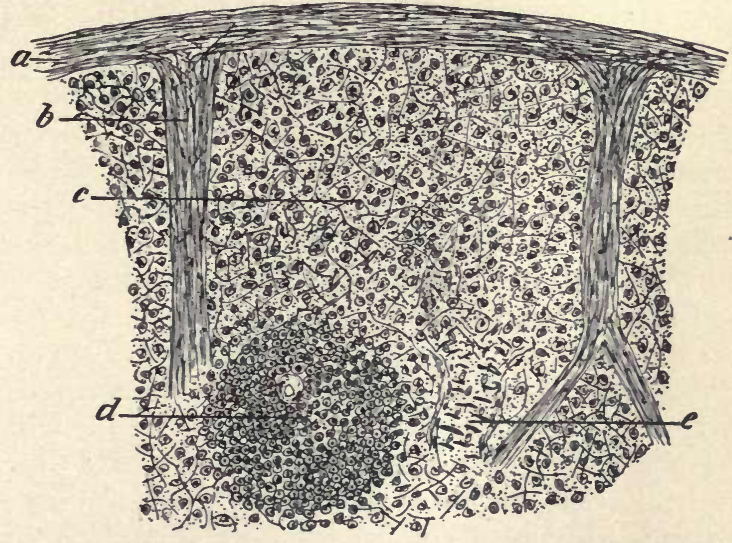

Portion of spleen. $a$, capsule; $b$, trabecula ; $c$, splenic pulp; $d$, Malpighian corpuscle; $e$, portion of artery.

and the larger branches lie in the trabecular tissue as they ramify through the organ. The terminal arteries and veins, instead of being connected by eapillaries, open into the retiform sinuses making up the splenic parenchyma, so that the blood-current percolates through this spongy tissue in its pas- 
sage from artery to vein. Toward the terminations of the bloodvessels, openings appear in their walls, and the vessels become lost in the sinuses, the endothelium of the tunica intima merging into the flat connective-tissue cells incompletely lining the reticulum.

The parenchyma of the spleen is mostly made up of the splenic pulp. 'This consists of the open network of sinuses filled with plasma and free cells, some of which are peculiar to this situation, others derived from the blood-stream passing through. The free elements of the pulp are : red blood-corpuscles in great abundance; lymphoid cells and leukocytes of all kinds ; large multinucleated amœboid granular cells ; and pigment-granules, free or within the leukocytes and amoboid cells, derived from the disintegration of the hæmoglobin of broken-down red blood-corpuscles.

The spleen is supplied with lymphatic vessels, which are much less conspicuous than the blood-supply.

Scattered about through the substance of the spleen are numerous spherical or oval nodules or follicles of typical lymphoid tissue, the Malpighian corpuscles. These occur surrounding small-sized arteries, which pierce them at or near their centres.

The function of the spleen is not thoroughly understood; it is concerned with the formation or purification of the blood. It may be a seat for the removal of worn-out red blood-corpuscles. Whether it contributes any corpuscular elements to the blood has not been settled.

The coccygeal gland (Luschka's gland) is a small, round pea-sized body, near the tip of the coccyx, in which the middle sacral artery terminates. It consists of a plexus of dilated capillaries, or even cavernous blood-sinuses, which are surrounded by masses of granular polyhedral connectivetissue cells. The gland is enveloped by a fibrous capsule, from which trabeculæ pass into the interior. It is plentifully supplied with nerves. Its function is unknown.

'The carotid glands are small bodies situated in the bifurcation of the common carotid arteries, one on each side. Their structure is similar to that of the coccygeal gland. 


\section{The Thyroid Gland.}

At an early embryonic period this gland opens into the pharynx by an outlet-duct; but this soon disappears, leaving the alveoli isolated and ductless.

The sustentacular connective tissue of the gland consists of a firm fibrous capsule enveloping the organ ; trabecule or septa (interlobular), of similar fibrous tissue, which divide the gland into lobes and lobules; and a delicate interalveolar or intralobular tissue between and supporting the secretory alveoli.

The glandular alveoli are spherical or oval saccules, without

Fig. 44.

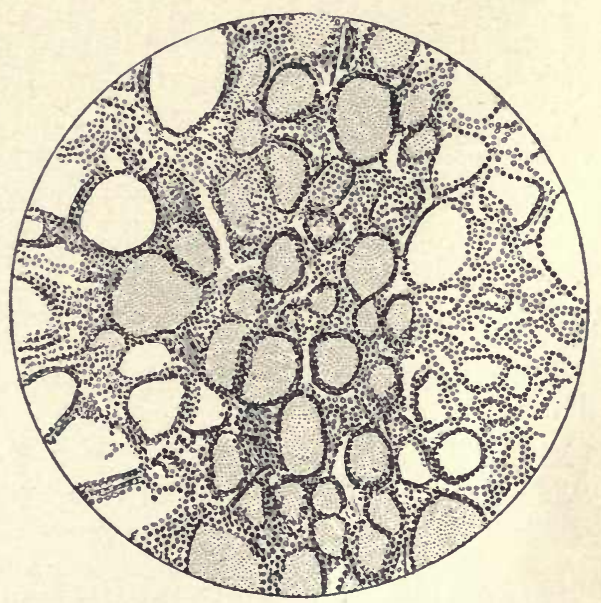

Alveoli of thyroid gland (Dunham).

outlet-ducts and entirely closed and isolated from one another (Fig. 44). They are lined with a single layer of cuboidal epithelium-cells with prominent nuclei. The cavities or lumina within the alveoli are of considerable size, are filled with secreted colloid substance, and their margins often have a scalloperl appearance.

The bloodvessels of the thyroid are exceedingly ample, and distribute a rich capillary network about the saccules. 
The lymphatics are also abundant, and through them the secretion of the gland is probably removed.

The function of the thyroid, as indicated by its rich bloodsupply and by the serious consequences of loss of the organ, is of great importance. Its secretion is entirely "internal," and by its action metabolism and cell-life are stimulated and hastened, obesity diminished, circulation is affected, and the cerebrum stimulated.

The parathyroids are small oval ductless glands about 6 to $10 \mathrm{~mm}$. long, situated near the thyroid, usually two on each side. They are enveloped by delicate fibrous tissue, and the epithelial columns and alveoli are separated by delicate trabeculæ of similar tissue. The connective tissue is very vasenlar. The parenchyma of the parathyroid bodies consists of polyhedral glandular epithelium-cells arranged in branching solid columns or hollow tubes, some of which occasionally contain secreted colloid material. The epithelium consists mainly of clear cells with large nuclei, not dissimilar to the cells of the thyroid gland. A few of the epithelial alveoli, however, consist of larger cells with granular oxyphile bodies, which look much like liver-cells; these cells are altogether different from those in the thyroid, and would seem to establish the individuality of the parathyroids as structures independent and distinct from the thyroid.

Little has yet been definitely ascertained as to the functions of the parathyroids, nor their genetic or vicarious relations to the thyroid.

\section{The Adrenal or Suprarenal Glands.}

General structure: These are ductless bodies made up of columns and masses of epithelium supported in sustentacular tissue, and abundantly supplied with bloodvessels and nerves (Fig. 45). The substance of the gland exhibits two parts, the cortex or peripheral portion, and the medulla or central portion.

The sustentacular connective tissue consists of a fibrous capsule enveloping the gland, and more delicate connective tissue permeating the interior and lying between the epithelial masses and vessels. 
The cortex consists of columns or nodules of epithelium (mostly without lumina), separated by delicate vascular connective tissue. The epithelium-cells are polyhedral, granular, and sometimes contain fatparticles. According to the arrangement of the epithelial masses, the cortex is divided into three zones : the zona reticularis, zona fasciculata, and zona glomerulosa, from within outward.

The zona reticularis, the innermost zone, consists of a network of epithelial columns anastomosing with one another. In the zona fasciculata, which comprises the largest part of the cortex, the epithelial columns are straight, parallel, radiating, and separate from one another. In the zona glomerulosa, which is next to the capsule, the epithelial columns are apparently coiled and convoluted, appearing on section as separate rounded nodules.

Capillaries and lymph-passages are abundant in the intercolumnar cortical tissue, in close relation with the epithelium-columns, which possess no basement-membranes.

The medulla of the adrenals

Fig. 45.

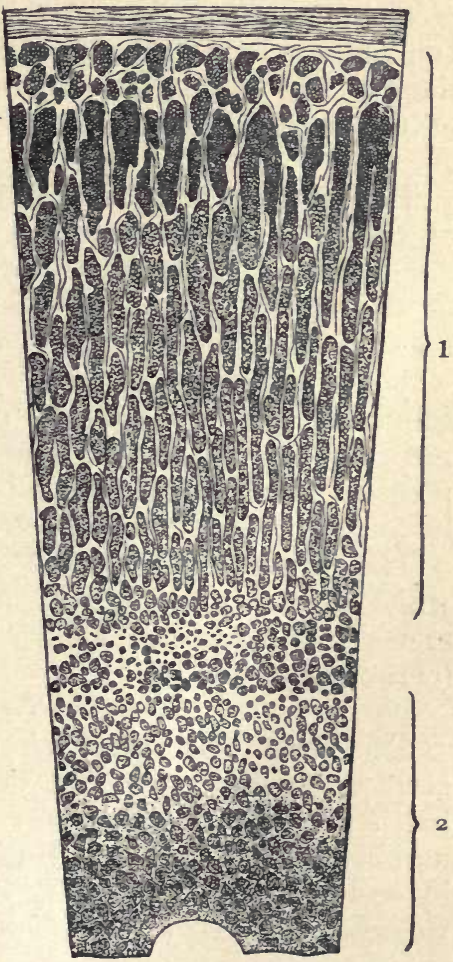

Section of human adrenal gland (Eberth). 1, cortex; 2, medulla. is quite distinct from the cortex and consists of epithelial masses and abundant bloodvessels, lymphatics, and nerves, supported in delicate connective tissue. The epithelium is arranged in branching and anastomosing nodules and columns or closed tubes; the cells are polyhedral or columnar. Capillaries are numerous and large venous sinuses are present. 
Nerve-fibres and small nerve-cells are abundant in the medulla.

The medullary portion of the adrenals is said to be of epiblastic origin, an outgrowth from the primitive spinal cord, while the cortex is derived from the mesoblast.

The internal secretion of the adrenals seems to be a powerful constrictor of the bloodvessels and is also probably a nervous stimulant. The organ is by some regarded as a part of the nervous system.

The pituitary body (hypophysis cerebri): The posterior lobe of this body is a nervous and fibrous structure, derived from and a part of the brain. The anterior lobe of the pituitary is of the nature of an epithelial ductless gland, being embryonically an epithelial outgrowth from the primitive pharynx, which is afterward cut off. It consists of columnar, closed tubular, or rounded acini of polyhedral epithelial cells, separated by fibrous septa. The acini are solid or a few may possess lumina containing colloid material. Most of the cells have clear bodies, but some are granular and oxyphile.

The pituitary is supposed to produce an internal secretion which has some influence on metabolism, as disease of it seems to be associated with peculiar anomalies of growth (acromegaly).

The pineal body (epiphysis cerebri), a small body near the corpora quadrigemina, has the structure of a ductless gland, but is probably a rudimentary sense-organ. It consists of a number of rounded acini lined by epithelium - cells, separated by and embedded in fibrous tissue. The acini contain gritty particles of earthy phosphates, the "brain-sand," or "acervulus cerebri." 


\section{CHA PTER X.}

\section{THE ALIMENTARY SYSTEM.}

THE alimentary tract is an apparatus in which food, the crude material of which the organism is built up, is received and digested by the action of various secretions, the elaborated products absorbed into the circulation, and the detritus discharged. It is a complex mechanism with many varied parts and functions, all working harmoniously together. It consists essentially of a long coiled muscular tube, the alimentary canal, opening at each end on the surface of the body, the walls of which contain glandular structures which secrete fluids and pour them into the canal to act on the raw food-materials, so as to fit them for absorption and nutrition. They also contain absorbent vessels by which the digested and elaborated food is introduced into the circulation.

The different parts and organs of this system are the mouth; teeth ; tongue; salivary glands ; tonsils ; pharynx ; œsophagus ; stomach ; small intestine; large intestine; rectum and anus; peritoneum ; liver and gall-bladder, and pancreas.

\section{The Mouth.}

The lining of the mouth consists of mucous membrane, which over the lips, floor of the mouth, cheeks, and soft palate rests upon the voluntary muscles in those situations; and over the bony parts, the maxillary and palate bones, rests upon the periosteum.

The mucous membrane of the mouth is continuous with that of the pharynx and tongue and with the skin, and in structure is similar to the latter. It consists of an epithelial lining, a tunica propria, and submucosa.

The epithelial layer is the representative of the epidermis of the skin, and consists of stratified squamous epithelium. 
The lower or germinal cells are rounded and spheroidal, but as the layers approach the surface the cells become gradually flatter and more squamous in character. This layer is very sinilar to the Malpighian layer of the skin. On its free surface the squamous cells approach a hard and horny condition and form an imperfect representative of the stratum corneum of the epidermis.

The epithelial layer rests on the tunica propria, a firm fibrous connective-tissue structure entirely analogous to the corresponding layer of the cutis vera. The outer surface of the tunica propria, as in the skin, is thrown up into numerous small rounded elevations, or papilloe, projecting upward into the epithelium; the intervals between the papilla are filled with epithelium-cells.

The submucosa consists of a variable amount of areolar and connective tissue extending from the tunica propria to the underlying structures. It contains numerous small racemose mucous glands, whose ducts open on the surface of the mucous membrane. Diffuse lymphoid tissue is present in the submucosa in places, especially in the vicinity of the fauces.

\section{The Teeth.}

General structure: The teeth present three divisions: the crown, projecting above the gum ; the fang, buried in the gum; and the neck, at the junetion of the two (Figs. 46 and 47 ).

Each tooth is made up chiefly of dentine, which is covered on the crown by encmel and on the fang by crusta petrosa or cement. The interior of the tooth is a cavity inclosed by the dentine, called the pulp-cavity, filled with a soft tissue or pulp.

The enamel is of epiblastic and epithelial origin ; the dentine and crusta petrosa are forms of connective tissue and are of mesoblastic derivation.

The fangs of the teeth rest in sockets of the jaw-bones lined by the periodontal membrane, which thus separates the tooth from the bone. This membrane is a vascular fibrous structure continuous with and similar in structure to the periosteum of the maxillary bones; it possesses transverse 
fibres, like the fibres of Sharpey, which penetrate both the bone and the crusta petrosa a short distance and hold the teeth firmly in place.

The enamel of the teeth is a very hard mineral (calcareous)

FIG. 46.

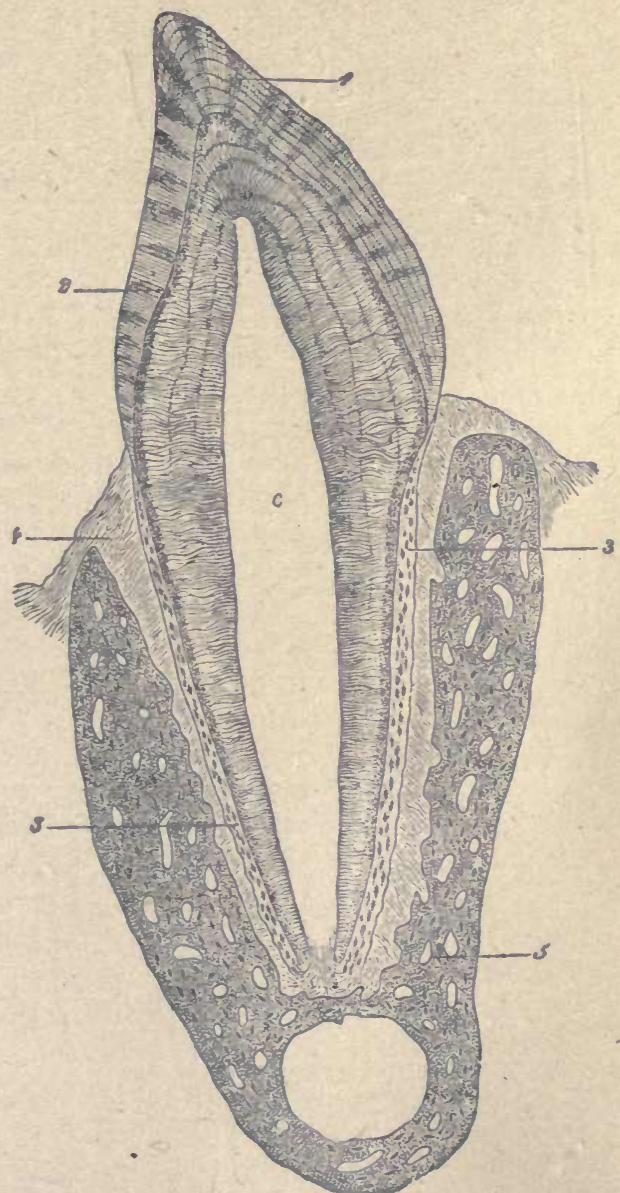

Section of tooth in situ (Waldejer). 1 , enamel; 2 , dentine; 3 , crusta petrosa ; 4 , periodontal membrane; 5 , maxillary bone; $c$, pulp-cavity.

8-Hist. 
substance, almost entirely free from organic matter, which invests the crown. It is disposed in slender straight or slightly wavy rods or prisms, of about six sides each, which are in general perpendicular to the surface of the dentine, and are held together by cement.

\section{FIG. 47.}

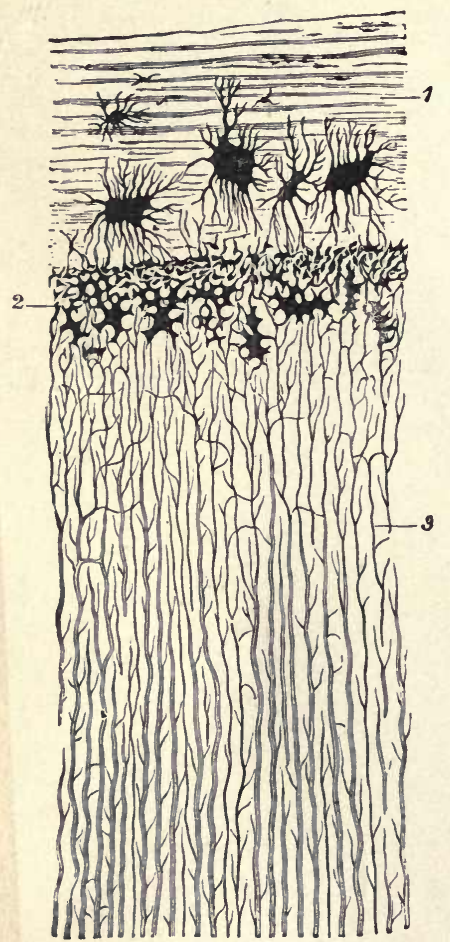

Section of portion of tooth (Waldeyer). 1, crusta petrosa, showing bony lacunxe and lamellæ; 2, interglobular spaces-granular layer of Purkinje; 3 , dentinal tubules. markings. At eruption and until worn off by attrition the enamel is covered with a thin epithelial cuticle, the membrane of Nasmyth, the remains of the enamel-organ.

The crusta petrosa is a thin layer of bone formed over the surface of the fang of the tooth by the osteogenetic action of the periodontal membrane which invests it. It is made up of bony lamellæ of the peripheric type, with the characteristic lacunæ and canaliculi.

The dentine, or ivory, which forms the bulk of the tooth, is a connective-tissue structure, impregnated and hardened by calcareous salts. It contains large numbers of minute channels or passages, the dentinal tubules, straight or slightly wavy, with short lateral branches; they are parallel to one another, run perpendicular to the surface of the dentine, and open into the pulp-cavity. Each tubule contains a dentinal fibre, a process from one of the odontoblasts or connectivetissue cells in the pulp-cavity. Surrounding each tubule is a dentinal sheath, a membranous portion of the calcified matrix especially resistant to the action 
of acid. The curves or undulations of the various tubules correspond to one another, and appear macroscopically as concentric lines, the lines of Schreger.

The peripheral portion of the dentine contains numerous stellate spaces communicating with the tubules, and in the fang with the canaliculi of the crusta petrosa; these are called interglobular spaces or dentinal lacunce, and from its granular appearance the zone where they occur is called the granular layer of Purkinje. These lacunæ are spaces left by incomplete calcification, and contain (at least at an early period) stellate connective-tissue cells.

The incremental lines of Salter are, roughly, concentric lines formed by interglobular spaces filled with an imperfectly calcified material; they indicate successive zones of calcification of the dentine.

The pulp-cavity is a space in the interior of the tooth entirely enclosed by the dentine, except at the apices of the fangs, where the vessels and nerves enter from the dental canals in the maxillary bones. The pulp consists of delicate connective tissue intermingled with bloodvessels and nerves. About the periphery of the pulp-cavity, lining the dentine which forms its wall, is a layer of modified connective-tissue ceils, called odontoblasts, long columnar or pyramidal cells lying perpendicular to the dentine and sending processes, the dentinal fibres, into the dentinal tubules.

Development of the teeth: The enamel is developed from epithelium, the remainder of the tooth from the subepithelial connective tissue. Morphologically teeth are usually regarded as modifications of the papilla of the mucous membrane. At the beginning of the development of the teeth, the epithelium of the mucous membrane on the edge of the jaw grows downward into the submucous tissue, dividing into a process for each tooth. These processes expand below and narrow above, forming hemispherical epithelial masses, called enamel-organs, whose connection with the surface-epithelium finally becomes entirely cut off or reduced to a mere thread of epithelial cells. Meantime beneath each enamel-organ the conneetive tissue becomes vascular and rich in cells, forming a papilla, which grows upward, pushing its way inte and invaginating the lower surface of the enamel-organ, so that the latter forms a 
sort of hood over it. The connective-tissue cells at the periphery of the papilla become modified and arranged in a layer of odontoblasts, which then produce the dentine.

The enamel-organ consists of epithelium cells at first like those of the lower or germinal layers of the mucous membrane. The outermost layer of cells of the enamel-organ become of a columnar or cuboidal type, while the interior cells degenerate into a pulpy mass of branched cells with anastomosing processes; the cells ("adamantoblasts") on the lower surface of the enamel-organ become especially long and columnar, and these cells secrete, or become transformed into, columns of enamel resting on the surface of the dentine.

The crusta petrosa is bony matter subsequently deposited on the fangs by the osteogenetic action of the periodontal membrane.

The permanent teeth are formed in a similar manner to the temporary teeth, their enamel-organs being developed from buds given off from the epithelial processes which form the enamel-organs of the temporary teeth.

\section{The Tongue.}

General structure : The tongue consists of a mass of voluntary muscle, covered with mucous membrane, and contains small glands, lymphoid tissue, blood and lymphatic vessels, the terminals of the special sense of taste, and other nerves.

The muscular substance of the tongue is made up of fasciculi of voluntary muscle-fibres interlacing and running in all directions, supported and bound together by connective tissue. A vertical fibrous septum divides the tongue into two lateral halves.

The mucous membrane, which is continuous with that of the mouth and pharynx, is made up of the usual three layers, of surface-epithelium, tunica propria, and submucous tissue.

The epithelial covering consists of stratified squamous epithelium, the surface-cells becoming rather horny; it is very similar to that lining the mouth and to the Malpighian layer of the skin. The tunica propria is a firm fibrous membrane supporting the epithelium and giving strength to the mucous membrane. The submucosa consists of areolar tissue con- 
tinuous with the connective tissue investing the muscle-fasciculi in the interior. The glands of the tongue are of two kinds, mucous and serous.

The mucous glands are small racemose glands, occurring in considerable numbers, and situated in the submucosa and between the muscular fasciculi.

The serous glands, or glands of Ebner, are small racemose glands occurring in the vicinity of the circumvallate papillæ and discharging their watery secretion into the furrows surrounding these papillæ.

Nodules and masses of lymphoid tissue are abundant, under-

FIg. 48.

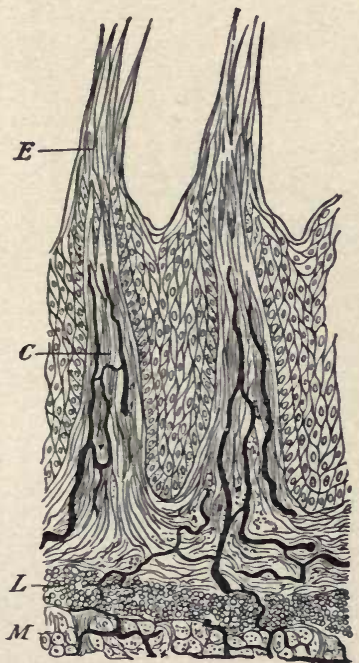

Fra. 49.

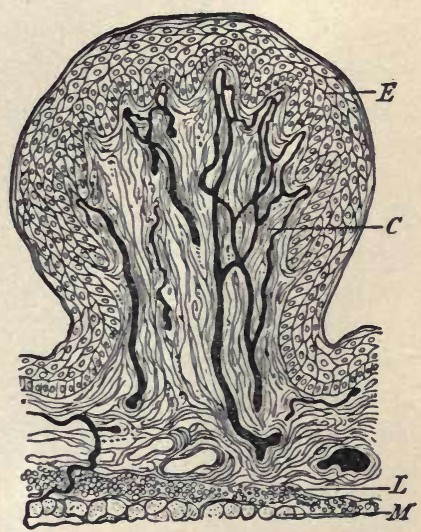

Papillæ of human tongue (Heitzmann).

Fig. 48.-Filiform papillæ.

Fig. 49.-Fungiform papilia. $E$, epithelium ; $C$, tunica propria, showing capillaries ; $L$, lymphoid tissue; $M$, muscle.

neath the epithelium, at the base of the tongue, forming the so-called lingual tonsil. The tongue is amply supplied with blood-and lymph vessels and nerves of motion, general sensation, and special taste-sense.

Papillæ of the tongue (Figs. 48, 49, 50) : On the upper sur- 
face of the tongue the epithelium and tunica propria are thrown up into papillæ, which are of three or four kinds-filiform, fungiform, circumvallate, and foliate.

The filiform papilloe are most numerous and thickly stud the upper surface of the tongue. They consist of slender papillary processes of the tunica propria, surmounted by slender conical projections of epithelium, the cells of which at the apex are horny and worn by attrition.

The fungiform papilloe are larger and much less numerous than the filiform, and are scattered about among the latter as visible red points. 'They are lower, broader, and have blunt

FIG. 50.

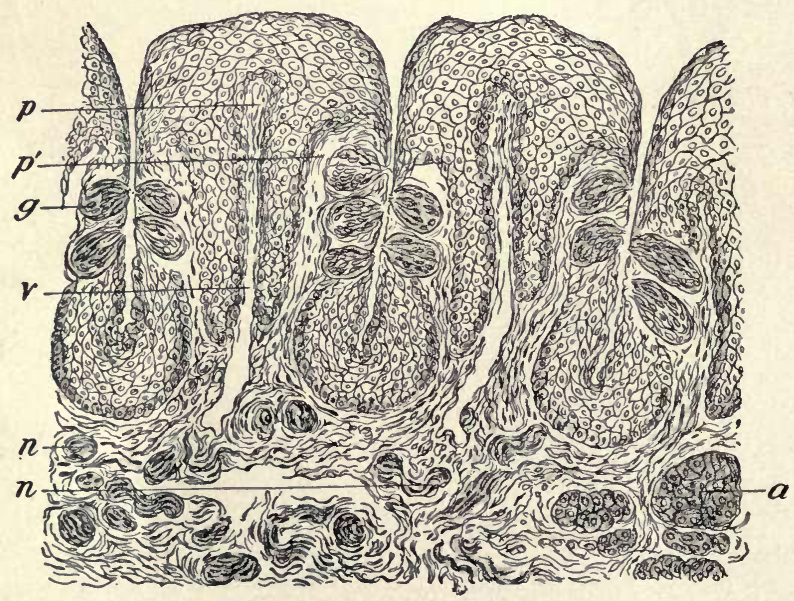

Circumvallate papillæ, of rabbit (Ranvier).

$p$, fibrous core of papilla; $g$, taste-buds ; $n$, nerves ; $a$, gland of Ebner.

rounded tops. They consist of a connective-tissue core projecting from the tunica propria, with minute secondary projections from its surface, all covered with a layer of epithelium.

The circumvallate papilloe are eight to twelve large broad papillæ arranged in a $\mathrm{V}$ shape at the base of the tongue. The papillæ are similar to the fungiform. but are larger, and each is surrounded by a deep furrow or diteh, so that the yapillæ 
themselves do not project much above the surrounding surface, but are depressed.

Scattered about in the epithelial covering of the sides of the furrow are numerous taste-buds or taste-goblets, oval bodies made up of modified epithelium and containing the terminals of the nerves for the special sense of taste.

The serous glands of Ebner open into the bottom of the furrows, their watery secretions apparently aiding the solution and dissemination of substances acting on the taste-terminals.

The foliate papilloe consist of a few parallel folds of the mucous membrane on each side of the base of the tongue, forming alternate ridges and furrows rather than papillæ. They are better developed in some of the other mammals, as the rabbit, than in man. The sides of their furrows contain taste-buds.

The taste-buds are terminals for the special sense of taste, and occur in the sides of the furrows of the circumvallate and foliate papillæ, with a few scattered about in the epithelial layer of the base of the tongue, the lingual surface of the epiglottis, and the soft palate. They are oval or egg-shaped bodies, with the long axis extending through the entire thickness of the epithelial layer, perpendicular or slightly oblique to the surface. They are made up of two kinds of modified epithelial cells, the external cortical cells, and the internal gustatory cells. The outer or cortical cells, also variously called protective, sustentacular, supporting, and tegmental cells, are long, slender, flattened, and fusiform cells, each with a prominent nucleus near its middle.

These cells are arranged longitudinally so as to form an oval body or covering for the gustatory cells, which lie in the axis of the taste-bud. The latter are very slender linear neuro-epithelium cells, with large nuclei near their middle causing a bulging at that point. They extend the entire length of the taste-bud, surrounded by the cortical cells. Their outer ends terminate in fine hairs, reaching the surface of the mucous membrane through a minute opening or pore at the apex of the taste-bud; their inner ends are slender or branched, and are supposed to communicate with fibres of the special nerves of taste. 


\section{The Salivary Glands.}

The salivary glands are the parotid, sublingual, and submaxillary, besides the numerous small mucous glands (labial, buccal, lingual, and palatal) distributed over the mucous membrane of the mouth and tongue.

General structure: They are racemose glands, consisting of saccules and ducts supported in sustentacular tissue, divided into lobes and lobules, and well supplied with bloodvessels, lymphatics, and nerves.

The sustentacular connective tissue consists of a firm fibrous capsule enveloping the entire gland, sending in trabeculæ (interlobular septa) which separate the various lobules and bear the larger vessels and nerves and the interlobular ducts, and of delicate intralobular tissue penetrating among the individual saccules, and forming basement-membranes for their support.

The ultimate saccules or alveoli, the secreting portions of the glands, are lined with polyhedral or spheroidal glandular epithelial cells of the serous or mucous type.

The interlobular ducts are lined with simple columnar epithelium-cells, which toward their attached bases are marked with longitudinal striæ or rods.

In the intralobular and intermediate ducts the epitheliumcells forming the lining become lower and more flattened.

The salivary glands are amply supplied with bloodvessels, a capillary plexus surrounding the various saccules. The lymphatics consist of lymph-spaces between the alveoli, and a system of vessels. Fine terminal nerve-filaments are supplied to the secretory-epithelium cells in great abundance, significant of the well-known great power which nervous influences exert on the secretary action of these glands.

The different salivary glands vary essentially only in the character of their secretory elements. The parotid is a serous gland, the sublingual is mucous, the submaxillary nixed.

In the parotid gland the secreting epithelium and alveoli are of the serous type. The saccules are lined with a single layer of spheroidal, granular, deeply staining, opaque cells, with rounded, central nuclei. In the resting stage, when charged with secretion, the cells are more distended, encroach 
on and tend to fill the lumen, contain more granules, and stain less deeply. In the exhausted condition, when the secretion is discharged, the cells are somewhat contracted and smaller, leaving the lumen larger, and appear darker, more opaque, and more deeply stained from the condensation of the protoplasm.

In the sublingual gland, which is of the mucous type, the alveoli are rather larger than in the serous parotid, and have a prominent basement-membrane. The cells lining the alveoli are of two kinds, the ordinary mucin-secreting (central) cells and the peripheral or demilune cells.

Frg. 51.

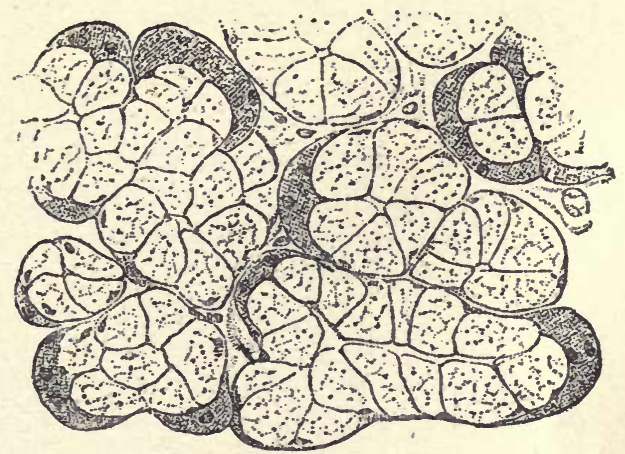

Mucous alveoli from submaxillary gland, showing mucinogenous cells and demilunes of Heidenhain (Ranvier).

The former make up much the larger part of the gland and form a single layer of spheroidal epithelium-cells lining the lumen, and (except where the demilunes are situated) resting upon the basement-membrane. These cells are of the type characteristic of mucous glands; when resting and distended with secretion they are swollen, and except where their bodyprotoplasm and nuclei are pressed toward the attached bases the cell-bodies are clear, transparent, and do not stain deeply. After exhaustion and discharge of the secretion by prolonged activity, the cells become smaller, less clear and transparent, and the more concentrated protoplasm stains more deeply.

In places between the mucinogenous or central cells are 
situated wedge-shaped, pyramidal, or crescentic bodies called the crescents of Gianuzzi or demilunes of Heidenhain, each of which is made up of a few opaque granular deeply staining dark cells. The significance of these demilune cells is quite unknown, though several views as to their nature have been propounded, some regarding them as younger germinal cells destined to develop into mucinogenous cells, others as old and exhausted cells which have not taken part in the secretory process, others still as being of the serous type.

The submaxillary gland is mixed, some of its alveoli being serous, like the parotid, others mucous and with demilunes, like the sublingual.

\section{The Tonsils.}

The tonsils (Fig. 52) are lymphoid glands located between the pillars of the fauces on each side. They consist of aggregations of lymphoid tissue enveloped by a fibrous capsule formed by a condensation of the submucous connective tissue. They are covered by the oral mucous membrane, and rest on the superior constrictor muscles of the pharynx.

The mucous membrane forming the surface of the tonsil dips down into a number (ten to fifteen) of blind depressions, or crypts, or follicles, each of which is lined with a continuation of the stratified squamous epithelium and surrounded by adenoid tissue.

Mucous glands are present, which discharge into the crypts. The lymphoid cells infiltrate the tunica propria of the mucosa so densely as to obscure it; they even penetrate among the epithelial cells, often making the boundary between the epithelial layer and the subjacent lymphoid tissue indistinct. The lymphoid cells make their way entirely through the epithelium in some number, and appear in the mouth as salivary corpuscles.

The lymphoid tissues in the tonsils, base of the tongue, palate, and pharynx form an adenoid ring about the fauces and pharynx. These structures in the tongue and pharynx are sometimes called the lingual and pharyngeal tonsils.

The saliva as secreted by the salivary glands, is a homo- 
geneous muco-serous fluid not exhibiting any features to the microscope; but as it occurs in the mouth it contains a few adventitious elements, those derived from the local tissues being epithelium-cells, leukocytes, and salivary corpuscles; while bacteria, fungi, food-particles, and other foreign objects

FIG. 52.

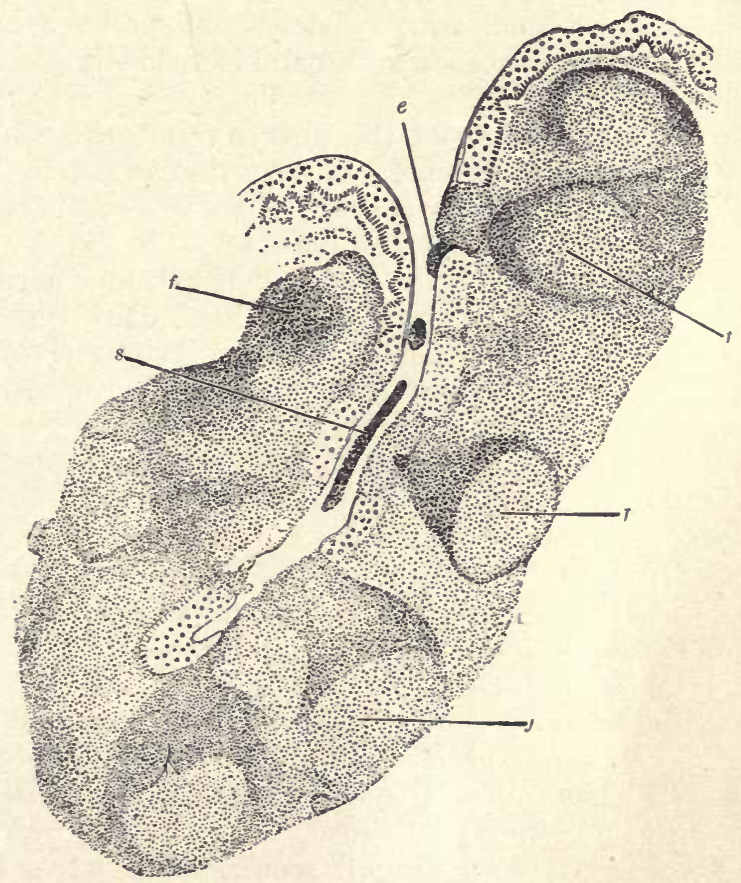

Section through crypt of tonsil (Stöhr). $e$, epithelium; $f$, lymphoid follicles; $\varsigma$, material within the crypt, composed partly of escaped lymphoid cells.

of extraneous origin may be present. The epithelial cells present are large transparent squamous cells with prominent nuclei and coarse granules in the body of the cell; their edges are often curled. The leukocytes that may be present are such as have wandered from the bloodvessels. The salivary corpuscles are lymphoid cells escaped into the mouth from the 
vicinity of the tonsils; after exposure to the saliva with its low specific gravity they become swollen and granular.

\section{The Pharynx.}

The walls of the pharynx consist of three layers, mucous membrane, a fibrous coat, and a muscular layer. The upper part of the pharynx, above the palate, belongs to the respiratory tract and is lined with eiliated epithelium; the lower part belongs to the alimentary tract and is lined with squamous epithelium.

The mucous membrane of the pharynx is continuous with that of the adjacent cavities, and consists of three layers, epithelium, tunica propria, and submucosa, and contains mucous glands and lymphoid tissue.

The epithelial lining in the upper or respiratory portion of the pharynx consists of stratified ciliated epithelium, continuous with that of the nasal fossæ and Eustachian tubes; in the lower or alimentary portion it consists of stratified squamous cells.

The tunica propria is a well-marked fibrous membrane, which in the lower part of the pharynx has papillæ projecting among the squamous epithelium.

The submucosa is a layer of areolar tissue connecting the mucosa with the fibrous and muscular tissues beneath, and containing numerous small racemose mucous glands and nodules of lymphoid tissue.

The lymphoid nodules often have crypts, like those of the tonsils. There is a special collection of lymphoid tissue on the posterior surface of the pharynx between the Eustachian tubes, called the pharyngeal tonsil.

The fibrous coat, or pharyngeal aponeurosis, is a firm fibrous membrane lying underneath the mucous membrane, thicker above, and becoming thinner below. Posteriorly it is thickened to form the median raphe.

The muscular layer, beneath the fibrous, is a sheet of voluntary muscle made up by the three constrictors and other muscles of the pharynx.

The soft palate consists of a thin layer of the voluntary palate muscles with fibrous aponeuroses, lined on each side by 
mucous membrane like that of the mouth and pharynx, of whose walls it forms a portion. The epithelial covering consists of squamous epithelium, except at the upper part of the posterior surface, where it is ciliated. Mucous glands and lymphoid tissue are abundant.

\section{The Esophagus.}

The esophagus is a tube whose walls consist of four coats, mucous, submucous, muscular, and fibrous, from within outward.

The mucous membrane, which when the osophagus is collapsed is thrown into longitudinal folds, consists of the epithelial covering, the tunica propria, and the muscularis mucosæ.

The epithelium lining the lumen of the tube (Fig. 11) is composed of stratified squamous cells, flattened on the surface and gradually becoming thicker and more spheroidal in the lower layers; it is similar to and continuous with the epithelium of the pharyngeal mucous membrane.

It is underlaid by the tunica propric, a dense fibrous layer from the surface of which papillæ project upward into the epithelium.

The muscularis mucosce is a thin layer of involuntary muscle-cells, longitudinally arranged, separating the mucosa from the submucosa. It is not present in the upper part of the œesophagus, but appears below in scattered strands, gradually increases downward, and in the lower part of the tube forms a well-marked complete ring.

The submucosa, next below, is a layer of loose areolar tissue, which permits the longitudinal folding of the mucous membrane. It contains small racemose mucous glands, discharging on the surface, blood and lymphatic vessels, and nerves.

The muscular layer, which lies beneath or outside of the submucous coat, is composed of two coats, an inner, in which the muscle-cells are arranged circularly or transversely; and an outer, in which they are longitudinal. In the upper portion of the osophagus the muscle is striated and voluntary, in the lower portion non-striated and involuntary ; in the middle it is mixed and exhibits a gradual transition from one kind to the other. 
The outer or fibrous investment of the osophagus consists of areolar tissue continuous with that surrounding the adjacent structures.

FIG. 53.

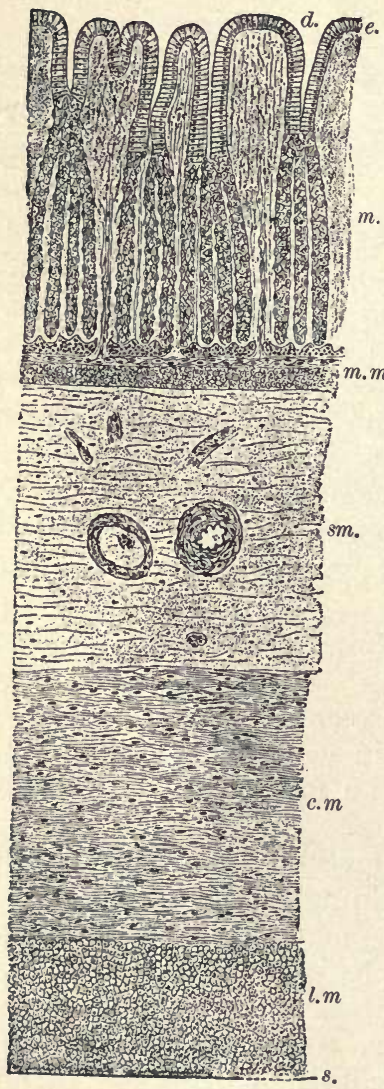

Diagrammatic section through coats of stomach (Mall). m, mucosa; $e$, epithelium; $d$, outlet of peptic gland; $m m$, muscularis mucosæ: $8 m$, submucosa ; $\mathrm{cm}$, inner circular muscular layer; $l m$, outer longitudinal muscular layer; 8 , peritoneum.

\section{The Stomach.}

The walls of the stomach (Fig. 53) may be divided into four coats, which are, from within outward, the mucosa (containing two kinds of glands, peptic and pyloric), the submucosa, the muscular layer, and the serous or peritoneal covering.

The mucosa consists of the epithelial covering, subepithelial connective tissue, muscularis mucosæ, lymphoid tissue, and the peptic and pyloric glands.

The epithelial lining of the stomach consists of a single layer of long columnar epitheliumcells, with their nuclei situated near their attached bases; among the columnar cells goblet-cells are abundant. The surface of the stomach dips down into closely crowded tubular depres'sions, the gastric glands, which are perpendicular to the surface and occupy the entire thickness of the mucosa. They are of two kinds, peptic and pyloric.

The peptic or cardiac glands occupy the cardiac portion and fundus of the stomach, yielding to the pyloric glands at the pyloric extremity. The peptic glands are simple or slightly divided tubnlar glands, often with the lower blind ends some- 
what bent and coiled. The ducts of these glands are short, and lined with a continuation of the surface columnar epithelium; the secreting portions of the tubules join the ducts at the necks of the glands. Besides occasional gobletcells, the fundus or secretory part of the peptic glands is lined with glandular epithelium of two kinds, central or chief cells, and parietal cells. The cential or chief cells are rather pale, faintly granular, columnar cells, which form a complete lining to the lumen of the glands; they are supposed to secrete pepsin. Between them and the basementmembrane, and often causing slight bulging of the latter, are scattered numerous larger oval prominent cells, the parietal cells, supposed to be connected with the secretion of hydrochloric acid; they do not form a complete layer, not coming into contact with one another, and do not usually abut on the lumen. Differences in the appearance of the cells when charged with secretion and when exhausted after digestion occur, analogous to the similar changes in other serous glands.

The pyloric glands (Fig. 54) occupy the pyloric end of the stomach. They are compound tubular glands, and are similar to the peptic glands, but have longer and wider ducts and shorter secreting portions, their lumen is opener and greater in diameter, they are more branched (more compound),

FIG. 54.

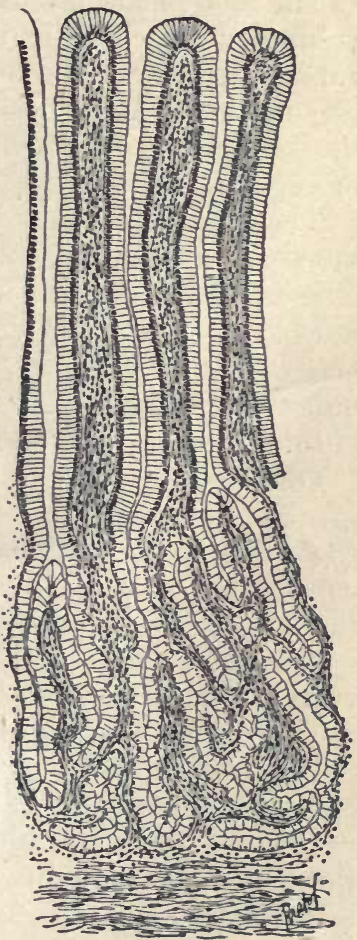

Pyloric glands (Böhm and Davidoff). and they contain no parietal cells, being lined with central cells only.

The subepithelial connective tissue of the gastric mucosa, the 
representative of the tunica propria, is a loose and delicate connective tissue extending between the glands and providing basement-membranes and sustentacular tissue for their support. It contains a rich capillary plexus enveloping the glands. Lymphoid cells are scattered about and diffuse lymphoid tissue occurs in places in the subepithelial tissue; lymphoid nodules occur especially in the pyloric region, where they are called lenticular glands.

The muscularis mucosce consists of two thin layers of involuntary muscle-cells, the outer longitudinal, the inner circular ; it separates the mucosa from the submucosa, also sending muscular branches up among the gland-tubes. When the stomach is contracted the mucosa is thrown into folds or rugæ, permitted by the loose nature of the submucosa.

The submucosa is a loose areolar tissue lying beneath the mucosa. It contains the larger blood and lymphatic vessels. The muscular coat of the stomach, lying beneath the submucosa, is a thick layer of involuntary muscle, divided in general into two parts, an inner arranged circularly and an onter longitudinal. At the pyloric orifices the circular muscle is thickened to form a sphincter.

Outside the muscular layer, and covering the outer surface of the stomach, is the serous or peritoneal coat, a portion of the peritoneun reflected over the surface of the organ. It consists of a thin fibrous membrane covered with endothelium.

The stomach is richly supplied with blood and lymphatic vessels, the larger trunks lying in the submucous tissue and giving off capillaries to form a rich network in the mucosa.

The gastric juice consists of a homogeneous serous secretion, containing leukocytes and epithelium-cells partly digested, "snail-shell-like" granules probably formed by the action of the juice on mucin, and extraneous food-particles and bacteria.

\section{The Small Intestine.}

The walls of the small intestine are composed of four layers, mucous, submucous, muscular, and peritoneal, from within outward. They are studded with papillary structures called 
villi, and contain two kinds of secretory glands, intestinal and duodenal, lymphoid structures, bloodvessels, lymphatics, and nerves.

The mucous membrane is thrown into transverse and oblique folds, the valvulce conniventes. Its free surface is raised in papillary projections, called villi, between the bases of which the intestinal glands dip down. The mucosa con-

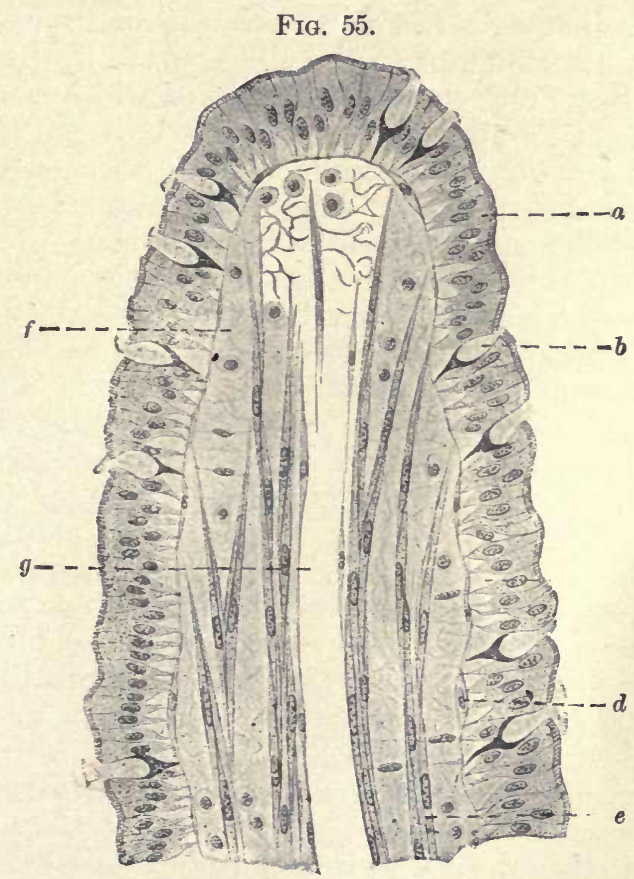

Axial section of villus (Kultschitzky). $a$, epithelium ; $b$, gob?et-cell ; $d$, connectivetissue cell of basement-membrane; $e$, smonth muscle-cells; $f$, reticulum of tunica propria; $g$, central lacteal.

sists of a lining of simple columanar epithelium, which differs somewhat in the villi and the glands; subepithelial connective tissue; adenoid tissue; bloor and lymph vessels, and the muscularis mucosæ.

The villi (Fig. 55) are minute club-shaped papillæ about a 9-Hist. 
millimetre long, which thickly stud the inner surface of the entire small intestine. In places they exhibit slight constrictions, called Watney's nodes. They are covered with a single layer of long columnar epithelial cells (Fig. 13), with their nuclei near their attached bases. The protoplasm of the free ends of these cells contains fine parallel longitudinal striæ, giving the appearance of a narrow striated border. Gobletcells are numerous among the columnar cells.

The epithelium-cells rest on a basement-membrane, and the framework in the interior of the villi is made up by an open connective-tissue reticulum, the meshes of which contain free lymphoid cells, forming a diffuse lymphoid tissue. The core or axis of the villus is formed by a small lacteal, a lymphatic capillary or radicle; this is a tube or sac formed by a single layer of endothelium-cells, with a blunt or slightly expanded blind extremity above (toward the lumen of the intestine), and emptying below into the larger lymphatic vessels of the submucosa. A minute artery enters each villus and breaks up into a rich capillary network which surrounds the lacteal. Muscular projections from the muscularis mucosæ extend up into the villi, and apparently serve by periodical contraction to empty them of their contents. The function of the villi is to absorb the digested food-materials from the intestinal ornal into the blood and lymph circulations. They especially absorb the insoluble and indiffusible fatty matters, which pass through the epithelium-cells in small granules, through the reticulum of the villus, and make their way into the lacteal.

The intestinal glands, or follicles of Lieberkïhn, are straight simple tubular glands between the bases of the villi, ard dip down to the muscularis mucosæ. They, and the surface between their orifices, are lined with a single layer of short granular columnar epithelial cells, with nuclei near their bases, which rest on a basement-membrane. Goblet-cells are interspersed among these columnar cells. These glands occur throughout the small and large intestines; they secrete the intestinal juice and also perhaps have absorptive functions.

The subepithelial connective tissue of the mucosa, the analogne of the tunica propria, consists of a delicate reticular tissue, embracing the glands and projecting up to form the framework of the villi, the surface condensed into a basement- 
membrane. It contains numerous lymphoid cells and diffuse lymphoid tissue, and is richly supplied with lymphaties and blood-capillaries. The muscularis mucosce consists of two thin layers of involuntary muscle, the inner circular, the outer longitudinal. It lies just below or outside of the extremities of Lieberkühn's glands, and separates the mucosa from the submucosa. It sends fibres up into the villi.

The submucosa of the small intestine is a loose areolar layer, permitting some play between the mucous and muscular coats. It contains the larger blood and lymphatic channels, which send capillaries into the mucosa, and a nerve-plexus, the plexus of Meissner.

The duodenal glands, or glands of Brunner, are rounded compound racemose or perhaps tubular glands, of the serents type, located in the submucosa of the duodenum. Each empties by a long outlet-duct which pierces the muscularis mucosæ and traverses the mucosa to the surface, parallel to the glands of Lieberkühn. Their cells are similar to those of the intestinal glands.

The lymphoid glands of the small intestine are definite and dense masses of lymphoid tissue, which occur in addition to the diffuse adenoid tissue of the mucosa; they are of two kinds, solitary and agminate. The solitary glands are spherical nodules or follicles of lymphoid tissue, occurring singly, and circumscribed by a condensation of the surrounding connective tissue. They are situated in the submucosa, or may extend into the mucosa and even to the epithelial covering. 'They occur throughout the small and large intestines, but are more numerous toward the lower part of the ileum and the beginning of the colon.

The agminate glands, or Peyer's patches, are aggregations of a number of solitary lymph-follicles placed in juxtaposition, and sometimes surrounded by looser adenoid tissue. They occupy the submucesa, some of the follicles in places also piercing the muscularis mucosæ and extending into the mucosa and to the surface-epithelium. They form longitudinal patches two to eight centimetres long opposite the mesenteric attachment of the intestine, and are most abundant in the lower portion of the ileum.

The muscular coat of the small intestine comprises two 
layers of involuntary muscle, a thick inner circular layer, and a thinner outer longitudinal layer. Between these two is the nerve-plexus of Auerbach.

The serous or peritoneal coat is the portion of the peritoneum reflected over the intestine to form its outer covering.

The intestine is well supplied with blood and lymphatic vessels, the larger channels of which are in the submucosa and send capillary plexuses into the mucosa. The lacteals constitute an important origin of the lymphatic system.

The intestine is also supplied with two plexuses of nerves; the plexus of Auerbach is a network of fibres with minute ganglia at their intersections, situated between the two layers of the muscular coat; the plexus of Meissner, situated in the submucous coat, is connected with and similar to that of Auerbach, but is made up of finer fibres and finer meshes.

\section{The Large Intestine.}

The large intestine has four coats, mucous, submucous, muscular, and serous, and contains glands of Lieberkïhn and solitary glands. It resembles the ileum in structure, but has no villi or Peyer's patches, and the outer muscular coat is differently arranged.

Its mucosa, lined with simple columnar epithelium, is everywhere studded with Lieberkühn's follicles, supported in a delicate subepithelial retiform tissue, which is well supplied with diffuse lymphoid tissue, capillaries, and lymphaties.

The glands of Lieberkühn are rather larger than in the small intestine, but are lined with similar cells, among which, however, goblet-cells are much more abundant.

The muscularis mucosce, vascular and nervous plexuses, submucous and peritoneal coats are much the same as in the small intestine, and solitary glands are abundant.

The muscular coat has the usual inner circular layer, rather thin in most places. The outer layer of longitudinal, nonstriated muscle, however, instead of forming a complete sheet, is limited to three longitudinal ' bands, which are shorter than the other structures, and thus cause the colon to be puckered or sacculated. 
The appendix vermiformis is similar in structure to the colon. The mucosa contains well-formed glands of Lieberkühn, and it and the loose submucosa contain numerous lymphoid follicles and diffuse lymphoid tissue. The muscular coat and peritoneal covering form the outer layers.

The rectum has a mucous and submucous layer similar to that of the large intestine. The covering of simple columnar epithelium changes at the anus into the stratified squamous variety.

The internal circular muscular layer is thickened to form the internal (involuntary) sphincter, over which the longitudinal muscular layer spreads out to form a complete investment (instead of consisting of three bands only). The external sphincter is a thin voluntary muscle surrounding the anus. The lower portion of the rectum has no peritoneal covering.

The peritoneum is a large serous or lymph sac, formed by a typical serous membrane covering the walls of the abdomen (parietal layer) and the abdominal viscera (visceral layer). It enables the latter to move freely.

It is mainly made up of a fibrous membrane containing lymphatics and bloodvessels, lined on the free surface with polygonal endothelium-cells, between which in places are lymphstomata.

Beneath it is a variable amount of areolar subserous or subperitoneal tissue, in which, especially in the parietal layers, mesenteries, and omenta, masses of fatty tissue often accumulate.

The blood, lymphatic, and nerve trunks which supply the intestines are situated in the mesenteries, which also contain numerous lymphatic glands.

\section{The Liver.}

The liver is usually classsed as a gland, producing the bile as a secretion (external). It is much more than this.

All the venous blood from the stomach and intestines, containing the products of digestion absorbed from the alimentary canal, is collected in the portal vein, redistributed in capillaries and passed through the liver as through a sieve, and is acted 
on by intimate contact with the liver-cells before being again collected in the hepatic vein and discharged into the general circulation.

In its passage through the liver the constituents of the blood are, in ways not well understood, altered by the action of the liver-cells for purposes useful to the animal economy. This action on the blood, of which the glycogenic and urea-forming functions are known instances, partakes of the nature of "internal" secretion. The liver also serves as a storehouse for certain substances. As a gland the liver is of the compound tubular type ; but in mammalia it is constructed more with reference to its functions bearing on the blood, and its glandular structure is masked and obscure.

General structure : The liver consists of masses of epithelial cells divided into small lobules or acini, supported in sustentacular tissue, and contains a network of outlet bile-ducts and

FIG. 56.

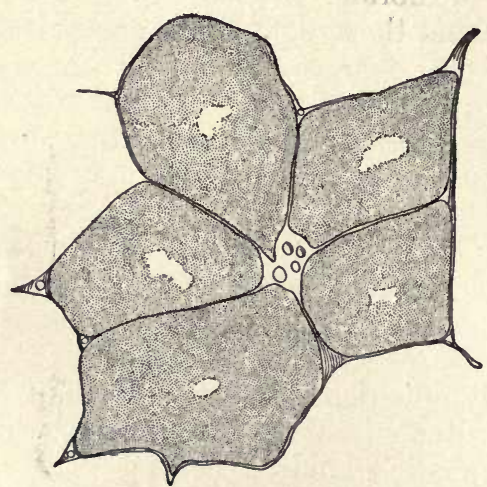

Lobules of liver of pig, showing interlobular septa and a portal tract (Klein).

three systems of bloodvessels : the hepatic artery, portal vein, and hepatic vein ; besides scanty lymphatics and nerves.

The sustentacular or connective tissue of the liver consists of the capsule (of Glisson), interlobular septa, and the "portal tracts."

The capsule of Glisson is a fibrous envelope investing the liver, beneath the peritoneum. The interlobular septa are 
delicate partitions, poorly marked in the normal human liver, which separate the hepatic lobules. 'The portal tracts are wellmarked processes of tibrous tissue passing into the liver with the portal vein, hepatic artery, and bile-ducts, and surround-

FIG. 57.

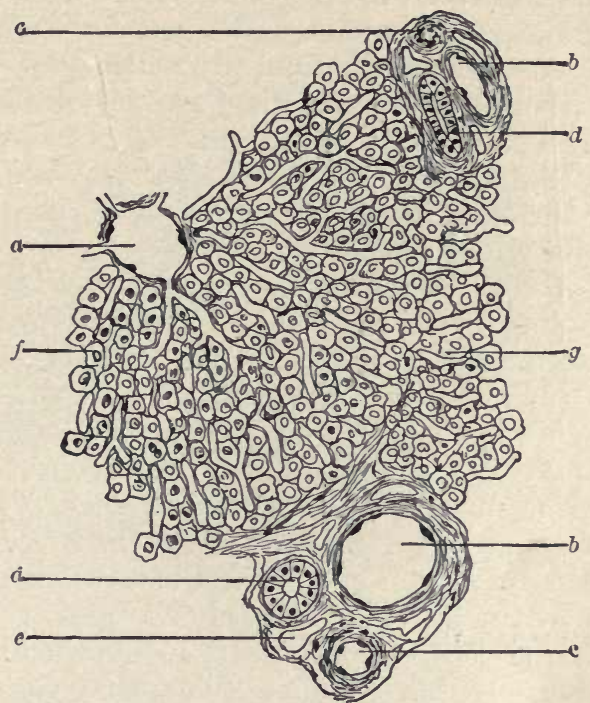

Diagram of portion of a lobule of the liver (Dunham). $a$, intralobular vein, receiving intralobular capillaries; $b$, branches of portal vein; $c$, branches of hepatic artery ; $d$, bile-ducts; $e$, lymph-vessel ; $f$, liver-cells ; $g$, junction of two lobules.

ing, accompanying, and supporting these vessels in all their subdivisions and ramifications throughout the organ. These tracts are situated at the junction of three or four lobules, and send lamellæ between the adjacent lobules continuous with their interlobular septa, indeed forming the only portions of these septa conspicuous in the normal liver.

In cross-section the portal tracts are therefore triangular or stellate, and in each may be seen three or more vessels, the largest a branch of the portal vein ; and a branch of the hepatic artery and a bile-duct, which are much smaller. Within the lobules of the liver connective-tissue elements are very scanty, being represented only by the endothelium of the capillaries. 
The hepatic artery is a small vessel, the branches of which accompany those of the portal vein in the portal tracts. It supplies blood for the nourishment of the connective tissue and vessels of the liver.

The bile-ducts collect and convey the bile from the secreting lobules to the hepatic duct, the general outlet-duct of the liver. They lie in the portal tracts. The smaller ducts are lined with simple columnar epithelium (by which they may be distinguished from the accompanying arteries of corresponding size), resting on a basement-membrane. As the ducts unite and become larger the connective tissue by which they are surrounded forms a thicker layer, in which elastic elements and involuntary muscle-cells appear.

The portal vein is the main afferent bloodvessel of the liver, conveying into it the venous blood from the digestive organs. Its branches follow the portal tracts, and are the largest and thinnest-walled of the vessels in the latter. As its branches lie always in the septa between the lobules, they are called the interlobular veins. They divide into capillaries, which penetrate and converge to the interior of the lobules, and there empty into the radicles of the hepatic vein.

The hepatic veins are the efferent bloodvessels of the liver. They begin by the blind hepatic radicles or intralobular veins, which lie in the centre or axis of the lobules and receive the intralobular capillaries. The intralobular veins empty into larger branches, the sublobular veins, the union of which forms the hepatic veins.

The lobules or acini of the liver are polyhedral in shape from mutual pressure, and about a millimetre in diameter. They are separated from one another by the interlobular septa of the sustentacular tissue, though in man these septa are incomplete and the boundaries of individual acini often difficult to determine. In cirrhosis of the liver and in some mammals the septa are complete and well marked.

Each lobule is made up of liver-cells, intralobular capillaries, bile-passages between the cells, and an intralobular vein in the centre or axis of the lobule. The acini are built around the intralobular veins as axes, and their bases abut on the sublobular veins into which these veins empty.

The liver-cells are polyhedral, nucleated, glandular epithe- 
lium-cells, which often contain granules and fat-particles. Differences as to granularity, etc., occur in the appearance of these cells in the various stages of digestion, as between fasting periods and after eating. The liver-cells fill the acini in a mass interrupted only by the radiating blood-capillaries and

FIG. 58.

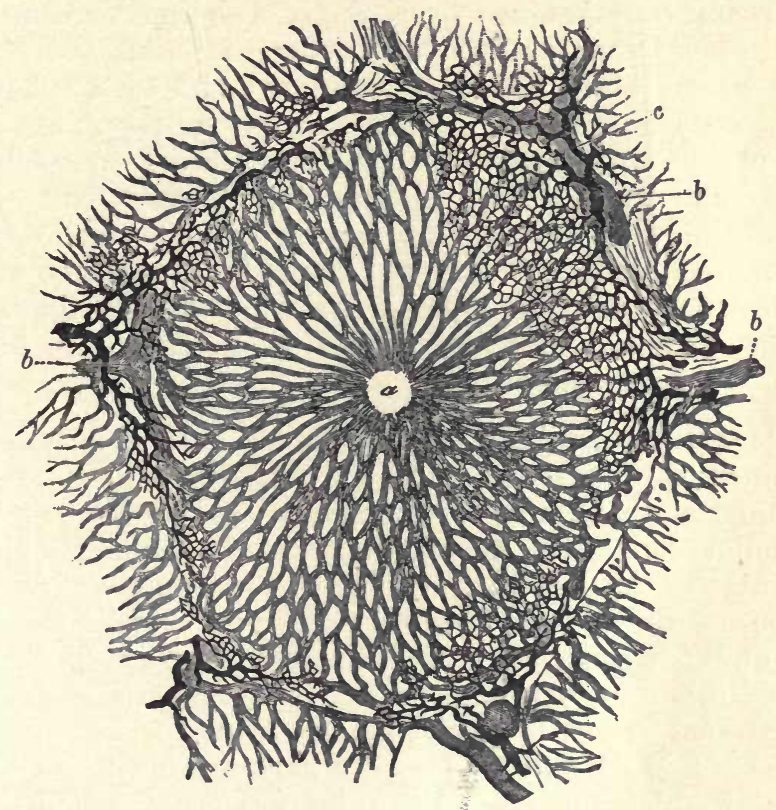

Vessels and bile-ducts of hepatic lobule (Cadiat). $a$, intralobular vein; $b$, interlobular veins, breaking up into intralobular capillaries: $c$, bile-duct with bilecapillaries (shown only at periphery of lobule).

the bile-passages. They are in contact with one another, and are not intermingled with connective tissue.

The intralobular veins, the ultimate radicles of the hepatic veins, occupy the centre or axis of the lobules.

The intralobular capillaries converge toward the intralobular vein from the periphery of the lobule, where they are formed by the breaking up of the interlobular or portal veins; in section they appear as conspicuous empty spaces or channels 
between rows of liver-cells, radiating from the intralobular vein in the centre. They are so arranged that each liver-cell abuts on at least one capillary. Through these capillaries the blood passes from the portal vein to the hepatic vein, coming into close relation with the liver-cells on the way. The capillaries and the intralobular veins are lined with delicate endothelium-cells which rest on the liver-cells.

The bile-passages are exceedingly fine canals formed by slight separations or grooves between adjacent liver-cells; short lateral blind branches sometimes also enter the bodies of the cells themselves. These passages unite to form a fine capillary network, which empties into the bile-ducts in the interlobular septa. Each bile-capillary is separated from the blood-capillaries by at least a portion of a liver-cell. These passages collect the bile secreted by the liver-cells, and though atypical they are the same in principle as the lumina of tubular glands. In some animals, as the frog, the tubular type is more apparent.

The gall-bladder and hepatic, cystic, and common ducts are lined with mucous membrane, consisting of simple long columnar epithelium with intermingled goblet-cells, and subepithelial connective tissue, in which mucous glands are present. In the gall-bladder this coat forms intersecting rugæ.

Beneath the mucosa is a fibro-muscular coat, composed of firm fibrous tissue containing involuntary muscle-cells. Outside of this coat, over the greater portion of the surface, is an external serous or peritoneal covering.

The bile in the gall-bladder is a dark opaque homogeneous secretion, thick and viscid from the presence of mucin, and is devoid of formed elements.

\section{The Pancreas.}

The pancreas (Fig. 59) is a racemose gland, which secretes the pancreatic fluid. It is made up of secreting alveoli and ducts, supported in connective or sustentacular tissue, which divides it into lobes and lobules, and is supplied with bloodvessels, lymphaties, and nerves.

The sustentacular or connective tissue consists of an areolar or fibrous capsule enveloping the gland; of trabeculæ or 
septa (interlobular) of similar tissue which traverse the pancreas and divide it into lobes and lobules; and of delicate connective tissue penetrating between the individual alveoli which make up the lobules, and providing them with basement-membranes.

The larger vessels, ducts, and nerves are conveyed through the organ in the interlobular connective tissue.

The larger (or interlobular) divisions of the ducts are lined

Fig. 59.

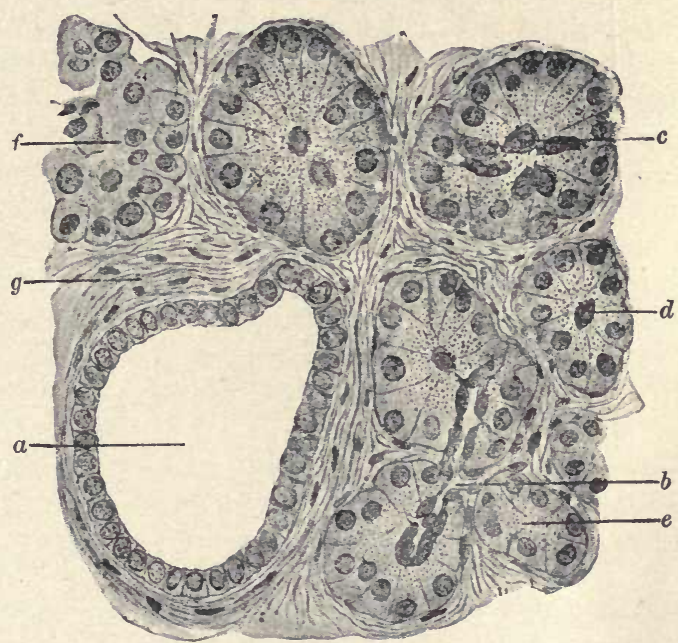

Portion of pancreas (Böhm and Davidoff), $a$, large duct: $b$, beginning ducts; $c, d, e$, secreting alveoli; $g$, sustentacular connective tissue.

with simple columnar epithelium; the small ductules with flattened epithelium-cells.

The secreting alveoli are rather tubular in form, of the serous type, and are lined with a single layer of spheroidal or columnar glandular epithelium-cells. The lumen of the alveoli also sometimes contains small cells, called centro-acinal cells.

The cells lining the alveoli vary according to the stage of secretion. Before secretion these cells are distended, and the zone toward the lumen is clear and contains coarse zymogen- 
granules, while the protoplasmic zone toward the attached bases of the cells, where the nuclei are situated, is dark, more opaque, and less (or more finely) granular. During secretion the zymogen-granules and clear material distending the cells are discharged; when exhausted by prolonged activity the cells are thus left shrunken, darker, opaque, and non-granular (or only finely granular).

Bodies of Langerhans: Between the lobules in places are rounded vascular aggregations of small cells, more open and less dense than the secreting alveoli which make up the bulk of the gland ; these are called the bodies of Langerhans, and are perhaps imperfectly developed lobules, though their nature is not entirely understood. 


\section{CHAPTER XI.}

\section{THE RESPIRATORY SYSTEM.}

\section{GENERAL CONSIDERATIONS.}

The lungs: One function of the lungs, analogous to that of the alimentary system, is to furnish to the circulation material (in this case oxygen) required in the nutrition of the body. Their other function, the excretion of carbon dioxide, is a glandular process, and in view of it the lungs can be regarded as a gland; they certainly approach the glandular type as closely as does the liver.

The structure of the lungs is strikingly that of racemose glands; the main departure from the ordinary glandular type is that the substances interchanged are gaseous rather than liquid or solid, and the medium of excretion consists not of spheroidal cells, but of squamous cells adapted to the exchange of gases.

The analogy of the lungs to a gland is increased if their alimentary or oxygen-furnishing function be regarded as a sort of internal secretory process, in which the separated product is discharged into the blood.

The other portions of the respiratory apparatus, the nose, pharynx, larynx, trachea, and bronchi (the ducts or air-passages of the lungs), and the pleura, for the most part simply serve tributary and subordinate purposes to the lungs.

\section{The Nasal Fossæ.}

The mucous membrane lining the nasal fossæ rests upon the periosteal and perichondrial coverings of the surrounding bones and the hyaline cartilaginous septum, and is continuous with the skin and the mucous membrane of the nasal ducts, pharynx, and Eustachian tubes. It is composed of two distinct areas, the respiratory and the olfactory. The olfactory area comprises the upper part of the fossæ, namely, the upper 
third of the septum, the roof of the nose, the superior and part of the middle turbinated bone, and the ethmoid in front of them. The remainder of the nasal fossæ and the accessory sinuses (maxillary, ethmoidal, sphenoidal, frontal) are covered by the respiratory portion of the mucosa.

The respiratory portion of the nasal mucous membrane, like the air-passages in general, is lined with stratified ciliated epithelium, among which are occasional goblet-cells. The epithelium rests on a firm tunica propria, beneath which is a variable amount of loose submucous areolar and connective tissue. The submucosa contains abundant and ample bloodvessels, nodules and diffuse masses of lymphoid tissue, and small racemose mucous glands with ducts lined by columnar epithelium, gradually merging into the ciliated cells of the surface. The vascularity of this region explains the frequency of nosebleed. Over the septum and turbinated bones the mucous membrane is thick, very vascular, and contains large numbers of the glands; in the accessory sinuses it is thin and less glandular and vascular.

The olfactory portion of the nasal mucous membrane contains the nerve-terminals for the sense of smell. It is thick, and mostly pigmented, of a yellowish color. Its superficial layer is composed of stratified columnar non-ciliated epithelium. The surface-cells are of two kinds, sustentacular and olfactory.

The sustentacular or supporting cells are long columnar cells, with deeply placed oval nuclei; their deep portions are branched and irregular, their free extremities are granular and exhibit the appearance of a superficial covering cuticle.

The olfactory cells are slender, elongated, linear cells, with large spherical nuclei which cause a marked bulbous swelling of the cell-body. They lie between the supporting cells, and are a form of neuro-epithelium, or, perhaps, actual nervecells. Their lower extremities are continuous with, or at least in intimate relation with, terminal filaments of the olfactory nerve. Their prominent round nuclei form a zone in the lower portion of the epithelial layer. The lowest epithelium. cells are spheroidal and germinal.

The epithelium rests upon a connective-tissue layer, the tunica propria and submucosa, which contain the terminal 
filaments into which the olfactory nerves divide, and large numbers of small mueous glands.

The pharynx: The upper portion of the pharynx, which has been already described (page 124), belongs to the respiratory tract, and is lined with ciliated epithelium, while the lower portion, lined with squamous epithelium, pertains rather to the alimentary canal.

\section{The Larynx.}

The larynx is a cartilaginous box surmounting the trachea and containing the vocal apparatus.

Its main cartilages, the thyroid, erieoid, and larger portions of the arytenoids, are of the hyaline variety, tending in old age to become ossified. The other cartilages, those of the epiglottis, certain processes of the arytenoids, the cornicula laryngis, and cuneiform cartilages (cartilages of Santorini and Wrisberg), are elastic cartilage.

The ligaments and membranes (thyro-hyoid and cricothyroid) which complete the framework of the larynx are fibro-elastic structures.

The interior of the larynx is lined with mucous membrane, consisting of an epithelial layer, tunica propria, and submucous tissue.

The epithelial lining of the larynx above the ventricles and over the true vocal cords consists of stratified squamous epithelium, continuous with that of the pharynx ; the ventricles and the portion of the larynx below the vocal cords are covered with stratified ciliated epithelium, like that of the trachea.

The tunica propria is a fibrous membrane, containing numerous elastic fibres.

The submucosa consists of areolar tissue, extending down to the perichondrial coverings, and containing the larger blood and lymphatic vessels, small racemose mucous glands, oceasional masses of lymphoid tissue, and the intrinsic voluntary muscles of the larynx.

The epiglottis contains a basis of elastic cartilage in its centre, covered on each side by submucous areolar tissue and 
a mucosa. The mucous membrane on the anterior (or lingual, or upper) surface of the epiglottis is a continuation of that of the tongue and is very similar to it.

The vocal cords are folds of the mucous membrane, with the tunica propria reinforced and strengthened by additional elastic fibres.

\section{The Trachea.}

The trachea is a rigid tube extending from the larynx to the upper part of the chest, where it divides into the two bronchi. Its walls are made up, from within outward, of mucous membrane, submucous tissue, cartilage and muscle, and fibrous tissue.

The mucous membrane of the trachea is lined with stratified ciliated epithelium-cells (Fig. 14), among which are occasional goblet-cells. The tunica propria underlying the epithelium is a firm supporting connective-tissue layer ; its most superficial layer forms a basement-membrane for the epithelium; below this the tunic is infiltrated with numerous lymphoid cells, and its deepest portion contains numerous longitudinal elastic fibres.

Outside the tunica propria is the submucosa, a layer of areolar and elastic tissue ; it contains the alveoli of numerous small racemose mucous glands, occasional lymphoid tissue, and the larger blood and lymphatic vissels.

Next outside the submucosa is a series of incomplete rings or horseshoe-shaped bodies of hyaline cartilage, defective posteriorly, which makes up the rigid incollapsible framework of the trachea. The connective tissue on the surfaces of the cartilage is condensed to form a perichondrium.

The hiatus at the posterior part of the cartilages is filled up partially by fibrous tissue, but chiefly by the trachealis muscle, a bridge of involuntary muscle which connects the ends and posterior surfaces of the cartilages; the larger portion of this muscle is arranged transversely, but externally there are a few longitudinal fibres.

There are some mucous glands located posterior or external to the trachealis muscle, whose ducts pierce the muscle and find their way to the lumen of the trachea. 
Ontside of the cartilaginous layer the trachea is surrounded by fibrous and areolar tissue continuous with that of the adjacent structures.

\section{The Lungs.}

The lungs are made up of air-vesicles or alveoli, the ultimate functional divisions of the organ; bronchial tubes, the air-passages leading to the air-vesicles; connective sustentacular tissue; an ample system of bloodvessels, besides lymphatics and nerves: and a serous covering derived from the pleura.

The lung-substance is more or less distinctly divided into pyramidal lobules, consisting of the groups of alveoli corresponding to terminal divisions of the bronchi and bloodvessels; the lobules are to a greater or less extent marked off from one another by septa, although closely packed together.

The connective tissue of the lung, its sustentacular framework, may be divided into the superficial investment of the organ and the interalveolar, peribronchial, and interlobular tissues. The surface of the lung is surrounded by elastic and areolar tissue, lying beneath the pleura and continuous with its subserous laver.

The free surface is covered by the visceral portion of the pleura.

The interalveolar connective tissue is a delicate tissue, mostly elastic, between, surrounding, and forming a supporting framework for the air-vesicles; it comprises the larger part of the pulnonary connective tissue, and to it are due the well-known elasticity and contractile tendency of the lungs.

The peribronchial connective tissue is a firmer tissue of the white-fibrous variety which forms definite sustentacular tracts accompanying, supporting, and surrounding in a single sheath the bronchi and their concomitant bloodvessels in all their ramifications and subdivisions throughout the lungs.

The interlobular connective tissue consists of more or less definite thin fibrous septa separating the lobules of the organ.

Pigment, usually black, is often abundantly deposited in patches in the interalveolar and interlobular tissue; it consists of particles inspired into the air-vesicles and removed into the interior by the lymphatics.

10-Hist. 
The bloodvessels of the lungs comprise two distinct sets of arteries, capillaries, and veins : the pulmonary vessels and the bronchial vessels.

The pulmonary vessels are far the larger of the two sets and carry out the respiratory function of the lungs. The pulmonary arteries convey the venous blood from the right ventricle of the heart, and break up into capillaries which

FIG. 60.

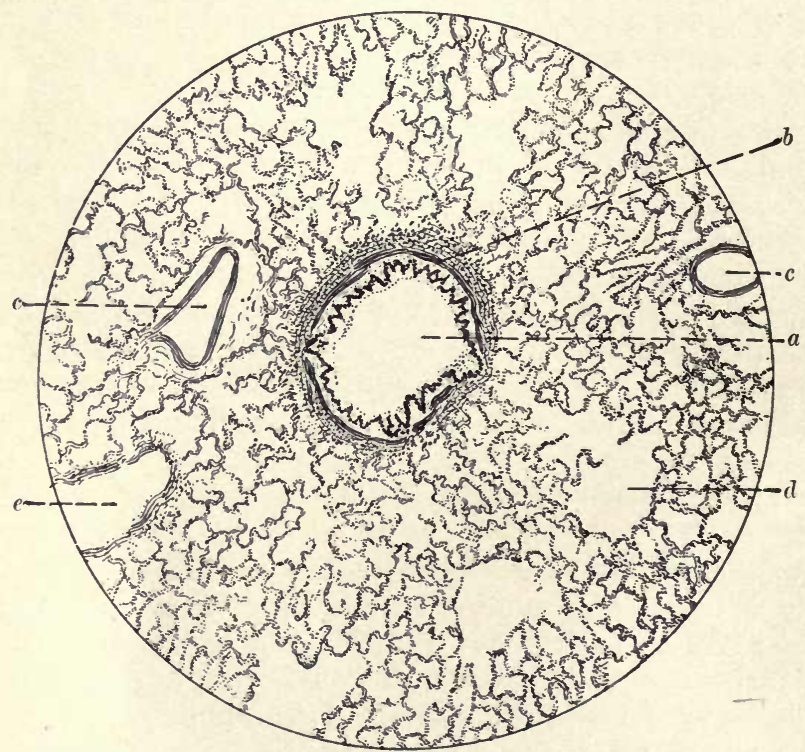

General appearance of lung under low magnification (Dunham). $a$, bronchus; $b$, muscularis mucosæ of bronchus: $c$, pulmonary veins; $d, e$, infundibula.

surround the air-vesicles in a close meshwork. The blood, aërated and arterialized in these capillaries, is collected from them and returned in the pulmonary veins to the left auricle.

The pulmonary arteries and veins accompany the bronchi in their course throughout the lungs, lying in the same sustentacular tract of peribronchial connective tissue, the bronchus in the centre, a pulmonary artery on one side, and a pulmonary vein, larger and with thinner walls than the 
artery, on the other. The size of the pulmonary vessels always corresponds to that of the accompanying bronchus, being not far from equal to it.

The bronchial vessels are much smaller than the pulmonary, and serve for the nutrition of the substance of the lung. The bronchial arteries are branches of the aorta. The capillaries from these arteries empty chiefly into the bronchial veins, but partly also into the pulmonary veins. The bronchial veins empty ultimately into the vena cava.

The bronchial vessels lie in the peribronchial connective tissue and accompany the bronchi and pulmonary vessels in their ramifications.

The lymphatics of the lungs comprise small channels origin-

Fig. 61.

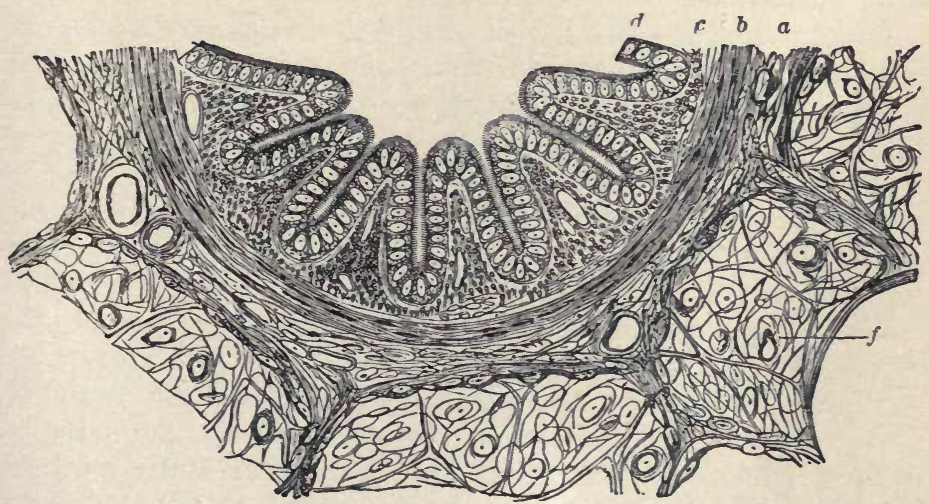

Portion of bronchiole (pig) in cross-section (Schultze). $a$, submucosa; $b$, muscularis mucosæ; $c$, tunica propria; $d$, epithelium; $f$, portions of neighboring air-vesicles.

ating from stomata in the air-vesicles, in the sustentacular and perivascular tissue, and from the pleural stomata.

The nerves of the lungs are small branches lying in the peribronchial tissue.

The bronchi are continuations of the trachea, and within the lung divide and subdivide dichotomously down to the terminal bronchioles (Fig. 61). They are the air-passages to 
the ultimate alveoli of the lungs, and are analogous to the ducts of glands. They are surrounded by a considerable amount of firm fibrous connective tissue, the peribronchial tissue ; each bronchus is accompanied in the same peribronchial sheath by correspondingly large pulmonary vessels, the artery on one side, the vein on the other, and by the smaller bronchial vessels and nerves, usually a bronchial artery, vein, and nerve being on each side of the bronchus.

The structure of the bronchi gradually changes as they vary in size. At the beginning they are about the same in structure as the trachea.

The medium-sized bronchi are made up, from within outward, of an epithelial layer, a tunica propria, muscularis mucosæ, and a submucosa, which contains plates of cartilage, mucous glands, and lymphoid tissue, and extends to the peribronchial tissue.

The bronchi are lined with stratified ciliated epithelium, underlying which is the tunica propria, consisting largely of elastic tissue. These two layers are thrown into longitudinal folds.

The muscularis mucose is a thin but well-marked layer or ring of involuntary muscle, arranged circularly ; it separates the tunica propria from the submucosa, and is not included in the longitudinal folds of the mucosa.

The submucosa consists of areolar tissue, merging into the firmer peribronchial connective tissue. It contains small racemose mucous glands, whose ducts discharge on the epithelial surface; occasional nodules of lymphoid tissue; and unequal curved plates of hyaline cartilage, the representatives of the tracheal rings, which in cross-sections appear as ares incompletely surrounding the bronchi.

Terminal bronchioles: As the bronchi decrease in size the cartilaginous plates diminish and finally disappear entirely; the stratified ciliated epithelium becomes reduced to a single layer of ciliated cells, and still later to a layer of low columnar non-ciliated cells; the connective-tissue layers become diminished in thickness, and the peribronchial tissue and mucons glands disappear. Thus, the terminal bronchioles are lined with a single layer of low columnar non-ciliated or flattened polygonal epithelium-cells, a slight amount of fibro-elastic 
connective tissue, and a thinned and interrupted muscularis mucosæ.

The infundibula : At their termination the bronchioles open into clusters of large elliptical cavities, called infundibula, the

FIG. 62.

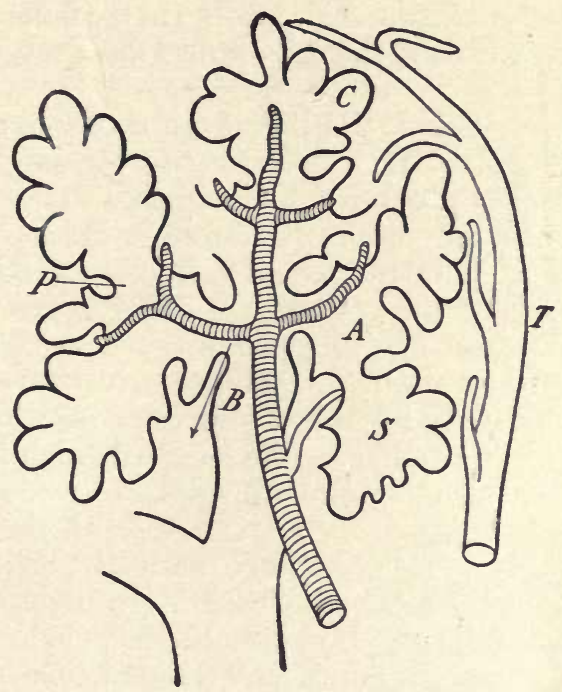

Diagram of air-vesicles and termination of bronchi (Miller). $B$, terminal bronchiole (the line leads from a terminal artery); $A, P$, alveolar passages; $S$, infundibulum; $C$, air-resicle; $T$, beginning vein.

walls of which are beset with air-vesicles. These infundibula branch off from the sides and ends of the final portions of the bronchioles, which are here somewhat expanded and lined with flattened polygonal epithelium-cells, and which are often called alveolar passages.

Each infundibulum is a blind elliptical sac, opening at one point into an alveolar passage, and with its walls formed entirely of air-vesicles.

The air-vesicles (air-sacs, air-cells, or alveoli) are small hemispherical recesses forming the walls of the infundibula and opening into the cavity of the latter. They make up the great bulk of the lung-substance, and are the ultimate func- 
tional divisions of the lungs, where the admission of oxygen into and the expulsion of carbon dioxide from the blood take place. They are lined with a single layer of large polygonal squamous epithelium-cells, at the junction of which appear occasional small intervals or stomata opening into lymphpassages. Among the squamous cells lining the vesicles occur here and there small spheroidal cells; in the fotal lung, before it is expanded with air, the cells lining the air-vesicles are all spheroidal.

Underneath the epithelial lining is a thin network of elastic tissue (the interalveolar connective tissue), which forms the framework and support for the vesicles. The walls of the alveoli also contain a close meshwork of blood-capillaries derived from the pulmonary artery, in close relation with the squamous lining of the vesicles, thus affording favorable conditions for the exchange of gases between the venous blood and the air in the alveoli. Adjacent air-vesicles are separated from each other only by thin septa, each of which contains but a single set of eapillaries, the loops of which lie in relation with the epithelial lining on both sides.

The pleura is a serous sac, the parietal portion of which lines the thoracic wall, and whose visceral portion forms a covering for the lung. It facilitates the movements of the lungs over the opposing surfaces. In structure it is a serous membrane, consisting of a fibrous stratum covered with polygonal endothelium-cells on the surface. Beneath it is the subserous or subpleural areolar tissue, which in the visceral portion of the sac is continuous with the superficial connective tissue of the lung. 


\section{CHAPTER XII.}

\section{THE SKIN.}

THE skin serves integumentary and protective purposes, is an important excretory organ, is the seat of sensory terminals, and possesses other functions. It consists of two parts, a thin outer epithelial layer, the epidermis or cuticle, and a thicker, lower connective-tissue layer, the cutis vera, or corium. Beneath it is a variable layer of subcutaneous tissue. The skin contains numerous glands of two kinds, sudoriparous and sebaceous, and also the hairs and nails; these are all derived from, or modifications of, the epidermis.

The epidermis, the thinner outer laver of the skin, consists of stratified squamous epithelium, and is of epiblastic origin. It varies in thickness and the development of its parts in different situations. It is divisible, in the first place, into two sharply distinct layers, an outer, hard, horny, layer, and a lower, soft protoplasmic layer, the "rete mucosum." Each of these is again divisible into other strata, which are, from the surface downward, the stratum corneum and stratum lucidum, making up the horny portion, the stratum granulosum and stratum Malpighii, making up the rete mucosum. In addition, the lowermost layer of (columnar) cells in the Malpighian stratum is almost disFig. 63 .

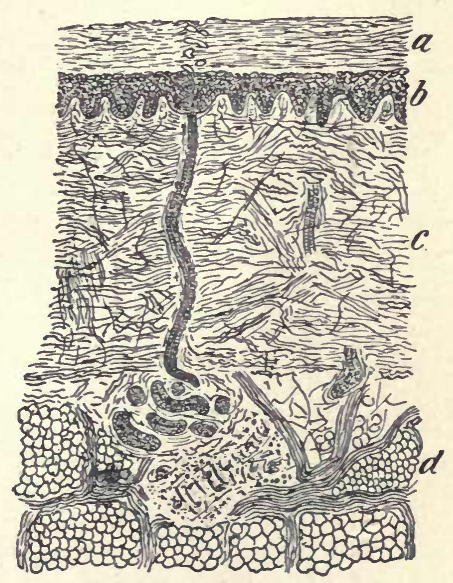
tinct enough to be differenSection of skin, showing sweat-gland. $a$,
stratum corneum; $b$, rete mucosum; $c$, corium; $d$, subcutaneous tissue. tiated as a special layer. Thesc layers may be exhibited thus : 
Horny portion of epidermis $\left\{\begin{array}{l}\text { Stratum corneum. } \\ \text { Stratum lucidum. } \\ \text { Rete mucosum . . . }\end{array}\right.$ Stratumum Malosum.
Stratum Malpii.

Of these layers the strata corneum and Malpighii are the thickest, most prominent, and always present; the strata lucidum and granulosum are much thinner, less conspicuous, and where the epidermis is thin may be quite indistinguishable. There are no bloodvessels or capillaries in the epidermis, but lymph is probably able to percolate between the cells.

The stratum Malpighii (or stratum germinativum) consists of about ten to twenty-five layers of deeply staining spheroidal epithelium-cells resting on the corium. Into this stratum project the papillæ which stud the surface of the corium, the intervals between the papillæ being filled with epithelial cells. The lowermost cells of the Malpighian stratum form a single layer of tall columnar cells arranged perpendicular to the surface of the papillæ and corium; this layer forms a quite distinct stratum. The other cells of the Malpighian layer, larger than the columnar cells, are spheroidal in shape, or polyhedral from mutual pressure, becoming somewhat flattened above. They are disposed irregularly, not in uniform layers, and have characteristic "vesiculated nuclei"; that is, their nuclei are surrounded by or seem to lie in a clear vesicular space. The surfaces of these cells are closely beset with ridges, or "intercellular bridges," causing the cells to appear in cross-section as if studded with projecting prickles or spines, whence they are often called prickle-cells. The ridges of adjacent cells unite edge to edge, and the minute communicating channels left between them probably enable lymph to pass between these cells for the nourishment of the non-vascular epidermis. The cells of the deeper portion of the stratum Malpighii contain pigment, which gives color to the skin, especially in freckles and dark-skinner races. The Malpighian layer contains terminal nerve-fibrils and tactile cells.

The stratum granulosum rests upon the stratum Malpighii, the two merging together without sharp dividing-lines. It is not always very distinet, but in thick epidermis it appears 
very prominently as consisting of three or four layers of flattened, lenticular, or (in cross-section) fusiform cells, which are dark, stain very deeply, and contain coarse granules of a substance called eleidin, supposed to be a precursor of the horny substance of the more superficial cells of the epidermis.

The stratum lucidum is a thin, translucent layer of horny, flattened cells, lying on the stratum granulosum, and forming the lowest layer of the horny portion of the epidermis. It is often indistinet.

The stratum corneum consists of numerous layers of flat, squamous cells, and forms the thick, outer, horny layer of the epidermis. The cells consist of a hard, horny substance, or keratin, into which the protoplasm of the underlying cells has become converted. There is practically no vitality left in these cells, but they are well adapted to protective purposes. In vertical section the cells appear as a succession of wavy lines, with occasional indistinct traces of the degenerated nuclei.

The corium, cutis vera, or dermis, is the connective-tissue portion of the skin, of mesoblastic derivation. It is a firm fibrous layer, corresponding to the tunica propria of mucous membranes, and is thicker than the epidermis; to it the firmness of the skin is due. It consists of an interlacing network of white and some elastic fibres, among which are scattered fusiform connective-tissue cells ; mingled with these are numerous capillaries, small lymph-vessels, and nervous elements.

The upper surface of the corium presents numerous oval papilloe projecting upward into the stratum Malpighii, which fills the intervals between them. Some of the papillæ are occupied by capillary loops, others by tactile nerve-terminals. In some situations the papillæ are closely crowded, in others more sparsely. The arrangement of the papillæ gives rise to the lines of the palm and sole, and to other superficial markings elsewhere.

The lower surface of the corium is plane and merges into the subcutaneous tissue.

The subcutaneous tissue (or superficial fascia) is a layer of loose areolar and adipose tissue which underlies the corium and extends to the subjacent structures. It varies in thickness in different parts of the body, and corresponds to the 
submucous layer of mucous membranes. This tissue contains the larger vascular and nervous branches, and Pacinian corpuscles; the hair-follicles and sweat-glands project down into it. Next to the corium the subcutaneous tissue is mostly loose and areolar, while deeper is a variable and often thick layer of fatty tissue, the panniculus adiposus.

The sudoriparous or sweat-glands (Fig. 63) occur in great abundance over the entire skin, and are derived from downgrowths of the epidermic epithelium. They are simple tubular glands of the serous type, consisting of single tubes lined by epithelium resting on a basement-membrane, and supported in the surrounding connective tissue. They extend from the surface downward into the subcutaneous tissue or lowest part of the corium, and their deeper portions are much convoluted. They consist of two parts, a secreting portion and an outlet-duct. The secreting portion consists of the lower part of the tube, sometimes somewhat branched, and is coiled and convoluted into a rounded nodule situated in the subcutaneous tissue. Its calibre is larger than that of the outlet portion, and it is lined with a layer of columnar epithelium. Between the epithelium-cells and their basementmembrane occur scattered involuntary muscle-cells.

The outlet-duct is a narrow tube of uniform size lined with two layers of spheroidal epithelium-cells. After leaving the convoluted secretory portion of the tube it pursues a straight or slightly wavy course to the epidermis, which it enters between the papilla. In the stratum Malpighii its lining is formed by the cells of that layer. Through the stratum corneum the duct passes to the surface in a spiral or corkscrew course through the horny substance. The ceruminous glands of the ear are modified sweat-glands.

The hairs occur in the skin over nearly the entire surface, being especially numerous and well developed in certain regions. 'They are composed of horny cells derived from the epidermis. The lower end of each hair exhibits a rounded enlargement, the bulb.

The root of the hairs, the portion imbedded in the skin, extends into the subeutaneous tissue, and is supported in and grows from a sheath, the hair-follicle, formed by a downgrowth of the epidermis and corium. 
A human hair is round or flattened an 1 made up of three parts, a cuticle, a cortex, and a medulla.

The cuticle is a thin outer covering composed of thin nonnucleated horny plates or cells, imbricated or overlapping; their edges form irregular transverse markings on the surface of the hair.

The cortex, making up the bulk of hair, consists of slender, elongated, fusiform, horny nucleated cells, arrangerl longitudinally and closely packed and cemented together.

The medulla is not always continuous or present; it consists of nucleated polyhedral cells loosely aggregated in the axis of the hair; these cells often contain air, giving them an opaque appearance.

Hairs often contain pigment, deposited in the cortex ; to this pigment and the air in the medulla the color of hair is due.

The hair-follicles (Fig. 64), which support the hairs and provide for their growth, are sheaths formed by downward projections of the epidermis and corial tissue. They lie perpendicular or oblique to the surface, and into their upper half the sebaceous glands open. A small band of involuntary muscle extends from the lower part of the follicle to the corium across the obtuseangled side, forming the erector
Fig. 64.

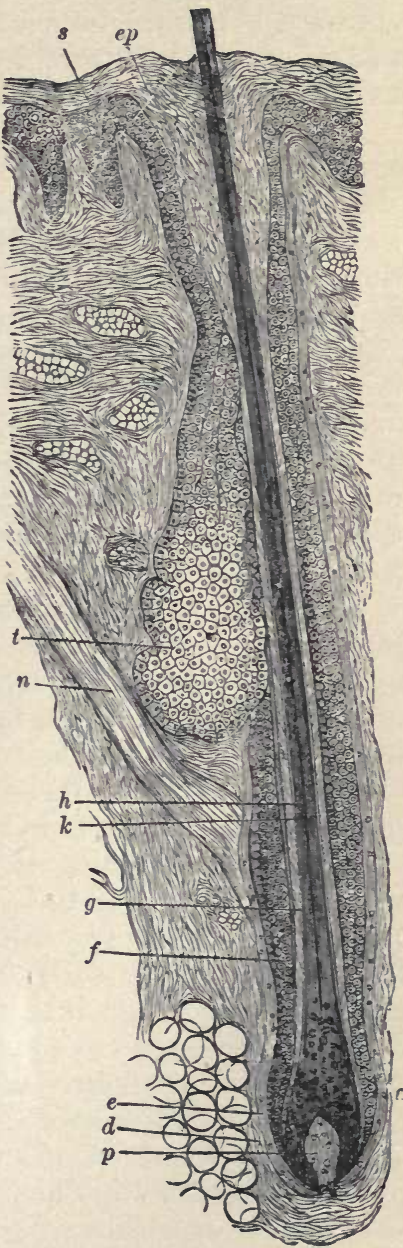

Longitudinal section of hair-follicle (Biesiadecki). c. bulb of hair; $d, e$, sheatbs derived from the corium; $f$, outer ront-sheath : $g$, inner rootsheath ; $h$, hair ; $k$, medulla of hair : $n$, erector pili muscle; $p$, papilla of hair ; $s$. rete mucosum ; $e p$, stratum corneum; $t$, sebaceous gland. 
pili muscles, which elevate the hairs and cause projections of the skin at their bases (cutis anserina). The hair-follicles consist of two parts-an outer fibrous sheath derived from the corium, and an inner epithelial portion continuous with the epidermis.

The outer fibrous sheath is a supporting investment of fibrous tissue, longitudinally and circularly arranged, continuous with the corium; its innermost layer, next to the epidernic sheath, is a translucent basement-membrane, the hyaline layer. At the lower end of the follicle the corial tissue sends a cellular and vascular projection, the papilla, into the hair-bulb.

The inner and more conspicuous epithelial portion of the hair-follicle is formed by a dipping down of the epidermis about the root of the hair. It corresponds mainly to the rete mucosum, the stratum corneum extending inward only a short distance near the mouth of the follicle. 'The epidermic portion of the follicle is usually described as consisting of two parts, an outer root-sheath and an inner root-sheath.

The outer root-sheath is a continuation of the stratum Malpighii, and consists of several layers of cells similar to those of that layer in the epidermis. The outermost layer of columnar cells, resting on the hyaline membrane, is very well marked. Alongside the expansion of the bulb of the hair the outer or Malpighian sheath becomes thinner. At the lower portion of the bulb this sheath curves around, upward, and inward, resting on the papilla, and becomes continuous with the cells of which the hair is composed, which are thus formed by gradual transition from the Malpighian cells; the innermost cells in this situation, next the papilla, become cuboidal and merge into the medulla of the hair, while the outer cells become gradually converted into the elongated horny cells of the hair-cortex.

The inner root-sheath lies between the hair and outer sheath along the lower half of the follicle; above it changes to a homogeneous sheath which fades gradually away, while below, in the lower part of the bulb, the inner sheath is gradually lost. It consists of three layers-Henle's layer, Huxley's layer, and the cuticular laver, from without inward.

The layer of Henle is a thin, translucent stratum composed of a single layer of flattened cells, next to the Malpighian sheath. Next within is the layer of Huxley, consisting of 
three or four strata of polyhedral cells, which stain deeply and are perhaps analogous to those of the stratum granulosum. The innermost layer is the cuticular layer, composed of a stratum of overlapping cells lying in close association with the cuticle of the hair.

The sebaceous glands are oval glandular saccules lying alongside the upper part of the hair-follieles, into which they empty. Some are simple or single; others divided into a few saccules. Their secretion, the sebum, is of a fatty nature, and serves to oil and soften the skin and hair. In some situations, as the labia minora and glans penis, they occur unassociated with hairs and open directly on the surface. Sebaceous glands are supported in the surrounding fibrous tissue, have short outlet-ducts, and are solid masses of cells without open lumina. The peripheral cells are smaller, spheroidal, and darker colored; the cells in the centre of the glands are much larger and clearer, being infiltrated with numerous droplets of fat. All the cells have well-marked nuclei.

The nails are modifications of the epidermis, consisting essentially of a much-thickened stratum lucidum. The layers on which the nail rests and to which it is attached form its bed. The nail grows only from the posterior or proximal part of the bed, or the matrix, the pale, visible convex portion of which is the lumula. The posterior edge of the nail, its root, rests in a groove formed by a fold of rete mucosum. The corium beneath the nail exhibits the usual fibrous structure ; its surface anterior to or beyond the matrix forms longitudinal ridges, representing papillæ; over the matrix are papillæ of the ordinary form. On the corium rests a stratum Malpighii of the usual type, filling the depressions between the ridges, and overlaid by a stratum granulosum. Upon this is the body of the nail (corpus unguium), a thick, horny layer derived from cells which have been transformed into keratin; it represents a highly developed stratum lucidum, and is formed only over the matrix, where continual growth keeps forcing it on ward over the remainder of its bed. There is no stratum corneum over the nails. 


\section{CHAPTER XIII.}

THE URINARY ORGANS.

THE action of the urinary organs is excretory, serving to remove from the blood waste katabolic products and superfluous ingested substances. The urinary apparatus consists of the kidneys, ureters, bladder, and urethra, the latter in the male being also utilized in the genital function.

\section{The Kidneys.}

General structure: The kidneys are compound tubular glands. They exhibit two parts, the parenchyma or secreting portion, and the sinus, a cavity containing the calices of the ureter in which the urine collects and is discharged into the ureter, which is the outlet-duct of the kidney. The sinus is situated within the kidney, of which the concave inner margin eonstitutes the hilum of the organ, where the ureter, renal artery, vein, lymphatics, and nerves enter. The renal parenchyma is arranged in a radiating manner about the sinus, and consists of two well-marked zones, an outer or cortex, and an inner or medulla.

The structural elements of the kidney are the uriniferous tubules (beginning with prominent rounded bodies, the Malpighian bodies), bloodvessels, and sustentacular connective tissue, besides inconspicuous lymphatics and nerves and the lining of the sinus. The kidneys, being derived from the foetal Wolffian body, are developed from the mesoblast, thus forming a notable exception to the usual epiblastic origin of epithelium.

The medulla of the kidney is composed of a number (eight to eighteen) of conical bodies, called the pyramids of Malpighi. These radiate about and from the renal sinus, with their bases outward, resting against the cortex, and their apices pointing in toward the renal pelvis (of the ureter), 
into the cavities of the subdivisions of which (calices) their apices project as papillæ. The pyramids (or the renal medulla) have a light-colored, shining, striated appearance, the striæ being radiating or nearly parallel. Two zones in the medulla are sometimes distinguished, an outer "boundary layer" and

Fig. 65.

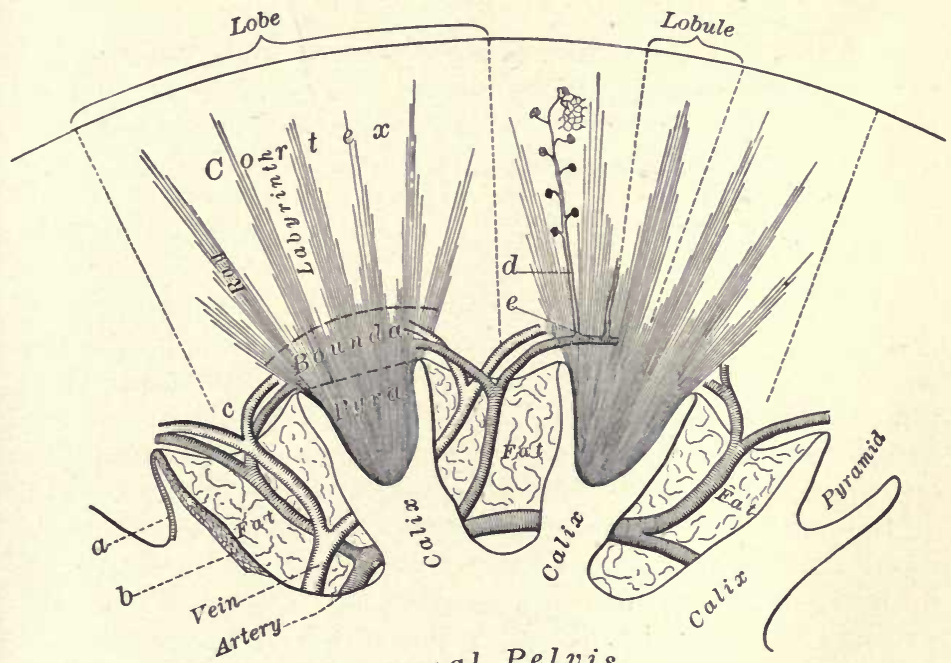

Renal Pelvis

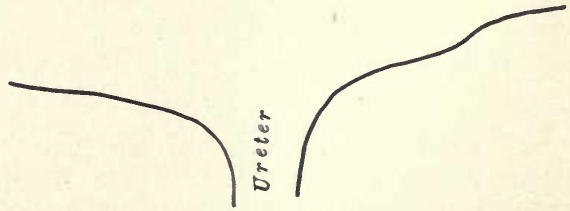

Diagrammatic sketch of structure of kidney (Dunham). $a, b$, epithelium lining the calix ; $c$, column of Bertin; $d$, interlobular artery ; $e$, arcuate artery.

an inner "papillary layer." The substance of the pyramids consists chiefly of straight urinary tubules converging to the apices; these produce the striated appearance of the pyramids.

The pyramids are often separated from one another by 
walls or processes of the cortical substance dipping down between them, called the columno Bertini; in these columns the bloodvessels pass into the substance of the kidney.

The adult human kidney is not normally divided into lobes; but in some mammals and in early human life the kidney is lobed, each Malpighian pyramid with the adjacent cortical substance forming a distinct lobe separated from other lobes by clefts in the renal substance.

The cortex of the kidney is a dark-red granular zone, much narrower than the medullary region, and forming the periphery, just underneath the capsule. It also sends processes down between the Malpighian pyramids, the columnoe Bertini mentioned above. The substance of the cortex is divided into the pyramids of Ferrein and the interpyramidal regions, which alternate with one another.

The pyramids of Ferrein, or medullary rays, or cortical pyramids, are narrow bundles or sheaves of straight tubules which radiate from the outer boundary of the Malpighian pyramids through the cortex nearly to the periphery. These cortical pyramids consist of straight portions of the uriniferous tubules, which are continuations of the radiating tubules of the Malpighian pyramids, each of the latter giving off a number of pyramids of Ferrein from its base. The tubules in the centre are longer than those on the outside of each pyramid of Ferrein, giving it the shape of a cone with a narrow base.

The intervals between the cortical pyramids are occupied by the interpyramidal substance (or labyrinth) of the cortex. This consists of the coiled and irregular portions of the uriniferous tubules, twisting in all directions, studded and intermingled with the numerous prominent round Malpighian bodies, which give the cortex its granular character.

The interpyramidal cortical regions comprise the active secretory parts of the kidney; the pyramids of Ferrein and Malpighi simply contain conducting tubules.

The sustentacular connective tissue of the kidney consists of the capsule, the intertubular or interstitial tissue, the perivascular tissue, and the connective tissue lining the wall of the renal sinus and continuous with the pelvis (ureter).

The capsule is a firm fibrous membrane enveloping the 
gland; it is connected with the delicate intertubular tissue of the interior; but as it does not send any firm trabeculæ FIG. 66.

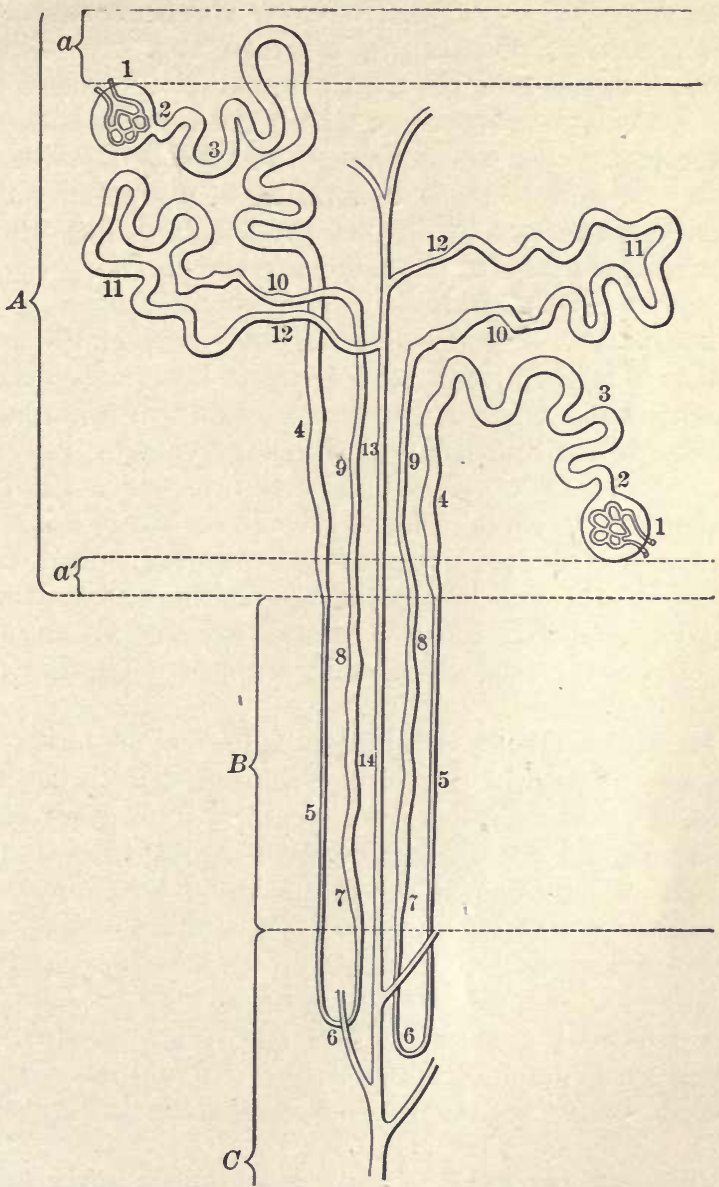

Diagrammatic representation of uriniferous tubules (Klein). $A$, cortex of kidney; $B$, boundary zone, and $C$, papillary portion of Malpighian pyramid; $a$, subcapsular portion, and $a^{\prime}$, inner portion of cortex destitute of Malpighian bodies; 1, Malpighian body, capsule of Bowman, and glomerulus; 2 , neck ; 3 , proximail convoluted tubule; 4, spiral tubule; 5, descending limb of Henle's loop; 6 , Henle's loop; 7, 8, 9, ascending limb of Henle's loop; 10, irregular tubule; 11 , distal convoluted tubule; 12 , curved tubule; 13,14 , collecting tubule.

11-IIist. 
into the gland it can normally be easily stripped off from the surface.

The connective tissue permeating the renal parenchyma (the intertubular or interstitial tissue) is a delicate tissue, very small in quantity, penetrating between and supporting the uriniferous tubules and vascular channels; it consists chiefly of the basement-membranes on which the tubules rest. Some connective tissue also accompanies the vessels entering at the hilum, and in and beneath the lining of the renal sinus are considerable quantities of fibrous, areolar, and adipose tissue.

The uriniferous tubules (Fig. 66) are the secreting or glandular elements of the kidney. They consist of long tubes, which (except the larger collecting-ducts) are single and unbranched. They are lined with a single layer of epitheliumcells resting on a basement-membrane, and in different parts of their course exhibit different divisions varying in arrangement, form, and structure. The parts into which the tubules are divided are, in their order, as follows: Malpighian body ; neck; proximal or first convoluted tubule; spiral tubule; the loop of Henle (consisting of a descending limb and ascending limb); irregular tubule; distal or second convoluted tubule; curved or arched tubule; straight collecting tubule, and tube of Bellini.

The Malpighian body is a hollow spherical structure or expanded sac forming the beginning of each uriniferous tubule. Its wall is formed by a membrane called the capsule of Bowman, and into its cavity, but covered by its epithelium, projects a tuft of capillary bloodvessels called the glomerulus.

The capsule of Bowman consists of a single layer of squamous epithelium resting on a basement-membrane. It forms a hollow spherical sac, the cavity of which at one point opens into the proximal convoluted tubule through a small neck. The layer of squamous epithelium is invaginated into the cavity so as to form a complete covering for the glomerulus (Fig. 67).

The glomerulus is a tuft of capillaries coiled into a spherical mass nearly filling the interior of the Malpighian body.

The capillaries arise from a single afferent vessel, and unite to form a single slightly smaller efferent vessel; these two vessels enter and leave the glomerulus together, forming a sort 
of stalk for the tuft, at a point opposite the neck or junction of the body with the uriniferous tubule.

The surface of the glomerulus is covered with a layer of squamous epithelium ("tuft-cells"), dipping down into the depressions between the capillary-loops; this epithelium is a

Frg. 67.

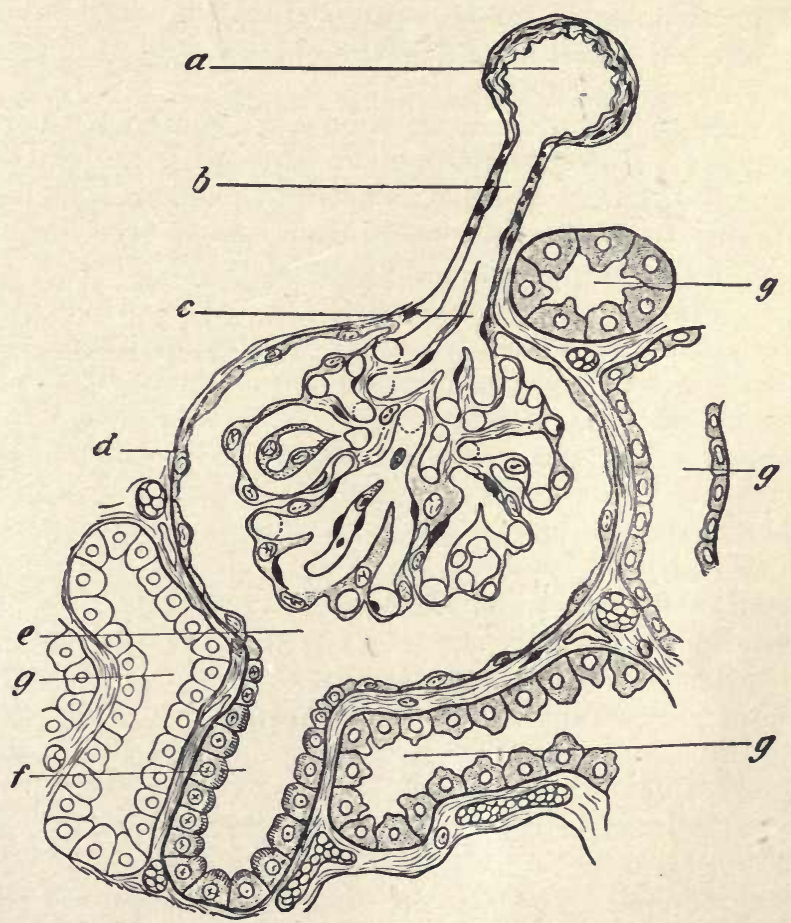

Sketch of Malpighian body (Dunham). $a$, interlobular artery; $b$, afferent vessel; $c$, capillary; $d$, capsule of Bowman; $e$, cavity of the capsule; $f$, beginning of proximal convoluted tubule; $g$, portions of convoluted tubules.

reflection of that lining the capsule of Bowman, with which it is continuous at the stalk of the glomerulus.

Thus the glomerulus is "outside" the capsule of Bowman just as the heart is outside the cavity of the pericardium. Malpighian bodies-arrangement and function: The Mal- 
pighian bodies are all situated in the interpyramidal portions of the cortex, which they stud as prominent granular points. The arrangement of the Malpighian body is adapted to secretory action; the expanded glomerulus affords a large surface over which fluid may transude from the blood circulating in the capillaries into the cavity of the capsule and uriniferous tubule. The slightly smaller size of the efferent vessel is explainable on the ground that the blood entering by the afferent vessel loses a portion of its fluid through the walls of the glomerulus.

The neck of the uriniferous tubule is a constricted portion at the junction of the Malpighian body with the proximal convoluted tubule. At this point the squamous cells of the capsule of Bowman become thicker and merge into the cuboidal cells that line the convoluted tubule.

The proximal convoluted tubule, the first portion of the uriniferous tube, is much twisted, contorted, and bent. It is lined with a single layer of columnar or spheroidal epithelium-cells, which are striated toward their attached bases; these cells are opaque, granular, somewhat irregular in shape and unequal in size, and of glandular type. The proximal convoluted tubules have a greater calibre than most portions of the uriniferous tubules; they are situated in the interpyramidal portions of the cortex, are surrounded by abundant capillaries, and are active secretory portions of the tubules.

The spiral tubule is the term applied to the portion of the uriniferous tubule that succeeds the proximal convoluted portion. It is straighter than the latter, having only a slight spiral twist, and lies in a pyramid of Ferrein, passing down to the medulla. It is lined with simple colunnar epithelium resembling that of the convoluted tubule, but rather lower and less striated.

The descending limb of Henle's loop begins at the junction of the cortex and medulla, where the spiral tubule merges into it, and lies in a Malpighian pyramid, passing downward to the loop of Henle. At the latter point the uriniferous tubule makes a sharp U-shaped bend on itself, turning upward in an ascending limb.

The descending limb is a straight, narrow tube lined with a single layer of flattened or lenticular epithelial cells, the 
nuclei of which cause a slight bulging at the centre of the cells. As the nuclei and bulging portions of the cells alternate on opposite sides of this tubule its lumen appears slightly sinuous.

The ascending limb of Henle's loop is situated partly in the Malpighian pyramid and partly in the pyramid of Ferrein. It is straight, parallel with the descending limb, and is lined with a single layer of columnar epithelium.

The irregular tubule is the portion of the uriniferous tubule succeeding the ascending limb, and is situated in the interpyramidal cortical substance. It pursues an irregular angular course, and is lined with striated, simple columnar epithelium.

The distal convoluted tubule succeeds the irregular portion, is situated in the interpyramidal cortex, and is similar in all respects to the proximal convoluted portion of the uriniferous tubule.

The curved or arched tubule is a short, curved tube, in which the distal convoluted tubule terminates. It passes into a pyramid of Ferrein and empties into one of the straight collecting tubules. It is lined with low cuboidal epithelium resembling that of the straight tubes.

The straight collecting tubes begin in the pyramids of Ferrein and pass in straight lines through the medulla of the kidney to the apices of the Malpighian pyramids, where, having successively united into a smaller number of ducts, of larger size, they empty into the calices as above stated. These largest discharging ducts are called the tubes of Bellini. Into each collecting tube opens, in the pyramids of Ferrein, a number of the curved tubules by which the secretory portions of the uriniferous tubules are connected with the collecting tubes.

In this manner the urine secreted by the secretory tubules is collecter and discharged into the pelvis of the kidney.

The collecting tubes have large open lumina, and are-lined with a single layer of clear columnar epithelium cells, which are shorter in the cortical portion of the kidney and become longer in the papillary region and tubes of Bellini.

The bloodvessels of the kidney (Fig. 68) are branches of the renal artery and vein. These vessels enter the kidney at its hilum and pass into the areolar tissue within and lin- 
FIg. 68.

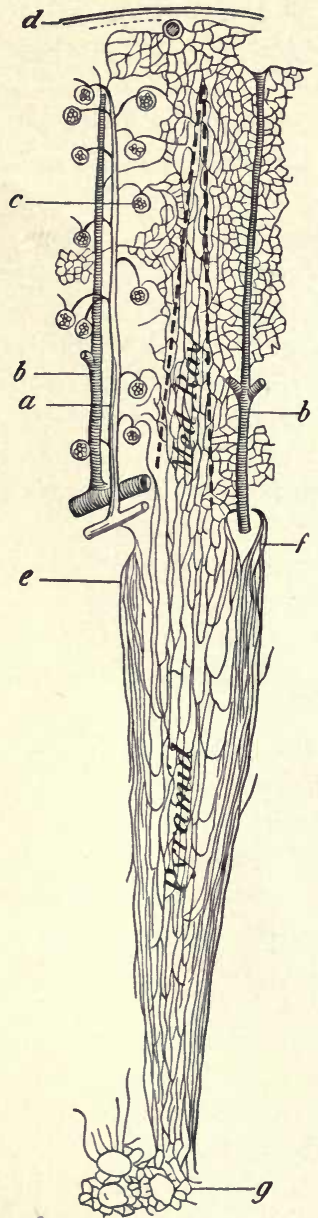

Course of bloodvessels in the kidney (I,udwig). $a$, interlobular artery; $b$, interlobular vein; $c$, Malpighian body, with afferent and efferent vessels and glom. erulus: $\boldsymbol{d}$, stellate vein; $e$, arteriæ rect: ; $f$, venæ rectæ ; $g$, capillaries about mouths of outlet-ducts. ing the sinus. They give off twigs which supply this region and divide into a number of branches, the arteria and vence proprioe renales, which pass outward in the columnæ Bertini, between the Malpighian pyramids.

On reaching the bases of these pyramids they divide into the arcuate arteries and veins, which follow the boundary between the cortical and medullary regions and, anastomosing with one another, form complete arterial and venous arches over the bases of the Malpighian pyramids. From these arches two sets of small vessels are given off, one the interlobular, passing radially outward into the cortex; the other, the vasa recta, converging inward to supply the medulla.

The interlobular arteries, ${ }^{1}$ or cortical arteries, are small straight branches from the arterial arches passing radially outward in the interpyramidal cortical regions, midway between the pyramids of Ferrein. They give off a small twig to each of the Malpighian bodies along their course, which enters the glomerulus as its afferent vessel. In the glomerulus the afferent vessel breaks up into a capillary tuft; the capillaries then reunite to form a single efferent vessel leaving the glomerulus. These efferent vessels then mostly

${ }^{1}$ Each pyramid of Ferrein, with the tributary interpyramidal tubules in its vicinity, may be regarded as a distinct section or physiological lobule of the renal cortex, though they are not marked off from one another by definite boundaries into distinct lobules. The cortical arteries pass between these sections or lobules, whence the term interlobular. 
break up into another capillary network enveloping the uriniferous tubules in the pyramids and interpyramidal regions of the cortex. These capillaries then unite again and empty into the interlobular veins, which occupy a situation corresponding to that of the interlobular arteries, and empty into the venous arches. The capillaries beneath the capsule unite in venous branches, the stellate veins, which meet in a starshaped manner and empty into the interlobular veins.

The arteriæ rectæ are small straight arterioles which arise in large numbers from the concave sides of the arterial arches and pass into the Malpighian pyramids, converging to the apices of the latter. They divide into elongated capillary meshes embracing the uriniferous tubules of the medulla. A few arteriolæ rectæ are also derived from afferent vessels of some of the glomeruli, which pass into the medulla, instead of breaking up into capillaries in the cortex. The blood from the medullary capillaries is collected by the vence recto, which are arranged similarly to the arteriæ rectæ and empty into the venous arches.

The structures composing the different parts of the kidney may be recapitulated as follows: the interpyramidal portions of the cortex contain the Malpighian bodies, the proximal and distal convoluted tubules, the irregular tubules, the curved tubules, and the interlobular vessels.

The pyramids of Ferrein contain the spiral tubules, part of the ascending limbs of Henle's loops, and portions of the straight collecting tubes.

The medulla contains the descending and part of the ascending limbs of Henle's loops, part of the straight collecting tubes, the tubes of Bellini, and the vasa recta.

The secretion of the urine is accomplished somewhat as follows: In the Malpighian bodies fluid transudes or filters from the blood through the thin capillary-walls and squamous cells into the cavity of the capsule of Bowman. The fluid thus separated is supposed to be mainly water, with very little solids. The glandular epithelium of other portions of the tubules, the convoluted tubules especially, then remove from the blood in the surrounding capillaries the solid constituents of the urine by a true secretory process ; these solids are dissolved by the fluid passing down the tubules. 
The pelvis of the kidney, centrally situated at one side, is continuous with or forms the upper extremity of the ureter.

This expanded upper end of the ureter divides into two or three divisions or infundibula, and these into calices into which the apices of the Malpighian pyramids project as papilla.

The wall of the renal pelvis consists of three layers, mucous (composed of epithelium and a tunica propria), muscular, and fibrous or areolar. The superficial layer of the mucosa consists of stratified squamous epithelium, of the variety called transitional, made up of a few layers of cells rapidly changing from the deeper spheroidal to the superficial squamous form. The epithelium rests on a fibrous tunica propria, beneath which is a fibro-muscular layer, containing involuntary muscle-cells. Underneath the latter is a broad layer of fibrous tissue. Within the renal sinus is a variable amount of areolar tissue, extending to the kidney-parenchyma, and usually containing considerable masses of adipose tissue. In this areolar tissue the larger arteries and veins of the kidney are situated and subdivide. The mucosa is said to contain a few small racemose glands, though the presence of such glands in the urinary tract is disputed.

The ureter is continuous with the renal pelvis, and its walls consist of three coats, mucous, muscular, and fibrous.

The mucous coat, which in the contracted ureter is thrown into longitudinal folds, consists of a lining of transitional epithelium, resting on a fibrous layer which represents a tunica propria and submucous layer. The mucosa is said to contain a few small racemose mucous glands.

Outside the mucosa is the muscular coat, composed of involuntary muscle in three layers, an inner longitudinal, a middle circular, and in the lower portion of the ureter a thin outer longitudinal layer. Surrounding the muscular coat is an areolar or fibrous layer.

\section{The Bladder.}

The walls of the bladder consist of three coats-mucous, muscular, and fibrous, from within outward.

The mucous coat, which is thrown into folds when the organ 
is contracted, consists of an epithelial layer, tunica propria, and submucosa.

The epithelium lining the bladder consists of a few layers of stratified squamous or transitional epithelium (Fig. 12), continuous with and similar to that lining the ureters and renal pelvis. The superficial layer of cells in the epithelium is a stratum of thick, squamous, or lenticular cells, possessing sometimes more than one nucleus each ; the lower surfaces of these cells exhibit indentations into which the underlying cells project. The cells beneath this outermost layer are oval or pyriform and perpendicular to the surface; the two or three layers beneath these are spheroidal or irregular germinal epithelial cells.

The epithelium rests on a firm fibrous tunica propria; beneath this is a layer of loose or areolar submucous tissue continuous with the fibrous tissue permeating the muscular coat. A few small racemose mucous glands are said to be present in the mucosa of the base of the bladder.

Outside the submucosa is the thick muscular layer, composed of fasciculi of involuntary muscle supported in fibrous tissue. The arrangement of the muscular fasciculi is rather indefinite, but in general three layers can perhaps be distinguished: an inner longitudinal layer; a middle circular layer, thickened at the neck of the bladder to form the sphincter vesicæ; and an outer longitudinal layer.

Outside of the muscular coat is a fibrous layer, of areolar tissue, which over a large portion of the fundus of the bladder is covered by peritoneum.

\section{The Urethra.}

The female urethra is lined internally with epithelium, stratified squamous, or sometimes, it is said, simple columnar. The epithelium rests on a firm tunica propria, which, especially toward the meatus, has papillæ projecting into the epithelium. Beneath the tunica propria is a loose submucosa, which contains expanded venous sinuses forming an erectile tissue. Small racemose mucous glands (urethral or Littrés glands) also occur in the submucosa, most numerous at the meatus. The mucosa, consisting of the above-mentioned layers, lies in 
longitudinal folds. Outside the submucous tissue is a wellmarked non-striated muscular coat, having an inner longitudinal and outer circular layer, and intermingled with elastic tissue. Outside the muscular coat is fibrous tissue, in which the urethra is imbedded.

The male urethra: The walls of the male urethra are composed of epithelium resting on a tunica propria (forming a mucous coat), submucous tissue, and muscular tissue, and contain numerous mucous glands.

The epithelium lining the male urethra is of the transitional variety at the vesical end, then changes to stratified columnar; throughout the middle and longest portion (penile) it is simple columnar, and for a short distance back of the meatus is of the stratified squamous type.

The epithelial layer rests on a fibrous tunica propria which toward the meatus sends papillæ into the epithelium.

These layers form the mucosa, which in the flaccid condition of the canal lies in longitudinal folds.

Outside the tunica propria is the submucous layer of areolar and loose fibrous tissue.

In the penile portion of the urethra the submucous tissue merges into the cavernous or erectile tissue of the corpus spongiosum, which is enveloped in a strong fibrous sheath.

The submucosa along the entire course of the male urethra contains small racemose mucous glands, the urethral glands or glands of Littré, lined with columnar epithelium and opening into the urethral canal; in the penile portion these glands are scattered throughout the erectile tissue.

The muscular coat of the urethra lies outside or within the submucous coat. In the prostatic and membranous portions the muscular layer lies beneath the submucosa, and consists of inner longitudinal and outer circular fasciculi of non-striated muscle.

Outside these muscular layers in the membranous urethra is an additional muscle, the voluntary compressor urethræ.

In the penile portion of the uretlira the muscular layer is imbedded in the submucous tissue. Near the bulb the -inner longitudinal and outer eircular layers of smooth muscle are still present; but anteriorly the circular layer disappears and the longitudinal fasciculi become thinned and scattered. 
The urethra is surrounded in its prostatic portion by the prostate ; in its penile portion by the corpus spongiosum ; between these two portions is a short interval, the membranous portion, in which the urethra is supported in fibrous tissue.

The urine is a clear, homogeneous, serous secretion, containing a slight amount of mucus. A very few adventitious elements may be normally present-detached epithelium-cells, mucous corpuscles, stray leukocytes, and spermatozoa. In abnormal conditions a variety of organic formed elements and crystalline or amorphous solid substances may appear. 


\section{CHAPTER XIV.}

THE REPRODUCTIVE SYSTEM.

THE consideration of the organs of reproduction naturally falls into two parts, those of the male and those of the female.

\section{MALE GENITAL ORGANS.}

The male genital organs consist of the testicles, the epididymis, the vas deferens, the seminal vesicles, the prostate, Cowper's glands, and the penis, besides vestiges of foetal structures. The main portion of these, the testes and seminal ducts, are derived from the embryonic Wolffian body, and their epithelium is therefore of mesoblastic origin.

The scrotum, the pouch containing the testicles, is covered externally with skin, thin and plicated. Beneath, and closely connected with the skin, is the dartos, a layer of vascular contractile tissue composed of areolar tissue and involuntary muscle. A septum from the dartos divides the serotum into lateral halves. Beneath the dartos are strata of areolar and fibrous tissue-the intercolumnar fascia, cremasteric fascia, and fascia propria ; the cremasteric fascia also contains strands of striated and non-striated muscle. Beneath these layers is the parietal layer of the tunica vaginalis, a closed serous sac derived from the peritoneum and invaginated into itself to form a covering for the testicle.

\section{The Testicle.}

The testicle is a compound tubular gland in which the spermatozoa are generated. Attached to the posterior aspect of the testicle is the epididymis, which consists of a body, an expanded upper extremity, or head, called the globus major, and a smaller lower extremity, or tail, the globus minor.

The testicle is essentially a congeries of tubules, secretory 
and conducting, called the seminiferous tubules, supported in a connective-tissue framework. It is well supplied with bloodvessels, and also has lymph-channels; its nerve-supply is inconspicuous and obscure.

The surface of the testis is covered with a serous membrane, the visceral layer of the tunica vaginalis; this sac is invaginated over the testis from behind, leaving a hiatus or hilum for the entrance of the elements of the spermatic cord.

The sustentacular connective tissue of the testis consists of the tunica albuginea, mediastinum testis, and interlobular and intertubular tissue.

The tunica albuginea is a dense fibrous capsule enveloping the organ, lying underneath and merging into the visceral layer of the tunica vaginalis. At the posterior part of the testis the tunica albuginea is thickened, forming a longitudinal ridge or mass called the mediastinum testis, or corpus of Highmore; this contains a system of communicating spaces, the rete testis.

In infancy numerous well-marked fibrous partitions (interlobular septa) radiate from the mediastinum testis and, extending across toward the tunica albuginea opposite, divide the testicle into a number of conical compartments or lobules, with their apices directed toward the mediastinum.

The adult and fully developed testicle, however, is often less completely and less conspicuously divided into lobules, the interlobular septa being occasional rather indefinite fibrous laminæ, a little thicker than the intertubular septa.

Lining the interlobular septa and the inner surface of the tunica albuginea is the tunica vasculosa, a layer of areolar tissue containing abundant bloodvessels.

The individual seminiferous tubules are separated from one another by the intertubular tissue, thin connective tissue partitions containing capillaries and providing basement-membranes for the tubules. In places within the intertubular tissue are situated groups of large spheroidal epithelioid (?) cells, interstitial cells.

The seminiferous tubules exhibit several divisions, which are, from their beginning onward, as follows: convoluted tubules, tubuli recti, rete testis, vasa efferentia, and epididymis. The convoluted portions generate the spermatozoa, the remaining 
seminal ducts are conducting-channels or secrete fluid constituents of the semen.

The convoluted tubules are single or branched tubes, of large diameter, coiled, bent, and twisted in intricate convolutions, closely packed together so as to fill practically the entire testicles. They are surrounded by well-marked basementmembranes, and their coils are separated from one another by the thin intertubular connective tissue. The tubules are lined by several layers of epithelium, from which the spermatozoa are generated.

The deepest or parietal layer of epithelium consists of large spheroidal spermatogenic cells ("spermatogonia") resting on the basement-membrane, among which are occasional long sustentacular cells ("cells" or "columns of Sertoli") projecting inward among the other cells toward the lumen.

Upon these parietal cells rests a layer or two of large spheroidal cells, mother-cells, derived from the "spermatogonia." Next internal to these are a greater number of smaller spherical-cells; these are called daughter-cells (also called "spermatids" and "spermatoblasts"), and are derived from the mother-cells.

The daughter-cells elongate and develop into the spermatozoa, (described below), which are located in clusters, their heads (until they become separated) being attached to the daughter-cells and their tails projecting into the lumen. The lumen of the tubules contains, besides the spermatozoa, a broken-down granular material.

Spermatogenesis: The development of the successive layers and generations of spermatogenic cells and the spermatozoa takes place by karyokinesis.

All parts of the seminiferous tract are not simultaneously engaged in active cell-division, but the different stages of the process are observable in different regions at the same time. While karyokinesis and the production of spermatozoa are actively going on in some places, in others the cells are quiescent and spermatozoa absent. Some of the details of the process of generation of the spermatozoa are still undetermined or in dispute. It is supposed that during their generation the number of chromosomes of the spermatozoa 
becomes "reduced" to half the number characteristic of the cells of the species (page 36), so that when joined to the female element, likewise reduced (page 39), the fertilized ovum possesses the normal number of chromosomes (sixteen in man).

As the spermatozoa are endowed with the power of transmitting to the offspring the characteristics of the parent, the process of spermatogenesis is a link in the chain of heredity. The mode by which the parent implants or involves his own characteristies within the minute compass of a spermatozoon, so that the latter in its further development reproduces its kind, is a biological question of great importance.

The characters of the parent are either congenital (racial, inherited) or acquired; the latter few in number, the former multitudinous. Many biologists contend that characteristics acquired by au individual subsequent to his conception and birth cannot be transmitted to his offspring; but supposing it possible, what mechanism exists in the genital organs by which the features of a distant part of the body can be impressed upon spermatozoa developing in the testicle so as to be carried on to the progeny? Obviously the only channels by which impressions or influences from other parts could be conveyed to the testicle are the circulation and the nerves. The testes are amply supplied with bloodvessels; not so well, perhaps, with nerves. We can conceive how, in conditions of toxæmia or generally lowered vitality, an impoverished or toxic blood could alter or lower the vitality of the spermatogenic cells and thus convey a deteriorating tendency to the offspring; and we are also familiar with trophic nervous effects; but aside from these, the manner in which bodily features might become impressed on the spermatogenic cells through nervous or circulatory influences is obscure.

Little is known likewise as to just how the congenital or inherited features of the parent, the fixed and stable characters of the race, are carried over from generation to generation. Amidst the vast multitude and diversity of cells making up the body, what line of cellular transmission of racial features connects the ovum, from which an organism develops, with the ovules or spermatozoa of the next generation which after a time appear in the body of the parent?

Many biologists regard the cells into which an ovum develops as of two fundamentally different kinds: somatic cells, making up the body generally; and germ-cells, to which the developmental powers of the ovum are transmitted. According to this theory, the spermatogenic cells of the testicle and the corresponding cells of the ovary are direct descendants of the orum through a succession of germ-cells (located in the sexual glands), which have carried on, in isolated continuity as it were, the reproductive potentialities inherent in the ovum.

The tubuli recti or straight tubules: The convoluted tubules converge toward the mediastinum testis, and at their proximal 
ends terminate in short straight tubules which empty into the channels forming the rete testis. These tubuli recti are narrower in calibre than the convoluted portions, and are lined with a single layer of low columnar or cuboidal epitheliumcells.

The rete testis is a network of anastomosing channels and sinuses formed by the union of the tubuli recti. These channels are situated in the substance of the mediastinum testis, and are lined with a single layer of flattened epithelial cells resting on the surrounding fibrous tissue.

The vasa efferentia: The seminiferous channels of the rete testis at its upper end continue upward in the form of ten to fifteen efferent vessels, the outlet-ducts of the testis, which pass through the tunica albuginea and enter the globus major, where they form the coni vasculosi. These vasa efferentia are lined with stratified columnar epithelium, partly ciliated ; the epithelium cells are thrown up into ridges and papillæ which project into the interior of the tubules and give the free surface a sinuous and undulating character. The epithelium rests on a basement-membrane and thick fibrous wallecontaining involuntary muscle-cells arranged transversely.

In the globus major the efferent tubules are coiled and convoluted, making up the coni vasculosi (or lobuli epididymidis), each tube forming a conical mass or lobule. The globus major is made up of these lobules.

The several tubes forming the coni vasculosi unite ultimately into one tube which continues downward and onward as the canal of the epididymis.

The epididymis consists macroseopically of a body, a head or globus major above, and a globus minor below. The globus major is intimately connected with the testis by the vasa efferentia ; the globus minor is attached to the testis by fibrous tissue; the body of the epididymis mainly by the tunica vaginalis.

The body and globus minor of the epididymis are made up of the windings of the canal of the epididymis, which is the continuation of the united vasa efferentia. "The canal of the epididymis is a single long continuous tube, coiled, convoluted, and packed into small compass; it is lined with stratified ciliated epithelium, resting on a basement-membrane and 
surrounded by fibrous tissue containing transverse involuntary muscle-cells. The coils of the canal are imbedded in and separated by areolar tissue, and the whole epididymis is enveloped in a fibrous capsule. At the lower end of the epididymis its canal becomes and continues onward as the vas deferens.

Various remnants of fœtal structures (vestiges of the Wolffian body) are found about the testicle.

The paradidymis, or organ of Giraldès, is situated in the connective tissue of the epididymis, and consists of a few closed convoluted tubules lined with epithelium. The ras aberrans is a tube similar to the vas deferens, and is sometimes found branching off from the lower part of the latter, or canal of the epididymis, passes up the spermatic cord for a variable short distance, and has a blind upper end. The hydatids of Morgagni are small pedunculated bodies, containing areolar, epithelial, or vascular tissue, attached to the upper part of the testis or epididymis.

The vas deferens, the continuation of the lower end of the canal of the epididymis, forms one of the constituents of the spermatic cord, and extends to the base of the bladder, where it becomes dilated (forming the "ampulla") and unites with the outlet of the seminal vesicle. It is a firm cordy tube, and its walls consist, from within outward, of a mucous, submucous, muscular, and fibrous coat.

The mucous coat is lined with stratified columnar epithelium, ciliated for a short distance at the beginning of the vas, but non-ciliated in the remaining greater portion of the canal; the epithelium rests on a tunica propria, which is surrounded by submucous areolar tissue. The mucosa is thrown into longitudinal folds in the vas, and in the ampulla into irregular rugre and plications. Small glands oceur in the mucosa in the ampulla.

The muscular coat is composed of an inner circular and outer longitudinal layer of involuntary muscle, intermingled with fibrous tissue. Outside the muscie is a layer of fibrous and areolar tissue.

The seminal vesicle is a sacculated pouch, largely of glandular character, branching off from the vas deferens. Its outlet 12-Hist. 
unites with the vas deferens to form the ejaculatory duct. Its walls are similar in structure to those of the vas. It is lined with columnar epithelium, which rests on a tunica propria, beneath which is submucous areolar tissue. Its mucosa is thrown into interlacing rugæ and plications, which form saccular and somewhat gland-like recesses; the mucous coat also contains small glands. Beneath the submucosa is an inner circular and outer longitudinal layer of involuntary muscle, outside of which is areolar tissue.

The ejaculatory ducts, one on each side, are formed by the union of the vasa deferentia with the outlets of the seminal vesicles. They are situated partly within the prostate, and empty into the prostatic urethra. 'They are lined with simple columnar epithelium, resting on connective tissue ; outside the latter are thin inner circular and outer longitudinal layers of involuntary muscle.

The prostate gland is a compound tubular gland, the mucous secretion of which enters into the composition of the semen. It surrounds a portion of the urethra and ejaculatory ducts. It is made up of sustentacular connective tissue, involuntary muscle, and secreting alveoli, besides vessels and nerves.

The sustentacular connective tissue consists of a firm fibrous capsule, septa traversing the organ in various directions, and interalveolar tissue. Intermingled with and acconpanying the connective tissue throughout the organ, forming in many places the greatest part of its framework, is a large amount of involuntary muscle, in the capsule and septa, between the alveoli, and surrounding the urethra.

The glandular alveoli of the prostate are of a racemose or compound tubular character, and are lined with columnar epithelium.

Their ducts unite to form twelve to twenty outlet-ducts opening into the urethra-i. e., the "prostatic sinus" on each side; the epithelium lining the ducts merges into the squamous type of the urethra. Many of the alveoli contain minute round, concentrically marked calculous granules, or prostatic concretions. From the lower surface of the urethra a short small cul-de sac extends into the prostate; this is called the sinus pocularis or uterus masculinus, and is homologous to the female uterus. 
Cowper's glands, situated beneath the membranous urethra between the layers of the deep perineal fascia, are two small racemose glands emptying into the urethra. The alveoli are lined with columnar epithelium apparently of the mucous type, though the purpose of the secretion is not well understood.

\section{The Penis.}

The penis consists of three longitudinal cylindrical compartments surrounded by fibrous sheaths-two corpora cavernosa filled with erectile tissue, and the corpus spongiosum containing erectile tissue and the urethra-all covered with skin

Fig. 69.

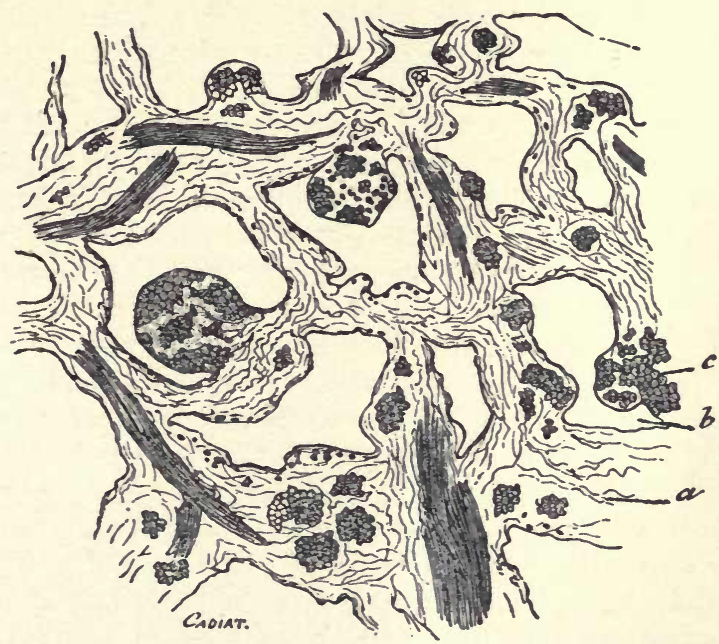

Cavernous or erectile tissue of penis (Cadiat). $a$, fibrous trabeculæ; $b$, cavernous sinuses; $c$, smooth muscle in cross-section.

and subcutaneous tissue. The skin is thin and movable. The subcutaneous areolar tissue is free from fat, contains vascular and nerve trunks, and by its looseness allows the skin much play.

The corpora cavernosa are surrounded by a common sheath of dense fibrous tissue, the tunica albuguinea. Each corpus 
cavernosum is also enveloped by a dense fibrous sheath of its own, lying just within the common sheath the septum ("pectiniform") thus formed between the two corpora is, however, incomplete and interrupted. From these sheaths fibrous trabeculæ are given off which traverse the interior of the corpora in all directions, branching and anastomosing so as to divide them into a spongy system of spaces or sinuses communicating freely with one another. The fibrous trabeculae contain some involuntary muscle and elastic tissue, and convey vessels and nerves. The sinuses formed by the septa are lined with endothelium, and into them open the bloodvessels, so that they form a system of cavernous spaces or vascular sinuses, through which the blood flows, from the arteries and into the veins (Fig. 69). This arrangement constitutes erectile tissue. When the venous outflow is cut off by muscular action blood accumulates in the sinuses, causing the penis to become turgid and erect.

The corpus spongiosum is a compartment filled with erectile tissue and containing the urethra. Its posterior end, slightly expanded, forms the bulb; its anterior end, the glans penis. It is enveloped in a firm fibro-elastic sheath containing a small amount of involuntary muscle. From the sheath fibro-clastic trabeculæ, containing occasional non-striated muscle, traverse the corpus and divide it into vascular sinuses, forming erectile tissue like that of the corpora cavernosa. Along the axis of the corpus spongiosum passes the urethra (more fully described above) surrounded by the erectile tissue, which merges into the submucous tissue of the urethra. The urethra is lined with columnar epithelium and into it open numerous mucous urethral glands (the "glands of Littré), which are scattered about in the erectile tissue of the corpus spongiosum.

The glans penis is an expansion of the anterior extremity of the corpus spongiosum, covered by closely adherent mucous membrane. The mucous membrane, continuous with that of the urethra, is lined with stratified squamous epithelium, resting upon a tunica propria, the surface of which is studded with papillæ containing numerous special sensory nerve-terminals. At the corona and base of the glans the mucous membrane contains abundant sebaceous glands, the glandula odoriferce or glands of Tyson, unassociated with hairs, the 
odorous secretion of which enters into the formation of the smegma. The mucous covering of the glans is reflected over the inner surface of the prepuce and at its margin merges into the skin.

The semen, as finally ejaculated, consists of an immense number of special cellular elements (spermatozoa) suspended in fluid (liquor seminis), together with granular, crystalline, and adventitious matter. The liquor seminis is derived from various parts of the seminal tract, especially the seminal vesicles, prostate, and Cowper's glands. It is a muco-albuminous fluid containing various chemical ingredients. It exhibits fine granular matter (cellular and protoplasmic débris), and, in small number, cast-off epitheliumcells, stray leukocy tes, clear " hyaline bodies" from the seminal vesicles, fat and lecithin particles, and the small prostatic concretions or "amyloid bodies." After standing, erystals are deposited in the semen, especially characteristic slender octahedral crystals of spermin phosphate.

The spermatozoa of different species of animals exhibit characteristic differences though the general structure remains similar; the same is the case with the homologous pollen of plants.

The spermatozoön of man (Fig. 70) is made up of a head 3 to $5 \mu$ long, a middle piece 5 or $6 \mu$ long, and a tail 40 to $50 \mu$ long. The head is of a flattened oval or pyriform shape, to the large end of which the middle Fig. 70.

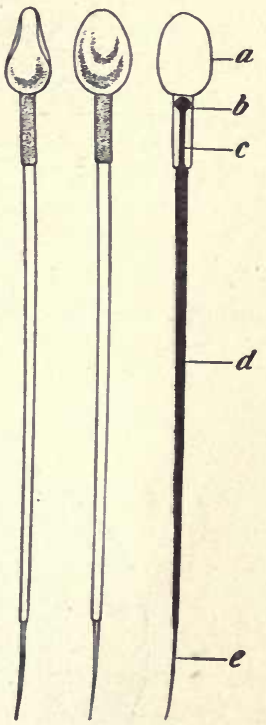

Human spermatozoa (Böhm and Davidoff). The left figure is a side view; the middle figure a top view. $a$, head; $b$, centrosome (?); $c$, middle piece: $d$, sheath about axial fibre $(e)$. piece or tail is attached. The middle piece is a cylindrical segment more or less distinctly marked off from the tail. The tail is a long fine filament or flagellum, tapering to a point; it possesses an "axial fibre" surrounded (except for a short dis- 
tance at the end) with a protoplasmic sheath. The tail exhibits rapid vibratory movements, by means of which the spermatozoön is propelled forward.

Spermatozoa are real cells, though of unusual type. The head is of the nature of a nucleus, while the tail and a possible protoplasmic envelope about the head may be taken to be cytoplasm. The chromosomes are "reduced." A very minute round body detected by some observers just posterior to the head is perhaps a centrosome.

\section{FEMALE GENITAL ORGANS.}

The female genital organs are the ovaries, oviducts, uterus, vagina, vulva, vestiges of foetal structures, and mammary glands. The pelvic organs are developed from the embryonic Wolffian body and duct of Müller and their epithelium is of mesoblastic origin.

\section{The Ovary.}

The ovary (Fig. 71) consists of a connective-tissue mass or stroma, in which are imbedded large numbers of developing

Fig. 71.

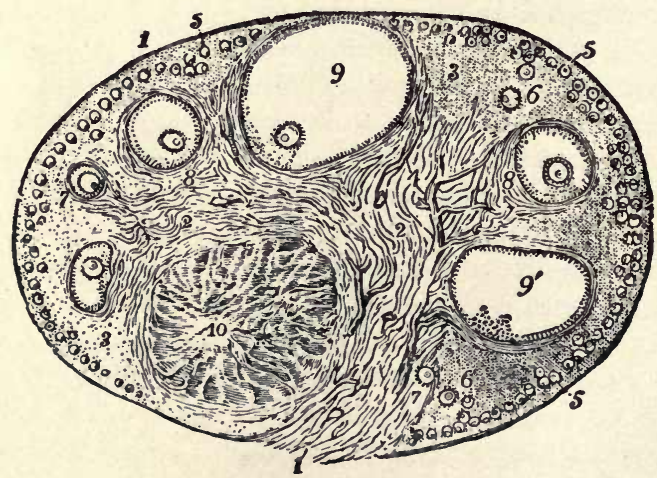

Section of ovary of cat (Schron). 1, epithelial covering; 1', hilum: 2 , medulla ; 3 , stroma of cortex ; 4 , bloodvessels; 5 , undeveloped ovisacs : $6,7,8$, developing Graafian follicles; 9 , mature Graafian follicles; 10 , corpus luteum.

ova, each in a structure called an ovisac or Graafian follicle; the whole organ being covered by an epithelial investment. 
The ova and Graafian follicles occupy the peripheral or cortical portion of the ovary (the "oöphoron"), leaving in the interior a medullary region continuous with the hilum, where the vessels enter.

The epithelial covering of the ovary is a single layer of cuboidal or low columnar cells, called "germinal epithelium" from the fact that the ova are developed from it during fetal life. This epithelium is continuous with the peritoneal endothelium, the glistening squamous cells of the latter being here replaced by columnar cells of less lustre.

The stroma of the ovary is a mass of connective tissue, made up in the cortical region largely of fusiform connectivetissue cells with scanty intercellular elements. On the surface, just beneath the epithelial covering, this tissue is condensed into a layer called the tunica albuginea, continuous and intimately connected with the stroma.

The medullary region and hilum contain the larger vessels, imbedded in connective tissue of a more ordinary kind, in which fibrous elements are more abundant.

Scattered about in the stroma (more abundantly in certain animals than in the human species) are large epithelioid (?) cells, the interstitial cells, similar to those of the testis. The ovary is well supplied with bloodvessels, and also possesses lymphatics and nerves.

The ova (called ovules before impregnation), which occupy the peripheral zone of the ovary, originate during fotal life from processes of the germinal epithelium which grow downward and are then cut off, learing isolated nodules of epithelial cells in the stroma. In each of these nodules one cell, destined to become the ovum, enlarges, while the other accompanying (indifferent) epithelial cells form a capsule about it and develop ultimately into the tunica granulosa of the mature Graafian follicle. The cortex of the ovary contains large numbers (estimated by some observers at about 70,000 in both ovaries) of these primitive ovisacs imbedded in the stroma, each consisting of a large ovule surrounded by a more or less definite envelope of small epithelial cells. In the course of ovulation and menstruation these primitive ovisacs develop as Graafian follicles, and successively mature and discharge the ovules from the ovary, leaving the remains 
of the follicles visible for a time as corpora lutea. At any one time the active ovary exhibits a number of Graafian follicles and corpora lutea in different stages of development, along with a large number of undeveloped ovisacs.

Graafian follicles (or vesicles): As the primitive ovisacs develop they enlarge and sink deeper into the ovary. The fully developed Graafian follicles are spherical or oval cysts about 4 to $10 \mathrm{~mm}$. in diameter. Each is surrounded by a condensation of the stroma in which it is imbedded, forming a vascular connective-tissue investment called the theca folliculi; this is usually described as consisting of two layers, an outer, ealled the tunica fibrosa, and an inner, ealled the tunica propria. The theca, be it noted, is not really a part of the Graafian follicle, but is a condensation of the ovarian stroma surrounding it.

The outer layer of the follicle proper, lying next to the tunica propria, is the membrana or tunica granulosa, composed of a few layers of small spheroidal nucleated epithelial cells. This tunic encloses a large cavity which is filled with an albuminous fluid, the liquor folliculi.

At one point in its circumference the tunica granulosa is thickened, having a mass projecting inward composed of cells identical with those which make up the tunic elsewhere; this mass or heap of cells is called the discus proligerus, or cumulus ovigerus. Within this cumulus the ovule is imbedded; the cells in a layer immediately surrounding the ovule are columnar and arranged in a radiating manner, forming the corona radiata.

The ovule, or ovum (Fig. 72), is a large spherical cell about $.2 \mathrm{~mm}$. in diameter ; each Graafian follicle contains one ovule, rarely two or three. The wall of the ovule is a thin clear hyaline membrane, showing indistinct radial markings; it is variously called the vitelline membrane, zona pellucida, zona radiata, and oölemma. The cell-body of the ovule, or vitellus, is a mass of protoplasm infiltrated with fat-particles. The nucleus of the ovule is a spherical body, often called the germinal vesicle; within it is a nucleolus, the germinal spot.

Ovulation: The Graafian follicle, when mature, makes its way to the surface of the ovary, causing a bulging at that point. The tissues overlying the follicle become thinned, 
and finally the vesicle ruptures, permitting the ovule to escape from the ovary and pass into the oviduct. The discharge of an ovule in this manner takes place regularly and periodically,

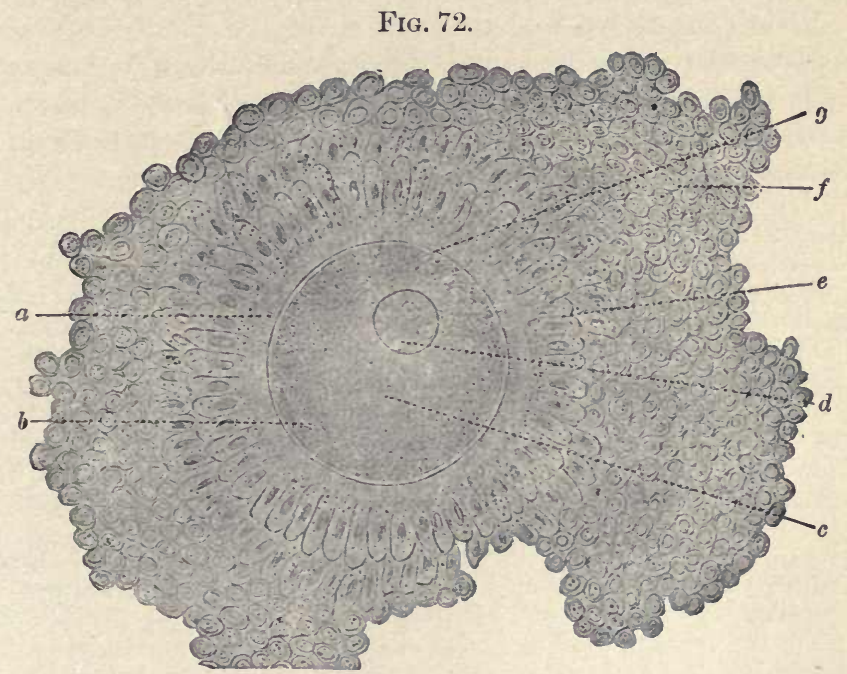

Human ovule and its surroundings in the ovary (Nagel). $a, g$, vitelline membrane (zona pellucida and zona radiata); $b$, vitellus or cy toplasm of ovule; $d$, nucleus or germinal vesicle; $e$, corona radiata; $f$, cells of cumulus ovigerus.

comprising the process called ovulation, which stands in a definite relation with menstruation.

Corpora lutea: The ruptured Graafian follicle after ovulation undergoes a series of retrograde changes, the resulting structure being called a corpus luteum.

The wound or cavity of the emptied Graafian follicle becomes filled with blood from the ruptured vessels, and the cells of the tunica granulosa proliferate abundantly. From the fatty degeneration of cells or the transformation of hæmoglobin into hæmatoidin the structure acquires a yellow color, whence its name. Into the mass of epithelial cells and bloodclot grow, from the ovarian stroma, vascular processes of connective tissue (granulations), giving the body a lobed or radiate appearance. By the continued growth and contrac- 
tion of the connective tissue, forming a sort of scar or cicatrix, the corpus luteum is finally obliterated.

The whole process is similar to that by which wounds in general undergo repair.

The corpora lutea which result when the discharged ovules are fecundated (the so-called "true" corpora lutea) attain a larger size and continue in existence a much longer time (for many months) than do those (the "false" corpora lutea) when pregnancy does not result.

Oögenesis: The ovary is similar in development to the testicle and complementary to it in function. Both are organs of high vital importance, being the essential genital glands and seats of reproduction.

The observations made above with respect to spermatogenesis apply also, mutatis mutandis, to oögenesis, the development of ovules.

The ovary, like the testicle, has an ample blood-supply and a less conspicuous imnervation. These two channels, circulatory and nervous, are the only apparent means of communication by which conditions in other parts of the maternal budy can influence the ovary and hence be transmitted to the offspring. That the ovary can in such way act in sympathy with organs not directly connected with it is shown by the relations between ovulation, menstruation, and pregnancy.

As to the transmission of racial and hereditary characteristics from the ovum of one generation to the ovules of the next through a succession or continuity of special germ-cells, the same considerations apply in oögenesis as in spermatogenesis. Some differences in action of the female and male genital glands may be noticed. All the ovules discharged by the human female exist in the ovary preformed from birth; consequently any acquired characters in the mother, if transmitted to offspring, would have to be impressed upon cells already formed rather than upon cells to be subsequently developed. The spermatozoa and spermatogenic cells, on the contrary, are being continually renewed and developed de novo. Of all the primitive ovules in the ovaries only a few hundred come to maturity in the lifetime of the human female; in contrast to this the male produces and disseminates millions of spermatozoa.

The oviducts, or Fallopian tubes, are conical tubes opening into the uterus by the small end and into the peritoneal eavity by the large end, which is fimbriated. The wall of the oviduct consists, from within outward, of mucous, submucous, muscular, and serous coats. The mucous coat is formed by a single layer of columnar ciliated epithelium-cells resting on a firm tunica propria. At the margins of the fimbrix the 
ciliated cells meet the squamous endothelium-cells lining the peritoneum. The mucosa is thrown into broad folds or plications, mostly branching, which project radially into the lumen of the tube. Between the mucosa and submucosa is a thin layer of involuntary muscle representing a muscularis mucosæ. The submucous coat is made up of areolar tissue containing the larger vessels. The oviducts contain no glands. Outside the submucous coat is a muscular coat, composed of a thick inner circular layer and a thinner outer longitudinal layer of involuntary muscle. The outermost or serous covering of the oviduct is formed by the peritoneum and subperitoneal tissue.

Remnants of fœtal structures: The parovarium is a series of tubules lined with simple columnar epithelium, situated in the broad ligament near the hilum of the ovary and between the latter and oviduct; they are remains of tubules of the embryonic Wolffian body. Other similar tubules, which have received special names, are also sometimes found in the broad ligament. Pedunculated hydatids (hydatids of Morgagni), small epithelial sacs, are sometimes attached to the ovary, parovarium, or a fimbria of the oviduct.

\section{The Uterus.}

The uterus is a hollow, muscular organ, the wall of which is composed chiefly of involuntary muscle, lined internally by mucous membrane and externally by serous membrane (Fig. 73).

The uterine mucous membrane consists of epithelium resting on a tunica propria which contains numerous tubular glands. The epithelium lining the uterus is a single layer of short columnar or cuboidal ciliated cells. The tunica propria is a layer of connective tissue of an embryonal or growing type, containing fusiform connective-tissue cells and lymphoid cells or leukocytes in abundance, with scanty intercellular elements; it contains abundant bloodvessels, extends downward to the muscular coat, and in it are imbedded the uterine glands.

The embryonic character of the connective tissue of the tunica propria is apparently due to the fact that the latter in 
the course of menstruation is at short intervals cast off and grows anew.

The uterine glands are numerous tubular glands, mostly simple and unbranched, extending perpendicularly from the surface of the mucosa to the muscular coat. Their deeper portions are convoluted, their superficial portions more

\section{FIG. 73.}

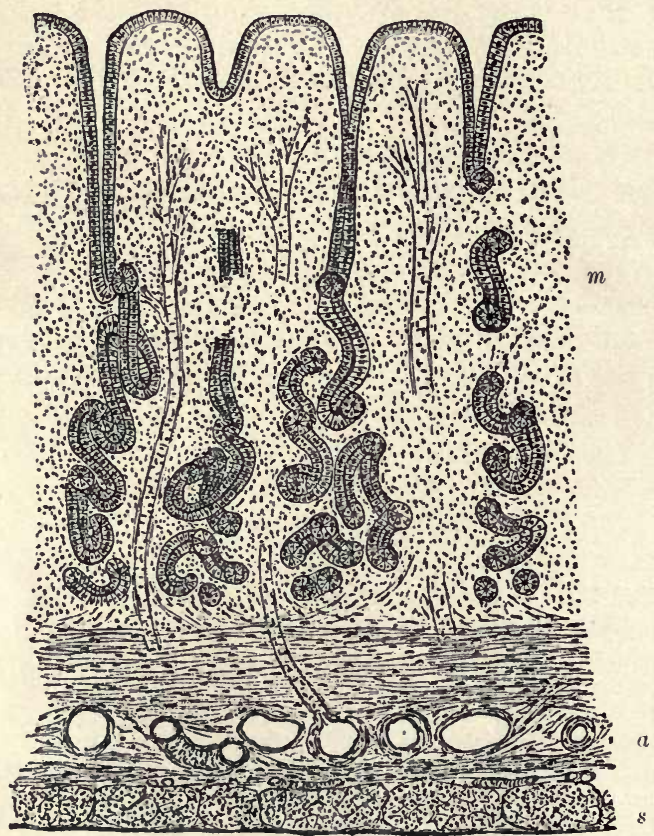

Section through wall of a rabbit's uterus (Schäfer). $m$, mucosa (the cilia of the epithelium are not shown); $a$, muscular coat ; $s$, peritoneum.

straight. They are lined by a continuation of the simple ciliated epithelium of the mucous surface. Their function is not so much secretory as to serve as foci for the regeneration of the epithelium after menstruation.

The muscular coat makes up the bulk of the uterine substance. It consists of layers and fasciculi of non-striated 
muscle, imbedded in connective tissue and intermingled with numerous large arterial and venous channels. The muscular tissue is arranged in general in three strata, an inner and an outer longitudinal and a middle circular layer; but these divisions are not very distinct and the arrangement is not very definite.

The outer surface of the uterus is covered with serous membrane, consisting of the folds of the peritoneum reflected over it. The bloodvessels of the uterus are unusually large, abundant, and tortuous.

The cervix of the uterus has a thicker mucosa, which is thrown into rugæ, and a more regular arrangement of the three muscle-layers than the body of the organ.

The upper portion of the cervical canal is covered with simple ciliated epithelium; but the lower portion is lined with stratified squamous epithelium, and the underlying tunica propria presents numerous projecting papillæ.

In the lower part of the cervix mucous glands are present, in addition to the uterine glands. From the closure of their outlet-ducts these mucous glands often become distended with secretion, forming small cysts called ovula of Naboth. The outer surface of the cervix uteri is lined by the vaginal mucous membrane.

During menstruation the mucous lining of the uterus becomes much thickened; the epithelium cells swell, the tunica propria becomes very vascular and increases in thickness, and the uterine glands enlarge. The mucosa becomes softened and degenerated, and is finally cast off, mingled with blood, as the menstrual discharge. After menstruation is over the mucous membrane is rapidly renewed, the surface epithelium being regenerated from the portions of the uterine glands remaining in the depths of the tunica propria.

During pregnancy there is a great hypertrophy or increased development of the mucous and muscular coats and the vascular system of the uterus. The mucosa undergoes special changes and to it become attached the placenta and fotal envelopes; it exhibits fusiform connective-tissue cells, large numbers of decidual cells (large brown pigmented, round, or polymorphous cells, sometimes multinucleated), dilated and compressed lumina of the uterine glands, and an increase of 
vascuiar channels, while the superficial and glandular epithelium becomes disintegrated or much altered. Its inner surface becomes intimately connected with the placenta and the chorion. Two layers are distinguished in the mucosa, a superficial "compact layer" and a deeper "cavernous" or "spongy layer"; the latter lies next to the muscular wall of the uterus, and is characterized by containing the distended deeper portions of the uterine glands.

This specially developed mucosa of the pregnant uterus is called the decidua, and is east off with the placenta and membranes at the birth of the foetus. The decidua exhibits three divisions: the decidua serotina, which enters into the formation of the placenta; the decidua vera, which lines the nonplacental portion of the inner surface of the uterus, and is cast off, united to the chorion, as part of the fotal membranes ; and the decidua reflexa, which in the early part of pregnancy grows out from the mucosa into the cavity of the uterus and surrounds the ovum and foetus, but at a later period degenerates and disappears. After labor takes place the hypertrophied uterus undergoes retrogressive changes and involution to its ordinary size, and its mucous lining is regenerated from the remnants of the tunica propria and epithelium left after the casting off of the decidua.

\section{Fœtal Appendages.}

The primitive appendages of the fotus are the amnion, chorion, allantois, and umbilical vesicle, all derived from the embryo, together with the decidua, which is of uterine origin. The umbilical vesicle is a rudimentary structure and soon disappears. The amnion, chorion, and decidua form, in general, from within outward, three envelopes about the fotus. As fully developed, at term, these structures form the "after-birth" or "secundines," consisting of the fotal membranes, the placenta, and the umbilical cord; these are delivered with the fœetus at birth.

The (internal or true) amnion, the innermost fœtal envelope, is a thin membranous closed sac filled with fluid in which the fœtus is immersed. It originates as an outgrowth from the embryo, and consists of two layers, the innermost (toward the fotus) an epithelial and epiblastic layer; the outer, a mesoblastic (somatopleural) connective-tissne layer. It is at first separate from the chorion, but afterward becomes united to it and lines the inner free surface of the fotal membranes and placenta.

The chorion, the middle envelope, in man originates chiefly from the 
external or "false" amnion (composed of epiblast and somatopleure) and over a small area from the allantois-besides the vitelline membrane of the ovum, which early disappears. The chorion exhibits externally (toward the uterine wall) an epiblastic epithelial layer, derived from the external amnion; and internally a connective-tissue layer of mesoblastic origin, derived from the amnion and allantois. The chorion is connected with the foetus by a vascular mesoblastic stalk (the umbilical cord) made up mainly of the allantois.

The allantois is a saccular outgrowth from the primitive intestine, extending to the chorion and uniting with the latter over a greater or less area. It consists of mesoblast (splanchnopleure) externally and internally has a cavity lined with hypoblast; in man the portion of the allantoic cavity beyond the abdominal wall consists only of a small tube extending into the umbilical cord. The umbilical bloodvessels are derived from the allantois. The mesoblastic tissues of the allantois enter into the formation of the chorion and umbilical cord.

At a very early period numerous vascular tufts, chorial villi, grow out from the chorion on all sides, projecting toward the uterine wall. A portion of the chorion, called the "chorion frondosum," enters into the formation of the placenta, the villi of this region undergoing great development and becoming intimately united with the corresponding portion of the decidua (the decidua serotina). Over the remainder of the chorion, the "chorion læve," the villi disappear, this portion of the chorion becoming united with the decidua vera to form (with the amnion) the foetal membranes.

The decidua, already described, is the modified uterine mucosa in three divisions-the serotina, vera, and reflexa.

The umbilical vesicle, or "yolk sac," in the higher mammals has little functional value, and soon disappears. It consists of a sac or vesicle situated between the amnion and chorion, and a pedicle or stalk in the umbilical cord. In origin it is continuous with the primitive intestine, and consists of a vascular mesoblastic (splanchnopleural) layer externally, and is lined with hypoblast internally.

The fotal membrane ("bag of waters") is a thin, translucent membrane given off from the margin of the placenta, forms a closed sac about the foetus, and is closely applied to the inner surface of the uterus. It is filled with a quantity of serous fluid, the liquor amnii, in which the foetus is immersed. It is formed, from within outward, of the amnion, chorion, and decidua vera.

The amnion is a thin, non-vascular membrane, lined on its free surface (that directed toward the fotus) by simple squamous epithelium, beneath which is a connective-tissue stratum composed of connectivetissue cells (chiefly flat, stellate or irregular, many with anastomosing processes), fibres, and gelatinous intercellular substance. In places the outer surface exhibits small hexagonal endothelioid cells. The amnion is loosely connected with the underlying chorion by fibrous strands, or very delicate muco-fibrous tissue, so that it has some play over the latter and can be stripped off.

The liquor amnii, which surrounds the fotus and fills the amniotic sac, is a serous fluid holding proteid and other substances in solution. 
The chorion, the middle constituent of the fotal membrane, exhibits two layers, an inner fibrous and an outer cellular layer. The fibrous layer is next to the amnion, with which it is loosely united; it is of mesoblastic origin and composed of fibrous tissue. The outer or "cellular layer" consists of several layers of large spheroidal prominently nucleated cells, apparently of epiblastic origin and epithelial nature.

Externally the chorion is closely united to the decidua vera (the decidua reflexa degenerating and disappearing by about the seventh month); the decidua vera, the mucosa of the pregnant uterus, at labor is cast off with the foetal membrane as its outermost lamina.

The placenta is formed by the union of the chorion frondosum and decidua serotina, and hence consists partly of fotal and partly of maternal tissues. It is essentially an intimate interlacement of fotal and maternal blood-channels, serving alimentary, respiratory, and excretory purposes for the fœtus. The placenta consists of four layers, which are, from the fotal surface outward, the amnion, the chorion, a broad layer of chorial villi separated by maternal blood-sinuses, and the decidua serotina.

The portion of the amnion lining the fretal surface of the placenta is continuous with that lining the fotal membranes, and is similar to the latter in its structure, having a superficial epithelial and deeper connective-tissue layer, and being connected with the chorion by delicate muco-fibrous tissue.

The placental portion of the chorion (chorion frondosum) presents toward the amnion a fibrous layer, and beneath this the epithelioid "cellular layer," as in the chorion læve, with which it is continuous; beneath the cellular layer is a coagulum-like stratum containing passages and spaces, called the "canalized fibrin."

The substance of the placenta is composed of a mass of "chorial villi," with intervening maternal blood-spaces, interposed between the chorion and decidua serotina. On following the fotal membranes into the margin of the placenta, the chorion and decidua separate, the interval between them being filled with the villi and sinuses. The umbilical vessels on reaching the placenta divide and subdivide and spread over its surface, running in the fibrous layer of the chorion. The branches of these vessels pass outward from the chorial membrane, toward the uterine wall, forming branching vascular tufts, the chorial villi, which are covered with an epithelioid investment from the outer epiblastic layer of the chorion.

The villi begin as large coarse trunks passing from the chorion toward the decidua; these divide into a great profusion of small cylindrical branches and twigs. The ends of some of the villi become attached to the decidua serotina, but most of the branches end in free or floating blunt, round extremities. The villi are composed of arterial and venous channels and capillary loops, imbedded in a lonse connective tissue, with their surface covered with tissue of epithelial origin. The foetal vessels are closed, and nowhere open into the maternal blood-channels.

The epithelioid tissue lining the surface of the villi consists variously of distinct cells, a protoplasmic lamina containing numerous nuclei, 
or projecting clusters of cells ; this epithelioid investment is derivedits deeper part at least-from the epiblastic outer layer of the chorion; partly also, perhaps, from the epithelium of the uterine mucosa. The twigs of the villi are separated by open spaces, "intervillous spaces," into which the maternal arteries and veins in the decidua open directly; these spaces thus form an extensive system of blood-sinuses, filled with maternal blood, into which the chorial villi project. By interchanges between the fotal blood circulating in the villi and the maternal blood which surrounds the villi the alimentary and excretory needs of the foetus are provided for.

The decidua serotina, which is continuous with the decidua vera, forms the deepest stratum of the placenta; it is a thin lamina, and has been already described as the mucosa of the pregnant uterus. Among its constituents the abundant "decidual cells" are notable. Septa from the decidua pass part way into the placenta and divide it into lobes or cotyledons. At the margin of the placenta these septa may reach the chorion and form in places a "subchorial decidual layer."

The umbilical cord is a vascular cord connecting the fotus with the placenta. It is formed by the stalk of the chorion and allantois, covered on the surface by the amnion. It consists of two arteries and one vein, twisted spirally, surrounded by gelatinous embryonic or young connective tissue ("Wharton's jelly"), and lined superficially with epithelium. The epithelium covering the surface of the cord is of the squamous variety, stratified or simple, and is continuous with the epithelium lining the amnion, also with the fotal epidermis.

In the earlier part of pregnancy the jelly of Wharton or connective tissue surrounding the umbilical vessels is a mucous tissue, composed of anastomosing mucous cells with the meshes and interstices between them filled with a soft mucinous or gelatinous intercellular substance; toward the close of pregnancy fibrous elements develop abundantly in the interstices of this tissue. The umbilical vessels possess thick walls, made up mainly of laminæ of involuntary muscle-cells arranged longitudinally, obliquely, and transversely; elastic fibres are scanty in the vessel-walls, and the adventitia merges into the surrounding Wharton's jelly.

At an early period the cord also contains the cavity of the allantois and the stalk of the umbilical vesicle, both being tubules lined with hypoblast; these structures later disappear, though traces of them may sometimes remain visible as columns or canals of epithelioid cells.

The vagina: The walls of the vagina are composed of mucous, submucous, muscular, and fibrous layers, from within outward. The inner surface is disposed in transverse rugæ. The mucosa consists of stratified squamous epithelium resting on a tunica propria. The latter contains abundant elastic fibres and diffuse lymphoid tissue, and its surface exhibits papillæ projecting into the epithelial layer. No glands are 13-Hist. 
present. The submucosa is a layer of loose connective tissue containing rich vascular plexuses, a sort of erectile tissue. The muscular coat consists in general of an inner circular and an outer longitudinal layer of involuntary musele. The outermost coat is fibrous, or areolar, connecting the vagina with the bladder anteriorly and with the peritoneum, rectum, and perineum posteriorly. Among the nervous elements of the vagina are special sensory terminals.

The vulva is covered with an integument continuous with the mucous membrane of the vagina and urethra and with the external skin. This integument consists of stratified squanıous epithelium, in places pigmented, resting on a papillated tunica propria, beneath which is a loose areolar submucous layer.

Opening on the surface are numerous small glands, both mucous and sebaceous; the latter oceur not only on the hairy portions of the labia majora, but also on the labia minora unassociated with hairs.

The glands of Bartholin are two macroscopic racemose glands, corresponding to Cowper's glands of the male, situated in the labia minora, one on each side of the outlet of the vagina. Special sensory nerve-terminals occur in the mucous membrane in this region.

Beneath the integument of the labia majora are masses of adipose, areolar, vascular, and muscular tissue; beneath that of the labia minora and vestibule is an especial abundance of vascular plexuses and sinuses, forming an erectile tissue. In the deeper strata of the genitalia are fasciculi of muscle, both striated and non-striated, variously arranged.

The hymen is a fold of the vaginal mucous membrane. The clitoris repeats in a rudimentary way the cavernous and erectile structure of the penis.

\section{The Mammary Glands.}

The mammary glands are fully developed only during their periods of functional secreting activity, the periods of lactation; at other times they are in an atrophic non-secreting condition.

When in the state of active secretion the mammary gland 
is a compound racemose gland, of the fatty type (secreting also a serous and albuminous fluid), and is situated in the subcutaneous tissue. It is divided by septa into fifteen to

FIG. 74.

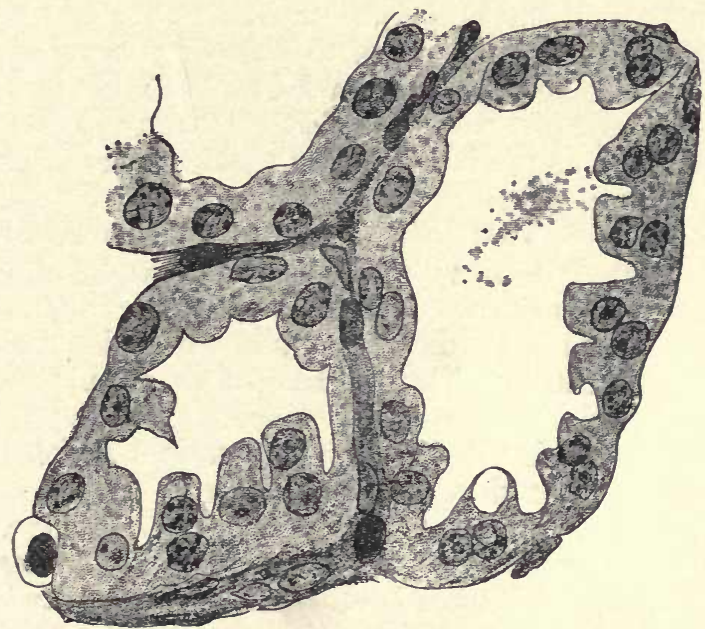

Secreting alveoli of mammary gland of guinea-pig during lactation (Michaelis).

twenty lobes, and these again into lobules, each of which is made up of a number of the ultimate alveoli (Fig. 74).

Each of the lobes has a separate outlet-duct opening at the nipple, each lobe thus being essentially a distinct gland.

The sustentacular connective tissue consists of interlobar septa, interlobular septa, and a small amount of interalveolar connective tissue lying between the alveoli. Adipose and areolar tissue is also abundant, filling the intervals between the lobes and lobules.

The ultimate secreting alveoli are large oval or elongated saccules, with large open lumina, and are lined with a layer of granular spheroidal epithelium-cells; which secrete the milk. Within these cells globules of oil develop and are discharged into the lumen to form the fat-particles of the secreted milk.

The alveoli empty into ducts, called lactiferous or galac- 
tophorous ducts, which are lined with a layer of low columnar epithelium and gradually increase in size as they unite. The ducts in each lobe unite to form a large outlet-duct, which pursues a separate course to the nipple, distinct from the other lobar ducts. These large lobar outlet-ducts have well-marked walls of fibrous tissue and some unstriped muscle; toward the nipple they are dilated so as to form ampulloc or sinuses, which serve as temporary receptacles for the milk, and open at the nipple by constricted orifices; they are lined with simple columnar epithelium nearly to the nipple, where the lining changes to stratified squamous.

The nipple is covered with a sort of mucous membrane, rosy or pigmented, lined with stratified squamous epithelium, and containing numerous special sensory nerve-terminals. Within the nipple is involuntary muscle, to which its erectility is due. Scattered about the base of the nipple and the surrounding areola are the small racemose glands of Montgomery, especially developed during lactation, which correspond to small projections on the surface.

When not in a condition of active secretion, the alveoli are smaller, less numerous, rudimentary or atrophic; instead of having a cavity their lumen is filled with cells, so that they are represented by small solid nodules of epithelium.

The system of ducts is present and well formed.

Areolar and adipose tissue is more abundant, taking the place of the undeveloped or degenerated alveoli, which are imbedded in this increased amount of connective tissue.

Such is the rudimentary and undeveloped structure of the mamma in females before the first pregnancy, and in males.

During pregnancy the gland undergoes active development, especially the secretory alveoli, which increase in number and size, while the intervening connective tissue is reduced, and the cells in the centre of the solid alveolar epithelial masses undergo fatty degeneration and at the beginning of lactation are perhaps cast off in the first milk as colostrum-corpuscles.

After lactation is over the gland undergoes retrogressive changes or involution to its former condition, the alveoli atrophying and being replaced by adipose and arcolar tissue.

Milk, the secretion of the mammary gland, consists micro- 
scopically of a homogeneous transparent liquid in which are suspended large numbers of minute round or oval globules of fat, varying in size from two to five $\mu$. These globules cause the opacity and white color of the milk; they are supposedly surrounded and kept intact by albuminous (casein) envelopes.

The milk secreted at the beginning of lactation, the colostrum, eontains, in addition to the constituents of ordinary milk, larger spherules of fat and colostrum-corpuscles. The latter are large cells filled with particles of fat, and are regarded by some as epithelium-cells; by others, as wandering cells which have undergone fatty degeneration. 


\section{CHAPTER X V.}

\section{MUSCULAR AND SKELETAL STRUCTURES.}

Is a logical analysis of the systems of the body, the motor structures (voluntary) and the framework of the body deserve equal and coördinate rank and consideration with the other systems. The elemental tissues which make up the greatest part of the muscular and skeletal structures have, however, been already described (Chapter IV.), so that only a few further points need attention.

The voluntary muscles are made up of striated muscle-cells or fibers, united by sustentacular connective tissue into fasciculi, and these again into entire muscles. They are richly supplied with capillaries. That portion of the sustentacular tissue enveloping the entire muscle is called the epimysium; that investing the several fasciculi is the perimysium; the delicate connective tissue within the fasciculi, among the individual muscle-cells, is the endomysium.

Tendons consist of white connective-tissue fibres cemented together in parallel non-vascular dense bundles; these are bound together into entire tendons by trabeculæ and sheaths of sustentacular tissue, which contains the vascular supply. In rows between the white fibres are the tendon-cells, specialized connective-tissue cells; they send flat lamellar projections between adjacent fibres, thus having a rectangular appearance from the side, stellate in cross-section. The nuclei of adjoining cells are often situated near each other, forming pairs. The surface of tendons is often lined with endothelium.

The connection between the tendons and the muscle-cells is in general established by the fibres of the endomysium; these are united on the one hand to the sarcolemma at the extremities of the muscle-cells, and on the other are connected with tendon-fibres. At the junction of muscles and tendons their connective-tissue elements are continuous and merge into each other. 
Tendons and ligaments at their insertion into bones become intimately blended with the periosteum, and their fibres often meet the bony substance obliquely or perpendicularly. Some of the fibres penetrate the bone for a short distance after the manner of Sharpey's fibres, so that the osseous tissue and the tendon-fibres dovetail into each other, affording a very firm

FIG. 75.

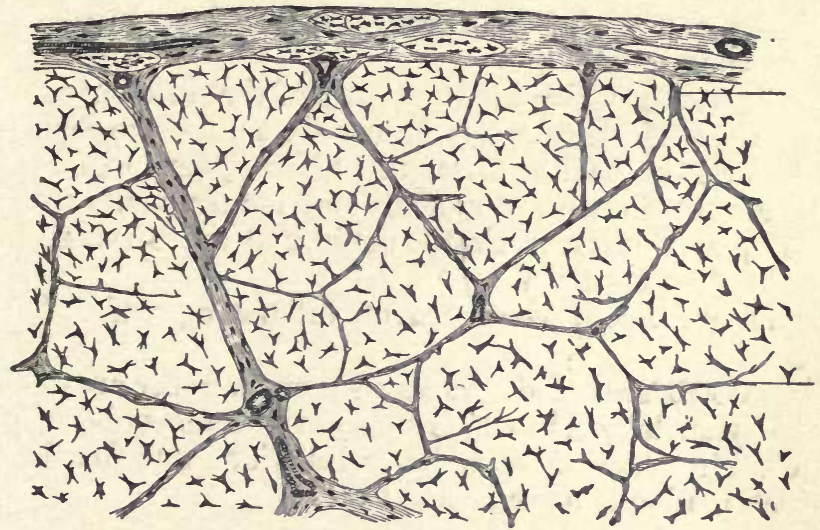

Cross-section of portion of tendon (Schäfer).

union. These fibrous insertions can be stripped from the bone only with difficulty, more so than the periosteum elsewhere, and the surface of the denuded bone at such points is rough and uneven.

Synovial membranes, allied to serous membranes, are thin fibrous membranes more or less completely covered on their free surface with flat connective-tissue or endothelium cells. They form sheaths or sacs in connection with moving surfaces, of three kinds-articular, vaginal, and bursal. The articular synovial membranes inclose joints; the vaginal form tendonsheaths; the bursal line the bursæ. They secrete, by a sort of glandular action, a glairy fluid which lubricates the opposed surfaces that move on each other.

Tendon-sheaths: The tendons in some situations, in the vicinity of joints, notably in the hand and foot, are sur- 
rounded by synovial sheaths or sacs in which they smoothly glide.

Bursæ are small synovial sacs situated at points exposed to friction; they facilitate movement by the gliding of their opposite surfaces over each other, lubricated by their glairy contents. They occur at exposed points underneath the skin, as over the patella, and interposed between tendons and bones, as over the bicipital tuberosity of the radius.

The structure of bones and cartilages, which make up the chief part of the skeletal framework, has been already sufficiently considered.

The ligaments, which bind the parts of the skeleton together, mostly consist of dense white fibrous tissue, and are characterized by firm, unyielding strength; a few ligaments, however, the ligamentum nuchæ of animals and the ligamenta subflava of the spine, consist of elastic tissue and are serviceable by virtue of their elasticity.

Fasciæ are chiefly composed of interlacing white fibrous tissue.

The articulations of the bones with one another are of three kinds-synarthroses, in which the joint is immovable ; amphiarthroses, in which there is limited motion; and diarthroses, in which motion is more or less free.

The synarthroses, or immovable joints, are exemplified by the bones of the skull and face, immovably united or dovetailed together by sutures.

In the amphiarthroses a layer of fibro-cartilage is interposed between the uniting bones (forming a "synchondrosis"), the articulation being reinforced by ligaments. There is no synovial joint-cavity and no surfaces moving on each other, the limited amount of motion possible being effected by the flexibility of the cartilaginous junction. The intervertebral junctions and the pubic and sacro-iliac symphyses are amphiarthrodial joints.

The intervertebral dises contain in their centre a pulpy reticular mass, the remains of the chorda dorsalis; outside of this is a broad zone composed of laminæ of fibro-cartilage, which merges at the periphery into dense white fibrous tissue.

The diarthroses, or ordinary joints, are characterized by the 
possession of synovial cavities and the movement of the ends of the bones on one another.

The articular ends of the bones entering into the formation of the joint are covered with a layer of hyaline cartilage, which takes the place of a periosteum. The articular surface of this cartilage is smooth, polished, and naked, not being covered by perichondrium, synovial membrane, or endothelium; the superficial cartilage-cells are flattened, the deeper ones often arranged in rows perpendicular to the surface; the deepest stratum of the cartilage is calcified.

At the margins of the joint the articular cartilages are continuous with synovial membrane, which closes the sides of the joint, and together with the cartilages completely incloses and lines the joint-cavity.

Beneath the synovial membrane is areolar and fatty tissue, filling in the spaces between the bony parts.

The synovial membrane in places projects into the cavity as vascular, fatty, or fibrous ridges, fringes, or villi.

The exterior of the joint, outside the subsynovial tissue, is firmly bound together by ligaments. In some joints the margins of the articulating surfaces are deepened by rims of fibrocartilage, as in the shoulder (glenoid ligament), hip (cotyloid ligament), and knee (semilunar cartilages).

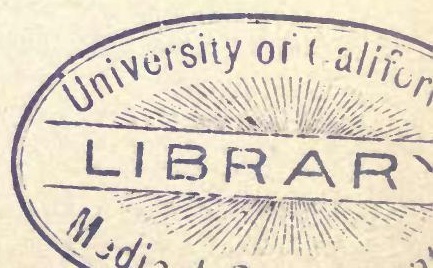




\section{CHAPTER XVI.}

\section{THE NERVOUS SYSTEM.}

THE nervous system is the seat and apparatus of sensation, consciousness, coördination, and volition, and its elements are distributed throughout all parts of the body. Nervous tissues are of epiblastic origin and constitute a class of tissues sui generis.

Division: The nervous system can for practical convenience be divided into the nerve-centres, nerve-trunks, and distal nerve-terminals; and the tissue-elements corresponding to these respectively are nerve-cells, nerve-fibres, and nerveterminals.

The cells and centres are concerned with the distribution and storage of nervous energy ; the fibres conduct nervous impulses; and the terminals are connected with the transformation of nervous force into other forms of energy, and vice versa.

According to present prevailing conceptions, the structural and functional units of the nervous system are elements called neurons, and the subject is here presented from this standpoint. A neuron is a single nerve-cell with all its parts and processes. The nerve-fibres are regarded as greatly elongated processes of nerve-cells, so that a neuron would include the body of the nerve-cell, its nearby processes, the nerve-fibre connected with it, perhaps extending a long distance to the periphery of the body, and the peripheral terminations of the nerve-fibre. A neuron therefore simply represents a single complete nerve-cell with all its parts.

\section{Nerve-cells.}

Nerve-cells (or ganglion-cells): In the full sense of the term "cell," a nerve-cell is the same as a neuron; but as ordinarily 
used the term nerve-cell is conveniently applied to the main body of these cells, regardless of the extended processes. Such a term as neurosome would, perhaps, be a better designation for the nerve-cell body. Nerve-cells are ordinarily of large size, and are situated in the ganglia and cerebro-spinal centres. Their cytoplasm usually consists of a network of fibrils (spongioplasm), in the meshes of which is a more fluid, homogeneous protoplasm (hyaloplasm), sometimes called neuroplasm.

The cell-bodies often contain granules (chromophilic granules) which stain deeply with various dyes, especially basic stains ; pigment is also sometimes present. There is no definite cell-envelope.

Nerve-cells possess each a large vesicular nucleus, usually poor in chromatin, and hence not staining deeply; within the nucleus is a large nucleolus. In shape nerve-cells vary, being

Fig. 76.

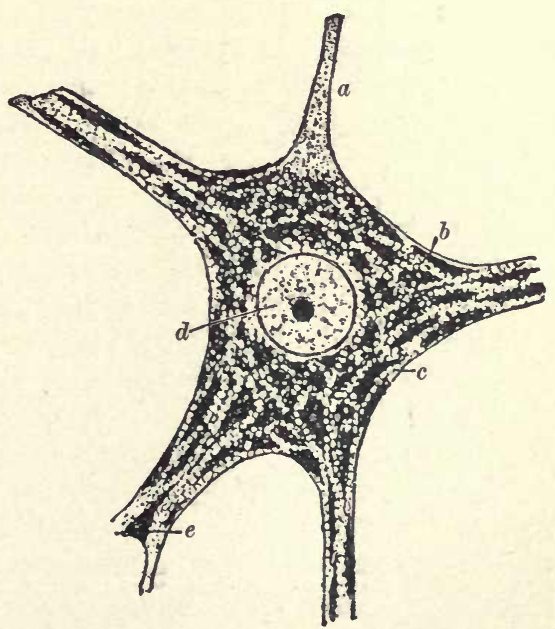

Body of multipolar nerve-cell from spinal cord (Nissl's stain), showing details of the structure (Ramon y Cajal). a, neurite; $b, c$, cytoplasm, containing coarse chromophilic granules; $d$, nucleus, with nucleolus; $e$, dendrite.

spherical (ganglia), oval, fusiform, pyramidal (cerebrum), or stellate (spinal cord). 
Nerve-cells are strongly characterized by the possession of processes, often greatly prolonged and very complex, ranging in number from one to many; at least one process is always present. The processes consist largely of prolongations of the spongioplastic fibrils of the cytoplasm.

Nerve-cells are classed as unipolar, bipolar, and multipolar according as they possess one, two, or several processes respectively. Another variety of ganglion-cell, which may

Frg. 77.

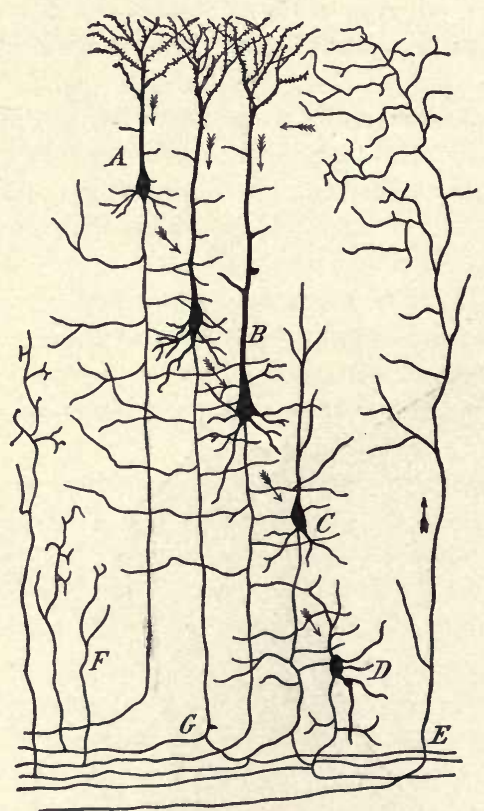

Nerve-cells from cerebral cortex (Ramon y Cajal). $A$, small pyramidal nerve-cell; $B$, large pyramidal nerve-cells; $C, D$, polymorphous nerve-cells; $G$, neurite; $E$ and $F$, fibres in white matter. The arrows indicate directions of nerve-impulses.

be called pseudo-unipolar, is apparently unipolar, but practically bipolar; it possesses a single process which soon divides like a ' $\mathrm{T}$ into two processes; embryonically this cell is bipolar. 
The processes of nerve-cells are of two very distinct kinds, dendrites or protoplasmic processes, and neurites or axiscylinder processes.

The dendrites, or protoplasmic processes, are long slender extensions or processes from the body of the nerve-cells, usually dividing and subdividing into branches like a tree, forming extensive "arborizations" in the vicinity of the cell. As they divide, the branches decrease in size to fine terminal twigs, which have either pointed or rounded ends. The terminals of dendrites have been called teledendrites. The number of dendrites given off by each nerve-cell varies; there may be none or several. The cerebro-spinal nerve-cells possess the most numerous and best developed dendrites, each having a number of such processes, while the cells of the ganglia are less abundantly supplied with them, and unipolar cells lack them altogether. The dendrites of neighboring nerve-cells interlace and lie in close relation with one another; but according to the neuron theory they are not actually united or structurally continuous together.

The neurites, axis-cylinder processes, or neuraxons, are slender filamentary processes from nerve-cells, which are prolonged as, and become continuous with, the axis-cvlinders of nerve-fibres, and as such are greatly prolonged, often extending to the periphery of the body. They acquire medullated envelopes at a short distance from the cells, and thus become converted into nerve-fibres. Sometimes, within the substance of the nerve-centres, neurites give off delicate, short lateral branches, the collateral fibres, but aside from these and except at their distal ends neurites do not branch, and their diameter is uniform throughout. All nerve-cells possess one neurite, sometimes more than one; if a cell has but one process, that process is a neurite.

At their distal ends neurites or nerve-fibres usually terminate in fine branches; these distal terminations have been called teleneurites. According to the length of the neurites and the situation of the teleneurites, two types of nerve-cells or neurons are recognized.

Nerve-cells of the first type have long neurites extending to the visceral, distal, or peripheral portions of the body, where their terminals or teleneurites are situated. 
Those of the second type have a short neurite which does not emerge from the nerve-centres, but not far from its origin divides into its terminal branches or fibrils,- - sometimes forming a basket-like network enveloping another nerve-cell. Neurons of the second type are situated entirely within the nerve-centres, those of the first type partly within the nerve-centres, partly without, in the general body-structures.

A neuron typically consists of a nerve-cell or cell-body, one or more dendrites, and a neurite prolonged as the axis-cylinder of a nerve-fibre to the distal part of the body (or to another part of the nerve-centres), where it has a special termination.

From the nature of nervous action, as we conceive it, a neuron must have a mechanism both for receiving nervous impulses and for sending them out again. Each neuron is supposed to be complete in itself, and to have no anatomical connection or continuity with other neurons; the processes of one nerve-cell are believed not to be united to those of other cells.

As it is evident that some communication of nervous impulses takes place from one neuron to another, it is supposed that such communication oecurs through contact of the processes or other parts of different cells with one another. If the cellprocesses are retracted (as by amoboid movement) or diseased, the communication may be eut off and altered nervous conditions result.

Another enceivable mode of transfer of nervous impulses from one cell to another, possible without direct entact or continuity, might be by a sort of induction, analogous to electric induction.

Function of dendrites and neurites : It is by some supposed that the dendrites are afferent or centripetal (cellulipetal) in their action, conducting impulses to the nerve-cell, and that the neurites are efferent or eentrifugal (cellulifugal), conveying impulses from the cell and degenerating when their connection . with the cell is severed; this supposition is clear enough with respect to motor and other efferent nerve-cells and fibres, but it is not so obvious in the case of sensory and afferent fibres, 


\section{Nerve-fibres.}

Nerve-fibres are regarded as processes of nerve-cells, being the continuations of neurites, enveloped by certain sheaths. They connect nervecells with nerve-terminals, and their function is the conduction of nervous impulses. Nerve-fibres are situated both within and without the cerebro-spinal centres, with certain differences in their envelopes in the two situations. In their passage through the body the fibres are united by sustentacular tissue into bundles, the nerves or nervetrunks. Nerve-fibres are of two kinds, medullated and non-medullated.

Medullated nerve-fibres (Fig. 78), also called myelinic or white fibres, make up the chief part of the cerebro-spinal nerves and occur also in large numbers in the cerebrospinal structures. Where most developed they consist of three parts-axis-cylinder; medullary sheath, and neurilemma. Within the cerebro-spinal centres there is no neurilemma, and at the terminations of the fibres both neurilemma and medullary sheath are wanting.

The axis-cylinder, or axon, the prolongation of a neurite, is a fine continuous filament, uniform in size, occupying the centre or axis of the fibres. In medullated fibres the axis-cylinder does not branch except for the "collateral fibres," and near its distal termination, where it may divide at a node of Ranvier. It exhibits fine longitudinal striations, apparently representing spongioplastic fibrillæ bound together by cement or Fig. 78.

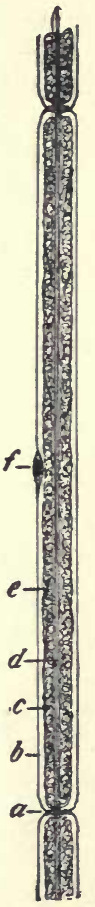

Segment of a medullated nerve-fibre. $a$, node of Ranvier; $b$, neurilemma: $c$, medullary sheath ; $d$, axis-cylinder; $e$, incisure of Schmidt; $f$, nucleus of neurilemma. protoplasm. It is the essential and conducting portion of the fibres, the medullary sheath and neurilemma serving as coverings for it. [At its central end the axiscylinder emerges as a process from a nerve-cell, and at its 
distal extremity usually breaks up into branches or fibrils connected with terminal structures. Axis-cylinders are of epiblastic origin, developing as outgrowths from the primitive nerve-cells.

The medullary sheath, or white substance of Schwann, is an envelope of semisolid material investing the axis-cylinder, the presence of which is the main feature distinguishing medullated from non-medullated nerve-fibres. It begins near the exit of the neurite rom the nervé-cell and extends nearly to the distal termination of the fibre. It protects and perhaps insulates the axis-cylinder. The medullary substance consists of an oily, refractile, semifluid substance called myelin, supposed to be supported in a reticulum or sheath of a horny substance termed neurokeratin.

At regular intervals the nerve-fibres exhibit constrictions, the nodes of Ranvier, dividing the fibres into internodal segments. At these nodes the continuity of the medullary sheath is interrupted, the neurilemma and axis-cylinder coming together at these points. In each internodal segment the medullary sheath exhibits a number of oblique or conical markings or divisions, called the incisures of Schmidt or of Lantermann; the nature of these is not definitely known.

The neurilemma, or sheath of Schwann, is a thin homogeneous membrane forming the outer covering of the nervefibre. It is present only in the portion of the fibres included in the nerve-trunks, being absent in the cerebro-spinal centres, where its place is taken by neuroglia. It also disappears at the nerve-terminations. At the nodes of Ranvier it comes into contact with and is cemented to the axis-cylinder, interrupting the continuity of the medullary sheaths at those points. At the middle of each internodal segment a nucleus surrounded by a small amount of protoplasm is situated beneath the neurilemma. This nucleus apparently belongs to the neurilemma, and each nucleated segment of the nenrilemma is commonly regarded as a specialized single connective-tissue cell.

Non-medullated nerve-fibres, also called amyelinic or gray fibres, or fibres of Remak (Fig. 79), possess no medullary sheath. The sympathetic nerves are made up chiefly of this kind of fibres. They consist in general of axis-cylinders 
covered apparently by a neurilemma which exhibits numerous nuclei. The axis-cylinders are similar to those of medullated fibres, except that they freely divide and anastomose with one another, forming plexuses. On their surface at intervals rest nuclei; these probably belong to a neurilemma, which, however, is so thin and delicate as to be difficult to distinguish.

\section{Nerve-centres.}

The nerve-centres are the cerebrum, cerebellum, and spinal cord, making up the cerebro-spinal structures ; and the numerous ganglia in different parts of the body.

The characteristic elements making up the central nervous structures are nerve-cells and processes, nerve-fibres, and a peculiar sustentacular tissue called neuroglia.

The pia mater also contributes a certain amount of ordinary connective tissue to the sustentacular framework.

Through differences in the distribution of these elements two parts are distinguishable in the substance of the cerebro-spinal structures, gray matter and white matter.

The gray matter consists of neuroglia, nerve-cells, ramifying den-

Frg. 79.

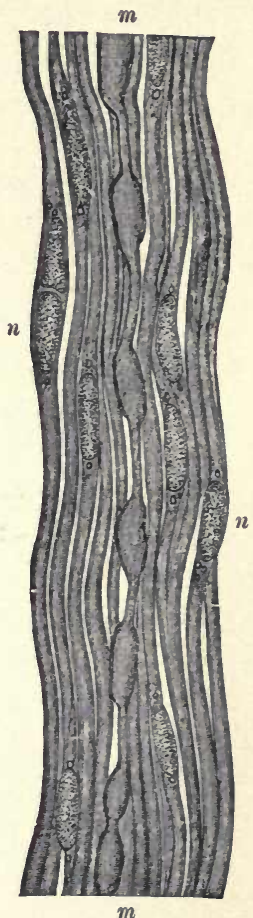

Non-medullated nerve-fibres (Key and Retzius). n, nuclei of neurilemma; $m$, a fibre which has a partial medullary sheath. drites, and scattered nerve-fibres, with and without medullary sheaths.

The white matter is an almost solid mass of parallel medullated fibres, supported by neuroglia.

Neuroglia, the chief sustentacular tissue of the cerebrospinal structure, is a sort of connective tissue, but is of epiblastic origin and derived from epithelial elements. It 14-Hist. 
forms a framework supporting the nervous elements. It

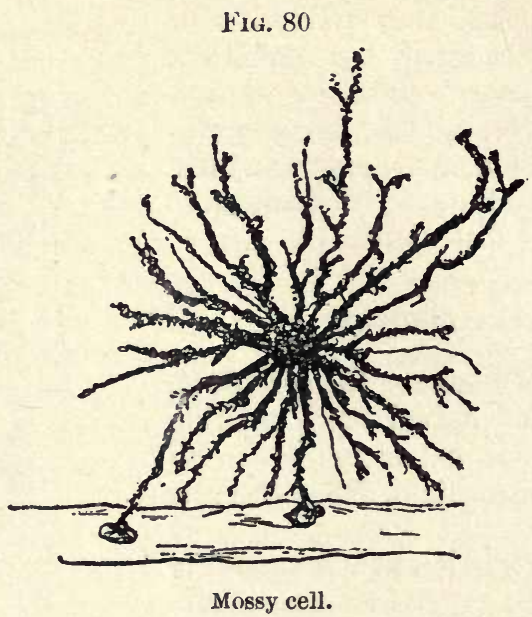

Frg. 81.

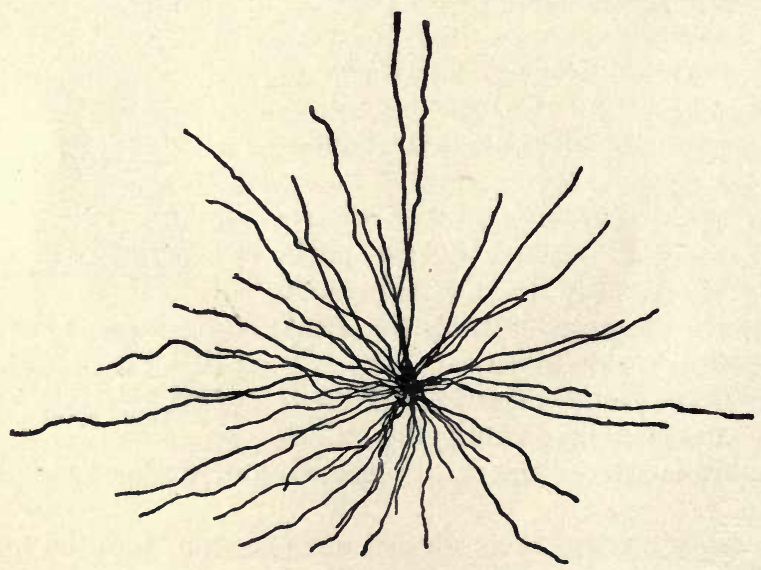

Spider-cell.

Neuroglia-cells, Golgi stain (Andriezen).

consists of numerous cells ("glia-cells"), possessing very 
many fine processes which interlace with one another to form a network. Some glia-cells, the "spider-cells," have long, fine unbranched processes ; others, the "mossy cells," have shorter, thicker, and branching processes (Figs. 80, 81). A homogeneous intercellular cement-substance is perhaps also present, contributing to the sustentacular framework.

The cerebro-spinal meninges are the dura mater, arachnoid, and pia mater.

The dura mater is a dense fibrous membrane forming the outer covering of the brain and spinal cord; its inner surface is lined with endothelium. Within the cranium it is closely applied to the cranial bones, forming their inner periosteum.

The arachnoid is a delicate connective-tissue lamina and reticulum. Externally it presents a continuous free surface lined with endothelium; this surface is opposed to the dura and separated from it by a large lymph-space, the subdural space. The deeper portion of the arachnoid is a network of delicate fibrous laminæ and cords, lined on their free surfaces with endothelium; this network is connected and continuous with the pia mater, and the cavities enclosed in it form a system of communicating lymph-spaces, called the subarachnoid space.

The pia mater intimately invests and follows the surface of the brain and cord; it consists of delicate vascular connective tissue, often containing irregular pigment-cells. At intervals it sends supporting fibrous trabeculæ inward into the substance of the brain and cord.

\section{The Cerebrum.}

The cerebrum consists of aggregations of white and gray matter.

The white matter is composed of radiating masses of medullated nerve-fibres, and occupies the interior and central portions of the organ.

The gray matter, containing the nerve-cells, covers the surface of the cerehrum and also occurs in the central and basal ganglionic tracts.

The gray matter of the cerebral cortex in general (though with variations in some situations) exhibits the following 
layers (Fị. 82), which are not sharply marked off from one another. Beneath the pia mater on the surface is a narrow molecular zone, consisting of neuroglia, glia-cells, terminal branches

FIG. 82.

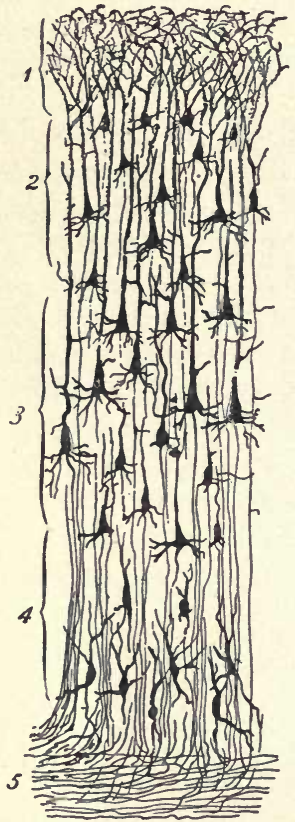

Nerve-cells of cerebral cortex (Cajal). 1, molecular zone; 2 , small pyramidal nerve-cells; 3 , large pyramidal cells: 4 , polymorphous cells; 5 , white matter. of nerve-cell processes, and fine medullated fibres running tangentially or parallel to the surface. Beneath this is a zone characterized by the presence of small pyramidal nerve-cells. These are long pyramidal cells, with their apices directed toward the surface. Each cell gives off an axiscylinder process from its base, running (for the most part) into the white matter beneath. From the angles at the apex and base of the cell are given off dendrites which divide into branches in the vicinity. This layer merges below into a broad zone characterized by large pyramidal nervecells, similar to those just described, but of larger size. Beneath this zone is a layer containing polymorphous nerve-cells of varions shapes-stellate, irregular, and fusiform. Neurogliacells are scattered about among the nerve-cells in the various zones.

The foregoing layers comprise the cortical gray matter. Beneath them is the white matter of the interior, which at regular intervals sends slender, tapering bundles of medullated fibres radiating into the lower layers of the gray matter.

The ganglionic parts of the cerebrum, as the corpus striatum, optic thalamus, corpora quadrigemina, olfactory bulb (or nerve), consist of gray and white matter variously distributed, with nerve-cells of various forms-multipolar, stellate, fusiform, etc. 
The crura cerebri, pons, and medulla oblongata are composed largely of white matter, or bundles of nerve-fibres passing into the cerebrum and cerebellum, intermingled with tracts of gray matter.

The ventricles of the brain, continuous with the central canal of the spinal cord, are mostly lined with "ependyma," a single layer of columnar epithelium, ciliated (especially in youth), resting upon a neurogliar basis. The columnar cells are related to the glia-cells.

In various parts of the brain are found corpora amylacea, minute rounded granules with concentric markings, resembling starch-granules in appearance and chemical reactions.

\section{The Cerebellum.}

The central portion of the cerebellum is a mass of white matter, within which is an irregular nodule of gray matter, the dentate body. Other nodules of gray matter occur in the upper part of the organ.

The superficial portion of the cerebellum is divided into branching laminæ, which in cross-section appear like the branches of a tree, whence they are called the arbor vitæ. These laminæ consist of a central core or axis of white matter, covered on each side with gray matter.

The gray matter of the cerebellar cortex exhibits two wellmarked layers, the nuclear layer and the molecular layer, between which is a stratum formed by the bodies of the cells of Purkinje.

The nuclear or granule layer is a broad zone lying next to and on each side of the central white matter, and contains a crowded mass of small multipolar nerve-cells with prominent deeply staining nuclei and small cell-bodies. Scattered about among these cells are a few larger multipolar nerve-cells and glia-cells, while medullated nerve-fibres passing into the central white matter are numerous throughout the nuclear layer.

The cells of Purkinje, or "antler-cells," are conspicuous nerve-cells, the bodies of which, separated by intervals, are disposed in a single layer at the junction of the nuclear and molecular layers. Their bodies are very large, rounded or flask-shaped, nucleated, nucleolated, and from their superficial 
aspect (that directed toward the surface) give off coarse protoplasmic processes which divide and subdivide (in narrow transverse planes) into extensive arborizations occupying the entire thickness of the molecular layer; from the deeper aspect each cell gives off an axis-cylinder process which passes inward through the nuclear layer.

The molecular layer is the superficial stratum of the cerebellar cortex, and consists chiefly, besides neuroglia, of the extensive interlacing ramifications of the dendrites of the cells of Purkinje and of the processes of other nerve-cells in the vicinity. This layer also contains scattered small multipolar nerve-cells; the neurites of some of these cells ("basket cells") run parallel to the surface and give off branches which break up into terminal basket-like networks of fibrils that surround the bodies of Purkinje's cells.

\section{The Spinal Cord.}

The spinal cord is divided into symmetrical lateral halves by the deep and narrow posterior median "fissure" (really a fibrous septum) and the broad but shallower anterior median fissure.

The periphery of the cord is formed by white matter, consisting of a dense mass of longitudinal medullated nervefibres, supported by neuroglia and narrow connective-tissue extensions from the pia mater passing radially inward. The narrow layer of white matter intervening between the gray commissure and the fundus of the anterior median fissure constitutes the "anterior" or "white commissure."

From physiological and pathological considerations the white fibres of the cord are divisible into definite longitudinal tracts, but these divisions are only partially apparent to sight in the normal cord (Fig. 84).

The interior of the cord is occupied by gray matter, which consists of two lateral parts, curved outward, joined by a transverse portion (the "gray commissure"), thus in crosssection roughly exhibiting an $\mathrm{H}$ shape.

The gray matter is composed of neuroglia, interlacing dendrites, nerve-fibres (with and without medullary sheaths), and nerve-cells. 
The portions of the gray matter which approach the surface of the cord anteriorly and posteriorly are called the anterior (or ventral) and posterior (or dorsal) horns or cornua; the anterior horns are broader and shorter; the posterior longer and narrower.

From these horns at intervals proceed the posterior (sensory) and anterior (motor) roots of the spinal nerves.

Fig. 83.

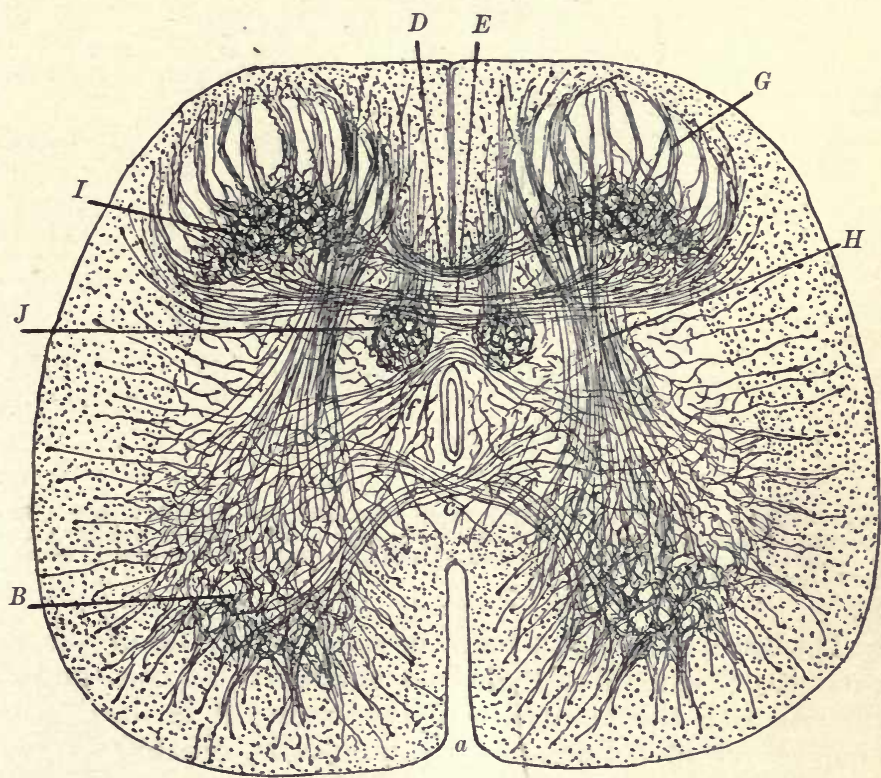

Cross-section of the spinal cord of a newborn child, showing the distribution within the gray matter of the collaterals from the neurites of the white matter (R. $\mathrm{J}$ Cajal). $a$, anterior fissure; B, pericellular branches of the collaterals from the anterior column: $C$, collaterals of the anterior commissure; $D$, posterior bundle of collaterals in the posterior commissure; $\mathrm{E}$, middle bundle of the posterior commissure : $f$, anterior bundle: $G$, collaterals from the posterior column; $H$, senso-motory collaterals from the posterior column; I, pericellular terminations of collaterals in the posterior horn; $J$, collateral terminations in the column of Clarke.

The nerve-cells of the cord (Fig. 76) are arranged in definite longitudinal series or columns, as follows: $(a)$ in the anterior horn are numerous very large stellate multipolar motor cells; 
(b) in the posterior horn are smaller and less numerous nervecells ; (c) the vesicular column of Clarke is a column of mediumsized nerve-cells, not continuous along the entire cord, on the inner sides of the posterior cornua; $(d)$ the intermedio-lateral column is a series of small nerve-cells laterally situated (in the "lateral horn") in the dorsal portion of the cord.

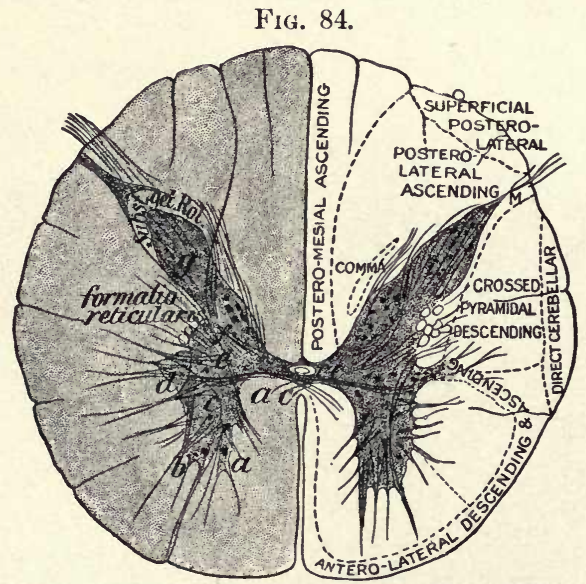

Cross-section of human spinal cord, middle dorsal region (Schäfer). $a, b \quad c$, nerrecells of anterior horn : $d$, intermedio-lateral column of nerve-cells ; $f$, nervecells of Clarke's column; $g$, nerve-cells of posterior horn; $c c$, central canal ; $a c$, anterior white commissure.

The central canal is a small tube in the middle of the gray commissure extending the whole length of the cord and continuous with the ventricles of the brain; it is lined with a single layer of columnar epithelium-cells, ciliated in youth, surrounded by neuroglia.

The neuroglia capping the posterior horns and surrounding the central canal-the "substantia gelatinosa"-is somewhat homogeneous and glistening.

\section{Ganglia.}

Ganglia are small macroscopic to microscopic aggregations of nerve cells and fibres, occurring abundantly in various parts of the body. Since they contain nerve-cells, their 
nature and function are essentially those of nervous centres. Some of them belong to the cerebro-spinal system of nerves -e.g., most of the ganglia connecterl with the cerebral nerves and those on the posterior roots of the spinal nerves; others pertain to the sympathetic system, as the lateral and anterior spinal and the visceral ganglia.

Typical ganglia are enveloped in a fibrous capsule continuous with the epineurium of the tributary nerves. The nervecells in the ganglia are aggregated in elongated or rounded clusters, separated by bundles of (medullated or non-medullated) nerve-fibres. Each nerve-cell is surrounded by a membranous nucleated capsule, composed of connective-tissue cells, continuous with the neurilemma of the issuing nervefibre.

The ganglia of the posterior roots of the spinal nerves consist of medullated fibres and large globular encapsulated nerve-cells, nucleated and nucleolated. In man these nervecells when mature possess a single process which at a short distance from the cell divides like a $T$ into two branches going in contrary directions; these cells are practically bipolar, as they actually are morphologically in some animals and the human embryo.

The sympathetic ganglia similarly consist of nerve-fibres (non-medullated and medullated) and nerve-cells. The latter are encapsulated, multipolar, often pigmented, occasionally in man (regularly in some animals) possessing two nuclei. Many of the sympathetic ganglia are very minute, imbedded in the substance of the viscera, as the heart and the intestines.

\section{The Nerves.}

The nerves, or nerve-trunks, consist of bundles of nerve-fibres bound together by sustentacular connective tissue.

'The cerebro-spinal nerves consist mainly of medullated fibres; the sympathetic nerves chiefly of non-medullated fibres.

The arrangement of nerves (especially the cerebro-spinal) corresponds to that of voluntary muscle. The individual fibres are grouped in bundles or "funiculi" ; and these again into the entire nerve-trunks (except in small nerves consisting of single funiculi). 'The entire nerve is enveloped in a con- 
nective-tissue sheath, called the epineurium. The separate funiculi are surrounded by similar fibrous sheaths, the perineurium, continuous with the epineurium. Within the funiculi is a delicate connective tissue, the endoneurium, penetrating among the individual nerve-fibres.

The larger blood and lymphatic vessels are situated in the perineurium and send capillaries into the endoneurium. In the small terminal nerves the epineurial covering consists of a layer of flattened connective-tissue or endothelioid cells, called the "sheath of Henle."

\section{Nerve-terminals.}

The axis-cylinders of nerve-fibres at their distal terminations lose their coverings and divide into fine terminal fibrils, or "teleneurites." Some of these terminals are situated in the gray matter of the nerve-centres, and are distributed in a manner analogous to the dendrites; others are distributed throughout the tissues of the body (somatic terminals), and are often associated with special structures exhibiting great diversity and specialization of function and form; some of them being highly developed and complex organs, such as the eye and ear.

From a functional standpoint these somatic terminals can be divided into two classes, afferent and efferent.

The afferent nerve-terminals have sensory functions and initiate nerve-impulses which travel along the fibres to the nerve-cells.

The efferent terminals receive impulses (motor, secretory, trophic, etc.) from the nerve-cells and impart them to the organs with which they are associated.

In general, the function of the somatic terminals is connected with the transformation of other forms of energy into the particular mode of molecular energy which constitutes nervous force; and with the retransformation of the latter into ordinary modes of motion.

Classes of nerve-terminals: Only the nerve-endings found in man are here considered; other terminals of similar general type occur in other animals.

The first division of teleneurites is into those situated in 
the nerve-centres (which do not here require further consideration), and those situated outside the nerve-centres (somatic).

The somatic nerve-terminals of man may be classified thus:

\section{Efferent:}

Terninal fibrils;

Motor end-plates of voluntary muscle.

Afferent:

Terminal fibrils;

Tactile cells;

Tactile corpuscles of Wagner (or Meissner); Touch, etc.

End-bulbs of Krause;

Corpuscles of Pacini (or Vater);

'Taste-terminals ;

Olfactory terminals;

Eye ;

Ear.

Terminal fibrils constitute the simplest form of nerve-endings, and are widely distributed, penetrating among the cells of the tissues involved (Fig. 85). They are continuations of the axis-cylinders of the nerve-fibres, which lose their coverings and break up into fine naked terminal fibrils. They often exhibit bulbous enlargements at intervals, and end in bulbs or points. They divide and branch, and in some places anastomose to form plexuses. They are either efferent or afferent.

The chief examples of efferent fibrillar nerve-endings are found in involuntary muscle, the heart, and secretory glands.

Non-striated muscle (as in the intestine and arteries) is usually associated with plexuses (sometimes gangliated) of nonmedullated fibres, delicate fibrils from which innervate the muscle-cells.

The heart-muscle is similarly innervated.

In many glands, as the salivary, delicate plexuses surround the acini and probably send fibrils (apparently efferent and stimulant to secretion) among the individual epithelium-cells. Afferent (sensory) terminal fibrils occur in the epidermis, 
mucous membranes, voluntary muscle, and doubtless in many other situations.

In the epidermis fibrils enter from the corium and are distributed among the cells of the Malpighian layer. Similar endings have been found in the epithelium of the mucous mem-

FIG. 85.

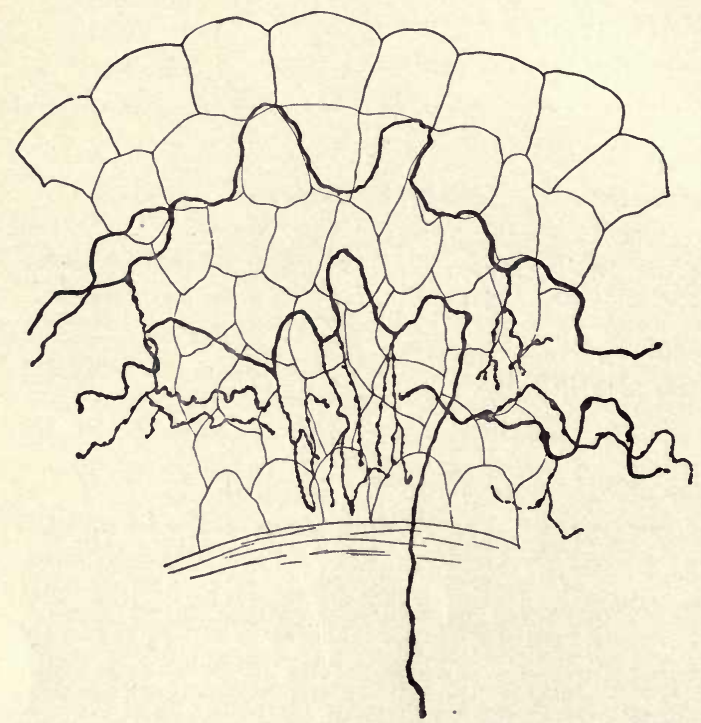

Terminal nerve-fibrils in epithelium of mucous membrane (bladder of rabbit) (Retzius).

brane of the mouth, and occur in mucous membranes elsewhere.

The cornea contains delicate terminal plexuses sending fibrils into the overlying conjunctival epithelium.

In voluntary muscles fine terminal nerve-fibrils of sensory nature have been demonstrated.

Motor end-plates of voluntary muscle (Fig. 86): The motor innervation of striated muscle is derived through "end-plates," which are terminals of medullated nerve-fibres.

The end-plate, of which each muscle-cell possesses one, exceptionally more, is closely attached to the surface of the 
muscle-fibre, in intimate relation with the muscle-protoplasm ; whether it lies underneath or external to the sarcolemma is unsettled. The end-plates are flattened and elliptical in form, and are made up of terminal branches of the axis-cylinder supported by a protoplasmic plate or "sole." The sole is a mass of granular protoplasm containing a number of nuclei, and forms a bed or basis for the terminal nerve-branches.

The nerve-fibre concerned, on reaching the end-plate, loses its medullary sheath, and its axis-cylinder divides into branches, enlarged and club-shaped, which ramify in an arborescent way over the end-plate, supported on or imbedded in the sole.

FIG. 86.

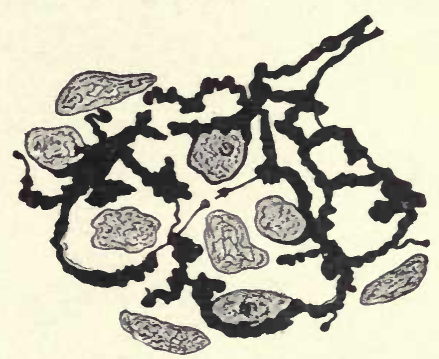

End-plate of striated muscle-fibre of a squirrel's tail (Galeotti and Levi).

Tactile cells occur scattered about in the lower part of the Malpighian layer of the epidermis, and have a tactile function. Each of them consists of an oval nucleated cell with its lower surface resting in a meniscus, a concavo-convex or saucer-shaped expansion of the end of a terminal nerve-fibril. Terminals of this type are sometimes called "simple tactile cells," in contradistinction to "compound tactile cells" (corpuscles of Merkel and of Grandry), not found in man, in which two or more cells are together associated with terminal nerveplates.

The cells of Langerhans, stellate cells occasionally found in the epidermis, are by some regarded as nerve-terminal cells, by others as wandering leukocytes.

Tactile corpuscles of Wagner (or Meissner) : These are elliptical structures situated in some of the papillæ of the corium of 
the skin (Fig. 87). They are associated with the sense of touch, and are most numerous in the hands and feet, where the tactile sense is delieate. The details of their structure are obscure. They are elongated, elliptical bodies, lying in the axis of the papillæ, and exhibit transverse or spiral markings. At their lower extremity they are joined by a medullated nervefibre (or sometimes more than one), the axis-cylinder of which, losing its coverings, enters the corpuscle, within which it winds

Fig. 88.

Fig. 87.

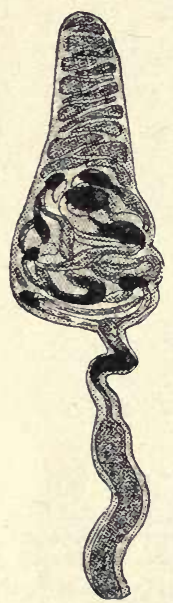

Tactile corpuścle of Wagner, from human corium (Böhm and Davidoff).

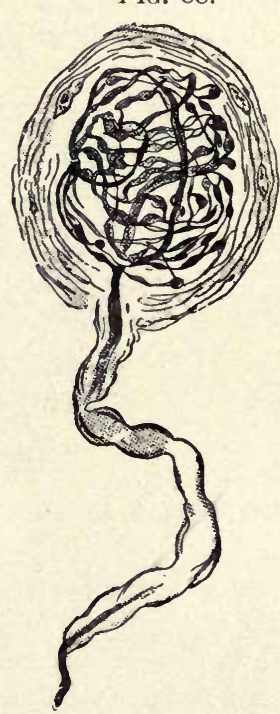

End-bulb of Krause, from human conjunctiva (Dogiel).

about and sends off lateral branches. Some authorities regard. these corpuscles as aggregations of transverse flat tactile cells, others consider them connective-tissue structures with transverse septa.

End-bulbs or tactile corpuscles of Krause (Fig. 88): These are small spherical structures in which medullated fibres terminate, and pertain to the sense of touch. They occur in the conjunctiva and adjacent portion of the cornea, in the corium 
of the glans penis and clitoris (genital corpuscles), in the vicinity of joints (articular corpuscles), and have been found in mucous membranes in other situations, as the lips, mouth, colon. They are enveloped in a delicate fibrous capsule containing nucleated endothelioid cells. Within the capsule is a core, of uncertain nature, in which the axis-cylinder of the tributary nerve-fibre forms an intricate convolution or divides into branches.

Corpuscles of Pacini (or Vater) (Fig. 89): These are oval or Fig. 89.

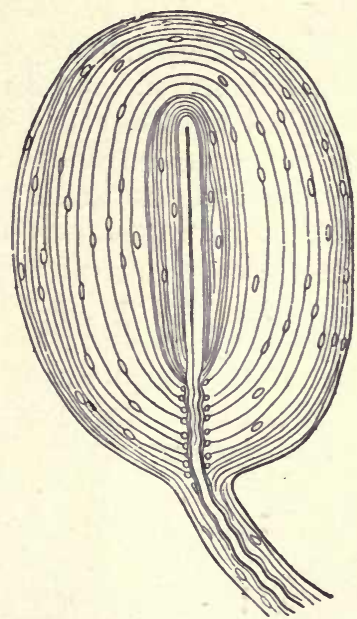

Pacinian corpuscle from mesentery of cat (Klein).

ellipsoidal structures, of tactile functions, two or three millimetres long and half as broad. They occur in the subcutaneous tissue (especially of the palms and soles), joints, periosteum, peritoneum, mesentery (especially near the solar plexus), and other situations; they are located especially along the course of nerves. They consist of the terminal axiseylinder, surrounded by a eylindrical core, and this again enveloped in a series of numerous concentric lamellæ or capsules containing lymph. The axis-cylinder pursues a straight course, lying in the long axis of the corpuscle ; sometimes it 
forks, or at its distal extremity may end in a bulb or divide into fibrils. The axis-cylinder is surrounded by a granular or perhaps multinucleated substance, which forms a cylindrical core or "inner bulb."

The bulk of the Pacinian corpuscle is chiefly made up of aseries of twenty-five to fifty concentric lamella, which envelop the core. Each lamella consists of a delicate connectivetissue membrane lined by endothelioid cells, the nuclei of which appear prominently in sections. The lamellæ are derived, partly at least, from the perineurial sheaths of the entering nerve-fibre, and they are separated by lymph-spaces filled with serum.

Taste-terminals: The taste-terminals are associated with specialized neuro-epithelial structures in the tongue, the "tastebuds," which are described at page 119. The nerves of taste end in terminal fibrils which are distributed through the epithelial lining of the mucous membrane in the vicinity of the tastebuds; some of the fibrils (intragemmal) ramify through the taste-buds; others (intergemmal) are distributed between them.

Olfactory terminals: 'The olfactory nerve-terminals consist of fine terminal fibrils distributed in the mucous membrane lining the upper part of the nasal fossæ, in association with specialized neuro-epithelial or neural elements, the "olfactory cells," which are described on page 142.

\section{The Eye.}

The eye (Fig. 90) is a highly specialized camera-like organ associated with the termination of the optic nerve. Its parts are: the conjunctiva, the sclerotic coat and cornea (tunica externa), the choroid coat, ciliary body and iris (tunica media or uveal tract), the aqueous humor, the crystalline lens and suspensory ligament, the vitreous body, the retina (tunica interna), optic nerve, and certain subsidiary structures.

The conjunctiva is a mucous membrane covering the front of the eye and inner surface of the eyelids. It consists of a tunica propria covered with stratified columnar epithelium, except that over and near the cornea the epithelium is of the stratified squamous variety and over the cornea there is no tunica propria. 
The sclerotic is the outermost tunic of the eye. Its anterior portion is modified to form the cornea. It is a thick, dense capsule, made up of laminæ of white fibres, between which are

FIg. 90.

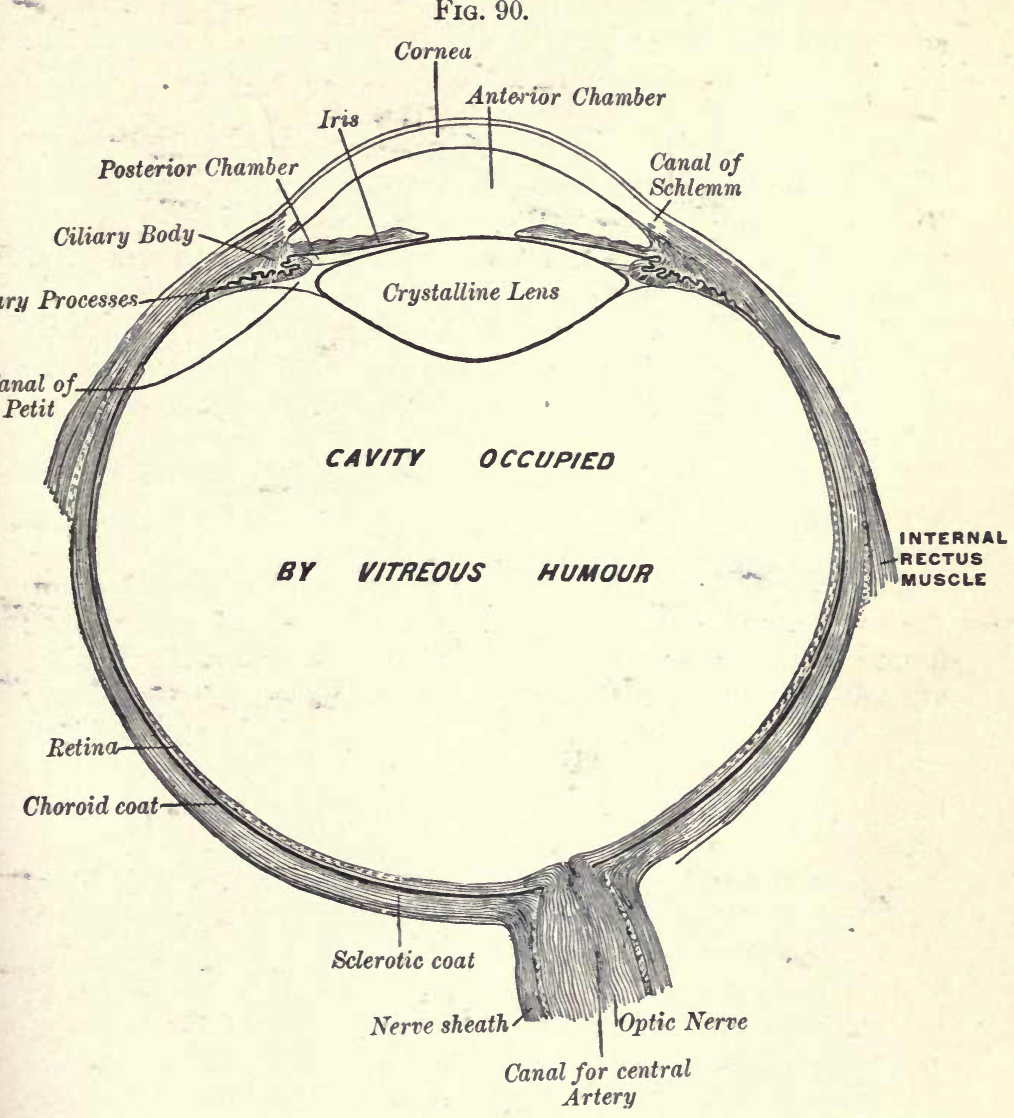

Horizontal section through eye (Allen).

flattened stellate connective-tissue cells and a few elastic fibres. Its vascular supply is scanty, but the communicating pericellular spaces form a network of lymph-passages. 
Between the sclerotic and choroid coats is a layer of loose fibro-elastic tissue containing irregular pigmented connectivetissue cells; the portion of this tissue applied to the sclerotic is called the lamina fusca, that next to the choroid the lamina suprachoroidea; in the midst of this tissue are ample lymphspaces (subscleral or perichoroidal) lined with endothelium. Externally the sclerotic rests in a sort of synovial sac, the capsule of Tenon.

The cornea is the modified transparent anterior portion of the sclerotic. It consists of five layers, which are, from before backward: epithelium, anterior elastic membrane, corneal substance proper, posterior elastic membrane, and posterior endothelium.

The epithelial layer covering the cornea anteriorly consists of a few layers of stratified squamous epithelium, and is the corneal portion of the conjunctiva; the lowermost cells are columnar.

The anterior elastic lamina (or "membrane of Bowman") is a thin, hyaline, apparently homogeneous layer, forming a basement-membrane for the epithelium.

The substantia cornece propria makes up the main part of the cornea, and consists of transparent laminæ of white fibres bound together by cement-substance. Between the laminæ are numerous stellate connective-tissue cells with anastomosing branches. These cells and their processes lie in spaces forming a network of passages through which lymph can pereolate for the nutrition of the cornea, which possesses no bloodvessels.

Behind the substantia propria is the posterior elastic lamina (or "membrane of Descemet"), a thin, homogeneous, hyaline layer like the anterior one. Upon the free posterior surface of this membrane is a single layer of endothelium-cells, continuous with those lining the anterior surface of the iris.

The cornea contains plexuses and terminals of nerve-fibrils. At the junction of the cornea and sclerotic, in the substantia propria, is a venous or lymph-passage, the canal of Schlemm, encircling the cornea.

The choroid is the thin, middle vascular tunic of the eye. Anteriorly it is continuous with the ciliary body and iris. The outermost layer of the choroid is the lamina supra- 
choroidea, similar to the lamina fusea of the sclerotic, from which it is separated by lymph-spaces. Next to this layer is the main stroma of the choroid, or vascular layer, which consists of an abundance of lymphatics and large bloodvessels imbedded in fibro-elastic tissue containing numerous pigmented stellate connective tissue cells.

FIG. 91.

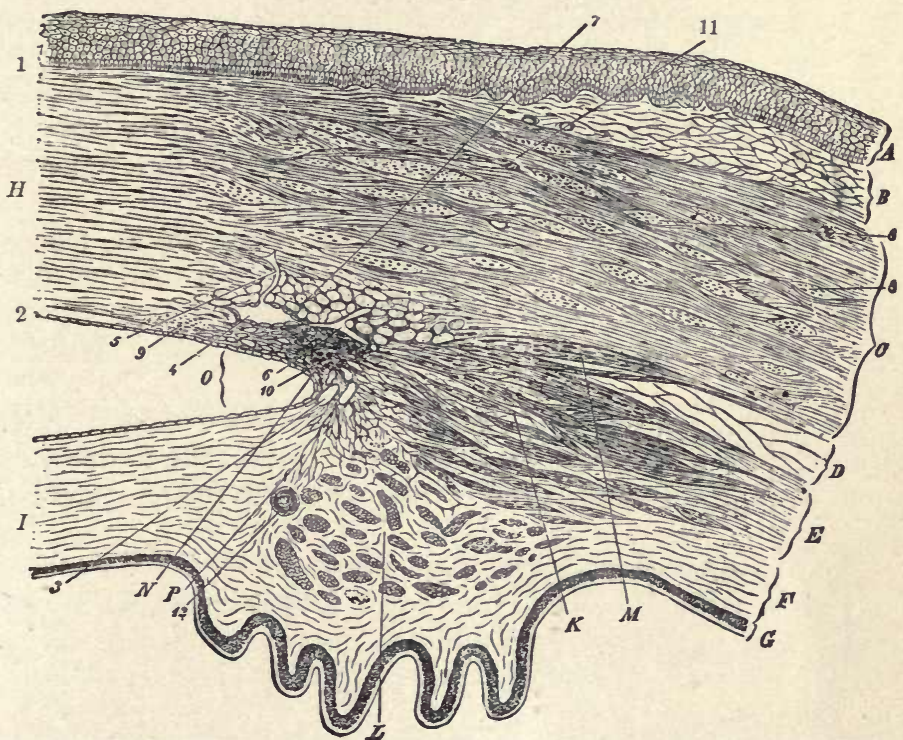

Section through ciliary body and tunics of eye at corneo-sclerotic junction (Waldeyer). $A$, epithelium of conjunctiva; $B$, tunica propria of conjunctiva; $C$, sclerotic; $D$, laminæ fusca and suprachoroidea; $E, F$, choroid; $G$, pars retinæ ciliaris and iridica; $H$, cornea; $I$, iris; $K, M$, radial and meridional portions of ciliary muscle ; $L$, circular portion of ciliary muscle; $N$, ligamentum pectinatum; 0 . margin of anterior chamber; 1 , anterior elastic lamina of cornea; 2 , posterior elastic lamina and endothelial layer of cornea; 3, spaces of Fontana; 4 , canal of Schlemm; 5,6 , vessels tributary to canal of Schlemm ; 7,8 , 9, fibrous bundles of sclerotic cut transversely; 10 , origin of meridional ciliary muscle; 11,12 , bloodvessels.

Next within this is the thin choriocapillary layer, a dense capillary network. Next to this is a thin, hyaline homogeneous vitreous layer (lamina basalis, membrane of Bruch), the innermost layer of the choroid and separating it from the retina. 
The ciliary body is a thickening of the choroid anteriorly, at its junction with the cornea, sclerotic, and iris. Its substance is similar to that of the choroidal stroma, consisting of vessels imbedded in connective tissue; the choriocapillary layer is wanting, the capillaries being more generally distributed.

Its posterior surface is thrown up into radiating processes, the ciliary processes, increasing in height toward the iris, at the periphery of which they abruptly terminate. The vitreous nembrane is continued over the surface of these processes.

Upon the posterior or inner surface of this membrane rests a layer of pigmented epithelium, and then a single layer of columnar epithelium; these two layers are an anterior continuation of the retina (pars ciliaris retince).

In the substance of the ciliary body is the ciliary muscle, fasciculi of involuntary muscle arising near the corneosclerotic junction and extending thence radially or meridionally outward and backward, together with some circular fasciculi.

The ciliary body at the margin of the iris is united to the corneo-sclerotic junction by fibrous processes, the ligamentum pectinatum, the interstices of which form the lymph-spaces of Fontana.

The iris is attached by its peripheral margin to the ciliary body at the corneo-sclerotic junction. It consists of six layers: from before backward, endothelium, anterior boundary layer, stroma, posterior boundary layer, pigment-layer, and limiting membrane.

The endothelium covering the anterior surface of the iris is continuous with that on the posterior surface of the cornea.

The anterior and posterior boundary layers and stroma of the iris are continuations of choroidal tissue.

The anterior boundary layer is a loose connective tissue containing lymphoid cells; it merges into the stroma.

The stroma or main portion of the iris consists chiefly of radiating bloodvessels and circular and radiating fasciculi of involuntary muscle imbedded in connective tissue. The connective-tissue cells of the stroma and anterior boundary layer contain pigment in varying amount, to which the color of the iris is partly due. 
The posterior boundary layer is a thin hyaline layer corresponding to the vitreous layer of the choroid.

The pigment-layer is a continuation of the retina (pars iridica retinæ), and consists of densely pigmented epitheliumcells, fusiform anteriorly, polygonal posteriorly.

The membrana limitans iridis is a delicate cuticular membrane covering the pigment-layer posteriorly.

The aqueous humor consists of lymph, containing a few leukocytes; it occupies the anterior chamber of the eye, which is essentially a large lymph-space communicating with the ocular lymphatics and is lined with endothelium, the same layer which covers the posterior surface of the cornea and anterior surface of the iris.

The crystalline lens consists of a mass of epithelioid lensfibres covered by a layer of epithelium anteriorly; the whole enveloped in a capsule.

The anterior epithelium is a single layer of low columnar cells covering the anterior surface of the lens beneath the capsule.

The lens-fibres, composing the bulk of the lens, are long curved hexagonal fibres cemented together, arranged somewhat concentrically and meridionally. Oval nuclei are present near the middle of the fibres at the equator of the lens, and in all the fibres when young. The lens-fibres are greatly elongated epithelium-cells, derived from the posterior epithelium of the embryonic lens. At the equator of the lens a transition from the anterior epithelium into the posterior epithelial fibres is observable.

The capsule is an elastic cuticular membrane enveloping the lens.

The suspensory ligament (zone of Zinn, zonula ciliaris) encircles and supports the lens, being attached to the capsule of the latter near the equator. It is a fibrous structure, radially plicated ; it is connected posteriorly with the hyaloid membrane of the vitreous body, and with the ciliary body and processes, so that contraction of the ciliary muscle relaxes it and permits the elastic lens to increase in convexity and refractive power. At its union with the lens it splits into two layers, anterior and posterior, with a lymph-space between them, called the canal of Petit. 
The vitreous body fills the large cavity of the eye. It consists of a soft gelatinous substance, scattered about in which are stellate connective-tissue cells and scanty fibrous elements -a sort of mucous tissue. It is surrounded by a homogeneous membrane, the hyaloid membrane, which separates it from the retina and lens.

The retina, the inner tunic of the eye, lies between the choroid and the vitreous. It is divisible into three parts, the optical, ciliary, and iridic portions; the two latter being rudimentary anterior portions.

The pars retince iridica covers the posterior surface of the iris, and consists of densely pigmented epithelium-cells covered by a limiting membrane.

The pars retina ciliaris covers the posterior surface of the ciliary body and processes, and consists of layers of pigmented and colunnar epithelium and an internal limiting membrane.

A short distance behind the ciliary body the pars retince optica or main functional portion of the retina, the retina proper, begins by an abrupt thickening or fold, the ora serrata.

The retina proper exhibits the following layers, from within outward (Fig. 92): internal limiting membrane; nerve-fibre layer ; ganglion-cell layer ; inner reticular layer ; inner nuclear layer; outer reticular layer; outer nuclear layer; external limiting membrane; layer of rods and cones, and pigmentlayer.

The elements making up the retina are nervous, neuroepithelium, pigmented epithelium, and sustentacular.

Conspicuous among the sustentacular elements are the radiating fibres of Miiller, specialized neuroglia-cells. Their inner ends are expanded and by their junction form the socalled internal limiting membrane of this portion of the retina; from these pyramidal ends the cells or fibres, more attenuated, pass perpendicularly outward through the various layers to the external limiting membrane, giving off lateral sustentacular branches and reticula; in the inner nuclear layer the fibre exhibits a nucleus, and externally the fibres terminate in fine processes which pierce the external limiting membrane and lie among the bases of the rods and cones. Other branched neuroglia-cells lie in the outer reticular layer.

The nerve-fibre layer is made up of non-medullated nerve- 
fibres derived and radiating from the optic nerve and also connected with the retinal nerve-cells.

The ganglion-cell layer consists of rather scattered large rounded nerve-cells with numerous branched processes.

Fig. 92.

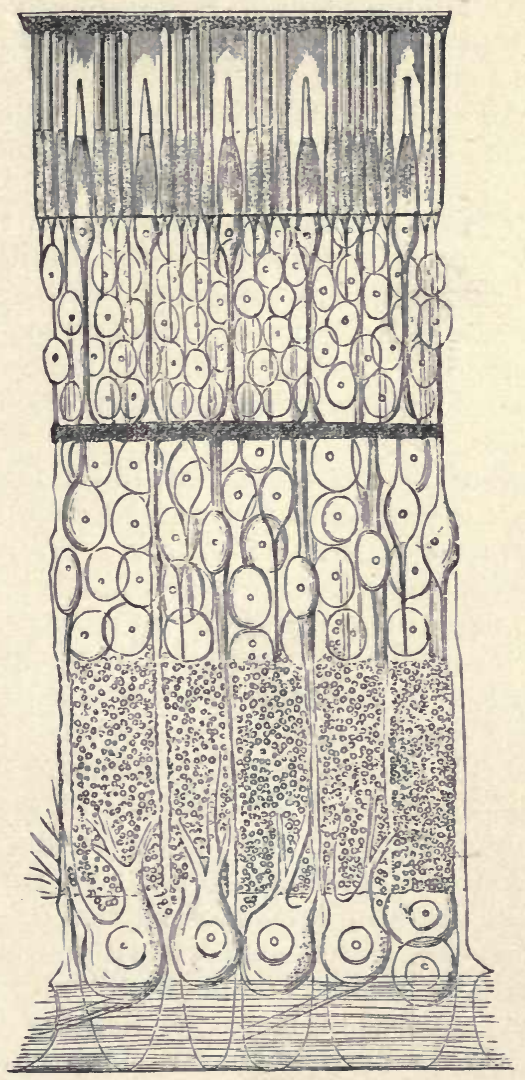

Diagrammatic section of human retina (Schultze).

The inner reticular (or molecular) layer is a broad nonnucleated zone formed by a delicate fibrous reticulum derived 
from the neuroglia-elements and the processes of neighboring nerve-cells.

The inner nuclear (or granular) layer is a narrower zone crowded with nerve-cells having prominent nuclei ; the nuclei of Müller's fibres are also in this layer.

The outer reticular (or molecular) layer is a narrow reticulum of neuroglia-fibres and nerve-cell processes.

The outer nuclear (or granular) layer is a broad zone crowded with the nuclei and the lower portions of the rod and cone cells.

The external limiting membrane is a thin perforated membrane, through the openings of which pass the rod and cone cells.

The layer of rods and cones is composed of the portions of the rod and cone cells external to the limiting membrane. The rod and cone cells are elongated neuro-epithelium cells, arranged perpendicularly, divided into two zones by the limiting membrane, their nucleated portions occupying the outer nuclear layer and their terminal portions forming the layer of rods and cones.

The portion of the rod-visual cells in the nuclear layer is a slender fibre, the "rod-fibre," with a bulbous expansion containing the transversely banded nucleus. The portion external to the limiting membrane, or "rod," consists of two segments, the inner one continuous with the rod-fibre being fusiform, and granular or fibrillated (especially in its outer part); while the outer segment is a slender cylinder containing rhodopsin and possessing special staining properties.

The portion of the cone-visual cells ontside of the limiting membrane, or the "cone," also consists of two segments, an outer part similar to the corresponding portion of the rods except that it is shorter, conical, and contains no rhodopsin; and an inner broad fusiform portion, fibrillated or granular externally. The portion of the cone-cell within the outer nuclear zone consists of a slender "cone-fibre" with an expansion, containing the nucleus, next to the limiting membrane and uniting with the fusiform segment. The rod-cells considerably outnumber the cone-cells.

The pigment-layer of the retina, lying next to the vitreous membrane of the choroid, consists of a single layer of hexagonal columnar epithelium-cells, with their nuclei next to the 
choroid, and their inner portions, adjoining and between the rods and cones, studded with pigment-granules.

In the macula lutea the rods are absent and some of the retinal layers are thickened and pigmented yellow. At the fovea centralis only the layer of cone-cells is present in full development, the layers in front of it-i. e., toward the centre of the globe-being greatly thinned and nearly abolished.

The eyelids (upper) are covered anteriorly with thin skin, beneath which is areolar subcutaneous tissue containing little or no fatty tissue. Next to this is a layer of striated muscle, the orbicularis palpebrarum. Posterior to this is a zone of loose connective tissue, the fascia palpebralis, into which the insertion of the levator palpebræ merges. Posterior to this and next to the conjunctiva is a dense fibrous plate, the tarsus, in which are imbedded a number of modified sebaceous glands, the Meibomian glands, each consisting of an elongated series of follicles opening into a duct which discharges at the margin of the lid.

In the upper part of the tarsal plate, especially toward the nose, are small accessory lachrymal glands. The posterior surface of the lid is covered by conjunctiva, composed of stratified columnar epithelium on a tunica propria, which lies next to the tarsus.

At the margin of the lid is a row of stiff hairs, the eyelashes, the follicles of which separate the margin of the orbicularis palpebrarum from the main portion of the muscle; behind the lashes are modified sweat-glands (Moll's glands); and still farther posteriorly are the openings of the Meibomian glands.

The lachrymal gland is a racemose gland of the serous type, the saccules of which empty into a number of independent and separate outlet-ducts. The lachrymal canals are tubes lined with mucous membrane connecting the conjunctivæ with the nasal fossæ.

\section{The Ear.}

The ear is a complicated structure associated with the termination of the nerves of hearing, and with the sense of position. It is divided into the external, middle, and internal ear. 
External ear: The auricle consists of a basis of elastic cartilage covered with subcutaneous tissue and skin-except the lobule, whose interior is occupied by fibrous tissue.

The outer (cartilaginous) portion of the external auditory canal is lined with skin, which is very thick and contains hairs, their sebaceous glands, and ceruminous glands, a modified form of sweat-gland. This portion of the canal has a basis of elastic cartilage beneath the subcutaneons tissue.

The inner (bony) portion of the auditory canal is mostly lined with thin skin containing no glands or hair.

The middle ear (or tympanum) is a cavity filled with air, communicating with the Eustachian tube anteriorly and the mastoid sinuses posteriorly, and crossed by a chain of ossicles connecting the tympanic membrane with the fenestra ovalis.

Its walls are formed by bone, the tympanic membrane, and the secondary tympanic membrane, all lined with mucous membrane.

The mucous membrane is thin and closely connected with the underlying periosteum; it is reflected over the surface of the ossicles and mastoid sinuses and is continuous with the mucous lining of the Eustachian tube. Its superficial layer consists of low columnar epithelium, in places ciliated, in other places non-ciliated. Anteriorly are scattered tubular glands.

The tympanic membrane is made up of an intermediate fibrous lamina covered externally with skin and internally with a portion of the mucosa lining the middle ear; the surface epithelium-cells of the latter are low and non-ciliated.

The secondary tympanic membrane closes the fenestra rotunda, and consists of an intermediate fibrous lamina, covered externally with mucous nembrane (having non-ciliated low epithelium) continuous with the mucosa of the middle ear, and internally with endothelium and subendothelial tissue continuous with that lining the internal ear.

The Eustachian tube is an open canal connecting the middle ear with the pharynx. Its walls are formed of bone, cartilage (partly hyaline, partly elastic), and fibrous tissue, lined with mucous membrane continuous with that of both the middle ear and pharynx. The surface cells of this mucosa are stratified ciliated epithelium. In its upper portion the mucous 
lining is thin ; in the lower or pharyngeal portion it is thicker, underlaid with areolar submucous tissue, and contains mucous glands and lymphoid tissue.

The internal ear (or labyrinth) contains the terminal apparatus of the auditory nerve, and occupies a tortuous bony cavity (the "bony labyrinth") in the temporal bone.

The bony labyrinth has three divisions-the cochlea, semicircular canals, and vestibule, the latter connecting the two others.

Within the bony labyrinth is a membranous sac, the "membranous labyrinth," containing "endolymph" and corresponding in general to the windings of the bony labyrinth.

The membranous labyrinth is separated from the bony walls in most places by a peripheral lymph-space, lined with endothelium and filled with "perilymph."

The bony labyrinth is lined with periosteum, covered in general with endothelium.

The membranous labyrinth consists of the utricle and saccule, situated in the vestibule, the membranous semicircular canals, and the scala media of the cochlea.

The neuro-epithelial terminals of the auditory nerve are situated on the inner surface of the membranous labyrinth, and consist of the maculoe acustice of the utricle and saccule, the cristce acustice of the ampullæ of the semicircular canals, and the organ of Corti.

The vestibule is a bony chamber connecting the semicircular canals with the cochlea. It contains the utricle and saccule, which are separated from the bony walls by the perilymphspace. This space is in relation with the middle ear by the fenestra ovalis, which is closed by the base of the stapes.

The utricle and saccule are rounded membranous sacs, communicating with each other by a small indirect canal, the "ductus endolymphaticus." Their walls are formed by a fibrous membrane lined internally with simple squamous epithelium, altered to columnar in the maculæ acusticæ ; externally they are covered with endothelium. They are connected with the periosteum of the bony walls by fibrous bands, which cross the perilymph-space and convey vessels and nerves. The utricle opens into the semicircular canals; the saccule communicates through the small "canalis reuniens" with the 
scala media, or cochlear portion of the membranous labyrinth.

The utricle and saccule each possesses on its inner surface a neuro-epithelial terminal, called the macula acustica.

The macula is an area covered with columnar epitheliumcells of two kinds: $(a)$ "sustentacular" or "fibre-cells," long, slender cells, between which are situated (b) "hair-cells," pyriform cells with filamentary processes or cilia projecting from their free ends. Terminal nerve-fibrils from the auditory nerve are in relation with the bases and sides of the hair-cells. The surface of the macula is covered with a matrix or layer containing minute calcium-carbonate crystals, or "otoliths."

The semicircular canals, opening from the utricle, are membranous tubes formed of a fibrous membrane lined internally by simple squamous epithelium (except over the cristæ) and externally with endothelium, and separated from the bony walls by the perilymph-space. Each membranous canal has an enlargement or ampulla at one of its junctions with the utricle, and on the inner surface of each of the three ampulla is situated a neuro-epithelial structure called the crista acustica.

These cristce are ridges covered with columnar epitheliumcells of two kinds, similar to those of the maculæ acusticæ: (a) "sustentacular" or "fibre-cells," long, slender columnar cells, between which are (b) "hair-cells," shorter cells with long hairs or cilia projecting from their free ends into the ampullæ. Nerve-fibrils terminate among the hair-cells. There is no layer of otoliths over the cristæ.

The cochlea consists of three passages, the scala vestibuli, scala media, and scala tympani, wound spirally two and a half turns around a central column or "modiolus" (Fig. 93).

The scala media is the cochlear portion of the membranous labyrinth; the other two scalæ represent the perilymph-spaces.

The seala vestibuli and scala tympani are lined with endothelium, and are separated from each other externally by the scala media and internally by the "lamina spiralis," a spiral bony ledge projecting from the modiolus.

The scala vestibuli is the uppermost of the three cochlear passages, and opens below into the perilymph-space of the vestibule; at the apex of the cochlea it communicates with the scala tympani. The lower end of the latter is blind, but 
communicates with the middle ear by the fenestra rotunda, closed by the secondary tympanic membrane.

The scala media (canalis or ductus cochlearis) is the middle passage of the cochlea ; it ends blindly above, and communicates below with the saccule by the small canalis reuniens.

FIส. 93.

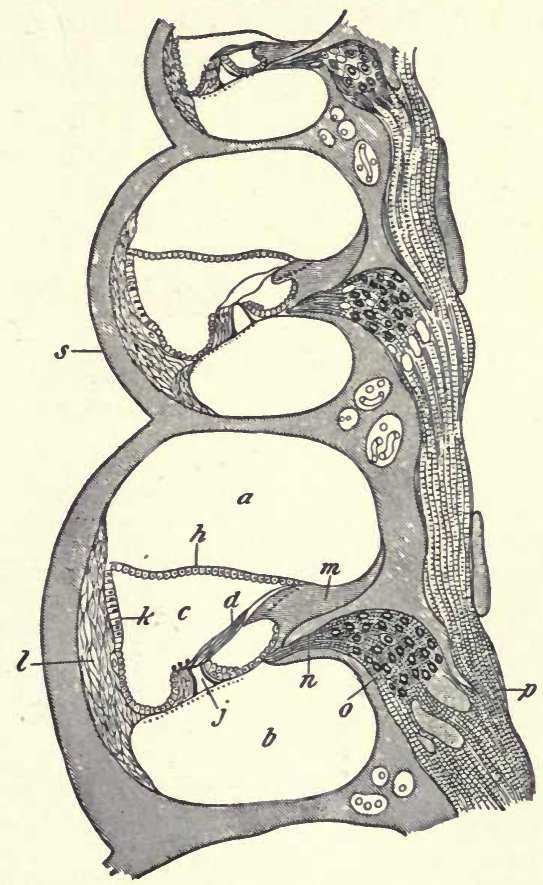

Radial section of portion of cochlea of guinea-pig (Klein). $a$, scala vestibuli; $b$, scala tympani : $c$, scala media : $d$, riembrana tectoria ; $h$, membrane of Reissner; $j$, organ of Corti, resting on basilar membrane; $k$, stria vascularis ; $l$, ligamentum spirale; $m$, limbus; $n$, nerve-fibres in lamina spiralis; 0 , spiral ganglion; $p$, nerve-fibres in modiolus; $s$, bony wall.

The upper wall of the scala media, separating it from the scala vestibuli, is formed by the membrane of Reissner, a very thin fibrous membrane lined on the vestibular surface with endothelium, and on the surface within the scala media with simple squamous epithelium. 
The outer wall of the scala media is lined with a single layer of epithelium-cells, varying from squamous to columnar, which rest on the ligamentum spirale, a fibrous cushion lying against the bony walls on the outer side of the cochlea; a portion of this outer wall ("stria vascularis") is very vascular.

The floor or lower wall of the scala media is formed by the margin or limbus of the lamina spiralis, the basilar membrane, and columnar and neuro-epithelium (the organ of Corti) resting on the latter. The margin of the bony spiral lamina is thickened by the development on its upper surface of a mass of fibrous tissue, the whole forming the limbus.

The outer projecting aspect of the limbus is hollowed into a groove, the sulcus spiralis, the upper lip of which is called the labium vestibulare, the lower lip the labium tympanicum.

The labium vestibulare is divided by clefts into fine processes called the "auditory teeth."

The membrana basilaris is a connective-tissue lamina extending from the labium tympanicum of the limbus across to the ligamentum spirale on the outer wall, shutting off the scala media from the scala tympani; it is lined underneath (within the scala tympani) with endothelium, while its upper surface is covered with columnar epithelium and the neuroepithelium constituting the organ of Corti.

The upper surface of the limbus is lined with simple squamous epithelium continuous with that lining the membrane of Reissner.

The sulcus spiralis is lined with a layer of columnar epithelium-cells, which extend to the inner hair-cells or inner margin of the organ of Corti.

The organ of Corti (Fig. 94): These inner hair-cells comprise a single row of columnar epithelium-cells, with a number of filaments or hairs projecting from their free ends; they are adjacent to and on the inner side of the pillars of Corti, their attached ends not extending to the basilar membrane.

The pillars or rods of Corti are two rows of elongated epithelial elements, the lower ends of which rest on the basilar membrane some distance apart, and whose upper ends are 
articulated together, meeting like the rafters of a roof; they inclose a lymph-space or passage, the "tunnel of Corti," which follows the spiral windings of the cochlea.

These pillar-cells, inner and outer, have slender shafts of a firm substance, with enlarged extremities, and with nuclei and some ordinary protoplasm at their lower ends.

External to the pillars of Corti are three or four rows of outer hair-cells, columnar epithelium-cells with hairs projecting from their exposed ends. They extend from the upper sur-

FIr. 94.

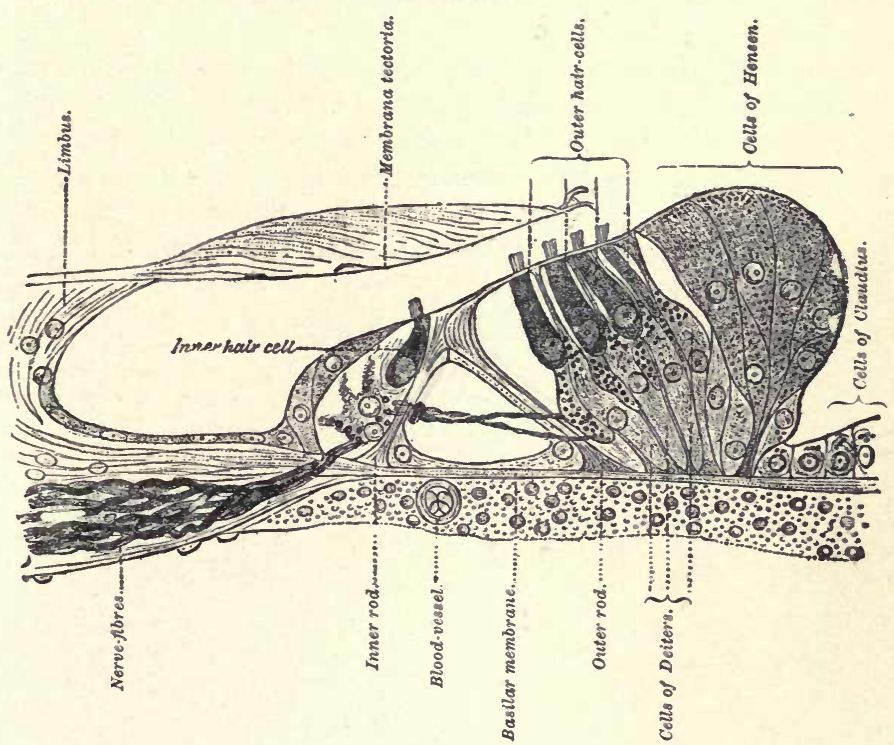

Organ of Corti, human, in cross-section (Retzius).

face of the organ of Corti only about half-way to the basilar membrane.

The outer hair-cells alternate with and are supported by the cells of Deiters, long columnar sustentacular epithelium-cells, somewhat like the pillar-cells, and with their bases separated by slight intervals, the "spaces of Nuel."

The upper ends of the pillar-cells and cells of Deiters 
exhibit a cuticular structure (the "membrana reticularis"), with openings for the ends of the hair-cells.

External to the outer hair-cells is a zone of long columnar epithelium-cells (cells of Hensen), which externally become shorter and merge into a zone of low columnar epithelium (cells of Claudius); the latter occupy the outermost part of the basilar membrane and are continuous with the squamous epithelium lining the outer wall of the scala media.

Attached to the labium vestibulare of the limbus is a soft structure, the membrana tectoria, which extends outward over the organ of Corti.

Nerve-fibrils from the cochlear branch of the auditory nerve terminate among the hair cells of the organ of Corti. The cochlear nerve lies in the modiolus, and sends branches laterally into the lamina spiralis, where they are associated with nerve-cells to form the spiral ganglion; from the ganglion the nerve-fibres pass out into the organ of Corti at the labium tympanicum. 


\section{PATHOLOGY.}





\section{PATHOLOGY.}

\section{INTRODUCTORY.}

Pathology ( $\pi \alpha 00_{5}$, disease, and lóros, discourse) is the science which treats of animal and vegetable life under abnormal conditions-i. e., the science of disease. It is that branch of medicine which considers abnormalities of structure and function, the causes thereof, and the relation of these changes to the clinical aspects of disease produced thereby.

Human pathology then includes the study of etiology or the causes of disease ; morbid anatomy-macroscopical and microscopical; and morbid or pathological physiology. With these must always go hand in hand clinical observation, in order that a true conception may be formed of disease-processes. As our knowledge of anatomy is more advanced than that of physiology, so our knowledge of morbid anatomy is more perfect than that of morbid physiology.

The importance of this branch of medicine cannot be overestimated by the student; it is the one sure basis for diagnosis, prognosis, and rational therapeusis. Some of the greatest additions to medical knowledge have been made by the study of morbid anatomy in conjunction with clinical observation. Apoplexy was supposed to be due to the interruption of certain imayinary spirits till Wepfer showed cerebral hemorrhage to be the cause in a large number of cases. In this manner Bright demonstrated the relation between certain alterations in the kidneys, and albuminuria and dropsy. Typhoid and typhus fevers were regarded as identical until 1836 , when a post-mortem study of the lesions present proved the contrary. The mediæval physician rarely endeavored to locate the seat of a discase-certainly not beyond assigning it to one of the larger cavities of the body, $i . e$. , the head, 
chest, or abdomen. In fact, but little was added to the teachings of Hippocrates (460 B. C.) and Galen (130-200 A. D.) until about 1500 , when the first post-mortems began to be made.

During the sixteenth century Vesalius, Sylvius, and their successors laid the foundation of modern medicine by the systematic study of anatomy, to which was added by Harvey and others during the seventeenth century such essential physiological data as the function of the heart, eirculation of blood, and the mechanism of respiration.

Morgagni (1682-1771) was the first to attempt to localize a disease-process in a particular organ and wrote the first great treatise on morbid anatomy. The first illustrated work on the subject was by Baillie (1799), who drew his inspiration and material from John Hunter. It was not until the early part of this century, however, that there was a real beginning in its study. But there were limitations to gross morbid anatomy.

Histology received its first impulse from Bichat, to whom is due also a step in advance in pathology; he declared that the ultimate seat of a disease might be a particular tissue of an organ. Though the microscope had been employed in the seventeenth century by Leuweenhoeck and Malpighi, it was as recent as 1847 that the foundation of normal histology was laid by the work of Schleiden on "Vegetable Pathology" and later of Sehwann on the "Comparison of the Cellular Structure of Vegetables and Animals."

Modern or cellular pathology dates from the teachings of Virchow (1858), to whom credit is due, more than to any other one man, for elevating the study of disease to a place in the science of biology. The cellular theory of life led naturally to the cellular theory of disease. A comparison of the lower forms of animal and vegetable life with the eells of higher ones convinced Virchow that if the former are indiciduals, the latter must be so regarded also. For the axiom of Harvey, "Omne vivum ex ovo," he substituted the dictum "Omnis cellula a cellula"; he applied the histological discoveries of Schleiden and Sehwann to pathology and showed that the essence of disease is the altered cell,-the histologieal and vital unit of all organized tissue,-and called attention to 
the minute changes which it undergoes in disease. The cellular theory has done for pathology what the atomic theory has for chemistry.

Medical knowledge has been advanced more in recent years by experimental pathology than by any other method of investigation. Though Galen is said to have used living animals for pathological experimentation, the foundation of these methods and the revival of pathology in general were due to John Hunter in the latter part of the last century. To bacteriology, one branch of experimental pathology, we owe our knowledge of the microbic cause of many of the infectious diseases - the germ-theory, in its practical results having almost revolutionized medicine and surgery within a quarter of a century.

Disease is a deterioration in or deviation from the normal standard called health. Probably all deviations of function from normal are dependent on some alteration in structure; when our present methods fail to discover such anatomical changes the disease is said to be functional, in contradistinction to organic disease, in which such alterations can be demonstrated. There is no sharp line of demarcation between health and disease, the one passing imperceptibly into the other.

Heredity plays a most important part in the causation of disease ; it is not uncommon to see reproduced in a family the same infirmities or diatheses for many generations-and the ill results of consanguineous marriage are a matter of common knowledge.

Susceptibility to various diseases varies greatly in different individuals - the determining factors being inherited in some cases and acquired in others, and temporary or permanent in their duration. The susceptibility of children of tubercular parents to tuberculosis is observed with great frequency; without such inherited tendencies however, during some temporary impairment of health, there may be an acquired susceptibility to the disease, which, for instance, is especially common after measles in children-the attack either preparing a soil particularly inviting to the tubercle bacilli or reducing the patient's resisting powers to such an extent that they are not able to cope with the invasion of these micro-organisms 
successfully, as would be the case were they not temporarily so reduced.

The well-known insusceptibility of the negro to yellow fever and malaria are examples of the comparative immunity which a race may enjoy; and among the lower animals the same thing is noted of different species-for instance, the frog is immune against infection with anthrax, while the mouse, guinea-pig, and many other animals are extremely susceptible.

The comparative insusceptibility of an individual to a second attack of such diseases as smallpox, scarlet fever, and measles is an example of acquired immunity. Such immunity may be conferred artificially, as is witnessed daily in the practice of vaccinating against smallpox.

Equally striking is the varying susceptibility of an individual at various times in his life to the same disease.

Exciting causes are those immediately responsible for the onset of the disease ; among these may be mentioned trauma, exposure to heat and cold, poisons, and, by far the most important of all, animal and, more especially, vegetable parasites - of which latter bacteria are the most important.

But a discussion of these topics would lead far beyond the limits assigned us and must be thus dismissed. The study of bacteriology has grown within a few years to large proportions-and volumes have been written concerning animal parasites.

Pathology, or pathological anatomy, with which the following pages are more especially concerned, may be divided for convenience into two sections-one, the general aspects of diseaseprocesses without reference to any individual part; and the other, diseases of special organs and systems. 


\title{
GENERAL PATHOLOGY.
}

\author{
CHA P TER I.
}

\section{PATHOLOGY OF THE BLOOD AND CIRCULATION.}

\section{INFLAMMATION.}

Inflammation is undoubtedly the most important and most common phenomenon in pathology. It is convenient to consider it as an entity rather than a series of morbid phenomena. No subject in medicine is more deserving the student's attention. It underlies a majority of the diseases to which man is heir. In all ages its consideration has so dominated the doctrines and medical philosophy of the time that it might well be said to form the basis of the theory and practice of medicine and surgery.

The term "inflammation" was created in the very infancy of science, indicating that the part involved seemed to burn-to be inflamed. The cardinal symptoms which are familiar to all to-day were described two thousand years ago by Celsus-tumor, rubor, calor, and dolor. The interpretation of these symptoms and conception of the underlying processes varied as this or that symptom was considered of the greatest importance. Humoral doctrines perished with Harvey's discovery of the circulation of the blood, the advances made in chemistry, and the systematic study of morbid anatomy. Since then the study of inflammatory lesions has followed step by step the progress in the biological sciences.

Definition: We may attempt to define inflammation as the ensemble of the degenerative and reactionary phenomena occurring in living tissues as the result of mechanical, infectious, and toxic injuries.

Early experiments: John Hunter, a century ago, was the first to throw experimental light upon this sulject of in- 
flammation. He froze the ear of a rabbit and then allowed it to thaw, thus exciting considerable inflammation. The animal was killed when the process was at its height, the bloodvessels of the head injected, and the ears removed and dried. The uninflamed ear was clear and transparent, and the bloodvessels easily seen; the inflamed ear was thick and opaque, and the arteries much enlarged.

A few years later very numerous indeed are the papers and monographs describing the changes in the size of the bloodvessels in an inflamed animal membrane studied under the microscope. A frog was restrained in a muslin bag and to the web of the foot-less frequently to its mesentery-or to the web of the foot of a young duck similarly restrainedvarious irritants were applied and the results noted under the microscope. The increased size in the eapillary bloodvessels was thus observed in the affected area, and further a marked slowing in the rapidity of the blood-eurrent.

By 1840 we find the description much more complete. Addison and others carefully described, in addition, an axial stream of red blood-cells and a much more slowly moving peripheral stream in which were found the leukocytes; and as the velocity of the circulation became less and less the great accumulation of the latter along the inner wall of the vessels was noted. Addison maintained the identity of the leukocyte and pus-cell ; and even the passage of the leukocytes through the bloodvessel-walls was described, but failed to attract much attention; a phenomenon which, since the work of Cohnheim, twenty-eight years later, has been studied with great interest.

The experiments of Cohnheim (1867), though they simply duplicated the experimental studies of earlier observers on living animal membranes, were of the greatest importance, for to him undoubtedly all credit is due for our knowledge of the migration of the leukocytes, the announcement of which came as a surprise to all.

Microscopical appearances of inflamed animal membranes: First stage: Dilatation of vessels; acceleration of blood-current. When the mesentery of a curarized frog is exposed under the microscope there is soon noted a dilatation of the arteries and then of the veins, and to a very much less degree of the capil- 
laries; by the end of fifteen or twenty minutes it may be very decided, most apparent, however, in the arteries. At the same time there is a marked acceleration of the blood-current, equally pronounced in arteries, veins, and capillaries. The stage of

Positions of the Corpuscles in Circulatixg Blood (Eberth and Schimmelbesch).

FIG. 95.

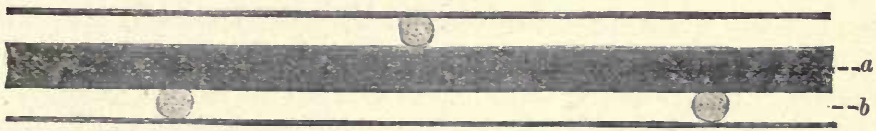

Appearance when the relocity of the circulation is normal. $a$, axial column of corpuscles, both red and white, in such rapid movement that individual corpuscles cannot be distinguished. Occasionally a white corpuscle is thrown from the axial mass and appears in the plasmic zone $b$.

Fig. 96.

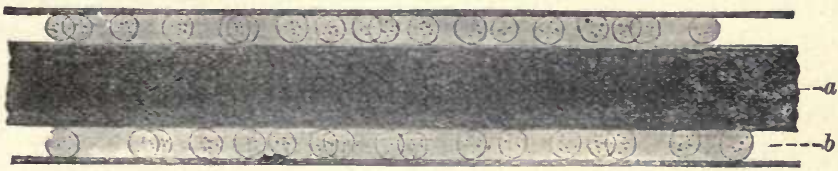

Appearance when the velocity of the circulation is moderately reduced. The zone $b$ contains numerous leukocytes.

FIG. 97.

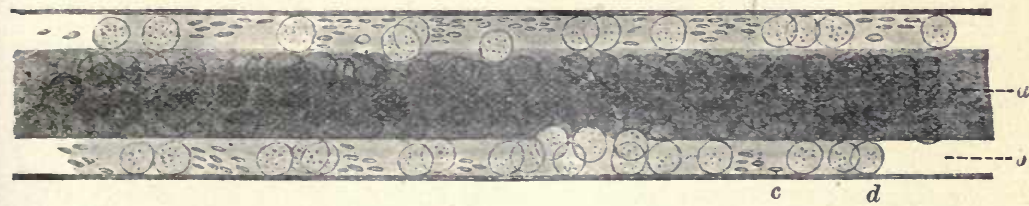

Appearance when the current of blood is sluggish. $a$, red corpuscles, still in the axis ; $b$, peripheral zone, containing leukocytes, $d$, and blood-plates, $c$.

When stasis is fully established the red corpuscles also invade the peripheral zone. The figures are from observations made on the vessels of a dog's omentum during life.

acceleration in the blood-current will never last more than one or two hours, by the end of which time the vessels have become twice their normal diameter.

Second stage: Retardation of blood-current; margination 
of the leukocytes. The acceleration of the blood-current, which may be only transitory, is followed by its progressive retardation until the vessels are engorged with blood. The current becomes slower and slower, until finally each individual red blood-cell can be recognized, and every heart-beat causes sudden jerks in the flow.

But soon one's attention is fixed upon the veins. Normally there are only a few leukocytes to be seen in the periphery of the current, where they are occasionally driven from the axial stream of red blood-cells by reason of their lower specific gravity. But now the number of leukocytes in this peripheral stream is rapidly increasing. They lag behind the still quite rapidly moving axial stream of red blood-cells, momentarily clinging from place to place as they are carried on. This same tendency is also seen in the capillaries.

In the arteries, with each cardiac diastole a number of leukocytes roll into the peripheral stream, only to be swept back into the axial stream with the next systole. Finally the inner surface of the walls of the veins is almost lined by a layer of leukocytes. This phenomenon of margination was described by all the earlier observers (Figs. 95, 96, and 97):

Third stage: Diapedesis; interstitial exudation. Margination is followed, sometimes immediately, sometimes only after an hour or more, by diapedesis or migration of the leukocytes. There appears on the external surface of the vessel-wall, usually on a vein, but sometimes on a capillary, a small button-like elevation or hump, which little by little grows gradually larger. This colorless protuberance is seen to undergo manifold variations in form, to throw out and retract little finger-like processes; it finally becomes pear-shaped with its point toward the vessel. The tapering end is gradually enlarged into a slender pedicle, which finally separates from the vessel-wall and is retracted into the body of the leukocyte, for this separated mass of protoplasm is now readily recognized as such. The migration of a leukocyte takes sometimes two hours. The same process is repeated at a large number of points around the veins and capillaries, so that by the end of six or eight hours an immense number of leukocytes have accumulated along the external surface of the vessel-walls. In the arteries this phenomenon is not noted. 
It does occur to a very considerable extent in the capillaries, and from them emigration of the red corpuscles also takes place. The leukocytes, as their number increases, do not remain in the neighborhood of the vessels from which they migrated; while the red globules, which have no power of amœboid movement, remain near the vessel from which they escaped. The migration of the leukocytes continues to such an extent that, as there is no room for them in the interstices of the tissue, some reach the free surface of the mesentery. By the end of six or seven hours the phenomenon is no longer to be seen.

The serous membrane becomes opaque, due to an exudate of serum from the bloodvessels, which, by coagulating and entangling in its meshes of fibrin white and red blood-cells, forms on the surface of the mesentery a sort of a pseudomembrane.

These blood-vascular changes have been observed in warmblooded animals; in the wing of a bat and the mesentery of a rabbit.

The dilatation of the bloodvessels was supposed by Cohnheim to be due to the direct injury of the vascular walls by the trauma, producing chemical and molecular changes of the greatest importance. These changes in the vascular walls, he suggested, caused an increased friction of the blood against the walls, which was supposed to explain the retardation of the current. A further consequence was the greatly increased permeability of the vessel-walls, permitting cellular and serous exudations. We cannot state with certainty the exact cause of the dilatation of the vessels. That it is not due to vasomotor paralysis is shown by the fact that when inflammation occurs in a part in which the vaso-motor nerves have been cut the vessels still further dilate.

The slowing of the current is an important factor in the subsequent phenomena of margination and diapedesis. This importance has been experimentally shown by the fact that artificial acceleration of the current induced by the intravenous injection of a 6 per cent. salt solution materially retards the development of these phenomena; and directly so in proportion to the degree of this acceleration.

An increased permeability of the vessel walls has been ex- 
perimentally demonstrated. A colloid liquid injected into the vessels traverses their walls even under feeble pressure. The microscope fails to find any lesion, though it is claimed by some that an increase in the size of the natural stomata between the endothelial cells can be made out.

Diapedesis: The demonstration of the migration of leukocytes threw a flood of light upon a very important questionthe source of inflammatory cells. The formation of pus-cells from connective-tissue cells had never been seen by any one. It became very evident that at least the great majority were migrated leukocytes, the resemblance of which to the cells crowding all inflammatory tissues had been commented upon by Virchow and other observers many years before. It is quite probable that their number is greatly augmented by their multiplication subsequent to migration. As an explanation of the phenomena of diapedesis two theories mainly are invoked - the theory of chemotaxis and the theory of phagocytosis.

Chemotaxis: It has been known for a number of years that vegetable plasmodia are attracted by certain substances and repelled by others: if placed on a surface at a little distance from a nutrient material, such as a deeoction of dead leaves, the organism moves toward it; while numerous other materials, such as a solution of salt and sugar, repel it. To these phenomena Stahl gave the name positive and negative trophotropism, for which Pfeffer substituted the terms positive and negative chemotaxis.

A similar ehemotactic sensibility has been demonstrated on the part of the white blood-cells toward various substances.

It has been shown that whereas some materials, such as quinine, alcohol, chloroform, repel the leukocytes, cultures of various micro-organisms and proteid substances which can be separated therefrom attract them. So, it has been suggested, the leukocytes are attracted by substances formed at the site of inflammation.

Phagocytosis : Metschnikoff, studying the phenomena of inflammation from the standpoint of a biologist, assigns to the leukocytes the main rôle. He attempts to elucidate these complex phenomena by producing them, as far as possible, in organisms of the most simple structure. We find that unicel- 
lular organisms which abound in media surrounding us are subject to infectious diseases. Infection, which is one of the most important causes of inflammation, is simply a struggle between a parasite and its host. We must regard the sum total of the pbenomena of inflammation in human beings as simply the efforts of nature to offset the effects of an injury. However simple the phenomena resulting from similar injury to a unicellular organism-though not constituting inflammation as we are clinically familiar with it-yet their significance is the same, and represents a primitive condition of this process which undergoes a slow evolution as we ascend in the animal scale. The power of locomotion and intracellular digestion is the amoeba's mode of defence.

Metschnikoff says: "If we take a specimen (such as the yellow plasmodium of Physarum) on our object-glass and touch its central part with a minute glass rod previously heated in a flame, we shall produce thermal excitation. Immediately after being touched the central part of the plasmodium dies and may be clearly distinguished from the living peripheral portions, which remain motionless as if nothing has occurred, and are unaffected by the necrosed portion. A few hours later, however, the plasmodium awakes from its passive condition and creeps away from the dead part. . . . . We thus see the irritating agencies excite in the plasmodium either a course of events similar to those which accompany the taking of solid nutriment, or a more or less marked repulsion. In attempting to produce a reaction which should correspond to inflammation in the higher animals, we have brought about the phenomena of attraction or repulsion which occur so frequently in the lives of plasmodia and the inferior animals generally."

In embryo sponges there are stages in which the organism is composed of but two layers, the inner of which is formed of amoboid cells, which have the power of englobing various solid bodies for the purpose of obtaining nutriment and for defence.

In adult sponges there are three layers-ectoderm, mesoderm, and entoderm, and now the function of digestion devolves entirely upon the entoderm, and the amoboid cells of the mesodern alone possess a phagocytic power. 
"It is apparent that the inflammation of vertebrates, in which the defending phagocytes emigrate from the vessels to proceed against offending bodies, is distinguished only quantitatively from the analogous phenomena in invertebrates, and must, therefore, be regarded as a reaction of the organism against deleterious agents. We must conclude that the essential factor, the primum movens of inflammation, consists in a phagocytic reaction on the part of the animal organism. All other phenomena are merely accessory, and may be regarded as a means to facilitate the access of phagocytes to the injured part. . . . The morbid phenomena, properly speaking, such as the primary lesion or necrosis, as well as the processes of repair, do not form part of the inflammation, and must not be confounded with it."

Function and fate of the cellular exudate: The phagocytic power of leukocytes is well recognized, though all varieties do not possess this function to the same degree; it devolving mainly upon the mononuclear and polynuclear forms, especially the neutrophiles. The lymphocytes are too young and have too little extranuclear protoplasm; nor are the eosinophiles, the protoplasm of which seems to be undergoing possibly a retrogressive change, ever seen to englobe red bloodcells, micro-organisms, or other foreign bodies. Young connective-tissue cells - fibroblasts - and endothelial cells of bloodvessels and perhaps of lymphatics, especially when rapidly proliferating, possess this function to a marked degree. Giant-cells are also occasionally phagocytic.

In acute inflammations the neutrophiles are the most active phagocytes, while in chronic inflammation the large mononuclear leukocytes are the most active. Besides this function as phagocytes at the site of inflammation, the migrated leukocytes undoubtedly aid in the restoration of the tissue. Ziegler has long maintained that they develop into connective-tissue cells, and Metschnikoff states that in the wounded tail of a batrachian tadpole, if watched for several days, he has seen the polynuclear cells converted by fusion of their nuclei first into mononuclear cells and then into typical branched connective-tissue cells. Also in rabbits all the transitional stages in the conversion of mononuclear leukocytes into epithelioid and giant-cells have been noted. 
The function of the serous exudate is quite probably primarily nutritive. Further, it frequently possesses bactericidal properties, and may be of value in diluting any bacterial poison present at the site of inflammation. On the other hand, however, the serum is often an extremely good culturemedium for various varieties of micro-organisms, so that its presence may not always result advantageously to the tissue.

Connective-tissue changes: ${ }^{1}$ We have noted that in addition to their phagocytic function the migrated leukocytes are capable of playing an important part in repair, by developing into connective-tissue elements; and further, on the other hand, that young connective-tissue cells and endothelial cells of bloodvessels, active proliferative changes in which are always noted from the very onset of all inflammatory processes, are markedly phagocytic, though this certainly cannot be looked upon as their sole function. It would seem that these are good arguments to extend our conception of inflammation beyond a simple phagocytic reaction on the part of the animal organism. There are examples of non-infectious inflammation where the leukocytic migration is most marked, though not necessarily phagocytic in its nature, in the sense in which the term is usually employed.

Synchronous with the blood-vascular alterations at the outset of inflammation, to which are always added more or less marked degenerative changes in, or even complete destruction of, certain of the cellular elements according to the severity of the irritant, active proliferative changes in the fixed connective-tissue cells occur, which must be regarded as a restorative as well as a defensive manifestation, and hence should be included in our conception of inflammation. We conclude that inflammation is a purposive reaction, having for its object the neutralization of the effects of an injury. In this sense, then, we should include under the head of inflammation the phenomena of repair. Undoubtedly their significance is the same; but in speaking of inflammation we have in mind a well-defined clinical picture which here is absent-the only difference in the phenomena, however, being one of degree.

Variations in the type of inflammation: In every inflamma-

${ }^{1}$ See under head of Repair. 
tion there are vascular and tissue changes; but there is a great variability in the character of inflammations resulting from differences in the nature of the irritants, their persistence, and in the tissues on which they act.

Irritants may be divided into organized and unorganized, the former including animal and vegetable parasites, and the latter mechanical, physical, and chemical influences. The migrating leukocytes play the more important part when the irritant is organized. There is a very considerable difference between a blister in which there is an enormous serous exudate containing but few leukocytes, and the slight serous but enormous cellular exudate produced by pyogenic cocci.

Though this property of inducing the migration of a large number of leukocytes is well recognized as belonging to certain micro-organisms, yet the various other factors concerned in bringing about such a result are not fully understood. Intense cold produces an inflammation which is accompanied by an excessive migration of leukocytes, while the inflammation produced by heat is accompanied by the emigration of but very few leukocytes.

Purulent or suppurative inflammation is a variety characterized by an excessive migration of polymorphonuclear leukocytes or neutrophiles, and a tendency to liquefaction of affected areas-i.e., by the formation of pus.

Pus is a creamy fluid, of specific gravity about 1030, and usually alkaline in reaction. If it be allowed to stand, it separates into two layers. The upper-the liquor puris-is a clear fluid, yellowish in tint, and resembling blood-serum. If a drop from the opaque lower layer be examined under the microscope, one will find suspended therein a great number of colorless, granular, round cells (see Fig. 98). Their peculiar horseshoe-shaped nuclei identify them as polymorphonuclear leukocytes. If the specimen is from an acute abscess, in a large number of the cells amoboid movements may be noted, especially if examined on a warm stage.

$\dot{A}$ circumscribed collection of pus in any tissue is known as an abscess. In addition to the excessive migration of leukocytes which characterizes purulent inflammation, there are also a necrosis and liquefaction of the tissue at a given point, setting free, as it were, the migrated cells within this 
area. In other words, an abscess is simply a liquefied inflammatory focus containing a large number of migrated

Fig. 98.

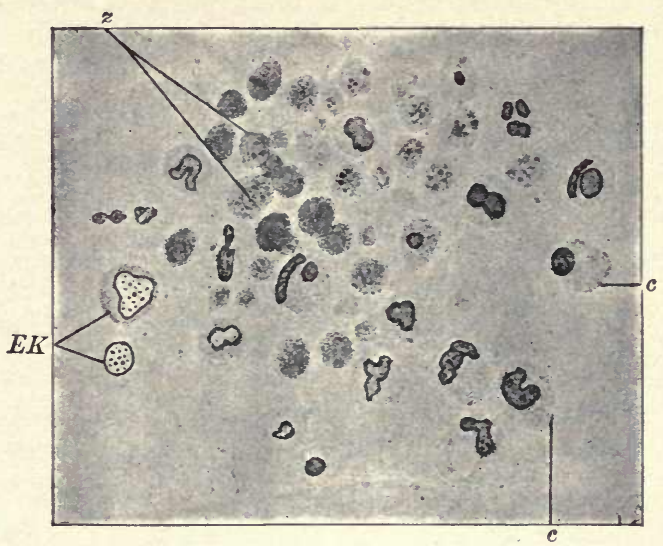

Pus from virulent abscess-formation (Grawitz). The leukocrtes show marked necrotic changes, chromolysis. $c$, $c$, well-preserved leukocytes; $E K$, connectivetissue cells from the neighboring granulations; $z$, similar cells necrosed.

leukocytes. In the greater number of cases the liquefaction of the tissues is a sort of peptonization brought about by fer-

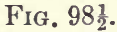

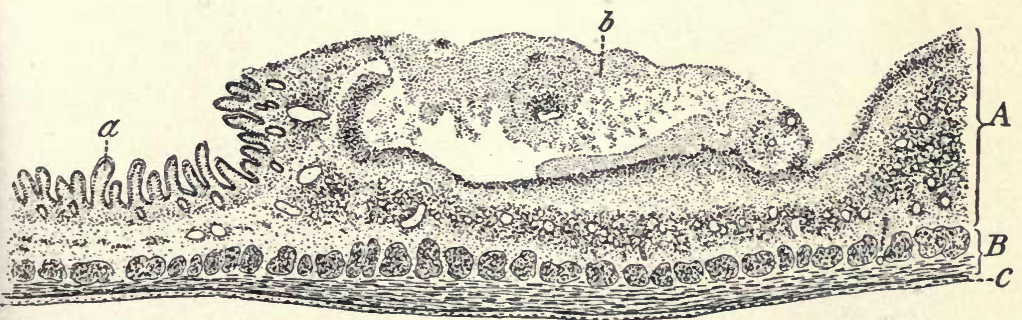

Typhoid ulcer of intestine, second week. $A$, mucosa and submucosa ; $B$, muscularis; $C$, serous coat; $a$, villi ; $b$, slough, on both sides the broad infiltrated border. $\times 12$ (Schmaus).

ments the product of bacterial growth. The organisms most frequently concerned in purulent inflammations are the pyogenic staphylococci and streptococci, though the bacillus 17-Hist. 
coli, gonococcus, and others occasionally prove pyogenic. Around the central fluid mass, the pus, there is first a layer which is soon to become pus, next a zone of inflammation and

Fig. 99.

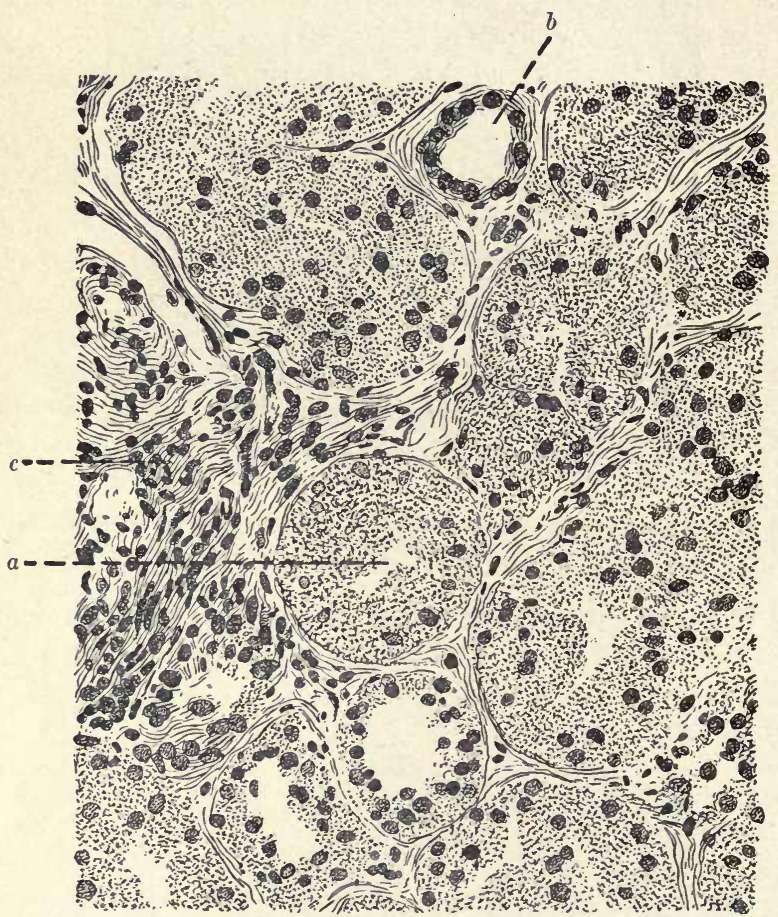

Parenchymatous nephritis. a, cross-section of a convoluted tubule of the kidney, the lining epithelium of which is the seat of albuminoid degeneration. The cells are swollen and their bodies filled with abnormally coarse granules. The cells to the left are so far disintegrated that the nuclei have lost most of their chromatin. Such cells cannot recover. The cells to the right are less profoundly altered and their nuclei retain sufficient chromatin to stain slightly. These cells might, perhaps, recover. Other convoluted tubules similarly affected are represented in oblique section. $b$, tubule with low, unaffected epithclium, the nuclei of which stain deeply ; $c$, round-cell infiltration of the interstitial tissue in the neighborhood of a Malpighian body, the edge of which is just above the line $c$. Section stained with hæmatoxylin and eosin.

repair, and then healthy tissue. If such an inflammatory process occurs on the skin or a mucous membrane, the result- 
ing superficial destruction of tissue and loss of substance

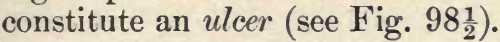

Parenchymatous and interstitial inflammation: In inflammation of glandular structures, in some instances, the degenerative or proliferative changes in the epithelial cells may be the more marked, while in others the changes in the interstitial tissue are the more pronounced, suggesting the term parenchymatous for the former and interstitial inflammation for the latter condition, though they cannot be strictly separated from each other (see Fig. 99). The same terms are sometimes employed in a somewhat similar manner in inflammations of muscle and nerve-tissue.

Productive inflammation (see Fig. 100) is a term occasionally employed when the proliferative changes predominate over the exudative and degenerative, due either to the nature of the cause or the extent of the injury. In the viscera, when the action of an irritant is long continued, such proliferative activity of the connective-tissue cells may lead to a marked increase in the sustentacular tissue and a secondary atrophy of the parenchyma, the term chronic interstititial inflammation being applied to the condition. Productive inflammation is well illustrated in the process of repair.

\section{REPAIR.}

Healing by first intention: Immediate reunion of an aseptic wound takes place with scarcely any vascular phenomena. The prompt juxtaposition of the lips of the wound leaves no opportunity for an exudation of any great extent. There is more or less hemorrhage, a small amount of the blood coagulating between the edges of the wound. The filaments of fibrin which form constitute a sort of scaffolding between the wounded surfaces.

By the end of twenty-four hours there is evidence of a superactivity on the part of the injured connective-tissue cells. They become hypertrophied, their divided prolongations grow and new ones are protruded, extending along the fibrin scaffolding, uniting with similar prolongations from other cells, and thus forming a second scaffolding firmer than the first. The cells then complete the formation of the cicatrix by the 
development of white fibrous and yellow elastic fasciculi. Though the migration of leukocytes may have been a little exaggerated, they have taken no active part in the reparative process.

We might say that the phenomena of inflammation have been reduced to their simplest terms-complete or partial destruction of some cells, and a marked functional super-

FIG. 100.

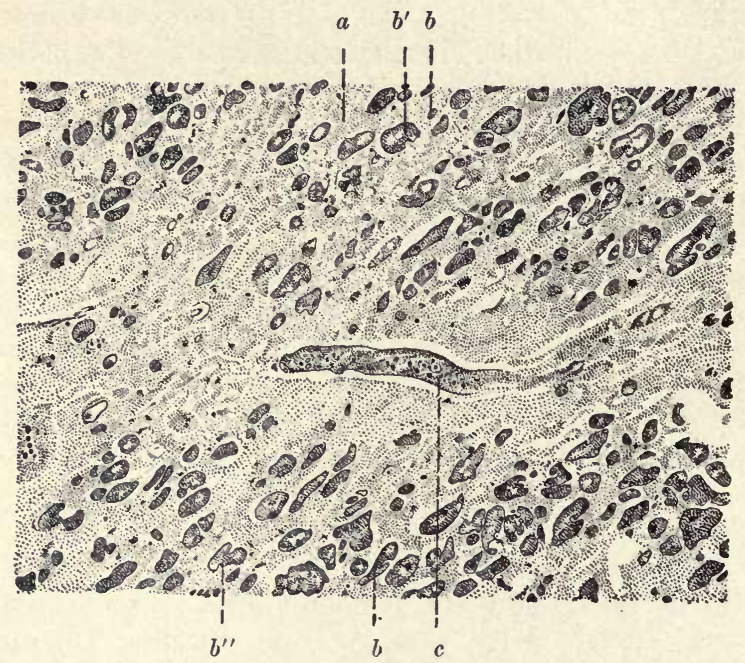

Chronic interstitial myocarditis, late stage. a, dense fibrous tissue, the final result of the interstitial inflammation; $b, b^{*}, b^{\prime \prime}$, atrophied cardiac muscle-cells; $b^{\prime}$, vacuolation of a less atrophic cell; $b^{\prime \prime}$. section showing anastomotic branch joining two cells; $c$, partially obliterated bloodvessels.

activity of other connective-tissue elements having for its object the complete restoration of the injured part.

When the irritant is greater the reparative process progresses with greater energy.

Healing by second intention: When the surfaces of a wound are not in apposition or are septic-i.e., infected by bacteriathe reactionary and reparative phenomena progress with greater energy because of the greater call upon the reparative efforts of the organism, or the greater severity of the irritant. 
effects of highly noxious chemical products of bacterial growth on the new proliferating cells.

If we examine an open wound-i.e., one which is healing by second intention-it will be noted that its surface is covered by papillary elevations about the size of pin-points. This is the type of tissue concerned in the repair of all such lesions, and on account of its appearance is called:

Fra. 102.

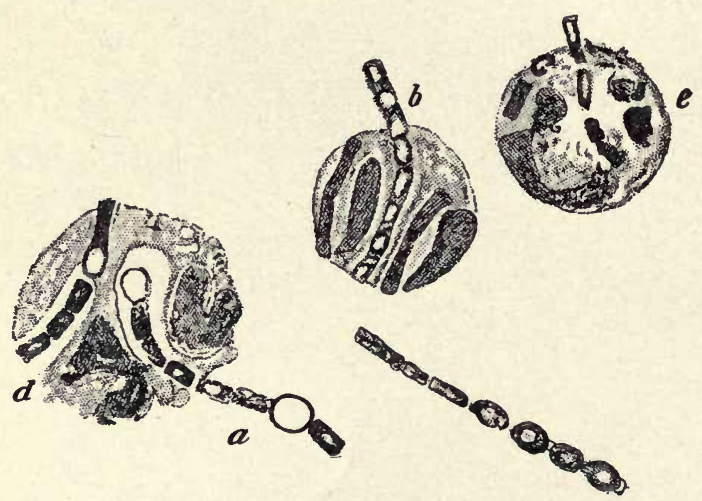

Phagocytes from granulations infected with virulent anthrax bacilli (Afanassieff). $a$, thread of bacilli, partly within and partly outside of a phagocyte; both portions show a vacuolation of the bacilli, indicative of their degeneration. $d$, thread almost entirely incorporated. Within the cell the incorporated bacilli lie in vacuoles in the cytoplasm, probably digestive vacuoles. In $b$ and $e$ similar appearances are presented. c, degenerating thread of bacilli from the fluid of the granulations. Vacuolation has also taken place in this thread, showing that the fluids of the granulations have a destructive influence upon the bacilli.

Granulation-tissue : On microscopical examination one finds these little elevations to be composed of loops of newly formed bloodvessels (see Fig. 101) surmounted by a mass of rapidly proliferating connective-tissue cells and migrated leukocytes. In addition there may be present certain larger cellular elements. One variety, the giant-cell, is often many times the size of a leukocyte, its chief characteristic being a variable number of nuclei--sometimes as many as one liundred, usually, however, from five to twenty-irregularly arranged, sometimes around the periphery of the cell, evenly distributed 
through the cell-protoplasm or clustered in one spot. These cells are markedly phagocytic, englobing and removing bacteria, fragments of the red blood-corpuscles and protoplasmic débris generally (see Figs. 102 and 103). Another variety is large and fusiform with an elongated nucleus.

In the midst of these innumerable cellular elements run newly formed capillary bloodvessels, the walls of which are formed of a single layer of cells scarcely differentiated from those surrounding. The exact method of the formation of these new bloodvessels is as yet obscure.

As the reparative process progresses the young spherical connective-tissue cells become more angular and later fusiform. As they often resemble the epithelial cells of mucous mem-

FIG. 103.

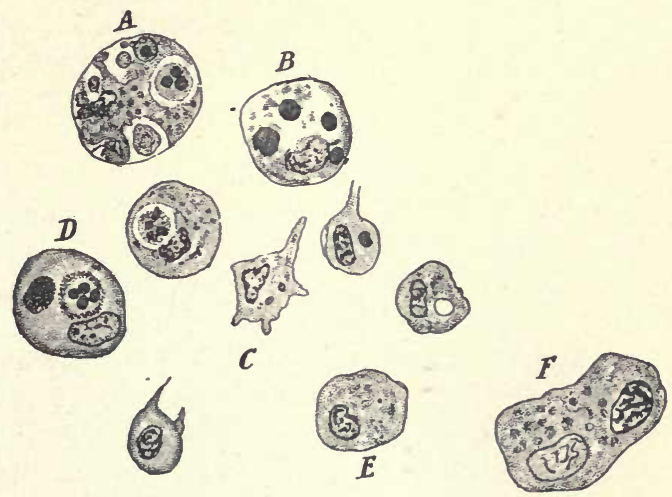

Phagocytes from aseptic granulations (Nikiforoff). $C$, phagocytes with pseudopodia ; $E$, without pseudopodia; $F$, proliferating, the daughter-nuclei in the spirem phase of karyokinesis ; $A, B, D$, with leukocytes, fragments of tissue, and red corpuscles in their cytoplasm.

branes they are sometimes called epithelioid. To these cells, which are especially consecrated to the restoration of connective tissue, Ziegler applies the term fibroblasts; or chondroblasts or osteoblasts if concerned in the regeneration of cartilage or bone. Still further changes in shape take place, until finally they become fully developed flat and slender connective-tissue cells.

Having thus rapidly attained their full development, 
evidences of advancing senile degeneration manifest themselves with equal rapidity, the numerous new bloodvessels undergoing atrophy in a similar manner, and thus is formed the cicatrix.

The formation of new fibrous tissue and regeneration of other connective tissues occur in much the same manner, their differentiation occurring secondarily.

\section{THE INFECTIVE GRANULOMATA.}

Under this head are usually described a number of infectious diseases all characterized by the formation of tumor-like nodular masses, microscopically somewhat resembling granulation-tissue in the number and character of their cells, which have, however, but little tendency to develop into a permanent tissue, and are very liable to undergo degenerative changes. They are forms of subacute inflammation.

\section{Tuberculosis.}

The term tubercle was at one time applied to any nodular growth. By Laennec it was employed mainly in connection with the large caseous yellow nodules observed in phthisical lungs. Baillie (1794) was the first to call attention to the small, gray, millet-seed-sized tubercles found in the lungs, and which are now regarded as the anatomical basis of the larger yellow nodules. But even the smallest "miliary tubercle" is found microscopically to be made up of still smaller cellular tubercles.

Though the histology of these cellular tubercles is quite distinctive, since the discovery of the specific microbic cause by Koch in 1882, the presence of this bacillus must be regarded as the essential characteristic of the disease; this then is the criterion, whatever the macroscopic or microscopic character of the lesion (see Figs. 104, 105).

Histological tubercle: The first effect of the presence of the tubercle bacillus in a tissue is the multiplication of its fixed connective-tissue cells, resulting in the formation of cells termed epithelioid on account of the resemblance to epithelial cells in their abundance of protoplasm. There is also 
excited a migration of leukocytes, and surrounding the epi-

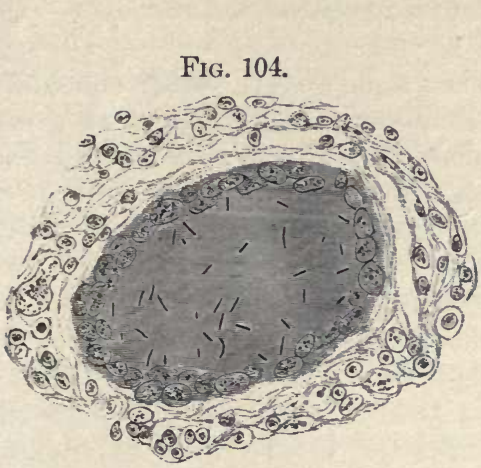

Tubercle bacilli in giant-cell (from tuberculosis of horse). $\times 600$ (Cheyne).
Fig. 105.

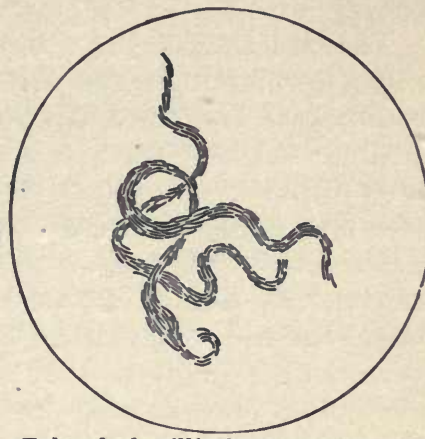

Tubercle bacilli (from a colony on blood-serum), showing the wavy parallel lines. $\times 500$ (after Koch).

Fia. 106.

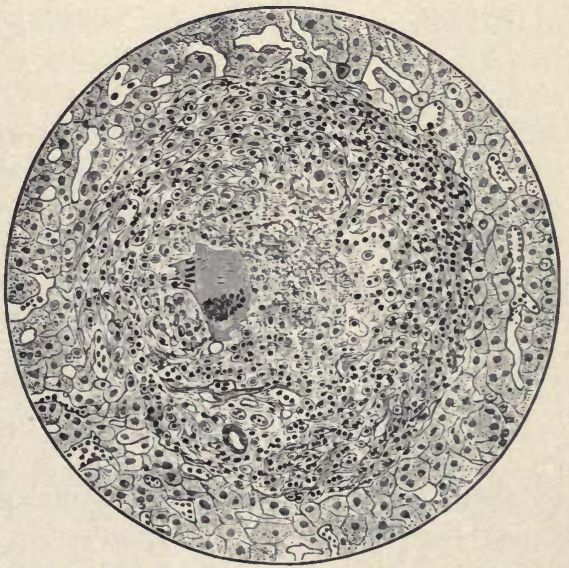

A tubercle from a case of tuberculosis of the liver. A multinucleated giant-cell occupies the centre. Around is an area of commencing caseation and outside this a zone consisting principally of fibroblasts, and, to a less extent, of leukocytes. The leukocytes are most numerous on the side where caseation is most advanced. $\times 250$.

thelioid cells we find a layer of smaller lymphoid cells, both often being supported by a fine connective-tissue reticu- 
lum. Such an arrangement of epithelioid and lymphoid cells, with often a large multinucleated giant-cell in their centre, constitutes the typical histological tubercle (see Fig. 106 ).

The structure of these tubercles, however, varies considerably; in some instances the leukocytic migration is so great as almost to hide the epithelioid cells. If properly stained, the tubercle bacillus may be found between the epithelioid cells first formed; later they are more numerous, many being within the cells, especially - within the giant-cells.

The giant-cell was at one time supposed to be typical of tuberculosis, but it is now known to be frequently found in other chronic inflammatory processes, and in some tumorformations, though neither so abundant nor so conspicuous. They often have as many as fifteen to twenty nuclei arranged. around the periphery of the cell or at either of its poles. By some observers the giant-cells are supposed to result from the rapid multiplication of the nuclei of the epithelioid cells without division of the cell-protoplasm ; according to Metschnikoff and others, they result from the running together of the phagocytic leukocytes and epithelioid cells, combining, as it were, for a common weal.

Caseation is a most characteristic change affecting the tubercle. It consists in the transformation of its centre, often, in fact, of the whole tubercle-depending on the abundance and virulence of the bacteria-into a structureless, opaque, and granular material, macroscopically resembling cheese. In part this is due to toxins resulting from the growth of the tubercle bacilli ; and in part is due to the entire absence of blood-supply, which can be demonstrated in properly injected specimens, the capillaries being traceable to the margin of the tubercle only.

Fibroid transformation: When the inflanmatory process is very chronic, and the bacilli few in number, in place of a necrotic and destructive process-caseation-the tissues seem to gain the upper hand, and by the active proliferation and development of the connective-tissue cells a new fibrous tissue is formed and thus the disease is limited; the tubercle is said to have undergone a fibroid change, and is replaced by scartissue. 


\section{Syphilis.}

The lesion which places syphilis among the infective granulomata is the gumma, which occurs in the late or tertiary stage of the disease.

Though the cause of the disease is not definitely determined, no one doubts the living nature of the contagium.

The gumma occurs in almost all the tissues of the body, most frequently, however, in the skin and subcutaneous cellular tissue; in bone (tibia, sternum, and skull); in the brain and its membranes (less frequently in the spinal cord); and in the liver, kidneys, and lungs. It presents as a firm yellowish white nodular mass varying in size from that of a hemp-seed to that of an apple. On section it has a gelatinous or gummy appearance. The centre is often yellow and cheesy from the necrosis it is prone to undergo. If the nodule is superficially situated, this necrotic softening frequently results in the formation of deep excavated ulcers.

Microscopically the gumma is composed of migrated leukocytes and proliferating connective-tissue cells of various forms. Epithelioid and giant-cells are less frequently seen than in tuberculosis. If caseation has begun, in the centre is found an opaque, homogeneous, and

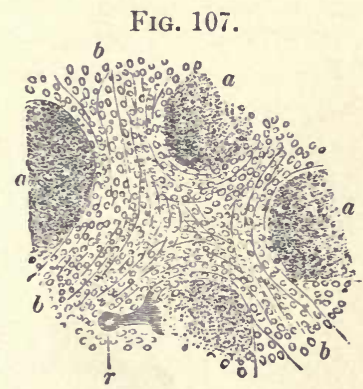

Gummy growth from liver. $a$, central portions of growth, consisting of granular débris; $b$, peripheral granulation-tissue; $r$, a bloodvessel. $\times 100$ (Cornil and Ranvier).

granular material. At the periphery there is a vascular newly-forming connective tissue (Fig. 107).

Leprosy, glanders, actinomycosis, and rhinoscleroma are also characterized by the formation of nodular masses of granulation-like tissue.

\section{FEVER.}

Accompanying inflammation there is often very considerable constitutional disturbance. The body-temperature may be elevated; the pulse and respirations accelerated; glandular secretions.altered; metabolism disturbed; definite anatomical 
changes in various tissues likewise accompanying these phenomena-all of which are included in the clinical term fever.

Rise of body-temperature is the most essential phenomenon to the existence of fever.

The normal constant temperature of the body depends on an equilibrium between the processes of heat-production and heat-dissipation, which is maintained through the influence of the nervous system; the exact nature of this mechanism, however, is not yet fully understood. In fever this equilibrium is disturbed, so that the processes of heat-dissipation and heat-production do not bear a normal relation to each other.

Etiology : Clinically fever has been observed to follow considerable extravasations of blood; experimentally it occurs after transfusion of blood from one animal to another and also after intraperitoneal injections of blood.

The demonstration of the fibrin-ferment as the pyogenic substance capable of exciting such a rise of temperature suggested the investigation of other ferments, and it was found that the intravenous injection of pepsin, trypsin, and papoid produced a well-defined fever. 'This type of fever' corresponds to that observed by surgeons after extensive aseptic wounds, as a subcutaneous fracture, the pyogenic material resulting from the disintegration of the injured tissues.

Most morbid processes with which fever is associated are due to micro-organisms, and it is consequently quite natural to turn to these elementary forms of vegetable life in endeavoring to elucidate this subject. Early investigators, less than fifty years ago, demonstrated that injections of putrefactive materials into the blood of animals were capable of producing marked febrile reactions, and a knowledge of the part played by micro-organisms in the production of putrefaction made quite evident the importance of their rôle in fever.

In 1863 Davaine described a rod-shaped organism in the blood of animals sick with splenic fever, and found that in a healthy animal, if inoculated with the blood of an animal so affected, the disease was reproduced. Subsequently Pasteur showed that if these organisms were removed by filtration through earthen cylinders, though the disease could not then 
be reproduced by inoculation, there resulted a febrile reaction, which could only be attributed to chemical poisons-called ptomains or toxins - the result of the growth of the bacteria.

These chemical substances may be the product of organisms which are not capable of existing in the living body, but only in dead vegetable or animal tissues-i.e., saprophytic bacteria, to which class belong the poisonous alkaloids which have been isolated from putrefying fish, meat, sausage, cheese, etc. But of far greater clinical importance in the etiology of fever are the products of pathogenic bacteria.

Undoubtedly febrile rise of temperature may be produced by other agencies, such as fear and affections of the nervous system; but here grave functional and anatomical disturbances are entirely absent.

The significance of fever is not known, though modern experimental evidence supports the theory that it is a conservative effort on the part of nature to conbat the noxious substances which give rise to it. In animals rendered hyperthermic by external heat or cerebral puncture and inoculated with various organisms-pneumococcus and others-the increase in body-temperature seemed to exert a most favorable influence on the course of the infection.

The anatomical changes in fever, due to the increased temperature alone, are few. It is difficult to separate the results produced by the high temperature from those produced by its exciting cause. Cloudy swelling and fatty degeneration of the heart, liver, and kidneys, are probably in part due to the fever itself, though in part undoubtedly to toxic substances circulating in the blood.

\section{THE CIRCULATORY APPARATUS.}

Hyperæmia is an increase in the amount of blood in a part: and is either actual, due to an increase of the flow to the part; or passive, due to an obstruction of the outflow.

Active hyperæmia: Blushing is a physiological example. Pathologically it is hardly met except associated with some of the phenomena characteristic of inflammation. A variety due to vaso-motor paralysis, as seen in experimental section of the cervical sympathetic in animals, is occasionally met with clinically, as after gunshot-wound of the same nerve. 
Passive hyperæmia is produced locally by thickening of venous walls, external pressure on the veins of a part by a tumor, gravid uterus, etc. Cardiac weakness produces a general venous congestion finally, if severe enough, of all the tissues of the body.

The consequences of venous congestion are more important than those of active congestion-the blood-serum transudes in greater quantities than can be removed by the lymphatics, and the tissues become odematous and swollen. In the internal organs there may result pressure-atrophy of the true parenchyma-cells and an increase of the connective-tissue elements.

\section{FIG. 108.}

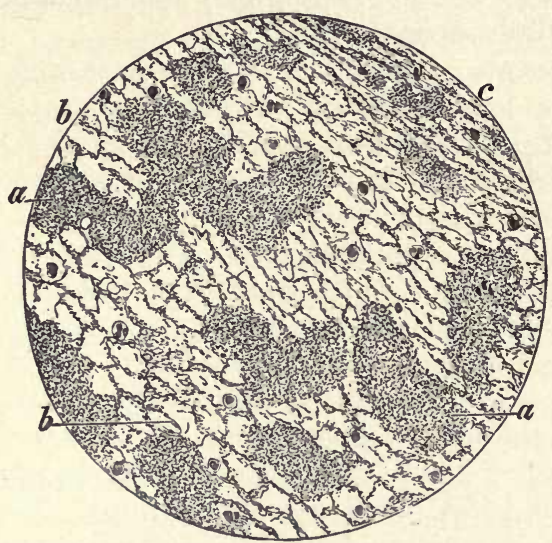

Section of white thrombus containing few leukocytes. $a$, granular masses; $b$, granular threads of fibrin arranged in a network ; $c$, parallel arranged threads of fibrin. $\times 200$ (Zeigler).

Local anæmia or ischæmia: This occurs where there is interference with the blood-supply of a tissue, and results in atrophy or some degenerative change, or if long continued in necrosis. It is produced by thrombosis or embolism, atheroma of the vessel-walls, arterial spasm, or external pressure.

Thrombosis is the coagulation of blood within the heart or bloodvessels during.life.

The appearance and structure of a thrombus depend on the rapidity with which it is formed; if slowly, it is composed of 
layers of fibrin containing blood-plates and leukocytes in its meshes and is of a grayish-white color ; if rapidly, as the result of the almost complete stoppage of the circulation, it is composed of fibrin and all the elements of the blood, is soft and red, resembling a post-mortem clot (Fig. 108).

The cause of thrombosis is found either in alterations in structure of the vessel-walls, in the composition of the blood or the rapidity of its flow. If the circulation is arrested at any point, coagulation ensues as the result of the altered nutrition of the vessel-wall, producing structural changes incompatible with preservation of the normal fluidity of the blood. Inflammatory and degenerative changes in the vascular walls, as well as alterations in the composition of the blood, for instance after a prolonged illness like typhoid fever, favor the same result.

The fate of a thrombus may be organization, calcification forming phleboliths, or fatty degeneration and liquefaction.

Embolism is the process of plugging or stopping up of a bloodvessel by foreign bodies of various description, carried in the blood-stream and too large to pass through the vessel at that point.

The plug or embolus may be a portion of a thrombus; of a diseased heart-valve or of a tumor; masses of bacteria and other parasites, and also pigment, as in malaria ; particles of fat; and in wounds of large veins, air, which may have gained entrance (Fig. 109). Its point of lodgement depends on its source; if from the systemic reins, it will be arrested by branches of the pulmonary artery; if from the pulmonary veins or left side of heart, it will occlude some systemic artery, most frequently in the spleen, kidneys, or brain; if from tributaries of the portal vein, it will be arrested by branches of that vein within the liver. If the obstructed artery is terminal-i.e., has no free anastomosesan infarct is the result.

Infarcts may be anæmic or hemorrhagic. The circulation beyond the embolus is arrested-a wedge-shaped anæmic area is produced in which later occur coagulative necrosis and caseation, the degenerated tissue being finally absorbed.

In some cases, however, the affected wedge-shaped area is 
engorged with extravasated blood supposed possibly to be due to a back flow from the veins, in which case the final remaining scar is pigmented (Fig. 110).

Edema: Normally a certain amount of fluid transudes from the capillary bloodvessels into the interstices of connective tissues and the various serous cavities of the body; when the amount of this fluid is increased or its removal by

Fig. 109.

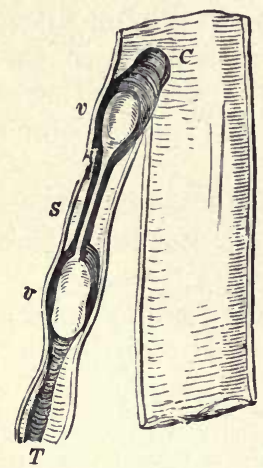

A thrombus in the saphenous vein, showing the projection of the conical end of the thrombus into the femoral vessel. $S$, saphenous vein; $T$, thrombus; $C$, conical end projecting into femoral vein. At $v, v$, opposite the valves, the
thrombus is softened (Virchow).
Fig. 110.

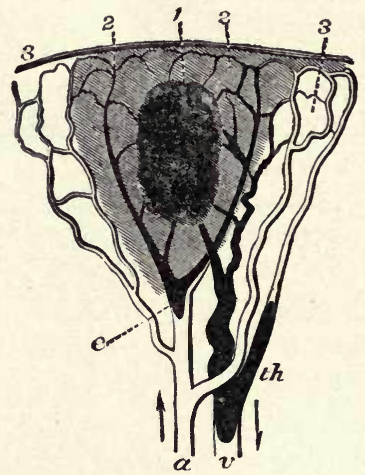

Diagram of a hemorrhagic infarct. $a$, artery obliterated by an embolus $(e) ; v$, vein filled with a secondary thrombus $(t h) ; 1$, centre of infarct which is becoming disintegrated ; 2 , area of extravasation; 3 . area of collateral hyperæmia ( $O$. Weber).

the veins and lymphatics is lessened the condition is known as oedema or dropsy. The increased transudation may be caused by arterial or venous hyperæmia, hydræmic states of the blood, and possibly by alterations in the walls of the bloodvessels.

Obstruction of the lymphatic circulation is never a powerful factor in the production of dropsy; but venous obstruction is a most important mechanical cause.

Clinically the chief varieties of oedema are cardiac, renal, cachectic, and angionenrotic. Cardiac dropsy is an example of increased transudation from passive hyperæmia, due to the 
inability of the heart to maintain the normal force of the circulation. Renal dropsy results from alterations in the blood as a consequence of its loss of albumin and possibly also from degenerative changes in the vascular walls. Cachectic odema is associated with almost any condition of impaired health accompanied by anæmia. Localized redemas are sometimes associated with lesions of the nervous system, as those attending neuritis, neuralgia, and occasionally hemiplegia.

18-Hist. 


\section{CH A PTER II.}

\section{PATHOLOGY OF NUTRITION.}

ALL pathological processes are primarily nutritive; but the term is usually restricted to necrosis, the degenerations and infiltrations, hypertrophy, atrophy, and tumors.

\section{Local Death.}

Necrosis is a local death of simple cells or groups of cells, in contradistinction to general or somatic death. It is due to mechanical, chemical, and thermic injuries; interference with the blood-supply; and to lesions of the central nervous system-i. e., trophic disturbances.

The mechanical causes of necrosis are numerous, such as the crushing of a finger by violence. External pressure, as from bandages or splints on bony prominences, may cause necrosis, as may also tension, or pressure from within, produced by inflammatory exudates. It is often difficult to distinguish between the direct injurious effect of mechanical, chemical, or thermic trauma on the tissue-cells and their indirect effect through circulatory disturbances, as the blood also undergoes changes resulting in coagulation and stasis in the capillaries. A temperature of $50^{\circ}$ to $68^{\circ} \mathrm{C}$., if continued for any length of time, causes necrosis and higher temperatures more quickly.

Obstruction of the circulation by a thrombus, embolus, or atheroma of the vessel-walls produces necrosis. A number of these causes may act together. If the vitality of a tissue has been reduced by long-continued passive hyperæmia, for cxample as the result of uncompensated valvular disease of the heart, it succumbs much more rapidly. A slight injury may suffice in the aged or in conditions like typhoid fever, where the vital forces are greatly depressed. In senile gangrene, to the lowered vitality of the tissue are added a weak heart and 
atheromatous vessels. In the so-called neuropathic necroses occurring in diseases of the nervous system an important part is undoubtedly played by trauma and circulatory disturbances.

Under the microscope it is noted that the cell-nucleus loses its affinity for nuclear dyes_-" chromolysis"-or the chromatoplastic substances are broken up into fragments- " karyolysis"-and the nucleus finally disintegrates and disappears, the cell-protoplasm becoming homogeneous in appearance. There

FIG. 111.

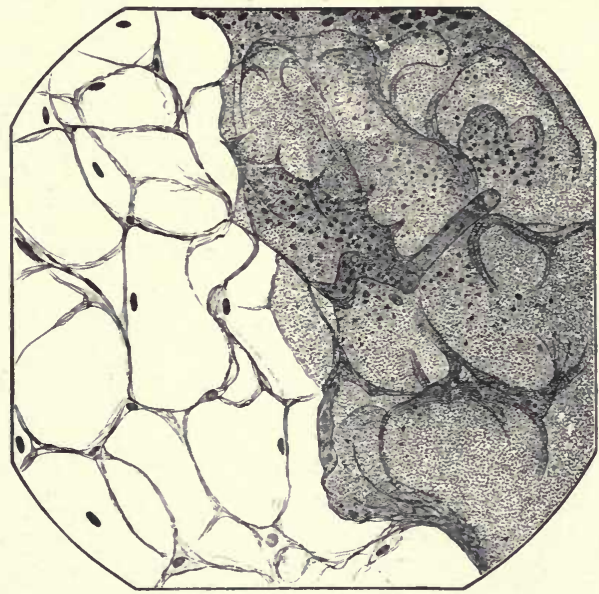

Fat-necrosis. The abrupt transition from the healthy cells on the left to the necrosed cells on the right is well marked. The contents of the affected cells are finely granular. (From a specimen by Dr. Rolleston.)

is always more or less inflammatory reaction in the surrounding tissues. Complete regeneration or cicatrization, calcification, or cystic formation may follow.

Several varieties of necrosis are usually described : Coagulation-necrosis is a variety in which there is coagulation of the fluids normally present-lymph-or of inflammatory exudates, and the cellular protoplasm of the tissue. Fibrin is deposited in the form of granules or fine fibrillæ; the cells lose their nuclei and their protoplasm becomes granular. The necessary ferment to produce this coagulation is derived either from the degenerating and disintegrating cells or from bacterial prod- 
ucts. Macroscopically the affected area is pale, opaque, waxy, and, later, gray in appearance. On mucous surfaces a false membrane is formed, as seen in diphtheria.

In liquefaction-necrosis there is a kind of peptonization of the tissue-elements, which break down and become fluid, the active agent being some bacterial or non-bacterial ferment.

The term caseation is used to describe a degenerative change where the tissue macroscopically has the appearance of hard or soft cheese, and is most frequently seen in connection with tubercular and syphilitic inflammations. Microscopically one finds simply a fine granular débris.

Fat-necrosis is a variety which affects fatty connective tissues. It is most frequently noted in the abdominal cavity in the subperitoneal fat, and generally is associated with some pathological change in the pancreas. It is characterized by the formation of opaque, white areas about the size of a pea. Microscopically the cell-contents are crystalline, opaque, or granular (Fig. 111).

Gangrene is the death of tissue en masse. There are two varieties, dry or mummification and moist or sphacelus. The former occurs when the part is so situated that rapid evaporation of the fluids present takes place, especially when the necrosis has been caused by interference with the blood-supply. The latter occurs where evaporation is hindered and where the blood reaching the part cannot escape owing to venous obstruction. The presence of moisture favors the multiplication of micro-organisms, resulting in putrefactive changes, the tissue becoming dark brown or a mottled greenish-black in color (due to disorganized blood-pigment), and distended with gases produced by the organisms.

\section{The Infiltrations and Degenerations.}

Though theoretically there is a difference between a degeneration and an infiltration - the former indicating a conversion of the cell-protoplasm into a less highly organized substance, rendering it less suited for the performance of its functions, while in the latter there is a substitution or deposit of some new substance in the cell-practically it is often difficult to distinguish between the two. 
Fatty infiltration may be a physiological or a pathological process. It occurs physiologically under the skin, between muscles and surrounding organs; pathologically in abnormal places, as between individual muscle-fibres, and in normal situations in great excess, as in general obesity. It is due to either the over-production of fat or its deficient consumption. Fatty infiltration of the liver may be produced experimentally in the lower animals by an excess of fatty food and restriction of oxidation by preventing muscular movement. In phthisis, cancer, and severe anæmias the explanation seems to be found in lessened oxidation. In chronic alcoholism the change often occurs, the alcohol being oxidized in place of the fat.

The fat-drops are larger than in fatty degeneration, and are found either between the tissue-elements or within the cells,

FIs. 112.

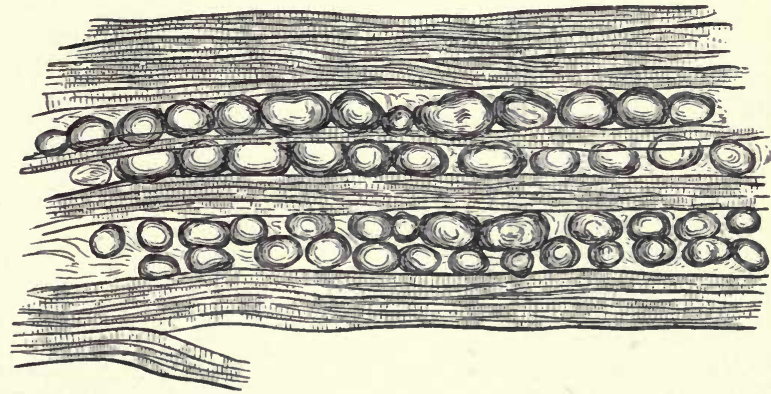

Fatty infiltration of muscle from a case of stiffening of the ankle by an epithelioma of the skin over it. The muscular fibres are narrowed, and adipose tissue appears between them. $\times 80$ (Coats).

the nucleus and protoplasm being pushed one side until the cell is like a vesicle filled with fat (Fig. 112). The liver is frequently affected in advanced phthisis. On section oil accumulates on the knife or may be scraped from the cut surface. The affected organ is enlarged, heavy but of less specific gravity, soft, less elastic and doughy, and of a light yellow color, or mottled, if the change is not uniform throughout. In the liver the change is particularly noted in the periphery of the acini, giving them a pale yellow margin. 
In fatty infiltration of the heart the muscle-fibres are not necessarily affected, and consequently there is little or no functional impairment, though by interfering with the local bloodsupply the condition may become one of fatty degeneration.

Fatty degeneration is caused by local interference with the blood-supply, anæmias, and high temperature. Poisons such as mercury, arsenic, lead, phosphorus, ether, chloroform, and, of still greater interest clinically, the toxins of micro-organisms are frequent causes. The cell is shrunken, not swollen, its outline irregular, and the nucleus destroved. The oil-drops are smaller and do not tend to run together as in fatty infiltration, appearing in the cell first as small shining granules near the nucleus. There is a gradual liquefaction of the cells, ending in their death.

Any tissue in the body, with the exception, in the blood, of the red globules, may be affected; but the parenchymacells of the liver, kidney, heart, and central nervous system most often-the interstitial tissue being possibly later affected.

The fat-drops are distinguished chemically by their insolubility in acetic acid and weak solutions of sodium and potassium hydroxide; and by their solubility in alcohol, ether, and chloroform, and by staining black with osmic acid. Fine needles in rosettes, sheaves of margaric acid, and rhombic plates of cholesterin are often found.

The organ affected is pale yellow or mottled, very anæmic, and feels greasy and soft.

Amyloid, lardaceous, or waxy degeneration is a very frequent retrograde change and next to fatty degeneration is the most common. It signifies the presence in the tissue of a colorless, firm, translucent, homogeneous, inelastic material of about the consistency of wax or solidified lard, to which, on account of its behavior toward indine, the term "starch-like" has been given; though we now know that it is not a carbohydrate, but a nitrogenous compound (Fig. 113). If a solution of iodine and iodide of potassum (Lugol's) be applied to the freshly cut surface of an affected area, there results a mahoganybrown coloring, the surrounding normal tissue being stained a canary-yellow. The addition of a 1 per cent. solution of sulphuric acid gives the amyloid material a bluish color. For microscopical sections a much more satisfactory staining reaction is 
found on the addition of methyl- or gentian-violet, producing a beautiful rose-pink, while the normal tissue stains blue.

The changes are frequently so extensive in all the organs in the body and the resulting symptoms so numerous that it is often referred to as lardaceous disease. 'The organs most

FIG. 113.

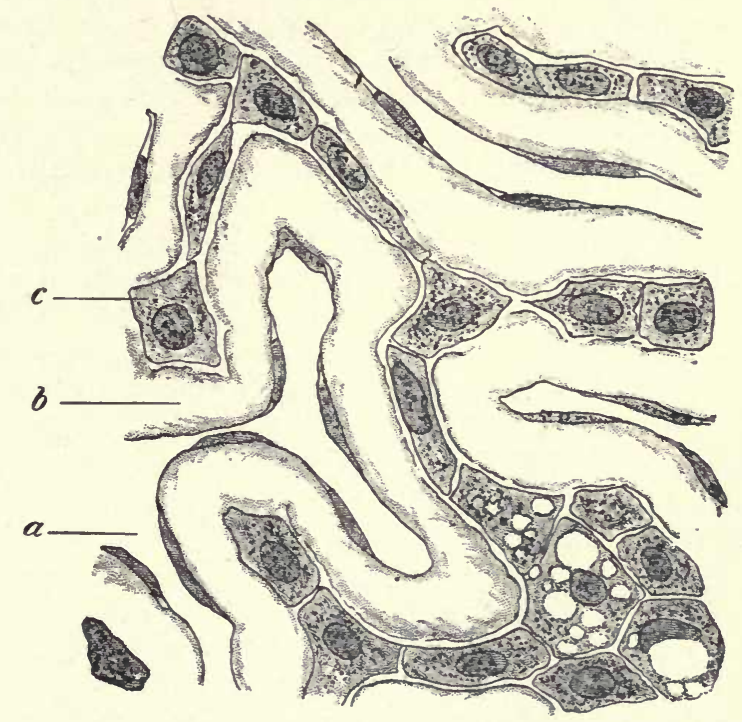

Amyloid infiltration in the liver (Thorna). $a$, lumen of an intralobular capillary, surrounded by the endothelial wall of the vessel ; $b$, amyloid substance immediately beneath the endothelium; $c$. epithelial cells of the hepatic parenchyma, some of which show a fatty infiltration.

frequently affected however are the liver, kidney, spleen, lymphatic glands, intestinal tract and suprarenal capsules. The changes begin in the inner coat of the smaller capillaries, and later affect the cells and interstitial substance of the surrounding tissue, extending along the course of the bloodvessels. If extensively infiltrated, the organ is increased in size; is pale, firm, and glistening in appearance. The change may be quite extensive and yet only noted on microscopical examination. The parenchyma-cells of the organ swell and become less angular. 
The function of the part is impaired by reason of the interference with the blood-supply and displacement of the proper tissue-elements. The spleen is usually the earliest organ involved, beginning in the Malpighian follicles, which become large, pale and translucent; and resemble grains of sago-hence the term sago-spleen.

The condition occurs most frequently in connection with long-continued suppurative processes ; with syphilis and tuberculosis; and not so frequently with other cachexias-cancer, leukæmia, dysentery, and malaria, and in a few instances without any apparent cause.

Colloid, mucoid, and hyaline degenerations are all closely allied and but little understood.

a. Colloid: The prototype of this variety is the colloid material of the thyroid gland (Fig. 114), somewhat similar

F1G. 114.

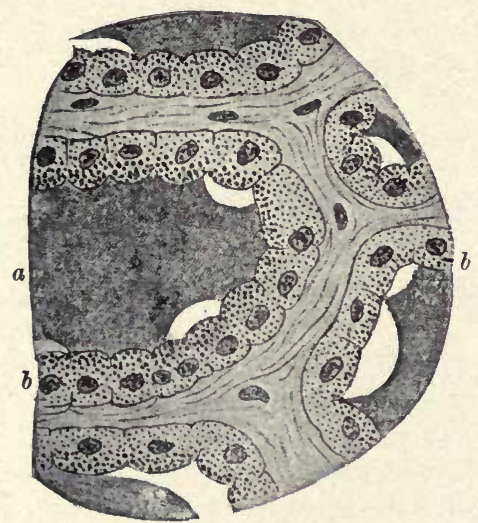

Section of thyroid gland, showing colloid degeneration. $a$, colloid; $b$, secreting cells with granules (after Pozzi).

in appearance to mucus, but firmer. The change affects mainly epithelial cells and is most often seen in cystic goitre and in new growths-e.g., colloid cancer-giving rise to a gelatinous appearance. In ovarian eysts the loculi are sometimes filled with colloid masses.

b. Mucoid: The physiological type of this change is found 
in the mucous secretions of many glands. The change affects both epithelial and connective-tissue structures, but the latter is of the greater importance pathologically. The affected area has a translucent, gelatinous, and swollen appearance.

The most remarkable condition in which it is found is in what is known as myxoedema, the chief clinical characteristic of which is a peculiar swelling of the skin and subcutaneous connective tissue, which differs from ordinary anasarca in not pitting on pressure.

c. Hyaline degeneration is very closely allied to amyloid. It occurs in connective tissue and the walls of bloodvessels. The material has a glistening waxy appearance but is less translucent than amyloid, the staining reactions of which are also absent.

Cloudy swelling, parenchymatous or granular degeneration, are terms used to describe a retrograde change in which the elements affected, especially epithelial and muscle cells, are swollen and cloudy and filled with minute albuminous granules. The outline of the cell becomes indistinctand distorted, the nucleus however remaining often but little altered. These granules are insoluble in ether, but soluble in dilute acids and

Fig. 115.

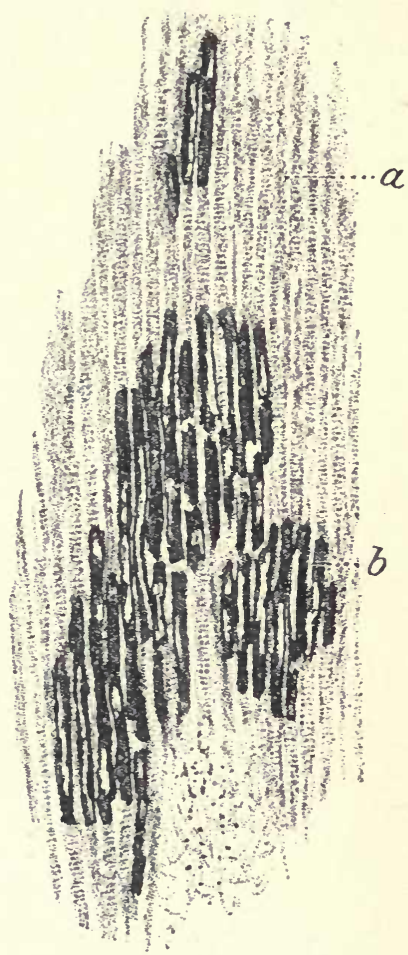

Calcareous infiltration of cardiac muscle (Langerhans). $a$, degenerated cardiac muscle; $b$, muscular fibres impregnated with lime-salts. The specimen was taken from a case of chronic lead-poisoning. The cells which are the seat of the calcareous infiltration must have been dead for a considerable time before the death of the individual.

caustic potash. The whole organ is swollen, less translucent, dull grayish, cloudy, and anæmic-looking as if it had been 
boiled. The change may occur in any organ in the body, but is most frequent in the liver, kidney, heart-muscle, and mucous membrane of the gastro-intestinal tract. It occurs in all inflammations, in acute infectious diseases, fever, after burns; and in poisoning from phosphorus, arsenic, and mineral acids. The cells either soon recover or the process quickly passes on to fatty degeneration.

Calcareous infiltration, or calcification, is the impregnation of tissues with the salts of lime in the form of the insoluble carbonate and phosphate. All parts are alike susceptible, both the cells and intercellular substance, though the latter to

Fig. 116.

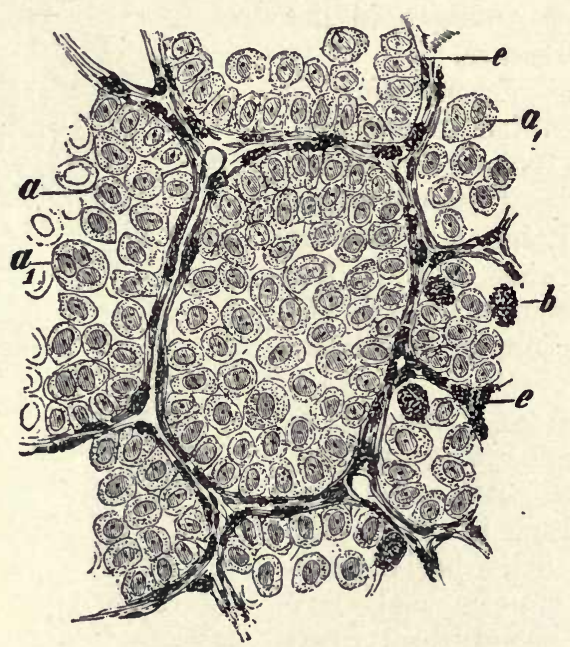

Melanotic alveolar sarcoma of the skin. $a$, mononuclear ; $\boldsymbol{a}^{\prime}$, polynuclear sarcomacells with epithelial character; $b$, pigment-cells; $c$, stroma with bloodvessels and pigment. $\times 300$ (Ziegler).

the greater degree. The reason of this deposit is not clear, but it is always connected with a lowered state of nutrition. The tissue appears dusted with little fine granules, which in the earliest stage might be mistaken for fat; but they dissolve on the addition of hydrochloric acid-and with effervescence if the carbonate is present (Fig. 115). 
These minute refractive granules enlarge and extend, and so form dark, opaque, granular patches-white, grayish, or yellow in color macroscopically. Physiologically the condition occurs in the pineal gland, choroid plexus, in cartilage in old age, and notably in the development of bone. The most frequent pathological sites are the valves of the heart, arterial walls, caseous lymph-glands, and in myoma uteri when the blood-supply is poor. It may be noted in necrotic tubercular foci and infarcts.

Pigmentary infiltration, or abnormal pigmentations, depend on both intrinsic and extrinsic sources of supply, the former being either hæmatogenous or non-hæmatogenous.

1. Intrinsic: $a$. Hæmatogenous. There are two varieties of pigment derived from hæmoglobin when red blood-cells are destroyed : hæmosiderin, which contains iron, and hæmatoidin, which does not.

Hæmosiderin gives the characteristic chemical reactions of iron, and occurs in minute granules in the liver-cells in

\section{Frg. 117.}

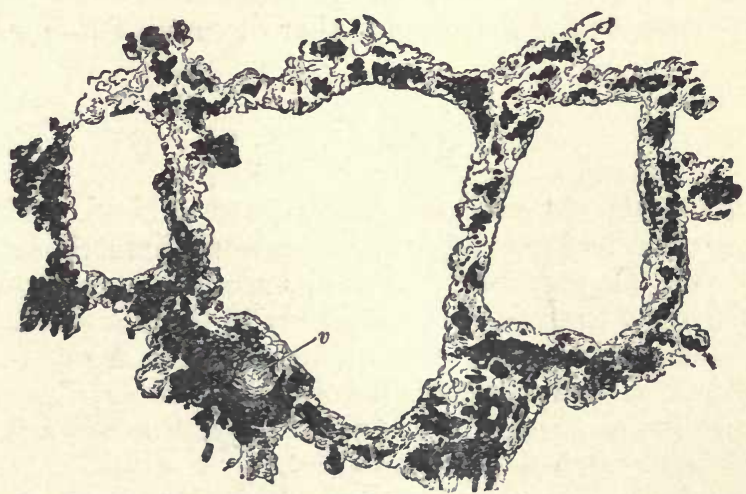

Pigmentation of the lung (from a woman, æt. sixty-five, with slight emphysema), showing the situation of the pigment in the thickened alveolar walls and around the bloodvessel, $v$. The walls of the latter are also thickened and its lumen diminished. $\times 75$ (Green).

pernicious anæmia; and is found also in the spleen, renal epithelium, and medulla of bone in this disease, in leukæmia, malaria, severe cachexias, in poisons, and septic processes. 
Hæmatoidin is found in rhombic crystals or granular in old blood-clots, free or within leukocytes and other cells.

$b$. Of non-hæmatogenous pigments, melanin is the most important. It is elaborated by living cells, and contains no iron. Granules of melanin vary in color from yellow to black. It is noted, among other instances, in melanotic sarcoma (Fig. 116).

2. Extrinsic: Extrinsic sources of pigment are very numerous, the material being absorbed either through the lungs or gastro-intestinal tract. Carbon is one of the most common varieties of extrinsic pigment. When coal-dust is inhaled it gives rise to deposits of pigment in the lungs, the condition being known as coal-miner's lung, or anthracosis (Fig. 117). Siderosis is similarly produced by the inhalation of fine particles of iron, the lungs becoming rusty in color. In chalicosis, stonemasons' lung, the particles are white and the lung consequently abnormally pale.

In argyria silver is absorbed from the gastro-intestinal tract and afterward deposited in the tissues as an albuminate. The condition was more often seen when silver was popular in the treatment of epilepsy and other diseases of the nervous system.

\section{Atrophy.}

The term atrophy means literally want of food. The affected organ undergoes a more or less uniform reduction in size and weight. It may be congenital-agenesia-or acquired. In true atrophy there is a decrease only in the size of the cells of the tissue, their number remaining normal. A decrease in the number of cells is sometimes spoken of as aplasia, or more generally as numerical atrophy. Hypoplasia is a failure of the cells to reach their natural size.

The condition is seen physiologically in the thymus gland in the second year of life, in the generative organs at the climacteric, and in the general atrophy of old age.

Pathologically deficient blood-supply, if long enough continued but not too severe, is an important cause. Under this head would come pressure-atrophy, - $e . g$., the absorption of the bodies of dorsal vertebræ by an aortic aneurism-and 
also atrophies from disease, as, for example, the gut below a lumbar colotomy, which becomes the size of one's little finger. There are many instances of atrophy due to disturbances of normal nerve-influences, as that resulting in the salivary gland after cutting off its nerve-supply.

Atrophy is usually associated with other degenerative changes, especially fatty degeneration and calcification. Accompanying the atrophy of the cells proper of a tissue there is often an increase in its connective tissue, as, for instance, in pseudohypertrophic paralysis. In the liver and kidney this increase in connective tissue-cirrhosis - is often looked upon as an evidence of chronic interstitial inflammation in contradistinction to parenchymatous inflammation.

\section{Hypertrophy.}

The term hypertrophy, which properly means over-feeding or over-nourishment, is applied to an overgrowth of an organ, without marked alteration from the normal structure. It implies more than mere enlargement. A carcinomatous liver is enlarged, but not hypertrophied.

In true hypertrophy there is an increase only in the size of the cells. Hyperplasia, or numerical hypertrophy, is an increase in their number, their size not being increased but even diminished. Physiologically this condition is seen in the enlargement of the uterus following impregnation, and gigantism is a remarkable example of general hypertrophy. Pathologically, in leukæmia, the spleen is found hypertrophied to several times its normal size and weight.

Causes: Increased functional activity, which means also increased nourishment, is a most important factor in the production of many hypertrophies. When inereased work is thrown upon the heart, for example as the result of valvular disease, it becomes hypertrophied. Stricture of the urethra causes hypertrophy of the walls of the bladder. When one kidney is removed there is a compensatory hypertrophy of its fellow. So also when part of a secreting gland, like the liver or pancreas, is removed there is often a hypertrophy of the remaining portion. 


\section{Fibrosis.}

In fibrosis, or fibroid substitution, there is a wasting of the true parenchyma-cells and an increase in the connective tissue. It is not, strictly speaking, a degeneration except in that the new material is considered inferior to the original. When the change takes place in the central nervous system it is spoken of as a sclerosis; in the liver and kidney, as cirrhosis. It is often regarded as an evidence of chronic inflammation, for we know that there often results a great increase in the amount of connective tissue in an area so affected. But the change often occurs where there has been no inflammation. In pseudohypertrophic paralysis, which is not usually regarded as an inflammatory process, the true muscle-cells are almost entirely atrophied, yet by reason of the increase in the connective tissue the calves of the legs are greatly enlarged. In fibroid heart and the various scleroses of the central nervous system there is considerable doubt about the inflammatory nature of the change. In the liver and kidneys, however, it is undoubtedly a frequent sequel of long-continued inflammations.

\section{TUMORS.}

Definition: A tumor is a non-inflammatory, circumscribed swelling which has no function.

Etiology: Though a great many theories have been advanced, we actually know but little as to the causation of tumors. The older authors referred to a peculiar constitutional dyscrasia in this connection. Virchow laid great stress upon some unusual irritant as the exciting cause, as, for instance, cancer of the breast following a blow or other injury to the part; or the development of an epithelioma of the tongue at the site of chronic irritation produced, for instance, by a jagged tooth. Cohnheim advanced the theory that "in an early stage of embryonic development more cells are produced than are required for building up the part concerned, so that there remains a certain number unappropriated, which, owing to their embryonic character, are endowed with a marked capacity for proliferation." Some irritant or injury causes a flux of blood to the part and determines the multiv 
plication of the cells, which until that time have remained dormant.

With regard to the parasitic origin of malignant tumors, the many points of similarity in their clinical history and that of certain infectious diseases, such as tuberculosis, are at least very suggestive. Should their parasitic origin be demonstrated they would no longer be classed with tumors, but with the infective granulomata, which they closely resemble in many respects. The parasite of cancer is described as a protozoon. The true significance of the bodies so described is as yet sub judice. An epithelioma has been successfully transmitted from one animal to another by artificial inoculation.

The predisposing influence of age is an undoubted factor, some tumors only occurring at an advanced age, while others occur preferably in the young.

Heredity plays a doubtful rôle, the importance of which has been greatly overestimated.

Classification: Tumors have been variously classified.

Clinically they are divided into benign and malignant. The former are not dangerous except by reason of pressure they may exert on vital structures, or by mechanically interfering with the function of a part, as, for instance, by a tumor of the brain or larynx.

The latter threaten life and are characterized by certain well-recognized criteria of malignancy :

First, a tendency of the rapidly multiplying cells of the tumor to break through normal barriers and spread locally, infiltrating surrounding tissues. This is especially true of the malignant variety of epithelial tumors-carcinoma-and to a less extent of sarcoma, the malignant variety of the connective-tissue group. An adequate explanation of this characteristic is entirely lacking. The imagination of one author goes so far as to enable him to see in the infiltrating cells of a carcinoma only a host of invading parasites. In a child some irritant, as dirty hands, may excite the multiplication of epithelial cells, which, piling up and cornifying, constitute a benign wart; while in one advanced in years a similar irritant induces a rapid multiplication of epithelial cells, which, instead of piling up on the surface, invade the underlying tissues, thus constituting a most malignant tumor, a carci- 
noma-e. $g$., chimney-sweeps' cancer, which seems to result from the accumulation of soot upon the scrotum.

Non-malignant tumors are circumscribed, often encapsulated-facilitating their complete removal. The absence of capsule in malignant tumors and their extensive infiltration of surrounding tissues make their complete removal impossible, which explains an important clinical characteristic-their tendency to recur locally.

Second, metastasis, or the occurrence of secondary growths in various parts of the body, is but an extension of the tendency of malignant tumors to spread locally. Tumor-cells are carried by way of the lymphatics or bloodvessels to distant parts, where they multiply, yet retaining all their original characteristics, such as the tendency to horny degeneration of the epidermic cells in some varieties of carcinoma. The number of these secondary nodules is often so great as to remind one of the lesions of general miliary tuberculosis. Benign tumors are usually single.

Third, cachexia: A condition of anæmia, feebleness, and general impairment of nutrition, which is quite comparable to that noted in tuberculosis and other chronic infectious diseases, is an evidence of malignancy.

Fourth, rapid growth.

Fifth: It may be said that the more cellular a tumor and the less its histological structure follows any given type of tissue-i. e. the more atypical - the greater the probability of its being malignant.

Histological classification: Inasmuch as the microscopical study of tumors shows them to be composed of no new tissueelements, and to conform more or less closely to types of tissue normally present in the body, it is quite natural to classify them according to their microscopic structure. In the study of normal histology we become acquainted with four types of tissue-connective, epithelial, muscle, and nervous-and with these four varieties correspond four types of tumors.

In the connective-tissue group there are the fibroma, composed of fibrous tissue; the chondroma, of cartilage ; the osteoma, of bone; the lipoma, of fatty tissue; the myxoma, of mucoid tissue similar to that of Wharton's jelly of the umbilical cord; the glioma of neuroglia; the angeioma of bloodvessels; and the 
lymphangeioma of lymphatics. Corresponding to the two types of muscle-tissue - the unstriped and the striped-we have the leiomyoma and the rhabdomyoma.

The neuromata find their physiological prototype in the nerve-fibres and ganglion-cells of the central nervous system.

Whereas connective-tissue tumors contain no epithelial tissue, in another-the epithelial-group, the neoplasm is formed almost entirely of epithelial elements, only supported by a framework of connective tissue. This group includes the papilloma, the microscopical structure of which closely resembles the papillæ of the skin or the villi of the intestines; and the adenoma, in which the epithelial cells are arranged in such a manner as to imitate normal glandular structures with a certain degree of exactitude.

On the other hand the microscopical structure of the malignant tumors - the sarcomata and carcinomata-does not correspond with any normal type of tissue, but is entirely atypical.

The sarcoma is a very cellular and highly vascular connective-tissue tumor which histologically resembles one or another stage of granulation-tissue, several varieties being described according to the character of the cells of which it is formed; the small round-celled sarcoma having the microscopical appearances of the earliest stage of granulation-tissue ; and the spindle-celled sarcoma, that of a later stage of granulationtissue as its development approaches fully formed fibroustissue.

The carcinoma, which is composed of epithelial cells atypically arranged in a more or less dense connective-tissue stroma, is likewise described according to the character of the epithelial cells of which it is composed, viz., the squamouscelled carcinoma, or epithelioma; the columnar-celled, or adeno-carcinoma, and the spheroidal-celled, or carcinoma simplex.

When a benign tumor becomes malignant it still conforms to its own type of tissue ; that is to say, one of the connectivetissue group becomes sarcomatous and not carcinomatous, and one of the epithelial group becomes carcinomatous and not sarcomatous. 
Table :

1. Of Connective Tissue:

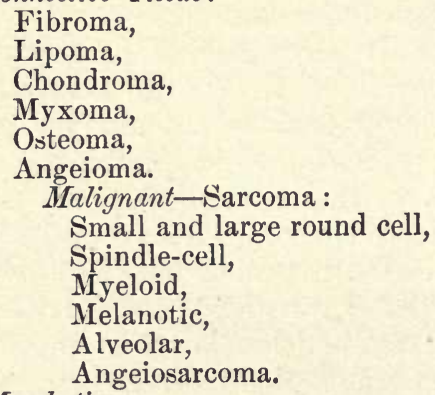

2. Of Muscle-tissue:

Leiomyoma, Rhabdomyoma.

3. Of Nervous Tissue:

Neuroma.

4. Of Epithelial Tissue:

Papilloma,

Adenoma.

Malignant-Carcinoma :

Squamous-celled-Epithelioma,

Cylindrical-celled-Adenocarcinoma,

Spheroidal-celled-Carcinoma Simplex $\left\{\begin{array}{l}\text { Scirrhous, } \\ \text { Encephaloid. }\end{array}\right.$

A fibroma is a tumor of fibrous connective tissue, according to the density of which hard and soft varieties are distinguished. The former is composed almost entirely of dense interlacing bundles of fibres and may be as hard as cartilage (Fig. 118). Between the fibres are a few compressed or spindle-shaped cells. In the soft variety round, spindle, and branching cells are numerous, and the fibres few in number with wider meshes. All gradations, however, are met with between the two, and in its cellular richness it may approach a fibrosarcoma.

These tumors always grow from preëxisting connective tissue. Their most frequent seats are the skin and subcutaneous connective tissue, the sheaths of nerves and tendons, and the periosteum of bones. They are also found growing from the connective-tissue framework of the mammæ, ovaries, 
uterus, and kidneys. They are liable to undergo mucoid and fatty degeneration.

Fig. 118.

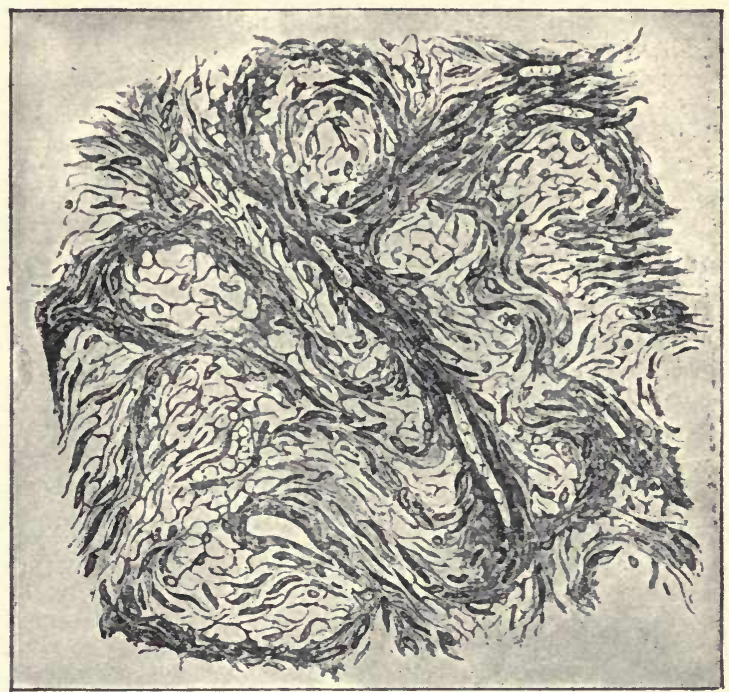

Section of a nodular fibroma (Birch-Hirschfeld). The dense fibrous tissue is in irregular nodules, between which are bands of less dense fibrous tissue containing bloodvessels.

A lipoma is a tumor composed of fatty tissue, similar to normal adipose tissue, except that the fat-cells are larger, contain more oil, and are not so regularly arranged. They are lobulated, often grow to enormous size, and sometimes are multiple (Fig. 119). They are most common in the subcutaneous fatty tissue of the back, shoulders, and buttocks. They are liable to myxomatous and cystic degeneration and calcification.

A myxoma is a tumor composed of mucous tissue-similar to that of Wharton's substance of the umbilical cord and the vitreous humor of the eye, which consists of a gelatinous, homogeneous, or slightly granular matrix crossed by fine fibrillæ, in which are imbedded irregular branching and 
anastomosing stellate or spindle-shaped connective-tissue cells (Fig. 120).

But few tumors are entirely myxomatous, being combined with other forms of connective tissue, constituting fibromyxoma, lipomyxoma, chondromyxoma, myxosarcoma, etc.

FI\%. 119.

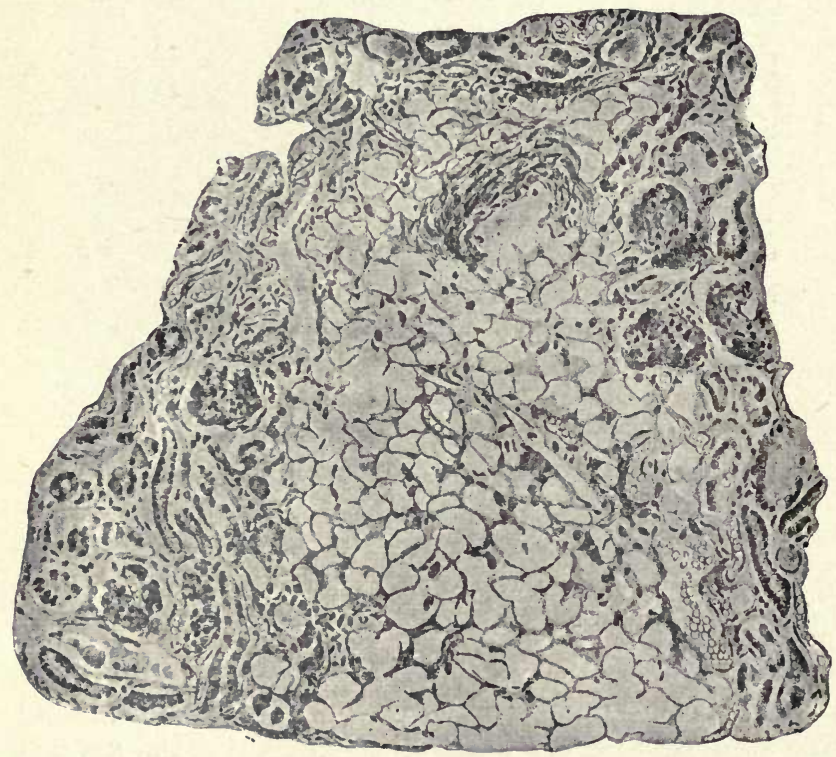

Lipoma of the kidney (Birch-Hirschfeld). The boundary between the adipose tissue of the tumor and the renal tissue is not sharply defined. The former occupies the middle of the section and extends to its lower edge.

They are found in submucous and subcutaneous tissue, in the mammary and parotid glands, and marrow of bone.

A chondroma is a tumor composed of hyaline, elastic, or fibro-cartilage or more frequently of a combination of these varieties of normal cartilage, differing therefrom in that the cells are much less regularly arranged. Hyaline cartilage is the form most frequently found (Fig. 121). If they occur in situations in which cartilage normally exists, they are called ecchondromata, or cartilaginous outgrowths. When they occur 
in other situations, as in the testicle, parotid and mammary glands, they are called enchondromata. They are liable to calcification and retrograde changes.

Fig. 120.

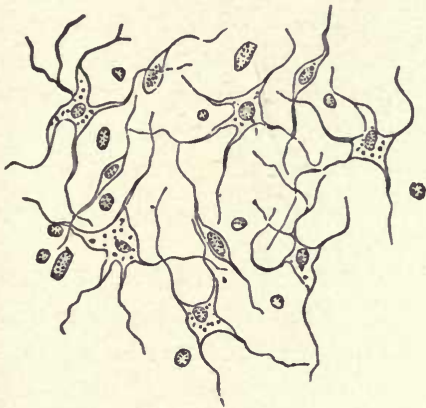

Myxoma (from the arm), showing the characteristic branched anastomosing cells, a few leukocytes, and one or two spindle-cells. $\times 200$ (Green).
FIG. 121.

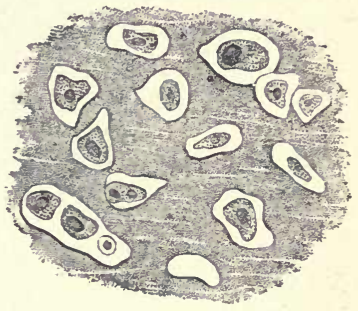

Hyaline chondroma. $\times 200$ (Green).

Fig. 122.

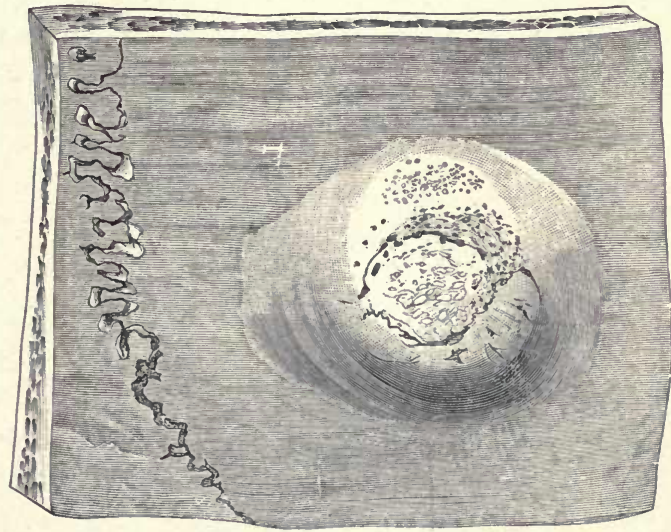

Ivory exostosis of the parietal bone, natural size (Ziegler).

An osteoma is a tumor composed of bone, and may be of either the dense or cancellous variety. It is said to be heterologus when occurring in organs unconnected with bone, 
as in the skin, lungs, meninges, testicle, and parotid gland. If occurring as an outgrowth from bone, they are known as exostoses (Fig. 122).

\section{Angeioma.}

An angeioma is a tumor composed mainly of bloodvessels. There are two varieties-simple and cavernous. In simple angeioma, or teleangiectasis, there is an abundant plexus of arteries, veins, and capillaries as found in the skin in novvi. They also occur on mucous surfaces and rarely in the internal organs.

Cavernous angeioma, which is the more common variety, resembles microscopically the tissue of the corpus cavernosum of the penis, being made up of large, irregularly shaped, intercommunicating spaces filled with blood, lined by endothelium, and separated from each other by connective-tissue partitions. They are most common in the liver, but are found also in the spleen, kidney, and uterus.

Lymphangeiomata are formed of dilated lymphatic vessels identical in structure with the hæmangeiomata. Cavernous lymphangeiomata have been occasionally found in the kidneys and lymphatic glands. In congenital enlargement of the tongue-macroglossia, and of the lips-macrocheilia, and in elephantiasis, there is a diffuse dilatation of lymphatic structures.

\section{Sarcoma.}

A sarcoma is a richly cellular malignant tumor of the connective-tissue group, which, histologically, resembles granulation-i. e., embryonic or undeveloped connective tissue. The cells, round and spindle-shaped, are greatly in excess of the intercellular substance. The more cellular its structure the greater the malignancy of the growth. Though the amount of the intercellular substance may be very scant, yet it may be of such quantity and character as to give rise to special varieties-fibrosarcoma, myxosarcoma, chondrosarcoma, and osteosarcoma. The bloodvessels, which in some instances are extremely abundant, are simple channels lined with a single layer of cells. 
Sarcomata are liable to retrograde metamorphoses-fatty degeneration, caseation, liquefaction, and ulceration. Accord-

FIG. 123.

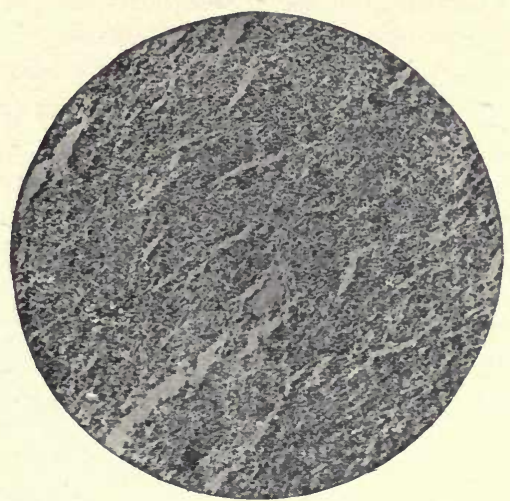

Small round-celled sarcoma of the neck. Section only moderately magnified, showing the extremely cellular character of the growth; the great friability of the tissue is owing to the minimal amount of intercellular substance it contains and the intimate relations between the tissue of the tumor and the walls of relatively large, thin-walled bloodvessels (Dunham).

ing to the character of the predominant cells, several varieties are described:

Round-celled sarcomata have their prototype in the early FIG. 124.

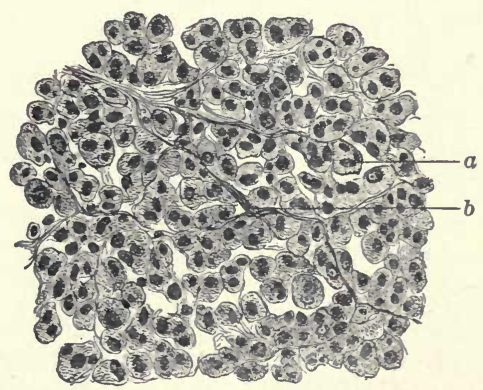

Large round-celled sarcoma of the tongue. $a$, large round cell containing three nuclei; $b$, delicate fibrous stroma supporting the cells of the growth. At the point $b$ this stroma contains a collapsed capillary bloodressel. The large round cells are probably of endothelial origin. The growth occurred in a man aged sixty-one years, and in the course of eight mouths had attained the size of a hickory-nut (Dunham). 
stage of granulation-tissue. The cells are about the size of leukocytes, with large nuclei and but little cytoplasm, though sometimes they are formed of larger spherical cells. There is scarcely any stroma between the cells (Figs. 123 and 124). The tumor is gray or pink in color, usually quite soft, and is found most commonly in the skin, testicle, eye, ovary, periosteum, bone, and subcutaneous connective tissue. When they occur in lymph-glands and adenoid tissue of mucous membranes they are designated lymphosarcomata, on account of the appearance of their supporting stroma. Metastasis takes place through the bloodvessels, secondary growths occurring most frequently in the lungs.

Spindle-celled sarcomata have their prototype in a more advanced stage of granulation-tissue-the transitional stage between granulation-tissue and young cicatricial tissue; the

FIG. 125.

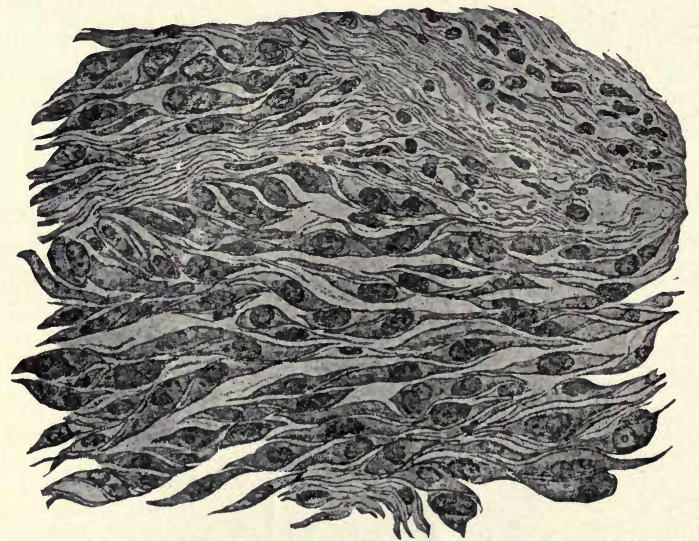

Large spindle-celled sarcoma (Birch-Hirschfeld).

cells are elongated or spindle-shaped and often irregular and provided with several processes (Fig. 125). They grow from dense connective tissues, as periosteum, intermuscular septa, fasciæ, and tendons, are firmer in consistency, and grow less rapidly than the round-celled variety. The amount and character of the intercellular substance in this and other types 
of sarcoma vary; they may be such as to suggest the term fibrosarcoma, myxosarcoma, chondrosarcoma, etc.

Giant-celled or myeloid sarcomata are characterized by the presence of large, flat, irregularly shaped, multinucleated cells,

Fig. 126.

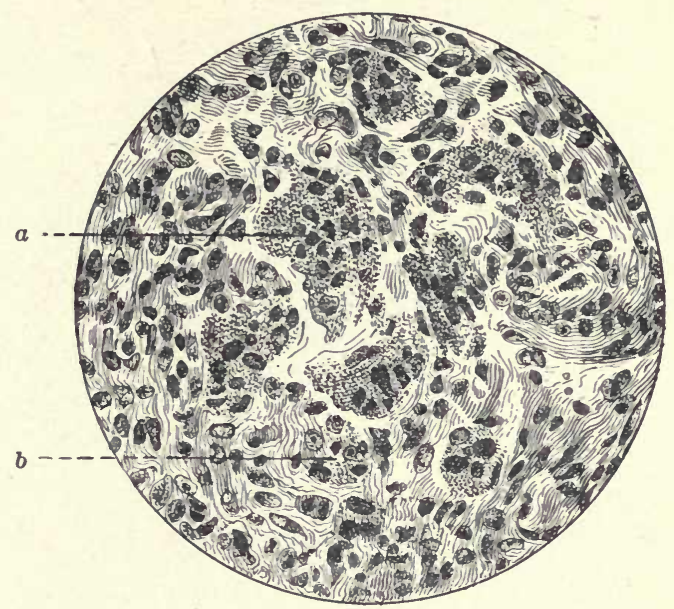

Giant-celled sarcoma of the superior maxilla : epulis. $a$, large giant-cell, with numerous nuclei; $b$, tangential section of a similar cell. Aside from the giant-cells, the growth is composed of spindle-cells and a moderate amount of a fibrous intercellular substance. The tumor was removed from a man forty-one years of age, and was of slow growth, having attained the size of a filbert in two and a half years (Dunham).

and arise most frequently from the narrow or periosteum of bone (Fig. 126). They are of slow growth and less malignant than the other varieties, though metastasis may occur.

Alveolar sarcoma is a very malignant variety, which bears a close resemblance to cancer in that the cells are arranged in an alveolar fibrous stroma. The cells, however, are not of an epithelial type, and are separated from each other by fine trabeculæ extending in from the alveolar walls (Fig. 127). They are most frequent in the skin, occurring also in lymphatic glands and serous membranes.

Angeiosarcomata arise from the adventitia of bloodvessels, which are surrounded by the masses of proliferating round 
cells. They occur in serous membranes, skin, brain, breast, and salivary glands. They are relatively benign, metastasis being very rare.

Endothelial sarcomata, or endotheliomata, are composed of concentrically arranged cells, strongly resembling the

FIG. 127.

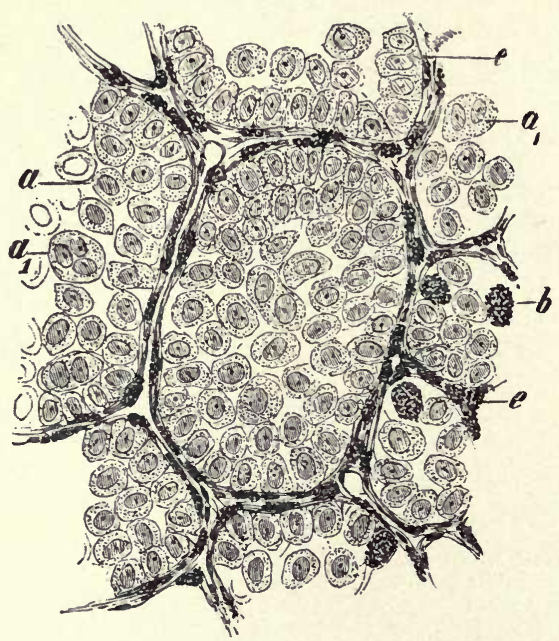

Melanotic alveolar sarcoma of the skin. $a$, mononuclear; $a^{\prime}$, polynuclear sarcomacells with epithelial character; $b$, pigment-cells; $c$, stroma with bloodvessels and pigment. $\times 300$ (Ziegler).

endothelial cells lining the lymph-vessels and spaces from which they have developed (Fig. 128). Degenerative changes and subsequent calcareous infiltration give origin to the so-called psammomata or "sand tumors" of the brain. They occur in the pleura, peritoneum, and meninges of the brain.

Melanotic sarcoma is an exceedingly malignant variety arising from pigmented tissues, such as the skin, choroid coat of the eye, and pia mater. Its structure may be that of any of the above varieties, though it is usually spindle-celled. 'The secondary metastatic growths are likewise pigmented and of similar structure. 


\section{Papilloma and Adenoma.}

A papilloma is a wartlike excrescence composed of papillæ resembling those of the skin and mucous membranes or the

FIG. 128.

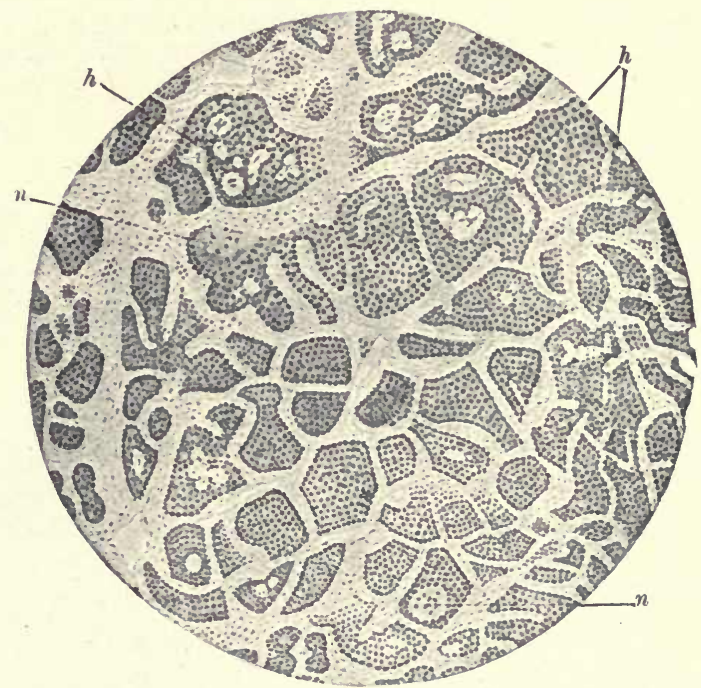

Endothelioma from the floor of the mouth (Barth). Older portion of the growth. This has a general alveolar structure, the alveoli being separated by a vascularized areolar tissue. $n, n$, necrosed groups of endothelial cells; $h, h$, similar necrosed masses that have undergone hyaline degeneration.

FIG. 129.

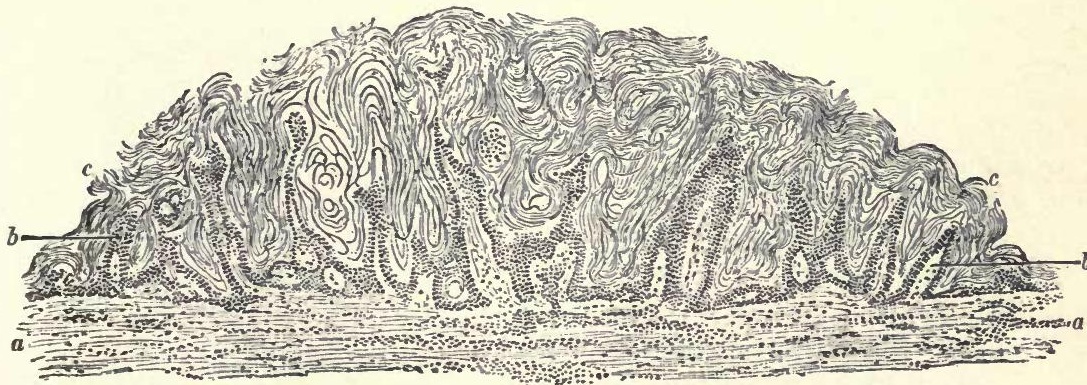

Section of wart of skin. $a$, corium; $b$, enlarged papillæ; $c$, stratified horny layer. $\times 40$ (Ziegler). 
villi of the intestines, and surmounted by epithelium. It may be a simple, smooth elevation, or have a cauliflower appearance (Fig. 129). They occur especially in the bladder, gastrointestinal tract, larynx, and nose.

An adenoma, as its name implies, is a tumor which, histologically, more or less closely resembles normal, tubular, or acinous glandular structures (Fig. 130). The absence of

FIG. 130.

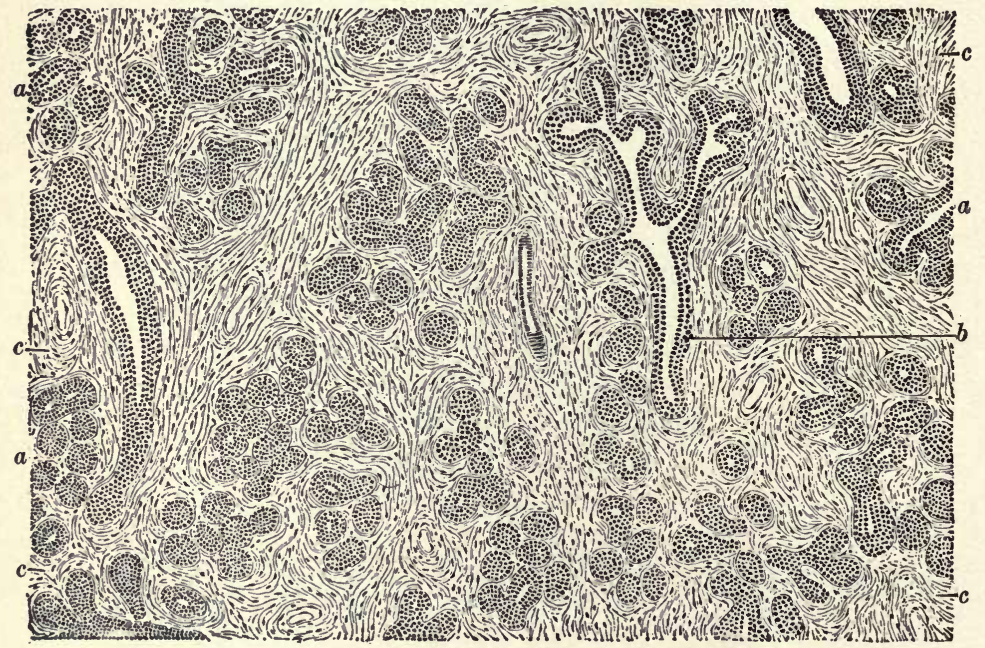

Adenoma of mamma. $a$, acini ; $b$, ducts; $c$, well-marked connective-tissue stroma. $\times 30$ (Ziegler).

excretory ducts often leads to cystic dilatation of the tubules or acini, from the accumulation of the secretions of the glandular elements, giving rise to the term cysto-adenoma. From the walls of such cysts papillary outgrowths may occur.

Adenomata are benign, though in some cases there may be metastasis. Their most frequent situations are the skin, mucous membranes, mammæ, kidneys, ovaries, liver, thyroid, and prostate gland. In the ovaries they are supposed to be the origin of ovarian cysts, which often reach an enormous size. 
Adenoma of the liver probably arises from the bile-ducts, as its epithelial elements differ in structure from the hepatic cells.

\section{Carcinoma.}

A carcinoma, or cancer, is the malignant variety of the epithelial-tissue group of tumors. The arrangement of the rapidly multiplying epithelial cells, of which the tumor is mainly composed, with reference to the connective-tissue stroma is atypical, peculiar to carcinoma-not seen in any other tissue in the body, though often suggestive of glandular structures. The cells originate from surface or glandular epithelium; breaking through normal anatomical boundaries, they infiltrate the surrounding connective tissue, which in its turn thus proliferates, and thus their mutual interpenetration gives rise to the microscopical structure which is always diagnostic of carcinoma-epithelial cells in an alveolar stroma. The cells are very apt to retain to a certain extent the characteristics of those from which they originated: if from one of the glands of the stomach, the new-formed cells also are cylindrical, and often seem to attempt to reproduce glandular tissue-i. e., there are variously shaped spaces more or less regularly lined by epithelium. If from the epidermis, the newly-formed squamous cells may show the same tendency to cornification.

The amount of the stroma varies; if considerable, the growth is hard-scirrhous; if scanty, it is soft and brain-like -encephaloid; further, it is often quite cellular from the presence of infiltrating leukocytes or newly-formed connectivetissue cells. Metastasis occurs through the lymphatics, while in sarcoma it occurs through the bloodvessels. The secondary growths have the same general structure as the primary.

Carcinoma occurs most frequently between the ages of forty-five and sixty-five, and is found most frequently in the uterus, gastro-intestinal tract, skin, mammary gland, ovary, and occasionally in the liver, kidney, thyroid, and prostate gland. It is quite liable to retrograde changes-fatty and colloid degeneration, necrosis, and ulceration.

Several varieties are described according to the character of the epithelial elements-squamous, cylindrical, - which compose the tumor: 
Carcinoma simplex, or spheroidal-celled cancer, arises in glands with spheroidal epithelium, as, for example, the mammæ. The acinous or glandular arrangement of the tissue from which it grows is soon obscured and replaced by the perfect alveolar structure typical of cancer. In the earliest stage of its growth there would be simply noted at a given point within an acinus a proliferation and piling up of the epithelial cells. Soon they break through the basement-membrane and infiltrate the surrounding connective tissue. The cells are generally spheroidal unless altered by mutual pressure. According to the density of the connective-tissue stroma, two varieties are distinguished, the scirrhous and encephaloid.

In the scirrhous variety the epithelial element is relatively small; the tumor is hard and grows slowly (Fig. 131). In

FIG. 131.

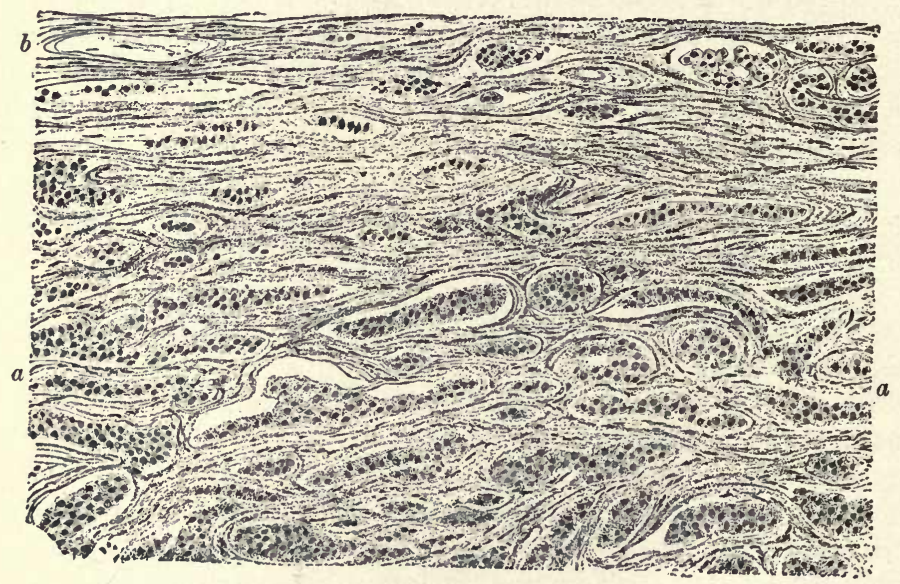

Scirrhous of mamma. $\times 100$ (Ziegler)

encephaloid the stroma is scanty, the tumor of soft consistency and rapid growth (Fig. 132).

Cylindrical-celled cancer, or adenocarcinoma, arises from surfaces covered with cylindrical epithelium, as from the glands of the gastro-intestinal tract. Especially in the early 
stage of development, its general structure is distinctly glandular, consisting of tubular or rounded gland-like spaces or alveoli lined with cylindrical epithelium. It is distinguished from simple adenoma, however, which it greatly resembles, by the irregular arrangement of the marginal epithelium, by the masses of epithelial cells which often completely fill the lumen of the alveoli, especially in the older parts of the

FIG. 132.

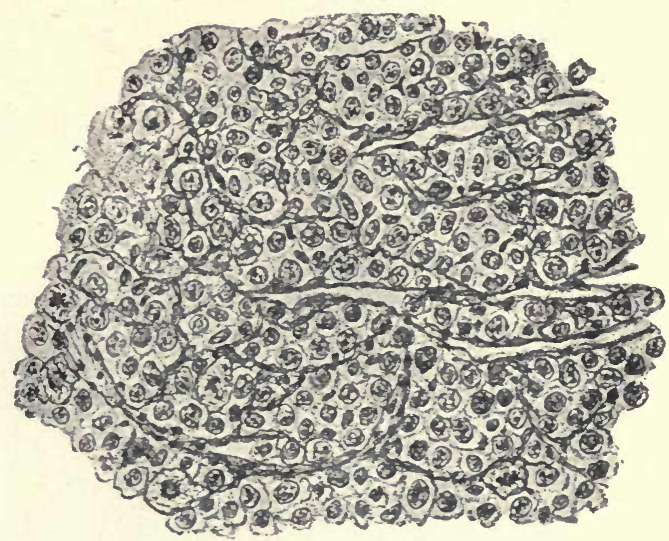

Medullary carcinoma of the mammary gland (Hansemann). The stroma of the tumor is here reduced to a minimal amount of areolar tissue containing the vascular supply of the growth.

tumor, and by epithelial cells which have broken through the basement-membrane and infiltrated the connective-tissue stroma between the alveoli.

Squamous-celled cancer, or epithelioma, arises from surfaces covered by stratified squamous epithelium, as from the skin, œsophagus, larynx, lips, etc. The proliferating epithelial cells show the characteristic tendency noted in all cancers to extend beyond normal limits and infiltrate adjacent tissues. The tumor is found to be composed of masses of flat epithelial cells grouped in conical or irregularly shaped processes or columns of variable length and breadth, simple or branching, and separated from one another by a scanty connective-tissue stroma. At many places in the deeper parts of the tumor, as 
the result of the pressure exerted by the rapid cell-proliferation and the lack of space, the flat cells are found on edge and concentrically arranged in whorls, which often have a glistening appearance as the result of a horny transformation or cornification-constituting the so-called "pearly bodies" (Fig. 133).

Epitheliomata appear as elevated nodular masses or diffuse infiltrations of rather slow growth, very prone to ulceration

FIG. 133.

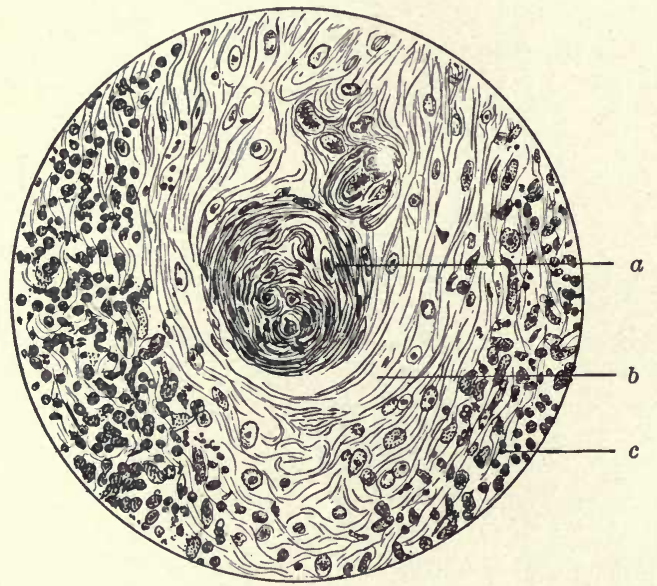

Epithelial pearl-body from an epithelioma of the lip. $a$, pearl-body; $b$, surrounding epithelium, forming one of the epitheliomatous tongues or columns; $c$, roundcelled infiltration of the contiguous fibrous tissue (Dunham).

and great destruction of tissue. Metastasis may occur, but is not common. The term colloid cancer is applied to any of the above forms which has undergone a colloid degeneration.

\section{Myoma.}

A myoma is a tumor composed of muscle-tissue. There are two varieties, corresponding to the two varieties of muscletissue-rhabdomyoma, formed of striped, and leiomyoma, of unstriped muscle-tissue.

Rhabdomyomata are exceedingly rare. In a few instances 
such tumors have occurred congenitally in the heart and caused death. Such tumors have also been found in the kidney, mixed with sarcomatous tissue and malignant in proportion to the amount of the latter (Fig. 134).

Leiomyomata are of much more common occurrence-the most frequent seat being the uterus, though they may occur wherever unstriped muscle-tissue is normally found. They are formed of bundles of muscle-cells, closely packed and inter-

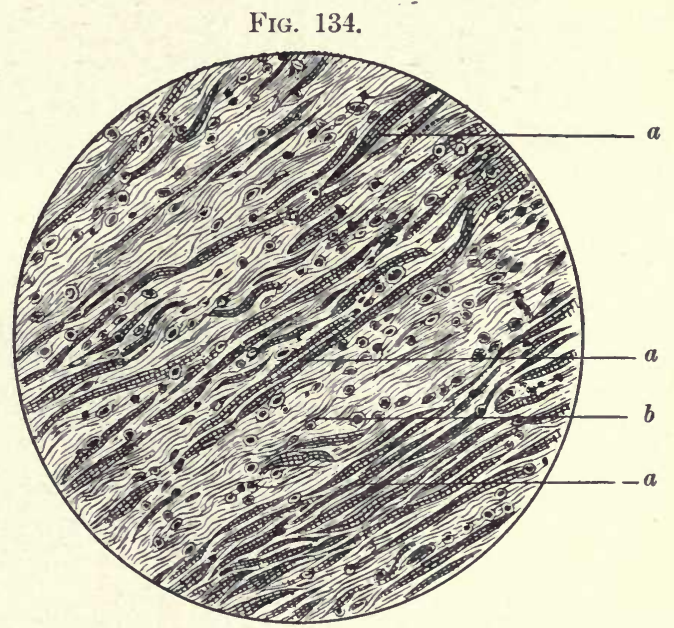

Rhabdomyosarcoma of the kidney. $a, a, a$, imperfectly developed striated musclefibres : $b$, tissue composed of small round and spindle-shaped cells, separated by considerable delicate fibrous intercellular substance. In other parts of the growth, which was the size of the fist, this tissue was more distinctly sarcomatous and the amount of muscular tissue smaller. The child from which this tumor was removed was about two years old.

lacing in every direction. When there is a large amount of connective tissue between the bundles they are termed fibromyomata. On account of the wavy course of the musclecells, in microscopical section they seldom are seen in their entire length, though they may be isolated by teasing out a portion of the tumor. The characteristic feature is the presence of the long rod-shaped nuclei-circular, however, if cut transversely (Fig. 135). Their appearance suggests spindle-celled sarcoma, but in the latter the cells are not 20-Hist. 
arranged in regular bundles and their nuclei are not oblong, but oval; sarcomatous transformation, however, may occur. Myxomatous degeneration and calcification are the most frequent retrograde changes to which they are liable.

\section{FIG. 135.}

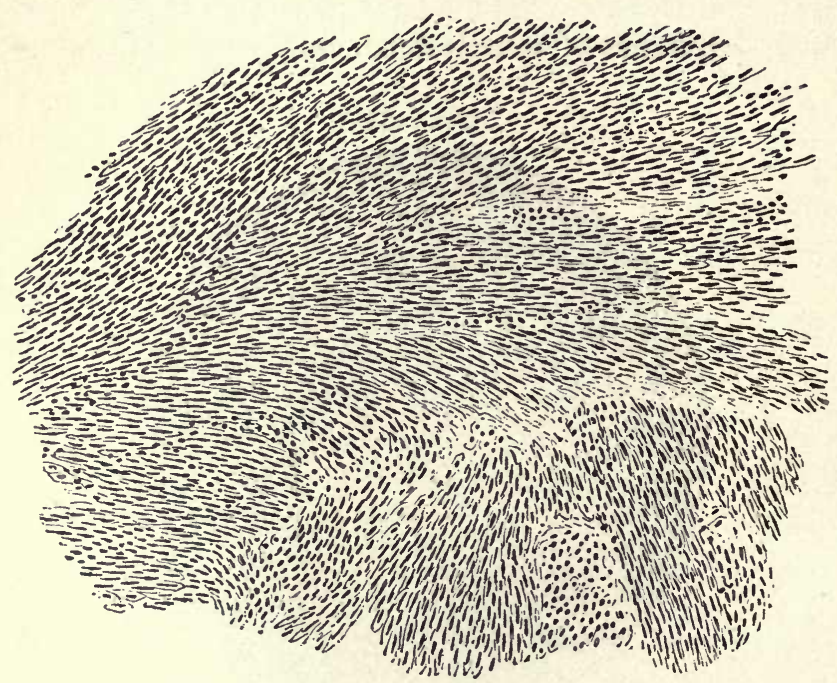

Leiomyoma of the uterus (Birch-Hirschfeld).

\section{Neuroma and Glioma.}

A true neuroma is a very rare tumor, composed of nervefibres and ganglion-cells. The so-called false neuromata are usually fibromata occurring in the course of a nerve and found also as bulbous swellings of the cut ends of the nerves in amputated limbs.

A glioma is a tumor composed of tissue similar to neuroglia, occurring in the brain and less frequently in the spinal cord and the retina of the eye. They are formed of a very delicate network of fibres, imbedded in which are numerous round or oval cells with very large nuclei. They are benign, though liable to undergo sarcomatous transformation. 


\section{Teratoma.}

Teratomata are tumors of complex structure, sometimes consisting of a mingling of several types of tissue as gland, nervous, muscle, and connective tissue ; sometimes being composed of more or less complete organs, as breasts, nerves,

\section{Fig. 136.}

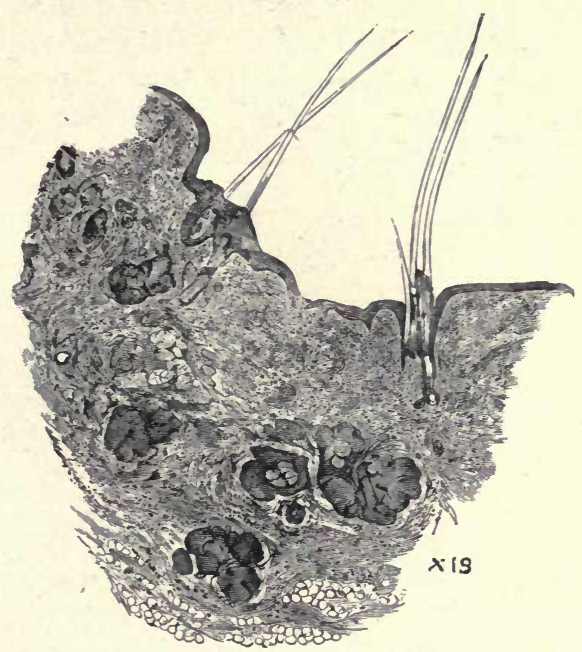

Dermoid cyst of the ovary, showing all the structures of true skin except sweatglands-viz., epithelium, rudimentary papillæ, fibrous tissue or cutis vera, hairfollicles, large sebaceous glands. $\times$ is (Boyd).

muscles, portions of intestine-constituting so-called dermoid tumors, which, however, usually present themselves as cysts filled with hair, teeth, and occasionally bone, muscle- and nerve-tissue. Teratomata also include certain malformations and monstrosities (Fig. 136).

\section{CYSTS.}

A cyst is a cavity, usually with a distinct membranous wall, which contains a fluid or semifluid material. They are not really tumors, though usually considered in connection with the latter. They are generally classified according to the method of their formation. 
Retention-cysts result from the accumulation or retention of the natural secretion of a gland, due to the obstruction of its duct. As examples may be mentioned sebaceous cysts or wens; and ranula, which results from obstruction of the ducts of the salivary gland. As the result of obstruction of a ureter the whole kidney may be converted into a cyst.

Disintegration-cysts result from degenerative softening and disintegration of normal or pathological tissues. This may occur after hemorrhage into the brain-apoplectic cyst. Parasitic cysts might be included under this head-for example, hydatid cysts. Tumors, especially sarcomata and chondromata, are liable to cystic degeneration. 


\title{
SPECIAL PATHOLOGY.
}

\author{
CHAPTER III. \\ DISEASES OF THE BLOOD.
}

\section{PLETHORA.}

THE condition of plethora, by which is understood an excess of blood in the body, was regarded by the older writers as of great importance, and most serious consequences were attributed to it. Clinically, its presence was supposed to be indicated by redness of the face and a vigorous circulation occurring in high livers.

Experimental evidence goes to show that if the amount of blood in the vessels is artificially increased the result is but temporary, as the excess is carried off by the kidneys in a few hours.

\section{OLIGEMIA.}

The term oligemia indicates a diminution in the total quantity of blood. This undoubtedly does occur after profuse hemorrhages, severe vomiting, and diarrhoea. There is reason to believe it exists in starvation and certain cachectic conditions.

\section{QUALITATIVE CHANGES IN THE BLOOD.}

Alterations in the plasma: The amount of fibrin formed from the blond was earefully investigated in the days of frequent venesection. It is greatly increased in certain acute inflammations, as pneumonia, pleurisy, and peritonitis. In some infectious diseases, as typhoid and smallpox, the amount of fibrin is not increased ; though if a pneumonia or pleurisy occurs as a complication such an increase is immediately noted. A reduction of the fibrin-forming property of the blood has been occasionally observed, as in purpura and 
scurvy. Our knowledge regarding chemical changes in the plasma is exceedingly meagre.

Alterations in the red corpuscles : Polycythomia or erythrocytosis, in which there is an actual increase in the number of red-cells in a given bulk of blood, is of relatively rare occurrence. Hayem states that they may be increased from the normal five million to six and a half million per cubic millimetre in the algid stage of cholera.

Oligocythomia, in which there is a reduction in the number of red cells, is very frequently noted. It may occur temporarily from a considerable loss of blood, or permanently as the result of interference with the formation of the red globules in the body. According to Hayem, if the condition is brought about rapidly, as by hemorrhage, a reduction in their number beyond one million per cubic millimetre is almost certain to prove fatal. When this reduction is progressive the body seems to adapt itself to these new conditions, patients being seen active and earning their living with less than two million red blood-cells per cubic millimetre. Extreme and fatal cases have been recorded in which there were less than three hundred thousand per cubic millimetre.

Alterations in the size and shape of the red corpuscles are frequent. Their normal average diameter is seven and a half microns-7.5 $\mu\left(\mu=\frac{1}{1000}\right.$ millimetre).

Microcytes are dwarf corpuscles two to five microns in diameter. Macrocytes, or megalocytes, are giant-cells nine to twenty microns in diameter. Poikilocytes are oval, pearshaped, or variously distorted corpuscles. Erythroblasts are nucleated red blood-cells, which are termed, according to their size, normoblasts, microblasts, and megaloblasts.

While the normoblasts occur normally in bone-marrow, and would seem to be present in the circulation in this immature form as the result of an unusual effort on the part of the blood-making organs to improve the blood, the megaloblasts are never present in healthy adult marrow, and, as they do not develop into mature corpuscles, they are looked upon as a degenerative type.

The microblasts are not so frequently met with as the other two varieties. Karyokinetic figures are sometimes seen in the nuclei of erythroblasts. 
Red blood-corpuscles have an especial affinity for eosin and other acid stains, and in a mixture of dyes, such as hæmatoxylin and eosin, stain only with one of them, the eosin, which property is termed monochromatophilia. Pathologically, however, they may develop an affinity also for basic dyes; when stained with hæmatoxylin and eosin mixture they present a violet or purple color, instead of the normal pinkthis is termed polychromatophilia.

Alterations in the leukocytes: Hypoleukocytosis or leukopenia - a deficiency in the number of leukocytes-is associated with certain conditions, as, long-continued fevers, like typhoid, when uncomplicated by inflammatory processes; with tuberculosis and inanition.

Leukocytosis-a transient increase in the number of leukocytes above the normal ten thousand per cubic millimetre-is noted in connection especially with inflammatory processes and infectious diseases. The degree of the leukocytosis in pneumonia and most inflammatory affections is regarded as an index of nature's power to combat the disease, its entire absence being an ill-omen. In typhoid fever, malaria, influenza, and acute miliary tuberculosis the leukocytes are not increased, which fact may at times be of some diagnostic value. Usually it is the polynuclear variety of lenkocyte which is increased. Malignant tumors, especially sarcomata, are accompanied by an increase in the number of leukocytes - even to the extent of fifty thousand per cubic millimetre or more-the small mononuclear being the variety mainly affected. In syphilis, rickets, and other cachexias there may be a similar leukocytosis. There is also an increase in the number of leukocytes after hemorrhage.

In leukcemia there is an enormous and permanent increase in the number of leukocytes-one to three hundred thousand per cubic millimetre in moderate cases.

Alterations in the blood-plates are as yet but little understood.

The amount of hæmoglobin contained in each red globule varies normally with the size of the corpuscle, though pathologically this relation does not exist. In most cases the amount of hæmoglobin in each globule is diminished; in some cases, however, there is an actual increase in the amount of 
hæmoglobin per red corpuscle-ascertained by comparing the number of red cells per cubic millimetre with the percentage of hæmoglobin.

\section{THE AN 压IAS.}

The term anæmia includes a variety of conditions in which there are qualitative alterations in the blood, the most conspicuous evidence of which, clinically, is pallor of the skin and mucous membranes, due to a reduction in the amount of hæmoglobin, whether from a reduction in the amount of blood, in the number of red corpuscles, or in the coloringmatter alone.

Reduction in the number of red corpuscles-oligocythomia, and of the amount of hæmoglobin-oligochromomia, are usually associated, though the latter may be present without alteration in the number of red cells, as is seen especially in chlorosis.

The anæmias are usually divided into two classes clinically -the primary or essential, and the secondary or symptomatic.

The former include simple primary anæmia, pernicious anæmia, chlorosis, leukæmia, and Hodgkin's disease, neither of which is dependent on any preceding affection.

The latter include those anæmias produced by disturbances outside of the hæmopoietic system-e.g., tuberculosis, malignant disease, etc.

\section{Primary Anæmias.}

Chlorosis is a primary anæmia, occurring principally in girls about the time of puberty, characterized by an excessive reduction in the amount of hæmoglobin and, in most cases, a peculiar sallow complexion having a somewhat greenish tinge, which has won for it the term green-sickness. The red corpuscles may be normal in number, but generally in prolonged cases there is a considerable reduction-never so great, however, as the percentage-reduction of hæmoglobin; in other words, the amount of coloring-matter in each individual corpuscle-la valeur globulaire-is reduced. This difference may be as great as 30 per cent.; while the number of corpuscles in a given case may be reduced to only two or three million per cubic millimetre, the quantity of hæmoglobin may be less than 20 or 30 per cent. of normal. 
When the red blood-corpuscles are reduced in number they are usually more or less altered in shape, according to the degree of the oligocythæmia. Poikilocytes, microcytes, and polychromatophiles are frequently present, and, when the condition is very severe, megalocytes may be quite numerous; nucleated red blood-corpuscles-usually normoblasts-are sometimes seen. The number and variety of leukocytes are normal in uncomplicated cases.

The only associated organic lesion which has been noted is a congenital smallness of the heart, bloodvessels, and genital organs.

Progressive pernicious anæmia is a severe anæmia which has no apparent cause and tends uninterruptedly toward a fatal issue. The number of red blood-corpuscles is reduced to a greater degree than in any other condition, sinking below five hundred thousand per cubic millimetre, even to one hundred and forty-three thousand in one case which has been recorded.

A characteristic feature is the average increase in size of the red corpuscles. Microcytes may occur, but are not common. Erythroblasts, poikilocytes, and polychromatophiles are frequently noted. The hæmoglobin is always reduced, but never to the same extent as the red blood-corpuscles. The leukocytes undergo no important change.

In some cases it has been possible to assign a cause. Atrophy of the gastro-intestinal mucous membrane and certain intestinal parasites, especially the anchylostoma duodenale, produce a secondary anæmia, often not distinguishable from progressive pernicious anæmia. The anæmia is usually looked upon as resulting from the excessive destruction of blood rather than from its defective formation. The pigmentation of various organs, the jaundice often present, and the excess of coloring-matter in the urine suggest this conception of its nature.

The various viscera are pale and bloodless, and in a more or less advanced stage of fatty degeneration.

A simple primary anæmia arising without any apparent cause is not recognized by many writers, yet cases do occur in which the characteristic features of both chlorosis and per- 
nicious anæmia are absent. The red blocd-corpuseles and hæmoglobin are usually reduced to the same degree ; the leukocytes undergo no important change either in number or kind.

Leukæmia or leukocythæmia: The term leukamia-white blood-was first proposed by Virchow, in 1845 , for cases in which there was an enormous increase in the number of leukocytes in the blood, associated with enlargement of the spleen and lymphatic glands. The condition had previously been regarded as an inflammation or suppuration of the blood.

The disease is well named, for at times the blood is so white it resembles milk or pus. The older writers were accustomed to speak of the relations existing between the number of white and red corpuseles; but as this depends also on fluctuations in the number of red corpuscles, it is more important to know the exact number of leukocytes per cubic millimetre. Instead of one leukocyte to five or six hundred red cells, the proportion is frequently one to eight, even one to two or three; in cases of moderate severity there are one to three hundred thousand per cubic millimetre-five hundred thousand per cubic millimetre not being infrequent.

Yet it is not so much the number of leukocytes, which is characteristic of this disease, as the character of the predominant variety; for the proportion of one to eight has been noted in non-leukæmic anæmias, and in some cases of leukoeytosis there may be as many as one hundred thousand white blood-corpuscles per cubic millimetre.

Virchow recognized two forms of the disease, one characterized by enlargement of the spleen, in which the blood showed an excessive number of large white cells ; and another characterized by enlargement of the lymphatic glands, in which the small variety of leukocyte predominated.

A third form of the disease was later described by Neumann, in which there were marked changes in the marrow of the bones.

Though it is customary to speak of lymphatic, splenic, and myelogenous varieties of the disease, these different forms are often associated. There is some doubt as to a pure splenic variety existing without accompanying changes in the bonemarrow. 
The spleno-medullary variety of leukomia is especially recognized by the presence in the blood of numbers of large-sized leukocytes, which have been called myelocytes on account of their supposed origin in bone-marrow. These cells are rarely if ever seen in normal blood; they are usually $16 \mu$ to $20 \mu$ in diameter, though occasionally not much larger than a red corpuscle, when they can only be distinguished from the mononuclear leukocytes by the presence of neutrophilic granules in their protoplasm, or sometimes of eosinophilic granules -eosinophile myelocytes. When the blood is stained with hæmatoxylin and eosin these neutrophilic granules cannot be seen ; but if Ehrlich's triple stain is employed they are readily recognized. In this variety of leukæmia the myelocytes often make up 30 to 50 per cent. of the total number of leukocytes, while in other diseases in which they rarely occur they never exceed 2 to 9 per cent.

The relative number of eosinophiles-normally constituting 2 to 4 per cent. of the white blood-cells-is not usually increased, though there is always an absolute increase in their number-50 to 80 per cent. of which are eosinophile myelocytes. It was formerly supposed that a relative increase in the eosinophiles was diagnostic of leukæmia; they are increased to a much greater extent, however, in some other affections-notably in asthma and discases of the skin-and often are even diminished in leukæmia. The number of polynuclear neutrophiles is relatively diminished, making up but 50 per cent.-in some cases even less than 10 per cent. of the total number of white blood-cells; an increase would suggest some inflammatory complication. The small lymphocytes are also relatively reduced in number, constituting but 3 to 5 per cent., instead of 15 to 25 per cent. of the total number of leukocytes.

Basophilic myelocytes and small leukocytes are occasionally noted, their protoplasm containing granules which have a selective affinity for basic dyes.

The red blood-corpuscles are usually reduced to two or three million per cubic millimetre, and proportionally likewise the hæmoglobin. Alterations in the size, shape, and staining properties of the red cells may be noted; and erythroblasts are always found at some stage of the discasemostly normoblasts, though some megaloblasts and micro- 
blasts. The presence of these nucleated red blood-cells is especially characteristic when the oligocythæmia is slightin fact, it is the only disease in which they are present under that condition. The manner in which this excessive accumulation of leukocytes is brought about, whether due to an increased proliferation or a diminished destruction of these elements, is not known. Some maintain that it is primarily a disease of the blood-making organs-the spleen, lymphatic glands, and bone-marrow-while others believe the changes in these organs to be secondary to the alterations in the blood. The enlargement of the spleen, which is nearly always present, is the result of an enormous increase of the lymphatic elements. The marrow of spongy and long bones becomes yellowish in color; microscopically the marrow-cells are found to be greatly increased in number. The lymphatic glands are also sometimes involved; in fact, any lymphatic tissue in the body may be affected.

In the lymphatic variety of leukcemia, which is of much less frequent occurrence, the enlargement of the lymphatic glands is especially marked. A pure lymphatic form is rare, usually there being some involvement of the spleen and bonemarrow. The lenkocytes are never increased to the same extent, the large and small mononuclear elements making up 95 per cent. of their number. Myelocytes are present in very small numbers only, if at all ; the eosinophiles and polynuclear neutrophiles are relatively and absolutely reduced.

The oligocythæmia is much more marked than in the splenomyelogenous variety; nucleated red blood-corpuscles, however, are extremely infrequent.

Hodgkin's disease, malignant lymphoma, lymphosarcoma, or pseudoleukamia, resembles leukæmia in all respects except in that there is no increase in the number of lenkocytes in the blood. The lymphatic glands throughout the body are enlarged as a result of a hyperplastic proliferation of the lymphatic elements. As in leukæmia, the spleen, bone-marrow, tonsils, lymphatic follicles of the intestinal mucous membrane, and all the other lymphatic tissues in the body may be affected. Nodules, from the size of millet-seed to that of a walnut, composed of lymphoid cells, may occur in the liver, kidneys, 
lungs and serous membranes; also, rarely, in the ovaries, testicles, heart, and brain.

At the beginning of the disease the blood is found to be normal, but soon the anæmia and cachexia become prominent. When the anæmia is pronounced, alterations in the size and shape of the red blood-corpuscles oceur, and a few nucleated erythrocytes-usually normoblasts-may be noted, as in other severe anæmias.

The percentage-reduction in hæmoglobin is often greater than that of the red corpuscles-a so-called chlorotic condition of the blood.

Cases are sometimes observed in which the spleen alone is involved, and are described under the term splenic anæmia.

\section{Secondary Anæmias.}

Whatever the cause of secondary or symptomatic anæmias, they have certain common characteristics. There is usually a greater percentage-reduction of hæmoglobin than of red blood-corpuscles; in mild eases the number of red cells is not reduced, though the hæmoglobin varies from 60 to 75 per cent. of normal. The individual corpuscular richness in hæmoglobin is never above par, as in pernicious anæmia. In severe cases the oligocythæmia may be almost as great as in the latter disease. Alterations in the size and form of the red cells occur; microcytes and megalocytes are sometimes noted, the former more frequently, however.

Poikilocytosis and degenerative changes-vacuolation and polychromatophilia-occur in the red blood-corpuscles; normoblasts, the presence of which may be regarded as evidence of a regenerative effort on the part of the blood-making organs, are likewise to be seen.

The number of leukocytes varies considerably ; if there is a leukocytosis, it eonsists of an increase in the polynuclear neutrophiles. 


\section{CHAPTER IV.}

\section{DISEASES OF THE CIRCULATORY SYSTEM.}

\section{HEART AND PERICARDIUM.}

\section{ATROPHY.}

Hypoplasia of the heart-a congenital smallness-is often associated with a similar malformation of the aorta and other bloodvessels, and of the generative organs. This condition has been noted especially in connection with chlorosis. The heart is uniformly affected in all its parts.

Brown atrophy, an acquired change associated with pigmentation, is seen in cases of wasting diseases, as tuberculosis and

Frg. 137.

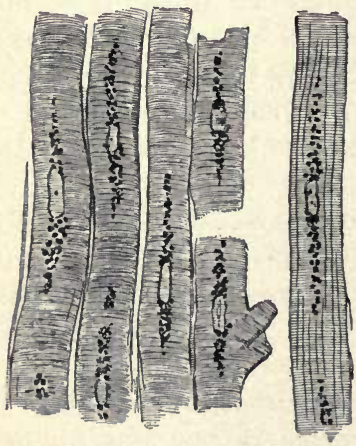

Brown or senile atrophy of the heart (Ribbert). The muscleat the ends of the nuclei are collections of pigment-granules. fibres are reduced in diameter; cancer; and especially also in old age. 'The heart is dark brown in color, and quite small, often not weighing more than a third of normal; its walls are thin and cavities contracted. The epicardium is frequently wrinkled, the coronary arteries prominent and tortuous, due to the loss of subpericardial fat. On microscopical section the muscle-fibres are found to be thinner than normal, and in the centre of each is a brown spot of pigment (Fig. 137). Similar pigmentation is also often associated with cardiac hypertrophy.

The resulting symptoms are not usually serious, as the lessened power of the cardiac muscle is but commensurate with the decreased demand made upon it. 


\section{HYPERTROPHY.}

Three varieties of cardiac hypertrophy are usually described, according to the size of the cavities associated with the increased thickness in the muscle-walls; simple, in which the cavities are of normal size; concentric, in which they are smaller than normal; and eccentric, in which they are dilated. In simple dilatation also the heart is enlarged, but not hypertrophied.

Causes: The hypertrophy is the result of increased demands made upon the heart-muscle. Stenotic and incompetent valves are a very frequent cause. General arterio-sclerosis, often

Fig. 138.

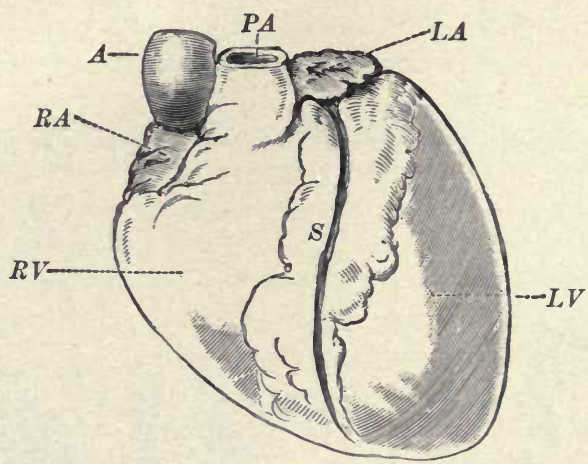

Hypertrophy of left ventricle (front view). Heart is elongated. Septum occupies middle of anterior surface. (From a case of granular kidney from a specimen in Charing Cross Hospital Museum (Green).)

associated with interstitial nephritis, greatly increases the work of the left ventricle and leads to its hypertrophy (Figs. 138 and 139). Obstruction to the pulmonary circulation, as, for instance, in fibroid phthisis and emphysema, leads to hypertrophy of the right ventricle (Figs. 140 and 141). Simple functional overactivity, as in hysteria and exophthalmic goitre, may lead to a uniform hypertrophy of the whole heart.

Dilatation occurs where the heart-muscle is exhausted and unable to overcome the impediment.

Microscopically is noted an increase in the size of the muscle-fibres; there is also probably an increase in their 
number. Fibroid and fatty degenerative changes are usually associated with dilatation. The size and weight of the organ

FIG. 139.

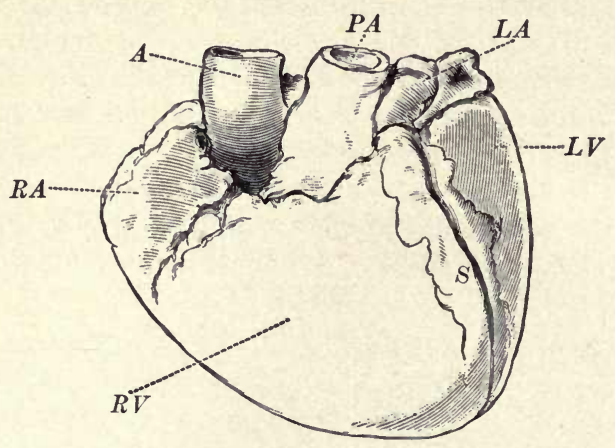

Hypertrophy of right ventricle (front view). Heart is quadrilateral and septum is displaced to the left. Right auricle is dilated. (From a case of chronic bronchitis and emphysema; specimen in Charing Cross Hospital Museum (Green).)

FIG. 140.

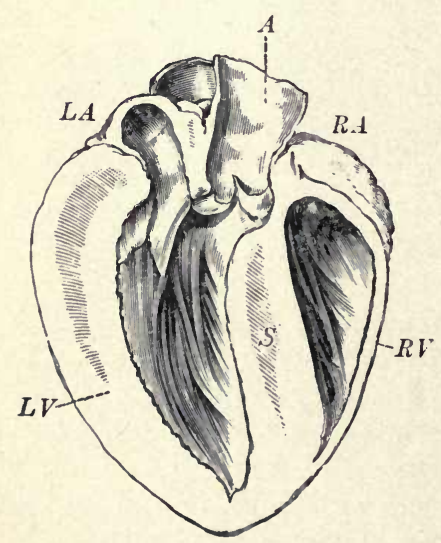

Anterior half of heart (Fig. 138), seen from behind. Left ventricle forms the whole of apex. Wall of $L V$ : wall of $R V:: 10: 2$ (normal proportion, 5:2) (Green).
FIG. 141.

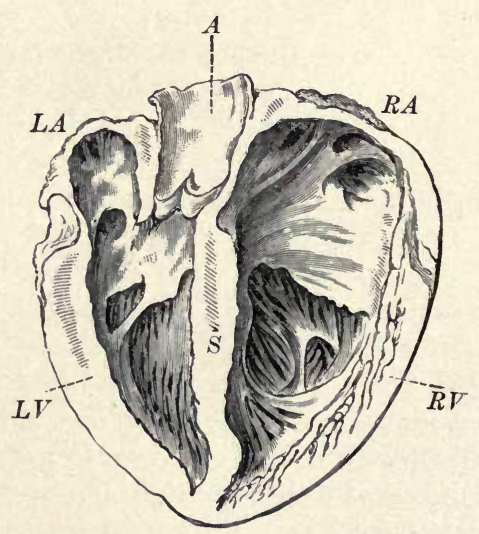

Anterior half of heart (Fig. 139), seen from behind. Right ventricle is seen to take greater share in formation of apex than left ventricle does. Wall of $R V$ is much thickened, but not so thick as that of the left. Tricuspid orifice and $R A$ are dilated (Green). 
are often so greatly increased as to justify the term "cor bovinum". As long as the hypertrophy is compensatory there are no symptoms; but when the cardiac muscle can no longer meet the demands made upon it, the results are most serious. If the left ventricle is at fault, there is a passive congestion of the pulmonary circulation. When the right ventricle is at fault, or in turn becomes involved, there occur passive congestion of the general systemic circulation and dropsy.

\section{DEGENERATIVE CHANGES.}

\section{Cloudy Swelling.}

Cloudy swelling occurs either as an early stage of inflammation or as a precursor of fatty degeneration. It is noted especially in the course of diphtheria, smallpox, scarlet fever, and other infectious diseases; also in rheumatism and after severe burns.

The myocardium is soft-even friable, pale, and looks as if it had been slightly boiled.

Microscopically the muscle-fibres are seen to be swollen-the spaces between the individual muscle-fibres being smaller than normal-and their transverse striations are indistinct. The fibres appear as though seen through ground glass or a layer of dust, due to the presence of innumerable fine albuminous granules.

\section{Fatty Infiltration.}

Normally there is a certain amount of adipose tissue beneath the epicardium, especially along the course of the coronary arteries, lower border of the left ventricle, and at the apex.

Pathologically the adipose tissue may not only form a thick coating over the entire surface, but even extend into the walls of the heart between the muscle-fibres, deposits even appearing at places under the endocardium. The muscle-fibres themselves are not involved, except that they are often atrophied as the result of pressure, when the accumulation of adipose tissue is excessive. Besides the cardiac weakness thus resulting, a fatty degeneration of the muscle-fibres may be induced. 


\section{Fatty Degeneration.}

In fatty degeneration the individual muscle-fibres are involved, the cell-protoplasm being replaced to a great extent by small globules of fat. These droplets appear first in the neighborhood of the nucleus; but may finally occupy the whole fibre, giving rise to an appearance somewhat resembling cloudy swelling; the granules are larger than in the latter condition, however, dissolve in alcohol, chloroform, or ether, and stain black with osmic acid (Fig. 142). The main factor

FIG. 142.

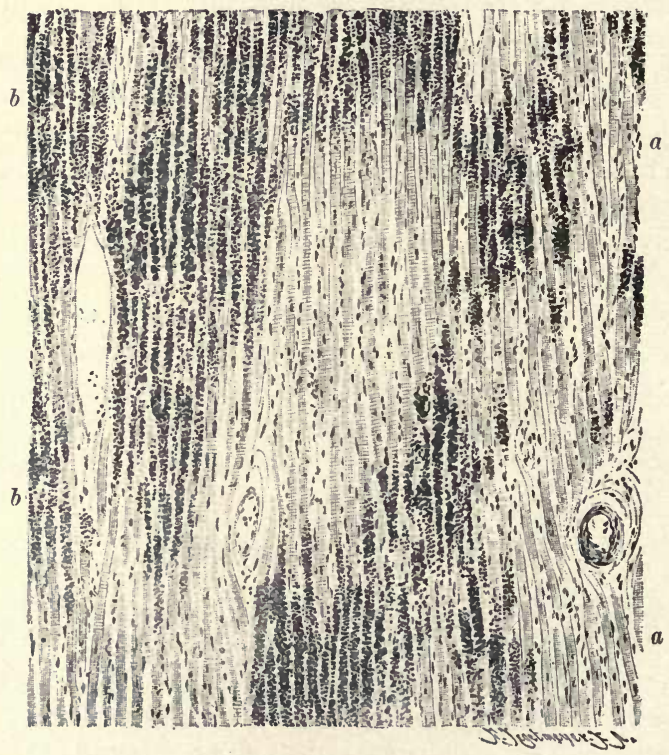

Well-marked chronic fatty degeneration of heart. $a$. healthy; $b$, fatty degeneration of muscle-fibres. $\times 80$ (Ziegler).

in its production is either a general or a local anæmia. The local anæmia may be brought about by sclerosis of the coro- nary arteries, or an improper circulation of the blood through them as the result of a valvular lesion. The condition is 
frequently associated with pernicious anæmia, leukæmia, phthisis and other wasting diseases ; and with arsenic- and phosphorus-poisoning.

The macroscopic appearance of the heart is quite characteristic. It is usually pale, flabby, and dilated. The degenerative change generally occurs in patches, the matted appearance produced thereby being most readily seen from within, beneath the endocardium. When the process is diffuse the cardiac muscle has a uniform yellowish hue.

Myomalacia cordis is a localized softening - a necrosis-of the heart-wall due to embolic or thrombotic obstruction of the coronary arteries. The area from which the blood is cut off is often wedge-shaped, pale yellow in color, and depressed below the surface.

Amyloid and hyaline degenerations are sometimes noted. Calcareous infiltration of the myocardium occurs, but is extremely rare.

\section{INFLAMMATIONS.}

\section{Myocarditis.}

Inflammation of the heart-muscle may be primary, but is much more frequently secondary to a peri- or endocarditis. It occurs in the course of acute infectious fevers, as typhoid, diphtheria, scarlet fever, smallpox, and puerperal septicæmia. The heart-muscle is friable and has a yellowish-red and matted appearance; the cavities are usually dilated. Microscopically, there is noted in addition to the cloudy swelling of the muscle-fibres a round-celled infiltration of the supporting connective-tissue framework; some are proliferated connective-tissue cells, the majority, however, being migrated leukocytes. Abscesses, appearing macroscopically on section of the heart-wall as yellowish-white spots, may occur. They are most frequent in the anterior wall of the left ventricle, and vary from the size of a pinhead to that of a cherry.

In fibrous or chronic myocarditis there is a great increase in the interstitial connective tissue. This fibroid change or substitution may be diffuse or circumscribed. Diffuse intermuscular sclerosis is often associated with general arterio- 
sclerosis and fibroid change in the kidneys and other organs. As a result, the heart may be hypertrophied by the increased work imposed upon it, but much more frequently the musclefibres are atrophied by the mechanical pressure of the newly formed fibrous tissue, and are found to have undergone granular and fatty degeneration. Circumscribed areas of fibroid substitution are often consecutive to degenerative changes produced by obstruction of the coronary arteries. A saccular dilatation-aneurism-may result from the thinned heart-wall giving way to pressure from within at such a point.

\section{Endocarditis.}

Inflammation of the endocardium usually affects the valves; when affecting the cavities of the heart it may be designated specially as mural endocarditis.

Acute endocarditis is rarely a primary affection. The most frequent cause is acute articular rheumatism. It occurs also

FIG. 143.

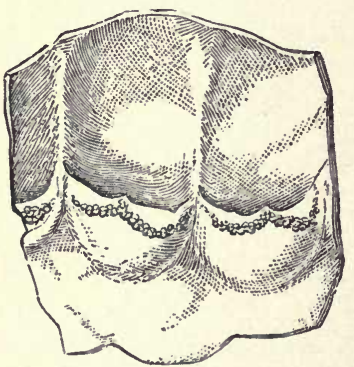

Inflammation of aortic valves, the earlier stage of the process, showing the situation of the inflammatory granulations (Green).
FIG. 144.

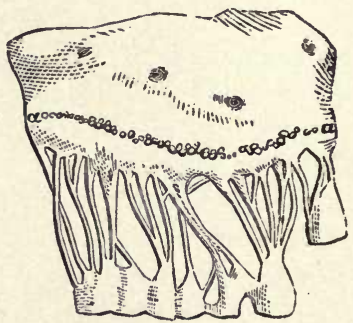

Inflammation of mitral valve, the earlier stage of the process. Valve seen from the auricular surface, showing the situation of the inflammatory granulations (Green).

in the course of pneumonia, puerperal septicæmia, scarlet fever, typhoid fever, gonorrhœa, and, in fact, in any acute infectious disease. It occurs also in Bright's disease, cancer, and diabetes.

The most characteristic lesions are the so-called warty vegetations formed along the line of contact of the valves, especially on the auricular aspect of the mitral, and in a row 
of bead-like elevations on the ventricular surface, some little distance from the margins of the cusps of the aortic (Figs. 143 and 144). These small warty elevations are less frequently seen on the tricuspid and pulmonary valves, and on the chordæ tendinex and parts of the mural endocardium. They crumble and can be easily detached by the finger, leaving behind a slightly roughened surface, or possibly a small ulcer the size of the base of the vegetation. The change in the endocardium produced by the inflammatory process and the extra irritation and friction at the line of contact of the valves on closure, invite the deposition of fibrin; there is an active proliferation of the endothelial cells, which, together with migrating leukocytes, infiltrate the layers of fibrin and tend to bring about the organization of the clot which has formed.

In some cases the inflammatory process is especially severe and there is a tendency to suppuration and ulceration. Small collections of pus may be scen at the base of the valves. The masses of fibrinous deposit are more abundant and irregular, occurring indiscriminately over the endocardial surface of the valves and heart-wall. Where there has been actual destruction of tissue there will be noted on section, microscopically, at the bases of these vegetations, typical granulation-tissue, to which the clot is firmly adherent. If the section is properly stained, micro-organisms may usually be seen in the superficial layers of the endocardium. The vegetations are liable to be broken off and carried in the circulation, as emboli, to distant parts. If the micro-organisms are likewise transported, metastatic abscesses in various organs are produced.

Chronic endocarditis results in various distortions of the affected leaflets of the valves, and, in consequence, serious disturbances in the circulation. The vegetations become fibrous and have a broad base. As in all chronic inflammations, there is a tendency toward the overproduction of connective tissue. The proliferating endothelial and connective-tissue cells and infiltrating leukocytes become organized into fibrous tissue. The valves are thickened, rigid, and eventually retracted and distorted. The opposed inflamed leaflets of the valve may become agglutinated or firmly adherent to the adjacent heartwall and cause still further deformity. The chordæ tendineæ 
also become thick and rigid, and interfere with the function of the valves. The dense cicatricial connective tissue may become infiltrated with lime salts.

In some cases the inflammatory process may be chronic from the first. This is especially liable to occur in persons of a dvanced years, and also in connection with chronic alcoholism, syphilis, and gout, which are active factors also in producing general arterio-sclerosis. The alterations in the valves lead to stenosis, an obstruction to the flow of blood; or insufficiency, when the valves cannot close, and thus prevent the regurgitation of the blood. A valve may be both stenotic and insufficient.

Either condition necessarily leads to imperfect circulation of the blood through the heart. The natural result of the increased work thrown upon the heart-muscle is hypertrophy. When this compensatory hypertrophy fails, most serious changes occur in the various organs. The lungs become œedematous and gradually indurated, as the result of an increased proliferation of the interstitial connective tissue. The pulmonary vessels are distended, even varicose; thrombi may form, as the result of the sluggish circulation. The liver, spleen and kidneys are enlarged by reason of their passive congestion. The countenance becomes cyanosed from the stagnation of the circulation and lessened oxidation; there are serous effusions into the peritoneal, pleural, and pericardial sacs and general œdema of the subcutaneous tissue and skin. The mucous membrane of the gastro-intestinal tract is swollen and odematous.

\section{Pericarditis.}

Inflammation of the pericardium may be a primary affection, occurring in connection with rheumatism and Bright's disease, and various acute infections fevers, as pneumonia, typhoid, scarlet fever, influenza, and puerperal septicæmia; or much less frequently it may be a secondary affection resulting from the extension of an inflammatory process from neighboring structures to the pericardium.

The membrane first becomes dull and lustreless. The character of the exudate varies, usually purely serous, but sometimes hemorrhagic or seropurulent. The opposed sur- 
faces of pericardium often look as if the two layers had rubbed. together, while the more or less marked deposit of fibrin was still in a soft plastic condition, giving them a rough, ragged, or hairy appearance, suggesting the term "cor villosum."

The parietal and visceral layers may become agglutinated, constituting what is known as adherent pericardium, which may lead to hypertrophy of the heart, by reason of the increased work thus imposed upon it.

Sclerotic areas, known as white spots, or milk-spots, are probably the result of a chronic localized inflammation; they are noted in about 50 per cent. of all post-mortems. They may be quite small, or large enough to cover the anterior surface of the heart. They occur most frequently on the right ventricle, next on the left ventricle, near the apex, and also on the posterior surface of the heart, near its base. They are formed simply of dense connective tissue, covered by endothelium. Their exact significance is not fully understood.

\section{THE BLOODVESSELS.}

\section{DEGENERATIONS.}

Fatty degeneration: Not infrequently there are seen in the aorta and other parts of the arterial system yellow spots or streaks slightly raised above the internal surface; under the microscope it is found that the endothelial cells of the intima have undergone fatty degeneration. The degeneration may involve also the muscle-cells of the media. The affected areas may soften and small superficial erosions result.

Calcareous infiltration most frequently affects the middle coat of the artery, occurring by preference in vessels of medium size. The greatly increased rigidity of the radials, often noted clinically, is generally due to this cause.

Amyloid degeneration usually begins in the capillary walls of the organ affected. The entire wall is involved.

\section{ARTERITIS.}

Acute inflammation of the walls of an aitery, independent of any neighboring inflammatory process, is very rare. 
Chronic arteritis, atheroma, or arterio-sclerosis, however, is of frequent occurrence. The aorta alone may be affected, but more commonly the whole arterial system is more or less uniformly involved; the veins even may be involved ("angiosclerosis"). It is usually associated with sclerotic changes in the heart and internal organs, and seems to be dependent upon the same causes-syphilis, gout, rheumatism, lead-poisoning, chronic alcoholism, Bright's disease, and old age. It sometimes follows acute infections, as typhoid and scarlet fever, and occasionally seems to be dependent on the cachexia of cancer and tuberculosis.

In the aorta at an early stage of the process there are pale pinkish, rounded, or oval patches or nodules beneath the smooth and unaltered intima, varying in size from a mere point to half an inch in diameter. On cutting into one of these patches it is found to be firm and fibrous; in the centre there may be a yellow focus of fatty degeneration. Microscopically the patch is composed of dense fibrous tissue which seems to have been produced by a proliferation of the subendothelial connective-tissue cells; at first many round, oval, and stellate cells can be readily seen, but later the structure of the affected area may be quite indefinite from advancing fatty degeneration. The calcareous plates often met with in the aorta are the result of the infiltration of such patches with the salts of lime; these plates may be so numerous as to alter completely the appearance of the vessel.

In the smaller vessels the changes are somewhat different. Similar patches of opaque and firm tissue, one-sixth to onethird of an inch in length, may occur at irregular intervals along the course of the vessels. On cross-section of the artery its lumen is seen to be considerably encroached upon at one side.

In other cases-endarteritis obliterans-these patches are entirely absent; the lumen of the vessel is uniformly encroached upon, sometimes almost obliterated by the increased thickness of the intima produced by an active proliferation of the endothelial and subendothelial connective-tissue cells. The musclefibres of the media are atrophied, and there is usually a marked thickening of the adventitia, the result of a round-celled infiltration which becomes more or less fully developed into fibrous tissue. 
Serious consequences may follow these changes in the arterial walls. In the coronary arteries the obstruction of the circulation may be the immediate cause of fatty degeneration of the heart-muscle and death. In the brain the anæmia resulting from such an obstruction may result in areas of softening. In the aorta aneurismal dilatation is a frequent result. The heart is generally hypertrophied in consequence of the obstruction to the circulation offered by the thickened and rigid arterial walls.

\section{Aneurism.}

An aneurism (Fig. 145) is a localized dilatation of an artery, resulting from any cause which weakens its walls-as arteritis and injury, and from increased arterial pressure. Aneurisms may be classified according to their shape into fusiform, cylindrical, and saccular.

In fusiform and cylindrical aneurisms there is a general dilatation of all the coats of the artery. A cirsoid aneurism is one in which the vessel is rendered tortuons and convoluted as the result of its elongation and unequal dilatation of different parts.

In sacculated aneurisms, the most important variety, there is a unilateral dilatation of the vessel. First, the media gives way, and then there is a dilatation of the intima and adventitia, and thus is formed a sac which communicates with the artery by a narrow opening. As the sac increases in size it may finally rupture. The blood may be retained subsequent to rupture by the surrounding tissues; the term false aneurism is used to designate such a cavity, communicating with an artery and containing blood, the walls of which are not formed by the coats of the vessel. A dissecting aneurism is one in which rupture of the intima occurs and the blood burrows a false passage between the coats of the artery.

The results of the continued presence of the aneurism may be extensive necrosis of neighboring tissues. Erosion of the vertebre and pressure of the aneurism on the spinal cord have been the unsuspected cause of a paraplegia. In a similar manner the sternum and ribs may be eroded and the aneurism project beneath the skin. 
FIG. 145.

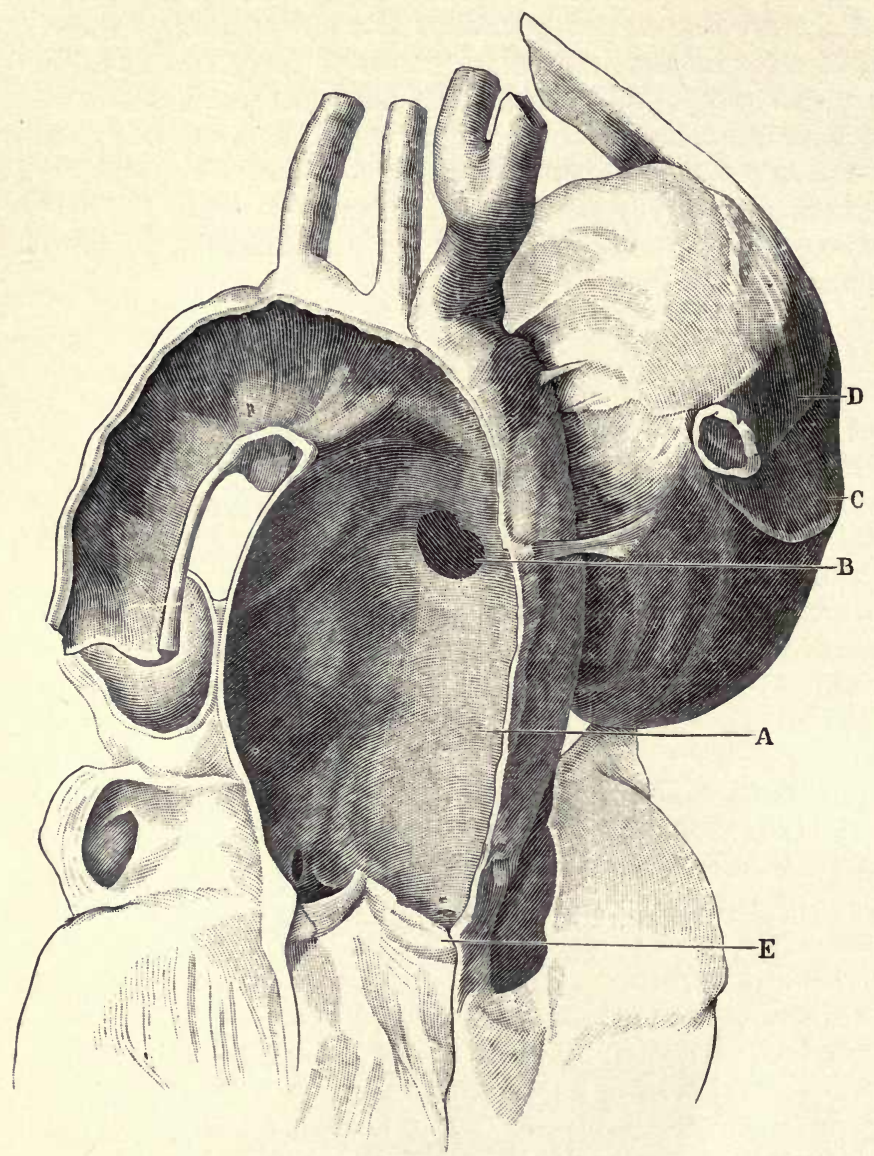

Aneurism of the ascending aorta (woman, aged 30 ). $A$, aorta ; $B$, orifice of communication; $C$, aneurismal sac; $D$, rib adherent to the sac; $E$, shrunken aortic valves (Ziegler).

\section{PHLEBITIS.}

Acute inflammation of a vein occurs either in connection with the formation of a thrombus-thrombo-phlebitis, or is associated with an inflammatory process in surrounding tissues, which may produce thrombosis also, secondarily. 
Chronic phlebitis may occur in association with arteriosclerosis, the changes being of the same character, but rarely so marked. Calcareous infiltration of the affected areas is much less common.

Varix is a dilatation of veins analogous to the aneurismal dilatation of arteries. The essential factor in its production is an increase in blood-pressure within the veins, as by passive hyperæmia due to heart disease, pressure of a tumor or the gravid uterus.

The veins are not only dilated but elongated, and thus become tortuous and convoluted; the circulation through them is greatly slowed, and it is not infrequent for thrombosis to occur in consequence. 


\section{CHA PTER V.}

\section{DISEASES OF THE RESPIRATORY ORGANS.}

\section{THE NASAL PASSAGES.}

Acute rhinitis, or coryza, inflammation of the mucous membrane of the nose, occurs most frequently as a simple "cold in the head" due to exposure; it may also accompany influenza, measles, typhoid fever, and other infectious diseases. Diphtheria may primarily involve, or extend to, the nasal passages. The inflammatory hyperæmia gives rise to a feeling of stuffiness in the nose, and the subsequent exudation to the mucopurulent discharge.

Chronic rhinitis supervenes as the result of repeated acute attacks. The mucous membrane becomes thickened and the nasal passages obstructed-hypertrophic rhinitis. Later there may be complete atrophy of the mucous membrane; in such cases there is usually an extremely fotid, purulent discharge -atrophic rhinitis or ozana.

Syphilis may affect the nose, in the tertiary stage, in the form of gummata, beginning in the mucous membrane, periosteum, or perichondrium. There may be extensive ulceration and destruction of tissue, resulting in serious deformities.

\section{THE LARYNX.}

Acute inflammation of the larynx-laryngitis-may be due to exposure to cold or inhalation of irritating vapors; and accompanies measles, whooping-cough, smallpox, typhoid fever, and other infectious diseases. In some cases there is such an nedematous infiltration of the loose submucous tissue over the aryepiglottic folds and at the base of the epiglottis as to give rise to serious, even fatal, obstruction.

The severe inflammatory process excited by the diphtheria bacillus is especially characterized by an exudate rich in fibrin, which coagulates and adheres to the larynx as a grayish 
or yellowish pseudomembrane. Microscopically this pseudomembrane is found to consist mainly of the fibrillæ of fibrin and migrated leukocytes.

Chronic laryngitis may follow repeated acute attacks, or result from overuse of the voice ; or it may be secondary to diseases of the nasopharynx. The mucous membrane is thickened, due mainly to an increase of connective tissue, which is characteristic of all chronic inflammations; it has quite frequently a granular appearance as a result of enlargement of the racemose glands of the larynx, and often there are superficial ulcerations or erosions.

Tuberculosis of the larynx is generally secondary to pulmonary tuberculosis, occurring in about 30 per cent. of these cases. The mucous membrane is thickened from the presence of characteristic tubercles or a diffuse infiltration; to this succeed caseation and ulceration, which may result in destruction of the vocal cords.

Syphilis also may cause deep infiltrations, necrosis, and ulceration; secondarily serious deformities may arise from contraction of the newly formed connective tissue.

Papillomata constitute more than one-half the tumors of the larynx. They are especially common in people whose profession requires the constant use of the voice. They grow most frequently from the squamous epithelium of the vocal cords.

Epithelioma is the variety of cancer generally met in the larynx. It appears first in the form of a nodular thickening of the mucous membrane, which later undergoes ulceration.

Sarcoma of the larynx, which is usually of the spindlecelled variety, is not of frequent occurrence.

\section{The Bronchial Tubes.}

Acute inflammation of the bronchial tubes-bronchitisresults frequently from the extension of a simple inflammatory process in the upper air-passages; it is also almost a constant manifestation of certain acute infectious diseases, as typhoid fever, measles, scarlet fever, whooping-cough, and influenza. The larger and medium-sized bronchi are involved; in children, however, the terminal bronchioles and surround- 
ing lung tissue, are specially liable to be affected, constituting lobular or eatarrhal pneumonia.

The mucous membrane of the bronchi is intensely red and swollen. Upon the character of the inflammatory exudate

FIg. 146.

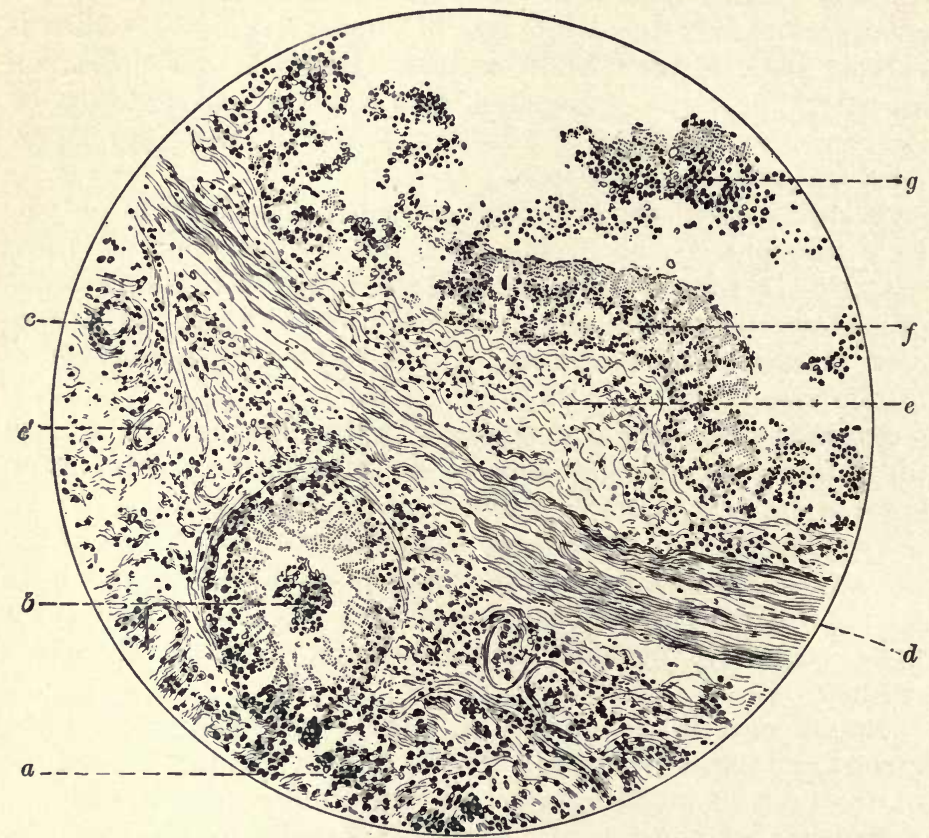

Catarrhal bronchitis. $a$, areolar tissue of the submucosa, infiltrated with serum and leukocytes; $b$, alveolus of a mucous gland, infiltrated at the periphery by leukocytes. The epithelium is undergoing colliquative necrosis, and in the centre of the lumen are a few leukocytes with fibrin. $c, c^{\prime}$, bloodvessels; $c^{\prime}$, shows an infiltration of the wall by emigrating leukocytes; $d$, muscularis mucosæ; $e$, subepithelial areolar tissue of the mucous membrane, infiltratcd with serum and leukocytes; $f$, columnar epithelium of the surface in a state of colliquative necrosis: $g$, exudate within the bronchus. In this portion of the bronchus the destructive processes are so acute that the epithelium is destroyed, instead of stimulated to the production of excessive mucus (Dunham).

depends the appearance of the sputum. At first it is a glairy, tenacious fluid containing a few leukocytes-simply an exaggeration of the normal secretion of the glands of the mucous membrane. Later it becomes yellowish in color, muco- 
purulent or purulent, and less tenacious, histologically resembling pus by reason of the large number of leukocytes present. When the amount of this secretion is excessive, especially in more chronic cases, the condition is known as bronchorrhoea; in some instances the odor of the sputum is very offensivefoetid bronchitis.

Microscopically the blood vessels are found to be dilated, and surrounding them and infiltrating the walls of the bronchi are numerous migrated leukocytes and young connective-tissue cells. The epithelial cells of the mucosa are degenerated and in many places desquamated (Fig. 146).

Chronic bronchitis occurs most commonly in the aged, who are often predisposed by reason of a passive congestion of the lungs, due to some form of cardiac weakness. It may follow a neglected acute attack or gradually develop after repeated subacute attacks. It is also frequently associated with emphysema or other diseases of the lungs, with gout and Bright's disease, and, in children, with rickets.

The bronchial walls are usually greatly thickened from new connective-tissue formation in the outer fibrous coat. The mucous membrane is generally atrophied; the epithelial cells are no longer columnar, but rounded or polygonal. In some cases, however, the mucous membrane is swollen and hypersemic, and dotted with little gray points-the mucous glands, which are greatly enlarged.

The cartilages of the bronchi and the muscular coat may be so far supplanted by newly forming connective tissue as greatly to weaken their walls. This in connection with increased pressure from within, due to the increased expiratory efforts of coughing, may cause fusiform or saccular dilatation of the bronchi in places, bronchiectasis.

Fibrinous bronchitis is a variety of chronic bronchitis in which there are expectorated at intervals, during months or years, branching fibrinous casts of the bronchi of a lobe. 'The exact pathology of the affection is not understood. Similar casts of the larger tubes may result from an extension of a diphtheritic inflammation of the larynx or trachea. 


\section{THE LUNGS.}

\section{ATELECTASIS.}

Atelectasis is a condition in which the lung is compressed or collapsed, so that there is an absolute absence of air from the air-vesicles.

Congenitally it occurs, especially posteriorly and at the base of the lungs, from some mechanical obstruction of the air-passages, as the presence of meconium; or an inability to take a full inspiration, as the result of extreme weakness. Under these circumstances the tern apneumatosis is sometimes applied. The affected areas are bluish, and so solid that if a portion is thrown into water it sinks.

Later in life a large pleuritic effusion, or a wound in the chest-wall, may result in the collapse of a whole lung. A lobular collapse occurs in broncho-pneumonia, and is supposed to result from a valve-like obstruction of a bronchiole by a plug of mucus, which cannot be dislodged by inspiration, but permits the air to escape from the lobules supplied. The tissue is dry and tough, has a firm, flesh-like feel, does not crepitate, and sinks if placed in water. If the condition persists, there is finally an entire and permanent obliteration of the air-vesicles by an overgrowth of connective tissue.

\section{EMPHYSEMA.}

Interstitial emphysema is a condition analogous to emphysema of subcutaneous tissues; there is an accumulation of air in the connective-tissue septa between the lobules of the lung as the result of rupture of an air-vesicle. It occurs most frequently in the course of whooping-cough or broncho-pneumonia in young children; and from excessive straining-efforts in parturition, defecation, coughing, etc.

By vesicular emphysema we understand a dilatation of the air-vesicles which occurs as the result of a weakened condition of the lung-tissue from inflammatory or degenerative processes, lessening its ability to resist increased pressure within, from such causes as chronic cough, blowing of windinstruments, etc. The lungs are considerably larger than normal; there is a gritty feeling on section, and the dilatation 
of the air-spaces may be so great as to be apparent to the naked eye.

Microscopically the air-vesicles are found to be rounded instead of polygonal, and are increased in diameter. The

FIG. 147.

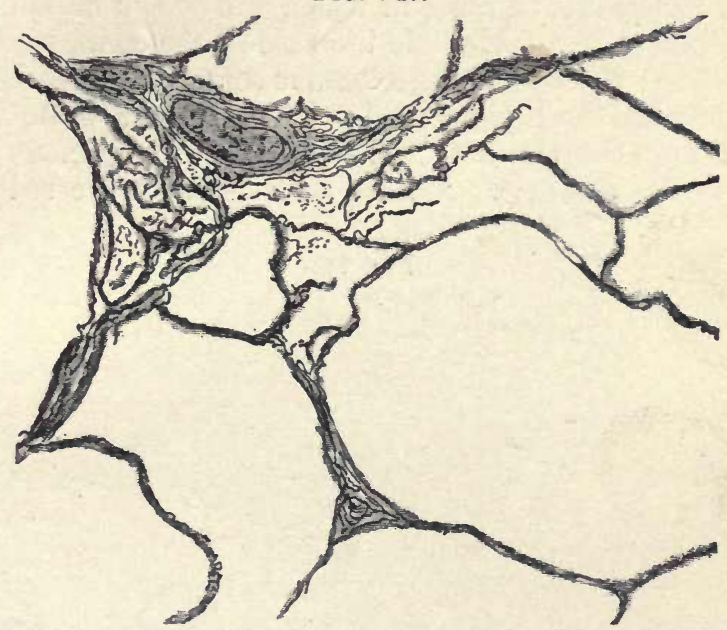

Section from an emphysematous lung (Ribbert). The pulmonary alveoli are enlarged; their walls are stretched and thinned; atrophied because of repeated excessive air-pressure within the alveoli. In more extreme cases of emphysema the atrophy of the alveolar walls may lead to their total destruction in places, so that the cavities of neighboring alveoli communicate. (Compare with Fig. 149.)

alveolar walls are thinned (see Fig. 147); and the bloodvessels, being stretched over a larger surface, are narrowed, the anæmia of the lung-tissue thus produced further favoring the atrophy and degenerative changes in the alveolar septa. As a remote consequence of the increased pulmonary blood-pressure there is hypertrophy of the right heart.

\section{PASSIVE HYPERÆMIA.}

Passive hyperæmia, brown induration, or brown sedema, results from some obstruction to the outflow of blood from the lungs, most frequently from some myocardial degeneration, or valvular lesion, especially mitral; though it may occur in connection with aortic stenosis, or regurgitation. 
The lungs are heavy and somewhat larger than normal. On section they are dark red in color, and on pressure a brownish-red serum exudes, frothy from admixture of airœdema. In other and more chronic cases the lung-tissue is dry and indurated.

Microscopically, in cases in which the brown induration is marked, there is considerable increase in the connective tissue of the lung; the pleura, interlobular septa, and alveolar walls are thickened and pigmented. The alveolar walls have a beaded appearance, due to the passive engorgement of the capillary bloodvessels. Within the air-spaces are red blood-

FIG. 148.

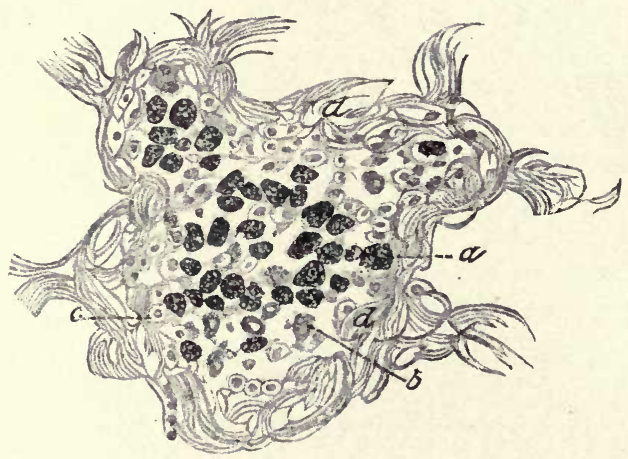

Brown induration of lung. $d$, prominent capillaries in alveolar wall ; $a$, cells containing pigment in lumen of alveolus; $b, c$, alveolar epithelium. $\times 250$ (Schmaus).

corpuscles, numerous lenkocytes, and desquamated epithelial cells-many of which contain pigment derived from the altered red blood-corpuscles which they have englobed and digested (see Fig. 148). Later, these cells having been taken into the lymphatic system, the pigment is thus distributed.

Ædema of the lungs may also result from active congestion, produced by inhalation of very hot or cold air, irritating gases, etc. Microscopically the alveoli are found to contain a few leukocytes, desquamated epithelial cells, and a granular debris representing the coagulated serous exudate. 


\section{HEMORRHAGIC INFARCT.}

Infarctions are usually multiple, and occur in the centre of the lower lobe, or near the root of the lung; when superficial they form a slight elevation beneath the pleura. They are wedge-shaped, firm, brown in color, and are separated by a distinct line of demarcation from the surrounding lung-tissue.

FIG. 149.

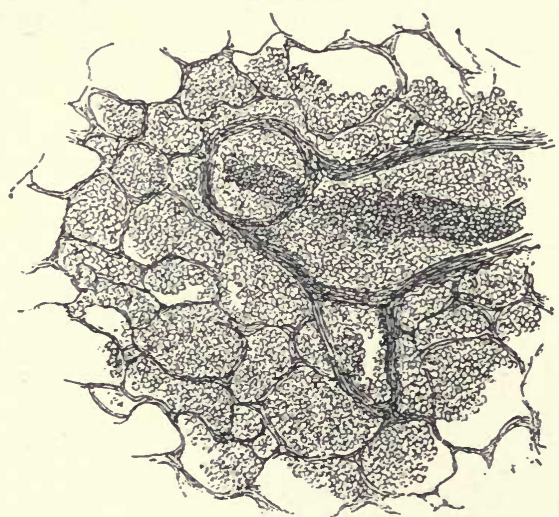

Hemorrhagic infarct of the lung (Kaufmann). The section contains a portion of the plinged vessel bevond the site of the embolus. It and the pulmonary alveoli are filled with blood, which, in the latter, has passed through the capillary walls, rendered pervious by malnutrition. This blood may be derived from the pulmonary vein and also from the bronchial artery, which communicates with the capillaries of the alreolar walls.

Microscopically the air-spaces are found to be crowded with red blood-corpuscles (see Fig. 149).

Pneumonia is the term employed to designate inflammation of the lung, in contradistinction to inflammation of the bronchi or pleura. Histologically and clinically there are several well-defined varieties of pneumonia :

\section{LOBAR PNEUMONIA.}

Lobar pneumonia is an acute, infectious, self-limited disease, with a definite clinical course, and characterized pathologically by an extensive inflammation of the lung, involving an entire lobe. 
Etiology: The diplococcus pneumonio is found in the inflammatory exudates and in the sputum; it is the micro-organism nost frequently associated with the disease, though the pneumococcus of Friedländer, streptococcus, staphylococcus, typhoid bacillus, and bacillus of influenza seem to be capable of producing similar lesions.

The lower lobe of the right lung is most frequently affected.

Division of process: The inflammatory process may be divided into three stages: engorgement, red and gray hepatization.

In the stage of engorgement, or congestion, the affected area is deep red in color, crepitates less and is somewhat firmer than normal, but is not solidified, does not sink if placed in water ; the overlying pleura is dull and lustreless. On section a bright red, frothy fluid exudes on pressure.

Microscopically the capillary bloodvessels of the alveolar walls are greatly distended with blood and encroach somewhat upon the air-spaces, within which are a large number of red blood-corpuscles, and a few leukocytes and desquamated epithelial cells. A similar exudate is seen in the smaller bronchi.

In the stage of red hepatization the inflammatory exudate which has poured into the air-spaces has coagulated and completely solidified the lung-tissue, giving it a liver-like consistency. On pressure the affected area does not crepitate, is firm to the touch, yet friable, and if placed in water sinks. It is swollen, and upon the external surface the ribs have left an imprint. The pleura is often coated with a fibrinous exudate. On section the surface is bright red, smooth and glistening; later, as the fibrinous plugs within the air spaces contract, the surface, especially if torn, has a granular appearance, and with a little care the projecting alveolar plugs may be lifted or scraped from the air-spaces, of which they form a perfect mould.

Microscopically the air-vesicles are completely filled, even distended, with the inflammatory exudate. The exuded bloodserum has coagulated, and the fine fibrillæ of fibrin entangle within their meshes a large number of red blood-corpuscles and migrated leukocytes and a few epithelial cells derived from the swollen and proliferating epithelium of the alveolar 
walls. The exudate in the bronchioles and on the pleural surface is composed of similar elements. The capillary bloodvessels of the delicate walls of the air-spaces are much less

Fig. 150.

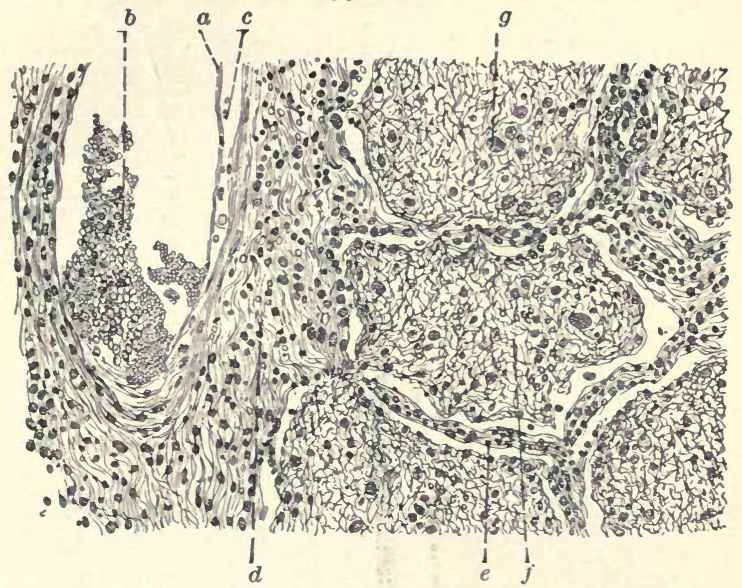

Section from lung in the second or exudative stage of croupous nneumonia. $a$, endothelial wall of a small vein: $b$, blood within the vein, unusually rich in leukocytes, which have collected during the slowing of the circulation. The line $b$ points to the nucleus of a leukocyte. Part of the blood has fallen out of the section during its preparation. $c$, leukocytes beneath the endothelium of the vascular wall; $d$, cedematous fibrous tissue surrounding the vessel. The fibres of the tissue have been separated by the exuded serum. This tissue is also moderately infiltrated with leukocytes that may have passed through the walls of the vein, and contains a few red blood-corpuscles. $e$, wall separating two pulmonary alveoli. This is also somewhat infiltrated with leukocytes, $f$, exudate within all alveolus, consisting of serum, fibrin, leukocytes, and red bloodcorpuscles; it also contains a few epithelial cells desquamated from the alveolar wall, $g$.

prominent than in the first stage, but are surrounded by migrated leukocytes and proliferating connective-tissue cells, forerunners of the hyperplasia of connective-tissue which occurs in some chronic pneumonic processes (see Figs. 150 and 151).

In the stage of gray hepatization the lung-tissue still remains solid, but becomes mottled in appearance, and finally a uniform gray in color.

Microscopically it is found that the red blood corpuscles have become decolorized or have disappeared, and the fibrin filaments are broken down and granular. The exudate does 
not now entirely fill the air-spaces; it is composed mainly of leukocytes, which have greatly increased in number and are now undergoing a fatty degeneration (see Fig. 152).

If the patient survives, the inflammatory process may terminate in resolution, abscess formation or gangrene.

FIG. 151. .

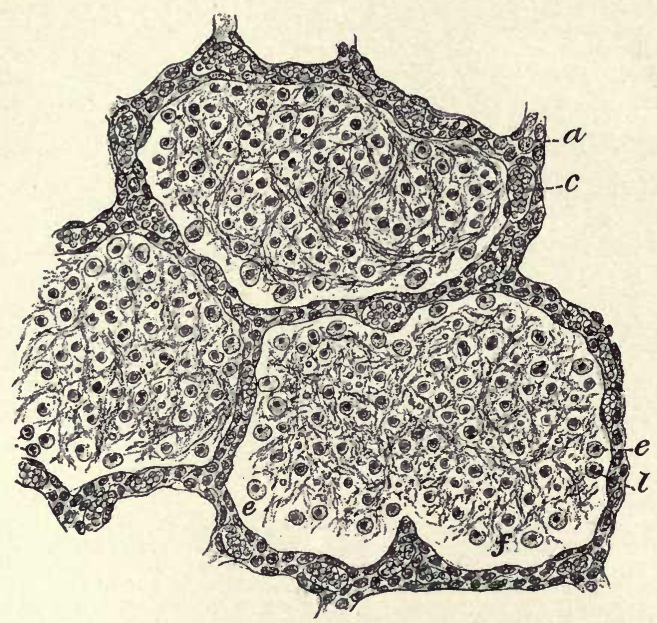

Croupous pneumonia-red hepatization. Three alveoli filled with fibrinous exudate. In the latter a mesh-work of fibrin $(f)$, desquamated alveolar epithelium $(e)$, leukocytes (l), and red blood-corpuscles, $a$, alveolar septa with capillaries. $\times 250$ (Schmaus).

Resolution is the most frequent termination, and is brought about by a fatty degeneration and liquefaction of the exudate, which is finally absorbed by the lymphaties, or expectorated.

Abscess-formation as the result of secondary infection with pyogenic micro-organisms is a rare termination. It occurs most frequently in the upper lobes.

Gangrene also is a rare termination. It is especially liable to occur in the drunkard, and in cases in which the circulation is exceedingly weak.

Associated lesions: Although the inflammatory process in the lungs is the characteristic feature of lobar pneumonia, not infrequently there are widespread pathological changes due to the general systemic intoxication. Circulatory failure due to 
degenerative changes in the myocardium is the most frequent cause of death.

FIG. 152.

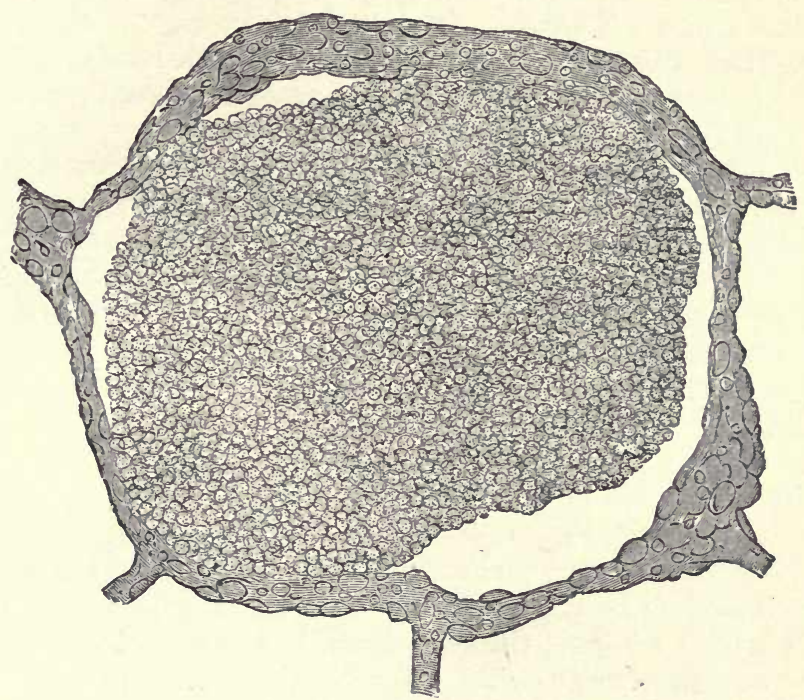

Croupous pneumonia-gray nepatization-showing the large accumulation of cellular elements within one of the pulmonary alveoli, which in some parts have undergone such extensive fatty degeneration that their distinctive outlines are no longer visible. $\times 200($ Greeu).

\section{LOBULAR PNEUMONIA.}

In lobular or bronchopneumonia the inflammatory process does not involve large areas of lung-tissue, though the coalescence of the smaller lobular areas of consolidation, which are more or less widely distributed throughout both lungs, may simulate a lobar pneumonia.

Etiology : Lobular pneumonia may be a primary affection ; but it is much more frequently secondary, occurring in the course of influenza, whooping-cough, measles, and other infectious diseases. In animals it can be experimentally produced by causing them to inhale steam or other irritating vapors; or by section of both vagi-the glottis being rendered insensitive, saliva and portions of food carrying harmful microorganisms are brought to the finer bronchi by inspiration. 
Bacteria play an important part in the production of catarrhal pneumonia, though no one organism is recognized as the specific cause. In a large percentage of cases Fränkel's pneunococcus is found.

Lesions: The inflammatory process seems always to begin in the terminal bronchioles, and to extend by contiguity and continuity to surrounding lung-tissue.

Scattered through both lungs there is a variable number of solid patches, some of which are due to collapse of the airvesicles, while some are the result of inflammatory exudation. The collapsed areas vary from one-tenth to one-fifth of an inch in diameter, and are depressed below the surface of the lung-tissue. The pneumonic patches vary from the size of a pea to that of a hazelnut or walnut, and are raised above the surface; the overlying pleura may be somewhat opaque from inflammatory exudation; the surrounding pulmonary tissue is often more or less emphysematous.

As a whole the lungs are congested but crepitant ; the solid patches, however, are airless and sink in water. On section the areas of collapse are found to be more or less conical in outline, with the apex directed toward the bronchi with which they are connected. The pneumonic patches are soft and friable, at first dark red, and later grayish-yellow in color; they are not separated by a sharp line of demarcation from the surrounding tissue. By the coalesence of such patches nearly a whole lobe may be involved, in which case it is often difficult to distinguish from the consolidation of a lobar pneumonia. The cut surface of such an area is smooth, however, not granular; irregular in outline; and there are neighboring smaller areas which have not as yet become fused to the larger one.

The inflammatory process is sometimes divided into two stages, red and gray splenization, corresponding to red and gray hepatization of the lobar variety.

Microscopically, in the early stage of red splenization, if a single lobule is examined, we note in the centre a small bronchus with infiltrated, thick walls; its lumen may be filled with leukocytes and desquamated epithelial cells. The interlobular connective tissue and alveolar walls are also infiltrated and thickened by proliferating connective-tissue 
cells and migrated leukocytes. The air-spaces immediately surrounding the bronchus are filled with a fibrillary network of fibrin, a variable number of red and white blood-corpuscles, and epithelial cells. A little further from the bronchus the air-spaces contain only the large, flat, rounded or oval cells derived from the proliferating epithelium which lines the alveolus.

In the later stage of gray splenization there are fatty degeneration and liquefaction of the alveolar exudate, part of which is finally expectorated and part absorbed by the lymphatics.

Resolution is the usual termination. With the absorption of the alveolar exudate the round-celled infiltration of the walls disappears; the whole process takes much longer, however, than in lobar pneumonia.

\section{CHRONIC INTERSTITIAL PNEUMONIA.}

Chronic interstitial pneumonia, or fibrosis, is characterized by an excessive formation of new fibrous tissue, involving the alveolar walls and the supporting connective-tissue framework of the lungs. It may be secondary to lobar or lobular pneumonia, or due to the inhalation of solid irritating patches -pneumokoniosis.

The affected lung is generally smaller than normal, and feels firm, fibrous, and elastic ; bronchiectatic cavities are frequently met. When due to the inhalation of particles of coal or carbon-anthracosis-the lungs are deeply pigmented; similar lesions occur in artificers in iron and steel-siderosisonly the coloration is brown instead of black; silicosis is the term applied to an analogous condition in quarrymen, in which the lungs have a marble-like or slate-like appearance, produced by inhalation of the dust-particles of dry silicious stone.

Microscopically there is noted thickening of the pleura, the interlobular septa continnous with its deeper layer, the peribronchial and perivascular tissue, and the interalveolar septa. The air-vesicles are compressed, their walls thickened and fibrous (Fig. 153). Following lobar pneumonia, cases have been described in which, in addition to the thickening of the walls of the air-spaces, there was organization of the 
alveolar exudate in places; appearing even a few days after the onset of the disease, were intra-alveolar plugs of newly

FIG. 153.

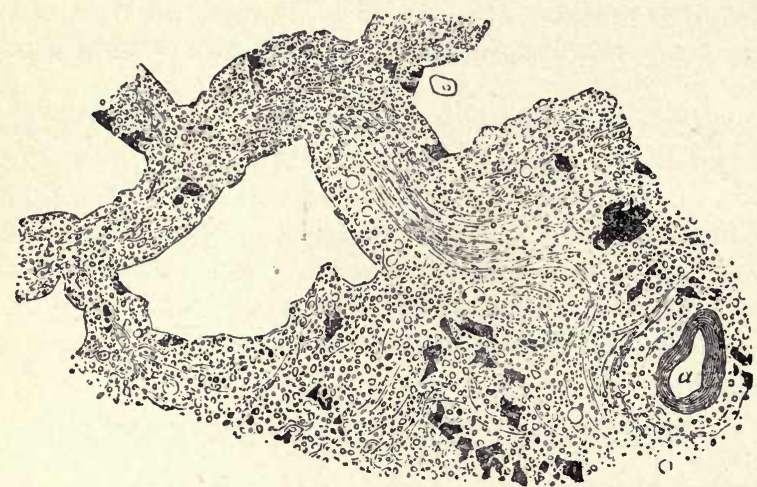

Interstitial pneumonia (from a case of unilateral "cirrhosis" of the lung). The bronchi were much dilated, and there was a complete absence of any caseous change. The drawing shows the new fibro-nucleated growth both in the alveolar walls and in the interlobular tissue, also the pigmentation. At $a$ a divided vessel is seen. With a higher power a delicate reticulum is visible between the cell-elements. $\times 100$ (Green).

formed fibrous tissue, well supplied with bloodvessels communicating with those of the alveolar walls.

\section{GANGRENE.}

Gangrene of the lungs occurs as the result of obstruction of the pulmonary or bronchial arteries, and in the course of typhoid and other infectious diseases. It may follow a pneumonia ; result from a wound of the lungs; or be due to pressure, as by an aneurism or by some foreign body in a bronchus. It may also result from the extension of necrotic processes from neighboring structures. There are two varieties: the circumscribed and the diffuse.

In the circumscribed variety there are usually one or more small foci in each lung, blackish, even greenish in appearance. These foci have a most offensive odor, are soft and pultaceous, and are surrounded by congested or hepatized lung-tissue.

Diffuse gangrenous areas may result from the coalescence 
of smaller foci, or may be diffuse from the first and involve even an entire lung.

The sputum is usually intensely foetid.

\section{THE INFECTIVE GRANULOMATA.}

\section{TUBERCULOSIS OF THE LUNGS.}

The pathological changes in the lungs produced by the tubercle bacillus vary somewhat with the different modes of infection, whether through the respiratory passages, the blood or lymph channels; also with the number and virulence of the bacilli, and more especially with the ability of the patient to withstand their invasion. This last depends not only on the state of one's general health, but also on one's inherited tendencies toward the disease, the importance of which cannot be overestimated. Just what is transmitted to an individual who inherits a predisposition to tuberculosis cannot be stated further than that there is a soil markedly favorable to the growth of the germ.

Besides the characteristic lesion-the histological tubercle - which is distinctive of the pathological changes produced by the tubercle bacillus, these organisms are capable of exciting tissue-changes quite similar to those of ordinary simple inflammation. As in simple inflammations, the more intense the irritant the more prominent the phenomena of cellular and serous exudation, while in inflammations of longer duration and less intensity the characteristic feature is the tendency toward organization and production of new fibrous tissue; so some tubercular inflammations are characterized by exudations and others by proliferative changes.

When the bacilli gain access by, and are distributed through the medium of the bronchial tubes in large numbers, the lesions are not so sharply circumscribed as they are when the organisms are deposited from the bloodvessels here and there in the pulmonary tissue in small numbers; nor are they of the same productive type, but are characterized by more extensive exudation and a greater tendency to coagulation-necrosis, caseation, and the involvement of larger areas.

In the bronchogenic tubercular inflammations of the lungs, in some instances there is a tendency for the areas of pneu- 
monic consolidation to conform to the distribution of the smaller bronchi-histologically the alveolar exudate resembling that of lobular pneumonia ; in others there is a diffuse hepatization of one or more entire lobes, the exudate consisting of fibrin, leukocytes, and epithelial cells, as in lobar pneumonia.

To those cases in which there is extensive pneumonic consolidation and subsequent caseation and destruction of lungtissue the term phthisis pulmonalis is often restricted. Originally the term was employed to designate a wasting of the whole body associated with disease of the lungs. The pulmonary lesions, later recognized as characteristic of such cases, were both "ulcerative" and nodular; gradually, however, smaller nodules-tubercles-were noted, and finally regarded as the starting-point of the older destructive lesions. In its modern acceptation the term phthisis has come to be used in connection with the wasting or destruction of the lungtissue rather than with the general wasting and emaciation of the whole body; so we find most authors describe separately phthisis pulmonalis and miliary tuberculosis.

In these bronchogenic forms of pulmonary tuberculosis the factors concerned in the production of a lobular distribution and catarrhal exudation at one time, and a lobar distribution and fibrinous exudate at another, may not be understood; the character of the associated secondary infection is, however, quite possibly one explanation. The histological tubercles which are often noted in connection with these pulmonic consolidations, especially at their borders, might be assumed to be due to the penetration of stray individual organisms between the epithelial cells to the perialveolar and peribronchial connective tissues. These pneumonic consolidations most frequently begin in the apices of the lungs, probably as the result of some impairment in the nutrition of the lung-tissue at this point, by reason of lessened aëration of the blood due to diminished range of the respiratory movements at the apices; general feebleness and cardiac weakness still further favoring the same results.

It has been stated that when the tubercle bacilli are deposited in small numbers in the pulmonary tissue from the bloodvessels, the lesions are different from those produced by the entrance of large numbers through the respiratory pas- 
sages. It might be assumed that where each organism is deposited from the blood there results the accumulation of epithelioid and lynıhoid cells, with possibly a giant-cell in their centre, which constitutes the typically histological tubercle; and that the "system" of such histological tubercles, of which even the smallest gray miliary tubercle visible to the naked eye is formed, results from the presence of as many organisms. Secondarily are noted the exudations into the surrounding air-spaces. The conditions which hold in the lungs differ from those in other organs of the body on account of the accessibility of the former not only to the tubercle bacilli in larger numbers, which is regarded as an explanation of the greater prominence of inflammatory exudations, but also to streptococci and other secondarily infecting organisms, which play a most important part in pulmonary tuberculosis.

\section{Acute Miliary Tuberculosis.}

Acute miliary tuberculosis occurs usually as part of a general miliary tuberculosis, which not infrequently results from the rupture of some tubercular focus- such as a caseous lymph-gland-into a bloodvessel ; or follows operations upon tubercular joints or bones. Clinically the disease has always had a peculiar interest on account of its resemblance to typhoid fever at certain stages.

The lungs are congested and studded throughout with small grayish, gelatinous-looking nodules about the size of millet seeds or small shot; at first they may be so small and translucent as to be scarcely visible to the naked eye, though finally becoming quite large, especially in the lungs of children. They may be widely separated or so closely set as to solidify the whole lung. Some may be yellowish from advancing caseation. Microscopically it is noted that these nodules are situated in the interlobular or interalveolar septa, and are composed of several histological tubercles (Fig. 154). In these acute processes giant-cells are not often seen, the centre and often the entire tubercle being caseons. The surrounding air-spaces may be more or less completely filled with inflammatory exudates, and the interalveolar septa considerably thickened. 
In more chronic cases, in which perhaps the bacilli are less virulent or are admitted more slowly to the lungs, the nodules are larger and not so numerous; the areas of caseous degeneration are much more extensive.

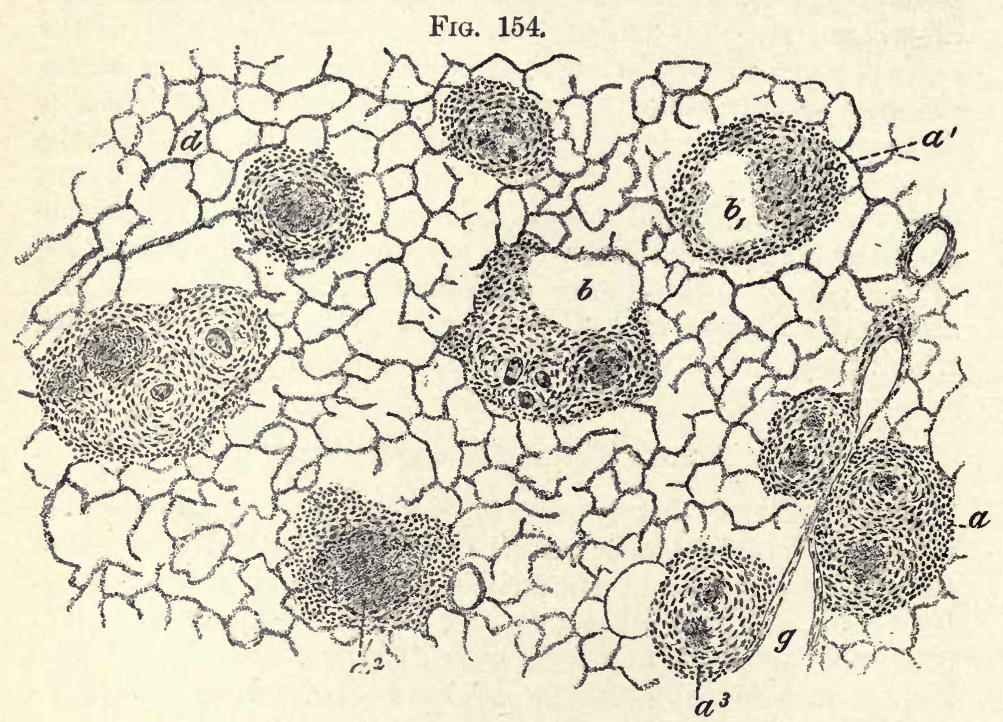

Acute miliary tuberculosis of the lungs. $a, a^{1}, a^{2}, a^{3}$, tubercles with central caseation,

in part in parenchyma on alveolar tubes $\left(b, b^{1}\right)$, in part situated on bloodvessels.

In many tubercles giant-cells. $\times 40$ (Schmaus).

\section{Acute Phthisis.}

As has already been pointed out, the main features of phthisis are the lobar and lobular consolidations, which are wont speedily to undergo caseation. The size of these pneumonic areas may vary greatly.

In the earlier stages grayish miliary patehes, one-twelfth to one-sixth of an inch in diameter, stand out prominently beneath the pleura or upon the congested cut surface of the lungs, in greatest number at the apices. Microscopically the patches are found to have each a caseous centre, which is structureless and granular in appearance-the outline of the air-spaces may, however, be faintly discernible; further away 
from this central zone the alveoli are filled with catarrhal or fibrinous exudations (Figs. 155 and 156). Near the centre of the patch may be noted a bronchiole, its lumen filled with desquamated epithelial cells and a granular debris. The walls of the air-vesicles and bronchioles are infiltrated and somewhat thickened.

At a later stage these areas of consolidation reach the size of a hazelnut or walnut, or they may involve an entire lobe or even the whole lung. Although such a diffuse consolidation

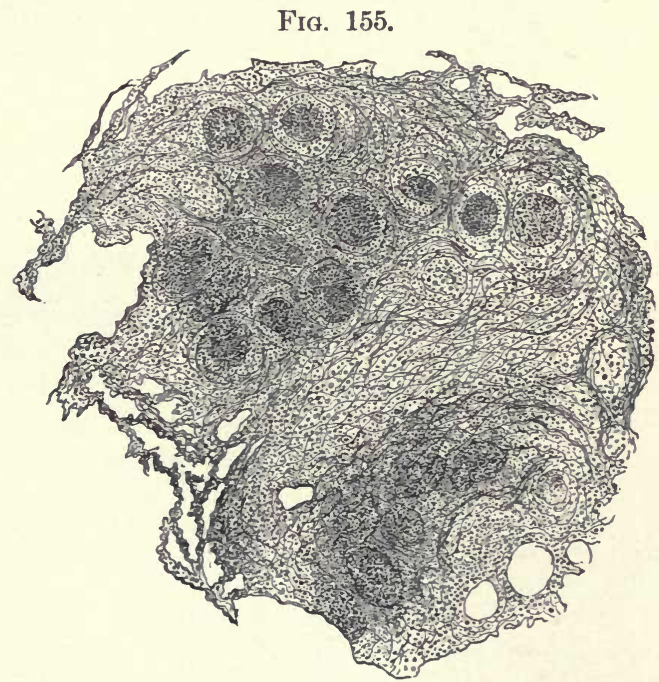

A small soft gray tubercle from the lung in a case of acute tuberculosis. The whole of the tubercle is shown in the drawing, and is largely constituted of intraalveolar products. $\times 100$, reduced to $\frac{1}{3}$ (Green).

may appear quite as uniform as that of lobar pneumonia, microscopically there is usually evidence of an original lobular character of the inflammatory process.

On section the surface of the affected areas, which have become yellowish in color as the result of extensive caseation, is generally smooth, though it may have a granular appearance when the character of the inflammatory exudate is fibrinous. The lungs may be fairly riddled with small, irregular 
cavities with cheesy walls, resulting from the breaking down and evacuation of the caseous material. 'The pleura is generally thickened, especially over the apices.

Microscopically it is noted that the areas of consolidation are formed of the smaller patches just described, each with a caseous centre and an outer zone of air-spaces filled by inflammatory exudations. The peribronchial connective tissue and alveolar septa are infiltrated as in lobular pneumonia, and occasionally typical histological tubercles may he seen here and there, especially at the periphery of the pneumonic areas.

FIG. 156.

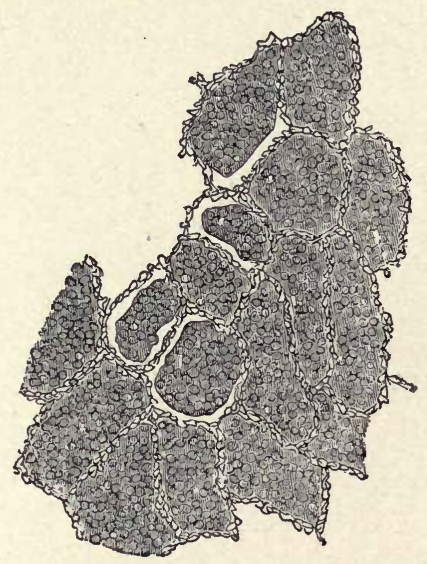

Section of lung from a case of acute phthisis, showing that the consolidation consists almost exclusively of products accumulated within the alveoli. In some parts a free space is seen between the alveolar walls and their contents; this is due simply to the shrinking of the latter caused by hardening of the specimen. $\times 50$ (Green).

Besides the cases which conform to a lobular type of pneumonia, others have been described which resembled lobar pneumonia, not only in the character of the inflammatory exudate, but also in its lobar distribution. In some instances of acute phthisis, tubercles may be entirely absent, or at least indistinguishable in consequence of the extensive caseous degeneration; though they can generally be found, if not in the lungs, on the pleura, or peritoneum, or elsewhere. 


\section{Chronic Phthisis.}

Chronic phthisis is the common form of pulmonary tuberculosis. The primary lesions are generally found in the apices of the lungs. In the great majority of cases the inspired air is the source of the infection, though occasionally without doubt the tubercle bacilli are introduced through the blood or

F19. 157.

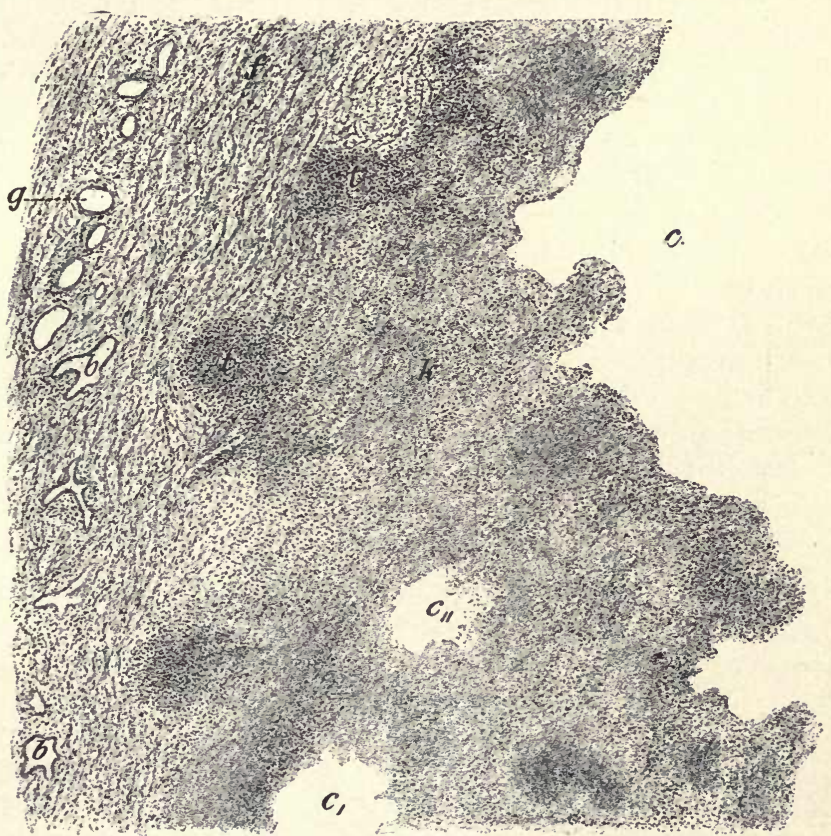

Wall of a tubercular cavity. $c$, cavity; $k$, cheesy wall $c^{\prime}, c^{\prime \prime}$, small recent cavities; $t$, tubercle. To the left, fibrous tissue in which are, $b$, bronchi ; $g$, vessels. $\times 100$ (Schmaus).

lymph channels - the further extension of the disease resulting from rupture of a caseous focus into a bronchus and aspiration of the infected material into other parts of the lungs.

The lungs are firmly adherent to the chest-wall ; the pleural cavities may be thus entirely obliterated. The pleuræ are 23-Hist. 
greatly thickened-even as much as a quarter of an inch at the apices-and fibrous, and may be studded with gray gelatinous nodules. On section a most characteristic lesion is noted : the cavity, which results from liquefaction of caseous areas (Fig. 157). They are most frequent in the upper lobes, and vary in size from that of a hazelnut to that of a small orange, or even much larger, and have glistening, firm fibrous walls. Especially numerous in the upper part of the lungs are noted irregulariy rounded, opaque, grayish or yellowish areas of consolidation.

The histological changes are similar to those in acute phthisis, except as modified by the longer duration of the inflammatory process. In the centre of these areas the tubercles caunot be distinguished on account of the caseous degeneration which has taken place; at the periphery, however, they may be. Here and there are nodules which are found to be composed of several histological tubercles. The centre of such a system of tubercles is generally caseous, though it may be firm and fibrous. These tubercles are situated in the interalveolar and interlobular septa, and may be seen in various stages of development. They are generally surrounded by patches of catarrhal or fibrinous exudation.

\section{Fibroid Phthisis.}

In fibroid phthisis, which is the most chronic form of the disease, the reparative efforts on the part of nature predominate over the destructive effects produced by the tubercle bacilli. An area of caseation is often found to be surrounded by a capsule of more or less fully developed fibrous tissue, and thus its further extension is arrested. There is always an especially marked inerease in the amount of interalveolar and interlobular connective tissue, which leads to extensive induration of the lung-tissue.

Tubereulosis is often engrafted upon simple chronic interstitial pneumonia.

The complications of phthisis are numerous and important. The pleura is nearly always implicated. In acute cases, over pneumonic areas, it is usually the seat of a seropurulent or fibrinous exudation, as in lobar pneumonia; or a diffuse 
acute inflammation may result from rupture of a tubercular focus into the pleural cavity. In more chronic cases there are almost constantly, extensive adhesions between the parietal and visceral lavers.

Extension of the tubercular process in the lung itself is caused by the aspiration of material from caseous foci to other parts. Sputum loaded with tuberele bacilli is very apt to infect the upper air-passages, and, if swallowed, the gastro-intestinal tract. A general miliary tuberculosis may result from secondary infection of the blood-through tubercular infiltration of the vessel-wall or rupture of a caseous focus into its lumen.

Pneumothorax is due to perforation of the pleura. The pleura is very liable to undergo necrosis at a given point as the result of an underlying focus of caseation in which are involved the bloodvessels which nourish it. If this occurs before pleuritic adhesions have been formed, perforation oceurs. In this manner are explained many eases in which an acute pleurisy seems to precede the development of the pulmonary affection.

Fatal hemorrhage may result from erosion of one of the branches of the pulmonary artery.

Ancemia, emaciation, and fever are constant accompaniments of phthisis.

Widespread amyloid changes in the viscera are present in a large number of cases.

\section{SYPHILIS OF THE LUNGS.}

Syphilitic inflammation of the lungs in adults is rare; though gummata, appearing as grayish or yellowish rounded nodules, are sometimes observed. They may undergo central necrosis, and thus result in the formation of cavities.

In congenital syphilis there is sometimes a consolidation of extensive areas of lung-tissue, to which the term white pneumonia has been given, on account of the pale and anæmic appearance of the affected areas. Microscopically there is noted a great increase in the connective tissue, as in chronic interstitial pneumonia, and a catarrhal exudate within the alveoli. 


\section{OTHER GRANULOMATA AND TUMORS.}

Actinomycosis is a rare affection of the lungs which clinically and pathologically may closely resemble tuberculosis. There are nodules of lobular pneumonia varying in size from that of a pea to a cherry, which later become confluent and undergo softening, resulting possibly in cavity-formation. Microscopically the fungus is readily recognized within these areas and in the sputum.

Glanders also rarely affects the lungs, producing either diffuse inflammation with abscess-formations or isolated characteristic nodules which microscopically are found to consist of masses of round cells.

In leprosy the lungs are frequently affected, the lesions resembling very closely those of tuberculosis.

Tumors : Fibromata, lipomata, chondromata, sarcomata, and carcinomata are sometimes oloserved in the lungs. Secondary sarcoma is quite common, and occurs much more frequently than primary. Secondary carcinoma is not so common as secondary sarcoma.

\section{THE PLEURA.}

Hæmothorax is a term indicating blood in the pleural cavity. It may result from rupture of an aneurism, or fracture of the ribs or other injury to the chest.

Hydrothorax signifies a non-inflammatory accumulation of serum in the pleural cavity. It is usually bilateral, occurring in the course of a general dropsy due to some chronic heart or kidney disease, or to changes in the composition of the blood. A slight serous effusion is not infrequent just before death. If the amount of the effusion is considerable, it may greatly interfere with respiration.

Pneumothorax: Air alone is rarely present in the pleural cavity. It is usually associated with a serous or purulent effusion-hydropneumothorax or pyopneumothorax. It may result from traumatic rupture or penetrating wounds of the pleura ; 90 per cent. of all cases, however, are due to pulmonary tuberculosis. 


\section{PLEURITIS.}

Pleuritis, pleurisy or inflammation of the pleura, is nearly always secondary to an adjacent inflammatory process, as pericarditis, pneumonia, gangrene or tuberculosis of the lungs. It may occur in the course of some general disease, as pyæmia, septicæmia, typhoid fever, influenza, and other infectious diseases, rheumatism, gout, and Bright's disease.

Several varieties are usually described according to the character of the inflammatory exudate: fibrinous, serofibrinous, and purulent.

The fibrinous variety is generally confined to circumscribed areas, though it may involve the entire pleura on one side. At first the membrane is congested and its normal glistening appearance is lost, due to the cloudy swelling of the endothelial cells, the exudations and other phenomena characteristic of the early stage of inflammation. The serous exudate, rich in fibrin, coagulates in the form of a thin white pellicle on the surface, varying in thickness in different cases. Where opposed surfaces have become agglutinated, on separation there is often a shaggy appearance somewhat similar to that produced by pressing together the buttered surfaces of two pieces of bread-"bread-and-butter" pleurisy.

Microscopically may be noted the dilated bloodvessels, surrounding which and infiltrating the connective tissue are numberless migrated leukocytes. The thin pellicle on the surface of the pleura consists of a delicate network of fibrinfilaments, containing in its meshes migrated leukocytes, red blood-corpuscles, and possibly a few desquamated endothelial cells, resembling thus very closely the alveolar exudate in the stage of red hepatization of pneumonia. At this stage opposed pleural surfaces, which are found adherent, can readily be separated. Later, however, on this temporary scaffolding of fibrin a new connective tissue is built, through the agency of the leukocytes and proliferating endothelial cells, which results finally in firm fibrous adhesions.

The serofibrinous variety may be simply a more advanced stage of the inflammatory process. The quantity of the serous exudate which accumulates in the pleural cavity varies greatly-it may exceed four litres. 
Microscopically the serous exudate is found to contain a few white and red corpuscles and desquamated endothelial cells. In chemical composition and physical appearances it resembles blood-serum. When the effusion is excessive the lung is compressed against the vertebral column, airless and almost bloodless.

Empyema: A purulent effusion into the pleural cavity results either primarily from the presence of some pus-producing micro-organism, or from secondary infection of a simple serous effusion. The micro-organisms most frequently found are the pneumococcus, streptococcus, staphylococcus and tubercle bacillus; occasionally the bacillus coli, typhoid and others. The pleura is greatly thickened, and on its surface are more or less abundant granulations. A localized necrosis may result in perforation of the pleura and discharge of the effusion externally through the thoracic wall, into the lungs, or through the diaphragm into the peritoneal cavity. 


\section{CHAPTER VI.}

\section{DISEASES OF THE GASTRO-INTESTINAL TRACT.}

\section{THE MOUTH.}

To inflammation of the nucous membrane of the mouth the term stomatitis is applied.

Catarrhal stomatitis is a simple inflammation unattended by ulceration. It occurs most frequently in children, and results from a great variety of causes, such as food which is too hot or too cold, lack of cleanliness, etc. The mucous membrane is congested and swollen, and either unduly dry or there is an increased production of its mucous secretion.

Croupous or pseudomembranous stomatitis is nearly always due to the extension of a similar inflammation in the pharynx, produced by the bacillus of diphtheria.

Ulcerous stomatitis occurs most frequently between the ages of four and ten years; though it may occur in adults in Iocalized epidemics, in camps and garrisons. Certain drugs, especially mercury, are capable of producing similar lesions. The inflammatory process begins on the border of the gums and extends to the lips, cheeks, and edges of the tongue. The uleers which form on the lips and cheeks may become quite large. Suppuration and destruction of tissue around the teeth may be so great that they finally become loosened and fall out.

Aphthous stomatitis also occurs most frequently in children. $\mathrm{Bad}$ hygiene, and debility resulting from prolonged illness, are predisposing causes. It is characterized by the presence of small yellowish-white patehes (aphthce), lying on an inflamed base, and varying in size from that of a hemp-seed to that of a split pea. The lesion first appears as a small red papule with a whitish centre. By the end of twenty-four hours this white central area of degenerated epithelial cells involves the whole patch. The inflammatory process seldom leads to ulceration. 
Gangrenous stomatitis, noma, or cancrum oris, is a rare affection, characterized by a spreading gangrene of the cheek, generally involving one side only; it is nearly always fatal. It occurs most frequently in weakly, ill-nourished children, especially after some exhausting acute infectious disease. The earliest lesion usually noted is a sloughing ulcer on the inside of the cheek, near the corner of the mouth. The mucous membrane becomes black and gangrenous, and soon the process involves the entire thickness of the cheek and extends in all directions.

Thrush, or muguet, is a mycotic stomatitis, due to a fungus, the oildium albicans, occurring especially in infants. It is characterized by the presence of white patches, resembling curdled milk, upon the mucous membrane. There is a tendency for these patches to extend and involve the pharynx, and even the gastro-intestinal tract. If a white patch is removed, the underlying mucous membrane is red, eroded, and may bleed. Microscopically the white deposit is found to be composed of the delicate filaments of the parasite and degenerated epithelial cells.

All the infectious granulomata may affect the mucous membrane of the mouth.

Tuberculosis is not often noted. It may be primary or secondary. The nodular masses undergo caseation, often resulting in the formation of ulcers closely resembling those of tertiary syphilis.

Syphilis affects the month either in the form of mucous patches in the secondary stage; or gummata varying in size from that of a pea to that of a hazelnut, in the tertiary. The gummata may break down and leave deep ragged ulcers in their site.

Tumors: Flat-celled carcinomata, epitheliomata, are of quite frequent occurrence. They appear first as a small nodule, or circumscribed infiltration, which soon tends to ulcerate and spread rapidly.

Sarcomata usually affect the gums, sarcomatous epulis. 


\section{THE PHARYNX.}

Simple catarrhal inflammation of the pharynx, or simple angina, is due to exposure to cold, or occurs in the course of acute infectious fevers. A more chronic inflammation may result from the excessive use of the voice, or be secondary to some disease of the nasal cavities. The mucous membrane is red and swollen. In some cases vesicles form, which burst and leave behind small superficial erosions. Not infrequently there are granulation-like projections above the surface of the mucous membrane, due to hypertrophy, or distention of the mucous glands, or to hyperplasia of the lymphatic follicles.

At first the mucous membrane is thickened, but later it often becomes atrophic.

Pseudomembranous pharyngitis is in most instances produced by the Klebs-Löffler bacillus diphtheriæ. Lesions in every other way similar are sometimes produced by other microorganisms, notably the streptococcus pyogenes. Such "diphtheritic" pseudomembranes are especially frequent in scarlatina and other acute infectious diseases, and may also follow the application of various irritants to the mucous membrane.

Diphtheria is an acute infectious disease characterized by a severe toxæmia, giving rise to an irregular fever, great prostration, often followed by cardiac and other localized or general paralyses; and locally by the formation of a pseudomembrane at the point of lodgment of the diphtheria bacilli, either on an abraded surface or a mucous membrane, generally that of the pharynx and upper air-passages.

The pseudomembrane is grayish-white, later yellowishwhite in color. Small patches appear on the posterior pharyngeal wall, the tonsils, soft palate, or nares, which tend rapidly to extend and coalesce, and thus may cover the whole pharyn ; less frequently on the buccal walls, the osophagus, stomach, vagina, uterus, and on surface abrasions. The pseudomembrane is more or less adherent, and if removed a raw bleeding surface may be left behind. The formation of this membrane is the result of the severe inflammatory process set up by the diphtheria bacilli. The serous exudate coagulates and entangles the other inflammatory products. Microscopically it is found 
to be composed of a network of fine fibrillæ, in the meshes of which are red blood-corpuseles, migrated leukocytes, desquamated epithelial cells, and masses of micro-organisms. The underlying mucous membrane is congested and infiltrated with leukocytes; its epithelial cells are undergoing granular degeneration or coagulation-necrosis (Fig. 158).

FIG. 158

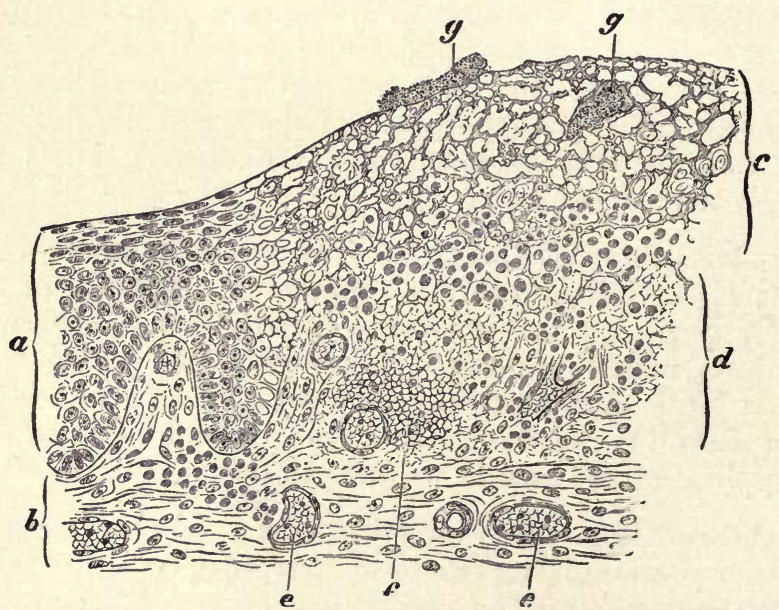

Edge of a diphtheritic membrane. Scction from the human uvula (Ziegler). $a$, normal stratified epithelium; $b$, subepithelial fibrous tissue of the mucous membrane; $c$, epithelium that has undergone coagulation-necrosis. Only remnants of cells remain in the coarse fibrinous meshwork. $d$, œedematous subepithelial fibrous tissue containing fibrin and leukocytes; $e$, bloodvessels; $f$, hemorrhage ; $g, g$, groups of the bacteria causing the necrosis.

The associated lesions are the result of the absorption of toxins-the bacilli themselves do not gain entrance to the circulation. The neighboring lymphatic glands are usually greatly swollen; the kidneys are nearly always affected, as shown clinically by the albuminuria which is present in the majority of cases-the renal epithelium becomes cloudy and swollen, and minute hemorrhages are sometimes seen. Not infrequently there are necrotic foei in the liver, and in nearly all cases some myocardial degeneration. The diphtheritic paralyses are due to degenerative changes in the nerve-centres and peripheral nerves. 
In tonsillitis the inflammation may be superficial and involve only the mucous membrane of the tonsil as part of a general pharyngitis. In other cases-lacunar or follicular tonsillitis-it involves, in addition, the mucous membrane lining the crypts, which become distended with inflammatory products and project above the surface as small yellow spots. The exudations at the mouth of adjacent lacunæ may coalesce,

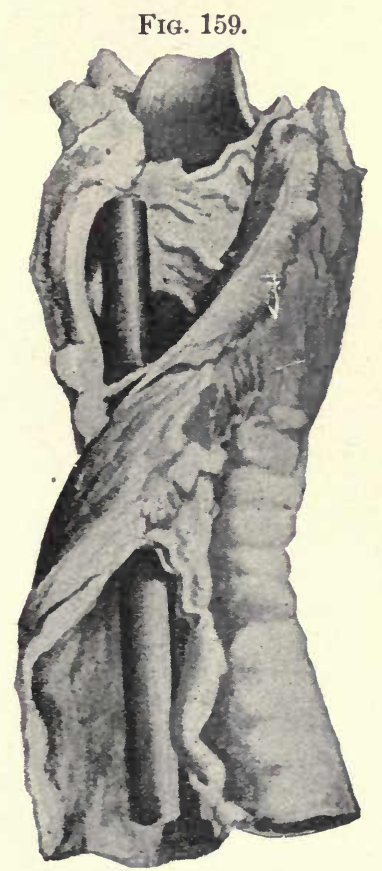

Cicatricial stricture of cesophagus (Warren Museum).
FIG. 160.

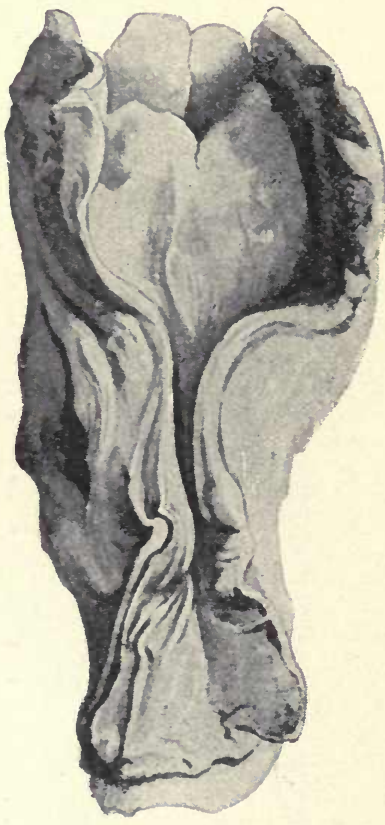

A very tight stricture of the cesophagus of many years' duration, apparently the result of chronic inflammatory action; small abscess on the left (Warren Museum).

and the resulting yellow patch somewhat resembles diphtheritic membrane. Ulceration of the walls of the crypts may produce quite extensive destruction of tonsillar tissue. In other cases the inflammatory process ends in the formation of an 
abscess-phlegmonous tonsillitis or quinsy-the tonsil becoming so large as to project beyond the median line. The uvula and epiglottis are often œelematous, and the submaxillary and cervical lymphatic glands enlarged.

FIG. 161.

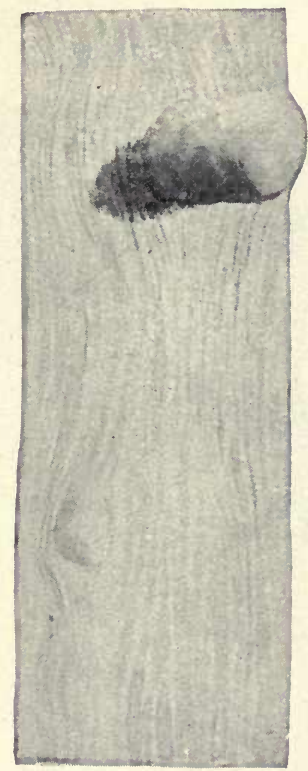

Traction diverticulum which was adherent to a cheesy bronchial gland. The cut shows the external œesophageal wall with the diverticulum artificially distended (Warren Museum).
FIG. 162

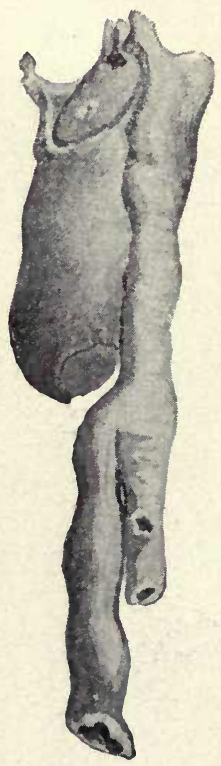

\footnotetext{
Congenital malformation of the cesophagus. Upper portion ends in a cul-de-sac; the lower portion opens into the trachea (Harvard Med. School, Warren Museum).
}

Chronic hypertrophy may follow repeated attacks of acute tonsillitis; usually, however, it is associated with hypertrophy of the lymphoid tissue of the naso-pharynx - "adenoids." Microscopically there is noted an increase in the connective-tissue stroma and of the lymphoid elements. 


\section{THE SALIVARY GLANDS.}

Inflammation of the salivary glands may occur in the course of varions acute infectious diseases, as septicæmia, typhoid, etc.; or result from a special infection, when it is known as mumps - the parotid gland being most frequently affected.

Mumps rarely terminates in the formation of an abscess in the affected gland, while this is the characteristic tendency in the secondary infections, or metastatic mumps.

\section{THE OESOPHAGUS.}

Inflammation of the œsophagus may be due to ingestion of acids or alkalies, food or drink too hot or too cold, the lodgment of foreign bodies, etc. The inflammatory process may end in ulceration and cicatricial stenosis (Figs. 159 and 160).

Cylindrical dilatation of the œsophagus results from longcontinued stenosis. Sacculated dilatations result either from. pressure from within or traction from without, as by contracting adhesions (Figs. 161 and 162).

Squamous-celled carcinoma is the most frequent affection of

FIG. 163.

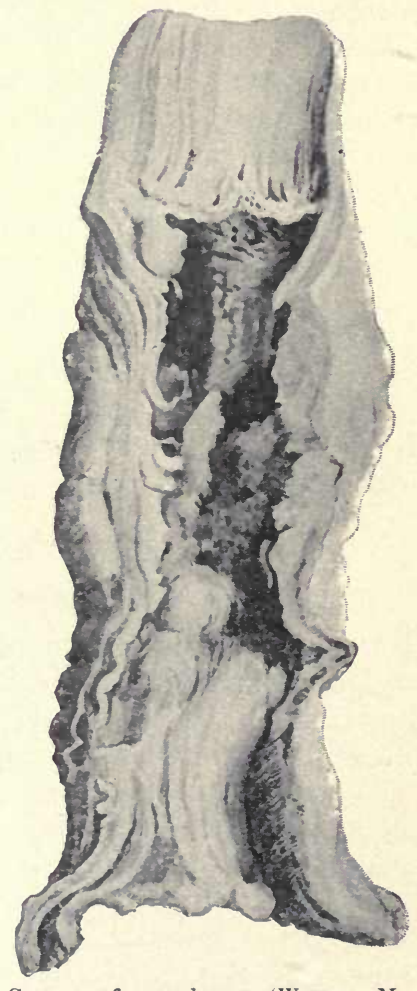

Cancer of cesophagus (Warren $\mathrm{Mu}$. seum). the esophagus (Fig. 163). It generally oceurs in the lower third, though it may be found in any part, and gradually produces a complete stenosis. 


\section{THE STOMACH.}

\section{ALTERATIONS IN POSITION AND SIZE.}

Gastroptosis, or total descent of the stomach, is the most frequent displacement. It may be associated with similar displacements of the other abdominal organs-enteroptosis, or Glénard's disease.

Gastrectasia, or dilatation of the stomach, may result from pyloric obstruction produced by the cicatrix of a healing ulcer, carcinoma, pressure of a tumor, ete. ; or may occur in consequence of simple atony of the muscular walls brought about by overfeerling, inflammatory or degenerative changes. The resulting stagnation of the stumach-contents leads to abnormal fermentations and a train of symptoms due to autointoxication. When due to pyloric obstruction the dilatation may be enormous. At first there may be compensatory hypertrophy of the muscle-walls of the stomach; but later they become thin and atrophied, and the mucous membrane nonabsorptive.

\section{SIMPLE ACUTE GASTRITIS.}

Simple acute gastritis is an exceedingly common condition. In most cases it results from dietetic errors-excessive indulgence, ingestion of food which has undergone partial decomposition, or is too hot or too cold. It may also be produced by various chemical irritants, as arsenic, the iodides and salicylates, even in therapeutic doses. The mucous membrane is found to be congested, swollen, covered with a film of glairy mucus, especially in the pyloric region, and punctated here and there by small hemorrhagic patches. The gastric secretion is reduced in quantity and quality, a fact first observed by Beaumont, through a gastric fistula, in the case of the Canadian, Alexis St. Martin. The clinical opportunities for noting the marked change in the quality of the gastric juice-the hypochlorhydria, etc.-are numerous, through the use of the stomach-tube.

Microscopically both the parietal and the central cells of the gastric tubules are swollen and granular; the bloodvessels of the interglandular connective tissue are distended and sur- 
rounded by migrated leukocytes. At a later stage the leukocytic migration becomes more marked, either as a diffuse round-celled infiltration of the mucosa, and often also of the submucosa, or confined to more or less sharply circumscribed patches. The lymph-follieles are often hyperplastic.

\section{PURULENT GASTRITIS.}

Purulent or phlegmonous gastritis is a rare and almost invariably fatal affection. It occurs in the course of puerperal fever, pyæmia, smallpox and similar infections; or it may be a primary condition. The inflammatory process is either circumscribed or diffuse. In the former case there is generally but one abscess-most frequently situated near the pylorus, and varying in size from that of a hazelnut to that of a goose egg. In the latter case there is a diffuse purulent infiltration most marked toward the pylorus, involving first the submucosa; later the mucosa becomes riddled with perforations, through which pus wells up. The muscle and serous coats may be involved, and the serous surface covered with a purulent exudation.

\section{ACUTE TOXIC GASTRITIS.}

Acute toxic gastritis, or gastritis venenata, results from the ingestion of such poisons as alcohol, arsenic, phosphorus, bichloride of mercury, organic and inorganic acids, and caustic alkalies.

The lesions produced vary with the kind and quantity of the poison taken and the length of time elapsing before death ; they are associated with similar changes in the œsophagus, and, below the stomach, in the small intestine, even to the ileocrecal valve.

The inflanmatory process excited by dilute acids and alkalies may resemble that in simple acute gastritis. When concentrated they produce local death of the tissues; and if the patient survives, most serious defornities of the stomach may result. The slough involves the mucosa and often also the other coats of the stomach, frequently producing a perforation. Adjacent organs, especially the liver and spleen, are often corroded and discolored in a like manner. 
The sloughs produced by sulphuric and hydrochloric acids are dry and brittle, and ashy-gray in color, or black and charred in extreme cases; those of nitric acid are yellow. The surrounding tissue is the seat of an intense hemorrhagic inflammation. The sloughs produced by the caustic alkalies are not brittle but soft, and have somewhat the appearance of a soft membrane. Phosphorus, arsenic, and antimony in large doses produce more especially a fatty and mucoid degeneration of the epithelial cells of the gastric tubules, resulting in an opaque, yellowish-white appearance of the mucous membrane.

\section{CHRONIC GASTRITIS.}

The systematic clinical use of the stomach-tube for the purpose of obtaining the gastric secretions for study, within recent years, has thrown much light upon the pathological physiology of digestion. And the microscopical examination of pieces of the gastric mucosa found in the washings from the stomach has added greatly to our knowledge of the anatomical lesions associated with the well-defined fundamental types of chronic gastritis recognized clinically.

The character of the exciting cause seems to bear no relation to the resulting lesion. Evidences of chronic gastritis are found so frequently at autopsy that a terminal gastritis must be looked upon as an almost constant accompaniment of the final stages of all chronic diseases.

More or less pronounced inflammatory changes are associated with organic lesions of the stomach, as ulcer and cancer. They are secondary also to dilatation and atony of the musclewalls, and the irritation produced by the resulting stagnation of the stomach-contents and abnormal fermentations which take place. In fact, the possible causes of gastritis are so numerous that it is surprising a normal stomach should be found after middle life.

The most frequent causes of chronic gastritis as an independent and primary affection of the stomach are dieteticnot only improper food, but improper methods of eating it; bolting the food, with insufficient mastication, and large draughts of liquid which are often unduly hot or cold; the excessive use of condiments, etc. 


\section{Gastritis Mucipara.}

As in acute gastritis, the changes are most marked in the region of the pylorus. In early stages of the inflammatory process there is a diffuse redness of the mucous membrane, which later becomes a mottled pale gray. The mucosa is swollen and covered by a tenacious layer of tough mucus. Here and there are small cysts, produced by occlusion of the gastric tubules. Occasionally it is studded with papillary projections about the size of a pea. As in other chronic inflammations, there may be a marked increase in the connective-tissue stroma, which results finally in atrophy of the glandular tissue.

It is only on microscopic examination, however, that the distinctive features of this variety of chronic gastritis can be noted. The number of gastric tubules in a given field is much less than normal. Most characteristic is the mucoid degeneration of the chief and border cells of the tubules, extending to the fundus of the gland. In addition to these parenchymatous changes, there is a more or less pronounced cellular infiltration of the interglandular connective tissue. As would naturally be expected, the gastric secretions are greatly reduced in quantity and quality, and in late stages even entirely wanting.

\section{Gastritis Glandularis Prolifera.}

This variety of chronic gastritis stands in marked contrast to the one just described. The distinctive feature here is the proliferative activity of the chief and parietal cells lining the gastric tubules, the parietal or acid-producing cells especially being greatly increased in size and number and lying in close juxtaposition. These oxyntic or parietal cells may also be found in the tubules of the pyloric region, where they are not normally present. In some cases there seems to be a proliferation of the glandular tubules, the entire gland becoming elongated and tortuous. 'There may be associated with these parenchymatous changes more or less cellular infiltration of the interglandular connective tissue.

The cylindrical surface-epithelium is often desquamated 24-Hisț. 
over quite extensive areas, and ulcer is not an infrequent complication.

As might be expected, the secretory activity of the gastric tubules is greatly increased. Not only is the quantity of the gastric juice augmented, but it is much richer in pepsin and hydrochloric acid.

\section{Gastritis Glandularis Atrophica.}

Gastritis glandularis atrophica may oceur as a terminal stage of other varieties of chronic gastritis, as a senile change, or complicating various chronic diseases, and rarely as a primary affection. It is characterized by a degeneration of both the cylindrical cells of the surface of the mucous membrane and vestibular alveoli, and the cells lining the gastric tubules. The former undergo a mucoid, and the latter a granular and fatty degeneration. In the tubules it becomes impossible, by reason of their loss of staining properties and indistinctness of outline, to distinguish between the chief and parietal cells.

Macroscopically the mucous membrane presents a smooth, thin, white surface. In addition to the glandular elements, the submucosa and muscular coats may be involved by the atrophic changes, a thin layer of fibrous tissue taking the place of the latter. In other cases a similar destruction of glandular tissue is the result of a proliferation of the interglandular connective tissue. Instead of a thinning of the stomach-wall, it may be greatly thickened by an overgrowth of connective tissue beneath the mucosa-often resulting in great reduction of the size of the organ, even to a capacity of a few ounces.

The natural result of these lesions is a total abolition of the secretory functions of the stomach. In spite of this, however, the patient may remain well nourished and free from other ill consequences as long as intestinal digestion is normal. The evil results produced are often alone due to a failure of such compensation on the part of the small intestine.

A purely interstitial inflammation of the stomach independent of lesions of the parenchyma is described by Hayem; but 
clinically has no distinctive feature by which it can be recognized.

\section{ULCER.}

Ulcer of the stomach may occur in the course of acute and chronic gastritis; or independently; not infrequently it is found post-mortem when no symptoms had existed during life to indicate its presence. It is stated that in 5 per cent. of deaths from all causes either an open ulcer or a resulting cicatrix can be found.

It is rare before the age of ten years, though it has been met with in infants at birth ; it is much more frequent in the female than male.

Circulatory disturbances-arteriosclerosis, thrombosis, and embolism - are undoubtedly important etiological factors, as are likewise general anæmia and malnutrition. The frequent association clinically of hydrochloric-acid superacidity-in 80 or 90 per cent. of cases - is suggestive of autodigestion, and it is maintained by many that without this excessive acidity of the gastric secretions ulcer does not occur. At least it must be admitted that it could make the production of ulcer easier when factors interfering with the nutrition of the stomachwall, at a given point, are present; and also might subsequently serionsly interfere with the healing of such a lesion. At first it was asserted by Rigel that this superacidity was constantly present in ulcer. The small percentage of cases in which it is not, might be accounted for by an associated gastritis that has gone on to glandular atrophy. On the other hand, it is intelligible that the superacidity may be excited by the ulcer-in a manner possibly analogous to the hypersecretion of tears produced by a corneal ulcer.

Trauma alone cannot produce gastric ulcer; other factors are necessary. It is generally conceded that gastric ulcer artificially produced in lower animals heals promptly; and in man pieces of the mucous membrane have been torn away by suction of the stomach-tube, the lesion healing without the formation of an ulcer. But if a high degree of anæmia is first produced in dogs by the gradual withdrawal of blood, ulcers ean be produced by various irritants, which heal very slowly; and so, clinically, ulcer is especially frequent in chlor- 
otic young girls, in whom, in addition to the anæmia, a hyperchlorhydria is often present.

There is generally but one ulcer, though there may be several, often developing successively.

FIG. 164.

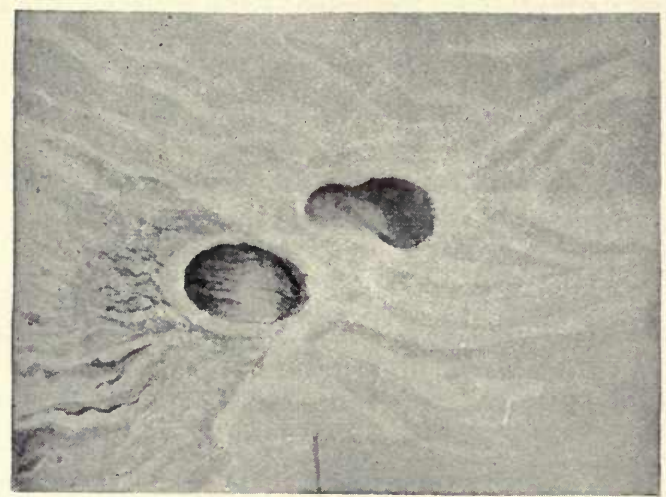

Two ulcers in the small curvature of the stomach, base formed by muscular coat (Warren Museum).

FIG. 165 .

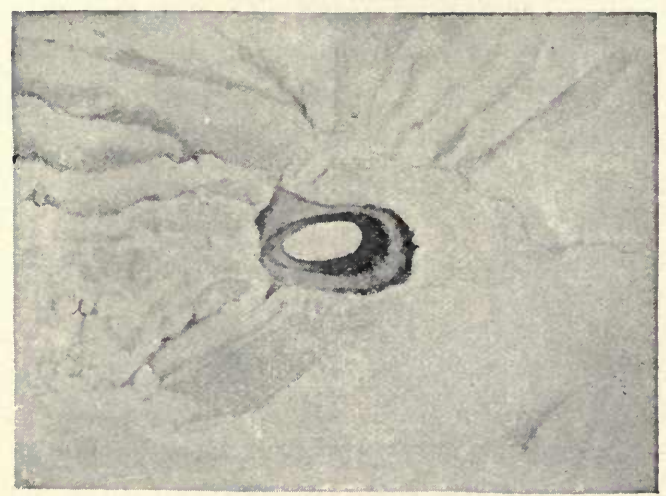

Perforating ulcer of the stomach (Warren Museum).

Gastric ulcers-macroscopic description: The lesion occurs most frequently along the lesser curvature and on the posterior 
wall near the pylorus (Fig. 164). In size it varies from onequarter of an inch to four or five inches in diameter; generally, however, from a half to two inches. In shape it is usually rounded or oval, and has a characteristic punched-out appearance, especially if the mucous membrane alone is involved. If it is deeper, the excavation may be somewhat funnel-shaped by reason of its sloping edges.

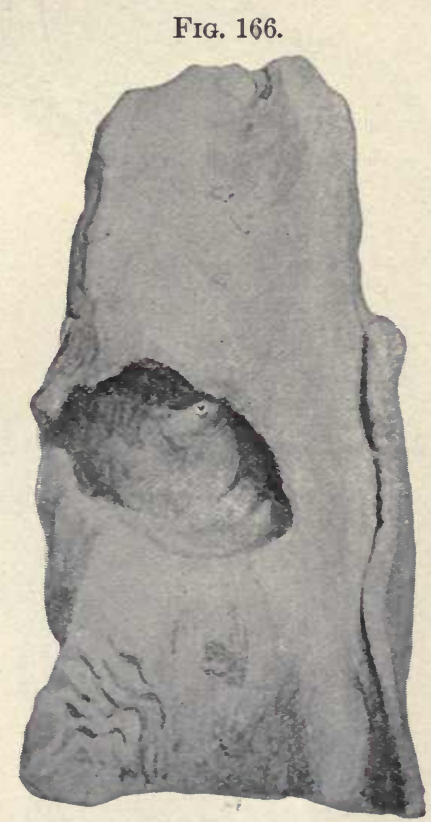

An ulccr of the stomach, showing at its base the open orifice of a vessel: death from hemorrhage (Warren Museum).

If all the coats of the stomach-wall are involved, as often happens, inflammatory adhesions may bind it to an adjacent organ, as the liver or pancreas, which thus comes to form the floor of the ulcer.

Microscopically, there is noted some round-celled infiltration in the immediate neighborhood of the ulcer. The walls of the adjacent bloodvessels are greatly thickened by an obliter- 
ating endarteritis - a state of affairs which protects largely against hemorrhage.

The dangers in gastric ulcer, which are always imminent, are perforation and hemorrhage.

FIG. 167.

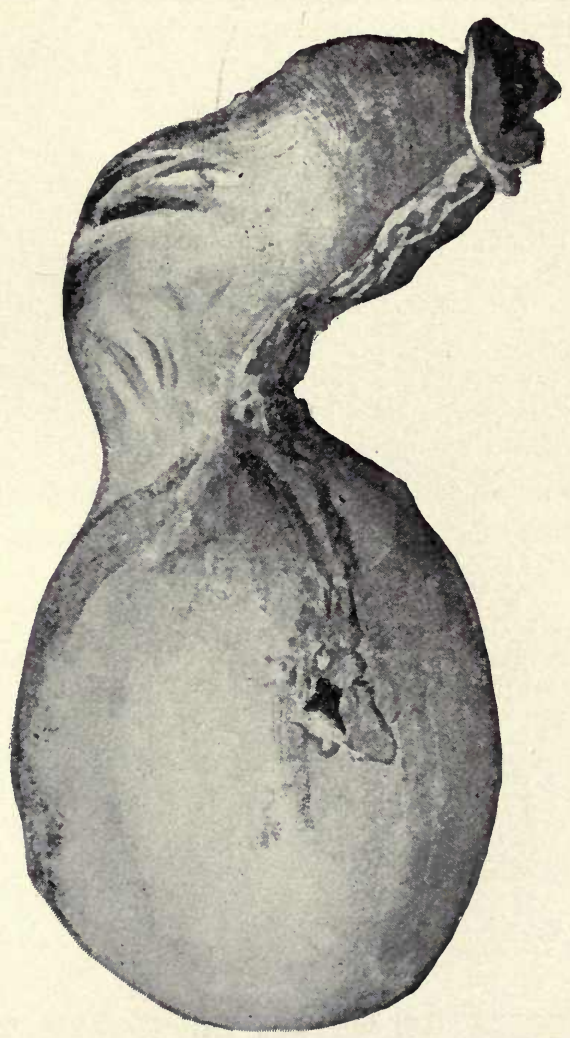

Hour-glass contraction of stomach (Warren Museum, Harvard Medical School).

Perforation (Fig. 165) and the escape of the stomachcontents into the peritoneal cavity cause death immediately from shock; or in a few days from a general peritonitis. This accident occurs most frequently when the ulcer is situated on 
the anterior wall of the stomach. It may also take place into the liver, spleen, or pancreas, with resulting abscess-formations; or into the pleura, pericardium, or lungs.

Hemorrhage, when a large vessel is eroded, may prove rapidly fatal (Fig. 166).

Cicatrization and consequent contractions of portions of the stomach-wall may cause considerable deformity-hourglass contraction, or pyloric or cardiac stenosis (Fig. 167).

\section{TUMORS.}

Carcinoma is the most common and most important tumor of the stomach. It is almost invariably a primary affection.

FIG. 168.

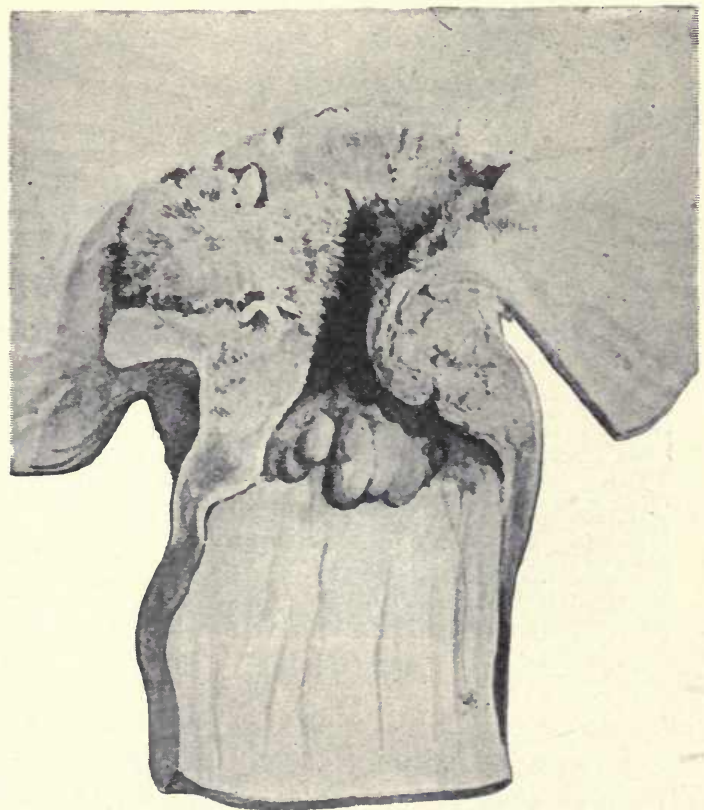

Cancer: a large fungous growth at the pylorus, with dilatation and hypertrophy of the walls (Warren Museum).

Secondary cancer is rare, though it may result by extension from adjacent organs; and in a few instances there seems to 
have been a direct implantation of eancer-cells upon the gastric mucosa from a primary lesion above-œsophageal, lingual, or facial.

It rarely occurs before thirty years of age, though in a few instances it has been noted in infants. From the age of fifteen years on, it rapidly increases in frequency, three-fourths of all cases occurring between the ages of forty and seventy years.

Fig. 169.

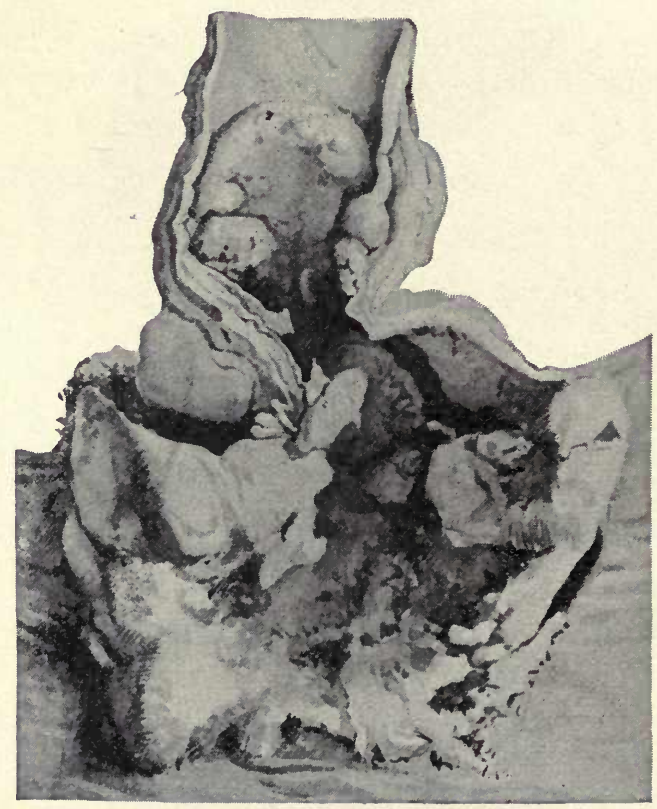

Cancer of the cardiac end of stomach and œsophagus (Warren Museum).

In women, cancer affects primarily the stomach in 20 to 30 per cent. of cases; and in men in 40 to 50 per cent. In about one-half the cases the neoplasm is situated at the pylorus, the posterior wall and lesser curvature coming next in frequency.

The position and shape of the stomach are often altered. 
When there is pyloric obstruction its capacity may be greatly increased (Fig. 168); or much diminished if such a condition exists at the cardia, or if there is a diffuse carcinomatous infiltration of the walls of the organ (Fig. 169). If the pylorus is not supported by adhesions, it may sink to the pelvis.

Metastases occur in one-half the cases; the liver is involved in over one-third of them. The neighboring lymphatic glands, especially those behind the lesser curvature of the stomach, become converted into large cancerous nodules, and the peritoneum is often involved by direct extension of the tumor-formation.

Histologically several varieties of the neoplasm are recognized :

Scirrhus is the most common, constituting about 75 per cent. of the cases. There may be a ring-like contraction at the pylorus, or a diffuse infiltration of the walls of the stomach without any nodular prominences, which condition might be confounded with a hyperplastic interstitial gastritis. Occasionally small, flat nodules occur on the serous surface over the region of the tumor. If the mucous membrane ulcerates, which is rare, it is only superficial. Scirrhus is almost invariably situated at the pylorus.

Encephaloid grows very rapidly, and forms large, soft, grayish-white, irregular, papillomatous masses, which project into the cavity of the stomach. It is so prone to undergo necrosis that it generally presents at the autopsy as a bowl-shaped, ulcerating depression, with irregular, ragged walls of varying height and thickness, formed by the surrounding tumor-mass. Perforation may result from extensive ulceration.

Adeno-carcinoma in general appearance resembles encephaloid, and tends to undergo extensive ulceration in a similar manner.

Squamous-celled cancer affects the region of the cardia, taking its origin from the flat cells of the osophagus.

Colloid carcinomata have a transparent jelly-like appearance, due to the degenerative change they have undergone. This degeneration may affect any form of cancer, but more especially adeno-carcinoma. 


\section{THE INTESTINES.}

\section{Congenital Deformities and Displacements.}

Absence of the whole, or large parts, of the intestinal tract is sometimes met with in monstrosities. Lesser developmental defects are not uncommon. The anus may be wantingimperforate anus-and likewise the rectum and lower part of the colon. Meckel's diverticulum (Fig. 170) is a remnant of

FrG. 170.

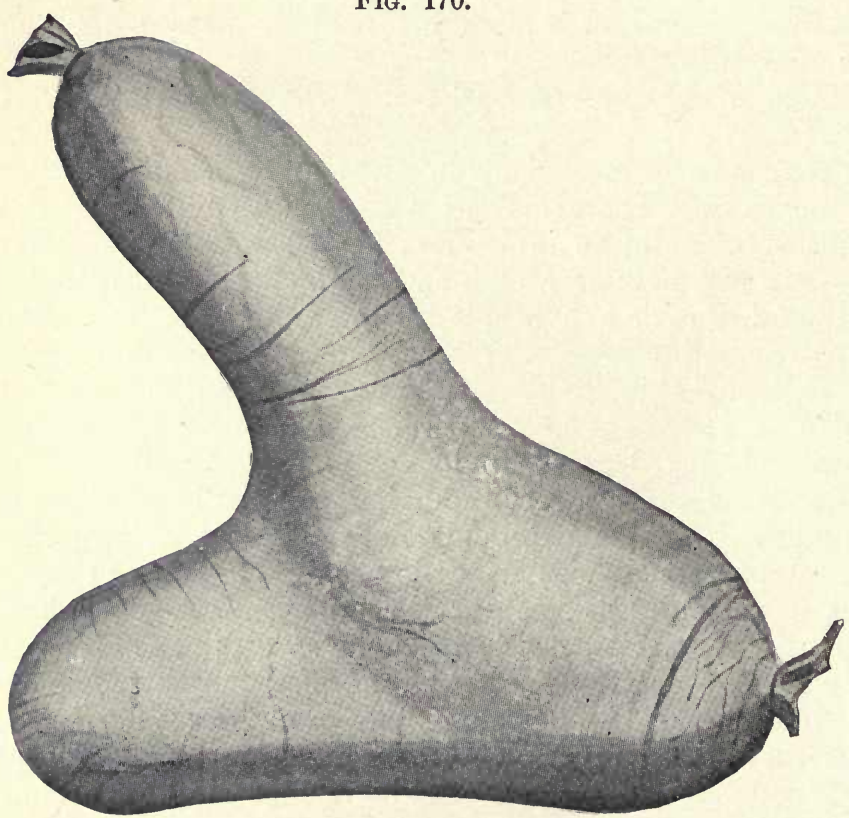

Meckel's diverticulum (Dennis).

the omphalo-mesenteric duct of foetal life. It is a cylindrical appendage, of the same structure as the small intestine, and arises from the latter, opposite the mesenteric attachment, about a meter above the ileo-creal valve. Such diverticula are sometimes the cause of intestinal obstruction (Fig. 171).

The cæcum and ascending colon are sometimes situated on 
the left side, and the descending colon and sigmoid flexure on the right. The transverse colon may be entirely absent, the ascending and descending colon lying side by side.

FIG. 171.

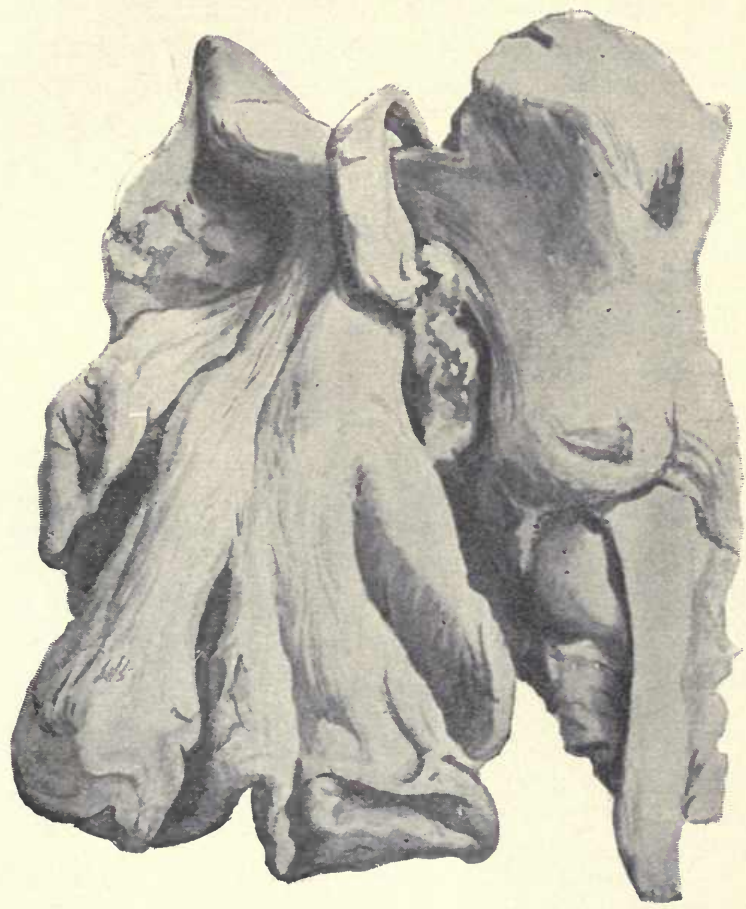

Internal strangulation by a divertículum (Warren Museum).

\section{Acquired Deformities and Displacements.}

Hernia is a term by which is generally understood a protrusion of the intestine through a natural but abnormally dilated opening in the abdominal wall. It pushes in front of itself the peritoneum, which thus forms the sac of the hernia. Strangulation is the most serious result of hernia, and occurs when the gut is so constricted or compressed that it is no longer 
pervious. By reason of the interference with its bloodsupply, if the constriction is not relieved, gangrene sooner or later ensues.

Volvulus is another frequent cause of intestinal obstruction. There is either a twist of the bowel on its long axis, or, more frequently, a loop of intestine is twisted around its mesenteric attachment. The sigmoid flexure is the most frequent seat of its occurrence.

Intussusception, or invagination, is more frequent in children than in adults, occurring usually at the ileo-cæcal valve. One part of the intestine slips over another, the length of the invagination, which is nearly always downward, varying from a few inches to several feet (Fig. 172). Thus the cæcum and

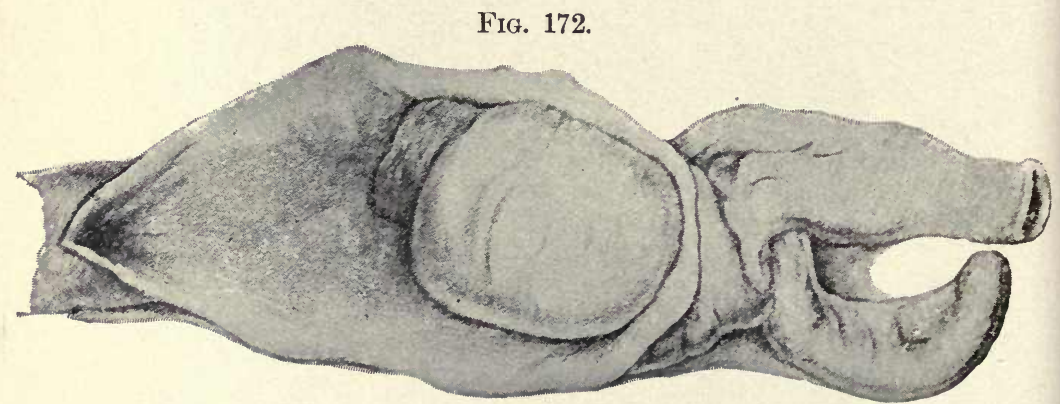

1leo-cæcal intussusception of minor degree. To the right is seen the appendix vermiformis just about to be swallowed (Hutchinson).

lower part of the ileum may be pushed into the colon until the ileo-cæcal valve even reaches the rectum. Gangrene and a fatal peritonitis are the usual termination.

\section{Inflammations.}

Inflammatory processes may involve more or less the entire length of the intestinal tract-entero-colitis-or be more particularly limited to one of its subdivisions, suggesting the terms duodenitis, colitis, ileo-colitis, typhlitis, proctitis, etc.

Enteritis-inflammation of the small intestine-is an 
exceedingly frequent affection, the most prominent clinical feature of which is diarrhoe. The watery consistency of the stools is due, not so much to the inflammatory serous exudation, as to the increased peristalsis, which permits of less time for the abstraction of the fluid constituents of the intestinal contents.

The inflammatory process is caused by improper food, especially that in which poisonous alkaloids have been formed during putrefactive changes; also by inorganic poisons, like arsenic and antimony; and it may occur secondarily in the course of various infectious diseases.

Lesions: The mucous membrane is red and swollen, often covered with mucus; and here and there may be small hemorrhagic spots. The solitary and agminated lymphatic follicles are swollen, and appear as small, dull gray prominences upon the mucous surface; small pitted, so-called follicular ulcers result if these lymphatic glands undergo necrosis. In severe cases the mesenteric glands are enlarged. When the inflammatory process becomes chronic, the muscularis is involved, as in gastritis, and often much hypertrophied. Sometimes polypoid elevations are produced by proliferation of glandular elements. In the later stages there may be atrophy of the mucous membrane alone, or involving, in addition, the muscularis.

A duodenitis may be associated with an inflammation of the stomach. It is exceedingly liable to produce an obstruction of the common bile-duct by inflammatory swelling of the mucosa and accumulation of mucus, thus giving rise to retention of bile and an obstructive or catarrhal jaundice.

Acute colitis is, in the great majority of instances, associated with the condition recognized clinically as dysentery. Sporadic cases result from ingestion of tainted food, from mercury and other poisons. The infectious nature of the epidemic form naturally suggests itself. The amoba coli, a unicellular amœboid body, measuring twenty to fifty microns in diameter, is found not only in the stools, but also in the intestinal wall adjacent to the dysenteric ulcers, and in the pus in hepatic abscesses secondary to dysentery. Typical dysentery has 
been produced in lower animals by the injection of dysenteric stools into their intestines.

The severity of the lesions noted varies greatly. In mild cases the mucous membrane is swollen and hyperæmic, and

FIG. 173.

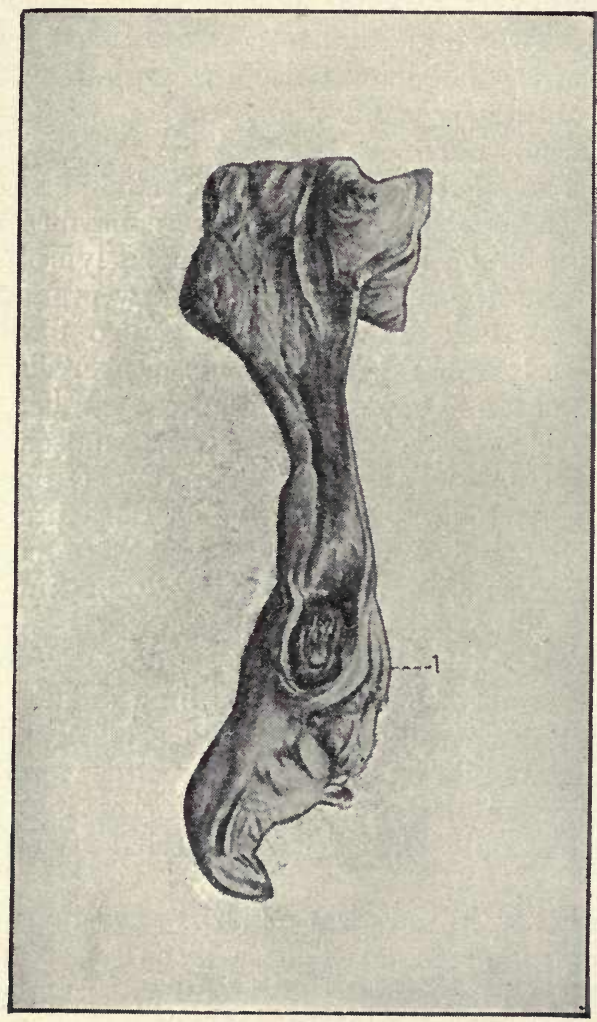

Appendix containing biliary calculus (Museum, Carnegie Laboratory). 1, biliary calculus.

covered with tenacious mucus. The solitary follicles are enlarged, and petechial hemorrhages are often seen. At a later stage and in more severe cases these follicles undergo 
necrosis ; the resulting ulcers may be superficial, or deep and ragged in outline, spreading rapidly, so that there is but little of the mucous membrane unaffected. In many cases the

FIG. 174.

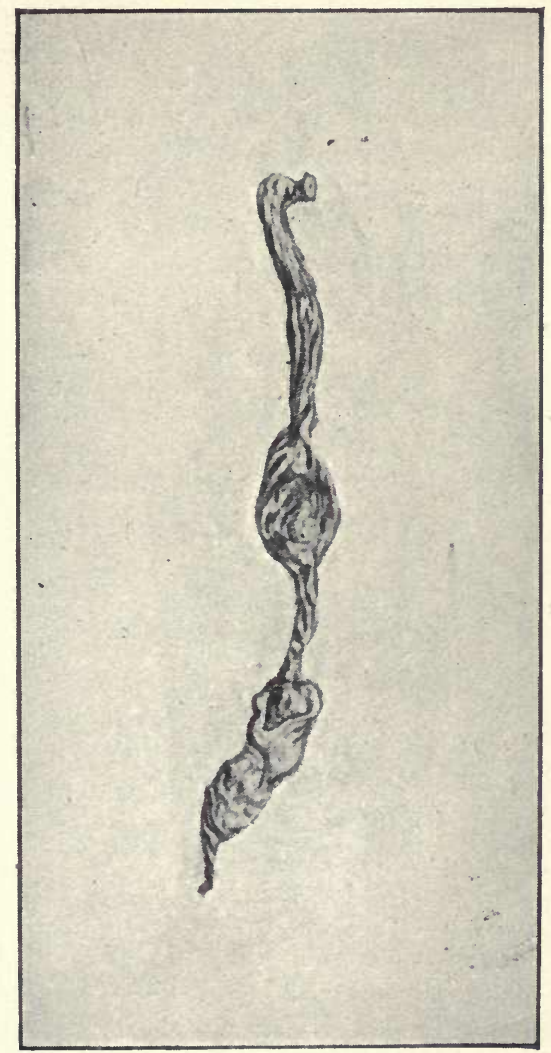

Appendix containing two fecal concrements: case of perforative appendicitis, the perforation opposite the distal concrement (Museum, Carncgie Laboratory).

mucous membrane is covered with a grayish or brownish pseudo-membrane, either in isolated patches or less frequently as a uniform coating-diphtheritic or croupous dysentery. 
384 DISEASES OF THE GASTRO-INTESTINAL TRACT.

FI(t. 175.

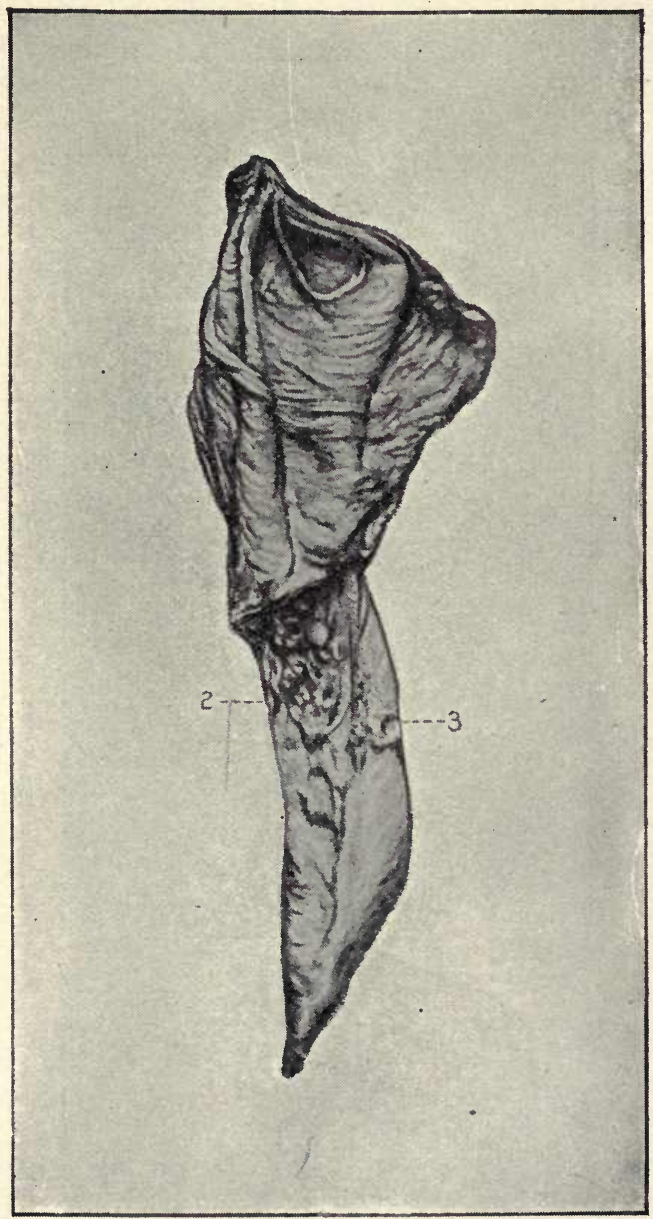

Suppurative appendicitis, abscess of appendicular wall, perforation, localized suppurative peritonitis; 2 , ruptured abscess of wall; 3 , extension of a suppurative focus (Museum, Carnegie Laboratory).

Sloughs of varying depth and sometimes of very considerable size may form, and, on separating, leave behind ragged ulcers. 
Chronic inflammation may succeed the acute. In some cases the inflammatory process is limited to the rectum-proctitis. Appendicitis - inflammation of the vermiform appendix-is now recognized as the cause of the set of symptoms which were at one time attributed to inflammation of the cæcumtyphlitis. On laying open the appendix, fæcal concretions are sometimes found, to which an etiological significance has been attributed (Figs. 173 and 174); but they are quite as

FIG. 176.

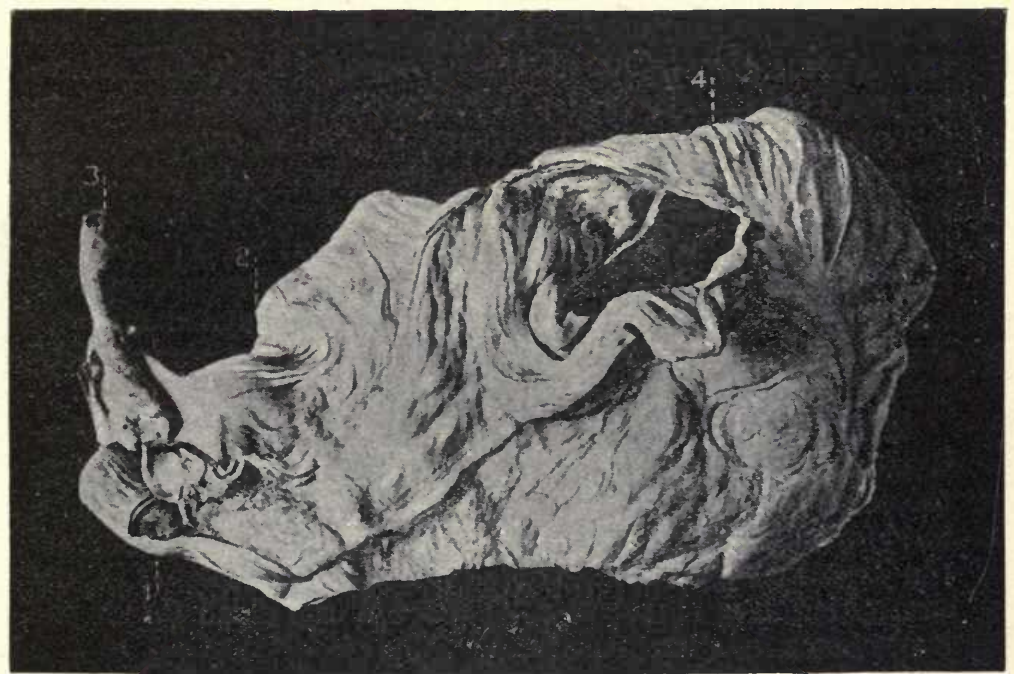

Funnel-shaped cæcum and appendix, gangrenous appendicitis: 1, fecal concrement; 2, mesenteriolum; 3 , appendix, situated behind and to inner side of cæcum ; 4, ilio-cæcal junction (Hartley).

likely the result as the cause of the condition, and formed by accumulations of mucus, desquamated epithelial cells and other inflammatory products. Undoubtedly the common colon bacillus, which is normally present, plays an important part, multiplying rapidly and gaining new virulence in the favorable nidus produced by the inflammatory process. The entire wall of the appendix may be involved, or the lining mucous membrane alone. Necrosis and ulceration may result in 
perforation and localized purulent collections, or a general septic peritonitis. Not infrequently the whole organ becomes almost immediately gangrenous (Figs. 175 and 176).

\section{Specific Inflammations of the Intestines.}

Asiatic cholera is an acute infectious disease characterized by an intense inflammation of, and a copious serous exudate from, the small and large intestine; due to the spirillum choleræ, first described by Koch. Clinically, also, the main features of the disease are referable to the intestinal tract, the constitutional disturbances being due to the absorption into the general circulation of toxins produced by the microorganisms there localized. The mucous membrane, particularly in the lower part of the ileum, is swollen, congested, and frequently the seat of ecchymoses; the solitary and agminated lymphatic follicles are often swollen, as may be likewise the mesenteric glands. The bowel is distended as if paralyzed, and filled, often with large quantities, of a serous transudation containing small white flakes of desquamated and degenerated epithelial cells, similar to the characteristic " ricewater" dejections.

Later in the disease, in some epidemics, the mucous membrane may be ulcerated or covered by a pseudo-membrane, the colon presenting an appearance similar to that in dysentery - possibly the result of secondary infections. Outside of the intestinal tract one of the most marked features of the disease is the pronounced thickening of the blood due to the enormous serous transudations.

The heart, liver, spleen, and kidneys may be the seat of parenchymatous degenerations characteristic of other toxæmias as well, however, and in no way distinctive of this disease.

Typhoid fever is an acute infectious disease produced by the bacillus of Eberth, the characteristic lesions being almost entirely confined to the lymphadenoid structures of the lower part of the ileum and upper part of the colon. Contaminated water or milk is the chief source of infection.

The morbid changes in typhoid fever may begin as a diffuse inflammation of the intestinal mucosa, but almost immediately, at least within twenty-four hours, the solitary follicles and 
Peyer's patches become infiltrated, intensely congested, swollen, and project into the lumen of the intestine. The largest patches may be as much as a quarter of an inch thick. The solitary follicles vary in size from that of a pin point to that of a pea (Fig. 177). By the end of the first week the number of patches and follicles involved is about as large as it will probably become, and the stage of inflammatory infiltration and hyperplasia has reached its height. These changes may affect only a few patches and follicles in the neighborhood of the cxcum or involve the entire intestinal tract.

Fig. 177.

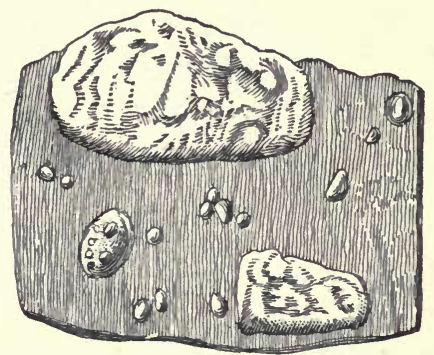

Swelling of Peyer's patches and solitary glands of the intestine, as seen in typhoid fever (Green).

Generally the swollen solitary follicles are not so numerous as the swollen Peyer's patches, though in some cases they are more prominent, the patches being but little affected. Cases of undoubted typhoid have been reported in which all intestinal lesions were absent.

Resolution now slowly takes place through the absorption of the inflammatory products; or a greater or less number of

FIG. 178.

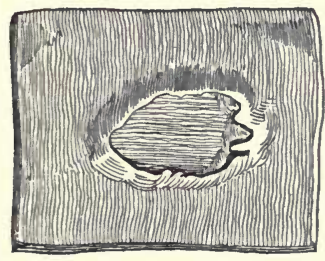

A typhoid ulcer of the intestine (Green). patches, according to the severity of the case, undergo necrosis as the result of interference with the bloodsupply by the pressure of the surrounding excessive infiltration. The patches become less congested, grayish in color, with possibly a yellowish or brownish necrotic centre. When the slough separates there is left behind a large ragged ulcer, which generally involves only the mucous membrane, but may extend even through the muscularis and rarely through the serosa, thus producing perforation. Hemorrhage may also occur with the separation of the slough. 
The resulting ulcers usually conform to the size and shape of the patch involved (Figs. 178 and 179) ; especially in the

FIG. 179.

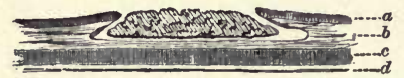

A typical ulcer of the intestine (diagrammatic), showing the undermined edges of the ulcer and the slough still adherent: $a$, epithelial lining; $b$, submucous tissue; $c$, muscular coat ; $d$, peritoneum (Green).

lower part of the ileum, however, the ulcers are liable to coalesce, and thus involve extensive areas of the mucous membrane. The solitary follicles undergo necrosis in a similar manner, resulting in the formation of rounded ulcers. By the end of the third week this stage of ulceration is generally complete, and during the fourth week cicatrization is in progress, finally leaving behind thin, transparent, flexible scars, which often can be recognized years afterward; the lymphatic elements are not replaced.

Microscopically the swelling of the patches and follicles is found to be due mainly to an active proliferation of their lymphoid cells; a few larger epithelioid cells are usually present.

Coincident with these intestinal lesions of typhoid, the mesenteric glands, in relation to the areas most affected, become swollen from a similar inflammatory hyperplasia, and to an extent in proportion to the severity of the intestinal lesion.

Enlargement of the spleen is also almost constantly presentproduced by its congestion and an increase of its lymphoid elements; large epithelioid cells are often present, as in the intestinal lesions and mesenteric glands, which are actively phagocytic, frequently having encysted within them fragments of red corpuscles.

The spleen, as well as the liver and kidneys, are often the seat of necrotic foci. The typhoid bacilli can usually be demonstrated in large clumps, in properly stained sections of the spleen (Figs. 180 and 181), though the whole organ may possibly be cut up in a vain search for them. They are also present in the intestinal lesions, mesenteric glands, liver and kidneys, in the dejecta, and can sometimes be found in the 
FIG. 180.

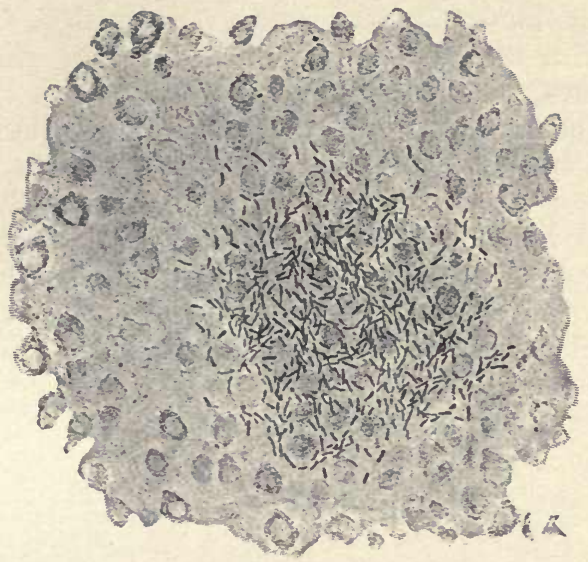

Human spleen, tenth day of enteric fever (Charcot).

FIg. 181.

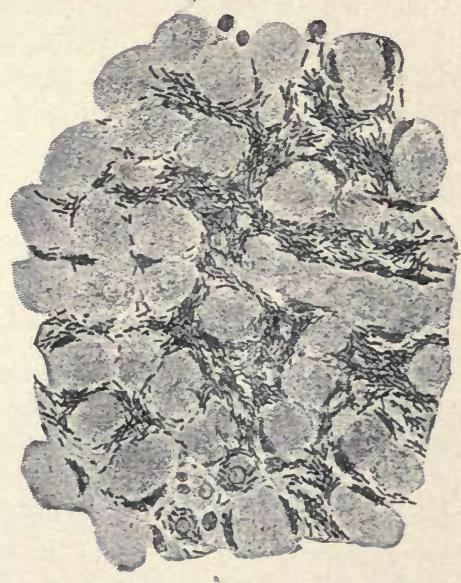

Human liver, tenth day of enteric fever.(Charcot).

blood. In the ahsence of a leukocytosis, the blood of typhoid presents a marked clinical contrast to the blood in other acute infectious diseases. 
The lesions of other organs are in no way characteristic of the disease. Parenchymatous or granular degenerations are found in all toxæmias of sufficient degree and duration; the liver, kidneys, and heart-walls are so affected.

Inflammation of the pericardium, endocardium, or myocardium is not uncommon.

A peculiar waxy or hyaline degeneration, which may occur also in other infectious diseases, involves the voluntary muscles, the fibres being converted into a homogeneous, colorless, shining mass resembling amyloid degeneration, but not giving its characteristic reaction with iodine and sulphuric acid, and affecting most frequently the abdominal muscles, adductors of thighs, the pectorals, the diaphragm, and sometimes the myocardium.

Bronchitis, lobar and lobular pneumonia are frequent complications; gangrene of the lungs a rare one. Occasionally there is an inflammation of the parotid gland, which tends to terminate in abscess-formation. A grave peritonitis is generally the result of perforation, though it may have its startingpoint in a deep ulcer which involves, but does not perforate the serosa; or it may be due to the inflammation involving the mesenteric glands.

An obliterating endarteritis may occur, especially during convalescence, leading to thrombosis and necrosis of the tissue supplied. Thrombosis may also occur in the veins, especially the femoral, rarely in the cerebral sinuses.

An orchitis occurring during convalescence, and frequently terminating in suppuration, has been described.

Tuberculosis, though scarcely ever noted in the osophagus or stomach, very frequently affects the intestinal tract-in fact, is one of the most common pathological conditions met in the intestine. While not necessarily confined to the lymphadenoid tissue, these structures are especially involved, the lesions being most numerous in the lower part of the ileum and in the colon. It may be primary - rarely in adults, though somewhat more frequently in infants; generally it is secondary to tuberculosis of the lungs, and the result of swallowing infected sputum.

The tubercle appears beneath the mucous membrane 
as a grayish nodule; soon it becomes yellowish from rapidly advancing caseation, and finally breaks down completely.

The resulting ulcer generally involves the muscularis; its floor is rough and nodular, from the presence of small tubercles, which can be seen beneath the serosa externally (Fig. 182). The ulcers at first resemble somewhat those of

FIG. 182.

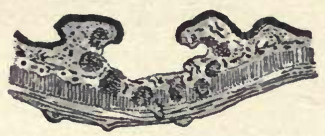

Tubercular ulcer of the intestine (Kaufmann). The cavity of the ulcer was formed through disintegration and removal of the cheesy matter formed in the earlier tubercles. Now the base of the ulcer is formed by necrosed and cheesy material, beneath which eight or nine distinct tubercles are distinguishable, those in the centre extending into the muscular coat of the intestine. The infection has also extended into the lymphatics beneath the serous coat, where three tubercles can be seen.

typhoid fever; but soon, as the result of breaking down of other newly-forming tubercles at their margins, the ulceration extends beyond the limits of the lymphoid tissue, no longer conforming to the size and shape of these structures. Fresh tubercles being formed in this way along the line of the lymphatic vessels, which run toward the mesentery, the long axis of the ulcer becomes transverse, a complete ring of mucous membrane being destroyed, in some cases, in this mannerannular ulcer. The border of the ulcer, like its base, is infiltrated here and there with small tubercular nodules. On the external serous surface radiating lines are noted, extending from the base of the ulcer around the intestine toward the mesentery, marking the tubercular infiltration of the subserous lymphatics. Perforation is rare, owing to this infiltration and thickening of the tissues. The corresponding mesenteric glands are generally affected. If the ulcer heals, which is infrequent, a puckered scar and possibly cicatricial deformities remain.

Microscopically, the characteristic feature is the presence of small tubercles in the floor and walls of the ulcer.

Syphilitic ulceration of the solitary follicles and Peyer's patches of the small intestine sometimes occurs in infants. 
In adults, though the small intestine and colon may rarely be involved, the rectum is the part of the intestinal tract most frequently affected.

\section{TUMORS.}

Carcinoma is the most important and most frequent tumor affecting the intestinal tract, though not nearly as common as

FIG. 183.

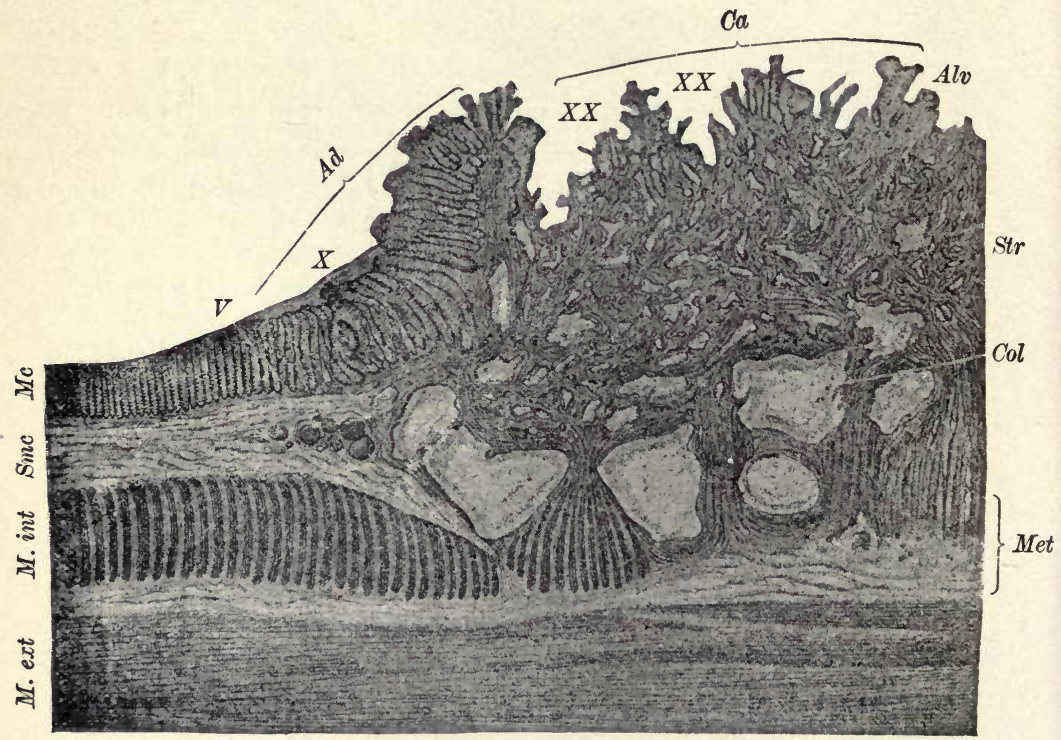

Section showing degenerative changes in carcinoma of the rectum : $M c$, mucosa; Smc, submucosa : M. int, inner muscular fibres (circular); $M$. ext, outer muscular fibres (longitudinal); $V$, bloodvessels; Ad, margin of growth, showing hypertrophied follicles and submucous tissue infiltrated with new adenoid tissue : $\boldsymbol{X}$, mucus; $C a$, ulcerated portion of the superficial surface of the growth ; $X \boldsymbol{X}$, remains of gland-follicles stlll recognizable; $A l v$, glandular recesses dilated into distinct alveoli; Col, large alveoli filled with mucoid or colloid material; Str stroma; Met, adenoid proliferation infiltrating deeper layers of bowel-wall (Esmarch).

in the stomach. In the small intestine it is rare, the duodenum near the opening of the bile-duct being the point at which it usually is situated. More frequently it occurs in the 
rectum, the cæcum, and flexures of the colon. It usually presents as a solitary, soft, often ulcerating, fungous mass, which projects into the lumen of the intestine (Fig. 183).

Microscopically, the adeno-carcinoma or cylindrically-celled cancer is the variety most frequently noted.

Connective-tissue tumors are not often found in the intestinal tract.

\section{THE LIVER.}

\section{PASSIVE HYPER}

Passive hyperæmia is produced by some obstruction to the circulation through the hepatic vein, as by a large pleuritic effusion; intrathoracic tumors, cirrhosis of the lungs; and especially valvular disease of the heart. At first the liver is often considerably enlarged; on section, it has a peculiar mottled or nutmeg appearance, the darker congested areas around the central hepatic vein of the lobule contrasting markedly with the lighter periphery, which may be yellowish or yellowish-white from advancing fatty degeneration. At a later stage the liver is decreased in size, from atrophy of the parenchyma-cells produced by the excessive pressure of the blood; and there may be very considerable hyperplasia of the connective tissue, giving the organ a slightly roughened or uneven surface.

Microscopically, the central hepatic vein and surrounding capillaries are greatly dilated and engorged with blood. In the centre of the lobule the rows of liver-cells between the dilated eapillaries are entirely atrophied. In the peripheral zone of the lobule there is often an advanced fatty degeneration.

\section{ACUTE YELLOW ATROPHY.}

Acute yellow atrophy of the liver occurs as an independent specific disease; or secondarily to phosphorus-poisoning, typhoid fever, puerperal septicæmia, and other infectious diseases. As an independent affection, however, it is exceedingly rare.

Anatomically, the characteristic feature of the disease is the 
rapid diminution of the liver to one-half, even one-quarter its natural size, as the result of extensive degenerative changes of the parenchyma. This reduction in size affects mainly its vertical diameter, the organ being flattened out against the spinal column to a few centimetres in thickness, and almost concealed by the diaphragm and superimposed coils of intestine. It is ochreous-yellow in color, soft and flabby, in places almost pulpy; or the yeilow alternates with relatively firmer red patches-so-called red atrophy-giving the surface a mottled appearance, especially on section. The capsule is wrinkled to such an extent that it can be picked up by the fingers.

Microscopically, the ontlines of the lobules are indistinguishable. The liver-cells are in various stages of degeneration or necrosis; in the yellow areas their outlines may be preserved, but their protoplasm contains numerous fat-globules and granules of yellow pigment; or the cells may be entirely replaced by a detritus of yellow pigment; and irregular collections of fat-globules, only the connective-tissue framework of the liver remaining. In the red areas the cells are entirely wanting, the tissue being colored by an infiltration with hematogenous pigments.

\section{INFLAMMATIONS.}

Acute purulent inflammation (Fig. 184) of the liver results in the formation of one or more circumscribed collections of pus. The avenues open to infection by the invasion of various micro-organisms are numerous: the hepatic artery, portal vein, bile-duct, and the patent umbilical vein in infants ; or infection may extend from neighboring organs through the lymphatics. The bacillus coli communis, staphylococci, and streptococei are the organisms which have been most frequently found associated with these purulent inflammations of the liver; in cases following dysentery the amoba coli is frequently found in the pus.

The single large so-called tropical abscess is a very common affection in the torrid zone, often as a sequela of dysentery. It may be associated with the presence of tumors or parasites in other cases. The abscess is generally situated in the right lobe, and varies in size from a man's fist to that of a child's 
head; an entire lobe or even the whole organ may be involved. It may rupture into the pleural or peritoneal cavities, into the stomach, intestine, or externally through the

FIG. 184.

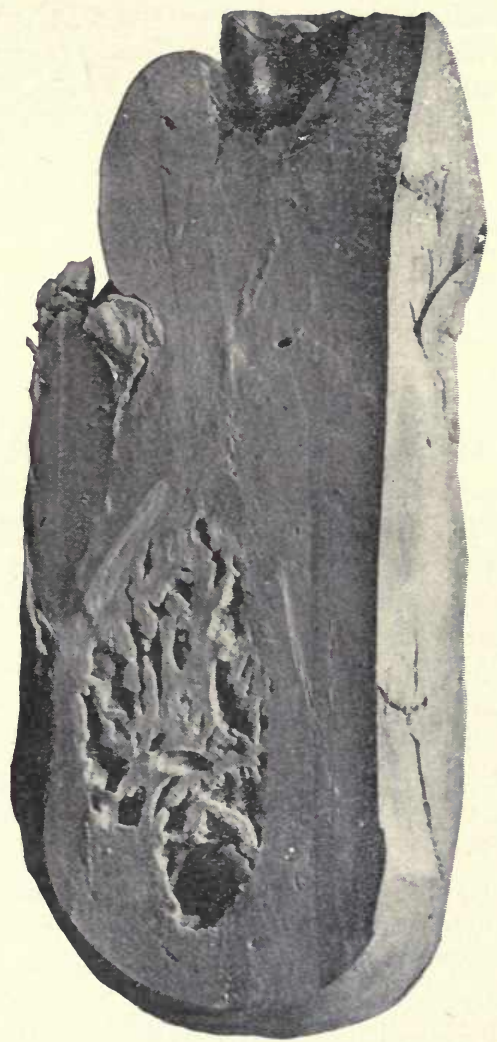

Abscess of liver in a case of pyæmic infection through a wound in the appendix vermiformis caused by lodgenient of a pin (Loomis and Thompson).

abdominal wall. As to the mode of their formation, but little is known ; undoubtedly they often result from the confluence of smaller abscesses.

The walls of the abscess are ragged-shreds of necrotic liver- 
tissue projecting into the cavity ; there is no trace of a membrane. The contents of the eavity have an offensive, sickly odor; are thick and creamy like pus, or thin and reddishbrown from the admixture of extravasated blood.

Microscopically, this fluid is found to contain pus-cells, fragments of degenerated liver-cells, shreds of connective tissue, and red blood-corpuscles. The abscess-wall shows but little, if any, inflammatory infiltration.

Small metastatic abscesses, varying in size from a pin-head to that of a walnut, are sometimes present to the number of forty or fifty, seattered throughout the liver. They occur in the course of pyæmia and septic inflammations of parts tributary to the portal vein.

Microscopically: In the early stage of their formation there is noted an area of necrosis, the centre of which may have become simply a granular detritus arising from the disintegration of the liver-cells. Surrounding the necrotic area is a zone of more or less marked inflammatory infiltration.

Chronic interstitial inflammation or cirrhosis of the liver is also known as "gin-drinkers" "liver, for at least two-thirds of the cases are due to chronic alcoholism. Certain acute infectious diseases, chiefly cholera, typhoid, and intermittent fever, are supposed by some to be important etiological factors. Among chronic infectious diseases, syphilis is an undoubted cause of cirrhosis. Gout and rheumatism seem to be responsible for some cases. Experimentally, the condition has been produced in lower animals by chronic poisoning with phosphorus and cantharides. Though in most instances hematogenous in its origin, in some it is biliary, due to an obstruction of the bile-ducts.

In the early stages the liver may be considerably hypertrophied ; finally, however, in the great majority of cases it is reduced in size even to one-third the normal. The longer the duration of the disease the greater the atrophy, and the tougher and more leathery the consistence of the organ. Its surface is rendered irregular by numerous nodular projections varying in size from a millet-seed to that of a hazelnut. The yellow color of these granulations suggested to Laënnec the term cirrhosis (from $x \iota j \rho \rho_{5}$, yellow). On section a grating sound is emitted, and the almost cartilaginous consistence of 
the organ may be noted; a network of connective-tissue bands, running in all directions-extensions from the thickened fibrous capsule of Glisson-is seen, which divides the parenchyma into small islets corresponding in size to the surfacegranulations. The yellow bile-stained groups of lobules and pink interlobular fibrous tissue gives the cut surface a mottled and granular appearance.

Microscopically, the chief characteristic is the chronic diffuse inflammatory hyperplasia of the interstitial connective tissue, and resulting atrophy of the parenchyma of the liver, at least a numerical atrophy, as the hepatic cells which remain in the projecting granulations or nodules frequently undergo a compensatory hypertrophy to twice their normal size. As long as the hyperplasia is in excess of the atrophic changes the organ is enlarged. As the newly formed connective tissue becomes more sclerotic, compressing the cells of the lobules and the vessels from which they obtain their nourishment, the atrophy which is produced, both by direct pressure and interference with the blood-supply, sooner or later results in a reduction in the size of the organ. The yellow appearance of the nodules is in part due to the obstruction, produced by the hyperplastic connective tissue, to the discharge of bile from the bile-capillaries into the interlobular ducts; and in addition there is generally an excessive accumulation of fat in the liver-cells, resulting not only from the lessened bloodsupply, but also probably from the fact that there are fewer cells in which to store the fat normally present in the liver.

The pathological chonges begin as a round-celled infiltration of the interlobular connective tissue. Through the further development of these migrated and proliferating cells dense fibrous tissue is formed, the cellular character of which is finally almost entirely lost, so that it comes to resemble cicatricial tissue (Fig. 185). 'The result of the obliteration of the portal capillaries is engorgement of the portal circulation, the most important aspect of which clinically is the enormous serous transudation into the peritoneal cavity, which is called ascites.

Hypertrophic or biliary cirrhosis differs from the ordinary atrophic variety, in that the connective-tissue hyperplasia, which is interlobular or monolobular, does not have the same 
tendency toward sclerosis and contraction, and as a result the atrophic changes in the parenchyma are not so marked. The term biliary is suggested by the fact that it generally arises

FIG. 185.

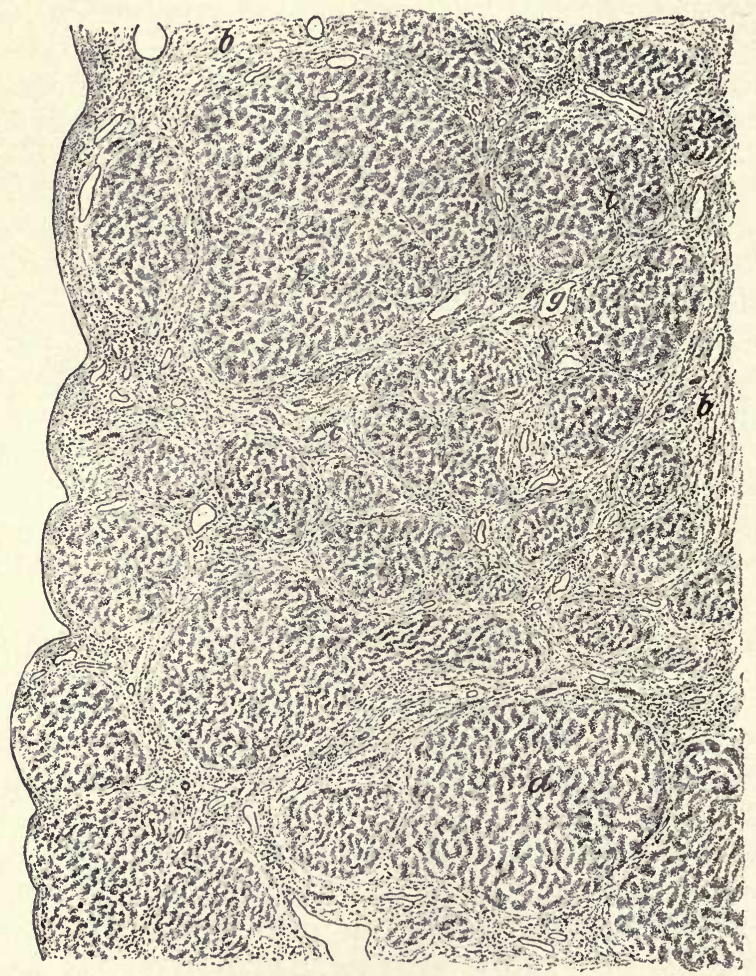

Atrophic cirrhosis of liver. $a$, acini, in parts much diminished in size; $b$, interacinous bands of connective tissue. $\times 250$ (Schmaus).

in connection with inflammation or obstruction of the bileducts.

The inflammatory hyperplasia begins around the interlobular bile-ducts, instead of around the branches of the portal vein. Experimentally, a monolobular cirrhosis can be pro- 
duced by obstruction of the bile-ducts, though in man it is by no means a constant accompaniment of such an obstruction. In some cases formation of new bile-capillaries is observed. The organ is enlarged, its surface finely granular, and on seetion yellow to dark green bile-stained patches are noted.

Clinically, jaundice is nearly always present, due to the obstruction of the bile-ducts by the newly forming connective tissue; the portal vein is not obstructed as in the atrophic variety, so that ascites is generally absent.

The specific granulomata: Syphilitic lesions of the liver occur generally as a diffuse cirrhosis, resembling the ordinary atrophic cirrhosis, except that the bands of newly formed connective tissue are much heavier. Characteristic gummata may be present also, or they may occur unassociated with connective-tissue hyperplasia; they appear as yellowish or grayish, firm rounded masses, with necrotic, cheesy centres, varying in size from a pea to that of a hen's egg.

Tubercular lesions of the liver are rarely primary. Diffuse miliary tubercles frequently occur, however, in the course of general miliary tuberculosis; in some instances there are a few large foci only. A diffuse interstitial connective-tissue hyperplasia is sometimes associated with the miliary tubercles.

\section{TUMORS.}

Primary connective-tissue tumors do not often occur in the liver; angiomata more frequently than any other. Melanotic sarcoma has been observed, the black nodules of the neoplasm scattered through the organ, varying in size from a milletseed to that of a man's fist; much more frequently, however, the tumor is secondary to melanotic sarcoma of the eye.

Primary epithelial tumors of the liver are also rare.

Primary carcinoma is exceedingly infrequent as compared with the secondary. It occurs either as a large mass, generally in the right lobe, with secondary nodules throughout the organ; or it may occur as a diffuse infiltration, which resembles atrophic cirrhosis of the liver by reason of the nodular appearance of the surface, and the anastomosing bands of fibrous tissue noted on section. But in the islets between 
them, however, there is seen under the microscope the atypical structure characteristic of the neoplasm, and not liver-tissue.

Secondary carcinoma of the liver is quite common, occurring generally in the form of rounded and umbilicated, pinkish nodules, varying in size from a pea to that of an apple, and often so numerous as to produce an enormous enlargement of the organ. The liver-cells surrounding such a nodule are greatly flattened and atrophied. Microscopically, their structure usually recalls that of the primary tumor.

Echinococcus cyst is the most important parasitic disease of the liver, and is produced by the larvæ of the tænia echinococcus. There may be one or more cysts, varying in size up to that of a man's head. They consist of a connective-tissue capsule, inside of which is the parasitic cyst-wall proper, from which originate smaller vesicles-brood-capsules-and the heads of the immature tapeworms. As long as the parasite is alive the cyst contains a clear non-albuminous fluid, in which are found scolices, the size of a millet-seed, and characteristic hooklets.

\section{THE BILE-DUCTS AND GALL-BLADDER.}

Inflammation of the bile-ducts is generally secondary to an inflammation affecting the mucous menbrane of the duodenum. It may also be produced by the presence of a gall-stone or an intestinal parasite which has crept into the common duct. The result is an obstruction to the outflow of bile, and consequent absorption of bile-pigment, giving rise to the condition known as obstructive jaundice. (A non-obstructive jaundice may arise from increased hæmolysis in various severe infectious diseases-as yellow fever.)

Inflammation of the gall-bladder is quite common, and has a tendency to end in suppuration. It may result from extension of an inflammation from the bile-ducts, or be due to the presence of gall-stones or retained bile.

Stenosis of the bile-ducts may arise from the pressure externally of an aneurism, a tumor, or an inflammatory process around the duct. Congenital atresia sometimes occurs.

Primary carcinoma of the gall-bladder is occasionally met; the irritation produced by the presence of gall-stones is looked upon as a possible cause. 


\section{THE PANCREAS.}

Atrophy of the pancreas is not infrequently found in connection with diabetes mellitus.

Inflammation of the pancreas is a rare condition. Cirrhosis is generally secondary to an inflammatory process in some adjacent tissue; occasionally it is seemingly due to alcohol and syphilis.

Carcinoma is the most important tumor found in the pancreas. It is generally situated at the head of the gland, though the whole organ may be involved.

\section{THE PERITONEUM.}

Inflammation of the peritoneum, or peritonitis, but rarely occurs as a primary affection, as compared to the frequency of this condition in the other large serous sacs, the pleural and pericardial; nearly always it is secondary. It may be acute or chronic; circumscribed or diffuse. So-called idiopathic cases occur at times in which there is no demonstrable cause. In a great majority of instances bacteria play a most important part in exciting the inflammatory process. They may gain direct access to the peritoneum from the blood in septicæmia, pyæmia, and other infectious diseases. Much more frequently, however, there is a local septic inflammation of some organ which the peritoneum covers-as of the uterus and its appendages, and of the appendix vermiformis. The bacillus coli communis has frequently been found in cases associated with affections of the intestinal tract. Micro-organisms met with in other instances are the streptococcus and staphylococcus progenes, diplococcus pneumoniæ, and gonococcus.

When the infection takes place gradually and the organisms are in small numbers, the inflammatory process may be localized-as in connection with appendicitis and affections of the pelvic viscera. The entire peritoneum may subsequently become involved, as the result of extension of the inflammation; in other cases it is immediately involved by the introduction of a large amount of infective material-as in perforation of the stomach, or intestine, or nupture $\mathrm{gf}_{\mathrm{f}} \mathrm{an}_{\mathrm{a}}$ abscess into the peritoneal cavity.

26-Hist. 
Chronic peritonitis is characterized by thickening of the peritoneum, and the formation of adhesions between opposed inflamed serous surfaces, as in pleurisy.

Tuberculosis, primarily affecting the peritoneum, is rare. The most frequent sources of infection secondarily are tubercular affections of the mesenteric and retroperitoneal lymphatic glands, of the vertebræ, and of the pelvic organs in the female; miliary tubercles may occur in general miliary tuberculosis. 


\section{CHA PTER VII.}

\section{DISEASES OF THE URINARY ORGANS.}

\section{THE KIDNEYS.}

\section{PASSIVE HYPER EMIA.}

Passive hyperæmia occurs in conjunction with valvular disease of the heart, and diseases of the lungs, which seriously impede the circulation-as fibroid phthisis and severe emphysema.

The kidneys are generally enlarged in the earlier stages, are firm and elastic; the capsule, which is not adherent, may readily be removed, exposing a smooth surface. On section, the cortex is found to be congested and swollen; the Malpighian bodies may be enlarged and stand out prominently; the pyramids are deeply congested, and have a striated appearance, produced by dilatation of their bloodvessels.

At a later stage, there may be a very considerable hyperplasia of interstitial connective tissue, and the organ consequently more or less decreased in size and dense-cyanotic induration.

Microscopically, the prominent features are the dilatation and overfilling of the bloodvessels; atrophy of the epithelium of the tubules, and a marked increase in the amount of interstitial connective tissue. Quite often there are small extravasations of blood within the capsule of the Malpighian bodies.

\section{BRIGHT'S DISEASE}

To bilateral inflammations of the kidneys, in which the irritant has gained access through the blood, the generic term Bright's disease is applied, after the clinician who first explored this important field of pathology, and called attention to the relation of certain structural changes in these organsthe inflammatory nature of which was later recognized-to albuminuria and general dropsy. 
There is nothing distinctive of inflammatory processes in the kidneys. As in other organs, though inflammation affecting either the parenchyma or interstitial connective tissue alone does not occur, the changes may be so much more conspicuous in one or the other as to suggest the term parenchymatous or interstitial inflammation.

In acute inflammation produced by intense irritants, the glomeruli and epithelium of the secreting tubules are mainly affected; while in other instances in which the irritant is less intense and acts over a prolonged period, the inflammatory changes predominate in the interstitial connective tissue.

\section{Casts.}

Casts of the uriniferous tubules have been objects of very considerable interest in connection with these pathological

FIG. 186.

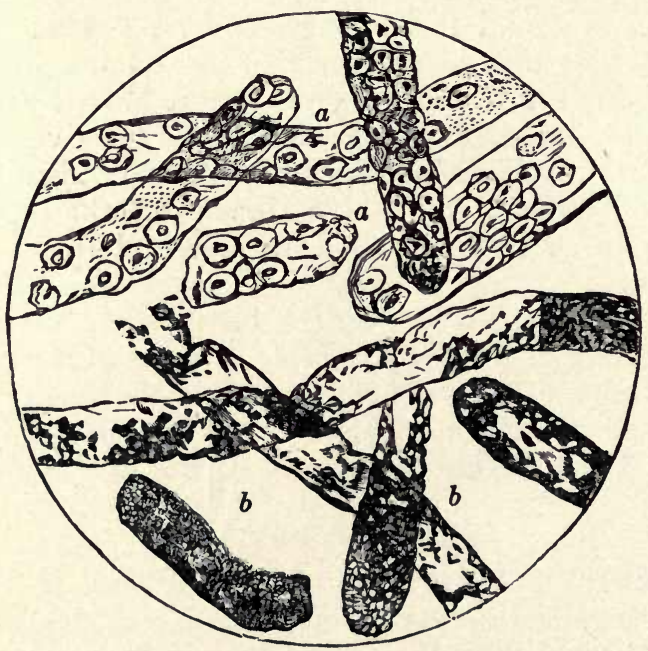

$a$, epithelial casts ; $b$, opaque granular casts (from a case of acute Bright's disease : Roberts).

changes since 1842 , when attention was first directed to them by Henle. They are found in the urine, and in the urinif- 
erous tubules at the site of their formation, in nearly all inflammations of the kidneys, and are but rarely present in normal urine.

The method of their formation is not perfectly understood -some probably by a species of secretion from the tubular epithelium, and others by coagulation of serous exudations into the tubules.

In size they vary according to the part of the kidney in which they are formed, measuring from 0.01 to 0.05 milli-

FIG. 187.

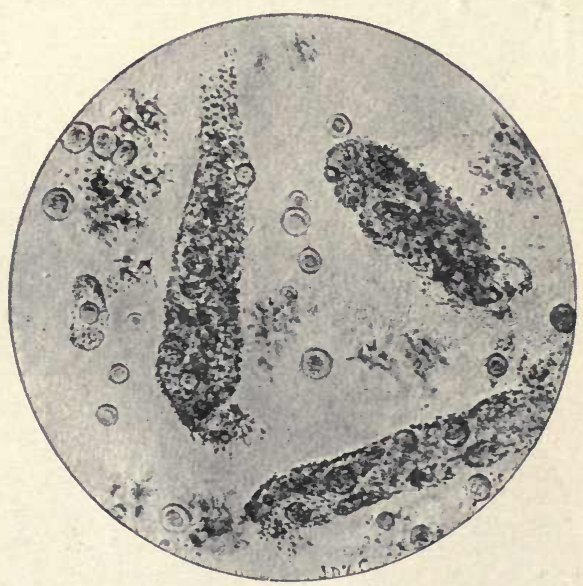

Granular casts (Musser).

metre in diameter, and one-tenth to a millimetre and more in length. Several varieties are distinguished.

Hyaline casts are homogeneous, transparent, and colorless. They are found in the urine from simply congested kidneys, and in transient albuminuria; often, moreover, in chronic parenchymatous and interstitial nephritis.

Granular casts are composed of a granular material resulting from the breaking down of the epithelial cells of the tubules. They are found especially in the urine from chronically inflamed kidneys. 
FIG. 188.

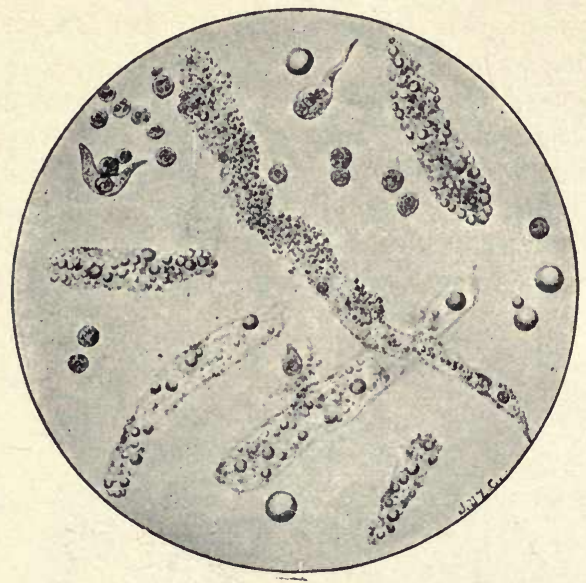

Fatty casts from a case of chronic parenchymatous nephritis (Musser).

FIG. 189.

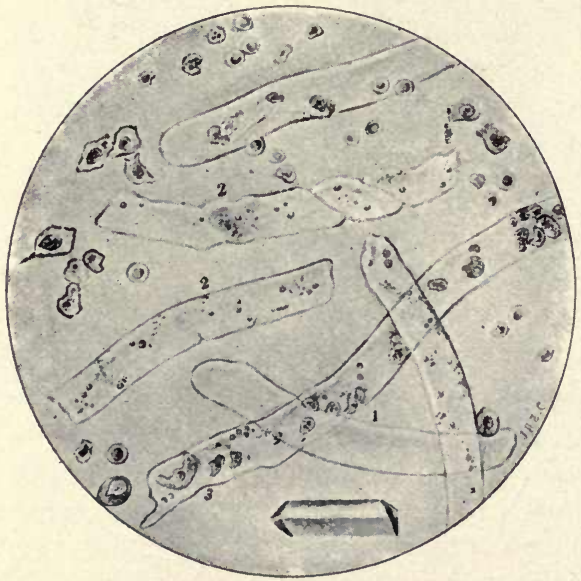

Hyaline casts from a case of acute nephritis: 1, plain hyaline cast : 2, granular deposits (hyaline casts); 3 , cellular deposit (blood and epithelium) (Musser).

Cellular casts occur when epithelial cells, or red and white blood-corpuscles cling to either of the above varieties. 
Waxy casts have a peculiar glistening appearance, which distinguishes them from the hyaline variety. The last two varieties occur in both acute and chronic nephritis (Figs. 186-190).

FIG. 190.

\section{ACUTE PARENCHYMATOUS NEPHRITIS.}

Acute parenchymatous nephritis may be a primary affection, occurring often without discoverable cause ; or secondary, as a rather frequent complication of scarlet fever, diphtheria, and other acute infectious diseases, and of pregnancy.

Clinically, the condition is characterized by acute onset, scanty, albuminous, and often bloody urine, dropsy, and uræmia ; the most prominent features are headache, coma, and possibly convulsions, due to retention in the blood of noxious substances, normally eliminated by the kidneys.

Anatomically, the appearance of the kidney varies considerably, according to the duration and intensity
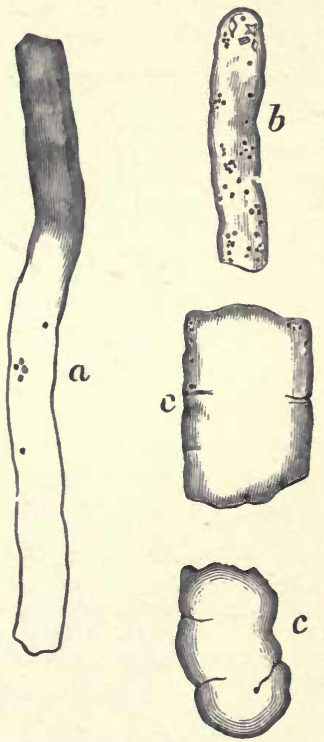

Different forms of waxy casts (v. Jaksch). • of the affection.

Macroscopically, in some instances of acute parenchymatous nephritis, especially where the inflammatory process is limited to the glomeruli-glomerulo-nephritis-further than a slight hyperæmia and swelling of the cortex, there may be but little evidence of the changes which have taken place. Generally, however, the organ is enlarged, often to twice its natural size, flabby and friable; its capsule is tense, gaping widely on being incised, and is easily detached, exposing a perfectly smooth surface. In the early stages the organ may be deeply congested; but later it is often pale-in proportion to the swelling and degenerative changes in the tubular epithelium, and the inflammatory cellular and serous exudations which express the blood from the interlobular vessels. 
On section, the swollen and rounded appearance of the kidney is seen to be due mainly to a marked increase in the thickness of the cortex. The cortex may be intensely hyper-

FIG. 191.

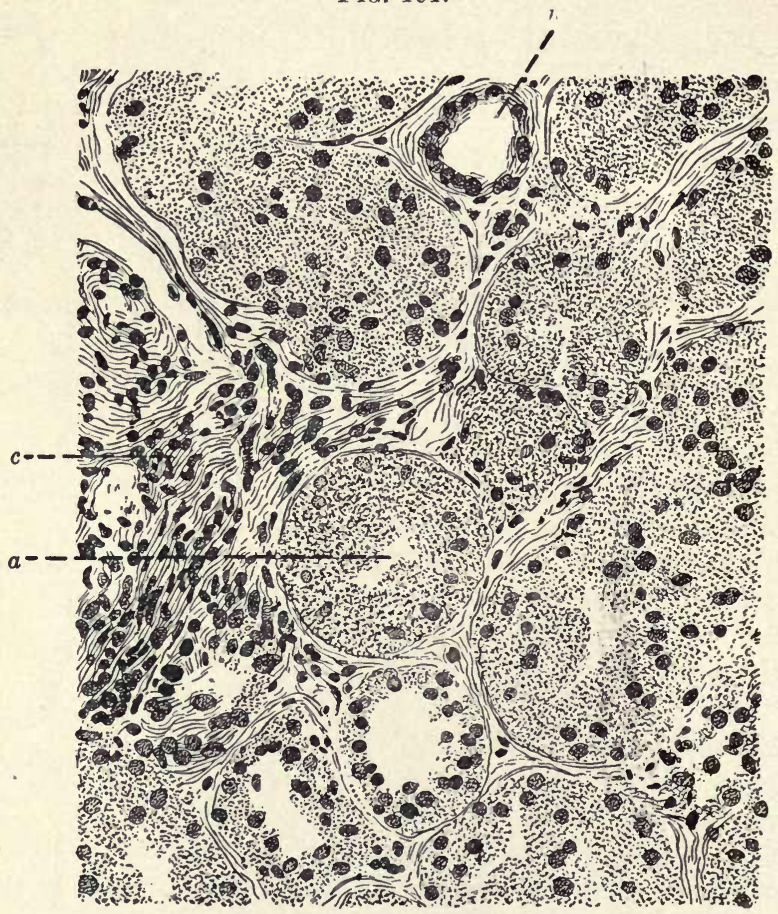

Parenchymatous nephritis. $a$, cross-section of a convoluted tubule of the kidney. the lining epithelium of which is the seat of albuminoid degeneration. The cells are swollen and their bodies filled with abnormally coarse granules. The cells to the left are so far disintegrated that the nuclei have lost most of their chromatin. Such cells cannot recover. The cells to the right are less profoundly altered and their nuclei retain sufficient chromatin to stain slightly. These cells might, perhaps, recover. Other convoluted tubules similarly affected are represented in oblique section. $b$, tubule with low, unaffected epithclium, the nuclei of which stain deeply; $c$, round-cell infiltration of the interstitial tissue in the neighborhood of a Malpighian body, the edge of which is just above the line $c$. Section stained with hæmatoxylin and eosin.

æmic, with here and there small punctiform hemorrhages, the distended glomeruli standing out prominently as dark red points; or mottled in appearance by yellow patches of fatty 
degeneration ; or of a uniform gray color in advanced stages, when the leukocytic infiltration has been excessive-contrasting strongly with the pyramidal portion, which is generally deeply congested.

Microscopically, also, the appearances presented in acute parenchymatous nephritis differ greatly both in the extent and character of the lesion. In some instances the changes are almost entirely confined to the tubular epithelium; resembling rather an acute degeneration than an inflammation. At other times there is a diffuse inflammatory infiltration, in addition to more or less marked changes in the tubular epithelium (Fig. 191); in still other cases, to which the term glomerulo-nephritis has been applied, the inflammatory changes begin in and are most marked around the glomeruli, though sooner or later becoming more general.

Just what the essential differences are, in the character of the exciting cause, which are factors in producing the varied lesions noted above, is not known; though it might be argued that, when the poisonous substance or irritant is being eliminated by the epithelial cells of the secreting tubules, the changes may be most marked there; but if of such a character as to be more readily eliminated with the watery constituents of the blood by way of the glomeruli, the primary inflammatory changes may be excited at that point.

The changes in the uriniferous tubules, most marked in the convoluted portions in the cortex, consist of a granular and fatty degeneration of the epithelial cells, some of which become desquamated and distend the tubules; at the same time the epithelial cells in places may show proliferative changes. The epithelium of the glomeruli may undergo similar degenerative and proliferative changes, and the whole tuft become enlarged from engorgement of its bloodvessels. Migrated leukocytes, and small masses of extravasated red blood-corpuscles, are often seen in and around the tubules and glomeruli. To this class of cases, in which the parenchymatous changes are the most marked feature, the term catarrhal or desquamative nephritis has been applied.

As has been noted, in some cases, especially those occurring in the course of scarlet fever, the inflammatory changes begin in the Malpighian bodies-glomerulo-nephritis. The glomeruli 
are swollen and infiltrated with leukocytes, and their capillary bloodvessels engorged. Bowman's capsule is thickened; its cells swollen, degenerated, and desquamated. The interlobular arteries are more prominent than normal, and are surrounded by migrated leukocytes. The leukocytic migration may be slight or excessive-occurring in foci or as a diffuse infiltration of the cortex. In some cases, even at an early date, there is noted a beginning organization of these migrated and proliferating cells into more or less highly developed connective tissue.

\section{CHRONIC NEPHRITIS.}

Chronic nephritis may follow an acute attack, but in the great majority of cases it arises insidiously as an independent affection. Clinically and anatomically, two varieties of chronic nephritis are recognized: one in which the changes are most marked in the parenchyma of the organ, and associated clinically with general dropsy and the passage of small quantities of urine of low specific gravity, loaded with albumin and casts-chronic parenchymatous nephritis; and the other, in which the increase in connective tissue is the most marked feature anatomically, and characterized clinically by the absence of dropsy, and passage of large amounts of pale urine containing comparatively small quantities of albumin and but few casts-chronic interstitial nephritis. These two varieties may be different stages of one affection, but, on the contrary, many cases of chronic parenchymatous nephritis never go on to cirrhosis; and often, in other instances, the connective-tissue hyperplasia is the most prominent feature from the earliest stages of the inflammatory process.

In chronic parenchymatous nephritis the kidney is generally enlarged, usually to a greater extent even than in acute nephritis. The capsule is readily removed, exposing a smooth surface, except, at a somewhat later stage, for slight irregularities here and there, where bits of the parenchyma have become adherent to the capsule and caused small lacerations on tearing it away. The surface is mottled and anæmic, its pale appearance earning for it the term large white kidney, though this term is applied to amyloid degeneration, and other conditions not included under the head of chronic parenchymatous nephritis. In consistency the organ is doughy. 
On section, the cortex is found to be two or three times its normal thickness, its mottled appearance being due to patches of fatty degeneration in the tubular epithelium.

The microscopical lesions are the same as in acute parenchymatous nephritis, only more marked. The most prominent feature is the degenerative changes in the epithelium of the convoluted tubules; the epithelial cells are swollen, granular, and many are in an advanced stage of fatty degeneration, finally breaking down completely, and filling the lumen of the tubule with a granular and fatty debris, the lining epithelium being entirely destroyed. Within the tubules may be also hyaline, granular, and fatty casts. There is generally some increase in the intertubular connective tissue. The changes in the glomeruli are equally marked, increased thickness of Bowman's capsule and of the connective tissue between the capillaries tending to convert them into atrophied fibrous bodies.

In chronic interstitial nephritis the kidney is usually greatly reduced in size-to less than two ounces in extreme cases-and is remarkably tough in consistency; its surface is of a reddishgray tint, and uneven from the presence of small nodules or granulations, fairly regular in size-about that of a milletseed. The capsule is thickened, opaque, and firmly adherent.

On section, the most noticeable feature is the marked atrophy of the cortex, appearing as a narrow rim but a few millimetres in thickness. The pyramids are also reduced in size, the atrophy of the intervening glandular tissue giving them the appearance of being closely packed together.

Microscopically, the prominent features are the atrophy of the glomeruli and uriniferous tubules, the tubular epithelium having undergone granular and fatty degeneration; and great increase in the connective-tissue stroma. The majority of glomeruli have become dense and fibrous nodules, but onehalf their normal size. Here and there may be well-preserved tubules and glomeruli. Occasionally tubules are met which seem to have undergone a sort of complementary dilatation, as the result of obliteration of tubules in other areas, becoming so exaggerated in some instances as to form cysts, large enough to be seen with the naked eye. Sometimes these cysts occur in rows, suggesting their origin from a single 
tubule; they may also result from the dilatation of Bowman's capsules, and are often filled with a colloid material. Hyaline and granular casts may be found within the tubules. At an early stage of the inflammatory process the interstitial tissue may be quite cellular, but the longer the duration of the affection the more fibrous and the greater the increase in the stroma ; from its contraction results the granular appearance of the surface. The walls of the bloodvessels are almost invariably thickened by an obliterating endarteritis

Associated with these lesions in chronic interstitial nephritis are nearly always a general arterio-sclerosis and a hypertrophied heart.

\section{DEGENERATIONS.}

Parenchymatous degeneration, or cloudy swelling of the kidneys, occurs in the course of diphtheria, scarlet fever, cholera, and other infectious diseases; and in poisoning from phosphorus, arsenic, and mercury. There is no sharp line of demareation between this condition and acute parenchymatous nephritis, in which it is liable to terminate, the changes varying with the virulence of the poison. The kidney is enlarged, rounded, and soft in consistency. On section, the cortex is thicker than normal, and pale or yellowish in appearance.

Microscopically, the principal changes are noted in the tubules of the cortex. The tubular epithelium is granular and swollen; the lumen of the tubule almost entirely obliterated, often appearing as little more than a stellate fissure. The cell outline is indistinct and its nucleus obscured.

Fatty degeneration very frequently follows cloudy swelling; it often occurs also in the course of long-continued wasting diseases, as cancer, tuberculosis, diabetes, and pernicious anæmia. The kidney is pale and extremely flabby, and often smaller than normal; on section the cortex is found to be of normal thickness, and of a diffuse yellowish or mottled, and greasy appearance.

Microscopically, the convoluted tubules are especially affected ; the cells are swollen, and near their bases are droplets of fat of various size.

Amyloid degeneration of the kidneys occurs in tuberculosis. 
syphilis and other cachexias; and is associated with similar changes in the liver, spleen, and abdominal lymphatic glands. It is generally accompanied by albuminuria, and in advanced stages by general dropsy.

At an early stage the kidney may appear perfectly normal to the naked eye. Later, however, it is enlarged, often to twice its normal size, pale, anæmic, and very firm and tough. The capsule can be readily removed, exposing a surface smooth, polished, and glistening. On section, the cortex is thickened and has a peculiar transparent, homogeneous, bacony appearance.

Microscopically, the changes are found to begin in the walls of the bloodvessels as in other organs, and hence are most readily recognized at an early stage in the Malpighian bodies, which appear, unstained, as prominent, transparent or translucent, polished clumps. The rose color imparted to the amyloid material by the use of methyl violet as a stain, gives the capillaries of the tufts the appearance of being injected. Later the changes are noted in the afferent and efferent vessels of the tufts, and arteriæ rectæ. Not infrequently the basenent-membrane of the uriniferous tubules may be similarly affected-the lining epithelium being in various stages of granular and fatty degeneration. This degenerative change is often associated with parenchymatous and interstitial nephritis.

\section{THE SPECIFIC GRANULOMATA.}

Syphilis in its early stages may give rise to a nephritis similar to that produced by other infectious diseases. In its later stages it is a very frequent cause of amyloid degeneration, and may also produce a chronic interstitial nephritis. Syphilitic gummata of the kidneys are very rare.

Tuberculosis occurs as a part of an acute miliary or chronic general tuberculosis, though cases are seen in which the disease starts primarily in the kidneys; infection may take place also along the ureters, secondarily to tuberculosis of the testicles, seminal vesicles, and bladder. The disease is generally more marked in one kidney than in the other, or may be entirely confined to one. In primary tuberculosis of the kidneys, at an early stage, the tubercular foci-small yellow 
caseous nodules-may be confined to the bases of the pyramids. Discharge of the necrotic tissue often occurs into the pelvis of the kidney, which is generally also involved-a tubercular pyelo-nephritis. At a later stage the whole organ is sometimes converted into a large sac, with caseous and ulcerating walls.

\section{TUMORS.}

Fibromata are not infrequently found in the kidney in the form of small, firm, white nodules about the size of a milletseed, though sometimes much larger.

Lipomata are rare, but the fatty external capsules of the kidney may be so greatly increased in thickness as to form practically a fatty tumor (Fig. 119).

Simple adenoma has been met with in the kidney, but is very rare. Small tumors sometimes occur, which are formed of a displaced remnant of the suprarenal capsule, and histologically resemble that gland.

Leiomyomata occasionally occur in the kidney, and are generally situated near the apices of the papillæ. A rare tumor, which is usually congenital, is the rhabdomyosarcoma, histologically found to be composed of round and spindlecells mixed with striated muscle-cells.

Primary sarcoma of the kidney is more frequent than primary carcinoma. Macroscopically, it varies considerably in size and appearance. Microscopically, the varieties most frequently met are the round and spindle-celled, the myxosarcoma, and myosarcoma.

Primary carcinoma of the kidney is rare. It generally begins in the cortex as an adeno-carcinoma.

Cysts occur frequently in the course of chronic interstitial nephritis, and even in the otherwise normal kidney, as the result of obliteration and dilatation of the uriniferous tubules and Bowman's capsule, and may attain sometimes a very considerable size. They may be so numerous as to cause surprise that the kidney should have been able to perform its functions at all. Congenitally, both kidneys may be transformed into masses of innumerable cysts of varying size, these organs being so greatly enlarged in some cases as even 
to interfere with parturition. Dermoid cysts are also found; echinococcic cysts are of occasional occurrence.

\section{PELVIS OF THE KIDNEY.}

In inflammation of the pelvis of the kidney-pyelitis-and the associated lesions in the kidney itself, infection takes place most frequently by way of the ureter, in connection with urethral stricture, enlarged prostate, vesical calculus, and cystitis. Though pyelitis may occasionally occur in the course of infectious diseases and in non-infectious cases, for example, as the result of the mechanical irritation produced by the presence of calculi, calculous pyelitis; yet in the majority of cases it is a septic or suppurative inflammation produced by micro-organisins. Sooner or later there generally follows a suppurative inflammation of the kidney itself-pyelo-nephritis.

FIG. 192.

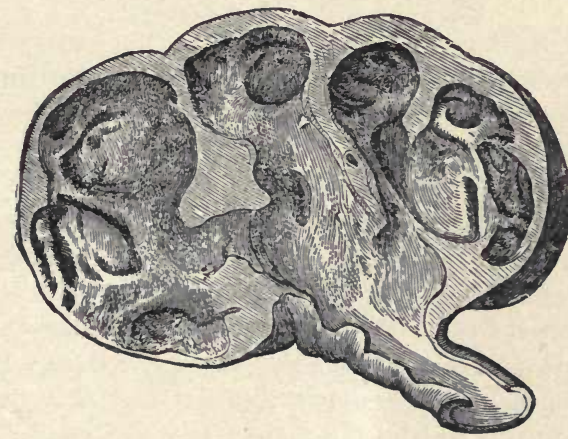

Hydronephrosis with granular atrophy of the kidney. The other kidney showed a marked compensatory hypertrophy (Virchow).

In pyelo-nephritis the kidney is swollen, soft, and friable, its surface dotted with small yellow elevations which contain pus. In size and number these abscesses may vary greatly. They may be few and confined mainly to the cortex, or the whole kidney may be fairly riddled with larger confluent abscesses, communicating with the pelvis of the kidney, the whole organ in some instances being converted into a large pus-sac-pyo-nephrosis. When obstruction of the ureter causes 
dilatation of the pelvis and calices of the kidney, hydronephrosis is the descriptive term (Fig. 192).

Calculi not infrequently occur in the pelvis of the kidney, sometimes attaining quite large dimensions; the term renal gravel is applied to the smallest gritty particles. During their formation the calculi often become more or less accurately moulded to the shape of the pelvis and the calices. They may be composed of uric acid, oxalate of lime, and phosphates, and rarely of cystin and xanthin. The results of their presence may be very serious. The smallest particles may pass through the ureter from time to time and give rise to attacks of excruciating pain-renal colic ; larger ones, by obstructing the ureter and the outflow of urine, may cause hydronephrosis. The continued irritation produced by a large calculus results generally in a pyelitis, and in some cases seems to be the exciting cause of cancer.

\section{URINARY BLADDER.}

Fissure of the bladder-a congenital malformation-is rather common, occurring as the result of a failure of the visceral arches to coalesce in front. The absence of the anterior wall of the abdomen and bladder exposes to view, protruding and filling the cleft, the inflamed mucous membrane of the posterior wall of the bladder which has been pushed forward by the abdominal viscera-exstrophy. The extent of such a fissure varies greatly. It may involve the pubic bones, and in the female, the vagina, clitoris, and urethra; or the bladder may be perfectly formed and the cleft confined, in the male, to the urethra, constituting an epispadias.

Hypertrophy of the muscle-walls of the bladder results from some obstruction either of the neck of the bladder or of the urethra-as by enlargement of the middle lobe of the prostate or a urethral stricture.

The mucous membrane of the bladder is raised into trabeculæ by the bundles of hypertrophied, interlacing musclefibres, giving rise to an appearance somewhat similar to the inner surface of the ventricle of the heart.

Inflammation of the bladder-cystitis-may be acute or 
chronic, and varies considerably in its severity. The inflammatory process may extend from the urethra in gonorrhoa; or result from the presence of vesical calculus; or occur in the course of acute infections diseases. The introduction of septic matter during catheterization is of common occurrence; and the decomposition of urine consequent upon the retention of urine in urethral stricture or hypertrophied prostate is also a frequent cause.

The mucous membrane in acute cases may be greatly swollen, and congested, and present numerous punctate hemorrhages and, at times, superficial sloughs; its surface is covered with a muco-purulent exudate, and in some cases a pseudo-membrane.

In chronic cases the mucous membrane is greatly thickened, and is often the seat of extensive ulceration; if there be some obstruction to the outflow of urine, the hypertrophied trabeculæ of muscle-fibres may give the surface a ribbed appearance.

In other cases, as the result of the organization and subsequent contraction of the inflammatory infiltration of its walls, the bladder is often much diminished in size. Inerustations of phosphates deposited from the alkaline urine may cover the surface of the mucous membrane.

The thick, tenacious, gelatinous material often present in the urine, in addlition to large quantities of migrated leukocytes, is not due to the presence of mucin, but is the result of the action of the alkaline urinary salts on the purulent exudate. Infection is liable to extend along the ureters to the kidneys.

Tuberculosis of the bladder may occur in phthisis, but is much more frequently secondary to tuberculosis of the kidney, uterus, prostate, epididymis, or seminal vesicles.

The lesion generally presents as a single large, or numerous small ulcers, most abundant at the base of the bladder and around the orifices of the ureters.

Tumors : Papillomata, varing in size from a pea to that of a pigeon's egg, and often having a cauliflower-like appearance, are of rather frequent occurrence. They are quite vascular and bleed readily. Carcinoma is nearly always 
secondary; the bladder is generally involved as the result of the extension of the new growth from the uterus, vagina, or rectum, or rarely by metastasis.

Calculi are frequently found in the bladder, varying greatly in size and number. In some cases there is a single calculus four or five inches in diameter; in others, small gravel-like particles.

The appearance of the larger stones varies according to their composition. The uric-acid calculus is generally round and hard, its surface smooth, and from a yellow to a red color. Mixed or triple-phosphate calculi are also frequent. They are white, soft, and friable, with roughened surfaces; or occur as incrustations on the mucous surface of the bladderwalls. Calcium-oxalate or mulberry calculi are brownish in color, hard, and rounded, with irregular nodular surfaces.

\section{THE URETHRA.}

Inflammation of the urethra, which is in most instances due to the gonococcus of Neisser, is a frequent and especially serious disease, in its consequences, in the male. In the great majority of cases the inflammatory process involves the entire extent of the urethra, though it may be confined to that portion anterior to the cut-off-muscle-anterior urethritis. The gonococci rapidly penetrate between the epithelial cells beyond the reach of antiseptics and astringents.

The mucous membrane is intensely congested and swollen, and from it comes a thick, purulent exudate.

If the posterior urethra is involved, the gonococci are very apt to invade the vas deferens, and excite an acute inflammation of the epididymis; the prostate gland may be likewise involved; the bladder, however, is but rarely affected.

The inguinal lymphatic glands are frequently enlarged, the inflammatory process sometimes terminating in suppuration.

Rarer complications of gonorrhœal urethritis are arthritis and inflammations of the pericardium and endocardium, and even at times of the pleura and the spinal meninges.

Chronic urethritis not infrequently follows the acute. Gonococci have been found as long as six years after an original 
infection in the purulent discharge from the urethra. Even after the disappearance of these organisms, lesions-postgonococci-remain, which may give rise to serious consequences. Chronic posterior urethritis is often responsible for functional derangements of the nervous system-sexual neurasthenia. In this chronic stage the inflammation is no longer diffuse, but circumscribed. The lesions consist of localized areas of submucous infiltration and erosions, or even definite ulcerations of the mucous membrane; at times some patches of granulation-tissue are noted.

Strictures often result at the site of these lesions from the formation of cicatricial tissue. As they gradually encroach upon the lumen of the canal and impede the outflow of urine, the bladder-walls become hypertrophied; but later this organ is dilated, as likewise may be the ureters and pelvis of the kidneys.

Tumors : Carcinoma of the urethra may result from extension of the new growth from adjacent organs, as the vulva, vagina, and glans penis; it is rarely primary, taking its origin from Cowper's gland.

Sarcoma of the urethra does occur, but is very rare. 


\section{CHAPTER VIII.}

\section{DISEASES OF THE NERVOUS SYSTEM.}

\section{THE MENINGES.}

Inflammation may involve both the cerebral and spinal meninges, or either separately. 'The dura alone may be affected - pachymeningitis; the pia and arachnoid are usually affected together-leptomeningitis, or simply meningitis.

In the cord, however, all three membranes are commonly involved at the same time, together with the periphery of the white matter of the cord itself, with which the meninges are intimately connected.

Pachymeningitis: In the brain inflammation of the outer layer of the dura-pachymeningitis externa-is generally secondary to disease or injury of the bones of the skull, of which it forms the periosteum. The inflammatory process is usually suppurative and confined to limited areas, though it may extend to the other membranes and the brain.

A suppurative pachymeningitis interna is generally associated with a leptomeningitis or a pachymeningitis externa.

In chronic pachymeningitis there is a diffuse or circumscribed thickening of the membrane, which is abnormally adherent to the cranium and to the arachnoid, and not infrequently an ossification of the outer layers of the membrane takes place.

Hemorrhagic pachymeningitis, or hæmatoma of the dura, occurs especially in chronic alcoholics and the insane. The condition is looked upon as a hemorrhagic inflammation of the dura. There first forms a highly vascular subdural pseudo-membrane, extending over the greater part of one or both hemispheres, the hæmatoma resulting secondarily from rupture of its vessels. This psendo-membrane presents the appearance, microscopically, of granulation-tissue, and contains many thin-walled bloodvessels. 
Leptomeningitis: In leptomeningitis the inflammatory process nearly always involves the arachnoid and pia of both brain and cord. The condition may occur as an epidemic cerebrospinal meningitis; or in the course of smallpox, scarlet fever,

FIG. 193.

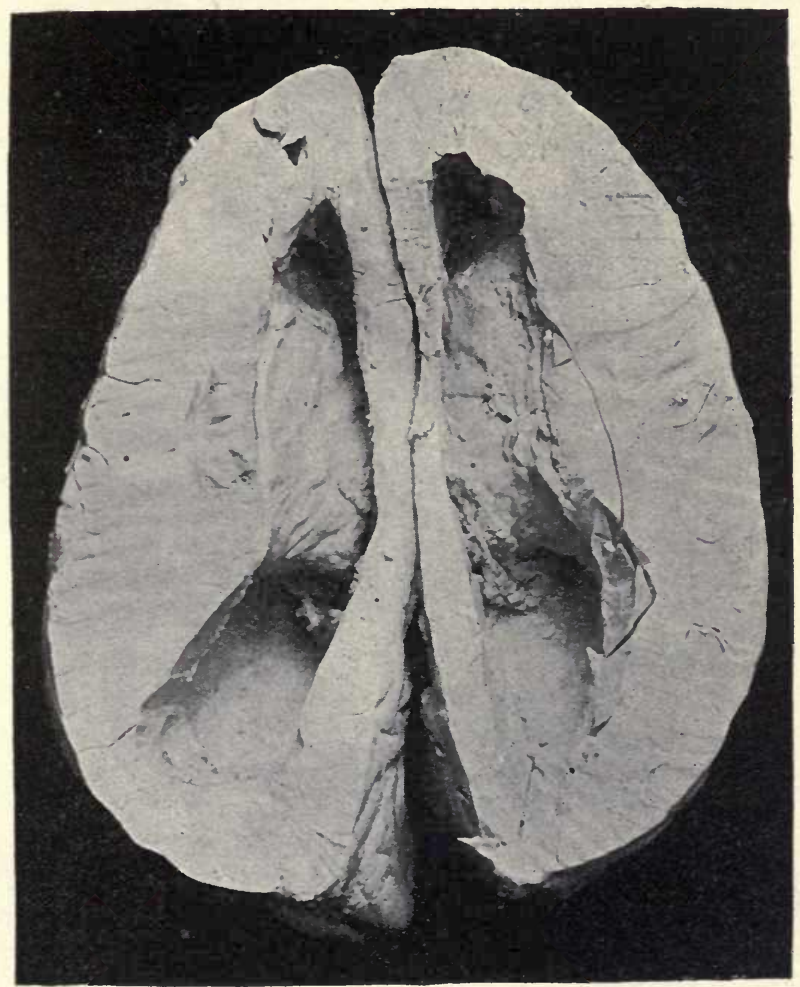

Hydrocephalus internus; dilated ventricles (U. S. A. Museum, No. 8233).

pneumonia, septicæmia, and other infectious diseases. The pneumococcus is the organism most frequently found associated with the disease, having been met with in 60 per cent. of all cases. 
In some cases the macroscopical changes are not marked, consisting simply of congestion, loss of normal lustre, and slight œdema. In more severe cases the exudate, which may be serous, sero-purulent, or purulent, becomes more marked, and even distends the subarachnoidal space, the cerebral convolutions being flattened and the brain compressed to such an extent as to give rise to coma. The ventricles may be likewise distended (Fig. 193). In some eases the exudate pre-

Fig. 194.

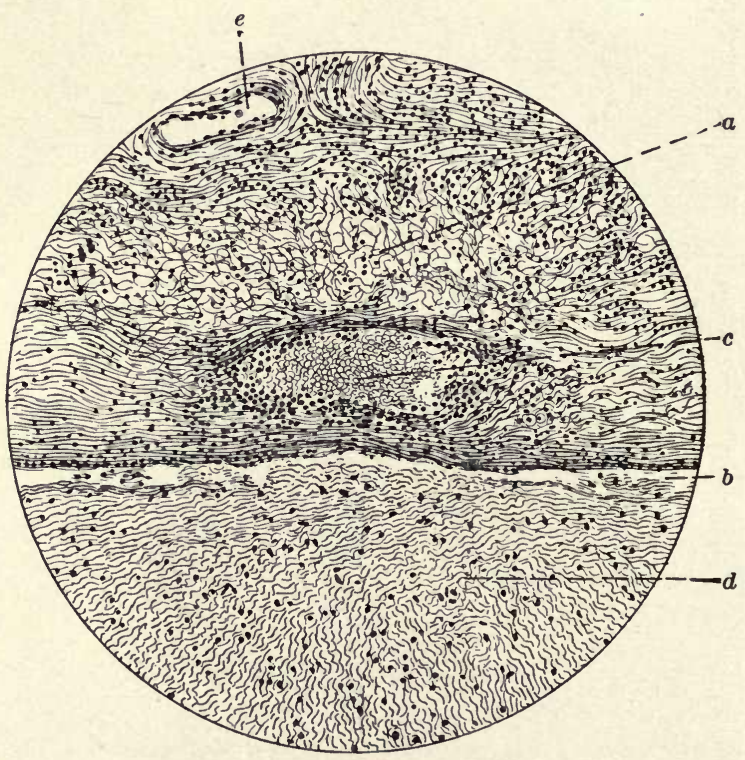

Fibrinous leptomeningitis. $a$, cerebral cortex; $b$, serum, with detritus, separating the braill from the pia mater; $c$, bloodvessel of the pia mater, the walls of which are infiltrated with emigrating leukocytes; $d$, fibrinous exudate ; $e$, smaller vessel of the pia (Dunham).

sents as an extensive, thick, opaque, fibrinous deposit on the under surface of the arachnoid. In other instances a greenish-yellow, purulent exudate may cover the surface of the brain to such an extent as to conceal almost entirely the convolutions of the cortex (Figs. 194 and 195). The inflamma- 
tory process may extend to the substance of the brain, varying from slight œdema of the cortex to abscess-formation.

Tubercular inflammation of the meninges is more common in children than in adults, and is usually part of a general miliary tuberculosis or secondary to tuberculosis of other

Fig. 195.

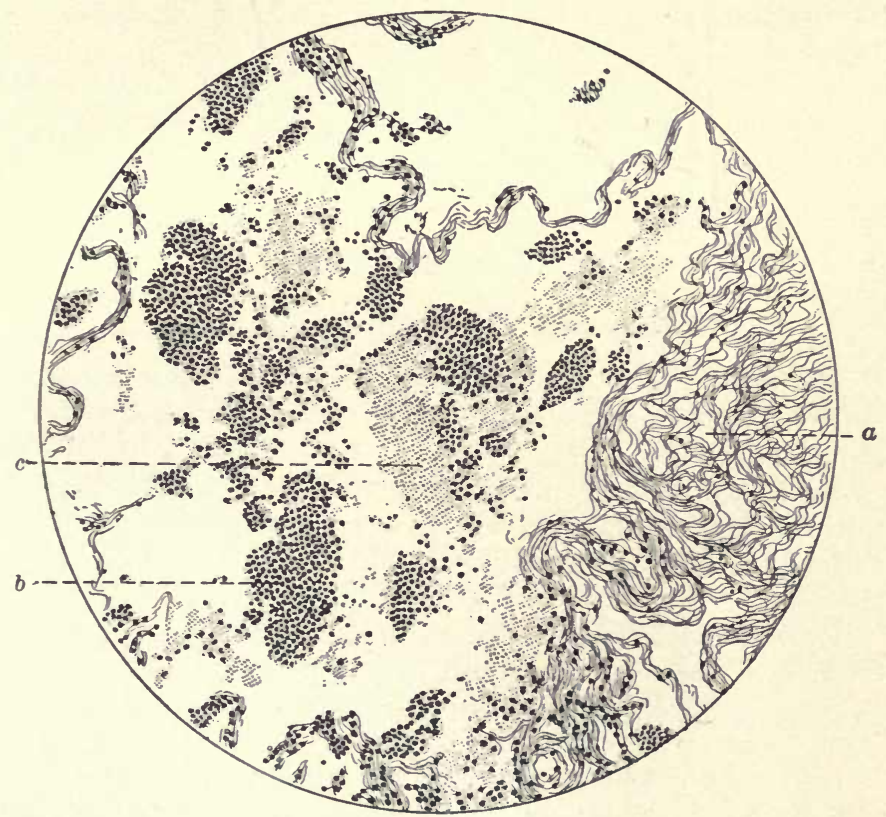

Serous leptomeningitis. $a$, odematous fibrous tissue of the pia mater, the fibrous elements of the tissue being separated by the serous exudate; $b$, group of leukocytes, probably held together in part by fibrin; $c$, granular fibrin and detritus ; $b$ and $c$ and other similar masses, lie in the serum, which occupies the whole field between the visible elements (Dunham).

regions of the body, most commonly the lungs. The miliary tubercles are most numerous in the pia at the base of the brain. The meninges are congested, and may be the seat of exudations similar to those occurring in simple meningitis. The ventricles of the brain are distended with a serous exudate and their walls studded with miliary tubercles. The 
cortex of the brain is congested and the convolutions flattened by pressure from within.

At times large caseous nodules are found in the pia mater and brain-tissue.

Syphilitic inflammation of the meninges is generally circumscribed. The gummata which form involve the pia and the cerebral cortex, or they may extend outward and involve the dura.

Tumors: Endothelioma is probably the tumor most frequently met in the meninges. Lipomata, fibromata, and myxomata are of rare occurrence.

\section{THE BRAIN.}

\section{THROMBOSIS AND EMBOLISM.}

The results of thrombosis and embolism in the brain may be most serious, producing death, or more or less extensive paralyses.

Emboli are brought most frequently from fibrinous vegetation on the cardiac valves, or from cardiac or aneurysmal thrombi. They generally lodge in the left middle cerebral artery.

Thrombosis is most frequent in the basilar artery, but may occur anywhere, as the result of the presence of an embolus, or some local inflammatory or degenerative alteration of the vessel-wall at the site of its formation.

In either case the result of this obstruction of the bloodsupply is a rapid necrotic softening of the area affectedencephalomalacia. The nerve-cells degenerate and lose their axis-cylinder processes; the myelin-sheaths of the nervefibres undergo fatty degeneration; and later the neurogliafibres a similar change. A more or less pigmented scar is finally formed, composed mainly of neuroglia-tissue. In the case of an infectious embolus, an abscess may form. These areas of softening are usually red from the extravasation of blood, as in infarcts in other organs; at a later stage when most of the pigment has been absorbed, or when little or no blood has been extravasated, they are yellow or white. 


\section{ANAMIA.}

Anæmia of the brain may depend on general anæmia, or in acute cases be due to severe hemorrhage. The condition is supposed to occur during sleep, in fainting spells and hysterical crises.

The gray and white matter on section appear much whiter than normal, and the small puncta vasculosa are almost entirely absent. A local anæmia may result from partial obstruction of the blood-supply by a thrombus or embolus, or the external pressure of a tumor.

\section{HYPER正MIA.}

An acute hyperæmia of the brain may be produced by excessive activity of the heart's action, and accompanies excessive brain-work, sunstroke, acute delirium, and some infectious diseases, as cholera and hydrophobia.

A passive hyperæmia may result from valvular disease of the heart and from interference with the return circulation through the jugular veins by pressure of a tumor, or by disease of the lungs - as extensive pneumonias and large pleuritic effusions. The brain is enlarged, the bloodvessels of the pia mater injected; the gray matter may have a diffuse rosy tint, and on section the puncta vasculosa are more conspicuous than normal. In passive hyperæmia, however, the gray matter has a slaty color and the sinuses and cortical veins are widely distended. Edema of the brain may result if the condition is long continued.

\section{CEREBRAL HEMORRHAGE.}

The clinical term apoplexy is still used synonymously with cerebral hemorrhage, of which it is the most striking symptom.

The most important factors in the production of cerebral hemorrhage are increased blood-pressure and the degenerative changes occurring in the bloodvessel-walls with advancing years, and in the course of various diseases, notably syphilis. The resulting clots vary greatly in size; they may be as small as a pea, or almost entirely occupy one hemisphere. Usually there is but one clot. They occur most frequently in the 
corpora striata and optic thalami, and the brain-tissue immediately surrounding.

If there is not a fatal termination of the hemorrhage, in a short time the clot undergoes complete liquefaction, and, having been surrounded by a fibrous-tissue capsule, a sort of cyst results; or later there may be simply a pigmented scar to mark the site of the hemorrhage.

Secondary degeneration of the direct and crossed pyramidal tracts results, when, as is often the case, the hemorrhage has occurred in the region of the internal eapsule; due, it is supposed, to the separation of these motor-nerve fibres from their trophic centres.

\section{INFLAMMATION.}

Acute inflammation of the brain-encephalitis-may occur in the course of various acute infectious diseases, as typhoid fever, septicæmia, and influenza. The condition is characterized by the formation of localized areas of softening, which are red or yellow in color, according to the amount of extravasated blood present.

Microscopically, in these areas, and also in others where softening is not yet manifest, the bloodvessels are dilated, engorged with blood, and surrounded with migrated leukocytes. The ganglion-cells and nerve-fibres undergo a granular and fatty degeneration. In the centre of the area the neuroglia undergoes a similar change; at the periphery, however, may be noted the formation of new neuroglia cells and fibres.

Suppurative inflammation of the brain is most frequently associated with a similar inflammation of the meninges. The condition may also occur in the course of pyæmia, ulcerative endocarditis, and other septic processes; and in influenza, pneumonia, and other infectious diseases; or it may follow direct injury, or septic inflammation of the bones of the skull.

The abscess-formations which characterize this condition may be quite small, or large enough to contain, in one case, as much as four hundred cubic centimetres of pus. In a large proportion of cases the abscess is solitary ; multiple abscesses are generally metastatic.

Chronic inflammation of the brain is characterized, like chronic inflammation of other organs, by an increase of the 
connective-tissue elements-the neuroglia, cells and fibres; and a partial or complete atrophy of the parenchyma-the ganglion-cells and nerve-fibres. The lesion may be confined to a lobe. In other cases, especially in drunkards and in general paralysis of the insane, the greater part of the brain

FIG. 196.

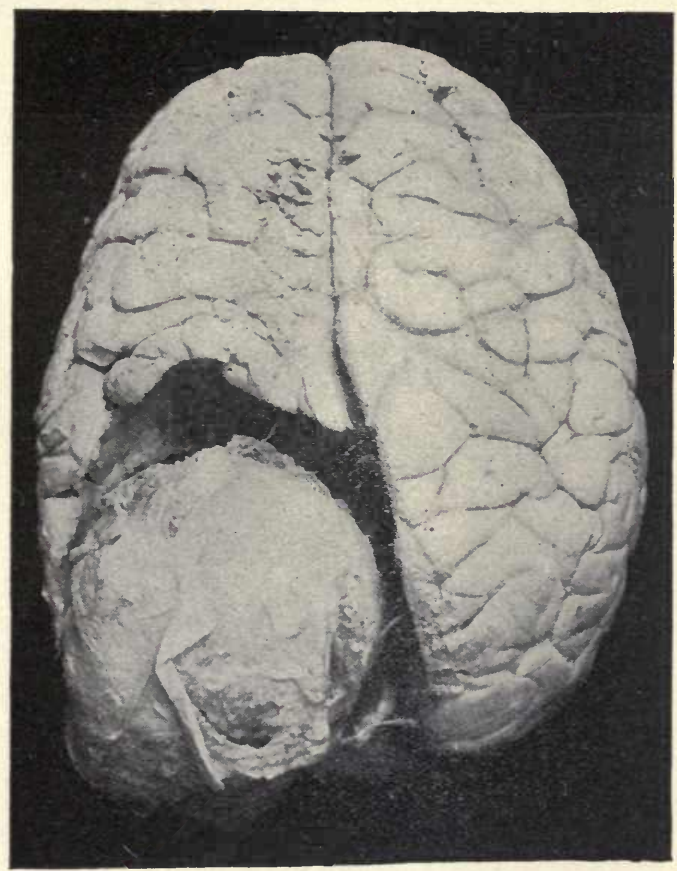

Sarcoma of brain (U. S. A. Museum, No. 7983).

may be involved. In still other instances there are multiple areas of sclerosis irregularly distributed throughout the brain and cord, the grayish or grayish-pink plaques varying in size from that of a pea to that of an almond.

Tuberculosis of the brain-the most frequent "tumor" formation-is a common lesion, presenting usually as caseous, circumscribed nodular masses the size of an egg or larger. 
The lesion exists most frequently in the cerebellum and is more common in the young than later in life.

Syphilis: Gummata of the brain nearly always originate primarily in the meninges. A syphilitic endarteritis may also produce diffuse or circumscribed changes in the brain-tissue.

Tumors of the brain are of especial importance by reason of the inflammatory and degenerative changes resulting from

FIG. 197.

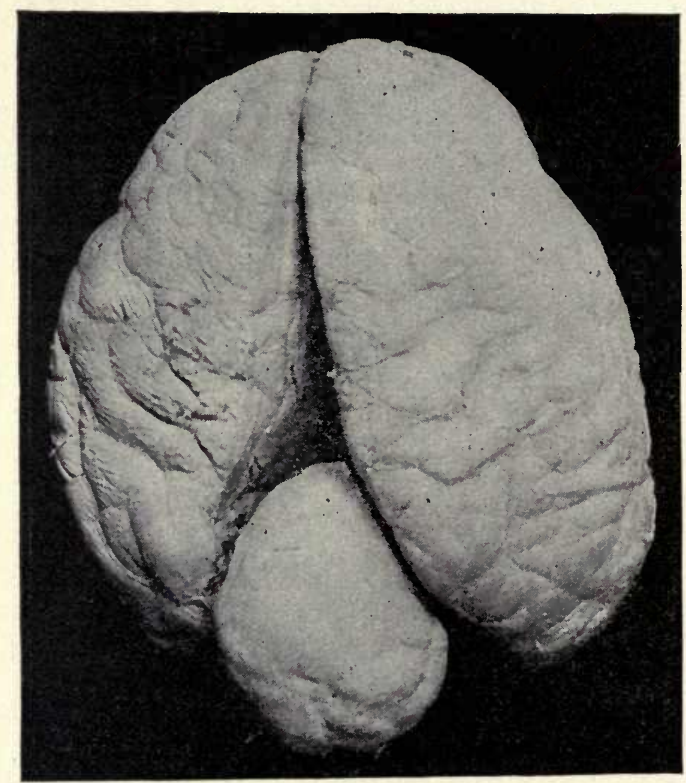

Sarcoma developed between the hemispheres, posteriorly (U. S. A.Museum, No. 8400).

their pressure on the brain-tissue. The glioma is the tumor most frequently met; it is generally solitary and grows always from the neuroglia-tissue-never from the pia. Sarcoma occurs next in frequency-generally the round-celled variety. (Figs. 196 and 197.) Carcinoma may occur as a metastatic growth in the brain, but never primarily. 


\section{THE CORD.}

\section{HEMORRHAGE.}

Hemorrhage is of much less frequent occurrence and much less extensive-the clot generally not more than one centimetre in diameter-than in the brain. The condition is generally the result of trauma, though it may occur spontaneously. When the central canal of the cord is dilated and filled with blood the term hoematomyelia is employed.

\section{DEGENERATIONS.}

FIG. 198.
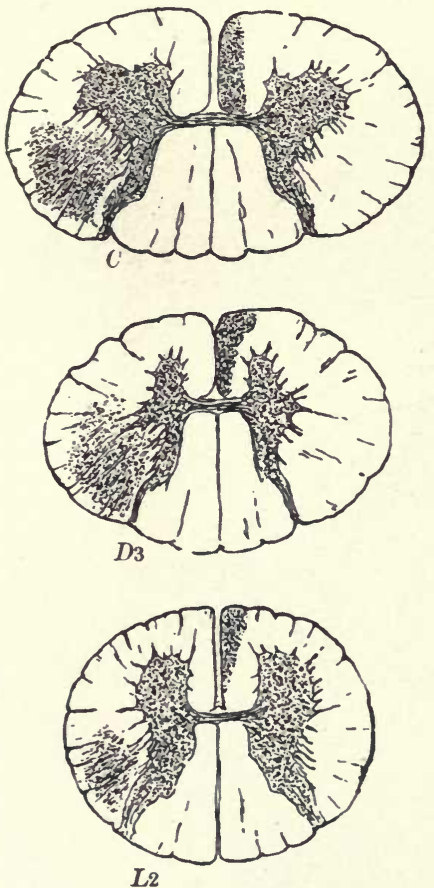

Descending degeneration in the pyramidal tract following hemorrhage into the internal capsule. The direct tract is murked, and is represented at a lower level than it is usually seen (F. W. Mott).
Secondary degenerations occur in the brain and cord as the result of any lesion interrupting the course of nerve-fibres in the brain, cord itself, or posterior nerve-roots. These changes are supposed to be due to the removal of normal trophic influences arising in the ganglioncells from which the nerve-fibres are cut off. The result is the destruction of the medullary sheaths of the fibres, to which is due their opaque white appearance. Tracts in the cord so

Fig. 199.

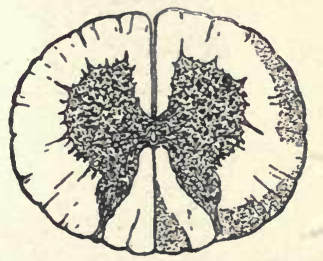

The ascending tracts of degeneration in the cervical enlargement after experimental hemisection of the spinal cord in the mid-dorsal region. The section shows well-marked degeneration of Goll's column, of the direct cerebellar tract, and of the autero-lateral tracts on the same side as the lesion (F. W. Mott). 
affected have a gray or yellowish-gray color in consequence, and hence the condition is often spoken of as gray degeneraFIG. 200.

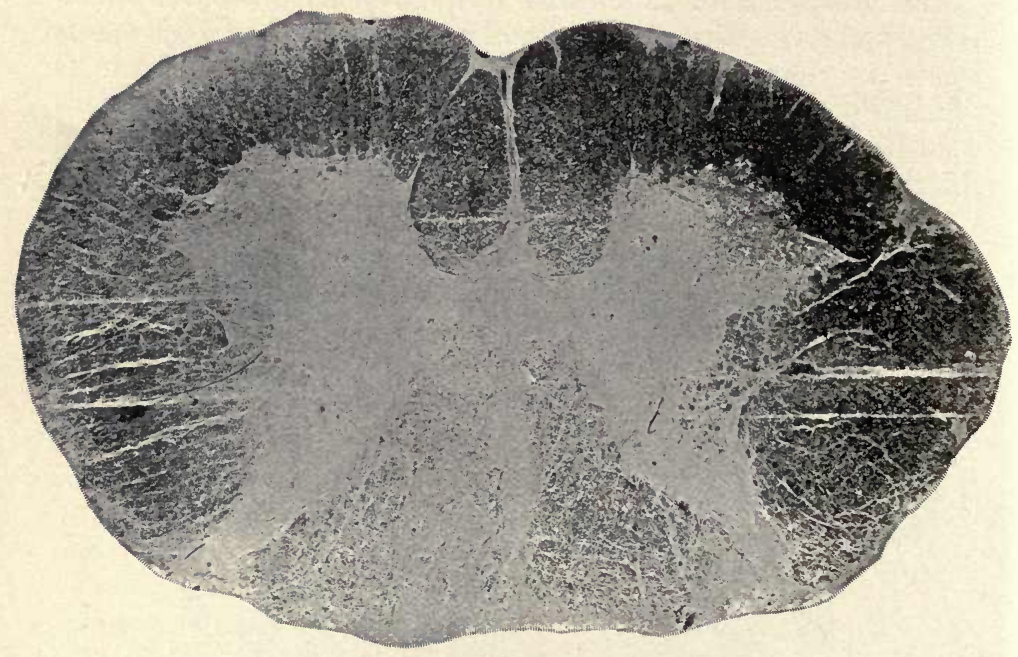

FIG. 201.

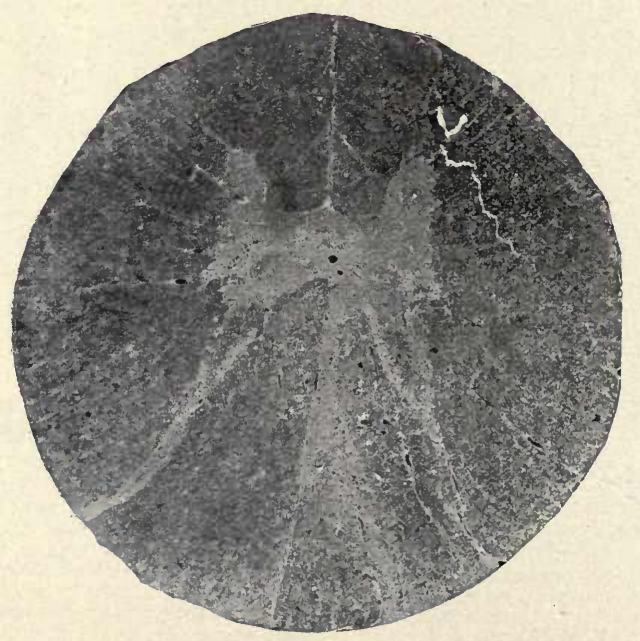




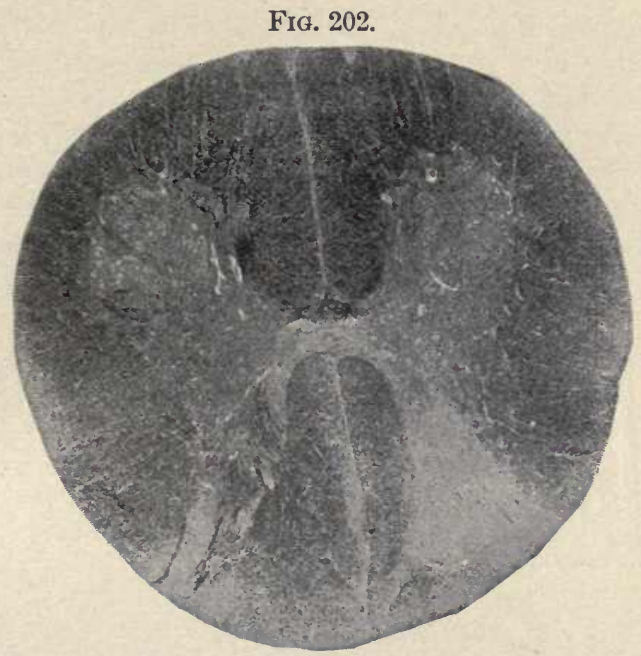

Sections of the spinal cord at the cervical (Fig. 200), dorsal (Fig. 201), lumbar (Fig. $202)$, levels showing ascending degeneration, unilateral, in the posterior column after a gumma involving the second and third lumbar nerve-roots. The relative extent of the degenerated fibres at the different levels is shown in the sections (Starr).

tion. In addition, microscopically, there is noted a reduction in the number of nerve-fibres, and sometimes a new formation of connective tissue.

A descending degeneration affecting the pyramidal tracts occurs as the result of hemorrhage or other lesion in the brain, interrupting the course of the motor fibres (Fig. 198).

Ascending degenerations occur after destruction of the cord or posterior spinal nerve-roots, or from any cause interrupting the course of the ascending sensory fibres of the cord, involving the column of Goll, direct cerebellar, and antero-lateral tracts (Figs. 199-202).

\section{Primary Degenerations.}

Sclerosis of the posterior columns, or locomotor ataxia, is the most important form of primary degeneration of the cord. Though the macroscopic and microscopic changes are marked, and have been carefully studied, the true nature of these 
FIG. 203.

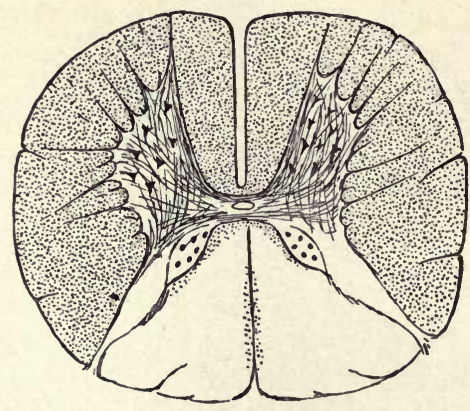

Diagrammatic representation of the supply of the groups of anterior-horn cells by the radicular branches of the anterior median arteries, showing one group of cells completely destroyed by occlusion of one of these small vessels, and thus explaining why in poliomyelitis there is usually permanent loss of movement in some one or more muscles (F. W. Mott).

FIG. 204.

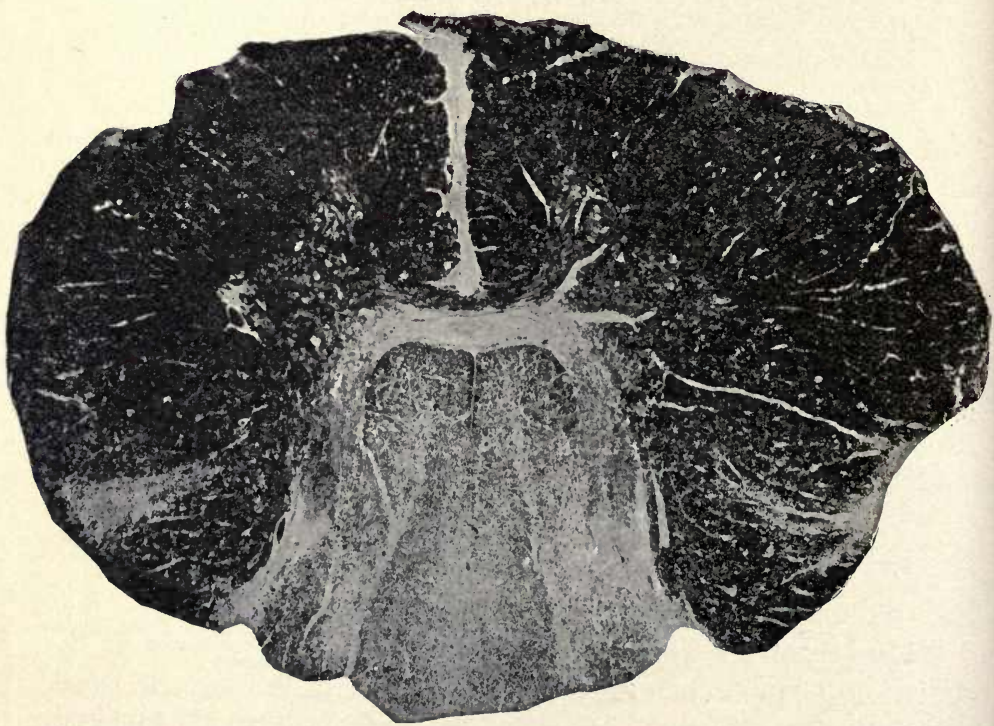

The lesion in the posterior columns at the first dorsal region in a case of locomotor ataxia (Starr). 
alterations is not fully understood. By a majority of pathologists, however, they are regarded as secondary to disease of the posterior roots or their ganglion-cells. By many, syphilis is regarded as the most frequent cause of this condition.

Macroscopically, in well-advanced cases the cord usually appears atrophied, especially in the region of the posterior white columns, which are narrow and sunken, and gray or grayish-yellow in color (Figs. 203 and 204). On trans-

Frg. 205.

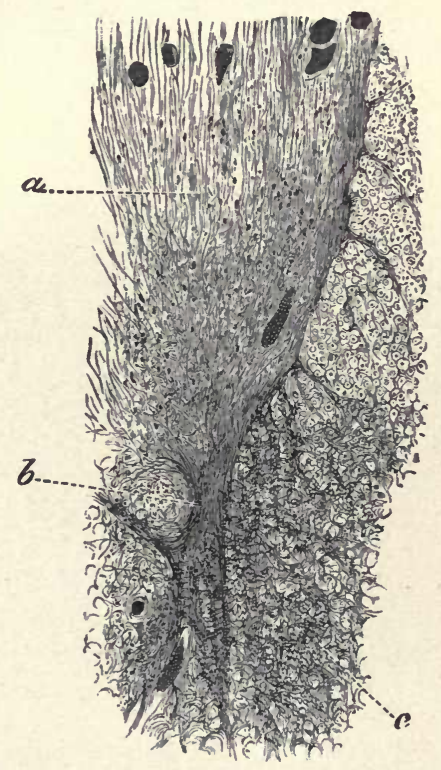

Section of spinal cord about the eighth dorsal segment (from a case of locomotor ataxia). There is sclerosis of the postero-external column and atrophy of the fine plexus of nerve-fibrils surrounding the cells of Clarke's column; moreover, a band of sclerosis is seen entering the column insterd of the bundle of nervefibres. The cells themselves are atrophied and their processes destroyed. This case was of interest because, in connection with these lesions, the patient had well-marked visceral symptoms-gastric crises, bladder troubles, and laryngeal crisis-in addition to the ordinary ataxic symptoms. $\times 100$ diameters $(\mathbf{F} . W$. Mott).

verse section these changes are found to be most marked in the lumbar or dorsal regions-the central gray matter is not generally affected.

28-Hist. 
Microscopically, the characteristic features are found to be an increase of the neuroglia-tissue and destruction of the myelin-sheaths, and later of the axis-cylinder-whether the nervous tissue or the neuroglia-tissue is primarily affeeted is not known (Fig. 205). Similar changes may occur in the cranial nerves, especially in the optic, and in the spinal nerves.

The prominent clinical feature-inco-ordination-is due to the interference with the centripetal, nerve-fibres, especially those coming from the muscles. This explains also the early loss of muscle-reflexes. Actual loss of muscle-power indieates implication of the motor tracts in the lateral columns; and

Fig. 206.

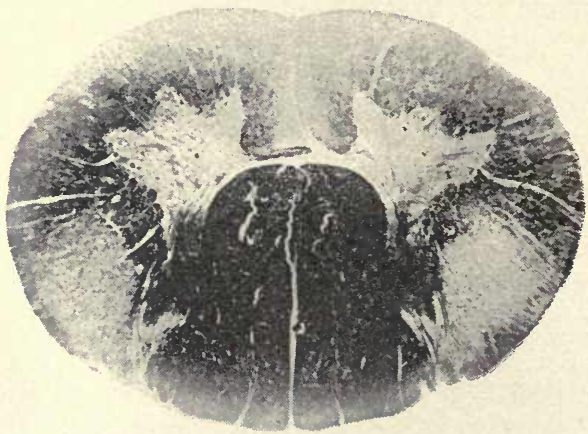

Photo-micrograph of a section of the cervical spinal cord (from a case of amyotrophic lateral sclerosis). Degeneration of the crossed pyramidal and direct tracts and the antero-lateral ground-fibres. The direct cerebellar traets, the antero-lateral ascending tracts, and especially the posterior columns, are unaffeeted. There was almost eomplete absence of cells and fine nerve-fibre reticulum in the anterior horns; this is observable by the difference in color as compared with the posterior horns (F. W. Mott).

localized atrophies, which may affect certain groups of muscles, if not due to such paralysis, results from the extension of the disease to the anterior cornua.

Friedreich's disease, or hereditary ataxia, oceurs at an early age, generally in several members of the same family. Anatomically, the characteristic features are degenerative changes in the posterior and lateral columns, involving the pyramidal and direct cerebellar tracts.

Spontaneous lateral sclerosis (Erb's spastic paraplegia) is a 
primary degeneration affecting the lateral columns, especially the pyramidal tracts. The changes usually begin in the lumbar cord, and are bilateral. The chief clinical characteristics are paralysis, muscular spasms, and exaggerated musclereflexes; the first being of course readily accounted for, and the two latter, in part at least, may be referred to the absence of the control exercised by the higher centres upon those in the cord, and transmitted normally through the tracts now degenerated.

In some cases, to which the term amyotrophic lateral sclerosis has been applied, the anterior cornua of gray matter are also involved, adding muscular atrophy to the symptoms already present (Figs. 206 and 207).

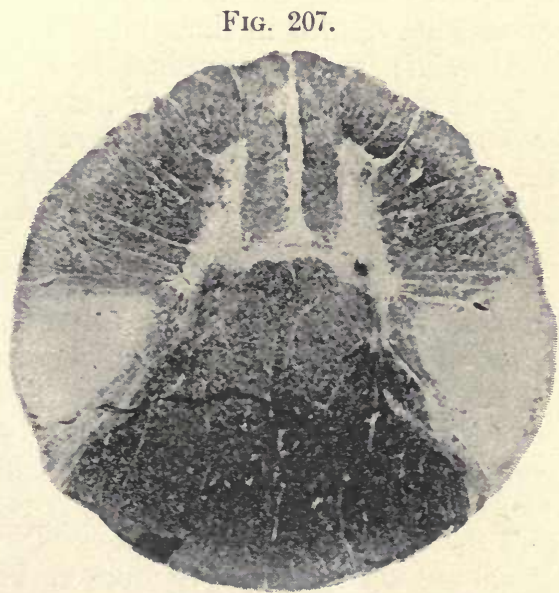

The same as previous figure, except that the section is of the seventh to eight dorsal segments. The pyramidal tracts are sclernsed, and there is considerable degeneration in the intermedio-lateral tract (F. W. Mott).

Progressive spinal muscular atrophy (chronic anterior poliomyelitis) is characterized by a gradual atrophy of the large ganglion-cells of the anterior cornua ; and clinically by progressive atrophy and consequent paralysis of the muscular system-commonly being first manifest in the hand. Though the degenerative changes affect mainly the ganglion-cells of the anterior cornua, the motor fibres arising from them, and 
probably, in most cases, both the direct and crossed pyramidal tracts are involved. In some instances the pyramidal tracts in parts of their course may be affected before the anterior cornua, which could account for the spasmodic rigidity in localized muscle-groups sometimes seen. Generally, however, there is no clinical evidence of the involvement of the pyramidal tracts.

\section{INFLAMMATION.}

Under the term myelitis-inflammation of the cord-is included, according to different authors, a great variety of affections. By many the primary degenerations just briefly described are included under this heading. To those cases in which the changes are limited in their longitudinal extent the term transverse myelitis has been applied, in contradistinction to others in which a considerable length of the cord is involved:

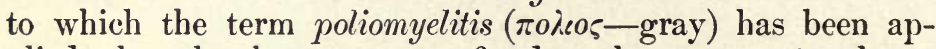
plied when the changes are confined to the gray matter alone; or leukomyelitis (isuxós-white), when confined to the white matter.

Acute transverse myelitis, resulting in softening of the cord and characterized elinically by paralysis of motion and sensation below the site of the lesion, may arise without assignable cause. It may be due to trauma, exposure to cold, or excessive venery, and certain of the acute infectious diseases have been regarded as exciting causes.

According to the color of the involved area, several stages are described-as red and yellow softening, and gray degeneration.

At the first stage the tissue is pink from the engorgement of the bloodvessels, and minute hemorrhages here and there.

Microscopically, the bloodvessels are dilated and surrounded by migrated leukocytes, the neuroglia-tissue is swollen and its cells sometimes increased in number. The ganglion-cells are likewise swollen, and show a diminished staining capacity, vacuolization, varicosity of their protoplasmic processes, and dislocation of their nucleus toward the periphery of the cell.

At a later stage the tissue is yellow, pressure-anæmia having 
given rise to fatty degeneration of the exudate and of the myelin-sheaths of the nerve-fibres. Microscopically, the nerve-fibres are found to be swollen and granular, and the myelin-sheaths filled with droplets of fat. Everywhere are numerous leukocytes, and possibly some neuroglia-cells which have taken up the disintegrated myelin, and on account of their appearance have been designated compound granular cells.

The myelitis rarely goes on to suppuration, though the affected area may undergo complete liquefaction (Fig. 208).

FIG. 208.

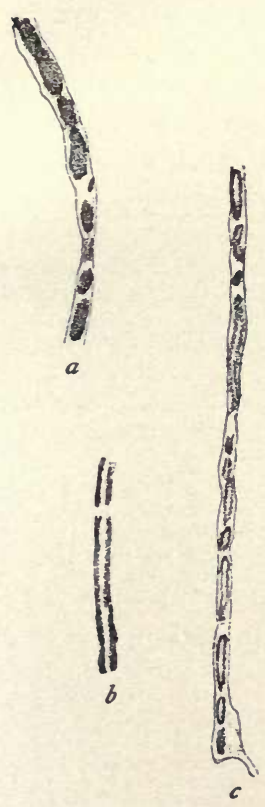

$a$, changes on the eleventh day after section of a frog's sciatic (Gray).

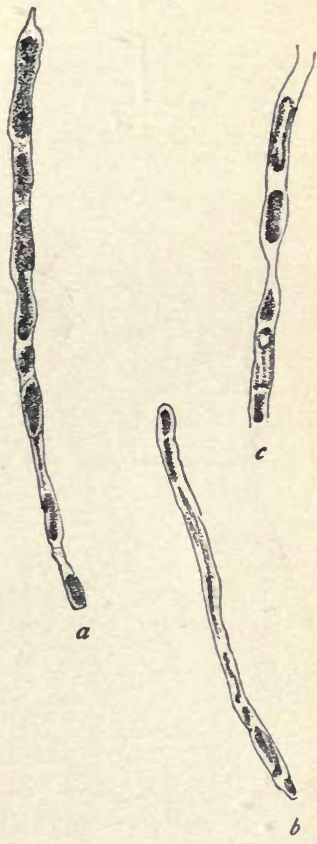

$b$, changes on the fifteenth day after section (Gray).

Usually, however, the fat is absorbed, and, the myelinsheaths having been entirely destroyed, a condition of gray degeneration or softening remains. 
Chronic transverse myelitis may follow an acute attack, the chief characteristic being a marked increase in the neurogliatissue in addition to the changes already noted. Similar changes may also be produced by chronic compression of the cord resulting, for instance, from the growth of a tumor in the meninges (Fig. 209), or the bony deformity produced by Pott's disease.

Acute anterior poliomyelitis is an acute inflammation of the anterior cornua of the gray matter of the cord accompanied

Fig. 209.

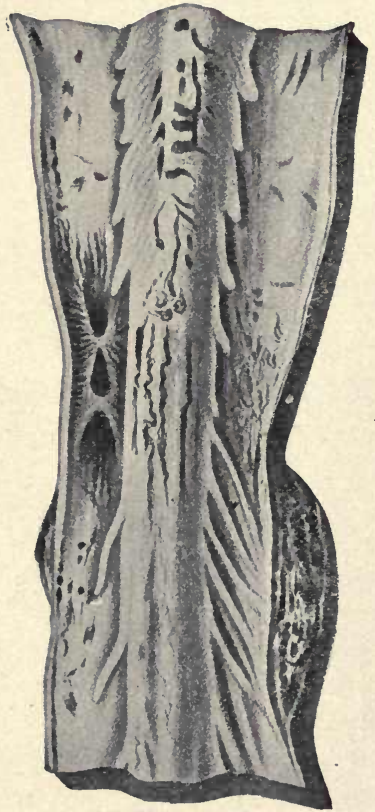

Meningitis and beginning compression-myelitis from an extradural tumor (Bock). by marked systemic disturbances. On account of its greater frequency in childhood, the condition is known clinically as "infantile paralysis," though the disease does occur in the adult, and is recognized as "acute spinal paralysis." Nothing definite is known as to its course, though by many it is regarded as an infectious disease, having a selective action on the tissues which are affected.

Macroscopically, characteristic alterations, which are most marked in the lumbar and cervical enlargements, may be entirely absent at an early stage of the disease, though the changes consecutive to the cessation of the acute inflammatory process are quite obvious, consisting of sclerosis and atrophy of the anterior gray coruna and the antero-lateral white columns.

Microscopically, at an early stage the bloodvessels of the anterior cornua are dilated and surrounded by migrated leukocytes, and there have also been noted swelling and granular degeneration of the large ganglion-cells. At a later stage, even within a few days of the onset of the disease, the ganglion-cells lose their 
FIG. 210.

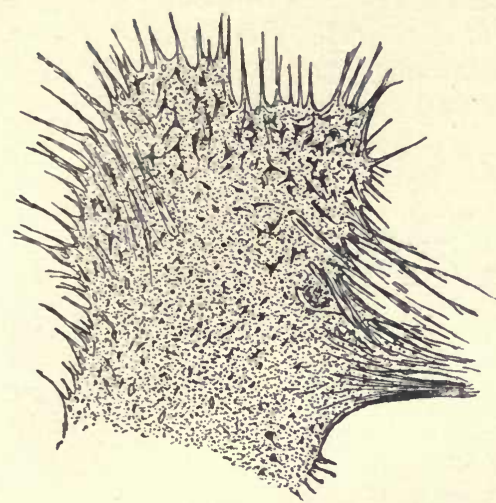

FIG. 211.

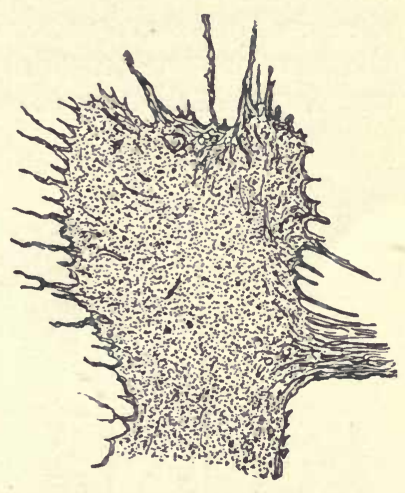

Anterior cornua from case of poliomyelitis, showing atrophy of the ganglioncells. For comparison the appearance of healthy cornua is shown. The small black triangles represent the cells as they appear under a low magnification (F. W. Mott).

FIG. 212.

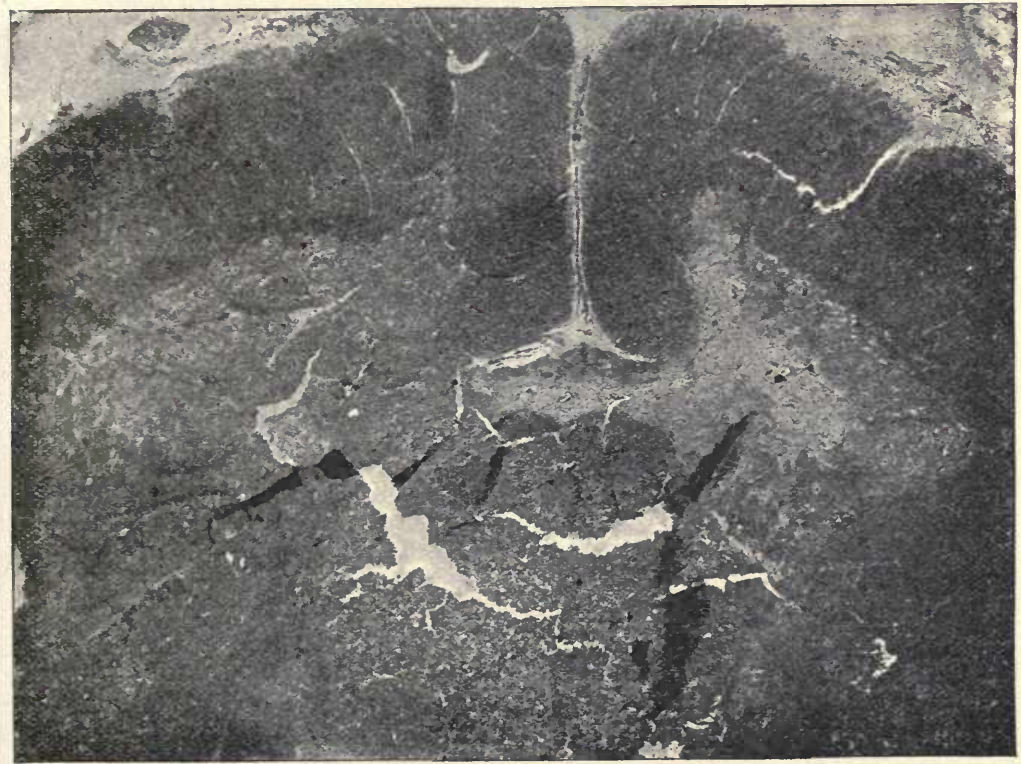

Spinal cord at sixth cervical level, from a case of infantile paralysis. The atrophy of the right anterior horn, the existence of sclerotic scar-tissue in the horn, as well as the absence of groups of cells, are shown. The left anterior horn is normal (Starr). 
nuclei and protoplasmic processes, and the cell-body becomes shrunken and finally entirely disappears. The nerve-fibres of the anterior roots show fatty degeneration of the myelin-sheaths and fragmentation of the axis-cylinders (Figs. 210-212).

FIG. 213.

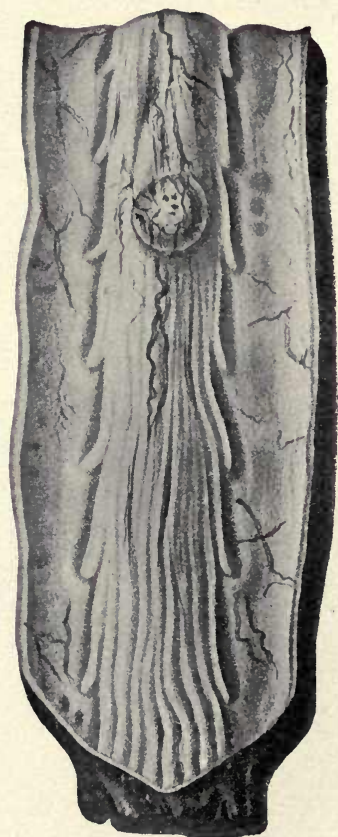

Sarcoma of the spinal cord (Bock).

Of primary tumors the glioma and sarcoma are the types most frequently encountered (Fig. 213). 


\section{INDEX.}

\section{A.}

Abscess of liver, 394

Acid stains, 25

Actinomycosis, 267

of lungs, 356

Adenocarcinoma, 302

Adenoid tissue, 5\%, 85

Adenoma, 299

simple, of kidney, 414

Adipose tissue, 52

Adrenal glands, 108

capillaries of, 109

cortex of, 109

zona fasciculata, 109

glomerulosa, 109

reticularis, 109

general structure of, 108

internal secretion of, 110

lymph-passages of, 109

medulla of, 109

sustentacular connective tissue of, 108

Agminate glands of small intestine, 131

Air-vesicles, 149

Alimentary system, 111

Allantois, 191

Alveolar sarcoma, 297

Alveoli, 195

Amnion, 190

Amoboid movement of cells, 30

Amphi-arthrosis, 200

Amyloid degeneration, 277, 323, 327 of kidney, 412

Anæmia, 312

of brain, 425

local, 270

pernicious, progressive, 313

primary, 312

simple, 313

secondary, 317
Aneurism, 329

cylindrical, 329

fusiform, 329

sacculated, 329

Angeioma, 294

cavernous, 294

lymphangeiomata, 294

Angeiosarcomata, 297

Aphthous stomatitis, 359

Apoplexy, 425

Appendicitis, 385

Appendix vermiformis, 133

Arachnoid, 211

Areolar tissue, 50

Arteries, $77-79$

terminal, 78

tunica adventitia, 78 intima, 77

media, 77

Arterio-sclerosis, 328

Arteritis, 327 chronic, 328

Asiatic cholera, 386

Atelectasis, 336

Atheroma, 328

Atrophy, 284 acute yellow, of liver, 393 of heart, 318

progressive spinal muscular, 435

Attraction-sphere, 27

Axis-cylinder, 207

processes, 205

Axon, 207

B.

Bacillus of Eberth, 386

Bacteria, pathogenic, 269

saprophytic, 269

Basement membranes, 52

Basic stains, 25

Bile-ducts, 136 S 
Bile-ducts, inflammation of, 400 -passages, 138 stenosis of, 400

Bladder, 168

calculi in, 418

coats of, 168

fibrous, 169

mucous, 168

muscular, 169

fissure of, 416

hypertrophy of, 416

inflammation of, 416

tuberculosis of, 417

tumors of, 417

Blastoderm, 40

Blood, 65, 91-103

circulation of, 75

-corpuscles, 91

red, 92

alterations in, 310

decay of, 95

difference of, in vertebrate, 96

functions of, 94

number of, 93

origin of, 95

structure of, 94

white, 97

consistency of, 97

number of, 97

shape of, 97

size of, 97

structure of, 97

surface of 97

diseases of, 309

anæmias, 312

plethora, 309

oligemia, 309

glands, 67, 104

-plasma, 91

-plates, 103

alterations in, 311

qualitative changes in, 309

Bloodvessels, dilatation of, 251

diseases of, 327

degenerations, 327

arteritis, 327

phlebitis, 330

of kidney, 165

of lung, 146

of salivary glands, 120

of small intestines, 132

of spleen, 105
Bloodvessels of stomach, 128

of thyroid gland, 107

Bone, 56-60

areolæ, 58, 59

primary, 58

secondary, 59

calcined, 56

cancellous, 56

-cells, 48, 56

compact, 56

decalcified, 56

development of, 58

cartilaginous, 58

membranous, 59

fibres of Sharpey, 57

lacunæ, 57

Howship's, 60

marrow, 57

function, 58

red, 57

cells, 57

yellow, 58

matrix, 56

lamellæ, 57

system, 57

Haversian, 57

intermediate, 57

peri-medullary, 57

peripheral, 57

perforating fibres, 58

tissues, 56

cancellous, 56

compact, 56

Bowman, membrane of, 126

Brain, diseases of, 424

anæmia, 425

cerebral hemorrhage, 425

embolism, 424

hyperæmia, 425 acute, 425

passive, 425

inflammations, 426

acute, 426

chronic, 426

suppurative, 426

syphilis, 428

thrombosis, 424

tuberculosis, 427

tumors, 428

ventricles of, 213

Bright's disease, 403

Bronchi, 147 
Bronchi, structure of, 148

Bronchial tubes, diseases of, 333

Bronchiectasis, 335

Bronchitis, 333

acute, 333

chronic. 335

fibrinous, 335

Bronchopneumonia, 343

Bronchorrhœa, 335

Brownian movements of cells, 31

Brunner, glands of, 131

Budding, 34

Bursæ, 200

\section{C.}

Cachectic oedema, 273

Calcareous infiltration, 282, 323, 327

Calcification, 28'

Calculi in bladder, 418

Canal of Petit, 229

Cancrum oris, 360

Capillaries, 80 stomata, 80

Capillary bloodvessels, 263

Capsule of Glisson, 134

Carcinoma, 301

of bladder, 417

of brain, 428

cylindrical-celled, 302

of intestines, 392

of liver, primary, 399

secondary, 400

of mouth, 360

of pancreas, 401

primary, of gall-bladder, 400 of kidrey, 414

simplex, 302

squamous-celled, 303

of stomach, 375

of urethra, 419

Cardiac dropsy, 272

hypertrophy, 319

Carotid glands, 106

Cartilage, 52

-cells 48

cellular, 55

hyaline, 53

of larynx, 143

perichondrium, 54

white fibro-, 54

yellow elastic, 55

\author{
Casts, 404 \\ cellular, 406 \\ granular, 405 \\ hyaline, 405 \\ waxy, 407
}

Catarrhal stomatitis, 359

Cavernous angeioma, 294

Cells, 20, 22

body, 24

$$
\text { contents of, } 24
$$

bone-, 48

cartilage-, 48

classes of, 22

differentiated or specialized, 23 connective tissue, 23

epithelial, 23

muscle, 23

nerve-, 23

free-living, 22

generalized or undifferentiated, 22

leukocyte, 23

ova, 23

single, 23

independent, 22

of Claudius, 240

connective-tissue, 23

connective tissue, 47

bone-, 48

cartilage-, 48

fat-, 48

flattened, 48

fusiform, 47

granular, 48

irregular, 48

lamellar, 48

mast-, 48

mucous, 47

neuralgia-, 48

odontoblasts, 48

pigmented, 48

plasma-, 48

round, 47

spherical, 47

stellate, 47

wandering, 49

cortical, 119

cyclosis, 30,31

definition of, 22

of Deiters, 239

epithelial, 23

fat, 48 
Cells, forms of, 28 disc-shaped, 28 flattened, 28 oval, 28 spherical, 28

free, 64

functions of, 29 motion, 29 nutrition, 29 sensibility, 29

ganglion, 202 granules, 25 gustatory, 119 of Hensen, 240 of Langerbans, 221 leukocytes, 23 lymphoid, 64 mast-, 48 movements of, 30 amœboid, 30 Brownian, 31 ciliary, 31 contraction, 30

mucous, 47

muscle, 23,60

nerve-, 23, 202

of cord, 215

neuralgia, 48

olfactory, 142

origin of, 33

ova, 23

plasma of, 48

processes, 28

properties of, 29

of Purkinje, 213

reproduction of, modes of, 33 budding, 34 direct division, 33

indirect division, karyokinesis or mitosis, 34

segmentation, 34

single, 23

size of, 29

spaces, 82

spores, 23

structure of, 23

body, 23

cell-wall, 24

centrosome, 23

intimate, 31

nucleus, 23

tactile, 221
Cells, vital properties of, 29 wall, 27 wandering, 49, 64

Cellular cartilage, 55 casts, 406

exudate, function and fate of, 254 fluids, 65

blood, 65

lymph, 65 semen, 65

Cement intercellular, 32

Centrosome, 27

Cerebellar cortex, gray matter of, 213

Cerebellum, 213 superficial portion, 213

Cerebral cortex, gray matter of, 211 hemorrhage, 425

Cerebro-spinal meningitis, 211

Cerebrum, 211 ganglionic parts of, 212

Chemotaxis, 252

negative, 102 positive, 102

Chlorosis, 312

Chondroma, 292

Chorion, 190

Choroid, 226

Chrematin, 36

Chromatin, 26

Chromophilic granules, 203

Chyle, 103

Cilia, 27 motion of, 28

Ciliary body, 228 movements of cells, 31

Circulatory apparatus, 269 anæmia, local, 270

embolism, 271

hyperæmia, 269 active, 269

passive, 270

ischæmia, 270

œdema, 272

thrombosis, 270

system, 75

arteries, 77 tunica adventitia, 78, 79 intima, 77,79 media, 77,79

capillaries, 80

diseases of, 318 arteritis, 327 
Circulatory system, diseases of, atrophy, 318 degenerations, 327 degenerative changes, 321 hypertrophy, 319 inflammations, 323 phlebitis, 330

heart, 75

vascular sinuses, 80 reins, 79

Circumvallate papillæ of tongue, 118 Cirrhosis of liver, 396

Clitoris, 194

Cloudy swelling degeneration, 281

Coagulation-necrosis, 275

Coceygeal gland, 106

Cochlea, 236

Cohnheim, experiments of, 248

fields of, 61

Colitis, acute, 381

Colloid degeneration, 280

Colostrum, 197

Columnar epithelium, 44

Common ducts, 138

Conjugation, 38

Conjunctiva, 224

Connective tissue, 47 cells. See Cells.

changes, 255

distribution, 47

extracellular elements, 47

of lung, 145

varieties of, 49-60

Cord, spinal, degenerations of, 429 ascending, 431 descending, 431 primary, 431 secondary, 429

hemorrhage of, 429 inflammations of, 436 tumors of 440

Corium, 153

Cornea, 226

epithelial layer of, 226

Corpora cavernosa, 179 lutea, 185

Corpus spongiosum, 180

Corpuscles, concentric. 89

of Hassall, 89

of Krause, 222

of Meissner, 222.

of Pacini, 223
Corpuscles, tactile, 221

Cortical cells, 119

Cowper's glands, 179

Crenation, 93

Croupous stomatitis, 359

Crura cerebri, 213

Crypts of tonsil, 122

Crystalliue lens, 229

Cutis vera, 153

Cylindrical-celled cancer, 302

Cystic ducts, 138

Cystitis, 416

Cystosome, 24

Cysts, 307

disintegration, 308

of kidney, 414

retention, 308

Cytolemma, 27

Cytoplasm, 2

D.

Decidua, 191

Degenerations, amyloid, $277,323,327$, 412

cloudy swelling, 281

colloid, 280

fatty, $277,327,412$

granular, 281

hyaline, 281,323

of kidneys, 412

lardacenus, 277

mucoid, 280

parenchymatous, 281, 412

of spinal cord, 429 ascending, 431

descending, 431

primary 431

secondiry, 429

waxy, 277

Degenerative changes, 321

Dendrites, 205

functions of, 206

Dentinal fibre, 114

sheath, 114

tubules, 114

Dermis, 153

Development, 33-41

of embryo, 40

Diapedesis, 252

Diath roses, 200

Differentiation, 40 
Diphtheria, 361

Disintegration cysts, 308

Ductless glands, 68, 104

Ducts, common, 71

ejaculatory, 178

interlobular, 70

intermediate, 70

intralobular, 70

Duodenal glands of small intestine, 131

Duodenitis, 381

Dura mater, 211

\section{E.}

Ear, 233

external, 234

middle, 234

Eberth, bacillus of, 386

Echinococcus cyst of liver, 400

Ectoplasm, 24

Ectosare, 24

Ejaculatory ducts, 178

Elements, extracellular, 20, 22, 32, 47 non-cellular, 20,22

ultimate, 19,22

Embolism, 271 of brain, 424

Embryo, development of, 40

Emphysema, 336 interstitial, 336 vascular, 336

Empyema, 358

Emulsions, 66

Encephalitis, 426

End-bulbs, 222

Endocarditis, 324 acute, 324 chronic, 325

Endocardium, 75 valves of, 75

Endomysium, 61

Endoplasm, 24

Endosarc, 24

Endothelial sarcomata, 298

Endothelioma of meninges, 424

Endothelium, 42, 46

Enteritis, 380

Eosinophile leukocytes, 100

Epiblast, 40 tissues developed from, 41 Epidermis, 151
Epididymis, 176 canal of, 176

Epiglottis, 143

Epimysium, 61

Epithelioma, 303

of larynx, 333

primary of liver, 399

Epithelium, 42-46

columnar, 44

ciliated, 45

simple, 45

stratified, 45

non-ciliated, 44 simple, 44

stratified, 44

function of, 43

glandular, 45

goblet, 45

location of, 42

neuro, 46

pigmented, 46

simple, 44

specialized, 46

neuro-, 46

pigmented, 46

squamous, simple, 43

stratified, 43

structure of, 42

transitional, 44

varieties of, 43

Erectile tissue, 81

Erythroblasts, 58

Erythrocytes, 92

Eustachian tube, 234

Exudate, cellular, function and fate of, 254

serous, function of, 255

Eye, 224

Eyelids, 233

\section{F.}

Fallopian tubes, 186

Fasciæ, 200

Fat-necrosis, 276

Fatty degeneration, 277, 322, 327 of kidney, 412

glands, 72

infiltration, 177

tissue, 52

Ferrein, pyramids of, 160

Fertilization of ovum, 39 
Fever, 267

anatomical changes, 269

etiology of, 268

significance of, 269

Fibres, 32

development of, 49

perforating, 57

of Sharpey, 57

white, 49

yellow elastic, 49

Fibroblasts, 47, 263

Fibroma, 290

of kidney, 414

Fibrosis, 286, 345

Filliform papillæ of tongue, 118

Fissure of bladder, 416

Flagella, 27 motion of, 28

Fluids, body, 64

cellular, 65

emulsions, 66

chyle, 66

milk, 66

homogeneous, 65

Fœtal membrane, 191

appendages, 190

allantois, 191

amnion, 190

chorion, 190

decidua, 191

foetal membrane, 191

placenta, 192

umbilical cord, 193

vesicle, 191

villi, 192

Foliate papillæ of tongue, 119

Follicles of Lieberkühn, 130

Fovea centralis, 233

Friedreich's disease, 434

Fungiform papillæ of tongue, 118

\section{G.}

Gall-bladder, 138

inflammation of, 400

primary carcinoma of, 400

Ganglia, 216

of posterior roots, 217

sympathetic, 217

typical, 217

Ganglion-cells, 202

Gangrene, 276
Gangrene of lung, 346

circumscribed, 346

diffuse, 346

Gangrenous stomatitis, 360

Gastrectasia, 366

Gastric ulcers, 372

dangers of, 374

Gastritis, 366

acute toxic, 367

chronic, 368

glandularis atrophica, 370 prolifera, 3069

mucipara, 369

purulent, 367

simple acute, 366

Gastro-in testinal tract, diseases of, 359

Gastroptosis, 366

Gemmation, 34

Genital organs, female, 182

mammary glands, 194

ovary, 182

oviducts or Fallopian tubes, 186

uterus, 187

vagina, 193

vulva, 194

male, 172

Cow per's glands, 179

epididymis, 176

penis, 179

prostate gland, 178

seminal resicles, 177

testicle, 172

vas deferens, 177

Giant-celled sarcoma, 297

Giraldès, organ of, 177

Gilanders, 267 of lungs, 356

Glands, 67

adrenal or suprarenal, 108

agminate, 90

of small intestine, 131

of Bartholin, 194

blood, 67,104

of Brunner, 131

carotid, 106

coccygeal, 106

corpuscles of Hassall, 89

cortex, 89

Cowper's, 179

ductless, 104

functions of, 104 
Glands, duodenal, of small intestine, 131

fatty, 72

lachrymal, 233

Luschka's, 106

lymphoid, 67

of small intestine, 131

mammary, 194

medulla, 89

Meibomian, 233

mucous, 71

of tonsils, 122

parotid, 120

prostate, 178

saccular, compound, 70

simple, 70

salivary, 120

inflammation of, 365

sebaceous, 157

secretory, 67

closed, 68

ductless, 68

morphology of, 70

open, 68

saccular, 70

compound, 70

simple, 70

tubular, 70

compound, 70

simple, 70

serous, 71

solitary, 90

sublingual, 121

submaxillary, 122

thymus, 88

thyroid, 107

of tongue, 117

tubular, compound, 70 simple, 70

of Tyson, 180

uterine, 188

Glandular epithelium, 45

Glioma, 306 of brain, 428

Glisson, capsule of, 134

Goblet epithelium, 45

Graafian follicles, 184

Granular casts, 405 degeneration, 281

Granulation tissue, 262

Granules, of cells, 25 basophile, 25
Granules, colorless, 25

Dobie's, 62

Ehrlich's division, 25

ëosinophile, 25

neutrophile, 25

oxyphile, 25

pigmentary, 25

Granulomata, infective, 264

actino mycosis, 267

glanders, 267

leprosy, 267

rhinoscleroma, 267

syphilis, 267

tuberculosis, 264

Granuloplasm, 24

Gustatory cells, 119

\section{H.}

Hæmaglobin, amount of, 311

Hæmothorax, 356

Hairs, 154

bulb, 154

cortex of, 155

cuticle, 155

follicle, 155

medulla, of, 155

roots, 154

Healing by first intention, 259

by second intention, 260

Heart, 75

and pericardium, diseases of, 318 atrophy, 318

degenerative changes, 321

hypertrophy, 319

inflammations, 323

valves of, 75

Hemorrhagic infarcts of lungs, 339

Henle, layer of, 156

Henle's loop, 164

ascending limb of, 165

descending limb of, 164

Hensen, line of, 62

Hepatic artery, 136 ducts, 138 veins, 136

Hernia, 379

Histologic structures, analysis of, 19 of man, 19 technique, 20

Homogeneous fluids, 65

Howship's lacunæ, 60 
Hyaline cartilage, 53 casts, 405

degeneration, 281, 323 matrix, 32

Hyaloplasm, 24

Hydronephrosis, 416

Hydrothorax, 356

Hymen, 194

Hyperæmia, 269

active, 269

of brain, 425

acute, 425

passive, 425

passive, 270,337

of kidneys, 403

of liver, 393

Hypertrophy, 285

of bladder, 416

cardiac, 319

causes of, 319

causes, 285

Hy poblast, 40

tissues developed from, 41

Hypoplasia, 284, 285

of heart, 318

\section{I.}

Infarcts, 271

Infective granulomata of lung, 347

Infiltration, calcareous, 282

and degenerations, 276

fatty, 277

pigmentary, 283

extrinsic, 284

intrinsic, 283

Inflammation, 247

of bile-ducts, 400

of bladder, 416

of brain, 426

acute, 426

chronic, 426

suppurative, 426

of cord, 436

definition of, 247

of gall-bladder, 400

interstitial, 259

of liver, 394

acute purulent, 394

chronic interstitial, 396

of meninges, 420

of pancreas, 401
Inflammation, parenchymatous, 259 of pelvis of kidney, 415

of peritoneum, 401

productive, 259

purulent, 256

suppurative, 256

of urethra, 418

variations in type of, 255

Interlobular ducts, 70 septa, 70

Intermediate duct, 70

Interstitial emphysema, 336 inflammation, 259

Intestines, acquired deformities and displacements of, 379

congenital deformities and displacements of, 378

inflammations of, 380

acute colitis, 381

appendicitis, 385

duodenitis, 381

enteritis, 380

specific, 386

Asiatic cholera, 386

syphilis, 391

tuberculosis, 390

typhoid fever, 386

large, 132

muscular coat of, 132

small, 128 .

agminate glands of, 131

blood vessels of, 132

duodenal glands of, 131

lymphatic vessels of, 132

ly mphoid glands of, 131

mucous membrane of, 129

muscular coat of, 131

muscularis mucosæ, 131

nerves of, 132

subepithelial connective tissue, 130

submucosa, 131

serous or peritoneal coat of, 132

walls of, 128

tumors of, 392

Intralobular capillaries, 137

ducts, 70

veins, 137

Intussusception, 380

Iris, 228

Irritants, 256

Ischæmia, 270 
K.

Karyokinesis, 26, 34

Kidneys, 158

bloodvessels of, 165

cortex of, 160

diseases of, 403

Bright's disease, 403

cysts, 414

degenerations, 412

amyloid, 412

fatty, 412

parenchymatous, 412

granulomata, specific, 413 syphilis, 413

tuberculosis, 413

hyperæmia, passive, 403

nephritis, acute parenchymatous, 407

chronic, 410

interstitial, 411

parenchymatous, 410

tumors, 414

general structure of, 158

medulla of, 158

pelvis of, 168

inflammation of, 415

wall of, 168

sustentacular connective tissue of, 160

uriniferous tubules of, 162

Krause, membrane of, 62

tactile corpuscles of, 222

\section{L.}

Lachrymal canals, 233 gland, 233

Lactation, 194

Langerhans' bodies, 140 cells of, 221

Lardaceous degeneration, 277

Laryngitis, acute, 332 chronic, 333

Larynx, 143 cartilages of, 143

diseases of, 332

epithelioma, 333

laryngitis, 332

papillomata, 333

sarcoma, 333

syphilis, 333

tuberculosis, 333
Larynx, ligaments of, 143

mucous membrane of, 143

epithelial lining, 143

submucosa, 143

tunica propria, 143

Leiomyona of kidney, 414

Lei myomata, 305

Leprosy, 267

of lungs, 356

Leptomeningitis, 421

Leukæmia, 314

Leukocyte, 23

Leukocytes, alterations in, 311

cell-bodies of, 98

nuclei of, 98

number of, 97

origin and development of, 102

structure of, 97

varieties of, 98

eosinophile, 100

large mononuclear, 99

polymorphonuclear or neutrophile, 100

small mononuclear, 99

transitional, 99

vital properties and functions of, 101

Leukocy thæmia, 314

Leukocytosis, 311

Lieberkühn, follicles of, 130

ligament, suspensory, 229

Ligaments of larynx, 143

Linin, 26

Lipoma, 291

of kidney, 414

Liquefaction-necrosis, 276

Liquor sanguinis, 91 seminis. 181

Liver, 133

-cells, 136

diseases of, 393

atrophy, acute yellow, 393

hyperæmia, 393

inflammations, 394

syphilis, 399

tuberculosis, 399

tumors, 399

general structure of, 134

lobules of, 136

sustentacular or connective tissue, 134

Localized œdemas, 273 
Locomotor ataxia, 431

Lungs, 145

bloodvessels of, 146

connective tissue of, 145

diseases of, 336

actinomycosis, 356

atelectasis, 336

emphysema, 336

gangrene, 346

glanders, 356

hemorrhagic infaret, 339

hyperæmia, passive, 337

leprosy, 356

cedema, 338

pneumonia, chronic interstitial, 345

lobar, 339

lobular, 343

syphilis, 355

tuberculosis, 347

tumors, 356

lymphatics of, 147

nerves of, 147

pyramidal lobules of, 145

Lisch ka's gland, 106

Lymph, 65, 82, 103

-cords, 87

-corpuscles, 103

follicles, 85

-sinuses, 89

medullary, 87

peripheral, 87

-spaces, 82

of Fontana, 228

Lymphangeioma, 294

Lymphatic glands, 86

capsule of, 87

circulation through, 88

cortex of, 88

elements of, 86

functions of, 90

medulla, 88

origin of, 83

vessels of, 87

afferent, 87

efferent, 88

system, 82

action of, 82

lacteals of, 84

morphologic elements of, 82

origin of, 83

vessels of, 82,84
Lymphatic system, vessels of, coats, 84 valves, 85

vessels, 84

of small intestine, 132

of spleen, 106

of stomach, 128

Lymphatics of lung, 147

of salivary glands, 120

of thyroid gland, 108

Lymphoid cells, 64 of tonsil, 122

glands, 67

of small intestine, 131

tissue of, 52, 8.5

cells of, 85

diffuse, 85

functions of, 90

meshes, 85

nodules, 85

I.

Macula lutea, 233

Malpighi, pyramids of, 158

Malpighian body, 162 arrangement and function, 163 corpuscles, 106

Mammary glands, 194

secretion of, 196

sustentacular connective tissue of, 195

Margination, 250

Marrow, function of, 58

red, 57

yellow, 58

Mast-cells, 48

Maturation of ovule, 38

Medulla oblongata, 213

Medullary sheath, 208

Medullated nerve-fibres, 207

Meibomian glands, 233

Meissner, tactile corpuscles of, 221

Melanotic sarcoma, 298

Membranes, 51

basement, 52

of Bowman, 226

distant, 51

of Krause, 62

mucous, 72

epithelium, 72

muscularia mucosæ, 72

secretion, 73 
Membranes, mucous, submucosa, 72 tunica propria, 72

of Nasmyth, 114

of Reissner, 237

serous, 73

connective tissue, 73

endothelium, 73

parietal, 73

subserous tissue, 73

visceral, 73

Meninges, diseases of, 420

leptomeningitis, 421

pachymeningitis, 420

chronic, 420

hemorrhagic, 420

syphilis, 424

tuberculosis, 423

tumors, 424

Mesoblast, 40

Metakinesis, 37

Micropyle, 41

Microscopical appearances of inflamed animal membranes, 248 first stage, 248

second stage, 249

third stage, 250

Mineral matters, 32

Mononuclear leukocytes, large, 99 small, 99

Mouth, 111

diseases of, 359

stomatitis, 359

syphilis, 360

thrush, 360

tuberculosis, 360

tumors, 360

mucous membrane of, 111

of epithelial layer, 111

of submucosa, 112

of tunica propria, 112

Mucoid degeneration, 280

Mucosa, 72

Mucous cells, 47

glands, 71

of tongue, 117

membranes 72

of larynx, 143

of mouth, 111

of nasal fossæ, 141

of œesophagus, 125

of pharynx, 124

of small intestine, 129
Mucous membranes of tongue, 116 of trachea, 144

of uterus, 187

Mumps, 365

Muscle cells, 60 cardiac, 62 involuntary or non-striated, 63 striated or striped, 60

voluntary, 198 motor end-plates of, 320

Muscular coat of large intestine, 132 of urethra, 170

structures, 198

substance of tongue, 116

Myelitis, acute transverse, 436 chronic transverse, 438

Myeloid sarcomata, 297

Myeloplaxes, 51

Myocarditis, 323

Myocardium, 75

Myoma, 304

leiomyomata, 305

rhabdomyomata, 304

Myomalacia cordis, 323

Myхoma, 291

\section{N.}

Nails, 157

root of, 157

Nasal fossæ, 141

mucous membrane of, 141 olfactory portion, 142 respiratory portion, 142

passages, diseases of, 332 rhinitis, 332 syphilis, 332

Nasmyth, membrane of, 114

Necrosis, 274

varieties of, 275

caseation, 276

coagulation, 275

fat, 276

gangrene, 276

liquefaction, 276

Nephritis, acute parenchymatous, 407

chronic, 410

interstitial, 411

parenchymatous, 410

Nerves, 217

arrangement of, 217

Nerve-cells, 202

of spinal cord, 215 
Nerve-centres, 209

-fibres, 207

medullated, 207

non-inedullated, 208

filaments of salivary glands, 120

of lung, 147

of small intestine, 132

-terminals, 218

classes of, 218

-tissue, 64

Nervous system, 202

diseases of, 420

division of, 202

Neuraxons, 205

Neurilemma, 208

Neurites, 205

function of, 206

Neuro-epithelium, 46

Neuroglia, 209

-cells, 48

Neurokeratin, 208

Neuroma, 306

Neurons, 64, 206

Neuroplasm, 203

Neurosome, 203

Neutral stains, 25

Neutrophile leukocytes, 100

Nipple, 196

Nodes of Ranvier, 208

Noma, 360

Non-medullated nerve-fibres, 208

Nuclear fibrils, 26

matrix, 26

membrane, 26

Nuclei, 23, 26

function of, 27

location of, 26

shape of, 26

size of, 27

structure of, 26

Nucleolus, 203

Nutrition, pathology of, 274

\section{O.}

Odontoblasts, 48

Edema, 272

of lungs, 338

varieties of, 272

cachectic, 273

cardiac, 272

localized, 273
Edema, renal, 273

Esophagus, 125

inflammation of, 365

mucous membrane of, 125

epithelium, 125

submucosa, 125

tunica propria, 125

muscular layer, 125

Olfactory cells, 142

terminals, 224

Oligemia, 309

Oögenesis, 186

Organs, 74

cells of, 74

of Corti, 235, 238

parenchyma 74

sustentacular tissue, 74

Ossification, 58

Osteoblasts, 59

Osteoclasts, 58

Osteoma, 293

Ova, 23, 64, 183

Ovary, 182

epithelial covering of, 183

stroma of, 183

Oviducts, 186

Ovulation, 184

Ovule, 38,184

fertilization of, 39

maturation of, 38

structure of, 38

Oxyphile leucocytes, 100

\section{P.}

Pachymeningitis, 420 chronic, 420

hemorrhagic, 420

Palate, soft, 124

Pancreas, 138

atrophy of, 401

carcinoma of, 401

connective tissue of, 138

inflammation of, 401

Papillæ of tongue, 117

circumvallate, 118

filiform, 118

foliate, 119

fungiform, 118

Papilloma, 299

of bladder, 417

Papillomata of larynx, 333 
Parathyroids, 108

Parenchymatous degeneration, 281 of kidney, 412

inflammation, 259

Parotid gland, 120

Pathogenic bacteria, 269

Pathology, human, 243 modern or cellular, 244

Pelvis of kidney, 168 wall of, 168

Penis, 179

Peptic or cardiac glands of stomach, 126

Pericarditis, 326

Pericardium, 76

fibrous, 76

serous, 76

Perimysium, 61

Periosteum, 57

Peritoneum, 133

inflammatisn of, 401

tuberculosis of, 402

Peritonitis, 401

chronic, 402

Peyer's patches, 131

Phagocytosis, 101, 252

Pharyngeal aponeurosis, 124 tonsil, 124

Pharyngitis, pseudomembranous, 361

Pharynx, 124, 143

diseases of, 361

diphtheria, 361

pseudomembranous pharyngitis, 361

simple catarrhal inflammation, 361

tonsillitis, 363

mucous membrane of, 124

epithelial lining, 124

lymphoid nodules, 124

submucosa, 124

tunica propria, 124

walls of, 124

Phlebitis, 330

chronic, 331

Phthisis, acute, 350

chronic, 353

fibroid, 354

Pia mater, 211

Pigmentary infiltration, 283

extrinsic, 284

intrinsic, 283
Pigmented epithelium, 46

Pineal body, 110

Pituitary body, 110 anterior lobe of, 110

posterior lobe of, 110

Placenta, 192

Plasma-cells, 48

Plethora, 309

Pleura, 150

diseases of, 356

empyema of, 351

hæmothorax, 356

hydrothorax, 356

pleuritis, 357

pneumothorax, 356

Pleuritis, 357

fibrinous, 357

serofibrinous, 357

Plexus of Auerbach, 132

of Meissner, 132

Pneumonia, 339

chronic interstitial, 345

lobar, 339

division of process. 340

etiology of, 340

lobular, 343

etiology of, 343

lesions of, 344

Pneumothorax, 356

Polar body, 49 corpuscle, 27

Poliomyelitis, acute anterior, 438 chronic anterior, 435

Polymorphonuclear leukocytes, 100

Polynuclear leukocytes, 100

Pons, 213

Portal vein, 136

Productive inflammation, 259

Pronucleus, female, 39

male, 40

Prostate gland, 178

sustentacular connective tissue, 178

Protoplasm, 22

granules, 24, 25

vacuoles, 24

Protoplasmic processes, 205

Pseudomembranous pharyngitis, 361 stomatitis, 359

Purkinje, cells of, 213

Purulent inflammation, 256

Pyelitis, 415 
Pyelo-nephritis, 415

Pyloric glands of stomach, 127

Pyonephrosis, 415

Pyramidal lobules of lung, 415

Pyramids of Ferrein, 160 of Malpighi, 158

\section{Q.}

Quinsy, 364

R.

Radiating fibres of Müller, 230

Ran vier, nodes of, 208

Rectum, 133

Red-marrow, 57

Renal calculi, 416

dropsy, 273

Repair, 259

Reproduction, 33, 41

of cells, 33

sexual, 38

Reproductive system, 172

Respiratory organs, diseases of, 332 system, 141

general considerations of, 141

Retention cysts, 308

Retiform tissue, 52

Retina, 230

Rhabdomyomata, 304

Rhinitis, 332

acute, 332

chronic, 332

Rhinoscleroma, 267

Round-celled sarcomata, 295

\section{S.}

Saccular glands, compound, 70 simple, 70

Saccule, 235

Saliva, 122

Salivary glands, 120

bloodvessels of, 120

ducts of, 120

general structure of, 120

inflammation of, 365

lymphatics of, 120

nerve filaments of, 120

sustentacular connective tissue of, 120

Salter, incremental lines of, 115
Saprophytic bacteria, 269

Sarcolemma, 60

Sarcoma, 294

alveolar, $29 \bar{i}$

angeiusarcomata, 297

of brain, 428

endothelial, 298

giant-celled or mveloid, 297

of larynx, 333

melanotic, 298

of mouth, 360

primary, of kidney, 414

round-celled, 295

spindle-celled, 296

of urethra, 419

Sarcoplasm, 61

Sarcous dises. 62

elements. 62

substance, 61

Scala media, 237

Schreger, lines of, 115

Sclerosis, amytrophic lateral, 435 of posterior columns, 431 spontaneous lateral, 434

Sebaceous glands, 157

Secretions, external, 68 internal, 68

Secretory glands, 67 morphology of, 70

Segmentation, 34

Semen, 65, 181

Semicircular canals, 236

Seminal vesicle, 177

Serous exudate, functions of, 255 glands, 71 of tongue, 117 membranes, 73

Sertoli, columns of, 174

Sharpey, fibres of, $\tilde{5} 7$

Sheath of Schwann, 208

Simple fluids, 65

Sinuses-lymph, 87 vascular, 80

venous, 79

Skeletal structures, 198

Skin, 151

Soft palate, 124

Specialized epithelium 46

Spermatogenesis, 174

Spermatozoa, 181

Spheroidal-celled cancer, 302

Spinal cord, 214 
Spinal cord, hemorrhage of, 429 nerve cells of, 215

Spindle-celled sarcoma, 296

Spiral ganglion, 240

Spleen, 104

bloodvessels of, 105

function of, 106

general structure, 104

lymphatic vessels of, 106

sustentacular framework of, 105

Splenic pulp, 105

Spongioplasm, 24

Spores, 23

Squamous-celled cancer, 303 epithelium, 43

Stains, acid, 24 basic, 24

neutral, 25

Stomach, 126

bloodvessels of, 128

diseases of, 366

gastrectasia, 366

gastritis, 366

acute toxic, 367

chronic, 368

purulent, 367

simple acute, 366

gastroptosis, 366

tumors, 375

ulcers, 371

lymphatic vessels of, 128

mucosa of, 126

epithelial lining, 126

muscular coat, 128

peptic or cardiac glands, 126

pyloric glands, 127

serous or peritoneal coat, $12 \mathrm{~s}$

subepithelial connective tissue of, 127

submucosa, 128

walls of, 126

Stomatitis, 359

aphthous, 359

catarrhal, 359

croupous, 359

gangrenous, 360

pseudomembranous, 359

ulcerous, 359

Stratum corneum, 153

granulosum, 152

lucidum, 153

Malpighii, 152
Sublingual gland, 121

Submaxillary gland, 122

Submucosa, 72

Suppurative inflammation, 256

Suprarenal glands. See Adrenal glands.

Suspensory ligament, 229

Sustentacular tissue, 87

Sweat-glands, 154

Synarthrosis, 200

Synovial membranes, 199

Syphilis, 267

of brain, 428

of intestines, 391

of kidneys, 413

of larynx, 333

of liver, 399

of lungs, 355

of meninges, 424

of mouth, 360

of nose, 332

T.

Tactile cells, 221

Taste-buds, 119 -terminals, 224

Technique, dyes, 20 general, 21

nuclear, 20

histological, 20

sections, 20

Teeth, 112

crusta petrosa, 114

dentine, 114

development of, 115

enamel of, 113

general structure of, 112

pulp-cavity, 115

Teledendrites, 205

Tendons, 198

Tendon-sheaths, 199

Teratoma, 307

Terminal bronchioles, 148

Testicle, 172

seminiferous tubules, 173

sustentacular connective tissue of, 173

Thoracic duct, 85

Thrombosis, 270

appearance and structure of, 270

of brain, 424 
Thrombosis, cause of, 271

Thrush, 360

Thymus gland, 88

Thyroid gland, 107

bloodvessels of, 107

functions of, 108

lymphatics of, 108

sustentacular connective tissue of, 107

Tissues, 20, 22, 42

adenoid, 85

adipose, 52

areolar, 50

classes of, 42

connective tissue, 47

endothelium, 42, 46

epithelium, 42

free cells and body fluid, 64

muscles. 60

nerve-, 64

connective, varieties of, 49

adipose, 52

areolar, 50

bone, 56

cartilage, 52

membranes, 51

basement, 52

distant, 51

mucous, 49

retiform, 52

white fibrous, 51

yellow elastic, 51

elements of, 21

erectile, 81

fatty, 52

lymphoid, 85

mucous, 49

nerve-, 64

retiform, 52

adenoid, 52

lymphoid, 52

sustentacular, 87

white fibrous, 51

yellow elastic, 51

Tongue, 116

general structure of, 116

glands of, 117

mucous, 117

serous, 117

lymplioid tissue of, 117

mucous membrane of, 116

epithelial covering, 116
Tongue, mucous membrane, submucosa, 116

tunica propria, 116

muscular substance of, 116

papillæ of, 117

circumvallate, 118

filiform, 118

foliate, 119

fungiform, 118

Tonsillitis, 363

Tonsils, 90, 122

crypts of, 122

lymphoid cells of, 122

mucous glands of, 122

Tracheal, 144

mucuus membrane of, 144

Transitional epithelium, 44

leukocytes, 99

Tuberculosis, 264

of bladder, 417

of brain, 427

caseation, 266

fibroil transformation, 266

giant-cell, $266^{\circ}$

histological tubercle, 264

of intestines, 390

of kidneys, 413

of larynx, 333

of liver, 399

of lungs, 347

acute miliary. 349

of meninges, 423

of mouth, 360

of peritoneun, 402

Tubular glands, compound, 70 simple. 70

Tumors, 286

of bladder, 417

of brain, 428

classification of 287

histological, $2 \diamond 8$

of cord, 440

definition of, 286

etiology of, 286

of intestines, 392

of kidneys, 414

of liver, 399

of lungs, 356

of meninges, 424

of mouth, 360

of stomach, 375

of urethra, 419 
Tunica adventitia, 78,79 intima, 77,79 media, 77, 79

Typhoid fever, 386

Tyson, glands of, 180

\section{U.}

Ulcer of stomach, 371

Umbilical cord, 193 vesicle, 191

Ureter, 168 coats of, 168 fibrous, 168 mucous, 168

Urethra, 169 muscular, 168

female, 169 inflammation of, 418 male, 170

muscular coat of, 170 stricture of, 419 tumors of, 419

Urethritis, acute, 418 chronic, 418

Urinary bladder, 416 organs, 158 diseases of, 403

Urine, 171 secretion of, 167

Uriniferous tubule, 162 neck of, 164

Uterine glands, 188

Uterus, 187 cervix of, 189 mucous membrane of, 187 muscular coat of, 188 Utricle, 235

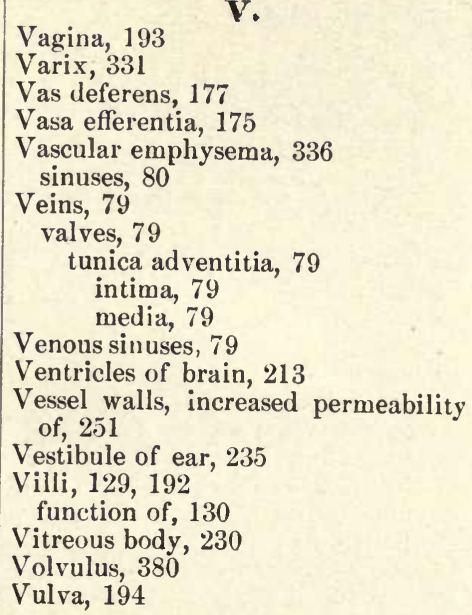

Wagner, tactile corpuscles of, 221

Wandering cells, 49, 64

Watney's nodes, 130

Waxy casts, 407 degeneration, 277

White fibres, 49 fibro-cartilage, 54 fibrous tissue, 51

\section{$\mathbf{Y}$.}

Yellow elastic cartilage, 55 fibres, 49 tissue, 51 marrow, 58 




\section{CATAlogue OF PUblications OF \\ LEA BROTHERS \& COMPANY,}

\%06, 708 \& 710 Sansom St., Pbiladelphia.

111 Fifth Ave. (Cor. 18th st.), New York.

The books in the annexed list will be sent by mail, post-paid, to any Post-Office in the United States, on receipt of the printed prices.

\section{IN口 $\mathrm{IX}$.}

ANATOMY. Gray, p. 11 ; Treves, 30 ; Gerrish, 11; Brockway, 4. DICTIONARIES. Dunglison, p. 8 ; Duane, 8 ; National, 4.

PHYSICS. Draper, p. 8 ; Robertson, 24 ; Martin \& Rockwell, 20.

PHYSIOIOGY. Foster, p. 10 ; Chapman, 5; Schofield, 25; Collins \& Rockwell, 6.

[Luff, 19 ; Remsen, 24.

CHEMISTRY. Simon, p. 26 ; Attfield, 3 ; Martin \& Rockwell, 20;

PHARMACY. Caspari, p. $5 . \quad$ [Bruce, 4 : Schleif, 25.

MATERIA MEDICA. Culbreth, p. 6 ; Maisch, 19 ; Farquharson, 9 ; DISPENSATORY. National, p. 21.

THER A PEUTICS. Hare, p. 13; Fothergill, 10 ; Whitla, 31 ; Hayem \& Hare, 14 ; Bruce, 4 ; Schleif, 25 ; Cushny, 6.

PRACTICE. Flint, p. 9; Loomis \& Thompson, 19 ; Malsbary, 20.

DIAGNOSIS. Musser, p. 21; Hare, 12; Simon, 25; Herrick, 15; Hutchison \& Rainey, 16 ; Collins, 6.

CLIMATOLOGY. Solly, p. 26 ; Hayem \& Hare, 14.

NERVOUS DISEASES. Dercum, p. 7 ; Gray, 11; Potts, 23.

MENTAL DISEASES. Clouston, p. 5 ; Savage, 24 ; Folsom, 10.

BACTERIOLOGY. Abbott, p. 2 ; Vaughan \& Novy, 30 ; Senn's

(Surgical), 25. Park, 22 ; Coates, 6. [Vale, 21.

HISTOLOGY. Klein, p. 17 ; Schafer's, 25 ; Dunham, 8 ; Nichols \& PATHOLOGY. Green, p. 12; Gibbes, 10; Coats, 6; Nichols \& Vale, 21.

SURGERY. Park, p. 22; Dennis, 7; Roberts, 24; Ashhurst, 3; Treves, 29; Cheyne \& Burghard, 5 ; Gallaudet, 10.

SURGERYั-OPERATIVE. Stimson, p. 27 ; Smith, 26 ; Treves, 29. SURGERY-ORTHOPEDIC. Young, p. 31 ; Gibney, 10.

SURGERY-MINOR. Wharton, p. 30. [Balledger \& FRACTURES and DISLOCATIONS. Stimson, p. 27. [Wippern, 3. OPHTHALMOLOGY. Norris \& Oliver, p. 21; Nettleship, 21; Juler, 17; OTOLOGY. Politzer, p. 23; Burnett, 5; Field, 9; Bacon, 4.

LARYNGOLOGY and RHINOLOGY. Coakley, p. 6 ;

DENTISTRY. Essig (Prosthetic), p. 9 ; Kirk (Operative), 17 ; American System, 2 ; Coleman, 6; Burchard 4.

URINARY DISEASES. Roberts, p. 24 ; Black, 4 ; Morris, 20.

VENEREAL DISEASES. Taylor, p. 28; Hayden, 14 ; Cornil, 6 ; Likes, 19.

SEXUAL DISORDERS. Fuller, p. 10 ; Taylor, 29.

DERMATOLOGY. Hyde, p. 16 ; Jackson, 16 ; Pye-Smith, 24 ; Morris, 20 ; Jamiesnn, 16 ; Hardaway, 12 ; Grindon, 12.

GYNECOLOGY. American System, p. 3; Thomas \& Mnndé, 29 Emmet, 9 ; Davenport, 7 ; May, 20 ; Dudley, 8 ; Crockett, 6.

OBSTETRICS. American System, p. 3 ; Davis, 7 ; Parvin, 22 ; Playfair, 23 ; King, 17 ; Jewett, 17 ; Evans, 9.

PEDIATRICS. Smith, p. 26 ; Thomson, 29 ; Williams, 31 ; Tuttle, 30.

HYGIENE. Egbert, p. 9 ; Richardson, 24 ; Coates, 6.

MEDICAL JURISPRUDENCE. Taylor, p. 28.

QUIZ SERIES, POCKET TEXT-BOOKS and MANUALS. Pp. 18, 25 and 27. 
ABBOTT (A. C.). PRINCIPLES OF BACTERIOLOGY: a Practical Manual for Students and Physicians. New (5th) edition thoroughly revised and greatly enlarged. In one handsome $12 \mathrm{mo}$. vol. of 585 pages, with 109 engrav., of which 26 are colored. Just ready. Cloth, $\$ 2.75$, net.

One of its most attractive charac- $\mid$ cessfully. To those who require a teristics is that the directions are so condensed yet nevertheless complete clearly given that anyone with a work upon Bacteriology we most moderate amount of laboratory train- cordially recommend it.-The Theraing can, with a little care as to peutic Gazette.

detail, make his experiments suc-

AMERICAN SYSTEM OF PRACTICAM MEDICINE. A SYSTEM OF PRACTICAL MEDICINE. In contributions by Various American Authors. Edited by ALFred L. Loomis, M.D., LL.D., and W. Gilman THompson, M. D. In four very handsome octavo volumes of about 900 pages each, fully illustrated. Complete work now ready. Per volume, cloth, $\$ 5$; leather, $\$ 6$; half Morocco, $\$ 7$. For sale by subscription only. Prospectus free on application.

Every chapter is a masterpiece of cine" is a work of which every completeness, and is particularly ex- American physician may reasonably cellent in regard to treatment, many feel proud, and in which every praccriginal prescriptions, formulæ, titioner will find a safe and trustcharts and tables being given for the worthy counsellor in the daily reguidance of the practitioner.

"The American System of Medi- Medical Journal.

AMERICAN SYSTEM OF DENTISTRY. In treatises by various authors. Edited by WILBUR F. LITCH, M.D., D.D.S. In three very handsome super-royal octavo volumes, containing about 3200 pages, with 1873 illustrations and many full-page plates. Per vol., cloth, $\$ 6$; leather, $\$ 7$; half Morocco, $\$ 8$. For sale by subscription only. Prospectus free on application to the Publishers.

AMERICAN TEXT-BOOKS OF DENTISTRY. In Contributions by Eminent American Authorities. In two very handsome octavo volumes, richly illustrated :

PROSTHETIC DENTISTRY. Edited by Cha RLEs J. Essig, M.D., D.D.S., Professor of Mechanical Dentistry and Metallurgy, Department of Dentistry, University of Pennsylvania, Philadelphia. 760 pages, 983 engravings. Cloth, $\$ 6$; leather, $\$ 7$. Net.

No more thorough production will It is up to date in every particular. be found either in this country or in It is a practical course on prosthetics any country where dentistry is un- which any student can take up durderstood as a part of civilization.- ing or after college.-Dominion DenThe International Dental Journal. tal Journal.

OPERATIVE DENTISTRY. Edited by FDWARD C. KIRK, D.D.S., Professor of Clinical Dentistry, Department of Dentistry, University of Pennsylvania. 699 pages, 751 engravings. Cloth, $\$ 5.50$; leather, $\$ 6.50$. Net. Just ready.

Written by a number of practitioners as well known at the chair as in journalistic literature, many of them teachers of eminence in our colleges. It should be included in the list of text-books set down as most useful to the college student.The Dental News.

It is replete in every particular and treats the subject in a progressive manner. It is a book that every progressive dentist should possess, and we can heartily recommend it to the profession.- The Ohio Dental Journal. 
AMERICAN SYSTEMS OF GYNECOLOGY AND OBSTETRICS. In treatises by the most eminent American specialists. Gynecology edited by Matrhew D. ManN, A. M., M. D., and Obstetries edited by BARTon C. Hirst, M.D. In four large octavo vólumes comprising 3612 pages, with 1092 engravings, and 8 colored plates. Per volume, cloth, $\$ 5$; leather, $\$ 6$; half Russia, $\$ 7$. For sale by subscription only. Prospectus free on application to the Publishers.

AMERICAN TEXT-BOOK OF ANATOMY. See Gerrish, page 11.

ALLEN (HARRISON). A SYSTEM OF HUMAN ANATOMY; WITH AN INTRODUCTORY SECTION ON HISTOLOGY, by E. O. ShaKesPEare, M.D. Comprising 813 double-columned quarto pages, with 380 engravings on stone, 109 plates, and 241 wood cuts in the text. In six sections, each in a portfolio. Price per section, $\$ 3.50$. Also, bound in one volume, cloth, $\$ 23$. Sold by subscription only.

A PRACTICE OF OBSTETRICS BY AMERICAN AUTHORS. See Jewett, page 17 .

A TREATISE ON SURGERY BY AMERICAN AUTHORS. FOR STUDENTS AND PRACTITIONERS OF SURGERY AND MEDICINE. Edited by Roswell Park, M.D. See page 22.

ASHHURST (JOHN, JR.). THE PRINCIPLES AND PRACTICE OF SURGERY. For the use of Students and Practitioners. Sixth and revised edition. In one large and handsome octavo volume of 1161 pages, with 656 engravings. Cloth, $\$ 6$; leather, $\$ 7$.

As a masterly epitome of what has text-book, we do not know its equal. been said and done in surgery, as a It is the best single text-book of succinct and logical statement of the surgery that we have yet seen in this principles of the subject, as a model country.-New York Post-Graduate.

A SYSTEM OF PRACTICAL MEDICINE BY AMERICAN AUTHORS. Edited by William PePPER, M.D., LL. D. In five large octavo volumes, containing 5573 pages and 198 illustrations. Price per volume, cloth, $\$ 5$; leather $\$ 6$; half Russia, $\$ 7$. Sold by subscription only. Prospectus free on application to the Publishers.

ATTFIELD (JOHN). CHEMISTRY; GENERAL, MEDICAL AND PHARMACEUTICAL. New (16th) edition, specially revised by the Author for America. In one handsome 12mo. volume of 784 pages, with 88 illustrations. Cloth, $\$ 2.50$, net.

It is replete with the latest inform- been adopted, bringing the work into ation, and considers the chemistry of close touch with the latest United every substance recognized officially States Pharmacopocia, of which it is or in general practice. The modern a worthy companion.-The Pittsburg scientific chemical nomenclature has Medical Reviev.

BALLENGER (W. L.) AND WIPPERN (A. G.). Shortly. A POCKET TEXT-BOOK OF DISEASES OF THE EYE, EAR, NOSE AND THROAT. In one handsnme $12 \mathrm{mo}$. volume of about 400 pages, with many illustrations. Cloth, $\$ 1.50$, net. Lea's Series of Pocket Text-books, edited by BerN B. Gallatdet, M. D. See p. 18.

BARNES (ROBERT AND FANCOURT). A SYSTEM OF OBSTETRIC MEDICINE AND SURGERY. Octaro, 872 pages, with 231 illus, Cloth, $\$ 5$; leather, $\$ 6$, 
BACON (GORHAM). ON THE EAR. One 12mo. volume, 400 pages, 109 engravings and a colored plate. Cloth, net, $\$ 2.00$. Just ready.

It is the best manual upon otology. dents of medicine--Cleveland JourAn intensely practical book for stu- nal of Medicine.

BARTHOLOW (ROBERTS). CHOLERA; ITS CAUSATION, PREVENTION AND TREATMENT. In one $12 \mathrm{mo}$. volume of 127 pages, with 9 illustrations. Cloth, $\$ 1.25$.

BARTHOLOW (ROBERTS). MEDICAL ELECTRICITY. A PRACTICAL TREATISE ON THE APPLICATIONS OF ELECTRICITY TO MEDICINE AND SURGERY. Third edition. In one octavo volume of 308 pages, with 110 illustrations.

BELL (F. JEFEREY). COMPARATIVE ANATOMY AND PHYSIOLOGY. In one $12 \mathrm{mo}$. volume of 561 pages, with 229 engravings. Cloth, \$2. See Students' Series of Manuals, page 27.

BULLINGS (JOHN S.). THE NATIONAL MEDICAL DICTIONARY. Including in one alphabet English, French, German, Italian and Latin Technical Terms used in Medicine and the Collateral Sciences. In two very handsome imperial octavo volumes containing 1574 pages and two colored plates. Per volume, cloth, $\$ 6$; leather, $\$ 7$; half Morocco, $\$ 8.50$. For sale by subscription only. Specimen pages on application to the publishers.

BLACK (D. CAMPBELL). THE URINE IN HEALTH AND DISEASE, AND URINARY ANALYSIS, PHYSIOLOGICALLY AND PATHOLOGICALLY CONSIDERED. In one 12mo. volume of 256 pages, with 73 engravings. Cloth, $\$ 2.75$.

A concise, yet complete manual, Concise, practical, clinical, well treating of the subject from a prac- illustrated and well printed.-Marytical and clinical standpoint.-The land Medical Journal.

Ohio Medical Journal.

BLOXAM (C. L.). CHEMISTRY, INORGANIC AND ORGANIC. With Experiments. New American from the fifth London edition. In one handsome octavo volume of 727 pages, with 292 illustrations. Cloth, $\$ 2$; leather, $\$ 3$.

BROCKWAY (F. J.). A POCKET TEXT-BOOK OF ANATOMY. In one handsome $12 \mathrm{mo}$. volume of about 400 pages, with many illustrations. Shortly. Cloth, $\$ 1.50$, net. Lea's Series of Pocket Text-books, edited by Bern B. Gallaudet, M. D. See page 18.

BRUCE (J. MITCHELL). MATERIA MEDICA AND THERAPEUTICS. New (6th) edition. In one $12 \mathrm{mo}$. volume of 600 pages. Just ready. Cloth, $\$ 1.50$, net. See Student's Series of Manuals, page, 27.

- PRINCIPLES OF TREATMENT. In one octavo volume. Preparing.

BRYANT (THOMAS). THE PRACTICE OF SURGERY. Fourth American from the fourth English edition. In one imperial octavo vol. of 1040 pages, with 727 illustrations. Cloth, $\$ 6.50$; leather, $\$ 7.50$.

BURCHARD (HENRY H.). DENTAL PATHOLOGY AND THERAPEU'TICS. Handsome octavo, 575 pages, with 400 illustrations. Just ready. Cloth, net, $\$ 5.00$; leather, net, $\$ 6.00$.

In the treatment of the subject is a valuable text-book on a subject the method pursued by the author which has heretofore not been adeis logical and sequential. The work quately represented.-Dental Cosmos. 
BURNETT (CHARLES H.)。 THE EAR: ITS ANATOMY, PHYSIOLOGY AND DISEASES. A Practical Treatise for the Use of Students and Practitioners. Second edition. In one 8vo. volume of 580 pages, with 107 illustrations. Cloth, $\$ 4$; leather, $\$ 5$.

CARTER (R. BRUDENELL) AND FROST (W. ADAMS). OPHTHALMIC SURGERY. In one pocket-size $12 \mathrm{mo}$. volume of 559 pages, with 91 engravings and one plate. Cloth, \$2.25. See Series of Clinical Manuals, page 25.

CASPARI (CHARLES JR.). A TREATISE ON PHARMACY. For Students and Pharmacists. In one handsome octavo volume of 680 pages, with 288 illustrations. Cloth, $\$ 4.50$.

The author's duties as Professor student who cannot understand must of Theory and Practice of Pharmacy be dull indeed. The book is full of in the Maryland College of Phar- new, clean, sharp illustrations, which macy, and his contact with students tell the story frequeutly at a glance. made him aware of their exact The index is full and accurate.wants in the matter of a manual. National Druggist.

His work is admirable, and the

CHAPMAN (HENRY C.). A TREATISE ON HUMAN PHYSIOLOGY. New (2d) edition. In one octavo volume of 921 pages, with 595 illustrations. Just ready. Cloth, $\$ 4.25$; leather, $\$ 5.25$, net.

In every respect the work fulfils mirable work of reference for the its promise, whether as a complete physician.-North Carolina Medical treatise for the student or as an ad-Journal.

CHARLES (T. CRANSTOUN). THE ELEMENTS OF PHYSIOLOGICAL AND PATHOLOGICAL CHEMISTRY. Octavo, 451 pages, with 38 engravings and 1 colored plate. Cloth, $\$ 3.50$.

CHEYNE (W. WATSON). THE TREATMENT OF WOUNDS, ULCERS AND ABSCESSES. In one 12mo. volume of 207 pages. Cloth, $\$ 1.25$.

One will be surprised at the need at any moment. The sections amount of practical and useful in- devoted to ulcers and abscesses are formation it contains; information indispensable to any physician.that the practitioner is likely to The Charlotte Medical Journal.

CHEYNE (W. W.) AND BURGHARD (F. F.) SURGICAL TREATMENT. In six octavo volumes, illustrated. Volume 1, 299 pages and 66 engravings, just ready. Cloth, $\$ 3.00$ net.

CLARKE (W. B.) AND LOCKWOOD (C. B.). THE DISSECTOR'S MANUAL. In one $12 \mathrm{mo}$. volume of 396 pages, with 49 engravings. Cloth, $\$ 1.50$. See Students' Series of Manuals, page 27.

CLELAND (JOHN). A DIRECTORY FOR THE DISSECTION OF THE HUMAN BODY. In one $12 \mathrm{mo}$. vol. of 178 pages. Cloth, $\$ 1.25$.

CLINICAL MANUALS. See Series of Clinical Manuals, page 25.

CLOUSTON (THOMAS S.). CLINICAL LECTURES ON MENTAL DISEASES. New (5th) edition. In one octavo volume of 750 pages, with 19 colored plates. Cloth, $\$ 4.25$, net. Just ready.

Folson's Abstract of Laws of U.S. on Custody of Insane, octavo, $\$ 1.50$, is sold in conjunction with Clouston on Mental Diseases for $\$ 5.00$, net, for the two works. 
CLOWES (FRANK). AN ELEMENTARY TREATISE ON PRACTICAL CHEMISTRY AND QUALITATIVE INORGANIC ANALYSIS. From the fourth English edition. In one handsome $12 \mathrm{mo}$. volume of 387 pages, with 55 engravings. Cloth, $\$ 2.50$.

COAKLEY (CORNELIUS G.). THE DIAGNOSIS AND TREATMENT OF DISEASES OF THE NOSE, THROAT, NASOPHARYNX AND TRACHEA. In one $12 \mathrm{mo}$. volume of about 400 pages, fully illustrated. Preparing,

COATES (W. E., JR.). A POCKET TEXT-BOOK OF BACTERIOLOGY AND HYGIENE. In one handsome 12mo, volume of about 350 pages, with many illustrations. Shortly. Cloth, $\$ 1.50$, net. Lea's Series of Pocket Text-books, edited by BERN B. GALLAUDET, M. D. See page 18 .

COATS (JOSEPH). A TREATISE ON PATHOLOGY. In one vol. of 829 pages, with 339 engravings. Cloth, $\$ 5.50$; leather, $\$ 6.50$.

COLEMAN (ALFRED). A MANUAL OF DENTAL SURGERY AND PATHOLOGY. With Notes and Additions to adapt it to American Practice. By Thos. C. StellwageN, M.A., M.D., D.D.S. In one handsome octavo vol. of 412 pages, with 331 engravings. Cloth, $\$ 3.25$.

COLLINS (C. P.). A POCKET TEXT-BOOK OF MEDICAL DIAGNOSIS. In one handsome $12 \mathrm{mo}$. volume of about 350 pages, with many illustrations. Shortly. Cloth, $\$ 1.50$, net. Leu's Series of Pocket Text-books, edited by Bern B. Gallaudet, M. D. See page 18.

COLLINS (H. D.) AND ROCKWELL (W. H.). A POCKET TEXT-BOOK OF PHYSIOLOGY. In one handsome 12mo. volume of about 300 pages, with many illustrations. Clcth, $\$ 1.50$, net. In press. Lea's Series of Pocket Text-books, edited by BERN B. GallaUdET, M. D. See page 18.

CONDIE (D. FRANCIS). A PRACTICAL TREATISE ON THE DISEASES OF CHILDREN. Sixth edition, revised and enlarged. In one large 8 vo. volume of 719 pages. Cloth, $\$ 5.25$; leather, $\$ 6.25$.

CORNIU (V.). SYPHILIS: ITS MORBID ANATOMY, DIAGNOSIS AND TREATMENT. Translated, with Notes and Additions, by J. Henry C. Simes, M.D. and J. William White, M.D. In one 8 vo. volume of 461 pages, with 84 illustrations. Cloth, $\$ 3.75$.

CROCKETT (M. A.). A POCKET TEXT-BOOK OF DISEASES OF WOMEN. In one handsome $12 \mathrm{mo}$. volume of about 350 pages, with many illustrations. Cloth,\$1.50, net. Shortly. Lea's Series of Pocket Text-books, edited by BERN B. Gallaudet, M. D. See page 18.

CROOK (JAMES K.) ON MINERAL WATERS OF THE UNITED STATES. Octavo, 575 pages. Just ready. Cloth, $\$ 3.50$, net.

CULBRETH (DAVU M. R.). MATERIA MEDICA AND PHARMACOLOGY. In one handsome octavo volume of 812 pages, with 445 illustrations. Cloth, $\$ 4.75$.

A thorough, authoritative and/adopted as the text-book in all colsystematic exposition of its most leges of pharmacy and medicine. important domain. - The Canada It is one of the most valuable works Lancet.

This work ought to be at once Medical Journal.

CUSHNY (ARTHUR R.). TEXT-BOOK OF PHARMACOLOGY. Handsome 8 vo., 728 pages, with 47 illus. Just ready. Cloth, $\$ 3.75$, net. 
DALTON (JOHN C.). A TREATISE ON HUMAN PHYSIOLOGY. Serenth edition. Octavo, 722 pages, with 252 engravings. Cloth, $\$ 5$; leather, $\$ 6$.

DOCTRINES OF THE CIRCULATION OF THE BLOOD. In one handsome $12 \mathrm{mo}$. volume of 293 pages. Cloth, $\$ 2$.

DAVENPORT (F. H.). DISEASES OF WOMEN. A Manual of Gynecology. For the use of Students and Practitioners. New (3d) edition. In one handsome $12 \mathrm{mo}$. volume of 387 pages, with 150 illustrations. Cloth, $\$ 1.75$, net. Just ready.

DAVIS (EDWARD P.). A TREATISE ON OBSTETRICS. FOR STUDENTS AND PRACTITIONERS. In one very handsome octavo volume of 546 pages, with 217 engravings and 30 full-page plates in colors and monochrome. Cloth, $\$ 5$; leather, $\$ 6$.

This work must become the prac-, books on the subject. It is exceptiontitioner's text-book as well as the ally useful from every standpoint.student's. It is up to date in every Nashville Jour. of Med. and Surgery. respect.-Va.Med. Semi-Monthly. $\quad$ From a practical standpoint the

A work unequalled in excellence. work is all that could be desired. A -The Chicago Clinical Review. thoroughly scientific and brilliant

Decidedly one of the best text-, treatise on obstetrics.-Med. Neros.

DAVIS (F. H.). LECTURES ON CLINICAL MEDICINE. Second edition. In one $12 \mathrm{mo}$. volume of 287 pages. Cloth, $\$ 1.75$.

DE IA BECHE'S GEOLOGICAL OBSERVER. In one large octavo volume of 700 pages, with 300 engravings. Cloth, $\$ 4$.

DENNIS (FREDERIC S.) AND BLLINGS (JOHN S.). A SYSTEM OF SURGERY. In contributions by American Authors. Complete work in four very handsome octavo volumes, containing 3652 pages, with 1585 engravings and 45 full-page plates in colors and monochrome. Per volume, cloth, $\$ 6.00$; leather, $\$ 7.00$; half Morocco, gilt back and top, \$8.50. For sale by subscription only. Full prospectus free on application to the publishers.

It is worthy of the position which American surgery and is thoroughly surgery has attained in the great practical.-Annals of Surgery.

Republic whence it comes. - The No work in English can be conLondon Lancet.

It may be fairly said to represent American Journal of the Medical the most advanced condition of Sciences.

DERCUM (FRANCIS $x_{\text {, }}$ EDITOR). A TEXT-BOOK ON NERVOUS DISEASES. By American Authors. In one handsome octavo volume of 1054 pages, with 341 engravings and 7 colored plates. Cloth, $\$ 6.00$; leather, $\$ 7.00$. Net.

Representing the actual status of our knowledge of its subjects, and the latest and most fully up-to-date of any of its class.-Jour. of American Med. Association.

The most thoroughly up-to-date treatise that we have on this subject. - American Journal of Insanity.

DE SCHWEINITZ (GEORGE E.). THE TOXIC AMBLYOPIAS. Their Classification, History, Symptoms, Pathology and Treatment. Very handsome octavo, 240 pages, 46 engravings, and 9 full-page plates in colors. Limited edition, de luxe binding, \$4. Net. 
DRAPER (JOHN C.). MEDICAL PHYSICS. A Text-book for Students and Practitioners of Medicine. In one handsome octavo volume of 734 pages, with 376 engravings. Cloth, $\$ 4$.

DRUTTT (ROBERT). THE PRINCIPLES AND PRACTICE OF MODERN SURGERY. A new American, from the twelfth London edition, edited by STANLEY BOYD, F.R.C.S. In one large octavo volume of 965 pages, with 373 engravings. Cloth, $\$ 4$; leather, $\$ 5$.

DUANE (ALEXANDER). THE STUDENT'S DICTIONARY OF MEDICINE AND THE ALLIED SCIENCES. New edition. Comprising the Pronunciation, Derivation and Full Explanation of Medical Terms, with much Collateral Descriptive Matter. Numerous Tables, etc. Square octavo of 658 pages. Cloth, $\$ 3.00$; half leather, $\$ 3.25$; full sheep, $\$ 3.75$. Thumb-letter Index, 50 cents extra.

Far superior to any dictionary for convenience and thoroughness. the medical student that we know of. Medical Record.

-Western Med. and Surg. Reporter. The best student's dictionary.-

The book is brought accurately to Canada Lancet.

date. It is a model of conciseness,

DUDLEY (E. C.). THE PRINCIPLES AND PRACTICE OF GYNECOLOGY. Handsome octavo of 652 pages, with 422 illustrations in black and colors. Cloth, $\$ 5.00$, net; leather, $\$ 6.00$, net. Just ready.

The book can be safely recom-| tice of modern gynecology.-Intermended as a complete and reliable national Medical Magazine. exposition of the principles and prac-

DUNCAN (J. MATTHEWS). CLINICAL LECTURES ON THE DISEASES OF WOMEN. Delivered in St. Bartholomew's Hospital. In one octavo volume of 175 pages. Cloth, $\$ 1.50$.

DUNGLISON (ROBLEY). A DICTIONARY OF MEDICAL SCIENCE. Containing a full explanation of the various subjects and terms of Anatomy, Physiology, Medical Chemistry, Pharmacy, Pharmacology, Therapeutics, Medicine, Hygiene, Dietetics, Pathology, Surgery, Ophthalmology, Otology, Laryngology, Dermatology, Gynecology, Obstetrics, Pediatrics, Medical Jurisprudence, Dentistry, etc., etc. By RoBley Dunglison, M. D., LL. D., late Professor of Institutes of Medicine in the Jefferson Medical College of Philadelphia. Edited by RICHARD J. DUNGlison, A. M., M. D. Twenty-first edition, thoroughly revised and greatly enlarged and improved, with the Pronunciation, Accentuation and Derivation of the Terms. With Appendix. In one magnificent imperial octavo volume of 1225 pages. Cloth, $\$ 7$; leather, $\$ 8$. Thumb-letter Index for quick use, 75 cents extra.

The most satisfactory and authori- scarcely be measured.-Med. Record. tative guide to the derivation, definition and pronunciation of medical terms.-The Charlotte Med. Journal.

Covering the entire field of medieine, surgery and the collateral sciences, its range of usefulness can

DUNHAM (EDWARD K.). MORBID AND NORMAL HISTOLOGY. Octavo, 450 pages, with 363 illustrations. Cloth, $\$ 3.25$, net. Just ready.

The best one-volume text or refer- $\mid$ of published in America.-Virginia ence book on histology that we know Medical Semi-Monthly.

EDES (ROBERT T.). TEXT-BOOK OF THERAPEUTICS AND MATERIA MEDICA. In one 8 vo. volume of 544 pages. Cloth, $\$ 3.50$; leather, $\$ 4.50$.

EDIS (ARTHUR W.). DISEASES OF WOMEN. A Manual for Students and Practitioners. In one handsome 8vo. volume of 576 pages, with 148 engravings. Cloth, $\$ 3$; leather, $\$ 4$. 
EGBERT (SENECA). A MANUAL OF HYGIENE AND SANITATION. In one $12 \mathrm{mo}$. volume of 359 pages, with 63 illustrations. Just ready. Cloth, Net, $\$ 2.25$.

It is written in plain language, ligence. The writer has adapted it and, while primarily designed for to American conditions, and his physicians, it can be studied with suggestions are, above all, practical. profit by any one of ordinary intel-1 - The New York Medical Journal.

ELLIS (GEORGE VINER). DEMONSTRATIONS IN ANATOMY. Eighth edition. Octavo, 716 pages, with 249 engravings. Cloth, $\$ 4.25$; leather, $\$ 5.25$.

EMMET (THOMAS ADDIS). THE PRINCIPLES AND PRACTICE OF GYN ACCLOGY. Third edition. Octavo, 880 pages, with 150 original engravings. Cloth, $\$ 5$; leather, $\$ 6$.

ERICHSEN (JOHN E.). THE SCIENCE AND ART OF SURGERY. Eighth edition. In two large octavo volumes containing 2316 pages, with 984 engravings. Cloth, $\$ 9$; leather, $\$ 11$.

ESSIG (CHARLES J.). PROSTHETIC DENTISTRY. See American Text-Books of Dentistry, page 2.

EVANS (DAVID J.). A POCKET TEXT-BOOK OF OBSTETRICS. In one handsome $12 \mathrm{mo}$. volume of about 300 pages, with many illustrations. Cloth, $\$ 1.50$, net. Shortly. Lea's Series of Pocket Text-books, edited by Bern B. Gallaudet, M. D. See page 18.

FARQUHARSON (ROBERT). A GUIDE TO THERAPEUTICS. Fourth American from fourth English edition, revised by FRANK WOODBURY, M.D. In one $12 \mathrm{mo}$. volume of 581 pages. Cloth, $\$ 2.50$.

FIELD (GEORGE P.). A MANUAL OF DISEASES OF THE EAR. Fourth edition. In one octavo volume of 391 pages, with 73 engravings and 21 colored plates. Cloth, $\$ 3.75$.

To those who desire a concise It is just such a work as is needed work on diseases of the ear, clear by every general practitioner. and practical, this manual com-American Practitioner and News. mends itself in the highest degree.

FLINT (AUSTIN). A TREATISE ON THE PRINCIPLES AND PRACTICE OF MEDICINE. Seventh edition, thoroughly revised by Frederick P. HENRY, M.D. In one large 8vo. volume of 1143 pages, with engravings. Cloth, $\$ 5.00$; leather, $\$ 6.00$.

The work has well earned its lead- medicine in the medical schools.ing place in medical literature.- Northwestern Lancet.

Medical Record.

The leading text-book on general

The best of American text-books on Practice.-Amer.Medico-Surgical Bulletin.

A MANUAL OF AUSCULTATION AND PERCUSSION; of the Physical Diagnosis of Diseases of the Lungs and Heart, and of Thoracic Aneurism. Fifth edition, revised by JAMES C. WILSON, M. D. In one handsome $12 \mathrm{mo}$. volume of 274 pages, with 12 engravings.

A PRACTICAL TREATISE ON THE DIAGNOSIS AND TREATMENT OF DISEASES OF THE HEART. Second edition enlarged. In one octavo volume of 550 pages. Cloth, $\$ 4$.

A PRACTICAL TREATISE ON THE PHYSICAL EXPLORATION OF THE CHEST, AND THE DIAGNOSIS OF DISEASES AFFECTING THE RESPIRATORY ORGANS. Second and revised edition. In one octavo volume of 591 pages. Cloth, $\$ 4.50$. MEDICAL ESSAYS. In one $12 \mathrm{mo}$. vol. of 210 pages. Cloth, $\$ 1.38$. - ON PHTHISIS : ITS MORBID ANATOMY, ETIOLOGY, ETC. A Series of Clinical Lectures. In one 8vo. volume of 442 pages. Cloth, $\$ 3.50$. 
FOLSOM (C. F.). AN ABSTRACT OF STATUTES OF U. S. ON CUSTODY OF THE INSANE. In one $8 \mathrm{vo}$. vol. of 108 pages. Cloth, $\$ 1.50$. With Clouston on Mental Diseases (new edition, see page 6) $\$ 5.00$, net, for the two works.

FORMULARY, POCKET, see page 32 .

FOSTER (MICHAEL). A TEXT-BOOK OF PHYSIOLOGY. New (6th) and revised American from the sixth English edition. In one large octavo volume of 923 pages, with 257 illustrations. Cloth, $\$ 4.50$; leather, $\$ 5.50$.

Unquestionably the best book that can be placed in the student's hands, and as a work of reference for the busy physician it can scarcely be excelled.-The Phila. Polyclinic.

This single volume contains all that will be necessary in a college course, and all that the physician will need as well.-Dominion Med. Monthly.

FOTHERGUL (J. MUNER). THE PRACTITIONER'S HANDBOOK OF TREATMENT. Third edition. In one handsome octavo volume of 664 pages. Cloth, $\$ 3.75$; leather, $\$ 4.75$.

To have a description of the clearly stated, cannot fail to prove normal physiological processes of an a great convenience to many thoughtorgan and of the methods of treat- ful but busy physicians. The pracment of its morbid conditions tical value of the volume is greatly brought together in a single chapter, increascd by the introduction of many and the relations between the two prescriptions--New York Med. Jour.

FOWNES (GEORGE). A MANUAL OF ELEMENTARY CHEMISTRY (INORGANIC AND ORGANIC). Twelfth edition. Embodying WATTS' Physical and Inorganic Chemistry. In one royal 12mo. volume of 1061 pages, with 168 engravings, and 1 colored plate. Cloth, $\$ 2.75$; leather, $\$ 3.25$.

FRANKLAND (E.) AND JAPP (F.R.). INORGANIC CHEMISTRY. In one handsome octavo volume of 677 pages, with 51 engravings and 2 plates. Cloth, $\$ 3.75$; leather, $\$ 4.75$.

FULLER (EUGENE). DISORDERS OF THE SEXUAL ORGANS IN THE MALE. In one very handsome octavo volume of 238 pages, with 25 engravings and 8 full-page plates. Cloth, $\$ 2$.

It is an interesting work, and one tive and brings views of sound which, in view of the large and pathology and rational treatment to profitable amount of work done in many cases of sexual disturbance this field of late years, is timely and whose treatment has been too often well needed.-Medical Fortnightly. fruitless for good. - Annals of

The book is valuable and instruc- Surgery.

FULLER (HENRY). ON DISEASES OF THE LUNGS AND AIR PASSAGES. Their Pathology, Physical Diagnosis, Symptoms and Treatment. From second English edition. In one 8vo. volume of 475 pages. Cloth, $\$ 3.50$.

GALLAUDET (BERN B.). A POCKET TEXT-BOOK ON SURGERY. In one handsome $12 \mathrm{mo}$. volume of about 400 pages, with many illustrations. Cloth, $\$ 1.50$, net. Shortly. Lea's Series of Pocket Textbooks, edited by Bern B. Gallaudet, M. D. See page 18.

GANT (FREDERICK JAMES). THE STUDENT'S SURGERY. A Multum in Parvo. In one square octavo volume of 845 pages, with 159 engravings. Cloth, $\$ 3.75$.

GIBBES (HENEAGE). PRACTICAL PATHOLOGY AND MORBID HISTOLOGY. Octavo, 314 pages, with 60 illustrations. Cloth, $\$ 2.75$.

GIBNEY (V. P.). ORTHOPEDIC SURGERY. For the use of Practitioners and Students. In one 8 vo. vol. profusely illus. Preparing. 
GERRISH (FREDERIC H.). A TEXT-BOOK OF ANATOMY. By American Authors. Edited by Frederic H. Gerrish, M. D. In one imp. octavo volume of 915 pages, with 950 illustrations in black and colors. Just ready. Clth, $\$ 6.50$; flexible waterproof, $\$ 7$; leath., $\$ 7.50$, net.

In this, the first representative treatise on Anatomy produced in America, no effort or expense has been spared to unite an authoritative text with the most successful anatomical pictures which have yet appeared in the world.

The editor has secured the co-operation of the professors of anatomy in leading medical colleges, and with them has prepared a text conspicuous for its simplicity, unity and judicious selection of such anatomical facts as bear on physiology, surgery and internal medicine in the most comprehensive sense of those terms. The authors have endeavored to make a book which shall stand in the place of a living teacher to the student, and which shall be of actual service to the practitioner in his clinical work, emphasizing the most important subjects, clarifying obscurities, helping most in the parts most difficult to learn, and illustrating everything by all available methods.

GOULD (A. PEARCE). SURGICAL DIAGNOSIS. In one $12 \mathrm{mo}$. vol. of 589 pages. Cloth, $\$ 2$. See Student's Series of Manuals, p. 27.

GRAY (HENRY). ANATOMY, DESCRIPTIVE AND SURGICAL. New and thoroughly revised American edition, much enlarged in text, and in engravings in black and colors. In one imperial octavo volume of 1239 pages, with 772 large and elaborate engravings on wood. Price of edition with illustrations in colors: cloth, $\$ 7$; leather, $\$ 8$. Price of edition with illustrations in black: cloth, $\$ 6$; leather, $\$ 7$.

This is the best single volume/ing, and especially the Surgical upon Anatomy in the English Anatomy.-Chicago Med. Recorder. language.-University Medical Mag- Holds first place in the esteem of azine.

Gray's Anatomy affords the student more satisfaction than any other treatise with which we are familiar. -Buffalo Med. Journal.

The most largely used anatomical text-book published in the English language.-Annals of Surgery.

Particular stress is laid upon the practical side of anatomical teachboth teachers and students. - The Brooklyn Medical Journal.

The foremost of all medical textbooks.-Medical Fortnightly.

Gray's Anatomy should be the first work which a medical student should purchase, nor should he be without a copy throughout his professional career.-Pittsburg Medical Review.

GRAY (LANDON CARTER). A TREATISE ON NERVOUS AND MENTAL DISEASES. For Students and Practitioners of Medicine. New (2d) edition. In one handsome octavo volume of 728 pages, with 172 engravings and 3 colored plates. Cloth, $\$ 4.75$; leather, $\$ 5.75$.

An up-to-date text-book upon measures which are often the physinervous and mental diseases com- cian's best reliance."-The Journal bined. A well-written, terse, ex- of the American Medical Association. plicit, and authoritative volume The descriptions of the various treating of both subjects is a step in diseases are accurate and the sympthe direction of popular demand.The Chicago Clinical Review.

"The word treatment," says the author, "has been construed in the broadest sense to include not only medicinal and non-medicinal agents, but also those hygienic and dietetic toms and differential diagnosis are set before the student in such a way as to be readily comprehended. The author's long experience renders his views on therapeutics of great value. - The Journal of Nervous and Mental Disease. 
GREEN (T. HENRY). AN INTRODUCTION TO PATHOLOGY AND MORBID ANATOMY. New (8th) American from the eighth London edition. In one handsome octavo volume of 582 pages, with 216 engravings and a colored plate. Cloth, $\$ 2.50$, net. Just ready.

A work that is the text-book of $/$ of the day-as much so almost as probably four-fifths of all the stu- Gray's Anatomy. It is fully up-todents of pathology in the United date in the record of fact, and so proStates and Great Britain stands in fusely illustrated as to give to each no need of commendation. The work detail of text sufficient explanation. precisely meets the needs and wishes The work is an essential to the pracof the general practitioner.-The titioner-whether as surgeon orphysAmerican Practitioner and News. ician. It is the best of up-to date Green's Pathology is the text-book text-books.-VirginiaMed.Monthly.

GREENE (WILLIAM H.). A MANUAL OF MEDICAL CHEMISTRY. For the Use of Students. Based upon BowMaN's Medical Chemistry. In one $12 \mathrm{mo}$. vol. of 310 pages, with 74 illus. Cloth, $\$ 1.75$.

GROSS (SAMUEL D.). A PRACTICAL TREATISE ON THE DISEASES, INJURIES AND MALFORMATIONS OF THE URINARY BLADDER, THE PROSTATE GLAND AND THE URETHRA. Third edition. Octavo, 574 pages, with 170 illustrations Cloth, $\$ 4.50$.

GRINDON (JOSEPH). A POCKET TEXT-BOOK OF SKIN DISEASES. In one handsome $12 \mathrm{mo}$. volume of 350 pages, with many illustrations. Shortly. Cloth, $\$ 1.50$, net. Lea's Series of Pocket Text-books, edited by Bern B. Gallaudet, M. D. See page 18.

HABERSHON (S. O.). ON THE DISEASES OF THE ABDOMEN Second American from the third English edition. In one octavo volume of 554 pages, with 11 engravings. Cloth, $\$ 3.50$.

HALL (WINFIELD S.) TEXT-BOOK OF PHYSIOLOGY. Octavo about 500 pages, richly illustrated. In press.

HAMUTON (ALLAN MCLANE). NERVOUS DISEASES, THEIR DESCRIPTION AND TREATMENT. Second and revised edition. In one octavo volume of 598 pages, with 72 engravings. Cloth, $\$ 4$.

HARDAWAY (W. A.). MANUAL OF SKIN DISEASES. New (2d) edition. In one $12 \mathrm{mo}$. volume of 560 pages, with 40 illustrations and 2 plates. Cloth, $\$ 2.25$, net. Just ready.

The best of all the small books to / day clinical experience. His great recommend to students and practi- strength is in diagnosis, descriptions tioners. Probably no one of our of lesions and especially in treatdermatologists has had a wider every- ment.-Indiana Medical Journal.

HARE (HOBART AMORY). PRACTICAL DIAGNOSIS. THE USE OF SYMPTOMS IN THE DIAGNOSIS OF DISEASE. New (4th) edition. In one octavo volume of 623 pages, with 205 engravings and 14 full-page colored plates. Cloth, $\$ 5.00$, net. Just ready.

It is unique in many respects, and he will become a better diagnostithe author has introduced radical cian. This is a companion to Pracchanges which will be welcomed by tical Therapeutics, by the same all. Anyone who reads this book author, and it is difficult to conceive will become a more acute observer, of any two works of greater practical will pay more attention to the simple utility.-Medical Review. yet indicative signs of disease, and 
HARE (HOBART AMORY). A TEXT-BOOK OF PRACTICAL THERAPEUTICS, with Special Reference to the Application of Remedial Measures to Disease and their Employment upon a Rational Basis. With articles on various subjects by well-known specialists. New (7th) and revised edition. In one octavo volume of 776 pages. Cloth, $\$ 3.75$, net; leather, $\$ 4.50$, net.

Its classifications are inimitable, it can be readily used in connection and the readiness with which any- with Hare's Practical Diagnosis. thing can be found is the most won- For the needs of the student and derful achievement of the art of in- general practitioner it has no equal. dexing. This edition takes in all -Medical Sentinel.

the latest discovered remedies.- The best planned therapeutic work The St. Louis Clinique.

The great value of the work lies in the fact that precise indications for administration are given. A complete index of diseases and remedies makes it an easy reference work. It has been arranged so that ical Review.

HARE (HOBART AMORY) ON THE MEDICAL COMPLICA TIONS AND SEQUELI OF TYPHOID FEVER. Octavo, 276 pages, 21 engravings and two full-page plates. Just ready. Cloth, $\$ 2.40$, net.

A very valuable production. One read with great profit.-Cleveland of the very best products of Dr. Journal of Medicine.

Hare and one that every man can

HARE (HOBART AMORY, EDITOR). A SYSTEM OF PRACTICAL THERAPEUTICS. In a series of contributions by eminent practitioners. In four large octavo volumes comprising about 4500 pages, with about 550 engravings. Vol. IV., just ready. For sale by subscription only. Full prospectus free on application to the Publishers. Regular price, Vol. IV., cloth, $\$ 6$; leather, $\$ 7$; half Russia, $\$ 8$. Price Vol. IV. to former or new subscribers to complete work, cloth, $\$ 5$; leather, $\$ 6$; half Russia, $\$ 7$. Complete work, cloth, $\$ 20$; leather, $\$ 21$; half Russia, $\$ 28$.

The great value of Hare's System of Practical Therapeutics has led to a widespread demand for a new volume to represent advances in treatment made since the publication of the first three. More than fulfilling this request the Editor has secured contributions from practically a new corps of equally eminent authors, so that entirely fresh and original matter is ensured. The plan of the work, which proved so successful, has been followed in this new volume, which will be found to present the latest developments and applications of this most practical branch of the medical art. The entire System is an unrivalled encyclopædia on the practical parts of medicine, and merits the great success it has won for that reason. 
HARTSHORNE (HENRY). ESSENTIALS OF THE PRINCIPLES AND PRACTICE OF MEDICINE. Fifth edition. In one $12 \mathrm{mo}$. volume, 669 pages, with 144 engravings. Cloth, $\$ 2.75$.

A HANDBOOK OF ANATOMY AND PHYSIOLOGY. In one $12 \mathrm{mo}$. volume of 310 pages, with 220 engravings. Cloth, $\$ 1.75$.

A CONSPECTUS OF THE MEDICAL SCIENCES. Comprising Manuals of Anatomy, Physiology, Chemistry, Materia Medica, Practice of Medicine, Surgery and Obstetrics. Second edition. In one royal $12 \mathrm{mo}$. vol. of 1028 pages, with 477 illus. Cloth, $\$ 4.25$; leather, $\$ 5$.

HA YDEN (JAMES R.). A MANUAL OF VENEREAL DISEASES. New (2d) edition. In one $12 \mathrm{mo}$. volume of 304 pages, with 54 engravings. Cloth, $\$ 1.50$, net. Just ready.

It is practical, concise, definite ticularly thorough, and may be and of sufficient fulness to be satis- relied upon as a guide in the manfactory.-Chicago Clinical Review. agement of this class of diseases.-

This work gives all of the prac- Northwestern Lancet. tically essential information about It is well written, up to date, and the three venereal diseases, gon- will be fonnd very useful.-Interorrhœa, the chancroid and syphilis. national Medical Magazine.

In diagnosis and treatment it is par-

HAYEM (GEORGES) AND HARE (H. A.). PHYSICAL AND NATURAL THERAPEUTICS. The Remedial Use of Heat, Electricity, Modifications of Atmospheric Pressure, Climates and Mineral Waters. Edited by Prof. H. A. HARE, M. D. In one octavo volume of 414 pages, with 113 engravings. Cloth, $\$ 3$.

This well-timed up-to-date volume recognition. Within this large is particularly adapted to the re- range of applicability, physical quirements of the general practi- agencies when compared with drugs tioner. The section on mineral are more direct and simple in their waters is most scientific and prac- results. Medical literature has long tical. Some 200 pages are given up been rich in treatises upon medical to electricity and evidently embody agents, but an authoritative work the latest scientific information on upon the other great branch of the subject. Altogether this work therapeutics has until now been a is the clearest and most practical aid desideratum. The section on climate, to the study of nature's therapeutics rewritten by Prof. Hare, will, for that has yet come under our obser- the first time, place the abundant vation.-The Medical Fortnightly. resources of our country at the in-

For many diseases the most potent telligent command of American remedies lie outside of the materia practitioners. - The Kansas City medica, a fact yearly receiving wider Medical Index.

HERMAN (G. ERNEST). FIRST LINES IN MIDWIFERY. In one $12 \mathrm{mo}$. vol. of 198 pages, with 80 engravings. Cloth, $\$ 1.25$. See Student's Series of Manuals, page 27.

HERMANN (L.). EXPERIMENTAL PHARMACOLOGY. A Handbook of the Methods for Determining the Physiological Actions of Drugs. Translated by Robert Meade Smith, M.D. In one 12mo, volume of 199 pages, with 32 engravings, Cloth, $\$ 1,50$. 
HERRICK (JAMES B.). A HANDBOOK OF DIAGNOSIS. In one handsome $12 \mathrm{mo}$. volume of 429 pages, with 80 engravings and 2 colored plates. Cloth, $\$ 2.50$.

Excellently arranged, practical, microscopical examination to be emconcise, up-to-date, and eminently ployed in each class. The technique well fitted for the use of the prac- of blood examination,including color titioner as well as of the student.- analysis, is very clearly stated. Chicago Med. Recorder.

This volume accomplishes its objects more thoroughly and completely than any similar work yet published. Each section devoted to diseases of special systems is preceded with an exposition of the methods of physical, chemical and Uranalysis receives adequate space and care.-New York Med. Journal.

We commend the book not only to the undergraduate, but also to the physician who desires a ready means of refreshing his knowledge of diagnosis in the exigencies of professional life.-Memphis Medival Monthly.

HILL (BERKELEY). SYPHILIS AND LOCAL CONTAGIOUS DISORDERS. In one 8 vo. volume of 479 pages. Cloth, $\$ 3.25$.

HILLEER (THOMAS). A HANDBOOK OF SKIN DISEASES. Second edition. In one royal $12 \mathrm{mo}$. volume of 353 pages, with two plates. Cloth, $\$ 2.25$.

HIRST (BARTON C.) AND PIERSOL (GEORGE A.). HUMAN MONSTROSITIES. Magnificent folio, containing 220 pages of text and illustrated with 123 engravings and 39 large photographic plates from nature. In four parts, price each, $\$ 5$. Limited edition. For sale by subscription only.

HOBLYN (RICHARD D.). A DICTIONARY OF THE TERMS USED IN MEDICINE AND THE COLLATERAL SCIENCES. In one $12 \mathrm{mo}$. volume of 520 double-columned pages. Cloth, $\$ 1.50$; leather, $\$ 2$.

HODGE (HUGH L.). ON DISEASES PECULIAR TO WOMEN, INCLUDING DISPLACEMENTS OF THE UTERUS. Second and revised edition. In one 8 ro. vol. of 519 pp., with illus. Cloth, $\$ 4.50$.

HOFFMANN (FREDERICK) AND POWER (FREDERICK B.). A MANUAL OF CHEMICAL ANALYSIS, as Applied to the Examination of Medicinal Chemicals and their Preparations. Third edition, entirely rewritten and much enlarged. In one handsome octavo volume of 621 pages, with 179 engravings. Cloth, $\$ 4.25$.

HOLMES (TIMOTHY). A TREATISE ON SURGERY. Its Principles and Practice. A new American from the fifth English edition. Edited by T. Pick Ering PICK, F.R.C.S. In one handsome octavo volume of 1008 .pages, with 428 engravings. Cloth, $\$ 6$; leather, $\$ 7$.

A SYSTEM OF SURGERY. With notes and additions by various American authors. Edited by JoHN H. PACKARD, M. D. In three very handsome $8 \mathrm{vo}$. volumes containing 3137 double-columined pages, with 979 engravings and 13 lithographic plates. Per volume, cloth, \$6; lesther, $\$ 7$; half Russia, $\$ 7.50$, For sale by mbecription only. 
HORNER (WULLAM E.). SPECIAL ANATOMY AND HISTOLOGY. Eighth edition, revised and modified. In two large 8vo. volumes of 1007 pages, containing 320 engravings. Cloth, $\$ 6$.

HUDSON (A.). LECTURES ON THE STUDY OF FEVER. In one octavo volume of 308 pages. Cloth, $\$ 2.50$.

HUTCHISON (ROBERT) AND RAINY (HARRY). CLINICAL METHODS. A GUIDE TO THE PRACTICAL STUDY OF MEDICINE. In one 12mo. volume of 562 pages, with 137 engravings and 8 colored plates. Cloth, $\$ 3.00$.

A comprehensive, clear and re- medical knowledge which receive markably up-to-date guide to clinical recognition, we mention Widal's diagnosis. The illustrations are test for typhoid and the Neuron plentiful and excellent. As exam- theory of the nervous system.ples of the more recent additions to Montreal Medical Journal.

HUTCHINSON (JONATHAN). SYPHILIS. In one pocket-size 12mo. volume of 542 pages, with 8 chromo-lithographic plates. Cloth, $\$ 2.25$. See Series of Clinical Manuals, p. 25.

HYDE (JAMES NEVINS). A PRACTICAL TREATISE ON DISEASES OF THE SKIN. New (4th) edition, thoroughly revised. In one octavo volume of 815 pages, with 110 engravings and 12 fullpage plates, 4 of which are colored. Cloth, $\$ 5.25$; leather, $\$ 6.25$.

This edition has been carefully re- as practical.-The American Jourvised, and every real advance has nal of the Medical Sciences.

been recognized. The work answers It is the best one-volume work the needs of the general practitioner, that we know. The student who the specialist, and the student. - The gets this book will find it a useful Ohio Med. Jour.

A treatise of exceptional merit characterized by conscientious care and scientific accuracy. - Buffalo Med. Journal.

A complete exposition of our knowledge of cutaneous medicine as it exists to-day. The teaching inculcated throughout is sound as well investment, as it will well serve him when he goes into practice.-Virginia Medical Semi-Monthly.

A full and thoroughly modern text-book on dermatology. - The Pittsburg Medical Review.

It is the most practical handbook on dermatology with which we are acquainted.- The Chicago Medical Recorder.

JACKSON (GEORGE THOMAS). THE READY-REFERENCE HANDBOOK OF. DISEASES OF THE SKIN. New (3d) edition. In one 12mo. volume of 637 pages, with 75 illustrations and a colored plate. Just ready. Cloth, $\$ 2.50$, net.

As a student's manual, it may be considered beyond criticism. The book is singularly full.-St. Louis Medical and Surgical Journal.

Without doubt forms one of the best guides for the beginner in dermatology that is to be found in the English language.-Medicine.

JAMIESON (W. ALLAN). DISEASES OF THE SKIN. Third edition. In one octavo volume of 656 pages, with 1 engraving and 9 double-page chromo-lithographic plates, Cloth, $\$ 6$, 
JEWETT (CHARLES). ESSENTIALS OF OBSTETRICS. In one $12 \mathrm{mo}$. volume of 356 pages, with 80 engravings and 3 colored plates. Cloth, $\$ 2.25$. Just ready.

An exceedingly useful manual for ing it in attractive and easily tangistudent and practitioner. The au- ble form. The book is well illusthor has succeeded unusually well trated throughout.-Nashville Jour. in condensing the text and in arrang- of Medicine and Surgery.

THE PRACTICE OF OBSTETRICS. By American Authors. One large octavo volume of 763 pages, with 441 engravings in black and colors, and 22 full-page colored plates. Just ready. Cloth, $\$ 5.00$, net; leather, $\$ 6.00$, net.

A clear and practical treatise upon the book abounds. The work is obstetrics by well-known teachers of sure to be popular with medical the subject. A special feature of students, as well as being of extreme this work would seem to be the value to the practitioner. - The excellent illustrations with which Medical Age.

JONES (C. HANDFIELD). CLINICAL OBSERVATIONS ON FUNCTIONAL NERVOUS DISORDERS. Second American edition. In one octavo volume of 340 pages. Cloth, $\$ 3.25$.

JULER (HENRY). A HANDBOOK OF OPHTHALMIC SCIENCE AND PRACTICE. Second edition. In one octavo volume of 549 pages, with 201 engravings, 17 chromo-lithographic plates, test-types of Jaeger anl Snellen, and Holmgren's Color-Blindness Test. Cloth, $\$ 5.50$; lealler, $\$ 6.50$.

The volume is particularly rich in / color blindness, etc. The sections matter of practical value, such as devoted to treatment are singularly directions for diagnosing, use of full and concise.-Medical Age. instruments, testing for glasses, for

KING (A. F. A.). A MANUAL OF OBSTETRICS. Seventh edition. In one $12 \mathrm{mo}$. volume of 573 pages, with 223 illustrations. Cloth, $\$ 2.50$.

From first to finish it is thoroughly cyclopedias. The well-arranged practical, concise in expression, well index renders the book useful to illustrated, and includes a statement the practitioner who is in haste to of nearly every fact of importance refresh his memory. - Virginia discussed in obstetric treatises or Medical Semi-Monthly.

KIRK (EDWARD C.). OPERATIVE DENTISTRY. Handsome octavo of 700 pages, with 751 illustrations. Just ready. See American Text-Books of Dentistry, page 2.

We have only the highest praise tempted. We can heartily recomfor this valuable work. It is replete mend it to the profession.-The in every particular, and surpasses Ohio Dental Journal. anything of the kind heretofore at-

KLEIN (E.). ELEMENTS OF HISTOLOGY. New (5th) edition. In one $12 \mathrm{mo}$. volume of 506 pages, with 296 engravings. Just ready. Cloth, $\$ 2.00$, net. See Student's Series of Manuals, page 27.

It is the most complete and con- This work deservedly occupies a cise work of the kind that has yet first place as a text-book on hisemanated from the press.-The Med- tology.-Canadian Practitioner. ical Age. 
LANDIS (HENRY G.). THE MANAGEMENT OF LABOR. In one handsome $12 \mathrm{mo}$. volume of 329 pages, with 28 illus. Cloth, $\$ 1.75$.

LA ROCHE (R.). YELLOW FEVER. In two 8vo. volumes of 1468 pages. Cloth, $\$ 7$.

LAURENCE (J.Z.) AND MOON (ROBERT C.). A HANDYBOOK OF OPHTHALMIC SURGERY. Second edition. In one octavo volume of 227 pages, with 66 engravings. Cloth, $\$ 2.75$.

LEA'S SERIES OF POCKET TEXT-BOOKS, edited by BERN B. Galladdet, M. D. Covering the entire field of Medicine in a series of 16 very handsome cloth-bound 12mo. volumes of $350-450$ pages each, profusely illustrated. Compendious, clear, trustworthy and modern, and issued at the very moderate price of $\$ 1.50$, net, per volume. The following volumes constitute the series.

Coates' Bacteriology and Hygiene. Brockway's Anatomy. Collins and RoCk well's Physiology. MARTIN and Rockwell's Chemistry and Physies. NiCHOLS and VALE's Histology and Pathology. SchleIF's Materia Medica, Therapeutics, Medical Latin, etc. MalsBARY's Practice of Medicine. Collins' Diagnosis. PoTTS' Nervous and Mental Diseases. Gallaudet's Surgery. LIKES' GenitoUrinary and Venereal Diseases. GRINDON's Dermatology. BALLENGER and WIPPERN's Diseases of the Eye, Ear, Throat and Nose. Evans' Obstetrics. CrocketT's Gynecology. Tutrle's Diseases of Children.

For separate notices see under various authors' names.

LEA (HENRY C.). A HISTORY OF AURICULAR CONFESSION AND INDULGENCES IN THE LATIN CHURCH. In three octavo volumes of about 500 pages each. Per volume, cloth, $\$ 3.00$.

CHAPTERS FROM THE RELIGIOUS HISTORY OF SPAIN; CENSORSHIP OF THE PRESS; MYSTICS AND ILLUMINATITHE ENDEMONIADAS; EL SANTO NIÑO DE LA GUARDIA; BRIANDA DE BARDAXI. 12mo., 522 pages. Cloth, $\$ 2.50$.

FORMULARY OF THE PAPAL PENITENTIARY. In one octavo volume of 221 pages, with frontispiece. Cloth, $\$ 2.50$.

SUPERSTITION AND FORCE; ESSAYS ON THE WAGER OF LAW, THE WAGER OF BATTLE, THE ORDEAL AND TORTURE. Fourth edition, thoroughly revised. In one handsome royal $12 \mathrm{mo}$. volume of 629 pages. Cloth, $\$ 2.75$.

STUDIES IN CHURCH HISTORY. The Rise of the Temporal Power-Benefit of Clergy-Excommunication. New edition. In one handsome $12 \mathrm{mo}$. volume of 605 pages. Cloth, $\$ 2.50$.

AN HISTORICAL SKETCH OF SACERDOTAL CELIBACY IN THE CHRISTIAN CHURCH. Second edition. In one handsome octavo volume of 685 pages. Cloth, $\$ 4.50$.

HEHMANN (C. G.). A MANUAL OF CHEMICAL PHYSIOLOGY. In one 8 vo, volume of 327 pages, with 41 engravings. Cloth, $\$ 2.25$. 
LIKES (SYLVAN H.). A POCKET TEXT-BOOK OF GENITOURINARY AND VENEREAL DISEASES. In one bandsome $12 \mathrm{mo}$. volume of about 350 pages, with many illustrations. Shortly. Cloth, \$1.50, net. Lea's Series of Pocket Text-books, edited by BERN B. Gallaudet, M. D. See page 18.

\section{LOOMIS (ALFRED L.) AND THOMPSON (W. GHLMAN,} EDITORS). A SYSTEM OF PRACTICAL MEDICINE. In Contributions by Various American Authors. In four very handsome octavo volumes of about 900 pages each, fully illustrated in in black and colors. Complete work now ready. Per volume, cloth, $\$ 5$; leather, $\$ 6$; half Morocco, $\$ 7$. For sale by subscription only. Full prospectus free on application to the Publishers. See American System of Practical Medicine, page 2.

LUFF (ARTHUR P.). MANUAL OF CHEMISTRY, for the use of Students of Medicine. In one $12 \mathrm{mo}$. volume of 522 pages, with 36 engravings. Cloth, \$2. See Student's Series of Manuals, page 27.

LYMAN (HENRY M.). THE PRACTICE OF MEDICINE. In one very handsome octavo volume of 925 pages, with 170 engravings. Cloth, $\$ 4.75$; leather, $\$ 5.75$.

Complete, concise, fully abreast of Practical, systematic, complete and the times and needed by all students and practitioners.-Univ. Med.Mag.

An exceedingly valuable text-book. well balanced.-Chicago Med. Recorder. 
MALSBARY (GEORGE E.). A POCKET TEXT-BOOK OF THEORY AND PRACTICE OF MEDICINE. In one handsome $12 \mathrm{mo}$. volume of about 350 pages. Cloth, $\$ 1.50$, net. Shortly. Lea's Series of Pocket Text-books, edited by BenN B. Gallaudet, M. D. See page 18.

MANUAuS. See Student's Quiz Series, page 27, Student's Series of Manuals, page 27, and Series of Clinical Manuals, page 25.

MARSH (HOWARD). DISEASES OF THE JOINTS. In one $12 \mathrm{mo}$. volume of 468 pages, with 64 engravings and a colored plate. Cloth, $\$ 2$. See Series of Clinical Manuals, page 25.

MARTIN (EDWARD). A MANUAL OF SURGICAL DIAGNOSIS. In one $12 \mathrm{mo}$. volume of about 400 pp., fully illustrated. Preparing.

MARTIN (WALTON) AND ROCKWELL (WM. H.). A POCKET TEXT-BOOK OF CHEMISTRY AND PHYSICS. In one handsome $12 \mathrm{mo}$. volume of about 350 pages, with many illustrations. Cloth, $\$ 1.50$, net. Shortly. Lea's Series of Pocket Text-books, edited by Bern B. Gallaudet, M. D. See page 18.

MAY (C. H.). MANUAL OF THE DISEASES OF WOMEN. For the use of Students and Practitioners. Second edition, revised by $\mathbf{L}$. $\mathrm{S}$. RAU, M. D. In one $12 \mathrm{mo}$. volume of 360 pages, with 31 engravings. Cloth, $\$ 1.75$.

MEDICAL NEWS POCKET FORMULARY, see page 32.

MITCHELL (S. WEIR). CLINICAL LESSONS ON NERVOUS DISEASES. In one $12 \mathrm{mo}$. volume of 299 pages, with 19 engravings and 2 colored plates. Cloth, $\$ 2.50$. Of the hundred numbered copies with the Author's signed title page a few remain; these are offered in green cloth, gilt top, at $\$ 3.50$, net.

The book treats of hysteria, recur- / contractions, rotary movements in rentmelancholia, disorders of sleep, the feeble minded, etc. Few can choreic movements, false sensations speak with more authority than the of cold, ataxia, hemiplegic pain, author.-The Journal of the Ameritreatment of sciatica, erythromelal- can Medical Association.

gia, reflex ocularneurosis, hysteric

MTCHELL (JOHN K.). REMOTE CONSEQUENCES OF INJURIES OF NERVES AND THEIR TREATMENT. In one handsome $12 \mathrm{mo}$. volume of 239 pages, with 12 illustrations. Cloth, $\$ 1.75$.

Injuries of the nerves are of fre- access to hospital records for the last quent occurrence in private practice, thirty years, as well as to the and often the cause of intractable government documents, and has and painful conditions, conse- skilfully utilized his opportunities. quently this volume is of especial -The Med. Age.

interest. Doctor Mitchell has had

MORRIS (MALCOLM). DISEASES OF THE SKIN. New (2d) edition. In one $12 \mathrm{mo}$. volume of 601 pages, with 10 chromo-lithographic plates and 26 engravings. Cloth, $\$ 3.25$, net. Just ready.

MÜLLER (J.). PRINCIPLES OF PHYSICS AND METEOROLOGY. In one large 8vo. vol. of 623 pages, with 538 cuts. Cloth, $\$ 4.50$. 
MUSSER (JOHN H.). A PRACTICAL TREATISE ON MEDICAL DIAGNOSIS, for Students and Physicians. New (3d) edition, thoroughly revised. In one octavo volume of about 1000 pages, with about 220 engravings and 48 full-page colored plates. In press.

Notices of previous edition are appended.

We have no work of equal value in English. - University Medical Magazine.

His descriptions of the diagnostic manifestations of diseases are accurate. This work will meet all the requirements of student and physician.-The Medical News.

From its pages may be made the diagnosis of every malady that afflicts the human body, including those which in general are dealt with only by the specialist.-North western Lancet.
It so thoroughly meets the precise demands incident to modern research that it has been adopted as a leading text-book by the medical colleges of this country.-North American Practitioner.

Occupies the foremost place as a thorough, systematic treatise.-Ohio Medical Journal.

The best of its kind, invaluable to the student, general practitioner and teacher.-Montreal MedicalJournal.

NATIONAL DISPENSATORY. See Stillé, Maisch \& Caspari, p. 27.

NATIONAL FORMULARY. See Stillé, Maisch \& Caspari's National Dispensatory, page 27.

\section{NATIONAL MEDIOAT DICTIONARY. See Billings, page 4.}

NETTLESHIP (E.). DISEASES OF THE EYE. New (5th) dmerican from sixth English edition, thoroughly revised. In one $12 \mathrm{mo}$. volume of 521 pages, with 161 engravings, and 2 colored plates, test-types, formulæ and color-blindness test. Cloth, $\$ 2.25$. Just ready.

By far the best student's text-book English language. - Journal of on the subject of ophthalmology and Medicine and Science.

is conveniently and concisely ar- The present edition is the result ranged.-The Clinical Review. of revision both in Englaud and

It has been conceded by ophthal- America, and therefore contains the mologists generally that this work latest and best ophthalmological for compactness, practicality and ideas of both continents.-The Phyclearness has no superior in the sician and Surgeon.

NICHOLS (JOHN B.) AND VALE (F. P.). A POCKET TEXTBOOK OF HISTOLOGY AND PATHOLOGY. In one handsome $12 \mathrm{mo}$. volume of about 350 pages, with many illustrations. In press. Cloth, $\$ 1.50$, net. Lea's Series of Pocket Text-books, edited by BERN B. Gallaudet, M. D. See page 18.

NORRIS (WM. F.) AND OLIVER (CHAS. A.). TEXT-BOOK OF OPHTHALMOLOGY. In one octavo volume of 641 pages, with 357 engravings and 5 colored plates. Cloth, $\$ 5$; leather, $\$ 6$.

A safe and admirable guide, well best, the safest and the most comrrequalified to furuish a working hensive volume upon the subject that knowledge of ophthalmology. - has ever been offered to the AmerJohns Hopkins Hospital Bulletin. ican medical public.-Annals of

It is practical in its teachings. Ophthalmology and Otology.

We unreservedly endorse it as the 
OWEN (EDMUND). SURGICAL DISEASES OF CHILDREN. In one $12 \mathrm{mo}$. volume of 525 pages, with 85 engravings and 4 colored plates. Cloth, $\$ 2$. See Series of Clinical Manuals, page 25.

\section{PARIK (ROSWELL). A TREATISE ON SURGERY BY AMERI-}

CAN AUTHORS. New and condensed edition. In press. In one royal octavo volume of about 1250 pages, with about 1000 engravings and many full-page plates. This work is also published in a larger edition, comprising two volumes. Volume I., General Surgery, 799 pages, with 356 engravings and 21 full-page plates, in colors and monochrome. Volume II., Special Surgery, 800 pages, with 430 engravings and 17 full-page plates, in colors and monochrome. Per volume, cloth, $\$ 4.50$; leather, $\$ 5.50$. Net.

The work is fresh, clear and practical, covering the ground thoroughly yet briefly, and well arranged for rapid reference, so that it will be of special value to the student and busy practitioner. The pathology is broad, clear and scientific, while the suggestions upon treatment are clear-cut, thoroughly modern and admirably resourceful.-Johns Hopkins Hospital Bulletin.

The latest and best work written upon the science and art of surgery. Columbus Medical Journal.

The illustrations are almost entirely new and executed in such a way that they add great force to the text.-The Chicago Medical Recorder.

The various writers have em. bodied the teachings accepted at the present hour.-The North American Practitioner.

Both for the student and practitioner it is most valuable. It is thoroughly practical and yet thoroughly scientific.-Medical News.

A truly modern surgery, not only in pathology, but also in sound surgical therapeuties. - New Orleans Med. and Surgical Journal.

PARK (WILLIAM H.). BACTERIOLOGY IN MEDICINE AND SURGERY. 12mo., about 550 pages, fully illustrated. In press.

PARRY (JOHN S.). EXTRA-UTERINE PREGNANCY, ITS CLINICAL HISTORY, DIAGNOSIS, PROGNOSIS AND TREATMENT. In one octavo volume of 272 pages. Cloth, $\$ 2.50$.

PARVIN (THEOPHILUS). THE SCIENCE AND ART OF OBSTETRICS. Third edition. In one handsome octavo volume of 677 pages, with 267 engravings and 2 colored plates. Cloth, $\$ 4.25$; leather, $\$ 5.25$.

In the foremost rank among the most practical and scientific medical works of the day.-Medical News.

It ranks second to none in the English language.-Annals of Gynecology and Pediatry.

The book is complete in every department, and contains all the necessary detail required by the modern practising obstetrician. - International Medical Magazine.

Parvin's work is practical, concise and comprehensive. We commend it as first of its class in the English language.-Medical Fortnightly.

It is an admirable text-book in every sense of the word.-Nashville Journal of Medicine and Surgery. 
PEPPER'S SYSTEM OF MEDICINE. See page 3.

PEPPER (A. J.). FORENSIC MEDICINE. In press. See Student's Series of Manuals, page 27.

SURGICAL PATHOLOGY. In one 12mo. volume of 511 pages, with 81 engravings. Cloth, \$2. See Student's Series of MIanuals, p. 27 .

PICIK (T. PICKERLNG). FRACTURES AND DISLOCATIONS. In one $12 \mathrm{mo}$. volume of 530 pages, with 93 engravings. Cloth, $\$ 2$. See Series of Clinical Manuals, page 25.

PLAYFAIR (W. S.). A TREATISE ON THE SCIENCE AND PRACTICE OF MIDWIFERY. Seventh American from the ninth English edition. In one octavo volume of 700 pages, with 207 engravings and 7 plates. Cloth, $\$ 3.75$ net; leather, $\$ 4.75$, net. Just ready.

In the numerous editions which, obstetrician. It holds a place among have appeared it has been kept con- the ablest English-speaking authoristantly in the foremost rank. It is ties on the obstetric art.-Buffalo a work which can be conscientiously Medical and Surgical Journal. recommended to the profession.- An epitome of the science and The Albany Medical Annals.

This work must occupy a foremost place in obstetric medicine as a safe guide to both student and practice of midwifery, which embodies all recent advances. - The Medical Fortnightly.

THE SYSTEMATIC TREATMENT OF NERVE PROSTRATION AND HYSTERIA. In one $12 \mathrm{mo}$. volume of 97 pages. Cloth, $\$ 1$.

POCIKET FORMULARY, see page 32 .

POCKET TEXT-BOOKS, see page 18.

POLITZER (ADAM). A TEXT-BOOK OF THE DISEASES OF THE EAR AND ADJACENT ORGANS. Second American from the third German edition. Translated by OSCAR DODD, M. D., and edited by Sir WILliam DALBY, F.R.C.S. In one octavo volume of 748 pages, with 330 original engravings. Cloth, $\$ 5.50$.

The anatomy and physiology of ment are clear and reliable. We each part of the organ of hearing can confidently recommend it, for it are carefully considered, and then contains all that is known upon the follows an enumeration of the dis- subject.-London Lancet.

eases to which that spccial part of A safe and elaborate guide into the auditory apparatus is especially every part of otology.-American liable. The indications for treat- Journal of the Medical Sciences.

POTTS (CHARLES S.). A POCKET TEXT-BOOK OF NERVOUS AND MENTAL DISEASES. In one handsome $12 \mathrm{mo}$. volume of about 450 pages. Cloth, $\$ 1.50$, net. Shortly. Lea's Series of Pocket Text-books, edited by Berx B. GallaUdet, M. D. See page 18.

PROGRESSIVE MEDICINE, see page 32.

PURDY (CHARLES W.). BRIGHT'S DISEASE AND ALLIED AFFECTIONS OF THE KIDNEY. In one octavo volume of 288 pages, with 18 engravings. Cloth, \$2. 
PYE-SMITH (PHULP H.). DISEASES OF THE SKIN. In one $12 \mathrm{mo}$. vol. of 407 pp., with 28 illus., 18 of which are colored. Cloth, $\$ 2$.

QUIZ SERIES. See Student's Quiz Series, page 27.

RALFE (CHARLES H.). CLINICAL CHEMISTRY. In one 12mo. volume of 314 pages, with 16 engravings. Cloth, $\$ 1.50$. See Student's Series of Manuals, page 27.

RAMSBOTHAM (FRANCIS H.). THE PRINCIPLES AND PRACTICE OF OBSTETRIC MEDICINE AND SURGERY. In one imperial octavo volume of 640 pages, with 64 plates and numerous engravings in the text. Strongly bound in leather, $\$ 7$.

REICHERT (EDWARD T.). A TEXT-BOOK ON PHYSIOLOGY. In one handsome octavo volume of about 800 pages, richly illustrated. Preparing.

REMSEN (IRA). THE PRINCIPLES OF THEORETICAL CHEMISTRY. New (5th) edition, thoroughly revised. In one $12 \mathrm{mo}$. volume of 326 pages. Cloth, $\$ 2$.

A clear and concise explanation that the work has met with general of a difficult subject. We cordially favor. This is further established recommend it.-The London Lancet. by the fact that it has been trans-

The book is equally adapted to the lated into German and Italian. The student of chemistry or the practi- treatise is especially adapted to the tioner who desires to broaden his laboratory student. It ranks unusutheoretical knowledge of chemistry. ally bigh among the works of this - New Orleans Med. and Surg. Jour. class. This edition has been brought

The appearance of a fifth edition fully up to the times.-American of this treatise is in itself a guarantee Mredico-Surgical Bulletin.

RICHARDSON (BENJAMIN WARD). PREVENTIVE MEDICINE. In one octavo volume of 729 pages. Cloth, $\$ 4$; leather, $\$ 5$.

ROBERTS (JOHN B.). THE PRINCIPLES AND PRACTICE OF MODERN SURGERY. New (2d) edition. In one octavo volume of about 800 pages, with about 500 engravings. Shortly.

- THE COMPEND OF ANATOMY. For use in the Dissecting Room and in preparing for Examinations. In one $16 \mathrm{mo}$. volume of 196 pages. Limp cloth, 75 cents.

ROBEPTS (SIR WLLLAM). A PRACTICAL TREATISE ON URINARY AND RENAL DISEASES, INCLUDING URINARY DEPOSITS. Fourth American from the fourth London edition. In one very handsome 8 vo. vol. of 609 pp., with 81 illus. Cloth, $\$ 3.50$.

ROBERTSON (J. MCGREGOR). PHYSIOLOGICAL PHYSICS. In one 12mo. volume of 537 pages, with 219 engravings. Cloth, \$2. See Student's Series of Manuals, page 27.

ROSS (JAMES). A HANDBOOK OF THE DISEASES OF THE NERVOUS SYSTEM. In one handsome octavo volume of 726 pages, with 184 engravings. Cloth, $\$ 4.50$; leather, $\$ 5.50$.

SAVAGE (GEORGE H.). INSANITY AND ALLIED NEUROSES, PRACTICAL AND CLINICAL. In one 12mo. volume of 551 pages, with 18, typical engravings. Cloth, \$2. See Series of Clinical Man. uals, page 25. 
SCHAFER (EDWARD A.). THE ESSENTIALS OF HISTOLOGY, DESCRIPTIVE AND PRACTICAL. For the use of Students. New (5th) edition. In one handsome octavo volume of 359 pages, with 392 illustrations. Cloth, $\$ 3.00$, net. Just ready.

Nowhere else will the same very The most satisfactory elementary moderate outlay secure as thoroughly text-book of histology in the Enguseful and interesting an atlas of lish language.-The Boston Med. and structural anatomy.-The American Sur. Jour.

Journal of the Medical Sciences.

A COURSE OF PRACTICAL HISTOLOGY. New (2d) edition.

In one $12 \mathrm{mo}$. volume of 307 pages, with 59 engravings. Cloth, $\$ 2.25$.

The book very nearly approaches overpraised. It bears eloquent tesperfection. Methods are given with timony to the wide knowledge and an accuracy of detail and prevision untiring industry of its author.of difficulties which can hardly be The Scottish Med. and Surg. Jour.

SCHLEIF (WILLIAM). MATERIA MEDICA, THERAPEUTICS, PRESCRIPTION WRITING, MEDICAL LATIN, ETC. $12 \mathrm{mo}$., 352 pages. Cloth, $\$ 1.50$, net. Just ready. Lea's Series of Pocket Text-books. Edited by Bers B. Gallatdet, M. D. See page 18.

SCHMTY AND ZUMPT'S CLASSICAT SERIES. Advanced Latin Exercises. Cloth, 60 cts. Schmidt's Elementary Latin Exercises. Cloth, 50 cents. Sallust. Cloth, 60 cents. Nepos. Cloth, 60 cents. Virgil. Cloth, 85 cents. Curtius. Cloth, 80 cents.

SCHOFIELD (ALFRED T.). ELEMENTARY PHYSIOLOGY FOR STUDENTS. In one 12mo. volume of 380 pages, with 227 engravings and 2 colored plates. Cloth, $\$ 2$.

SCHREIBER (JOSEPH). A MANUAL OF TREATMENT BY MASSAGE AND METHODICAL MUSCLE EXERCISE. Octavo volume of 274 pages, with 117 engravings.

SENN (NICHOLAS). SURGICAL BACTERIOLOGY. Second edition. In one octavo volume of 268 pages, with 13 plates, 10 of which are colored, and 9 engravings. Cloth, $\$ 2$.

SERIES OF CLINICAL MANUALS. A Series of Authoritative Monographs on Important Clinical Subjects, in $12 \mathrm{mo}$. volumes of about 550 pages, well illustrated. The following volumes are now ready: YEO on Food in Health and Disease, new (2d) edition, \$2.50; CARTER and Frost's Ophthalmic Surgery, $\$ 2.25$; HUTCHINSON on Syphilis, $\$ 2.25$; MARSH on Diseases of the Joints, $\$ 2$; OWEN on SurgicalDiseases of Children, $\$ 2$; PICK on Fractures and Dislocations, \$2; SAVAGE on Insanity and Allied Neuroses, $\$ 2$.

For separate notices, see under various authors' names.

SERIES OF STUDENT'S MANUALS. See page 27.

SIMON (CHARLES E.). CLINICAL DIAGNOSIS, BY MICROSCOPICAL AND CHEMICAL METHODS. New (2d) edition. In one very handsome octavo volume of 530 pages, with 135 engravings and 14 full-page colored plates. Cloth, $\$ 3.50$. Just ready.

This book thoroughly deserves its In all respects entirely up to date. success. It is a very complete, authentic and useful manual of the microscopical and chemical methods which are employed in diagnosis. Very excellent colored plates illustrate this work. - New York Medical In all respects enti
- Medical Record.

The chapter on examination of the urine is the most complete and advanced that we know of in the English language.-Canadian PracJournal. 
SIMON (W.). MANUAL OF CHEMISTRY. A Guide to Lectures and Laboratory Work for Beginners in Chemistry. A Text-book specially adapted for Students of Pharmacy and Medicine. New (6th) edition. In one $8 \mathrm{vo}$. volume of 536 pages, with 46 engravings and 8 plates showing colors of 64 tests. Cloth, $\$ 3.00$, net. Just ready.

It is difficult to see how a better the covers of this book.-The Northbook could be constructed. No man western Lancet.

who devotes himself to the practice Its statements are all clear and its of medicine need know more about teachings are practical._-Virginia chemistry than is contained between Med. Monthly.

SLADE (D. D.). DIPHTHERIA; ITS NATURE AND TREATMENT. Second edition. In one royal $12 \mathrm{mo}$. vol., $158 \mathrm{pp}$. Cloth, $\$ 1.25$.

SMUTH (EDW ARD). CONSUMPTION ; ITS EARLY AND REMEDIABLE STAGES. In one 8vo. volume of $253 \mathrm{pp}$. Cloth, $\$ 2.25$.

SMITH (J. LEWIS). A TREATISE ON THE DISEASES OF IN. FANCY AND CHILDHOOD. Eighth edition, thoroughly revised and rewritten and much enlarged. In one large 8vo. volume of 983 pages, with 273 engravings and 4 full-page plates. Cloth, $\$ 4.50$; leather, $\$ 5.50$.

The most complete and satisfactory text-book with which we are acquainted.-American Gynecological and Obstetrical Journal.

It truly is the most evenly balanced, clear in description and thorough in detail of any of the books published in this country on this subject.-Medical Fortnightly.

A treatise which in every respect

can more than hold its own against any other work treating of the same subject.-American Medico-Surgical Bulletin.

A safe guide for students and physicians.-The Am.Jour. of Obstetrics.

For years the leading text-book on children's diseases in America.Chicago Medical Recorder.

SMTTH (STEPHEN). OPERATIVE SURGERY. Second and thoroughly revised edition. In one octavo volume of 892 pages, with 1005 engravings. Cloth, $\$ 4$; leatber, $\$ 5$.

One of the most satisfactory works | dium for the modern surgeon.-Boson modern operative surgery yet ton Medical and Surgical Journal. published. The book is a compen-

SOLLY (S. EDWIN). A HANDBOOK OF MEDICAL CLIMATOLOGY. In one handsome octavo volume of 462 pages, with engravings and 11 full-page plates, 5 of which are in colors. Cloth, $\$ 4.00$. Just ready.

A clear and lucid summary of an accurate observer and practical what is known of climate in relation therapeutist.-Maryland Med. Jour. to its influence upon human beings. Every practitioner of medicine -The Therapeutic Gazette.

The book is admirably planned, clearly written, and the author speaks from an experience of thirty years as should possess himself of a copy and study it, and we are sure he will never regret it.-St. Louis Medical and Surgical Journal.

STILLÉ (ALFRED). CHOLERA ; ITS ORIGIN, HISTORY, CAUSATION, SYMPTOMS, LESIONS, PREVENTION AND TREATMENT. In one $12 \mathrm{mo}$. volume of 163 pages, with a chart showing routes of previous epidemics. Cloth, $\$ 1.25$.

THERAPEUTICS AND MATERIA MEDICA. Fourth and revised edition. In two octavo volumes, containing 1936 pages. Cloth, $\$ 10$; leather, $\$ 12$. 
STULÉ (ALFRED), MAISCH (JOHN M.) AND CASPARI (CHAS. JR.). THE NATIONAL DISPENSATORY: Containing the Natural History, Chemistry, Pharmacy, Actions and Uses of Medicines, including those recognized in the latest Pharmacopœias of the United States, Great Britain and Germany, with numerous references to the French Codex. Fifth edition, revised and enlarged, including the new U. S. Pharmacopoia, Seventh Decennial Revision. With Supplement containing the new edition of the National Formulary. In one magnificent imperial octavo volume of about 2025 pages. with 320 engravings. Cloth, $\$ 7.25$; leather, $\$ 8$. With ready reference Thumb-letter Index. Cloth, $\$ 7.75$; leather, $\$ 8.50$.

Recommended most highly for the amount of information contained in physician, and invaluable to the this work is made available is indidruggist.- Therapeutic Gazette.

It is the official guide for the Medical and Pharmaceutical professions. - Buffalo Med. and Sur. Jour.

cated by the twenty-five thousand references in the two indexes.-Boston Medical and Surgical Journal.

Should be recognized as a national

The readiness with which the vast standard.-North $\mathrm{Am}$. Practitioner.

STIMSON (LEWIS A.). A MANUAL OF OPERATIVE SURGERY. New (3d) edition. In one royal $12 \mathrm{mo}$. volume of 614 pages, with 306 engravings. Cloth, $\$ 3.75$.

A useful and practical guide for The book is worth the price for the all students and practitioners.-Am. illustrations alone.-Ohio Medical Journal of the Medical Sciences. Journal.

\section{STMMON (LEWIS A.). A TREATISE ON FRACTURES AND}

DISLOCATIONS. In one handsome octavo volume of 831 pages, with 326 engravings and 20 plates. Just ready. Cloth, $\$ 5.00$, net; leather, $\$ 6.00$, net.

Prëeminently the authoritative Taken as a whole, the work is the text-book upon the subject. The best.one in English to-day.-St. vast experience of the author gives Louis Medical and Surgical Journal. to his conclusions an unimpeachable Pointed, practical, comprehensire, value. The work is profusely il- exhaustive, authoritative, well writlustrated. It will be found indis- ten and well arranged.-Denver pensable to the student and the prac- Medical Times.

titioner alike.-The Medical Age.

STUDENT'S QUIZ SERIES. Thirteen volumes, convenient, authoritative, well illustrated, handsomely bound in cloth. 1. Anatomy (double number); 2. Physiology; 3 . Chemistry and Physics; 4. Histology, Pathology, and Bacteriology; 5. Materia Medica and Therapeutics ; 6 . Practice of Medicine ; 7. Surgery (double number); 8. GenitoUrinary and Venereal Diseases; 9 . Diseases of the Skin; 10. Diseases of the Eye, Ear, Throat and Nose; 11. Obstetrics; 12. Gynecology; 13. Diseases of Children. Price, $\$ 1$ each, except Nos. 1 and 7 , Anatomy and Surgery, which being double numbers are priced at $\$ 1.75$ each. Full specimen circular on application to publishers.

STUDENT'S SERIES OF MANUALS. 12mos. of from $300-540$ pages, profusely illustrated, and bound in red limp cloth. HermaN's First Lines in Midwifery, $\$ 1.25$; LUFF's Manual of Chemistry, $\$ 2$; BRUCE's Materia Medica and Therapeutics (sixth edition), \$1.50. net. BELL's Comparative Anatomy and Physiology, \$2; ROBERTson's Physiological Physics, \$2; GouLD's Surgical Diagnosis, \$2; KLEIN's Elements of Histology (5th edition), $\$ 2.00$, net; PEPPER's Surgical Pathology, \$2; Treves' Surgical Applied Anatomy, \$2; RALFE's Clinical Chemistry, $\$ 1.50$; and Clarke and LOCKWOOD's Dissector's Manual, $\$ 1.50$. The following is in press: PePper's Forensic Medicine.

For separate notices, see under various author's names. 
STURGES (OCTAVIUS). AN INTRODUCTION TO THE STUDY OF CLINICAL MEDICINE. In one $12 \mathrm{mo}$. volume. Cloth, $\$ 1.25$.

SUTTON (JOHN BLAND). SURGICAL DISEASES OF THE OVARIES AND FALLOPIAN TUBES. Including Abdominal Pregnancy. In one $12 \mathrm{mo}$. volume of 513 pages, with 119 engravings and 5 colored plates. Cloth, $\$ 3$.

TAIT (LAWSON). DISEASES OF WOMEN AND ABDOMINAL SURGERY. In two handsome octavo volumes. Vol. I. contains 546 pages and 3 plates. Cloth, $\$ 3$.

TANNER (THOMAS HAWKES) ON THE SIGNS AND DISEASES OF PREGNANCY. From the second English edition. In one octavo volume of 490 pages, with 4 colored plates and 16 engravings. Cloth, $\$ 4.25$.

TAYLOR (ALFRED S.). MEDICAL JURISPRUDENCE. New American from the twelfth English edition, specially revised by CLARK BELL, EsQ., of the N. Y. Bar. In one 8 vo, vol. of 831 pages, with 54 engrs. and 8 full-page plates. Cloth, $\$ 4.50$; leather, $\$ 5.50$ Just ready.

To the student, as to the physician, nesses, it strongly behooves them to we would say, get Taylor first, and then add as means and inclination enable you.-American Practitioner and News.

It is the authority accepted as final by the courts of all Englishspeaking countries. This is the.important consideration for medical men, since in the event of their being summoned as experts or wit-

be prepared according to the principles and practice everywhere accepted. The work will be found to be thorough, authoritative and modern.-Albany Law Journal.

Probably the best work on the subject written in the English language. The work has been thoroughly revised and is up to date.Pacific Medical Journal.

ON POISONS IN RELATION TO MEDICINE AND MEDICAL JURISPRUDENCE. Third American from the third London edition. In one octavo volume of 788 pages, with 104 illustrations. Cloth, $\$ 5.50$; leather, $\$ 6.50$.

TAYLOR (ROBERT W.). THE PATHOLOGY AND TREATMENT OF VENEREAL DISEASES. New (2d) edition. In one very handsome octavo volume of about 700 pages, with about 200 engravings and 6 colored plates. In press.

Notices of previous edition are appended.

By long odds the best work on venereal diseases. - Louisville Medical Monthly.

In the observation and treatment of venereal diseases his experience has been greater probably than that of any other practitioner of this continent.-New York Medical Journul.

The clearest, most unbiased and ably presented treatise as yet published on this vast subject.-The Medical News.

Decidedly the most important and authoritative treatise on venereal diseases that has in recent years appeared in English.-American Journal of the Medical Sciences.

It is a veritable storehouse of our knowledge of the venereal diseases. It is commended as a conservative, practical, full exposition of the greatest value.-Chicago Clinical Review.

The best work on venereal diseases in the English language. It is certainly above everything of the kind.-The St. Louis Medical and Surgical Journal. 
TAYLOR (ROBERT W.). A PRACTICAL TREATISE ON SEX. UAL DISORDERS IN THE MALE AND FEMALE. In one 8 vo. vol. of 448 pp., with 73 engravings and 8 colored plates. Cloth, $\$ 3$. Net.

It is a timely. boon to the medical the female is presented in an exhausprofession that an observer of Dr. tive manner, all of the causes proTaylor's skill and experience has ducing it being described. The written a work on this hitherto author has presented to the profesneglected and little understood class sion the ablest and most scientific of diseases which places them on a work as yet published on sexual scientific basis and renders them so disorders, and one which, if carefully clear that the physician who reads followed, will be of unlimited value its pages can treat this class of to both physician and patient.patients intelligently. Sterility in Medical News.

A CLINICAL ATLAS OF VENEREAL AND SKIN DISEASES. Including Diagnosis, Prognosis and Treatment. In eight large folio parts, measuring $14 \times 18$ inches, and comprising 213 beautiful figures on 58 full-page chromo-lithographic plates, 85 fine engravings and 425 pages of text. Complete work now ready. Price per part, sewed in heavy embossed paper, $\$ 2.50$. Bound in one volume, half Russia, $\$ 27$; half Turkey Morocco, $\$ 28$. For sale by subscription only. Address the publishers. Specimen plates by mail on receipt of ten cents.

TAYLOR (SEYMOUR). INDEX OF MEDICINE. A Manual for the use of Senior Students and others. In one large 12mo. volume of 802 pages. Cloth, $\$ 3.75$.

THOMAS (T. GATLLARD) AND MUNDÉ (PAUL F.). A PRAC TICAL TREATISE ON THE DISEASES OF WOMEN. Sixth edition, thoroughly revised by PAUL F. MUNDE, M. D. In one large and handsome octavo volume of 824 pages, with 347 engravings. Cloth, $\$ 5$; leather, $\$ 6$.

The best practical treatise on the This work, which has already gone subject in the English language. through five large editions, and has It will be of especial value to the been translated into French, Gergeneral practitioner as well as to the man, Spanish and Italian, is the specialist. The illustrations are very most practical and at the same time satisfactory. Many of them are new the most complete treatise upon the and are particularly clear and attrac- subject.-The Archives of Gynecoltive.-Boston Med. and Sur. Jour.

THOMPSON (SIR HENRY). CLINICAL LECTURES ON DISEASES OF THE URINARY ORGANS. Second and revised edition. In one octavo vol. of $203 \mathrm{pp}$., with 25 engravings. Cloth, $\$ 2.25$.

THE PATHOLOGY AND TREATMENT OF STRICTURE OF THE URETHRA AND URINARY FISTULA. From the third English edition. In one octavo volume of 359 pages, with 47 engravings and 3 lithographic plates. Cloth, $\$ 3.50$.

THOMSON (JOHN). DISEASES OF CHILDREN. In one crown octavo volume of 350 pages, with 52 illus. Cloth, $\$ 1.75$, net. Just ready.

TODD (ROBERT BENTLEY). CLINICAL LECTURES ON CERTAIN ACUTE DISEASES. In one 8 vo. vol. of $320 \mathrm{pp}$., cloth, $\$ 2.50$.

TREVES (FREDERICK). OPERATIVE SURGERY. In two 8 vo. vols. containing 1550 pp., with 422 illus. Cloth, $\$ 9$; leath., $\$ 11$.

A SYSTEM OF SURGERY. In Contributions by Twenty-five English Surgeons. In two large octavo volumes. Vol. I., 1178 pages, with 463 engravings and 2 colored plates. Vol. II., 1120 pages, with 487 engravings and 2 colored plates. Complete work, cloth, $\$ 16.00$. 
TREVES (FREDERICK). SURGICAL APPLIED ANATOMY. In one $12 \mathrm{mo}$. volume of 540 pages, with 61 engravings. Cloth, $\$ 2$. See Student's Series of Manuals, page 27.

TUTTLE (GEORGE M.). A POCKET TEXT.BOOK OF DISEASES OF CHILDREN. In one handsome $12 \mathrm{mo}$. volume of about 300 pages, with many illustrations. Cloth, $\$ 1.50$, net. Shortly. Lea's Series of Pocket Text-books, edited by BERN B. Gallaudet, M. D. See p 18.

VAUGHAN (VICTOR C.) AND NOVY (FREDERICK G.). PTOMAINS, LEUCOMAINS, TOXINS AND ANTITOXINS, or the Chemical Factors in the Causation of Disease. New (3d) edition. In one $12 \mathrm{mo}$. volume of 603 pages. Cloth, $\$ 3$.

The work has been brought down to date, and will be found entirely satisfactory.-Journal of the American Medical Association.

The most exhaustive and most recent presentation of the subject.American Jour. of the Med. Sciences.

The present edition has been not only thoroughly revised throughout but also greatly enlarged, ample consideration being given to the new subjects of toxins and antitoxins.Tri-Strute Medical Journal.

VISITING LIST. THE MEDICAL NEWS VISITING LIST for 1899. Four styles: Weekly (dated for 30 patients); Monthly (undated for 120 patients per month); Perpetual (undated for 30 patients each week); and Perpetual (undated for 60 patients each week). The 60patient book consists of 256 pages of assorted blanks. The first three styles contain 32 pages of important data, thoroughly revised, and 160 pages of assorted blanks. Each in one volume, price, $\$ 1.25$. With thumb-letter index for quick use, 25 cents extra. Special rates to advance-paying subscribers to THE MEDICAL NEWS or THE American Journal of the Medical Sciences, or both. See p. 32.

WATSON (THOMAS). LECTURES ON THE PRINCIPLES AND PRACTICE OF PHYSIC. A new American from the fifth and enlarged English edition, with additions by H. HARTSHORNE, M. D. In two large 8vo. vols. of 1840 pp., with 190 cuts. Cloth, $\$ 9$; leather, $\$ 11$.

WEST (CHARLES). LECTURES ON THE DISEASES PECULIAR TO WOMEN. Third American from the third English edition. In one octavo volume of 543 pages. Cloth, $\$ 3.75$; leather, $\$ 4.75$.

ON SOME DISORDERS OF THE NERVOUS SYSTEM IN CHILDHOOD. In one small 12mo. volume of 127 pages. Cloth, $\$ 1$.

WHARTON (HENRY R.). MINOR SURGERY AND BANDAGING. New (4th) edition. In one $12 \mathrm{mo}$. vol. of about 600 pages, with about 500 engravings, many of which are photographic. Shortly.

Notices of previous edition are appended.

We know of no book which more / work of ready reference for surthoroughly or more satisfactorily covers the ground of Minor Surgery and Bandaging.-Brooklyn Medical Journal.

Well written, conveniently arranged and amply illustrated. It covers the field so fully as to render it a valuable text-book, as well as a geons.-North Amer. Practitioner. The part devoted to bandaging is perhaps the best exposition of the subject in the English language. It can be highly commended to the student, the practitioner and the specialist.-The Chicago Medical Recorder. 
WHITLA (WHLLAM). DICTIONARY OF TREATMENT, OR THERAPEUTIC INDEX. Including Medical and Surgical Therapeutics. In one square octavo volume of 917 pages. Cloth, $\$ 4$.

WILLIAMS (DAWSON). THE MEDICAL DISEASES OF CHILDREN. In one $12 \mathrm{mo}$. volume of 629 pages, with 18 illustrations. Just ready. Cloth, $\$ 2.50$, net.

The descriptions of symptoms are diagnoses, prognosis, complications, full, and the treatment recommended and treatment. The work is up to will meet general approval. Under date in every sense.-The Charlotte each disease are giren the symptoms, Medical Journal.

WILSON (ERASMUS). A SYSTEM OF HUMAN ANATOMY. A new and revised American from the last English edition. Illustrated with 397 engravings. In one octavo volume of 616 pages. Cloth, $\$ 4$; leather, $\$ 5$.

THE STUDENT'S BOOK OF CUTANEOUS MEDICINE. In one $12 \mathrm{mo}$. volume. Cloth, $\$ 3.50$.

WINCKEL ON PATHOLOGY AND TREATMENT OF CHILDBED. Translated by JAMES R. CHADwICK, A. M., M.D. With additions by the Author. In one octavo volume of 484 pages. Cloth, $\$ 4$.

WÖHLER'S OUTLINES OF ORGANIC CHEMISTRY. Translated from the eighth German edition, by IRA REMSEN, M.D. In one $12 \mathrm{mo}$. volume of 550 pages. Cloth, $\$ 3$.

YEAR-BOOK OF TREATMENT FOR 1892, 1893, 1896,1897 and 1898. Critical Reviews for Practitioners of Medicine and Surgery. In contributions by 25 well-known medical writers. $12 \mathrm{mos}$., about 500 pages each. Cloth, $\$ 1.50$. In combination with THE MEDICAL NEwS and The American Journal of the Medical Sciences, 75 cents.

YEO (I. BURNEY). FOOD IN HEALTH AND DISEASE. New (2d) edition. In one $12 \mathrm{mo}$. volume of 592 pages, with 4 engravings. Cloth, \$2.50. See Series of Clinical Manuals, page 26.

We doubt whether any book on work of Dr. Yeo's. The value of dietetics has been of greater or more the work is not to be overestimated. widespread usefulness than has this -Nevo York Medical Journal.

much-quoted and much-consulted

A MANUAL OF MEDICAL TREATMENT, OR CLINICAL THERAPEUTICS. Two volumes containing 1275 pages. Cloth, $\$ 5.50$.

YOUNG (JAMES K.). ORTHOPEDIC SURGERY. In one 8 vo. volume of 475 pages, with 286 illustrations. Cloth, $\$ 4$; leather, $\$ 5$.

In studying the different chapters, | surgical specialty and every page one is impressed with the thorough- abounds with evidences of pracness of the work. The illustrations ticality. It is the clearest and most are numerous - the book thoroughly modern work upon this growing depractical-Medical Neros.

It is a thorough, a very comprehensire work upon this legitimate partment of surgery.-The Chicago Clinical Review. 


\section{PERIODICA LS.}

\section{PROGRESSIVE MEdICINE.}

A Quarterly Digest of New Methods, Discoveries, and Improvements in the Medical and Surgical Sciences by Eminent Authorities. Edited by Dr. Hobart Amory Hare. In four abundantly illustrated, cloth bound, octavo volumes, of 400-500 pages each, issued quarterly, commencing March 1st, 1899. Per annum (4 volumes), $\$ 10.00$ delivered.

\section{THE MEDICAL NEWS.}

Weekly, \$1.00 per Annum.

Each number contains 32 quarto pages, abundantly illustrated. A crisp, fresh weekly professional newspaper.

\section{THE AMERICAN JOURNAL OF THE MEDICAL SCIENCES.} Monthly, \$ 1.00 Per Annum.

Each issue contains 128 octavo pages, fully illustrated. The most advanced and enterprising American exponent of scientific medicine.

\section{THE MEDICAL NEIVS VISITING LIST.}

Four styles, Weekly (dated for 30 patients); Monthly (undated, for 120 patients per month); Perpetual (undated, for 30 patients weekly per year); and Perpetual (undated, for 60 patients per year). Each style in one wallet-shaped bonk, leather bound, with pocket, pencil and rubber. Price, each, $\$ 1.2$ i. Thumb-letter index, 25 cents extra.

\section{THE MEDICAL NEWS POCKET FORMULARY.}

Containing 1600 prescriptions representing the latest and most approved methods of administering remedial agent\%. Strongly bound in leather; with pocket and pencil. Price, $\$ 1.50$, net.

\section{COMBINATION RATES:}

American Journal of the

Medical Sciences,

M. $\cdot \quad \$ 4.00$

Medlcal News . . . . . . $\quad 4.00$

Progressive Medicine . . . . 10.00

Medical News Visiting List . . . 1.25

Medical News Formulary

1.50 net,

In all $\$ 20.75$ for $\$ 16.00$

First four above publications in combination . . \$15.75

All above publications in combination . . : $\quad 16.00$

Other Combinations will be quoted on request.

Full Circulars and Specimens free.

\section{LEA BROTHERS \& CO., Publishers,}

706, 708 \& 710 Sansom St., Philadelphia.

111 Fifth Avenue, New York. 






\section{DAY USE}

\section{RETURN TO DESK FROM WHICH BORROWED}

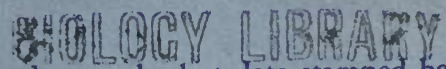

This book is due on the last date stamped below, or on the date to which renewed.

Renewed books are subject to immediate recall.

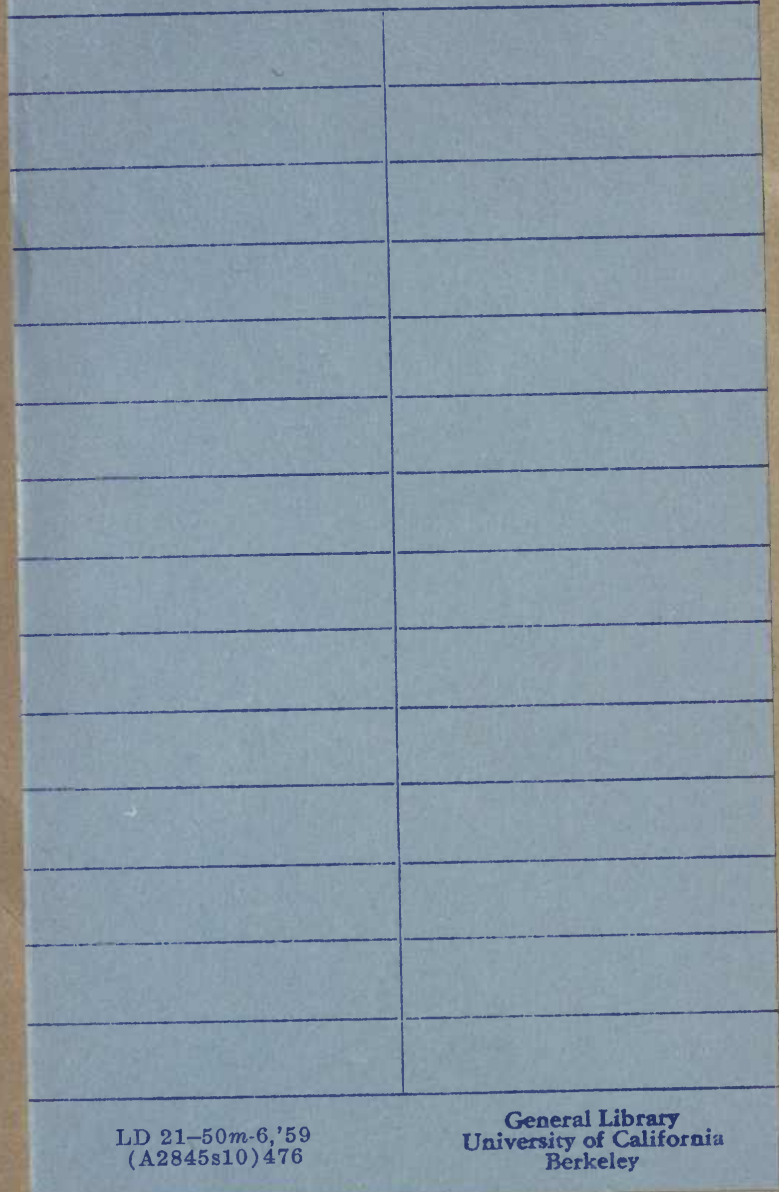




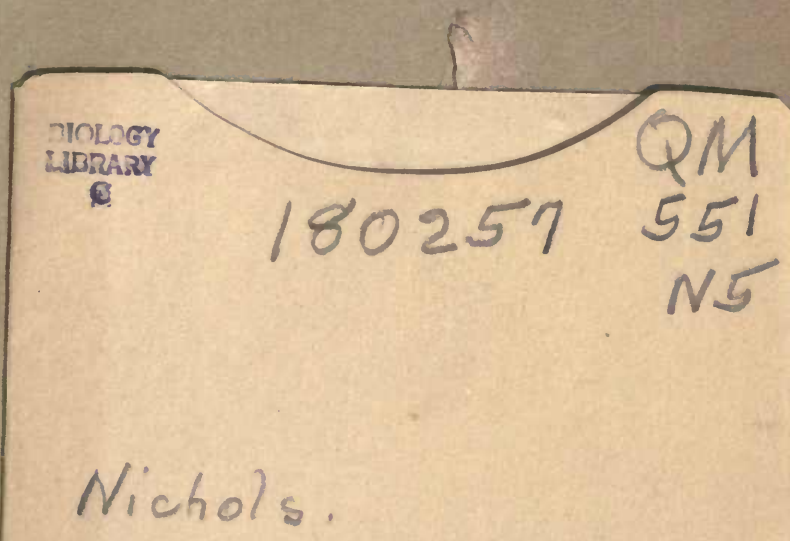

THE UNIVERSITY OF CALIFORNIA LIBRARY 

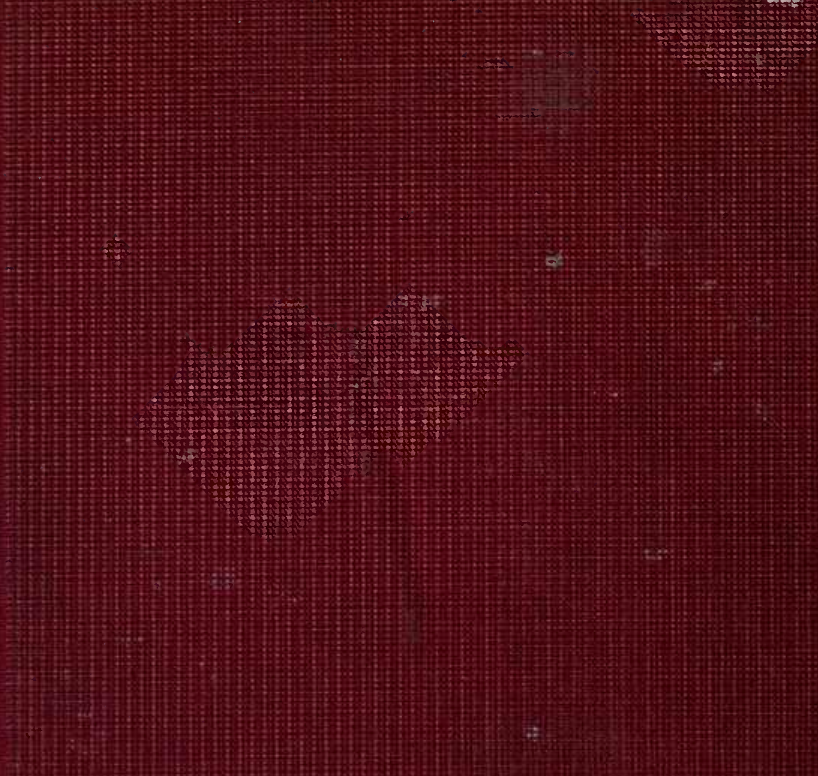